Georg Hirte

Effizienzwirkungen von Finanzausgleichsregelungen 


\section{Georg Hirte}

\section{Effizienzwirkungen von Finanzausgleichsregelungen}

In derbundesdeutschen Debatte über ein wünschenswertes Finanzausgleichssystem werden Effizienzaspekte weitgehend vernachlässigt. Es läßt sich jedoch zeigen, daß Finanzausgleichsregelungen Allokationsstörungen hervorrufen. Dies wird in dieser Arbeit anhand einer theoretischen Analyse demonstriert. In einer Secondbest-Welt können First-best-Betrachtungen jedoch zu Fehleinschätzungen führen. Deshalb werden die Wohlfahrtseffekte des bundesdeutschen Finanzausgleichssystems und einiger Reformvorschläge mit Hilfe einer Empirischen Allgemeinen Gleichgewichtsanalyse ermittelt. Unter anderem wird dabei der Einbezug der Neuen Bundesländer in den Finanzausgleich und der Vorschlag eines Einkommensteuerzuschlages der Bundesländer untersucht.

Georg Hirte wurde 1960 in Bamberg geboren. Er studierte Theologie und Volkswirtschaftslehre an der Universität Regensburg. Von 1989 bis 1995 war er wissenschaftlicher Mitarbeiter von Prof. Dr. J. Genosko an der Universität Hohenheim und der Katholischen Universität Eichstätt, wo er 1995 promovierte. 
Effizienzwirkungen von Finanzausgleichsregelungen Eine Empirische Allgemeine Gleichgewichtsanalyse für die Bundesrepublik Deutschland 


\section{FINANZWISSENSCHAFTLICHE SCHRIFTEN}

Herausgegeben von den Professoren

Albers, Krause-Junk, Littmann, Oberhauser, Pohmer, Schmidt

Band 76

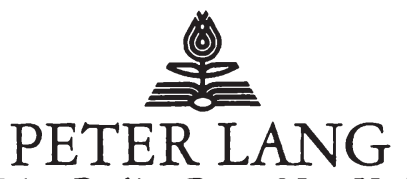

Frankfurt am Main - Berlin - Bern - New York · Paris - Wien

Georg Hirte - 978-3-631-75212-8

Downloaded from PubFactory at 01/11/2019 07:02:26AM

via free access 


\section{Georg Hirte}

\section{Effizienzwirkungen von Finanzausgleichsregelungen \\ Eine Empirische Allgemeine \\ Gleichgewichtsanalyse für die \\ Bundesrepublik Deutschland}

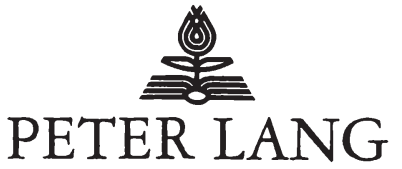

Europäischer Verlag der Wissenschaften

Georg Hirte - 978-3-631-75212-8

Downloaded from PubFactory at 01/11/2019 07:02:26AM

via free access 
Die Deutsche Bibliothek - CIP-Einheitsaufnahme

Hirte, Georg:

Effizienzwirkungen von Finanzausgleichsregelungen : eine empirische allgemeine Gleichgewichtsanalyse für die Bundesrepublik Deutschland / Georg Hirte. - Frankfurt am Main ; Berlin ; Bern ; New York ; Paris ; Wien : Lang, 1996

(Finanzwissenschaftliche Schriften ; Bd. 76)

Zugl.: Eichstätt, Kath. Univ., Diss., 1996

ISBN 3-631-49368-1

Open Access: The online version of this publication is published on www.peterlang.com and www.econstor.eu under the international Creative Commons License CC-BY 4.0. Learn more on how you can use and share this work: http://creativecommons.org/ licenses/by/4.0.

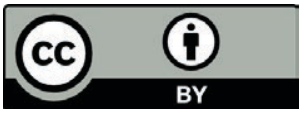

This book is available Open Access thanks to the kind support of ZBW - Leibniz-Informationszentrum Wirtschaft.

\author{
D 824 \\ ISSN 0170-8252 \\ ISBN 3-631-49368-1 \\ ISBN 978-3-631-75212-8 (eBook) \\ (C) Peter Lang GmbH \\ Europäischer Verlag der Wissenschaften \\ Frankfurt am Main 1996 \\ Alle Rechte vorbehalten.
}

Das Werk einschließlich aller seiner Teile ist urheberrechtlich geschützt. Jede Verwertung außerhalb der engen Grenzen des Urheberrechtsgesetzes ist ohne Zustimmung des Verlages unzulässig und strafbar. Das gilt insbesondere für

Vervielfältigungen, Übersetzungen, Mikroverfilmungen und die Einspeicherung und Verarbeitung in elektronischen Systemen.

Printed in Germany 123457 


\section{Inhaltsverzeichnis}

Einleitung 1

I Das bundesdeutsche Finanzausgleichssystem 5

1. Überblick und Diskussion 5

1.1. Regelungen vor $1995 \ldots \ldots \ldots 6$

1.1.1. Steuerverteilung 6

1.1.2. Länderfinanzausgleich (LFA) 7

1.1.3. Bundesergänzungszuweisungen (BEZ) 8

1.2. Die Auseinandersetzung um eine Neuregelung. . . . . . . . . . 8

1.3. Regelungen nach $1995 \quad 9$

2. Vorschläge zu einer Neuordnung . . . . . . . . . . . . . . . . 10

2.1. Überblick über Regelungen und Vorschläge . . . . . . . . . . . . . . 15

3. Regelungen und Vorschläge im Einzelnen . . . . . . . . . . . . . 18

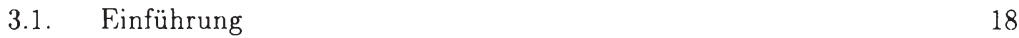

3.2. Bundesdeutsche Regelungen vor $1995 \ldots$. . . . . . . . . . . 19

3.2.1. Die Verteilung der Gewerbesteuer . . . . . . . . . . 20

3.2.2. Verteilung der Einkommenssteuern . . . . . . . . . 21

3.2.3. Umsatzsteuerverteilung 22

3.2.4. Länderfinanzausgleich 26

3.2.5. Bundesergänzungszuweisungen 34

3.3. Bundesdeutsche Regelungen nach $1995 \ldots \ldots 35$

3.3.1. Umsatzsteuerverteilung 35

3.3.2. Länderfinanzausgleich 36

3.3.3. Bundesergänzungszuweisungen (BEZ) 42

3.3.4. Zusätzliche Abgaben der Länder 42

3.4. Der Vorschlag des Wissenschaftlichen Beirats (1992) . . . . 43 
3.4.1. Einkommenssteuerverteilung und Einnahmenautonomie . . 43

3.4.2. Umsatzsteuerverteilung 43

3.4.3. Horizontaler Finanzausgleich 45

3.5. Der Vorschlag von Buhl/Pfingsten [1991] . . . . . . . . . . . . . 49

3.6. Der Vorschlag von Föttinger/SPahn [1993] . . . . . . . . . 49

3.7. Der Vorschlag von Fuest/Lichtblau [1991] 51

3.8. Der Vorschlag eines Verzichts auf die Umverteilung . . . . . . . . 52

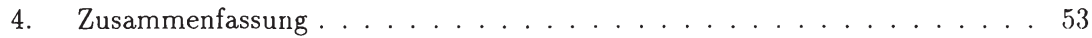

II Theoretische Grundlagen 55

1. Einleitung .......................... 55

2. Partialanalyse: Regionale Budgetgleichungen . . . . . . . . . . 56

2.1. Analyse des öffentlichen Budgets 57

2.2. Substitutionalität im bundesdeutschen Finanzausgleich . . . . . . 58

2.2.1. Umsatzsteuerverteilung $\quad 59$

2.2.2. Länderfinanzausgleich $\quad 59$

2.2.3. Gesamte Einnahmenveränderung 61

2.3. Zwischenbemerkungen 64

3. Exkurs: Grundlegendes Partialmodell . . . . . . . . . . . . . . . . 64

3.1. Grad der Finanzautonomie der Regionen . . . . . . . . . . . 65

3.2. Trennung von privater und öffentlicher Entscheidung 66

4. Fehlende Finanzautonomie . . . . . . . . . . . . . . . 68

5. Ausgabenautonomie der Länder . . . . . . . . . . . . . . . . 74

$\begin{array}{ll}\text { 5.1. Theorie der Finanzzuweisungen } & 75\end{array}$

5.1.1. Allgemeine Zuweisungen ohne Auflagen . . . . . . . . 76

5.1.2. Zweckgebundene Zuweisung ohne Eigenleistung . . . . . 76

5.1.3. Finanzzuweisungen mit Empfangsauflage/-bedingung . . . 78

5.1.4. Zuweisungen mit Eigenbeteiligung 82

5.1.5. Fliegenpapiereffekt (flypaper-effect) 85 
5.2. Finanzzuweisungen im bundesdeutschen Finanzausgleich

5.3. Zusammenfassende Bemerkungen zur Theorie der Finanzzuweisungen 90

6. Volle Finanzautonomie der Regionen 91

6.1. Verzerrungen der relativen Güterpreise . . . . . . . . . . . . . 91

6.2. Verzerrungen der Optimalsteuersätze . . . . . . . . . . . 92

7. Zusarnmenfassung der Partialanalyse . . . . . . . . . . . 95

$\begin{array}{ll}\text { III Wohlfahrtseffekte } & 97\end{array}$

1. Bedingungen für ein Pareto-Optimum . . . . . . . . . . . . . 97

2. Optimalbedingungen bei Staatseingriffen . . . . . . . . . . . . 102

2.1. Optimalbedingungen bei Ausgabenautonomie . . . . . . . . . 103

2.2. Optimalbedingungen ohne Finanzautonomie . . . . . . . . 107

2.3. Optimalbedingungen mit Finanzautonomie . . . . . . . . . . 108

3. Allokationsverzerrungen durch Staatseingriffe . . . . . . . . . . . . . . . 109

3.1. Produktionssteuern . . . . . . . . . . . . 110

3.2. Faktorsteuern . . . . . . . . . . . . . . . 110

3.3. Einkommenssteuern . . . . . . . . . . . . . . . 111

3.4. Konsumsteuern . . . . . . . . . . . . . . 112

3.5. Effizienzwirkungen von Finanzausgleichsregelungen . . . . . . . . 112

4. Wohlfahrtswirkungen .................... 114

4.1. Interregionale Wohlfahrtseffekte durch Transfers . . . . . . . . . 115

4.2. Interregionale Wohlfahrtseffekte durch ToT-Veränderungen . . . . . 117

4.2.1. Allgemeine Auswirkungen von ToT-Veränderungen . . . . 117

4.2.2. Auswirkungen der Veränderung von Einkommenssteuerzuschlägen . . . . . . . . . . . . . . . . 119

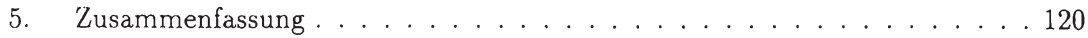

$\begin{array}{ll}\text { IV IDas EAG-Modell } & 123\end{array}$

1. Einleitende Bemerkungen . . . . . . . . . . . . . . 123

2. Theoretisches Modell . . . . . . . . . . . . . . . . . 125 
2.1. Regionale Produktionssektoren . . . . . . . . . . . . . . . 127

2.1.1. Besteuerung der Produktionssektoren . . . . . . . . . 127

2.1.2. Die Technologie und die Entscheidung der Sektoren . . . 128

2.2. Regionaler privater und öffentlicher Bereich . . . . . . . . . 132

2.2.1. Besteucrung der Nachfrageseite . . . . . . . . . . 132

2.2.2. Endnachfrage der Regionen ... . . . . . . . . 134

2.3. Steuereinnahmen und öffentliche Haushalte .......... 141

2.4. Regionaler Außenhandel ................. 142

2.5. Nationaler Außenhandel . . . . . . . . . . . . . . . . 143

2.6. Gleichgewichtsbedingunger _................ 144

3. Datensatz für das EAG-Modell . . . . . . . . . . . . . . . . . . 146

3.1. Struktur der benötigten Multiregionalen Tabelle . . . . . . . . 147

3.2. Auswahl des Referenzjahres . . . . . . . . . . . . . 148

3.3. Grundsätzliche Vorgehensweise . . . . . . . . . . . . . . 149

3.4. Interregionale 'Tabelle für die Westländer f . . . . . . . . 150

3.4.1. Randdaten der regionalen Input-Output-'Tabelle . . . . 150

3.4.2. Primärfaktormatrix ............. . . . . . . . . .

3.4.3. Vorleistungsmatrix . . . . . . . . . . 158

3.4.4. Endnachfragematrix . . . . . . . . . . 159

3.4.5. Sonstige Größen . . . . . . . . . . . . . 165

3.4.6. Ergänzung um das aggregierte Ostland . . . . . . . 166

4. Berechnung der Modelparameter: Kalibrierung . . . . . . . . . . . . 170

4.1. Vorgabe der Elastizitäten ................ 171

4.2. Berechnung der Parameter . . . . . . . . . . . 172

4.2.1. Produktionssektor . . . . . . . . . . 172

4.2.2. Haushaltssektor . . . . . . . . . . . . 174

$\begin{array}{ll}\text { V Simulationen } & 177\end{array}$

1. Finführung zu den Berechnungen . . . . . . . . . . . . 177 
1.1. Überprüfung des EAG-Modells . . . . . . . . . . . . . . . . 177

1.2. Grundsätzliche Vorgehensweise in den Simulationen . . . . . . . . 178

1.3. Konzepte zur Beurteilung der Ergebnisse . . . . . . . . . . . . . . . 179

1.3.1. Wohlfahrtsmessung .............. 180

1.3.2. Verteilungsmessung ............... 181

2. Hilfsgrößen zur detaillierteren Untersuchung . . . . . . . . . . . . 181

2.1. Terms of Trade Definition . . . . . . . . . . . . . . . 182

2.2. Steuerexporte und interregionale Transfers . . . . . . . . . 182

2.2.1. Effektiver Kapitalsteuergehalt der Produzentenpreise . . . 183

2.2.2. Effektiver Produktionssteuergehalt der Produzentenpreise 184

2.2.3. Nettotransfers . . . . . . . . . . . . . 185

2.3. Sensitivitätsanalysen . . . . . . . . . . . . . . 185

3. Die Ergebnisse im Einzelnen . . . . . . . . . . . . . . . . . 187

3.1. Vorbemerkungen zu den Berechnungen . . . . . . . . . 187

3.2. Einbezug der Ostländer zum 1.1.1995 . . . . . . . . . . . . . . 188

3.2.1. Übergang bei Beibehaltung der alten Regelungen . . . . . 189

3.2.2. Übergang mit den neuen Regelungen . . . . . . . . . . . . 194

3.3. Wechsel der Finanzausgleichsregelungen nach 1995 . . . . . 196

3.3.1. Wissenschaftlicher Beirat [1992] . . . . . . . . . 196

3.3.2. Buhl/Pfingsten [1991] . . . . . . . . . . 206

3.3.3. Föttinger/Spahn [1993] . . . . . . . . . . 210

3.3.4. Fuest/Lichtblau [1991] . . . . . . . . . . . . . . 214

3.3.5. Abschaffung der horizontalen Umverteilung . . . . . . 218

3.3.6. Ein Vergleich der Vorschläge. . . . . . . . . . 220

3.4. Finanzautonomie: Wissenschaftlicher Beirat [1992] . . . . . . . . . . 222

3.4.1. Einkommenssteuerzuschläge ... . . . . . . 222

3.5. Zusammenfassung der Ergebnisse . . . . . . . . . . . 227

$\begin{array}{ll}\text { VI Schlußbemerkungen } & 231\end{array}$ 
Abkürzungsverzeichnis

Verzeichnis der Variablen 236

Literaturverzeichnis 248 


\section{Abbildungsverzeichnis}

II.1 Optimale Wahl bei Rationierung $\quad 69$

II.2 HEV bei Rationierung . . . . . . . . . . . . . . . . . . 74

II.3 Doppelter Tangentialpunkt bei Zuweisungen mit Empfangsauflage . . . . . 79

II.4 Wohlfahrtsverlust bei Zuweisungen mit Empfangsauflage . . . . . . . . . . 80

II.5 Budgetsegmente bei LFA vor 1995, Ausschnitt . . . . . . . . . . . . . . . 89

III.1 Wohlfahrtseffekte bei Preiserhöhungen im Inland . . . . . . . . . . . . . . 118

IV.1 Struktur der Technologie . . . . . . . . . . . . . . . . . . . . . . . 129

IV.2 Nutzenbaum . . . . . . . . . . . . . . . . . . . . . . 135

IV.3 Bestimmung der sozialen Grenzkosten des öffentlichen Gutes . . . . . . . . 139 
Georg Hirte - 978-3-631-75212-8

Downloaded from PubFactory at 01/11/2019 07:02:26AM

via free access 


\section{Tabellenverzeichnis}

I.1 Regelungen und Vorschläge zur Umsatzsteuerverteilung . . . . . . . . . . 16

I.2 Vorschläge und Regelungen des Länderfinanzausgleichs . . . . . . . . . . . 17

I.3 Regelungen und Vorschläge zu den BEZ $\quad 18$

I.4 Vorschläge und Regelungen des Länderfinanzausgleichs . . . . . . . . . . . 54

II.1 Veränderung der Einnahmen durch die Umsatzsteuerverteilung . . . . . . . 60

II.2 Veränderung der Einnahmen durch den LFA . . . . . . . . . . . . 61

II.3 Gesamte Einnahmenveränderung nach Steuerverteilung, LFA und BEZ . 62

IV.1 Interregionale Input-Outputtabelle der technolog. Verflechtung . . . . . 147

IV.2 Schema für Übergang von VGR auf Input-Output-Konzept . . . . . . . . . 151

IV.3 BPW nach funktionellem I/O-Konzept . . . . . . . . . . . . . . . 152

IV.4 Schema des Übergangs von Konsum nach VGR auf IO-Konzept . . . . . . 153

IV.5 Berechnung der Importe nach dem IO-Konzept . . . . . . . . . . . 155

IV.6 Berechnung der Exporte nach dem IO-Konzept . . . . . . . . . . . . 155

IV.7 Schätzergebnisse des Gravitationsmodells 1982 . . . . . . . . . . . . . . 164

IV.8 Vorgegebene Elastizitäten und Kalibrierung . . . . . . . . . . . . . . 172

V.1 Übergang mit alter Regelung, Ausgabenautonomie . . . . . . . . . . . . . . 190

V.2 Übergang mit alter Regelung, keine Autonomie . . . . . . . . . . . . . . 192

V.3 Übergang mit alten Regelungen, Ausgabenautonomie, Variation der Bezugnachfrageelastizität . . . . . . . . . . . . . . . 193

V.4 Übergang mit neuer Regelung, Ausgabenautonomie . . . . . . . . . . . . . 194

V.5 Übergang mit neuer Regelung, keine Autonomie . . . . . . . . . . . . . . 196

V.6 Wiss. Beirat I, Ausgabenautonomie . . . . . . . . . . . . . . 197

V.7 Wiss. Beirat IV, Ausgabenautonomie . . . . . . . . . . . . . . 198

V.8 Wiss. Beirat II, Ausgabenautonomie . . . . . . . . . . . . . . . 198 
V.9 Wiss. Beirat III, Ausgabenautonomie . . . . . . . . . . . . . . . 199

V.10 Wiss. Beirat I, Ausgabenautonomie, mit und ohne Freizeitwahl . . . . . 200

V.11 Wiss. Beirat I, keine Autonomie . . . . . . . . . . . . . . . . 201

V.12 Wiss. Beirat II, keine Autonomie . . . . . . . . . . . . . . . . . 201

V.13 Wiss. Beirat III, keine Autonomie . . . . . . . . . . . . . . . . . 202

V.14 Wiss. Beirat IV, keine Autonomie . . . . . . . . . . . . . . . . . 202

V.15 Wiss. Beirat, Variante I, Ausgabenautonomie, Variation der Substitutionselastizitäten zwischen Kapital und Arbeit . . . . . . . . . . . . . . . . . 204

V.16 Wiss. Beirat, Variante I, Ausgabenautonomie, ohne Kapitalmobilität . . 205

V.17 Wiss. Beirat, Variante I, Ausgabenautonomie, Variation der Substitutionselastiziäten des Vorleistungseinsatzes, $\rho=3,0 \ldots 207$

V.18 Buhl/Pfingsten, Ausgabenautonomie . . . . . . . . . . . . . . . 207

V.19 Buhl/Pfingsten, keine Autonomie . . . . . . . . . . . . . . . . 208

V.20 Buhl/Pfingsten Ausgabenautonomie, mit fixem Arbeitsangebot . . . 210

V.21 Föttinger/Spahn I, Ausgabenautonomie . . . . . . . . . . . 211

V.22 Föttinger/Spahn II, Ausgabenautonomie . . . . . . . . . . . . . . . . 212

V.23 Föttinger/Spahn, keine Ausgabenautonomie (beide Fälle) . . . . . . . . 213

V.24 Föttinger/Spahn II, Ausgabenautonomie, fixes Arbeitsangebot . . . . . 215

V.25 Fuest / Lichtblau, Ausgabenautonomie . . . . . . . . . . . . . 215

V.26 Fuest /Lichtblau, keine Autonomie . . . . . . . . . . . . . . . . 216

V.27 Fuest/Lichtblau Ausgabenautonomie, Variation der Substitutionselastizitäten zwischen Kapital und Arbeit . . . . . . . . . . . . . . . 217

V.28 Abschaffung der Umverteilung, Ausgabenautonomie . . . . . . . . . . . 218

V.29 Abschaffung der Umverteilung, keine Autonomie . . . . . . . . . . . . . 219

V.30 Vergleich der Vorschläge zur Neuregelung bei Ausgabenautonomie . . . . . 221

V.31 Wiss. BeIRAT, I, Steuerautonomie bei gesenktem $\tau^{Y}$ und diff.Inzidenz . . 223

V.32 Wiss. BeIRat I, diff.Inzidenz, Finanzautonomie, mit $\tau_{L d}^{Y}$ bei quasifixem Arbeitsangebot . . . . . . . . . . . . . . . . . 225

V.33 Wiss. Beirat, I, Steuerautonomie bei fixen $\tau^{Y}$ und diff.Inzidenz . . . . 226 


\section{Einleitung}

Durch den Beitritt der Neuen Bundesländer zum Bundesgebiet am 3.10.1990 wurde die Diskussion um das bundesdeutsche Finanzausgleichssystem neu entfacht. Obwohl in der Zwischenzeit der Bund-Länder-Kompromiß zur Neuregelung der Umsatzsteuerverteilung, des Länderfinanzausgleichs und der Bundesergänzungszuweisungen verabschiedet wurde (Gesetz zur Umsetzung des Föderalen Konsolidierungsprogramms - FKPG), der zum 1.1.1995 zum vollen Einbzug der Neuen Bundesländer in den Finanzausgleich führte, ist ein Ende der Auseinandersetzung um das Finanzausgleichssystem nicht abzusehen. Vielmehr ist angesichts der erheblichen finanziellen Belastungen der Westländer zu erwarten, daß sich in Zukunft die Forderungen nach einer grundsätzlichen Neugestaltung des bundesdeutschen Finanzausgleichssystems verstärken werden.

Man kann vermuten, daß sich die in der politischen Diskussion auf den Tisch kommenden Neuordnungsvorschläge mehr oder weniger auf bereits bekannte Vorschläge stützen werden. Um jedoch aus der Vielzahl von bisher vorliegenden Reformvorschlägen eine optimale Auslese treffen zu können, entsteht die Notwendigkeit, entsprechende Auswahlkriterien zu bestimmen. Studiert man zu diesem Zweck die Literatur, dann bieten sich mehrere Kriterien an:

- Zum einen kann die Auswahl aufgrund der erzielten Verteilungswirkungen und Budgetveränderungen erfolgen. Entsprechende Entscheidungshilfen stellen die Vielzahl von Berechnungen der Finanzausgleichsströme dar (siehe z.B. GotTFried/WiEGARD [1992]).

- Zum zweiten können Finanzausgleichssysteme rein nach systematischen Gesichtspunkten, wie etwa "Widerspruchsfreiheit” oder "Aufrechterhaltung der Finanzkraftreihenfolge", abgeleitet und begründet werden (siehe z.B. BUHL/PFInGSTEN [1990 oder 1991], TAUBE [1990 oder LENK [1992]).

- Zum dritten können Effizienzaspekte ein Beurteilungskriterium für unterschiedliche Finanzausgleichssysteme darstellen (siehe z.B. Bös [1971 und 1978]).

Durchforstet man die Fülle der Veröffentlichungen im deutschsprachigen Raum, so zeigt sich, daß zu den ersten beiden Aspekten eine große Zahl von Arbeiten vorliegen. Dagegen erhält man den Eindruck, daß Finanzausgleichsregelungen keine verzerrenden Einflüsse hervorrufen und daher allokationsneutral sind. Die entsprechende Argumentation 
stützt sich auf die Vermutung, daß der bundesdeutsche Finanzausgleich nur zu allgemeinen Zuweisungen führt (siehe z.B.FISCHER [1988]). ${ }^{1}$ Wäre dies der Fall, dann entstünden nur Einkommenseffekte und es würde zur Bestimmung der aus dem Finanzausgleich resultierenden Wohlfahrtseffekte ausreichen, die Finanzströme zwischen den Regionen zu berechnen (siehe z.B. GotTfried/Wiegard [1992] "Gewinner sind die alten Länder”).

Ein weiterer Bereich allokativer Aspekte bezieht sich auf die Anreizwirkung der Finanzausgleichsregelungen bezüglich der Ausschöpfung der regionalen Einnahmenquellen (siehe z.B. Musgrave [1961] oder Bös [1971 und 1978]). Da jedoch im Finanzsystem der Bundesrepublik der Bund die Gestaltungshoheit ${ }^{2}$ für fast alle Steuerarten besitzt ${ }^{3}$ ist es den Ländern verwehrt, ihre Einnahmen über Veränderung von Steuersätzen zu beeinflussen. ${ }^{4}$ Aus diesem Grund sind scheinbar bei der Untersuchung des Finanzausgleichssystems etwaige Anreize zur Ausschöpfung der Steuerkraft ebensowenig von Bedeutung, wie die Effizienzwirkungen der einzelnen Steuerarten (siehe z.B. Bös [1978]).

Allerdings deuten neuere Arbeiten darauf hin, daß sich die Ökonomen einer Irrelevanz der Effizienzaspekte bezüglich des Finanzausgleichs nicht mehr ganz sicher sind. Die Aufsätze von Wahl, Frischmuth [1990], Richter/Wellisch [1993] und Homburg [1993] geben erste Hinweise darauf, daß die Rolle der Finanzautonomie der Länder, die Verwendungsmöglichkeit eines Finanzausgleichssystems zur Reduktion externer Effekte von Mobilität oder der Beachtung intertemporaler Effizienzbedingungen, zu einer Neubewertung von Effizienzaspekten führen könnten. ${ }^{5}$

Grundlegende Absicht der vorliegenden Arbeit ist es daher, durch eine genaue Analyse der Effizienzwirkungen von Finanzausgleichssystemen einen Beitrag zu einer Neubewertung

\footnotetext{
${ }^{1}$ Dies hieße, daß die „Theorie der Finanzzuweisungen” für den bundesdeutschen Finanzausgleich keine weitere Relevanz hätte (zur "Theorie der Finanzzuweisungen” siehe z.B. OATEs [1975] oder FISCHER [1988]).

${ }^{2}$ Die Gestaltungshoheit bezüglich des Steuersystems umfaßt im wesentlichen die genaue Abgrenzung der Bemessungsgrundlage, das Ausmaß der Steuerpflicht und die Festlegung des jeweiligen Steuertarifs und ist Teil der Gesetzgebungshoheit, die zusätzlich die Objekthoheit, d.h. das Steuerfindungsrecht umfaßt (ZimMERMANN/HeNKE [1994], S.183).

${ }^{3}$ Ausgenommen sind einige örtliche Aufwand- und Verbrauchsteuern für die die Länder und/oder Gemeinden die Gesetzgebungshoheit besitzen und die eingeschränkte Gestaltungshoheit der Gemeinden in Form des Hebesatzrechtes bei den Gewerbe- und Grundsteuern.

${ }^{4}$ Sogar die Aufkommen der wenigen Steuerarten über die die Länder eine Gesetzgebungshoheit besitzen, nämlich einige örtliche Verbrauchs- und Aufwandsteuern, stehen nicht den Ländern, sondern den Gemeinden zu. Eine genaue Auflistung aller Steuerarten, der Gesetzgebungshoheit und Ertragshoheit bezüglich dieser Steuern haben z.B. Zimmermann/Henke ([1994], S.132-139) zusammengestellt.

${ }^{5}$ Bezüglich des kommunalen Finanzausgleichs bzw. des kommunalen Zuweisungssystems siehe KUHN [1991/92] oder NAPP [1994].
} 
des bundesdeutschen Finanzausgleichs bezüglich allokativer Aspekte zu leisten, und damit die in der deutschsprachigen Literatur vorhandene „Effizienz-Lücke” ein Stück mehr zu schließen.

Die Untersuchung beginnt in Kapitel I mit einem kurzen Überblick über die aktuelle Diskussion zur Neuordnung des bundesdeutschen Finanzausgleichssystems. Darin sollen die Grundzüge der gesetzlichen Regelungen, sowohl vor 1995, als auch ab 1995, sowie einige, meiner Meinung nach wichtiger Neuordnungsvorschläge dargestellt werden. Obwohl dieser Überblick im wesentlichen zum Verständnis der weiteren Arbeit ausreicht, werden anschließend die Regelungen und Neuvorschläge in ihren Einzelheiten formal präzisiert. Dies soll nicht nur einen genauen Eindruck von der Komplexität der gesetzlichen Regelungen geben, sondern auch als Grundlage für einige der nachfolgenden Überlegungen dienen, in denen speziell auf die Einzelheiten der Regelungen und Vorschläge Bezug genommen wird.

In den nachfolgenden zwei Kapiteln erfolgt dann die theoretische Analyse der Effizienzwirkungen von Finanzausgleichsregelungen.

Im ersten Teil (Abschnitt 2. von Kapitel II) werden, in Anlehnung an Bös [1978], anhand einer einfachen Analyse der öffentlichen Budgetgleichungen erste grundlegende Einsichten abgeleitet. Es wird gezeigt, daß die Ausgestaltung der Finanzausgleichsregelungen einen Einfluß auf die Anreize zur Ausschöpfung der Steuerquellen der einzelnen Länder besitzt. Anschließend wird das das deutsche Finanzausgleichssystem auf dessen Anreizwirkung hin untersucht.

In den nachfolgenden Abschnitten werden die Auswirkungen von Finanzausgleichsregelungen, insbesondere die Verzerrungen auf der Nachfrageseite, unter Verwendung eines einfachen Entscheidungsmodells diskutiert, in dem unterschiedliche Grade der Finanzautonomie der Länder beachtet werden. Immer wieder wird dabei auch auf die Implikationen der Ergebnisse für die konkreten bundesdeutschen Regelungen eingegangen.

Da jedoch für die Wohlfahrtswirkungen nicht nur die Verzerrungen auf der Konsumseite von Relevanz sind, werden im darauffolgenden Kapitel III die Pareto-Bedingungen für ein allgemeines Gleichgewichtsmodell entwickelt. Daran schließt sich die Entwicklung der Optimalbedingungen einer Marktökonomie mit Staatstätigkeit an. Durch Vergleich mit den Pareto-Bedingungen lassen sich erste Schlüsse für die sich im Totalmodell durch die Staatstätigkeit ergebenden Verzerrungen gewinnen. Dabei sollen wiederum die Auswirkungen der Finanzausgleichsregelungen von besonderem Interesse sein. Nach dieser gesamtwirtschaftlichen Wohlfahrtsbetrachtung wird die Möglichkeit der Entstehung von regionalen Wohlfahrtsgewinnen untersucht, die auf Kosten anderer Regionen erzielt werden können. Dies soll bereits auf die Interpretation der empirischen Analyse vorbereiten. 
Die in der theoretischen Analyse herausgearbeiteten Rückwirkungen und Effizienzaspekte können u.a. wegen der bestehenden second-best Problematik nur in einem umfassenden allgemeinen Gleichgewichtsmodell unter Kenntnis der wirtschaftlichen Daten genauer bestimmt werden. In der Finanzwissenschaft hat sich mittlerweile das Instrumentarium der Empirischen Allgemeinen Gleichgewichtsanalyse (EAG-Analyse), deren hier verwendete Ausprägung auf Shoven/Whalley [1972, 1973, 1977] zurückgeht, als sehr hilfreich bei der Untersuchung derartiger Probleme erwiesen. Aus diesem Grund wird die theoretische Analyse im zweiten Teil der Arbeit durch eine EAG-Analyse für die Bundesrepublik Deutschland ergänzt.

Begonnen wird die empirische Untersuchung mit der Präzisierung des formalen allgemeinen Gleichgewichtsmodells (Abschnitt IV.2.). Daran schließt sich die Beschreibung der Datenbasis für die Bundesrepublik Deutschland (Abschnitt IV.3.) sowie die Darstellung der Kalibrierung (Abschnitt IV.4.) an, in der die wesentlichen Parameter des Modells bestimmt werden. In den anschließenden Simulationen (Kapitel V) werden die Wohlfahrts- und Verteilungswirkungen der gesetzlichen Regelungen zum Finanzausgleich in der Bundesrepublik sowie einiger Neuvorschläge berechnet. Dies sind die Empfehlungen des Wissenschaftlichen Beirats [1991], die von Buhl/Pfingsten [1990, 1991], FötTINGER/SPAhn [1993] und Fuest/Lichtblau [1991] sowie einer Abschaffung der horizontalen Umverteilungselemente in der Umsatzsteuerverteilung, dem Länderfinanzausgleich und den Ergänzungszuweisungen. Neben der Untersuchung der Auswirkungen des Einbezugs der Neuen Länder ab 1995 stehen dabei vor allem die Effekte eines Übergangs zu einem der anderen Finanzausgleichregimes im Mittelpunkt.

Die Ergebnisse der Berechnungen werden anschließend, so weit möglich, unter Verwendung der Erkenntnisse des Theorieteils ökonomisch interpretiert und anhand einer Reihe von Sensitivitätsanalysen auf ihre Verläßlichkeit hin "getestet”. Am Ende steht eine Zusammenfassung der wichtigsten Ergebnisse und Folgerungen für die Finanzausgleichsforschung und die Neuordnung des bundesdeutschen Finanzausgleichssystems (Kapitel VI). 


\section{Kapitel I}

\section{Das bundesdeutsche Finanzausgleichssystem}

\section{1. Überblick und Diskussion}

Das bundesdeutsche System des „aktiven" Finanzausgleichs ${ }^{1}$ regelt die Aufteilung der Gemeinschaftssteuern auf Bund, Länder und Gemeinden (vertikale Steuerverteilung), die Verteilung der Länderanteile an den Steueraufkommen auf die einzelnen Länder und/oder Gemeinden (horizontale Steuerverteilung) und schließlich die horizontalen Ausgleichszahlungen zwischen den Ländern in Form des Länderfinanzausgleichs (LFA). Außerdem enthält es Bestimmungen über die Bundesergänzungszuweisungen (BEZ) des Bundes an die Länder. Diese drei Typen von Finanzbeziehungen, nämlich Steuerverteilung, Länderfinanzausgleich und Ergänzungszuweisungen, sollen im weiteren als Grundelemente eines Finanzausgleichssystems verstanden werden. Dabei wird der Begriff „Finanzausgleich”, im Unterschied zum Begriff "Länderfinanzausgleich”, auf das gesamte System bezogen und umfaßt damit alle drei Komponenten des aktiven Finanzausgleichs. Sämtliche Aspekte der Aufgabenverteilung zwischen Bund und Ländern (passiver Finanzausgleich) werden dagegen außer Acht gelassen.

Die folgende Darstellung der Diskussion um den bundesdeutschen Finanzausgleich beginnt mit einem kurzen Überblick über die gesetzlichen Regelungen, die bis zum 1.1.1995 galten. Daran schließt eine kurze Zusammenfassung der Urteile des Bundesverfassungsgerichtes vom 24.6.86 und 27.5.92 an, die einen engen Rahmen für die Neuordnung des Finanzausgleichssystems vorgeben. Mit der Vorstellung der gesetzlichen Regelungen, die seit dem 1.1.1995 gelten und den vollständigen Einbezug der Neuen Länder regeln, wird die kurze Darstellung des bundesdeutschen Finanzaugleichssystem abgeschlossen.

\footnotetext{
${ }^{1}$ Dem Begriff „aktiver Finanzausgleich" wird die Verteilung der Einnahmen der Gebietskörperschaften auf Bund, Länder und Gemeinden zugeordnet. Demgegenüber bezieht sich der Begriff „passiver Finanzausgleich" auf die Verteilung von Aufgaben, Ausgabenerfordernissen und Einnahmemöglichkeiten auf Bund, Länder und Gemeinden. Zur Abgrenzung der relevanten Begriffe siehe die grundlegende Darstellung von Peffekoven ([1980], S.608-610).
} 
Diese gesetzlichen Regelungen sind Ausgangspunkt für die Diskussion über mögliche Neuordnungsvorschläge, die schließlich in der Auswahl von sechs Empfehlungen mündet. Diese werden etwas ausführlicher dargestellt und dabei die wesentlichen Unterschiede zu dem geltenden System herausgearbeitet.

\subsection{Regelungen vor 1995}

Im folgenden werden die gesetzlichen Regelungen im Überblick dargestellt, wie sie vor dem 1.1.1995 in Kraft waren. ${ }^{2}$

\subsubsection{Steuerverteilung}

Die Steuerverteilung in der Bundesrepublik, die in ihrer bisherigen Form durch die Finanzreform von 1969 festgelegt wurde, wird im Art.106 Abs.3 bis 5 des Grundgesetzes hinsichtlich der Verteilung der Gemeinschaftssteuern grundsätzlich geregelt. Neben der Aufteilung der Einnahmen aus den Gemeinschaftssteuern wird zur Steuerverteilung in der Bundesrepublik auch die Verteilung der Gewerbesteuern gezählt.

\section{(1) Verteilung der Gewerbesteuern}

Die Aufkommen aus den Gewerbesteuern stehen prinzipiell den Kommunen zu. Allerdings müssen sie aus diesen Aufkommen eine Gewerbesteuerumlage an Bund und Länder abführen. Von dieser Umlage erhalten Bund und Länder jeweils $50 \%$. Der Länderanteil wird anschließend nach dem Prinzip der regionalen Steueraufkommen auf die Länder verteilt.

\section{(2) Einkommens- und Körperschaftssteuerverteilung}

Vom dem Aufkommen aus der Lohnsteuer und den veranlagten Einkommenssteuern erhalten die Gemeinden $15 \%$. Der verbleibende Rest wird ebenso wie die Körperschaftssteueraufkommen und die Aufkommen aus Kapitalertragssteuern jeweils zur Hälfte auf den Bund und die Länder verteilt. Die Aufteilung der jeweiligen Anteile der Länder bzw. der Gemeinden der jeweiligen Länder erfolgt nach dem Prinzip des örtlichen Aufkommens, dessen Höhe bei der Lohnsteuer durch eine Zerlegung nach dem Wohnsitzprinzip und bei der Körperschaftssteuer nach dem Betriebsstättenprinzip bestimmt wird.

\footnotetext{
${ }^{2}$ Eine exakte und detaillierte Präsentation der Regelungen und Neuordnungsvorschläge erfolgt im Abschnitt 3 .
} 


\section{(3) Umsatzsteuerverteilung}

Der Anteil des Bundes am Umsatzsteueraufkommen wurde von 1978 bis 1991 stufenweise von $67,5 \%$ auf $65 \%$ abgesenkt. Der restliche Teil der Aufkommens wird entsprechend der Einwohnerzahl in einen West- und einen Ostteil aufgeteilt. Die weitere Verteilung wird für die West- und die Ostländer getrennt durchgeführt. $75 \%$ des Länderanteils wird nach Bevölkerungsrelationen auf die Länder verteilt (Vorabverteilung). Einen Teil der verbleibenden $25 \%$ erhalten die finanzschwachen Länder (Ergänzungsanteile) unter Berücksichtigung einiger Garantieklauseln. Der Rest wird nach Bevölkerungsrelationen unter den finanzstarken Ländern aufgeteilt (Restverteilung). Dabei sichern einige Garantieklauseln, daß die finanzstarken Länder nicht unter die länderdurchschnittliche Finanzkraft fallen.

\subsubsection{Länderfinanzausgleich (LFA)}

Der bundesdeutsche Länderfinanzausgleich (LFA) ist ein allgemeiner Finanzkraftausgleich in dem der Finanzbedarf der einzelnen Länder nur indirekt durch die Bevölkerungsanteile und der Bevölkerungsveredelung berücksichtigt wird.

\section{(1) Ermittlung der Finanzkraft der Länder}

Zur Ermittlung der gesamten (regionalen) Finanzkraft eines Landes werden die aus den Einnahmen der Landesregierung berechnete Finanzkraft des Landes und die nur zur Hälfte berücksichtigte Finanzkraft der Gemeinden des Landes, die sich aus den normierten Gemeindesteuereinnahmen und Einnahmen aus der Einkommenssteuerverteilung ergeben, verwendet. Die Einnahmen der Nordländer werden jedoch um die Sonderbelastungen aus dem Unterhalt der Seehäfen vermindert. Dividiert man die Einnahmen durch die Bevölkerungszahl ergibt sich die jeweilige (Pro-Kopf-)Finanzkraft. Die Einwohnerzahlen der Stadtstaaten werden dabei stärker gewichtet (Veredelung), so daß deren angerechnete Finanzkraft abgesenkt wird. Diese Veredelung wird auch bei der Berechnung der Bevölkerungszahlen der Gemeinden durchgeführt.

Als zweite Maßgröße wird die Ausgleichsmeßzahl ermittelt. Sie gibt die durchschnittliche Finanzkraft an, die ein Land hätte, wenn es durchschnittliche Pro-Kopf-Steuereinnahmen hätte. Diese Größe dient als Vergleichsmaßstab für die Finanzkraft der Länder.

(2) Finanzausgleichszuweisungen an die finanzschwachen Länder

Die Länder, deren regionale Finanzkraft geringer als die durchschnittliche Finanzkraft aller Länder ist (Ausgleichsmeßzahl), erhalten Finanzausgleichszuweisungen von den finanzstarken Ländern. Die genaue Höhe der Zahlungen berechnet sich nach einem zweistufigen 
Tarif, der sicherstellt, daß die besonders finanzschwachen Länder unter Einberechnung der LFA-Zuweisungen mindestens $92 \%$ der durchschnittlichen Länderfinanzkraft erreichen.

\section{(3) Ermittlung der Finanzbeiträge der finanzstarken Länder}

Die Finanzbeiträge der finanzstarken Länder werden nach einem dreistufig gestaffelten Tarif ermittelt. Dieser führt normalerweise dazu, daß die am wenigsten finanzstarken Länder von Zahlungen befreit sind, wohingegen die finanzstärksten Länder einen überproportional starken Beitrag zu den LFA-Zuweisungen leisten.

\section{(4) Garantieklauseln}

Zusätzlich enthält das Verfahren drei Garantieklauseln, die sicherstellen, daß die finanzschwachen Länder mindestens $95 \%$ der durchschnittlichen Finanzkraft der Länder (nicht der regionalen Finanzkraft und ohne veredelter Bevölkerung) erreichen. Die beiden anderen Garantieklauseln betreffen die finanzstarken Länder und sollen sicherstellen, daß diese durch ihre Finanzbeiträge nicht unter die durchschnittliche Finanzkraft der Länder fallen. Um dies zu garantieren, werden notfalls auch die LFA-Zuweisungen an die finanzschwachen Länder gekürzt.

\subsubsection{Bundesergänzungszuweisungen (BEZ)}

Die Bundesergänzungszuweisungen stellen allgemeine Zuweisungen des Bundes an die Länder dar. Sie dienen ebenfalls dem Zweck, die Finanzkraft der leistungsschwachen Länder anzuheben. Sie können aber auch zum Ausgleich bestimmter Sonderlasten der Länder verwendet werden. Ihre Gesamthöhe ist an den gesamten Umsatzsteueraufkommen orientiert. Aufgeteilt werden die BEZ zum einen in Form fester Beträge und zum anderen als teilweiser Ausgleich, der nach dem LFA noch verbleibenden Finanzkraftdefizite der finanzschwachen Länder.

\subsection{Die Auseinandersetzung um eine Neuregelung.}

Die Finanzausgleichsregelungen bestimmen einen Großteil der den Ländern zur Verfügung stehenden Finanzmittel. Es ist daher nicht verwunderlich, daß es immer wieder Versuche einzelner Länder gab, die geltenden gesetzlichen Regelungen über Gesetzesanträge im Bundesrat zu verändern. ${ }^{3}$

\footnotetext{
${ }^{3}$ Siehe z.B. den Gesetzesantrag der vier norddeutschen Bundesländer, Nordrhein-Westfalens und des Saarlandes, den sogenannten „Albrecht-Plan"(BundesRat [1988]) zur Übernahme der-Hälfte der Sozialhilfeausgaben durch den Bund, der von KLANBerg/Prinz [1988] diskutiert wird.
} 
In den letzten Jahren wurde darüber hinaus der Gang zum Bundesverfassungsgericht zunehmend das Hauptinstrument beim Versuch der Länder, sich eine bessere finanzielle Ausstattung zu sichern. ${ }^{4}$ Das Bundesverfassungsgericht bestätigte jedoch in seinen verschiedenen Urteilen weitgehend die Struktur und die Regelungen des bundesdeutschen Finanzausgleichs (BVerfGE 72, S.330-436, BVerfGe 86, S.198-279, siehe auch HeILMANN [1992], S.53-59).

Zu den Kritikpunkten des Verfassungsgerichts gehörte die bis 1987 geltende Berechnung der Finanzkraft der Länder, die nur aus der Steuerkraft bestand und andere Einnahmen der Länder nicht berücksichtigte. Dagegen wurde die Anrechnung der Seehafenlasten und die Einwohnerveredelung grundsätzlich als zulässig erklärt. ${ }^{5}$

Außerdem wurde die Rolle der Bundesergänzungszuweisungen (BEZ) neu bestimmt. Zusätzlich zu ihrer bisherigen Rolle als Ergänzung des LFA sollen diese nun auch dazu eingesetzt werden können, je nach Bedarf, die Finanzausstattung einzelner Länder gezielt zu verbessern. ${ }^{6}$

Zudem wurde vom Gericht moniert, daß in der Berechnung der Garantieklausel des $\S$ 10 Abs. 3 FAG (FAG'87) nicht die zur Ermittlung der LFA-Zuweisungen verwendete Finanzkraft berücksichtigt wird (siehe РеFfEKoven [1992], S.350).

\subsection{Regelungen nach 1995}

Die prinzipielle Bestätigung der geltenden Regelungen durch das Verfassungsgericht ließen auch für die Neuregelung des Finanzausgleichssystems zum 1.1.1995 kaum allzugroße Veränderungen der Bestimmungen erwarten. Daher überrascht es nicht, daß die Neuordnung im "Gesetz zur Umsetzung des Föderalen Konsolidierungsprogramms“(FKPG) vom 26.Juni 1993 nur einige Korrekturen enthält. Neben dem vollen Einbezug der Neuen Länder in einen gemeinsamen LFA sind die Vereinfachung der Umsatzsteuerverteilung und die Einführung einer zusätzlichen Garantieklausel im LFA, die sicherstellen soll, daß die Reihenfolge der Finanzkraft der Länder durch den LFA nicht verändert wird, die auffallendsten Veränderungen.

\footnotetext{
${ }^{4}$ Zuletzt die Normenkontrollanträge von Hamburg, Bremen, Saarland, Schleswig-Holstein in den Jahren 1988-1990 und Baden-Württemberg 1992. Eine Diskussion der Normenkontrollanträge findet sich bei Bartsch/Probst [1988], Dohnanyi [1986], Posser [1986] und Palm [1986].

${ }^{5}$ Zugleich sollte der Gesetzgeber die Einwohnerveredelung überprüfen. Da das ifo-Institut in dem entsprechenden Gutachten die Einwohnerveredelung auch in ihrer Höhe als durchaus gerechtfertigt ansah, wurde die bis dahin geltende Veredelung von $135 \%$ nicht verändert (siehe Hummel/Leibfritz [1987]).

${ }^{6}$ Zur Darstellung und Beurteilung des Urteils von 1986 siehe z.B. Geske [1986], Frey [1988], PEFfekoven [1988, 1990] oder Heilmann [1992], zum Urteil von 1992 siehe Peffekoven [1992].
} 
In der Umsatzsteuerverteilung wurde der Länderanteil auf $44 \%$ erhöht. Allerdings müssen die Westländer, incl. Westberlin, einen Teil dieser höheren Einnahmen an den Bund abführen. Da nun alle Länder gleichermaßen in die Verteilung einbezogen werden, entfällt die Vorwegaufteilung in einen Ost- und einen Westteil. In der horizontalen Umsatzsteuerverteilung wird die Garantieklausel für die finanzstarken Länder gestrichen und die Restverteilung dahingehend modifiziert, daß in ihr jetzt alle Länder berücksichtigt werden.

In der Ermittlung der Finanzkraft im Länderfinanzausgleich wird die Bevölkerungsveredelung auch auf Berlin und die Anrechnung der Seehafenlasten auf MecklenburgVorpommern ausgedehnt.

Die Berechnung der LFA-Zuweisungen und -Beiträge erfolgt nach wie vor mit Hilfe eines zwei- bzw. dreistufigen Tarifes. Allerdings werden in der Berechnung der Finanzbeiträge die Grenzen zwischen den Stufen verändert. Dies hat zur Folge, daß nun alle finanzstarken Länder zur Finanzierung der LFA-Zuweisungen herangezogen werden.

In den Garantieklauseln wird nun, durch ein recht kompliziertes Verfahren, die Beibehaltung der Finanzkraftreihenfolge gesichert. Darüber hinaus wird die Mindesthöhe des Finanzkraftausgleichs reduziert.

Die Bundesergänzungszuweisungen werden ebenfalls neu geregelt. Sie werden zum Teil zweckgebunden zur Haushaltssanierung besonders verschuldeter Länder gewährt. Der restliche Teil wird zur Reduktion der Finanzkraftfehlbeträge (nach LFA) an die leistungsschwachen Länder ausgeschüttet.

Die starke Kontinuität in den bundesdeutschen Finanzausgleichsgesetzen hält damit trotz der immensen Aufgabe der Eingliederung der Neuen Länder an. Allem Anschein nach ist der Gesetzgeber der Überzeugung, daß sich das bundesdeutsche Finanzausgleichssystem bewährt hat, sonst hätte er sich wohl an eine grundsätzlichere Reform herangewagt. Mit dieser Ansicht stünde der Gesetzgeber weitgehend alleine, wie die lange Debatte über eine Neuordnung des Finanzausgleichssystems deutlich zeigt.

\section{Vorschläge zu einer Neuordnung}

Im folgenden soll eine Reihe von Empfehlungen zu einer Neuregelung des Finanzausgleichssystems dargestellt werden. Dabei ist keineswegs beabsichtigt, sämtliche Vorschläge zu präsentieren. ${ }^{7}$ Es sollen vielmehr die wesentlichen Kritikpunkte an den gesetzlichen Regelungen herausgearbeitet werden.

\footnotetext{
${ }^{7}$ Einen Überblick über verschiedene Vorschläge bietet HeILmanN [1992].
} 
Die wohl stärkste Kritik am Finanzausgleichssystem besteht in der Infragestellung, der Notwendigkeit einer horizontalen Umverteilung zwischen den Ländern. Gerade eine unterschiedliche Finanzausstattung könnte im Sinne des Tiebout-Ansatzes zu einer höheren Wohlfahrt führen, da die Individuen in die Region wandern könnten, deren Kombination von Steuersätzen und öffentlichen Leistungen ihren Präferenzen am meisten entspricht („voting by feet”, TiEBout [1956]). Auf diese These stützen sich weitgehend die Ideen des kompetitiven Föderalismus (siehe z.B. BRENnAN/BUCHANAN [1980], S.173-186 oder KenYon/KincaID [1988]). ${ }^{8}$ Dies setzt allerdings voraus, daß die einzelnen Regionen in der Lage sind, ihre eigenen Einnahmen, z.B. durch die Erhebung eigener Steuern, zu beeinflussen. In stark abgeschwächter Form finden sich diese Vorstellungen in dem Vorschlag des Wissenschaftlichen BeIRATS [1992], der eine stärkere Steuerautonomie der Länder, bei gleichzeitig geringerem Finanzkraftausgleich, empfiehlt (siehe auch PEFFEKoven [1987, 1994], SAChVERSTÄndigenrat [1993] oder WAHL/Frischmuth [1990]). Eine Reduktion des Finanzkraftausgleichs ohne entsprechend verbesserter Steuersetzungsrechte der Länder fordern z.B. Fuest/LichtblaU [1991]. Die extremste Version dieser Kritik wäre die völlige Abschaffung der umverteilenden Elemente der Umsatzsteuerverteilung, wie sie z.B. HomBURG ([1994], S.327) in Erwägung zieht.

Eine Kritik im eher entgegengesetzten Sinn setzt an der Verwendung der Finanzkraft als alleinigem Maß zur Bestimmung der Ausgleichshöhe an. Im Prinzip müßte zusätzlich zur Einnahmensituation der Länder auch berücksichtigt werden, welchen Bedarf an öffentlichen Ausgaben sie tatsächlich besitzen. Ein unterschiedlicher Bedarf kann sich z.B. infolge regional differierender Kosten der Bereitstellung der öffentlichen Güter ergeben. Ein weiterer Grund könnten besondere Belastungen sein, wie z.B. einer besonders schlechte Grundausstattung mit öffentlichen Leistungen. Einen Vorschlag, der auf die unterschiedlichen Kosten abhebt, präsentieren FöTtingER/SPAHN [1993], die die Berücksichtigung der realen Finanzkraft der Länder fordern. In Berechnung dieser Größe gehen die unterschiedlichen Kosten der öffentlichen Güter sowie die bereitgestellte Menge der öffentliche Leistungen ein. Damit enthält der Vorschlag im Prinzip eine Anrechnung des regional unterschiedlichen Angebots an öffentlichen Leistungen. HOMBURG [1993] präsentiert eine Neuordnungskonzeption in der die Infrastrukturausstattung Grundlage für den Finanzbedarfsausgleich darstellt. Eine Abschaffung des Finanzkraftausgleichs und eine alleinige Ausrichtung an der Ausgabenbelastung pro Kopf wäre eine extreme Variante einer Bedarfsorientierung.

Kleinere Korrekturen der Ermittlung der Finanzkraft werden in einer Reihe von Arbeiten empfohlen. Insbesondere soll die Finanzkraft der Gemeinden vollständig berücksichtigt

\footnotetext{
${ }^{8}$ Eine kritische Auseinandersetzung mit den Ansätzen des fiscal federalism findet sich z.B. in GroENEWEGEN [1988].
} 
werden, da diese ebenso wie die Einnahmen der Länder die Höhe der regionalen Ausstattung mit öffentlichen Leistungen bestimmt (siehe z.B. WISSENSCHAFTLICHER BEIRAT [1992]). Umstritten ist zudem die Einwohnerveredelung der Bevölkerung der Stadtstaaten und die Berücksichtigung der Seehafenlasten als Sonderbedarfe (siehe z.B. PEFFEKOVEN [1987]). ${ }^{9}$

Eine weitere Kritik an der Systematik des Finanzausgleichs setzt an den Umverteilungselementen (Ergänzungsanteilen) der Umsatzsteuerverteilung an. Mit dem Argument, daß sämtliche umverteilenden Maßnahmen im LFA konzentriert werden sollten, wird eine starke Vereinfachung der Umsatzsteuer gefordert (siehe dazu vor allem PEFFEKOvEN [1985], aber auch Wissenschaftlicher Beirat [1992], Föttinger/Spahn [1993], SachVERSTÄNDIGENRAT [1992] u.v.a.). Außerdem ist die Verteilung der Steueraufkommen nach Bevölkerungrelationen umstritten. Fuest/LichtBLAU [1991] empfehlen, stattdessen das Volkseinkommen als Indikator für das Steueraufkommen der jeweiligen Bundesländer $\mathrm{zu}$ verwenden.

Die Konzentration der Umverteilungsmaßnahmen im LFA führt zu einer deutlichen Vereinfachung der Umsatzsteuerverteilung. Damit wird ein weiteres Ziel einer Reihe von Autoren schon beinahe erreicht. Sie fordern eine deutliche Vereinfachung des gesamten Finanzausgleichsverfahren. Dies könnte beispielsweise durch die Einführung eines einfachen linearen Umverteilungstarifs im LFA erreicht werden, durch den die meisten Garantieklauseln überflüssig werden würden (siehe z.B. BUHL/PFINGSTEN [1986, 1990, 1991] oder FöTtingER/SPAHN [1993]).

Ein weiteres Ziel von Neugestaltungswünschen sind die Bundesergänzungszuweisungen. Entsprechende Forderungen reichen von einer völligen Abschaffung der BEZ, über eine ausschließlich zweckgebundene Gewährung der BEZ, bis hin zu einem Einsatz der BEZ als Ergänzung des LFA (siehe z.B. Buhl [1986], Bartsch/Probst [1988], Peffekoven [1987] oder PALM $[1986,1987])$.

Aus der Vielzahl der Vorschläge zu einer Neukonzeption des bundesdeutschen Finanzausgleichssystems, die bei weitem nicht alle aufgelistet wurden, werden im folgenden fünf herausgegriffen. Mit der Auswahl sollen die oben dargestellten Empfehlungen weitgehend abgedeckt werden.

\footnotetext{
${ }^{9}$ Vorschläge zu einer Abschaffung der Seehafenlasten sind z.B. der von BuHL [1986] oder von Bartsch/Probst [1988]. Eine Erhöhung der Anrechnung der Sonderbedarfe fordern dagegen u.a. Breuel [1987], Posser [1986, 1987], Gobrecht [1987], von Dohnanyi [1986], Bartsch/Probst [1988], BreUel [1988] (siehe auch Bundesrat [1988]). Eine ausführliche Diskussion der entsprechenden Vorschläge findet sich bei KLANBERg/Prinz [1988].
} 
Eine Reihe von Vorschlägen bleibt gezwungenermaßen unberücksichtigt. Dazu gehören z.B. die von HENKE [1985] und FÄrBer [1992, 1993], die sehr interessante Anregungen bieten, aber nicht konkret ausformuliert sind und in ihren Einzelelementen in den hier berücksichtigten Vorschlägen auftauchen. ${ }^{10}$ Ebenso wird nicht näher auf den Vorstoß von HомвuRg [1993] eingegangen, der eine Koppelung der LFA-Zuweisungen an den Infrastrukturbestand anregte. Diesen Vorschlag kann man nur mittels eines dynamischen Modells untersuchen, das jedoch zur Analyse der anderen hier präsentierten Ansätze nicht benötigt wird. Ähnliches gilt für die Idee von RICHTER/WELLISCH [1993], für die der LFA den Abfluß der Renteneinkommen aus immobilen Faktoren in andere Regionen ausgleichen soll. Die Berücksichtigung von Unternehmens- und Haushaltswanderungen sowie von differenzierten Eigentumsverhältnissen erfordern in diesem Fall eine Modellstruktur, die zur Untersuchung der anderen Ansätze nicht notwendig ist. Ebenso wird der Vorschlag zu einer Neugliederung der Bundesländer nicht näher verfolgt (siehe HANSMEYER, KOPS [1990]), da er das Finanzausgleichssystem unberührt läßt.

Im folgenden soll mit Hilfe des Vorschlages des Wissenschaftlichen Beirats [1992] der Fall einer höheren Steuerautonomie der Länder untersucht werden. Die Ausrichtung am Finanzbedarf ist eine der Forderungen von FöTtINGER/SPAHN [1993], deren Empfehlung aus diesem Grund aufgegriffen wird. Eine sehr starke Vereinfachung des LFA-Verfahrens schlagen Buhl/PFingsten [1990, 1991] vor. Ihr Vorschlag, der aus systematischen Überlegungen heraus entwickelt wurde, dient als Prototyp eines einfachen Finanzausgleichsverfahrens. ${ }^{11}$ Schließlich wird noch der Ansatz von Fuest/LichtBLAU [1991] untersucht. Das Reizvolle an ihrer Lösung ist, daß die Umsatzsteuerverteilung nicht nach Bevölkerungsgrößen (Bedarf), sondern nach der Höhe des Volkseinkommen (Steueraufkommen) vonstatten gehen soll. Als Extremfall soll zusätzlich eine Abschaffung der horizontalen Umverteilungselemente in der Umsatzsteuerverteilung und des LFA diskutiert werden. Dieser Fall läßt auf die grundsätzlichen Wohlfahrtswirkungen der Umverteilungsmaßnahmen schließen.

Im folgenden sollen diese ausgewählten Vorschläge kurz dargestellt werden.

\footnotetext{
${ }^{10}$ HenKE [1985] fordert eine höhere Einnahmenautonomie der Länder und eine Abschaffung der BEZ. FÄRBER ([1993], S.307, S.312-313) schlägt z.B. vor, die BEZ an Sonderbelastungen auszurichten und kritisiert die umverteilende Wirkung der Orientierung der Umsatzsteuerverteilung an den Bevölkerungszahlen.

${ }^{11}$ Zugleich steht dieser Vorschlag für einen Versuch, ein optimales Finanzausgleichsverfahren aus systematischen Überlegungen heraus, abzuleiten. Weitere Arbeiten in diese Richtung sind die von LENK [1992] oder TAube [1990].
} 


\section{(1) Der Vorschlag des Wissenschaftlichen Beirats}

Die Empfehlung des Wissenschaftlichen Beirats [1992] liegt in drei Varianten vor, die sich in der Frage unterscheiden, an welcher Stelle des Finanzausgleichs eine Mindestauffüllung der Finanzkraft stattfinden soll, und inwieweit sich der Bund an diesem Mindestausgleich beteiligen soll.

In allen drei Vorschlägen wird eine Vereinfachung der Umsatzsteuerverteilung vorgeschlagen, die, für den Fall, daß die Mindestauffüllung nicht in der Umsatzsteuerverteilung vorgesehen ist, nur nach Bevölkerung durchgeführt werden soll. Der eigentliche Finanzausgleich soll in allen Varianten in Form eines linearen Tarifes mit einem Ausgleichssatz zwischen 50 und 60 Prozent vonstatten gehen. Dies entspricht einer Gleichbehandlung der Länder ungeachtet ihrer Finanzkraft. Zudem werden Sprünge in den Budgetgeraden vermieden.

Zusätzlich empfiehlt der Beirat eine Reduktion des Finanzkraftausgleichs bei gleichzeitiger Erhöhung der Steuerhoheit der Länder. Die daraus erzielten Einnahmen sollten entweder gar nicht oder nur in normierter Form, auf Basis eines durchschnittlichen Zuschlages, in der Finanzkraft der Länder berücksichtigt werden.

\section{(2) Die Empfehlung von Buhl/Pfingsten [1991]}

Der Vorschlag von BuhL/Pfingsten $[1990,1991]$ ist nur auf den LFA und die BEZ ausgerichtet. Der Länderfinanzausgleich soll ausschließlich nach einem linearen Umverteilungstarif durchgeführt werden. Die Ergänzungszuweisungen sollen nur nach Bedarfsverhältnissen verteilt werden, was in ihrer Arbeit durch Bevölkerungsverhältnisse ausgedrückt wird.

\section{(3) Der Vorschlag von Föttinger/Spahn [1993]}

In diesem Vorschlag soll die Umsatzsteuer ausschließlich nach Bevölkerungsrelationen verteilt werden. Der $L F A$ soll sich an der realen Finanzkraft orientieren und damit die regional unterschiedlichen Kosten der öffentlichen Güter mitberücksichtigen. Zu diesem Zweck wird für die öffentlichen Güter eine Art Preisindex gebildet, mit dem anschließend die Finanzkraft gewichtet wird. Der Finanzkraftausgleich erfolgt dann über einen linearen Umverteilungstarif.

\section{(4) Der Vorschlag von Fuest/Lichtblau [1991]}

Fuest/LichtblaU [1991] fordern die Orientierung der horizontalen Umsatzsteuerverteilung an der Relation der Volkseinkommen. Der Länderfinanzausgleich mit einem linearen Finanzausgleichstarif wird ergänzt durch Bundesergänzungszuweisungen, die das 
Erreichen einer bestimmten Mindestfinanzkraft garantieren sollen. Eine zweite Art der Ergänzungszuweisungen soll in Form zweckgebundener Zuweisungen vergeben werden.

\section{(5) Der Vorschlag einer Abschaffung der horizontalen Umverteilung}

Mit der Absicht, eine extreme Position zu beziehen, soll hier die Abschaffung der horizontalen Umverteilungselemente im Finanzausgleichssystem diskutiert werden (vgl. HoMBURG [1994], S.327). ${ }^{12}$

Es sei eine ausschließlich nach Bevölkerungrelationen durchgeführte Umsatzsteuerverteilung vorgesehen. Zudem sollen sowohl der Länderfinanzausgleich als auch die Bundesergänzungszuweisungen ersatzlos gestrichen werden.

\section{1. Überblick über Regelungen und Vorschläge}

Diese Vorschläge sollen nun kurz in einem Überblick zusammengestellt werden, um nochmals die Gemeinsamkeiten und Unterschiede zwischen den einzelnen Empfehlungen gegenüberzustellen. In der Tabelle I.1 werden die Regelungen bezüglich der Umsatzsteuerverteilung, in der Tabelle I.2 die zum Länderfinanzausgleich und in der Tabelle I.3 die zu den Bundesergänzungszuweisungen präsentiert.

Zum Verständnis soll folgender Hinweis genügen: Ein Querstrich bedeutet, daß eine derartige Regelung überhaupt nicht vorgesehen ist. Ansonsten sind die Tabellen weitgehend selbsterklärend.

Die kurze Darstellung der einzelnen gesetzlichen Regelungen und der Neuordnungsvorschläge sollte dazu dienen, ein ungefähres Bild der Besonderheiten der Vorschläge zu geben. Im folgenden wird diese Darstellung vertieft, da eine detaillierte Kenntnis der jeweiligen Vorschläge notwendig ist, um einzelne Aspekte der Effizienzwirkungen genauer untersuchen zu können. Z.B. können nur bei einer differenzierten Formalisierung der Vorschläge, die Ableitungen der einzelnen Einnahmenkategorien ermittelt werden, die zur Betrachtung bestimmter Effekte nötig sind.

Zum Verständnis der Ausführungen zu den Effizienzwirkungen, zu der Präsentation des EAG-Modells sowie zur Interpretation der Simulationsergebnisse sind diese genauen Detailkenntnisse jedoch nicht unbedingt notwendig. ${ }^{13}$

\footnotetext{
${ }^{12}$ Genaugenommen besitzt die Umsatzsteuerverteilung in ihrer Orientierung an den Bevölkerungsrelationen ebenfalls Umverteilungscharakter. Hier sollen jedoch nur die deutlich als UmverteilungsmaBnahmen charakterisierten Elemente gestrichen werden.

${ }^{13}$ Zur Programmierung des EAG-Modells sind sie allerdings unentbehrlich, da sie dort in dieser detaillierten Form implementiert werden sowie deren Ableitungen verwendet werden.
} 


\begin{tabular}{|c|c|c|c|c|c|c|c|c|c|}
\hline Umsatzsteuer & $<95^{a}$ & $>95^{b}$ & $\mathbf{B} / \mathbf{P}^{c}$ & $\mathbf{W}-\mathbf{2}^{d}$ & $\mathbf{F} / \mathbf{L}^{e}$ & $\mathbf{W}-\mathbf{1}^{f}$ & $\mathbf{W}-\mathbf{3}^{g}$ & $\mathbf{F} / \mathbf{S}^{h}$ & $\mathrm{Um} .^{i}$ \\
\hline Anteil Länder & $37 \%$ & $46 \%$ & \multicolumn{7}{|c|}{ wie gesetztliche Regelung } \\
\hline $\begin{array}{l}\text { (Vorab)verteilung } \\
\text { von ?\% Umst. }{ }^{j}\end{array}$ & \multicolumn{3}{|c|}{$75 \%$} & keine & \multicolumn{5}{|c|}{$100 \%$} \\
\hline Vorabverteilung (nach) & \multicolumn{3}{|c|}{ Bevölkerung } & keine & $\begin{array}{l}\text { Volks } \\
\text { eink. }\end{array}$ & & \multicolumn{3}{|c|}{ Bevölkerung } \\
\hline Ergänzungsanteile & \multicolumn{4}{|c|}{ an arme Länder } & \multicolumn{5}{|c|}{ keine } \\
\hline Restverteilung an & $\begin{array}{l}\text { reiche } \\
\text { Länder }\end{array}$ & \multicolumn{3}{|c|}{ alle Länder } & \multicolumn{5}{|c|}{ keine } \\
\hline Garantieklausel an & $\begin{array}{l}\text { reiche } \\
\text { Länder }\end{array}$ & \multicolumn{8}{|c|}{ keine } \\
\hline horizontale Vert. ${ }^{k}$ & \multicolumn{4}{|c|}{ ja } & \multicolumn{5}{|c|}{ nein } \\
\hline
\end{tabular}

${ }^{a}$ Regelungen vor 1995

${ }^{b}$ Regelungen nach 1995

${ }^{c}$ Buhl/Pfingsten [1991]

${ }^{d}$ Vorschlag des Wissenschaftlichen Beirats [1992], Variante II

${ }^{e}$ Fuest/Lichtblau [1991]

${ }^{f}$ Wissenschaftlichen Beirat [1992], Variante I

${ }^{g}$ WissenschaftLichen Beirat [1992], Variante III

${ }^{h}$ FötTINGER/SPAHN [1993]

${ }^{i}$ Die Abschaffung der Umverteilungselemente.

${ }^{j}$ Wieviel Prozent der Umsatzsteueranteile der Länder wird als Vorabverteilung verteilt?

${ }^{k}$ Ist im Vorschlag in der Umsatzsteuerverteilung eine horizontale Umverteilung vorgesehen?

Tabelle I.1: Regelungen und Vorschläge zur Umsatzsteuerverteilung 


\begin{tabular}{|c|c|c|c|c|c|c|c|c|}
\hline Finanzausgleich & $<95 \mid>95$ & W-1 & $\mathbf{W}-\mathbf{3}$ & W-2 & $\mathrm{B} / \mathrm{P}$ & $\mathbf{F} / \mathbf{L}$ & $\mathbf{F} / \mathbf{S}$ & $\mathrm{Um}$ \\
\hline LFA orientiert an & \multicolumn{6}{|c|}{ Finanzkraft, teilw. normiert } & $\begin{array}{l}\text { reale } \\
\text { Fin. }{ }^{a}\end{array}$ & - \\
\hline Veredelung Länder & ja & \multicolumn{6}{|c|}{ eventuell } & - \\
\hline $\begin{array}{l}\text { Gemeindesteuern }{ }^{b} \text { an- } \\
\text { gerechnet } \mathrm{zu}\end{array}$ & $50 \%$ & \multicolumn{3}{|c|}{$100 \%$} & \multicolumn{3}{|c|}{ nicht klar } & - \\
\hline Sonderbedarfe & ja & \multicolumn{3}{|c|}{ nein } & \multicolumn{3}{|c|}{ unklar } & - \\
\hline $\begin{array}{l}\text { Ersatzgröße für An- } \\
\text { rechnung des Finanz- } \\
\text { bedarfs }\end{array}$ & Bev & \multicolumn{4}{|c|}{ Bev, wenn veredelt } & nein & $\begin{array}{l}\mathrm{G}^{c} / \mathrm{Bev} \\
\text { reale } \\
\text { Kosten }^{d}\end{array}$ & - \\
\hline Tarif für $\mathrm{FZ}^{e}$ & zweistufig & \multicolumn{6}{|c|}{ linear, einstufig } & - \\
\hline Mindestgarantie & \multicolumn{3}{|c|}{ ja } & nein & \multicolumn{3}{|c|}{ ja } & - \\
\hline Tarif für $\mathrm{FB}^{f}$ & dreistufig & \multicolumn{6}{|c|}{ linear, einstufig } & - \\
\hline
\end{tabular}

${ }^{a}$ Finanzkraft.

${ }^{b}$ Einnahmen aus den Gemeindesteuern

${ }^{c}$ Öffentliche Ausgaben.

${ }^{d}$ Reale Kostenunterschiede der öffentlichen Leistungen.

${ }^{e}$ Finanzzuweisungen.

${ }^{f}$ Finanzbeiträge.

Tabelle I.2: Vorschläge und Regelungen des Länderfinanzausgleichs 


\begin{tabular}{||l||c|c|c|c|c|c|c|c|c||}
\hline \hline Ergänzungszuw. & $<95$ & $>95$ & $\mathbf{B} / \mathbf{P}$ & $\mathbf{W}-\mathbf{2}$ & $\mathbf{F} / \mathbf{L}$ & $\mathbf{W}-\mathbf{1}$ & $\mathbf{W}-\mathbf{3}$ & $\mathbf{F} / \mathbf{S}$ & $\mathbf{U m}$ \\
\hline \hline BEZ & \multicolumn{2}{|c||}{ Allgemeine BEZ } & Zweckgebundene BEZ & nein \\
\hline nach Finanzkraft & ja & nein & $\mathrm{ja}$ & \multicolumn{3}{|c||}{ nein } \\
\hline nach Finanzbedarf & nein & $\mathrm{ja}$ & \multicolumn{2}{|c|}{ nein } & ja & nein \\
\hline \hline
\end{tabular}

Tabelle I.3: Regelungen und Vorschläge zu den BEZ

\section{Regelungen und Vorschläge im Einzelnen}

\subsection{Einführung}

Will man die ökonomischen Auswirkungen der Finanzausgleichsregelungen genauer untersuchen, ist es notwendig, sich eine relativ genaue Kenntnis der einzelnen Regelungen zu erarbeiten. Da für eine ökonomische Analyse letztlich Zusammenhänge zwischen den Finanzausgleichszuweisungen und den wesentlichen Variablen des ökonomischen Systems, wie z.B. öffentliche Ausgaben, privater Konsum, Einkommen, Output etc. von Interesse sind, sollen diese Interdependenzen im folgenden, mit Hilfe einer formalen Darstellung der gesetzlichen Regelungen und der ausgewählten Vorschläge dargestellt werden. ${ }^{14}$

Ausgehend von den vor 1995 geltenden Regelungen werden anschließend die für die Zeit nach 1995 getroffenen Regelungen dargestellt. Die Formalisierung der Empfehlungen zu einer Neugestaltung des Finanzausgleichssystems werden dieses Kapitel abrunden.

Vorab einige Bemerkungen zu den Notationen:

Mit $S T$ werden die ursprünglichen Steueraufkommen und mit $T$ die Steuereinnahmen nach der Steuerverteilung bezeichnet. Sollen weitere, die Steuereinnahmen betreffende Größen notwendig sein, wie z.B. fiktiv berechnete Steueraufkommen zur Ermittlung der Gemeindefinanzkraft, so werden sie mit $F T$ geschrieben. Alle den LFA betreffende Variablen beginnen mit $F$. Die hochgestellten Indizes ordnen, fast immer, die Variablen bestimmten Teilen des Finanzausgleichssystems zu, wie z.B. $C$ der Umsatzsteuerverteilung, $E Z$ den

\footnotetext{
${ }^{14}$ Die Einnahmen des Bundes, die sich als Summe der Aufkommen der jeweiligen Steuerart abzüglich der Anteile der Länder ergeben, werden in dieser Darstellung nicht explizit aufgeführt, da sie für die weitere Untersuchung keine wesentliche Rolle spielen.
} 
Ergänzungszuweisungen. Die weiteren zumeist tiefgestellten Indizes bezeichnen entweder Regionen ( $s$ oder $r$ ) oder Güter bzw. Sektoren ( $i$ oder $j$ ). Der Bund wird mit $B d$, die Länder mit $L d$ und die Gemeinden mit $G m$ gekennzeichnet. Feste Parameter sind allgemein mit $\pi$ und Hilfsgrößen mit $\kappa$ notiert. $E W$ sind Bevölkerungsangaben. Wird eine Mengenbezeichnung (z.B. $\mathcal{S}$ oder $\mathcal{R}$ ) als Index verwendet, dann ist die entsprechende Variable die Summe über alle Größen der jeweiligen Menge.

Die Menge aller Länder sei

$$
\mathcal{R}=\{S H, H H, H B, N S, N W, H E, R P, S A, B W, B Y, O L\} .
$$

Die Ostländer $(O L)$ bestehen aus den fünf neuen Ländern sowie Berlin. ${ }^{15}$

Mit dem Begriff "Steueraufkommen" werden die in den einzelnen Ländern vor der Steuerverteilung eingegangenen Steuerzahlungen, sowie mit der Zuordung "Steuereinnahmen" die Einnahmen der Länder aus Steuermitteln nach der Steuerverteilung bezeichnet.

Zur klareren Abgrenzung der uneindeutigen Schreibweise der Gesetztestexte, die unter den Einnahmen eines Landes zum einen die Einnahmen des Landes, die im Landeshaushalt erscheinen, und zum anderen die Einnahmen aller Gebietskörperschaften eines Landes, also die Einnahmen des Landes und der Gemeinden, bezeichnen, wird folgende Konvention getroffen. Soweit die Einnahmen aller Gebietskörperschaften eines Landes gemeint sind, wird von "regionalen Einnahmen" gesprochen. Die Einnahmen der Landesregierung werden als "Einnahmen eines Landes" bezeichnet.

Die nachfolgenden Darstellung enthalten bereits einige kleine Vereinfachungen, die im Hinblick auf eine einfachere Formalisierung und die spätere Programmierung im Empirischen Allgmeinen Gleichgewichtsmodell gewählt wurden. An den entsprechenden Stellen wird dies jeweils vermerkt.

\subsection{Bundesdeutsche Regelungen vor 1995}

Die Darstellung der vor 1995 geltenden Regelungen ${ }^{16}$ enthält nur wenige der zwischen 1990 und 1995 eingeführten Veränderungen, die im Prinzip nur kosmetische Korrekturen der ursprünglichen Regelungen darstellten. Diese Übergangsregelungn werden für die Neuen Bundesländer nur insofern berücksichtigt, als sie eine grundsätzliche Verteilung

\footnotetext{
${ }^{15} \mathrm{Im}$ einzelnen steht $S H$ für Schleswig-Holstein, $H H$ für Hamburg, $H B$ für Bremen, $N S$ für Niedersachsen, $N W$ für Nordrhein-Westfalen, $H E$ für Hessen, $R P$ für Rheinland-Pfalz, $S A$ für das Saarland, $B W$ für Baden-Württemberg, $B Y$ für Bayern und $O L$ für die Ostländer.

${ }^{16}$ Die formale Präsentation der Regelungen lehnt sich sehr eng an die von GotTFRIEd/WIEg ARD [1992] vorgestellte Formalisierung an, der im Prinzip nichts hinzuzufügen ist.
} 
zwischen Ost- und Westländern vorsehen. Demnach ist zwar die nach Bevölkerungsanteilen vorgenommene Aufteilung des Umsatzsteueranteils der Bundesländer auf einen Westund Ostteil in der nachfolgenden Darstellung enthalten, nicht jedoch die Aufschlüsselung dieses Anteils auf die einzelnen Ostländer. Da die Ostländer $(O L)$ im Vergleich zu den alten Ländern eine relativ homogene Finanzsituation besitzen, werden sie im weiteren Gang der Untersuchung als ein aggregiertes Land betrachtet. Dies ermöglicht es auch, auf die Besonderheiten der Datenlage Rücksicht zu nehmen. ${ }^{17}$

\subsubsection{Die Verteilung der Gewerbesteuer}

Seit der Finanzreform von 1969 müssen die Gemeinden einen Teil des Steueraufkommens der Gewerbesteuer auf Ertrag und Kapital $S T_{s}^{G w}$, in Form einer Umlage $S T^{U m}$, an Bund und Länder abführen. $\mathrm{Zu}$ deren Berechnung werden die Gewerbesteueraufkommen der Gemeinden durch den jeweiligen Gewerbesteuerhebesatz dividiert. Der dadurch gebildete Steuermeßbetrag $\widehat{S T}_{s}^{G w}$ wird anschließend mit dem Vervielfältiger $h^{U m}$ multipliziert (siehe ausführlich HaverKamp [1988], S.68-69). Auf diese Weise werden Unterschiede in den Hebesätzen nivelliert. Dies führt zu folgender Formel für die Gewerbesteuerumlage:1819

$$
S T_{s}^{U m}=\frac{S T_{s}^{G w}}{h_{s}^{G w}} h^{U m}=h^{U m} \widehat{S T}_{s}^{G w}
$$

Von der Summe der Gewerbesteuerumlagen, $S T_{\mathcal{R}}^{U m}=\sum_{s \in \mathcal{R}} S T_{s}^{U m}$, erhalten Bund und Länder jeweils die Hälfte ( $§ 106$ Abs. 6 Satz 3 und 4 GG). Bezeichnet man den Anteilssatz der Länder ganz allgemein mit $\pi_{L d}^{U m}$, so erhält man als Länderanteil an der Gewerbesteuerumlage

$$
T_{L d}^{U m}=\pi_{L d}^{U m} S T_{\mathcal{R}}^{U m}
$$

Dieser wird gemäß der Anteile der Gewerbesteueraufkommen auf die Länder verteilt (§3 FAG). Die Einnahmen eines Landes $s$ aus der Gewerbesteuerumlage sind dann

$$
T_{s}^{U m}=\frac{S T_{s}^{G w}}{S T_{\mathcal{R}}^{G w}} T_{L d}^{U m}
$$

$\operatorname{mit} S T_{\mathcal{S}}^{G w}=\sum_{r \in \mathcal{R}} S T_{r}^{G w}$.

\footnotetext{
${ }^{17}$ Eine genaue, formalisierte Darstellung der Regelungen für die Ostländer findet sich bei GoTTFRIED/WIEGARD [1992].

${ }^{18}$ Soweit nicht anders angegeben, beziehen sich im weiteren alle Formeln mit regionalem Index auf alle Länder $s \in \mathcal{R}$.

${ }^{19} h_{s}^{G w}$ ist hier der durchschnittliche Hebesatz der Gemeinden eines Landes. In der gesetzlichen Regelung werden jeweils die kommunalen Hebesätze verwendet.
} 
Dieser Umlageanteil des jeweiligen Landes addiert mit den Einnahmen der Gemeinden des Landes aus der Gewerbesteuer

$$
T_{G m, s}^{G w}=S T_{s}^{G w}-S T_{s}^{U m}=\left(h_{s}^{G w}-h^{U m}\right) \widehat{S T}_{s}^{G w} .
$$

ergibt die gesamten regionalen Gewerbesteuereinnahmen nach der Gewerbesteuerverteilung

$$
T_{s}^{G w}=\left[h_{s}^{G w}\left(1+\pi_{L d}^{U m} \frac{S T_{\mathcal{R}}^{U m}}{S T_{\mathcal{R}}^{G w}}\right)-h^{U m}\right] \widehat{S T}_{s}^{G w} .
$$

\subsubsection{Verteilung der Einkommenssteuern}

In einem ersten Schritt werden die Aufkommen aus den Einkommenssteuern ${ }^{20}$ gemäß dem Prinzip der Steuerzerlegung auf die Länder und Gemeinden zugerechnet. ${ }^{21}$ Dies wird hier nicht näher spezifiziert. Es werden vielmehr die nach der Steuerzerlegung ermittelten Aufkommen berücksichtigt.

Als zweites erfolgt die Verteilung der Gemeindeanteile an den Lohn- und veranlagten Einkommenssteuern auf die Gemeinden nach dem Prinzip des örtlichen Aufkommens

$$
T_{G m, s}^{Y}=\pi_{G m}^{E k} S T_{s}^{E k}
$$

wobei der gemeindliche Anteilssatz an den Lohn- und veranlagten Einkommenssteuern $15 \%\left(\pi_{G m}^{E k}\right)$ beträgt (StÄndG 1979).

Vom verbleibenden Einkommenssteueraufkommen erhalten der Bund und die die Länder jeweils $50 \%\left(\pi_{L d}^{Y}\right)^{22}$ Als Einkommenssteueranteile der Länder erhält man damit

$$
T_{L d}^{Y}=\pi_{L d}^{Y}\left[S T_{\mathcal{R}}^{E k}\left(1-\pi_{G m}^{E k}\right)+S T_{\mathcal{R}}^{K}\right],
$$

\footnotetext{
${ }^{20}$ Obwohl die Körperschafts- und die Kapitalertragssteuer im Modell als Faktorsteuer modelliert werden, werden sie hinsichtlich der Finanzausgleichsregelungen als Einkommenssteuer behandelt.

${ }^{21}$ Die Lohnsteueraufkommen werden dabei den Ländern zugerechnet, in denen der jeweilige Lohnsteuerschuldner seinen Wohnsitz besitzt (Wohnsitzprinzip). Da die Lohnsteuer jedoch in der Regel vom Arbeitgeber abgeführt wird und Firmensitz und Wohnsitz voneinander abweichen können, ist in diesen Fällen eine Korrektur zwischen den Ländern vorgesehen. Ähnliches gilt für die Zurechnung der Aufkommen aus den Körperschaftssteuern, die nach dem Betriebsstättenprinzip den Länder zugerechnet werden soll. Die entsprechenden Einzelheiten der Lohnsteuer- und Körperschaftssteuerzerlegung werden im Zerlegungsgesetz geregelt.

Zur Vereinfachung der weiteren Darstellung wird hier auf die explizite Darstellung der Zerlegung verzichtet. Dies entspricht dem Vorgehen in der später durchgeführten EAG-Analyse. Die genaue Form der Einkommenssteuerverteilung unter Berücksichtigung der Zerlegung formalisieren GoTTFRIED/WIEGARD ([1992], S.137-138).

${ }^{22}$ D.h., vom Aufkommen der Lohn- und veranlagten Einkommenssteuer erhalten Bund und Länder jeweils 42,5\% des gesamten Aufkommens und vom Aufkommen der Kapitalertrags- und Körperschaftssteuer je $50 \%$.
} 
mit $S T_{\mathcal{R}}^{E k}$ als Aufkommen aller Länder aus der Lohn- und veranlagten Einkommens-, bzw. $S T_{\mathcal{R}}^{K}$ als Aufkommen aus der Kapitalertrags- und Körperschaftssteuer.

Dieser Betrag wird, nach erfolgter Korrektur durch die Steuerzerlegung, gemäß dem Prinzip des örtlichen Aufkommens auf die einzelnen Länder verteilt (Artikel 107, Absatz 1, Seite 1, GG). Die Verteilung der Einkommenssteueranteile der Gemeinden, $T_{G m}^{Y}$, auf die einzelnen Länder erfolgt ebenfalls nach dem Prinzip des örtlichen Aufkommens.

Die regionalen Einnahmen aus allen Einkommenssteuerarten nach der Steuerverteilung berechnen sich dann gemäß:

$$
T_{s}^{Y}=\underbrace{\pi_{L d}^{Y}\left[S T_{s}^{E k}\left(1-\pi_{G m}^{E k}\right)+S T_{s}^{K}\right]}_{T_{L d, s}^{Y}}+\underbrace{\pi_{G m}^{E k} S T_{s}^{E k}}_{T_{G m, s}^{Y}},
$$

und setzen sich aus den Steuereinnahmen des Landes $T_{L d, s}^{Y}$ und den Einnahmen der Gemeinden $T_{G m, s}^{Y}$ aus allen Einkommenssteuerarten zusammen.

\subsubsection{Umsatzsteuerverteilung}

Das Verfahren zur Verteilung der Umsatzsteuer ist deutlich komplizierter als die anderen Steuerverteilungsregelungen, da es seit der Finanzverfassungsreform von 1969 auch als Instrument zum horizontalen Finanzausgleich eingesetzt wird.

Der Anteilssatz $\pi_{L d}^{C}$ der Bundesländer zur Berechnung des Länderanteils an allen Umsatzsteuereinnahmen $\left(S T_{\mathcal{R}}^{C}\right)$

$$
\widehat{T}_{L d}^{C}=\pi_{L d}^{C} S T_{\mathcal{R}}^{C}
$$

wurde seit 1978 von $67,5 \%$ auf $63 \%$ (1994) verringert.

Die Aufteilung dieses Länderanteils erfolgt in einem mehrstufigen Prozeß. Als erstes wird der Anteil der Neuen Länder bestimmt (1), anschließend eine Vorabverteilung nach den Bevölkerungsverhältnissen vorgenommen (2), dann die steuerschwachen Länder mit Ergänzungsanteilen finanziell bessergestellt (3) und schließlich der verbleibenden Rest auf die steuerstarken Länder verteilt (4).

\section{(1) Anteil der Neuen Länder}

Zu Beginn der Umsatzsteuerverteilung wird der Länderanteil an der Umsatzsteuer gemäß der relativen Bevölkerungsstärke der Länder in eine West- und Ostteil aufgeteilt (§ 2 Abs.4 FAG). Als Umsatzsteueranteil der Ostländer erhält man damit

$$
T_{O L}^{C}=\frac{E W_{O L}}{E W_{\mathcal{R}}} \widehat{T}_{L d}^{C}=\kappa_{\mathcal{R}}^{O L} \pi_{L d}^{C} S T_{\mathcal{R}}^{C}
$$


mit $E W_{r}$ als Bevölkerungszahl der jeweiligen Länder und $\kappa_{\mathcal{R}}^{O L}$ als prozentualer Anteil der Bevölkerung der Ostländer an der Summe der Einwohnerzahl aller Länder $\left(E W_{\mathcal{R}}\right)$.

Alle weiteren Regelungen der Umsatzsteuerverteilung und auch des Länderfinanzausgleichs schließen die Ostländer aus. Zur Vereinfachung wird daher eine neue Menge $\mathcal{S}$ der Länder definiert, die alle Westländer umfaßt:

$$
\mathcal{S}=\{S H, H H, H B, N S, N W, H E, R P, S A, B W, B Y\} .
$$

\section{(2) Vorabverteilung nach Bevölkerung}

Vom übriggebliebenen Länderanteil an der Umsatzsteuer,

$$
T_{L d}^{C}=\widehat{T}_{L d}^{C}-T_{O L}^{C}=\left(1-\kappa_{\mathcal{R}}^{O L}\right) \pi_{L d}^{C} S T_{\mathcal{R}}^{C},
$$

werden $75 \%\left(\pi_{V A}^{C}\right)$ nach der Einwohnerzahl auf die Länder aufgeteilt

$$
V A_{s}^{C}=\frac{E W_{s}}{E W_{\mathcal{S}}} \pi_{V A}^{C} T_{L d}^{C}=\kappa_{\mathcal{S}}^{s} \pi_{V A}^{C} T_{L d}^{C} .
$$

$\kappa_{\mathcal{S}}^{s}$ ist dabei der prozentuale Bevölkerungsanteil des Landes $s$ an der Bevölkerung der Westländer $\left(E W_{\mathcal{S}}\right)$. Die verbleibenden $25 \%$ des Länderanteils an der Umsatzsteuer werden als Ergänzungsanteile (Vorwegausgleich) an die finanzschwachen Länder und als Restverteilung an die finanzstarken Länder ausgeschüttet.

\section{(3) Vorwegausgleich bzw. Vorwegauffüllung}

Die als Maßgröße verwendeten Pro-Kopf-Steuereinnahmen eines Landes setzen sich aus den Ländereinnahmen aus allen Einkommenssteuern $\left(T_{L d, s}^{Y}\right)$, den ländereigenen Steuern $\left(T_{s}^{R e g}\right)$ und der Gewerbesteuerumlage zusammen und belaufen sich auf

$$
t_{L d, s}^{1}=\frac{1}{E W_{s}}\left(T_{L d, s}^{Y}+T_{s}^{R e g}+T_{s}^{U m}\right)=\frac{1}{E W_{s}} T_{L d, s}^{1} .
$$

Das durchschnittliche Pro-Kopf-Steuereinkommen der Länder $\bar{t}_{L d}^{1}$ ist dann

$$
\bar{t}_{L d}^{1}=\frac{\sum^{r \in \mathcal{S}}\left(T_{L d, s}^{Y}+T_{s}^{R e g}+T_{s}^{U m}\right)}{E W_{\mathcal{S}}}=\frac{T_{L d, \mathcal{S}}^{1}}{E W_{\mathcal{S}}} .
$$

Vor der Ermittlung der weiteren Komponenten der Umsatzsteuerverteilung werden, einer Idee von GotTFRIED/WiEgard ([1992], S.139) folgend, die Länder in zwei Teilmengen zerlegt. Die erste Teilmenge $\mathcal{S}^{-}$erfaßt die Länder mit unterdurchschnittlichen Pro-KopfSteuereinnahmen und die zweite Teilmenge $\mathcal{S}^{+}$die restlichen, finanzstarken Länder:

$$
\mathcal{S}^{-}=\left\{s \mid t_{L d, s}^{1}<\bar{t}_{L d}^{1}, s \in \mathcal{S}\right\} \quad \mathcal{S}^{+}=\left\{s \mid t_{L d, s}^{1} \geq \bar{t}_{L d}^{1}, s \in \mathcal{S}\right\} .
$$


Die Pro-Kopf-Steuereinnahmen der finanzschwachen Länder werden mit Hilfe von Ergänzungsanteilen auf derzeit $92 \%\left(\pi_{E A}^{C}\right)$ der durchschnittlichen Pro-Kopf-Steuereinnahmen angehoben ( $\S 2$ Abs. 2 Satz 1 FAG). Die Ergänzungsanteile $E A_{s}^{C, 0}$ sollen dabei mindestens dem Anteil entsprechen, der den finanzschwachen Länder bei einer Verteilung nach Bevölkerungsrelationen zustünde $\left(E A_{s}^{E W}\right)$. Dies führt zu folgender Gleichung:

$$
E A_{s}^{C, 0}=\max \left[E A_{s}^{E W} ; E A_{s}^{M A}\right]
$$

mit

$$
E A_{s}^{E W}=\frac{E W_{s}}{E W_{\mathcal{S}}}\left(1-\pi_{V A}^{C}\right) T_{L d}^{C}=\kappa_{\mathcal{S}}^{s}\left(1-\pi_{V A}^{C}\right) T_{L d}^{C} \quad \forall s \in \mathcal{S}^{-}
$$

als garantierte Mindestergänzungsanteile und

$$
E A_{s}^{M A}=\max \left[0 ; E W_{s}\left(\pi_{E A}^{C} \bar{t}_{L d}^{1}-t_{L d, s}^{1}\right)\right] \quad \forall s \in \mathcal{S}^{-}
$$

als Mindestauffüllquote, die nur ungleich Null ist, wenn das finanzschwache Land weniger als $\pi_{E A}^{C}$, derzeit $92 \%$, der durchschnittlichen Steuereinnahmen der Länder erreicht.

Definiert man für die finanzschwachen Länder die Menge $\mathcal{S}_{-}^{-}\left(\mathcal{S}_{+}^{-}\right)$der Länder, deren Mindestergänzungsanteile $E A_{s}^{E W}$ kleiner (größer gleich) als die Mindestauffüllquote $E A_{s}^{M A}$ sind

$$
\mathcal{S}_{-}^{-}=\left\{s \mid E A_{s}^{E W}<E A_{s}^{M A}, s \in \mathcal{S}^{-}\right\} \quad \mathcal{S}_{+}^{-}=\left\{s \mid E A_{s}^{E W} \geq E A_{s}^{M A}, s \in \mathcal{S}^{-}\right\},
$$

so vereinfacht sich die weitere Darstellung.

Falls nun die Summe der Ergänzungsanteile insgesamt mehr als $\left(1-\pi_{V A}^{C}\right)$, derzeit $25 \%$, des Länderanteils an den Umsatzsteuern ausmacht, werden die Ergänzungsanteile, "die den Mindestanteil nach der Einwohnerzahl übersteigen" $\left(E A_{s}^{M A}-E A_{s}^{E W} \geq 0\right)$, proportional gekürzt (§ 2 Abs. 2 und 3 FAG), und man erhält, analog zu GoTTFrIED/WiEGARD ([1992], S. 140), als Formel für die gesamten Ergänzungsanteile

$$
E A_{s}^{C}= \begin{cases}E A_{s}^{E W} & \text { für } s \in \mathcal{S}_{+}^{-} \\ E A_{s}^{M A} & \text { für } s \in \mathcal{S}_{-}^{-} \text {und } \sum_{r \in \mathcal{S}} E A_{r}^{C, 0} \leq\left(1-\pi_{V A}^{C}\right) T_{L d}^{C} \\ E A_{s}^{E W}+\kappa_{p}^{C}\left(E A_{s}^{M A}-E A_{s}^{E W}\right) & \text { für } s \in \mathcal{S}_{-}^{-} \text {und } \sum_{r \in \mathcal{S}} E A_{r}^{C, 0}>\left(1-\pi_{V A}^{C}\right) T_{L d}^{C}\end{cases}
$$

mit dem proportionalen Kürzungsfaktor $\kappa_{p}^{C}$. Dieser entspricht dem Verhältnis des über den Pflichtanteil nach Bevölkerung hinausgehenden Verteilungsspielraums zum zusätzlich, 
aufgrund der $92 \%$ Klausel, über diesen Anteil hinausgehenden Bedarf an Finanzmitteln

$$
\kappa_{p}^{C}=\frac{\left(1-\pi_{V A}^{C}\right) T_{L d}^{C}-\sum^{r \in \mathcal{S}^{-}} E A_{r}^{E W}}{\sum_{r \in \mathcal{S}_{-}^{-}}\left(E A_{r}^{M A}-E A_{r}^{E W}\right)}=\frac{\kappa_{\mathcal{S}}^{\mathcal{S}^{+}}\left(1-\pi_{V A}^{C}\right) T_{L d}^{C}}{\sum_{r \in \mathcal{S}_{-}^{-}}\left(E A_{r}^{M A}-E A_{r}^{E W}\right)} \leq 1 .
$$

Dabei ist $\kappa_{\mathcal{S}}^{\mathcal{S}^{+}}$der prozentuale Anteil der Bevölkerung der finanzstarken Länder $\left(s \in \mathcal{S}^{+}\right)$ an der Bevölkerung aller Westländern.

(4) Verteilung der Restbeträge $(R V)$ an $s \in \mathcal{S}^{+}$

Die nach Abzug der Vorabverteilung und der Ergänzungsanteile vom Länderanteil verbleibenden Umsatzsteueraufkommen $\left(\left(1-\pi_{V A}^{C}\right) T_{L d}^{C}-\sum_{r \in S} E A_{r}^{C}\right)$ werden, vorausgesetzt sie sind größer Null, gemäß ihrer Einwohnerzahl auf die Länder mit überdurchschnittlichen Pro-Kopf-Steuereinnahmen verteilt ( $§ 2$ Abs. 3 FAG). Die entsprechenden Restverteilungsbeträge sind

$$
R E_{s}^{C}=\kappa_{\mathcal{S}^{+}}^{s}\left[\left(1-\pi_{V A}^{C}\right) T_{L d}^{C}-\sum_{r \in \mathcal{S}} E A_{\tau}^{C}\right] \quad \text { für } s \in \mathcal{S}^{+},
$$

mit $\kappa_{\mathcal{S}^{+}}^{s}=E W_{s} /\left(\sum_{\tau \in \mathcal{S}^{+}} E W_{r}\right)$.

Nun werden die finanzstarken Länder eingeteilt in die Länder, deren Pro-Kopf-Steuereinnahmen, unter Berücksichtigung der Umsatzsteueranteile

$$
t_{L d, s}^{2}=\frac{1}{E W_{s}}\left(T_{L d, s}^{1}+R E_{s}^{C}+V A_{s}^{C}\right)=\frac{1}{E W_{s}} T_{L d, s}^{2} \quad \forall s \in \mathcal{S}^{+},
$$

die durchschnittlichen Pro-Kopf-Steuereinnahmen aller Westländer

$$
\bar{t}_{L d}^{2}=\sum_{r \in \mathcal{S}} T_{L d, r}^{2} / E W_{\mathcal{S}}
$$

nicht erreichen $\left(\mathcal{S}_{-}^{+}\right)$, und die Länder, deren Steuereinnahmen diesen Durchschnitt nicht unterschreiten $\left(S_{+}^{+}\right)$(vgl. GotTFRIEd/WiEgaRd [1992], S.141)

$$
\mathcal{S}_{-}^{+}=\left\{s \mid t_{L d, s}^{2}<\bar{t}_{L d}^{2}, s \in \mathcal{S}^{+}\right\} \quad \mathcal{S}_{+}^{+}=\left\{s \mid t_{L d, s}^{2} \geq \bar{t}_{L d}^{2}, s \in \mathcal{S}^{+}\right\} .
$$

Erreicht, nach der Berechnung der Ergänzungsanteile und der bevölkerungsgemäßen Aufteilung der restlichen Umsatzsteueraufkommen, ein finanzstarkes Land mit seinen Steuereinnahmen je Einwohner - inclusive des Umsatzsteueranteils $\left(V A_{s}^{C}+R E_{s}^{C}\right)$ - nicht den Durchschnitt, der die Umsatzsteueranteile einschließenden Steuereinnahmen der Länder, wird sein Umsatzsteueranteil entsprechend erhöht (Garantieklausel)

$$
R G_{s}^{C}=E W_{s}\left(\bar{t}_{L d}^{2}-t_{L d, s}^{2}\right) \quad \text { für } s \in \mathcal{S}_{-}^{+} .
$$


Finanziert wird diese Erhöhung durch eine Verringerung des Anteils der anderen finanzstarken Länder an der Verteilung der Restbeträge (vgl. GotTfried, WiEgARD [1992], S. 141). Dies ergibt eine neue Aufteilung der Restverteilung in

$$
R V_{s}^{C}= \begin{cases}R E_{s}^{C}+R G_{s}^{C} & \text { für } s \in \mathcal{S}_{-}^{+} \\ R E_{s}^{C}\left(\frac{\sum_{+}^{r \in \mathcal{S}_{+}^{+}} R E_{r}^{C}-\sum_{r \in \mathcal{S}_{+}^{+}}^{r \in \mathcal{S}_{-}^{+}} R G_{r}^{C}}{\sum_{r}^{C} R E_{r}^{C}}\right) & \text { für } s \in \mathcal{S}_{+}^{+} .\end{cases}
$$

Reichen allerdings diese Beträge nicht aus, so erfolgt eine prozentuale Korrektur von $R G_{s}^{C}$, die im FAG nicht präzisiert ist ( $§ 2$ Abs. 3 FAG), aber z.B. so dargestellt werden könnte:

$$
R V_{s}^{C}=R E_{s}^{C}+R G_{s}^{C} \frac{\sum_{r \in \mathcal{S}_{+}^{+}} R E_{r}^{C}}{\sum_{r \in \mathcal{S}_{-}^{+}} R G_{\tau}^{C}} \quad \text { für } s \in \mathcal{S}_{-}^{+} .
$$

Dies stellt im Prinzip eine Garantieklausel für die finanzstarken Länder dar, die allerdings wie MiChaLK ([1989], S. 446-448) zeigt, keineswegs garantiert, daß alle ursprünglich finanzstarken Länder mit ihren Steuereinnahmen die länderdurchschnittlichen Einnahmen erzielen. ${ }^{23}$

Insgesamt erhält man als Umsatzsteuereinnahmen der Länder

$$
T_{s}^{C}= \begin{cases}V A_{s}^{C}+E A_{s}^{C} & \forall s \in \mathcal{S}^{-} \\ V A_{s}^{C}+R V_{s}^{C} & \forall s \in \mathcal{S}^{+} \\ \kappa_{\mathcal{R}}^{O L} \widehat{T}_{L d}^{C} & \text { für } s=O L\end{cases}
$$

\subsubsection{Länderfinanzausgleich}

Der horizontale Länderfinanzausgleich soll die unterschiedliche Finanzkraft der Bundesländer in etwa ausgleichen ( $§ 107$ Abs.2 GG), und wird durch finanzielle Zuweisungen der finanzstarken an die finanzschwachen Bundesländer durchgeführt. Allerdings werden die

\footnotetext{
${ }^{23}$ GotTFRIED/WIEgARD ([1992], S. 141) weisen deshalb auch darauf hin, daß in solchen Fällen bei der konkreten Berechnung eine weitere Korrektur erfolgt. Die unter den Durchschnitt gesunkenen ursprüglich steuerstarken Länder (aus $\mathcal{S}_{+}^{+}$) erhalten zusätzliche Anteile an der Umsatzsteuer, die von den anderen über dem Durchschnitt liegenden Ländern aus $\mathcal{S}_{+}^{+}$proportional aufgebracht werden. Dies wird hier jedoch nicht modelliert.
} 
Neuen Länder nach einer Vereinbarung des Einigungsvertrages erst zum 1.Januar 1995 am gemeinsamen Finanzausgleich beteiligt. Bis dahin wird für sie ein eigener Länderfinanzausgleich durchgeführt. Dieser wird im folgenden allerdings nicht dargestellt, verläuft jedoch im Prinzip analog zum LFA unter den Westländern.

Geregelt ist der horizontale Finanzausgleich zwischen den Bundesländern im Finanzausgleichsgesetz (FAG). Beiträge im Länderfinanzausgleich müssen alle die Länder leisten, „deren Steuerkraftmeßzahl in dem Rechnungsjahr (...) ihre Ausgleichsmeßzahl übersteigt" und Zuweisungen erhalten "die Länder, deren Steuerkraftmeßzahl im Ausgleichsjahr ihre Ausgleichsmeßzahl nicht erreicht"(§ 5 FAG 1969) ${ }^{24}$.

Die regionale Finanzkraftmeßzahl $M F_{s}$ setzt sich aus der Finanzkraftmeßzahl der Länder $M F_{L d, s}$ und der nur zu $50 \%\left(\pi_{G m}^{F A}\right)$ anzurechnenden Finanzkraftmeßzahl der Gemeinden $M F_{G m, s}$ zusammen:

$$
M F_{s}=M F_{L d, s}+\pi_{G m}^{F A} M F_{G m, s} .
$$

Die Finanzkraftmeßzahl der Länder entspricht den Einnahmen aus den Einkommenssteuern (incl. Körperschaftssteuern), den Umsatzsteuern, der Gewerbesteuerumlage und den anrechenbaren Ländersteuern ${ }^{25}\left(T_{s}^{\text {Reg }}\right)$. Für "Sonderbelastungen" durch den Unterhalt der Seehäfen, werden von den Steuereinnahmen der Länder Bremen, Hamburg und Niedersachsen im FAG festgelegte Beträge als Sonderlasten $S B_{s}$ abgezogen ( $₹ 7$ Abs. 3 FAG). Daraus ergib sich als Finanzkraftmeßzahl $M F_{L d, s}$ der Länder

$$
M F_{L d, s}=T_{L d, s}^{F}-S B_{s}=T_{L d, s}^{1}+T_{s}^{C}-S B_{s}=T_{L d, s}^{F A} .
$$

Die Finanzkraftmeßzahl der Gemeinden besteht aus den Einkommenssteuereinnahmen, den Steuerkraftzahlen der Grundsteuern ${ }^{26} F T_{s}^{G r}$ und der Gewerbesteuer $F T_{s}^{G w}$, beide aus dem Vorjahr, die um die im Ausgleichsjahr abgeführte Gewerbesteuerumlage $S T_{s}^{U m}$ vermindert sind ( $\S 8$ Abs. 1 FAG):

$$
M F_{G m, s}=T_{G m, s}^{E k}+F T_{s}^{G w}+F T_{s}^{G r}-S T_{s}^{U m}
$$

Die Steuerkraftzahl der Gewerbesteuer wird durch eine $250 \%$ ige Anrechnung der Grundbeträge der Gewerbesteuer vom Ertrag und Kapital, $\widehat{S T}_{s}^{G w}$, ermittelt ( 88 ABs. 2, Satz 3 FAG vom 1.11.1969).

\footnotetext{
${ }^{24}$ Die Jahreszahl gibt das Jahr an, in dem das jeweilige Gesetz im Bundesgesetzblatt veröffentlicht wurde.

${ }^{25}$ Diese bestehen aus der Vermögens-, der Erbschafts-, der KFZ-, der Bier-, der Rennwett- und Lotteriesteuer ohne der Totalisatorsteuer, der Grunderwerb-, der Feuerschutzsteuer und der Spielbankabgabe ohne der Sonderabgabe und der Troncabgabe sowie den Einnahmen aus der bergrechtlichen Förderabgabe des Landes ( 6 Abs. 1 FAG 1987).

${ }^{26}$ Diese sind im Vorgriff auf das EAG-Modell zu einer Größe zusammengefaßt.
} 
Die Steuerkraftzahl der Grundsteuern wird nach einem ähnlichen Verfahren bestimmt. Zur Vereinfachung wird hier die gesamte Grundsteuer mit dem länderdurchschnittlichen Hebesatz $h_{s}^{G r}$ dividiert, und der dadurch gewonnene Grundbetrag $\widehat{S T}_{s}^{G r}$ mit einem Gewichtungssatz von $250\left(\pi_{h}^{G \tau}\right)$ multipliziert. ${ }^{27}$ Dies führt zu

$$
\begin{gathered}
F T_{s}^{G w}=\frac{S T_{s}^{G w}}{h_{s}^{G w}} \pi_{h}^{G w}=\pi_{h}^{G w} \widehat{S T}_{s}^{G w} \\
F T_{s}^{G r}=\frac{S T_{s}^{G r}}{h_{s}^{G r}} \pi_{h}^{G r}=\pi_{h}^{G r} \widehat{S T}_{s}^{G r} .
\end{gathered}
$$

Bei der Ermittlung der Einwohnerzahlen der Länder werden die Einwohnerzahlen von Bremen und Hamburg statt mit $100 \%$ mit $135 \%$ gewichtet ( 9 FAG). Die Bevölkerungsgrößen im Finanzausgleich sind daher

$$
E W_{L d, s}^{F A}=\left(1+\pi_{L d, s}^{E W}\right) E W_{s},
$$

mit $\pi_{L d, s}^{E W}$ als Veredelungsfaktor der Einwohnerzahlen.

Die Einwohnerzahlen der Gemeinden werden je nach Gemeindegröße unterschiedlich gewichtet. Je größer die Gemeinde ist, um so höher ist der Prozentsatz. Eine detaillierte Aufschlüsselung wird hier allerdings nicht vorgenommen. Vielmehr wird ein durchschnittlicher, regionaler Veredelungsfaktor $\left(\pi_{L d, s}^{E W}\right)$ unterstellt, so daß sich die finanzausgleichsrelevanten Einwohnerzahlen der Gemeinden als

$$
E W_{G m, s}^{F A}=\left(1+\pi_{G m, s}^{E W}\right) E W_{s}
$$

schreiben lassen.

Zur Ermittlung der relativen Finanzstärke der Regionen wird deren Finanzkraftmeßzahl $M F_{s}$ mit der Ausgleichsmeßzahl $M A_{s}$ verglichen. Die Ausgleichsmeßzahl eines Landes (Region) ist die Summe der Steuereinnahmen, die ein Land hätte, wenn es bundesdurchschnittliche Pro-Kopf-Steuereinnahmen hätte; also im Prinzip eine Art Maß für die durchschnittliche Pro-Kopf-Finanzkraft der Länder. Die regionale Finanzkraftmeßzahl

$$
M A_{s}=M A_{L d, s}+\pi_{G m}^{F A} M A_{G m, s}
$$

ergibt sich aus der Summe der getrennt berechneten Ausgleichsmeßzahl des Landes

$$
M A_{L d, s}=E W_{L d, s}^{F A} \frac{\sum_{r \in \mathcal{S}}^{r \in \mathcal{S}} T_{L d, r}^{F A}}{\sum_{L d, r} E W_{L A, F}^{F A}}=\kappa_{\mathcal{S}, F A}^{L d, s} T_{L d, \mathcal{S}}^{F A}
$$

\footnotetext{
${ }^{27}$ Der Gewichtungssatz der Grundsteuer A ist nach § 8 Abs. 2 Satz 1 FAG vom 1.11.1969 180. Die gesetzlichen Vorschriften für die Grundsteuer B sind relativ kompliziert. Deren Grundbeträge werden je nach Höhe der Einnahmen der Gemeinden unterschiedlich gewichtet.
} 
mit $\kappa_{\mathcal{S}, F A}^{L d, s}$ als veredelter Bevölkerungsanteil des Landes an der veredelten Bevölkerung aller Länder, und der zu $50 \%\left(\pi_{G m}^{F A}\right)$ angerechneten Ausgleichsmeßzahl der Gemeinden

$$
M A_{G m, s}=E W_{G m, s}^{F A} \frac{\sum_{r \in \mathcal{S}}^{r \in \mathcal{S}} T_{G m, r}^{F A}}{\sum_{G m, r} E W_{G, F A}^{F A}}=\kappa_{G m, \mathcal{S}}^{G m, s} .
$$

Zur Vereinfachung werden nun die Länder in zwei Teilmengen aufzuspalten, deren erste, $\mathcal{S}^{-}$, die finanzschwachen und somit ausgleichsberechtigten und deren zweite Teilmenge, $\mathcal{S}^{+}$, die finanzstarken, d.h. ausgleichspflichtigen Länder umfaßt (GoTTFRIED und WIEGARD [1992], S. 144)):

$$
\mathcal{S}^{-}=\left\{s \mid M F_{s} \leq M A_{s}, s \in \mathcal{S}\right\} \quad \mathcal{S}^{+}=\left\{s \mid M F_{s}>M A_{s}, s \in \mathcal{S}\right\} .
$$

\section{(1) Berechnung der Finanzausgleichszuweisungen}

Die finanzschwachen Länder erhalten nach $\S 10$ Abs. 1 FAG die volle Summe des ihrer Finanzkraft $\left(M F_{s}\right)$ zum Erreichen von $92 \%\left(\pi^{M}\right)$ ihrer Ausgleichsmeßzahl $\left(M A_{s}\right)$ fehlenden Betrages. Liegt ihre Steuerkraft zwischen $92 \%$ und $100 \%$ ihrer Ausgleichsmeßzahl, dann erhalten sie nur 37,5\% $\left(\pi^{N}\right)$ des Fehlbetrages. Die Summe dieser Zuweisungen ist

$$
F A Z_{s}= \begin{cases}\left(\pi^{M} M A_{s}-M F_{s}\right)+\pi^{N}\left(1-\pi^{M}\right) M A_{s} & \\ =-M F_{s}+\left[\pi^{M}+\pi^{N}\left(1-\pi^{M}\right)\right] M A_{s} & \text { für } s \in \mathcal{S}_{-}^{-}, \\ \pi^{N}\left(M A_{s}-M F_{s}\right) & \text { für } s \in \mathcal{S}_{+}^{-}\end{cases}
$$

mit der Einteilung der Länder aus $\mathcal{S}^{-}$in die zwei disjunkten Teilmengen

$$
\begin{gathered}
\mathcal{S}_{-}^{-}=\left\{s \mid M F_{s}<\pi^{M} M A_{s}, s \in \mathcal{S}^{-}\right\} \\
\mathcal{S}_{+}^{-}=\left\{s \mid \pi^{M} M A_{s} \leq M F_{s} \leq M A_{s}, s \in \mathcal{S}^{-}\right\} .
\end{gathered}
$$

Der Einfachheit halber wird an dieser Stelle die Garantieklausel für die finanzschwachen Länder berücksichtigt, obwohl sie erst nach den Formeln (I.25) bis (I.26) vorgesehen ist. Dieses Vorgehen ist jedoch völlig äquivalent zu einer gesetztestreueren Darstellung, wie sie GotTfried, Wiegard ([1992], S.149) wählen.

Falls die Zahlungen nicht ausreichen, um für ein finanzschwaches Land einen Anteil von mindestens $95 \%\left(\pi^{S}\right)$ an der durchschnittlichen Steuer- bzw. Finanzkraft, ohne Berücksichtigung der Gemeindesteueranteile, zu erreichen, erhält es zusätzliche Zuweisungen $F Z Z_{\text {s }}$ von den ausgleichspflichtigen Ländern (§ 10 Abs. 3 FAG). 
Diese weiteren Zuweisungen $F Z Z$, berechnen sich aus der Differenz zwischen der durchschnittlichen Pro-Kopf-Steuerkraft der Länder, bezogen auf die tatsächlichen Bevölkerungszahlen ${ }^{28}$, und der Pro-Kopf-Finanzkraft, bezogen auf die tatsächliche Bevölkerungszahl des Landes nach Erhalt der LFA-Zuweisungen. Spaltet man die Menge der finanzschwachen Länder $\mathcal{S}^{-}$in zwei Teilmengen $\mathcal{S}^{\mp}$ und $\mathcal{S}^{=}$

$$
\begin{gathered}
\mathcal{S}^{\mp}=\left\{s \mid \pi^{S} \kappa_{\mathcal{S}}^{s} T_{L d, \mathcal{S}}^{F}-\left(T_{L d, s}^{F}+F A Z_{s}\right) \leq 0, s \in \mathcal{S}^{-}\right\} \\
\mathcal{S}^{=}=\left\{s \mid \pi^{S} \kappa_{\mathcal{S}}^{s} T_{L d, \mathcal{S}}^{F}-\left(T_{L d, \mathcal{S}}^{F}+F A Z_{s}\right)>0, s \in \mathcal{S}^{-}\right\}
\end{gathered}
$$

auf, so erhält man für $F Z Z_{\text {s }}$

$$
F Z Z_{s}= \begin{cases}\pi^{S} \kappa_{\mathcal{S}}^{s} T_{L d, \mathcal{S}}^{F}-\left(T_{L d, s}^{F}+F A Z_{s}\right) & \text { für } s \in \mathcal{S}^{=} \\ 0 & \text { für } s \in \mathcal{S}^{\mp} .\end{cases}
$$

Die Summe der Zuweisungen an die finanzschwachen Länder beträgt damit insgesamt

$$
F Z_{s}^{1}=F A Z_{s}+F Z Z_{s} \quad \forall s \in \mathcal{S}^{-},
$$

bzw.

$$
F Z_{s}^{1}= \begin{cases}\pi^{S} \kappa_{\mathcal{S}}^{s} T_{L d, \mathcal{S}}^{F}-T_{L d, s}^{F} & \text { für } s \in \mathcal{S}^{=} \\ F A Z_{s} & \text { für } s \in \mathcal{S}^{\mp} .\end{cases}
$$

\section{(2) Berechnung der Finanzausgleichsbeiträge}

Die Länder, deren Finanzkraft $\left(M F_{s}\right)$ zwischen $100 \%$ und $102 \%\left(\pi^{1}\right)$ ihrer Ausgleichsmeßzahl $\left(M A_{s}\right)$ liegen, werden nicht zu den Zahlungen herangezogen $\left(\pi^{\min }\right.$ als entsprechender Multiplikator ist gleich 0). Erst die Länder mit einer höheren Prozentzahl sind ausgleichspflichtig. Ihnen werden $70 \%\left(\pi^{m i t}\right)$ der Einnahmen, die zwischen $102 \%$ und $110 \%\left(\pi^{2}\right)$ des Durchschnitts und $100 \%$ der Einnahmen, die über $110 \%$ der länderdurchschnittlichen Finanzkraft liegen, als ausgleichspflichtige Beträge angerechnet (§ $10 \mathrm{Abs.} 2$ FAG). Die genaue Höhe der Zahlungen entspricht der Summe der Ansprüche und wird als Ausgleichsbetrag von den ausgleichspflichtigen Ländern, proportional zu ihren jeweiligen ausgleichspflichtigen Beträgen $\left(F P_{s}\right)$, aufgebracht.

\footnotetext{
${ }^{28}$ Die Regelungen im LFA beziehen sich sowohl bezüglich Bevölkerungszahlen als auch Steuerkraftzahlen auf sehr unterschiedliche Konzepte, auf deren Unterscheidung man sehr genau achten sollte.
} 
Die Länder, deren Finanzkraftmeßzahl ihre jeweilige Ausgleichsmeßzahl übertreffen, werden, analog zu Gotrfried/Wiegard ([1992], S. 146), entsprechend der drei Anrechnungszonen in drei Teilmengen eingeteilt:

$$
\begin{gathered}
\mathcal{S}_{1}^{+}=\left\{s \mid M A_{s}<M F_{s} \leq \pi^{1} M A_{s}, s \in \mathcal{S}^{+}\right\} \\
\mathcal{S}_{2}^{+}=\left\{s \mid \pi^{1} M A_{s}<M F_{s} \leq \pi^{2} M A_{s}, s \in \mathcal{S}^{+}\right\} \\
\mathcal{S}_{3}^{+}=\left\{s \mid \pi^{2} M A_{s}<M F_{s}, s \in \mathcal{S}^{+}\right\} .
\end{gathered}
$$

Dabei ist die Finanzkraft des Landes um so höher, je höher der tiefgestellte Index der Menge der jeweiligen Länder ist.

Die ausgleichspflichtigen Einnahmen der finanzstarken Länder berechnen sich dann (mit $\pi^{m i n}=0$ ) wie folgt:

$$
F P_{s}= \begin{cases}\left(M F_{s}-\pi^{2} M A_{s}\right)+\pi^{m i t}\left(\pi^{2}-\pi^{1}\right) M A_{s} & \text { für } s \in \mathcal{S}_{3}^{+} \\ \pi^{m i t}\left(M F_{s}-\pi^{1} M A_{s}\right) & \text { für } s \in \mathcal{S}_{2}^{+} \\ 0 & \text { für } s \in \mathcal{S}_{1}^{+} .\end{cases}
$$

Da allerdings die Möglichkeit besteht, daß bei vorgegebenen $\pi^{m i t}=\pi_{0}^{m i t}=0,7$ und $\pi^{m i n}=\pi_{0}^{m i n}=0$ die Summe der FP kleiner als die benötigten Ausgleichszahlungen $\left(\sum F Z_{r}\right)$ sind, werden die Beträge im zweiten Segment, d.h. oberhalb von $\pi^{1}$ und unterhalb von $\pi^{2}$, stärker angerechnet, höchstens jedoch zu $100 \%$. Falls dies nicht ausreicht, erfolgt zusätzlich eine Anrechnung des ansonsten beitragsfreien Bereiches des untersten Segmentes. Diese Beträge werden ebenfalls höchstens zu $100 \%$ berücksichtigt.

Für $s \in \mathcal{S}_{3}^{+}$oder $s \in \mathcal{S}_{2}^{+}$gilt:

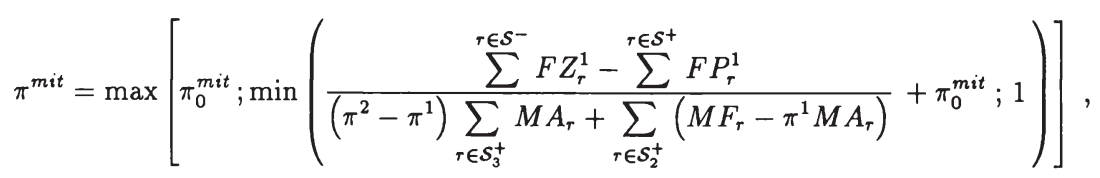

mit $F P_{\tau}^{1}$ als Höhe der insgesamt finanzausgleichspflichtigen Beträge bei $\pi_{0}^{m i t}$ bzw. $\pi_{0}^{\min }$. Den letzten Koeffizienten erhält man nach Definition der Menge

$$
\mathcal{S}_{*}^{+}=\left\{s \mid s \in \mathcal{S}_{2}^{+} \vee s \in \mathcal{S}_{3}^{+}\right\}
$$


als

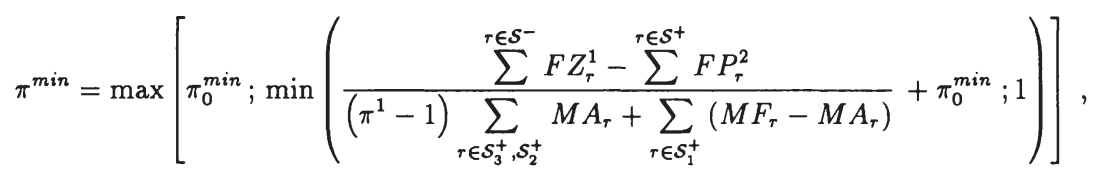

mit $F P_{r}^{2}$ als Höhe der ausgleichspflichtigen Beträge bei $\pi^{m i t}=1$ und $\pi_{0}^{m i n}=0$. Der Wert von $\pi^{\text {min }}$ liegt im Bereich zwischen $\pi_{0}^{m i n}$ und 1. Er wird dann erhöht, wenn nach Bestimmung von $\pi^{m i t}$ immer noch ein Fehlbetrag besteht.

Die Finanzbeiträge der ausgleichspflichtigen Länder entsprechen dann genau ${ }^{29}$

$$
F B_{s}^{1}=\frac{F P_{s}}{\sum_{r \in \mathcal{S}^{+}} F P_{r}} \sum_{r \in \mathcal{S}^{-}} F Z_{r}^{1} \quad \forall s \in \mathcal{S}^{+} .
$$

1.Garantieklausel: Fällt nun, nach Berechnung der zu leistenden Finanzbeiträge, ein ausgleichspflichtiges Land in seiner Finanzkraft, die nun die Finanzbeiträge enthält, unter den Durchschnitt aller Länder (§ 10 Abs. 3 FAG), werden seine Zahlungen um den Fehlbetrag reduziert und die der anderen ausgleichspflichtigen Länder proportional erhöht. ${ }^{30}$ Zur Darstellung dieser Korrektur wird die neue Steuerkraft der finanzstarken Länder unter Berücksichtigung der Finanzausgleichsbeiträge

$$
t_{L d, s}^{3}=\frac{1}{E W_{s}}\left(T_{L d, s}^{F}-F B_{s}^{1}\right) \quad \forall s \in \mathcal{S}^{+}
$$

und die durchschnittliche Steuerkraft aller am Finanzausgleich beteiligten Länder ohne Berücksichtigung der Finanzausgleichsströme

$$
\bar{t}_{L d}^{3}=T_{L d, \mathcal{S}}^{F} / E W_{\mathcal{S}}
$$

bestimmt. Unter Verwendung dieser Größen erhält man dann als Fehlbetrag der ausgleichspflichtigen Länder:

$$
F E_{s}^{1}=\max \left[E W_{s}\left(\bar{t}_{L d}^{3}-t_{L d, s}^{3}\right) ; 0\right] \quad \forall s \in \mathcal{S}^{+} .
$$

\footnotetext{
${ }^{29}$ Der Fall, daß nach Anpassung der Koeffizienten $\pi^{m i t}$ und $\pi^{m i n}$ die ausgleichspflichtigen Beiträge immer noch nicht ausreichen, um die Zuweisungen zu finanzieren, ist im Gesetz nicht vorgesehen. Wie er formalisiert werden kann zeigen GoTTFRIED/WIEGARD [1992].

${ }^{30}$ Die im Gesetz zum föderalen Konsolidierungsprogemm (FKPG) vom 26.6.1993 veränderten Garantieklauseln des $§ 10$ Abs. 3 FAG für die Jahre 1993 und 1994 werden in der hier dargestellten Regelung nicht berücksichtigt.
} 
Analog zu GotTfried/Wiegard ([1992], S. 141) werden nun die ausgleichspflichtigen Länder in die Menge der unter den Durchschnitt gerutschten und die Menge, der nach wie vor über der durchschnittlichen Steuerkraft liegenden Länder eingeteilt:

$$
\mathcal{S}_{-}^{+}=\left\{s \mid F E_{s}^{1}>0, s \in \mathcal{S}^{+}\right\} \quad \mathcal{S}_{+}^{+}=\left\{s \mid F E_{s}^{1}=0, s \in \mathcal{S}^{+}\right\} .
$$

Die neuen Finanzbeiträge der jetzt finanzschwach gewordenen ausgleichspflichtigen Länder ergeben sich dann durch eine Reduktion der bisher ermittelten Finanzbeiträge $F B_{s}^{1}$ um deren Fehlbeträge $F E_{s}^{1}$. Entsprechend werden die Finanzbeiträge der finanzstark bleibenden Länder proportional erhöht, um die Fehlbeträge zu finanzieren. Es ergibt sich damit

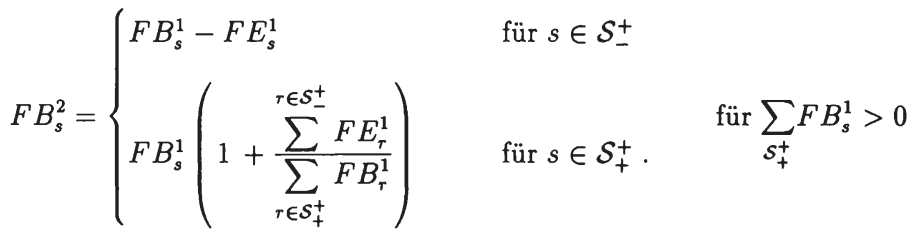

und $^{31}$

$$
F Z_{s}^{3}=F Z_{s}^{2} \quad \forall s \in \mathcal{S}^{-}
$$

2.Garantieklausel: Falls nun eines oder mehrere der finanzstarken Länder $\left(s \in \mathcal{S}_{+}^{+}\right)$, durch die zu leistenden Finanzbeiträge $F B_{s}^{2}$, unter die durchschnittliche Finanzkraft der Länder fällt (fallen), tritt die seit der Novellierung des FAG vom 26.4.1990 geltende Garantieklausel in Kraft. Diese sieht vor, daß die abgesunkenen Länder einen Ausgleich ihrer Fehlbeträge erhalten und alle anderen Ausgleichszuweisungen und Ausgleichsbeiträge entsprechend verändert werden ( $§ 10$ Abs.3, Satz 3 FAG). Die entsprechenden Fehlbeträge sind

$$
F E_{s}^{2}=\max \left[\kappa_{\mathcal{S}}^{s} T_{L d, \mathcal{S}}^{F}-\left(T_{L d, s}^{F}-F B_{s}^{2}\right) ; 0\right] \quad \forall s \in \mathcal{S}_{+}^{+} .
$$

Nach Aufteilung der Menge der finanzstarken Länder, die keinen Fehlbetrag $F E_{s}^{1}$ hatten, in

$$
\mathcal{S}_{+}^{+,-}=\left\{s \mid F E_{s}^{2}>0, s \in \mathcal{S}_{+}^{+}\right\} \quad \mathcal{S}_{+}^{+,+}=\left\{s \mid F E_{s}^{2}=0, s \in \mathcal{S}_{+}^{+}\right\},
$$

\footnotetext{
${ }^{31}$ Der Fall, daß diese Korrektur nicht möglich ist, da keines der Länder aus $\mathcal{S}_{+}^{+}$noch positive Finanzbeiträge leistet $\left(\sum_{r \in \mathcal{S}_{+}^{+}} F B_{r}^{1}=0\right)$ ist im Gesetz nicht vorgesehen, trat aber bei der Berechnung des LFA bereits auf und wird von GotTfried/Wiegard ([1992], S.150), unter Berufung auf den Schnellbrief VA6-FV3110-2/91, Anlage 2, formalisiert.
} 
ergeben sich als korrigierte Finanzausgleichszuweisungen und -beiträge

$$
F Z_{s}^{3}=F Z_{s}^{2}-\frac{T_{L d, s}^{F}+F Z_{s}^{2}}{\sum_{r \in \mathcal{S}^{*}} T_{L d, r}^{F}-\sum_{r \in \mathcal{S}_{+}^{+,+}} F B_{r}^{2}+\sum_{r \in \mathcal{S}^{-}} F Z_{r}^{2}} \sum_{r \in \mathcal{S}_{+}^{+,-}} F E_{r}^{2} \quad \forall s \in \mathcal{S}^{-}
$$

und

$$
F B_{s}^{3}= \begin{cases}F B_{s}^{2}-F E_{s}^{2} & \text { für } \forall s \in \mathcal{S}_{+}^{+,-} \\ F B_{s}^{2}+\frac{T_{L d, s}^{F}-F B_{s}^{2}}{\sum_{r \in \mathcal{S}^{*}} T_{L d, r}^{F}+\sum_{r \in \mathcal{S}_{+}^{+,+}} F B_{r}^{2}+\sum_{r \in \mathcal{S}^{-}} F Z_{r}^{2}} \sum_{r \in \mathcal{S}_{+}^{+,-}} F E_{r}^{2} & \text { und } \forall s \in \mathcal{S}_{-}^{+} \\ & \mathcal{S}_{+}^{+,+} .\end{cases}
$$

Die Menge $S^{*}$ setzt sich dabei aus der Menge der Länder aus $S^{-}, S_{-}^{+}$und $S_{+}^{+,+}$zusammen. Sind dagegen keine Fehlbeträge $F E_{s}^{2}$ aufgetreten, wird die Garantieklausel nicht relevant und man erhält anstelle obiger Gleichungen die Ausdrücke

$$
\begin{array}{cc}
F Z_{s}^{3}=F Z_{s}^{2} & \forall s \in \mathcal{S}^{-} \\
\text {und } & \\
F B_{s}^{3}=F B_{s}^{2} & \forall s \in \mathcal{S}^{+} .
\end{array}
$$

Die gesamten Finanzausgleichsströme einer Region, $F A_{s}$, ergeben sich schließlich aus den von den anderen Bundesländern erhaltenen LFA-Zuweisungen, $F Z_{s}^{3}$, abzüglich der Finanzbeiträge der Region an die anderen Regionen, $F B_{s}^{3}$ :

$$
F A_{s}=F Z_{s}^{3}-F B_{s}^{3}
$$

\subsubsection{Bundesergänzungszuweisungen}

Von den gesamten Umsatzsteueraufkommen sind $2 \%\left(\pi^{E Z}\right)$ für Ergänzungszuweisungen vorgesehen, die sich in 1993 und 1994 um genau bestimmte Beträge erhöhten (§ 11a, Abs.1a FAG, geändert im FKPG). Nach Verteilung eines Vorabbetrages $E Z_{s}^{F}$ an einige Länder, wird der Rest den leistungsschwachen Ländern, nach Maßgabe ihrer nach Durchführung des Länderfinanzausgleichs verbleibenden Fehlbeträge gegenüber der Ausgleichsmeßzahl, gewährt (§ 11a Abs.2 Satz 1, FAG 1987).

Als Ergänzungszuweisungen ergeben sich damit

$$
E Z_{s}=\pi_{s}^{E Z}\left(\pi^{E Z} S T_{\mathcal{R}}^{C}-\sum_{r \in \mathcal{R}} E Z_{r}^{F}\right)+E Z_{s}^{F},
$$


mit dem Verteilungssatz für die finanzschwachen Länder $\left(s \in \mathcal{S}^{-}\right)$in Höhe von

$$
\pi_{s}^{E Z}= \begin{cases}\frac{\max \left[M F_{s}+F Z_{s}^{3}-M A_{s} ; 0\right]}{\sum_{r \in \mathcal{S}^{-}} \max \left[M F_{r}+F Z_{r}^{3}-M A_{r} ; 0\right]} & \text { für } \\ \max \left[M F_{s}+F Z_{s}^{3}-M A_{s} ; 0\right] & \geq \pi^{E Z_{T}} T_{B d}^{C}-\sum_{S} V A^{E Z_{r}} \\ \text { sonst }\end{cases}
$$

und $\quad \pi_{s}^{E Z}=0 \quad$ für $s \in \mathcal{S}^{+}$.

\subsection{Bundesdeutsche Regelungen nach 1995}

Die infolge der Vereinigung notwendig gewordene Neuordnung des bundesdeutschen Finanzausgleichs wurde, nach langer Diskussion, im Gesetz zur Umsetzung des Föderalen Konsolidierungsprogramms (FKPG) vom 26.Juni 1993 abschließend geregelt.

Die Bestimmungen zur Verteilung der Gewerbe- und Einkommenssteuern bleiben weitgehend unverändert. Es gelten weiterhin die Gleichungen (I.2) und (I.3). Nur der Vervielfältiger zur Berechnung der Gewerbesteuerumlage wird im Standortsicherungsgesetz (StandOG) neu bestimmt. Für die Ostländer wird er ab 199538 und für die Westländer 56 betragen, wobei er zur Berechnung der für den Länderfinanzausgleich zugrundeliegenden Höhe der Gewerbesteuerumlage einheitlich auf 38 reduziert wird (Art.15 $§ 6$ Abs.2 und 3 StandoG vom 13.9.1993).

\subsubsection{Umsatzsteuerverteilung}

Im Gegensatz zur geltenden Regelung werden nun alle Länder einbezogen, so daß jeweils die Menge $\mathcal{S}$ durch die Menge aller Länder $\mathcal{R}$ ersetzt wird. Entsprechend entfällt die Vorabzuweisung an die Neuen Länder.

Der Anteil des Bundes an den Umsatzsteuereinnahmen wird ab 1995 auf $56 \%$ gesenkt. Von ihrem höheren Umsatzsteueranteil müssen die Länder jährlich 2,1 Mrd DM sowie $50 \%$ der Bundeszuschüsse zum Fonds Deutsche Einheit an den Bund abführen.

\section{(1) Vorabverteilung nach Bevölkerung}

Die Verteilung von $75 \%\left(\pi_{V A}^{C}\right)$ des Länderanteils an der Umsatzsteuer auf die einzelnen Länder bleibt unverändert bei (§ 2, Abs. 1 FAG 1993)

$$
V A_{s}^{C}=\kappa_{\mathcal{R}}^{s} \pi_{V A}^{C} T_{L d}^{C} .
$$




\section{(2) Ergänzungsanteile an die steuerschwachen Länder}

Die Berechnung der Ergänzungsanteile an die steuerschwachen Länder wird vereinfacht. Diese müssen nun nicht mehr mindestens so groß wie bei einer Verteilung nach den Bevölkerungrelationen sein. Ergänzungsanteile werden gewährt, wenn die Steuereinnahmen der Länder, vor der Umsatzsteuerverteilung, weniger als $92 \%$ der länderdurchschnittlichen Steuereinnahmen ausmachen. Wenn jedoch die Summe der Ergänzungsanteile mehr als $25 \%$ der Umsatzsteuereinnahmen der Länder umfaßt, werden die Ergänzungsanteile proportional gekürzt. Dies führt für $s \in \mathcal{R}_{-}^{-}$zu

$$
E A_{s}^{C}= \begin{cases}E A_{s}^{M A}=E W_{s}\left(\pi_{E A}^{C} \bar{t}_{L d}^{1}-t_{L d, s}^{1}\right) & \text { für } \sum_{r \in \mathcal{R}} E A_{r}^{C, 0} \leq\left(1-\pi_{V A}^{C}\right) T_{L d}^{C} \\ \frac{E A_{s}^{M A}}{\sum_{\tau \in \mathcal{R}^{-}} E A_{r}^{M A}}\left(1-\pi_{V A}^{C}\right) T_{L d}^{C} & \text { für } \sum_{r \in \mathcal{R}} E A_{r}^{C, 0}>\left(1-\pi_{V A}^{C}\right) T_{L d}^{C} .\end{cases}
$$

\section{(3) Verteilung der Restbeträge}

Die, nach Abzug der Vorabverteilung und der Ergänzungsanteile vom Länderanteil verbleibenden Umsatzsteueraufkommen werden nun nicht mehr nur an die finanzstarken, sondern an alle Länder entsprechend ihrer Einwohnerzahlen verteilt (§ 2 Abs. 2 Satz 2 FAG 1993)

$$
R V_{s}^{C}=\kappa_{\mathcal{R}}^{s}\left[\left(1-\pi_{V A}^{C}\right) T_{L d}^{C}-\sum_{r \in \mathcal{R}_{-}^{-}} E A_{r}^{C}\right] .
$$

Weggefallen ist die Garantieklausel für die finanzstarken Länder des § 2 Abs. 3 FAG 1988. Insgesamt erhält man also als Umsatzsteuereinnahmen der Länder

$$
T_{s}^{C}=V A_{s}^{C}+E A_{s}^{C}+R V_{s}^{C} .
$$

\subsubsection{Länderfinanzausgleich}

Die Bestimmung der Finanzkraft, d.h. der regionalen Finanzkraftmeßzahl $M F_{s}$ und der regionalen Ausgleichsmeßzahl $M A_{s}$, werden fast unverändert beibehalten. Sie werden zum einen dahingehend geändert, daß auch die Bevölkerung von Berlin veredelt wird ( 9 Abs. 2 FAG 1993) und bei den Sonderbedarfen für Mecklenburg-Vorpommern die Seehafenlasten von Rostock mit einbezogen werden ( 99 Abs. 3 des FAG vom 1993). Zum zweiten werden sowohl bei der Ermittlung der LFA-Zuweisungen, als auch bei der Berechnung der Finanzausgleichsbeiträge immer die veredelten Einwohnerzahlen zugrundegelegt. Schließlich wird nun in allen Schritten die Finanzkraft der Länder verwendet, die bei der Ermittlung der 
Finanzkraftmeßzahl des LFA definiert wird. Allerdings wird zum Teil die Finanzkraft der Länder und zum Teil die regionale Finanzkraft verwendet.

\section{(1) Berechnung der Finanzausgleichszuweisungen}

Die finanzschwachen Länder erhalten den vollen Betrag, der ihnen zum Erreichen von $92 \%\left(\pi^{M}\right)$ ihrer regionalen Ausgleichsmeßzahl fehlt, und 37,5\% $\left(\pi^{N}\right)$ des zwischen $92 \%$ und $100 \%$ ihrer regionalen Ausgleichsmeßzahl liegenden Betrages als LFA-Zuweisungen. Die Formel (I.19) gilt damit, bis auf die Neudefinition der Menge der Länder, in der nun auch die Neuen Länder enthalten sind $\left(r \in \mathcal{R}^{-}\right)$, unverändert.

Den finanzschwachen Ländern wird jetzt allerdings nicht mehr das Erreichen von $95 \%$ der durchschnittlichen Einnahmen zugesichert, da sie nur mehr die Hälfte $\left(\pi_{z}^{F}=0,5\right)$ des Fehlbetrages, um den ihre regionale Finanzkraft einschließlich der Ausgleichszuweisungen $F A Z_{s}$ unterhalb von $95 \%$ ihrer Ausgleichsmeßzahl liegt, zusätzlich zugewiesen bekommen (§ 10 Abs. 3., Satz 1 FAG 1993) $)^{32}$ :

Nach Einteilung der Menge der finanzschwachen Länder in

$$
\begin{aligned}
& \mathcal{R}_{-}^{-}=\left\{s \mid \pi^{S} \kappa_{\mathcal{R}, F A}^{s} T_{L d, \mathcal{R}}^{F A}-\left(T_{L d, s}^{F A}+F A Z_{s}\right) \leq 0, s \in \mathcal{R}^{-}\right\} \\
& \mathcal{R}_{-}^{-}=\left\{s \mid \pi^{S} \kappa_{\mathcal{R}, F A}^{s} T_{L d, \mathcal{R}}^{F A}-\left(T_{L d, s}^{F A}+F A Z_{s}\right)>0, s \in \mathcal{R}^{-}\right\}
\end{aligned}
$$

erhält man für $F Z Z_{s}$

$$
F Z Z_{s}= \begin{cases}\pi_{z}^{F}\left[\pi^{S} \kappa_{\mathcal{R}, F A}^{s} T_{L d, \mathcal{R}}^{F A}-\left(T_{L d, s}^{F A}+F A Z_{s}\right)\right] & \text { für } s \in \mathcal{R}_{-}^{-} \\ 0 & \text { für } s \in \mathcal{R}_{+}^{-} .\end{cases}
$$

Die Summe der Zuweisungen an die finanzschwachen Länder beträgt somit, vorbehaltlich einer etwaigen Änderung aufgrund der Garantieklausel in § 10 Abs. 4 Satz 3 FAG 1993,

$$
F Z_{s}^{1}=F A Z_{s}+F Z Z_{s} \quad \forall s \in \mathcal{R}^{-},
$$

bzw.

$$
F Z_{s}^{1}= \begin{cases}\pi_{Z}^{F}\left(\kappa_{\mathcal{R}, F A}^{s} \pi^{S} T_{L d, \mathcal{R}}^{F A}-T_{L d, s}^{F A}\right)+\left(1-\pi_{z}^{F}\right) F A Z_{s} & \text { für } s \in \mathcal{R}^{=} \\ F A Z_{s} & \text { für } s \in \mathcal{R}^{\mp}\end{cases}
$$

Diese Finanzzuweisungen werden den finanzschwachen Ländern dann gekürzt, wenn die finanzpflichtigen Beiträge der finanzstarken Länder nicht zur Finanzierung ausreichen. Die entsprechende Korrektur erfolgt jedoch erst nach Berechnung der finanzpflichtigen Beiträge (§ 10 Abs. 4 Satz 3 FAG vom 26.6.1993).

\footnotetext{
${ }^{32}$ Dies entspricht der Garantie, daß die finanzschwachen Länder mindestens $93,5 \%$ der durchschnittlichen Finanzkraft erreichen.
} 


\section{(2) Berechnung der Finanzausgleichsbeiträge}

Zur Finanzierung der Finanzuweisungen werden alle über dem Durchschnitt der Finanzkraft liegenden Beträge herangezogen. Es gibt damit keine ausgleichsfreie Zone mehr. Die dreistufige Einteilung wird beibehalten, jedoch die starken Unterschiede in der Heranziehung der einzelnen Stufen abgemildert.

Die Beträge, die zwischen 100 und $101 \%\left(\pi^{1}\right)$ (nicht mehr $102 \%$ ) der regionalen Ausgleichsmeßzahl liegen werden nun zu $15 \%\left(\pi^{\min }\right)$, die zwischen 101 und $110 \%\left(\pi^{2}\right)$ liegen zu $66 \%\left(\pi^{m i t}\right)$ und die über $110 \%$ der regionalen Ausgleichsmeßzahl liegenden Beträge zu $80 \%\left(\pi^{\max }\right)$ zur Berechnung der Finanzpflichtigkeit herangezogen ( $§ 10$ ABs. 2 Satz 1 FAG vom 26.6.1993). Demnach erhält man als Menge der in die einzelnen Abschnitte fallenden Länder

$$
\begin{gathered}
\mathcal{R}_{1}^{+}=\left\{s \mid M A_{s}<M F_{s} \leq \pi_{1} M A_{s}, s \in \mathcal{R}^{+}\right\} \\
\mathcal{R}_{2}^{+}=\left\{s \mid \pi_{1} M A_{s}<M F_{s} \leq \pi_{2} M A_{s}, s \in \mathcal{R}^{+}\right\} \\
\mathcal{R}_{3}^{+}=\left\{s \mid \pi_{2} M A_{s} \leq M F_{s}, s \in \mathcal{R}^{+}\right\} .
\end{gathered}
$$

Die ausgleichspflichtigen Einnahmen der finanzstarken Länder berechnen sich demnach wie folgt:

$$
F P_{s}=\left\{\begin{array}{cl}
\pi^{\max }\left(M F_{s}-\pi^{2} M A_{s}\right)+\pi^{m i t}\left(\pi^{2}-\pi^{1}\right) M A_{s} & \\
+\pi^{m i n}\left(\pi^{1}-1\right) M A_{s} & \text { für } s \in \mathcal{R}_{3}^{+} \\
\pi^{m i t}\left(M F_{s}-\pi^{1} M A_{s}\right)+\pi^{m i n}\left(\pi^{1}-1\right) M A_{s} & \text { für } s \in \mathcal{R}_{2}^{+} \\
\pi^{\min }\left(M F_{s}-M A_{s}\right) & \text { für } s \in \mathcal{R}_{1}^{+},
\end{array}\right.
$$

mit $\pi^{\max }=0,8, \pi_{0}^{m i t}=0,66$ und $\pi^{\min }=0,15$.

Eine Erhöhung der Anrechnungsgrößen ist im neuen FAG nur für $\pi^{m i t}$ vorgesehen, das bis auf 0,8 erhöht werden kann ( $§ 10$ Absatz 4 FAG). Dies wird allerdings erst am Ende der LFA-Berechnungen berücksichtigt.

Die Finanzbeiträge der ausgleichspflichtigen Länder berechnen sich unverändert proportional zu den finanzpflichtigen Beiträgen:

$$
F B_{s}^{1}=\frac{F P_{s}}{\sum_{r \in \mathcal{R}^{+}} F P_{\tau}} \sum_{r \in \mathcal{R}^{-}} F Z_{\tau}^{1} \quad \forall s \in \mathcal{R}^{+} .
$$

Im Gegensatz zur alten Regelung wird zusätzlich der Fall berücksichtigt, daß die Finanzbeiträge noch immer nicht zur Finanzierung der Finanzzuweisungen ausreichen ( $§ 10 \mathrm{Abs}$. 4 Satz 3 FAG 1993). Dies wird allerdings erst nach einigen Garantieregeln beachtet. 


\section{(3) Garantieklauseln}

Fällt, unter Berücksichtigung der zu leistenden Finanzbeiträge, ein ausgleichspflichtiges Land mit seinen im Finanzausgleich zu berücksichtigenden Einnahmen je (veredeltem) Einwohner unter den Durchschnitt der Einnahmen aller Länder, so ist, gemäß $§ 10$ Abs. 3 Satz 2 bis Satz 4 FAG 1993,

„der Fehlbetrag dieses Landes zu einem Viertel, höchstens jedoch bis zur Höhe seiner Ausgleichsleistungen nach Absatz 2 und Absatz 3 Satz 1 von den anderen ausgleichspflichtigen Ländern im Verhältnis der Ausgleichsbeiträge nach Absatz 2 zu übernehmen. Zur Sicherung der Finanzkraftreihenfolge ist der Ausgleich des Fehlbetrags auf den Betrag zu begrenzen, mit dem das begünstigte Land die Finanzkraftrelation des nächststärkeren Landes erreicht, höchstens jedoch auf den Betrag, der sicherstellt, daß ein an der Aufbringung beteiligtes Land in seiner Finanzkraftrelation nicht unter die des nächstschwächeren Landes sinkt. Kommt Satz 3 zur Anwendung, ist das nächststärkere Land nach Satz 3 von der Aufbringung des Fehlbetrags ausgenommen. “

Zur Darstellung dieser Garantieklauseln wird zuerst die neue Pro-Kopf-Steuerkraft der finanzstarken Länder unter Berücksichtigung der Finanzausgleichsbeiträge, $t_{L d, s}^{3}$, und die durchschnittliche Pro-Kopf-Steuerkraft aller am Finanzausgleich beteiligten Länder ohne Berücksichtigung der Finanzausgleichsströme, $\vec{t}_{L d}^{3}$,

$$
t_{L d, s}^{3}=\frac{1}{E W_{s}^{F A}}\left(T_{L d, s}^{F A}-F B_{s}^{1}\right) \quad \forall s \in \mathcal{R}^{+} \quad \text { und } \quad \bar{t}_{L d}^{3}=T_{L d, \mathcal{R}}^{F A} / E W_{\mathcal{R}}^{F A}
$$

bestimmt. Unter Verwendung dieser Größen erhält man dann als Fehlbetrag der ausgleichspflichtigen Länder:

$$
F E_{s}^{1}=\max \left[E W_{s}^{F A}\left(\bar{t}_{L d}^{3}-t_{L d, s}^{3}\right) ; 0\right] \quad \forall s \in \mathcal{R}^{+} .
$$

Entsprechend kann man die finanzstarken Länder in zwei Teilmengen aufteilen

$$
\mathcal{R}_{-}^{+}=\left\{s \mid F E_{s}^{1}>0, s \in \mathcal{R}^{+}\right\} \quad \mathcal{R}_{+}^{+}=\left\{s \mid F E_{s}^{1}=0, s \in \mathcal{R}^{+}\right\} .
$$

Unter Einbezug der in $\S 10$ Abs. 4, Satz 3 und Satz 4 des FAG vom 26.6.1993 enthaltenen Garantieklausel, erhält man folgendes Verfahren zur Ermittlung der anrechenbaren Fehlbeträge und der Finanzbeiträge, die zur Finanzierung der Fehlbeträge benötigt werden.

Die Fehlbeträge werden zu $25 \%\left(\pi_{1}^{F}\right)$, höchstens jedoch in Höhe der Finanzbeiträge $F B_{s}^{1}$ durch die fehlbetragsfreien finanzstarken Länder ausgeglichen. Ist dieser Ausgleich nur durch die Veränderung der Finanzkraftreihenfolge möglich, so sind die Regelungen des $\S 10$ Abs. 4, Satz 3 und Satz 4 zu beachten, die allerdings einiger Interpretationshilfen bedürfen. 
Im Prinzip besagen diese Regelungen, daß die Länder mit Fehlbeträgen dann geringere Ausgleichszahlungen erhalten, wenn sie bei vollem Ausgleich die Finanzkraft des nächststärkeren Landes übertreffen würden. Die Zuweisungen werden dann genau auf den Betrag $F E_{s}^{2}$ beschränkt, bei dem sie gerade die Finanzkraft des nächststärkeren Landes erreichen. Insgesamt ergibt sich als Fehlbetragsausgleich:

$$
F E_{s}^{3}=\min \left[\pi_{1}^{F} F E_{s}^{1} ; F B_{s}^{1} ; F E_{s}^{2}\right] \quad \forall s \in \mathcal{R}_{-}^{+} .
$$

Diese Fehlbeträge werden von den Länder aus $\mathcal{R}_{+}^{+}$aufgebracht, wobei wieder eine Garantieklausel zu beachten ist.

Fällt ein finanzstarkes Land, das an der Finanzierung der Fehlbeträge beteiligt ist, in seiner Finanzkraft unter die Finanzkraft eines finanzschwächeren Landes, so muß es keine Beiträge zum Fehlbetragsausgleich leisten $\left(F B E_{s}^{1}=0\right)$.

Die Beiträge der anderen finanzstarken Länder, deren Finanzkraftreihenfolge nicht verletzt wurde (die Menge $\mathcal{R}_{*}^{+}$), finanzieren sämtliche Fehlbeträge und sind daher

$$
F B E_{s}^{1}=\frac{F B_{s}^{1}}{\sum_{r \in \mathcal{R}_{*}^{+}} F B_{r}^{1}} \sum_{r \in \mathcal{R}_{-}^{+}} F E_{\tau}^{2} \quad \forall s \in \mathcal{R}_{*}^{+} .
$$

Dieses Verfahren ist recht umständlich und führt zu einem mehrfachen Durchlauf, bis schließlich keine der Bedingungen mehr verletzt ist. Als Ergebnis erhält man die endgültigen $F E_{s}^{3}$ und $F B E_{s}^{1}$.

Schließlich ergeben sich die neuen Finanzbeiträge als

$$
F B_{s}^{2}= \begin{cases}F B_{s}^{1}-F E_{s}^{3} & \text { für } s \in \mathcal{R}_{-}^{+} \\ F B_{s}^{1}+F B E_{s}^{1} & \text { für } s \in \mathcal{R}_{+}^{+} .\end{cases}
$$

Nun werden noch die Garantieklauseln des $\S 10$ Absatz 4 überprüft. Die Garantieklauseln der Satzes 1 und 2 betreffen nur die finanzstarken Länder und stellen sicher, daß ihre Finanzausgleichsbeiträge nicht ihre höchstmögliche Finanzpflichtigkeit übersteigen.

Sind für mindestens ein Land die Finanzbeiträge $F B_{s}^{2}$ größer als sein maximaler finanzpflichtiger Beitrag $F P_{s}^{1}$, wobei der Index 1 angibt, daß die zwischen $101 \%$ und $110 \%$ der Finanzkraft liegenden Beträge der regionalen Ausgleichsmeßzahl mit $\pi_{1}^{\text {mit }}=0,8$ angerechnet werden, so werden seine Finanzbeiträge auf $F P_{s}^{1}$ begrenzt und durch die anderen finanzpflichtigen Länder ausgeglichen (§ 10 Abs. 4 Satz 1 FAG 1993). Dies führt zu

$$
F B_{s}^{3}= \begin{cases}F P_{s}^{1} & \text { für } r \in \mathcal{R}^{+,-} \\ F B_{s}^{2}+\frac{F B_{s}^{2}}{\sum_{r \in \mathcal{R}^{+,+}} F B_{r}^{2}} \sum_{r \in \mathcal{R}^{+,-}}\left(F B_{r}^{2}-F P_{r}^{1}\right) & \text { für } r \in \mathcal{R}^{+,+}\end{cases}
$$


mit

$$
\mathcal{R}^{+,+}=\left\{s \mid F P_{s}^{1}-F B_{s}^{2}>0, s \in \mathcal{R}^{+}\right\} \quad \mathcal{R}^{+,-}=\left\{s \mid F P_{s}^{1}-F B_{s}^{2} \leq 0, s \in \mathcal{R}^{+}\right\}
$$

und

$$
F P_{s}^{1}= \begin{cases}\pi^{\max }\left(M F_{s}-\pi^{2} M A_{s}\right)+\pi_{1}^{m i t}\left(\pi^{2}-\pi^{1}\right) M A_{s}+\pi^{m i n}\left(\pi^{1}-1\right) M A_{s} & \text { für } s \in \mathcal{R}_{3}^{+} \\ \pi_{1}^{\text {mit }}\left(M F_{s}-\pi^{1} M A_{s}\right)+\pi^{m i n}\left(\pi^{1}-1\right) M A_{s} & \text { für } s \in \mathcal{R}_{2}^{+} \\ \pi^{\min }\left(M F_{s}-M A_{s}\right) & \text { für } s \in \mathcal{R}_{1}^{+} .\end{cases}
$$

Dieser Prozeß wird solange wiederholt bis für kein finanzstarkes Land seine Finanzbeiträge höher als seine maximal möglichen finanzausgleichspflichtigen Beträge sind (§ 10 Abs. 4 Satz 2 FAG 1993).

Da die zulässigen Finanzbeiträge höchstens der Summe der finanzpflichtigen Beträge $F P_{s}^{1}$ entsprechen, muß ein eventueller Überschuß der Summe der ermittelten Finanzzuweisungen über diese $F P_{s}^{1}$ korrigiert werden. Dies ist im $\S 10$ Abs. 4 Satz 3 FAG 1993 wie folgt geregelt:

„Übersteigt die Summe der Ausgleichszuweisungen nach Absatz 1 und Absatz 3 Satz 115 vom Hundert der Finanzkraft, die zwischen 100 und 101 vom Hundert der Ausgleichsmeßzahl liegt, sowie vier Fünftel der 101 vom Hundert der Ausgleichsmeßzahl übersteigende Finanzkraft der ausgleichspfichtigen Länder, so ist der Fehlbetrag von allen Ländern im Verhältnis ihrer Finanzkraft unter Berücksichtigung der Ausgleichsbeiträge und Ausgleichszuweisungen nach den Absätzen 1 bis 3 und Absatz 4 Satz 1 und 2 aufzubringen."

Dies heißt, daß die Summe der Überschüsse $\sum_{\mathcal{R}^{-}} F Z_{\tau}^{1}-\sum_{\mathcal{R}^{+}} F P_{\tau}^{1}$ zum Teil durch eine Reduktion der Finanzzuweisungen und zum Teil durch eine Erhöhung der Finanzbeiträge ausgeglichen wird. Die Erhöhung der Finanzbeiträge erfolgt proportional nach Finanzkraft, in der die Finanzausgleichszuweisungen und Finanzausgleichsbeiträge berücksichtigt werden. Ist $\sum_{\mathcal{R}^{-}} F Z_{\tau}^{1}>\sum_{\mathcal{R}^{+}} F P_{r}^{1}$ dann gilt daher

$$
F Z_{s}^{2}=F Z_{s}^{1}-\frac{T_{L d, s}^{F A}+F Z_{s}^{1}}{\sum_{r \in \mathcal{R}}\left(T_{L d, r}^{F A}+F Z_{\tau}^{1}-F B_{r}^{3}\right)}\left(\sum_{r \in \mathcal{R}^{-}} F Z_{r}^{1}-\sum_{r \in \mathcal{R}^{+}} F P_{r}^{1}\right) \quad \forall s \in \mathcal{R}^{-},
$$

und wegen $F B_{s}^{3}=F P_{s}^{1}$ schließlich

$$
F B_{s}^{4}=F B_{s}^{3}+\frac{T_{L d, s}^{F A}-F B_{s}^{3}}{\sum_{r \in \mathcal{R}}\left(T_{L d, r}^{F A}+F Z_{r}^{1}-F B_{r}^{3}\right)}\left(\sum_{r \in \mathcal{R}^{-}} F Z_{r}^{1}-\sum_{r \in \mathcal{R}^{+}} F P_{r}^{1}\right) \quad \forall s \in \mathcal{R}^{+}
$$


Ist dagegen $\sum_{\mathcal{R}^{-}} F Z_{r}^{1} \leq \sum_{\mathcal{R}+} F P_{r}^{1}$ dann gilt

$$
F B_{s}^{4}=F B_{s}^{3} \quad \forall s \in \mathcal{R}^{+} \quad F Z_{s}^{2}=F Z_{s}^{1} \quad \forall s \in \mathcal{R}^{-} .
$$

Somit erhält man als Finanzausgleichsbeträge insgesamt

$$
F A_{s}= \begin{cases}F Z_{s}^{2} & \forall s \in \mathcal{R}^{-} \\ F B_{s}^{4} & \forall s \in \mathcal{R}^{+}\end{cases}
$$

\subsubsection{Bundesergänzungszuweisungen (BEZ)}

Für eine Reihe von Sonderlasten werden den Bundesländern der Höhe nach festgelegte Bundesergänzungszuweisungen $\left(E Z_{s}^{F}\right.$ ) gewährt (§ $11 \mathrm{FAG}$ vom 26.6.1993). Diese müssen im Falle der Zuweisungen zum Zweck der Haushaltssanierung an Bremen und das Saarland zweckgebunden zur Schuldentilgung verwendet werden (§ 11 Abs. 6 FAG 1993).

Darüber hinaus werden BEZ an die leistungsschwachen Länder „in Höhe von $90\left[\pi^{E Z}\right.$, d.Verf.] vom Hundert ihrer nach Durchführung des Länderfinanzausgleichs verbleibenden Fehlbeträge der [regionalen, d.Verf.] Finanzkraftmeßzahlen gegenüber den [regionalen, d.Verf.] Ausgleichsmeßzahlen des Ausgleichsjahres"überwiesen (§ 11 Abs. 2 FAG 1993)

$$
E Z_{s}=E Z_{s}^{F}+\pi^{E Z} \max \left[M A_{s}-\left(M F_{s}+F A_{s}\right) ; 0\right] .
$$

\subsubsection{Zusätzliche Abgaben der Länder}

Die alten Bundesländer incl. Berlin(West) müssen nun aus ihrem jeweiligen Umsatzsteueranteil „50 vom Hundert der Bundeszuschüsse nach $\S 6$ Abs. 2 des Gesetzes über den Fonds 'Deutsche Einheit' zuzüglich eines Betrages von 2,1 Milliarden DM jährlich"an den Bund abführen ( 11 Abs. 2 Satz 1 FAG 1993). Dieser Beitrag $Z A^{C}$ wird je zu $50 \%$ nach Bevölkerung und nach dem regionalen Finanzkraftverhältnis nach LFA aufgebracht (§ 1 Abs. 2 Satz 2 FAG 1993), woraus sich folgende Gleichung ergibt:

$$
Z A_{s}^{C}=0,5 Z A^{C} \frac{E W_{s}}{\sum_{r \in \mathcal{W}} E W_{r}}+0,5 Z A^{C} \frac{M F_{s}+F A_{s}}{\sum_{r \in \mathcal{W}}\left(M F_{r}+F A_{r}\right)},
$$

mit $\mathcal{W}$ als Menge der Westländer incl. Westberlin.

Diese Beträge werden zusätzlich für die relativ reichen Westländer um vorgegebene Summen erhöht und für die andern Länder verringert. Die entsprechenden Beträge werden schrittweise bis zum Jahr 2005 vermindert (§ 1 Abs. 3 FAG 1993). 


\subsection{Der Vorschlag des Wissenschaftlichen Beirats (1992)}

Der Wissenschaftliche Beirat beim Bundesminister der Finanzen [1992] empfiehlt in seinem Gutachten über den Länderfinanzausgleich drei sich allerdings weitgehend deckende Vorschläge zur Neuregelung des Länderfinanzausgleichs.

\subsubsection{Einkommenssteuerverteilung und Einnahmenautonomie}

Grundsätzlich fordert der Beirat eine höhere Einnahmenautonomie der Länder. Dazu schlägt er explizit ein Zuschlagsrecht der Länder (und der Gemeinden) zur Lohn- und veranlagten Einkommenssteuer sowie, trotz eher gegenteiliger Analyse (WISSENSCHAFTLICHER BEIRAT [1992], S. 99), auch zur Körperschaftssteuer auf nichtausgeschüttete Gewinne vor (Wissenschaftlicher Beirat [1992], S. 97-99 und S.103).

Die Empfehlung sieht vor, daß der Bund vorab die jeweiligen nationalen Steuersätze senkt, und im Anschluß daran die Länder und der Bund die Möglichkeit erhalten sollten, einen nach oben begrenzten Zuschlag auf die gesenkten Steuersätze zu erheben. Eine denkbare Alternative wäre, nach Meinung des Beirats, die Gewährung von $\mathrm{Zu}$ - oder Abschlägen auf die jeweilige Steuerschuld. In beiden Verfahren müssen allerdings spezielle Regelungen bezüglich der Steuerzerlegung eingeführt werden. Die Einnahmen aus den Zuschlägen, gemäß dem ersten Verfahren, sollten im Finanzausgleichsverfahren nicht in der Finanzkraft berücksichtigt werden. Bei der Erhebung von $\mathrm{Zu}$ - oder Abschlägen auf die Steuerschuld, sollten dagegen die entsprechenden Einnahmen normiert, auf Basis der Steuerschuld, in die Finanzkraft eingehen (Wissenschaftlicher Beirat [1992], S.97104).

Die Länder erhalten in beiden Verfahren die gesamten Aufkommen $S T_{s}^{E k, Z}$ aus ihren regionalen Zuschlägen als Einnahmen $\left(T_{s}^{E k, Z}=S T_{s}^{E k, Z}\right)$ Dementsprechend erhält jedes Bundesland als Einnahmen aus den Einkommenssteuern

$$
T_{s}^{Y}=T_{L d, s}^{Y}+T_{G m, s}^{Y}+T_{s}^{E k, Z}
$$

Die Verteilung der sonstigen Einnahmen aus der Körperschaftssteuer und der Lohn- und Einkommenssteuer sollten weiterhin nach den geltenden gesetzlichen Regelungen vorgenommen werden (vgl. WisSENSCHAFTLICHER BEIRAT [1992], S.100).

\subsubsection{Umsatzsteuerverteilung}

Der BEIRAT präsentiert zur Garantie einer bestimmten Mindestfinanzkraft drei alternative Verfahren. Der Kern des Länderfinanzausgleichs, nämlich der lineare Umverteilungstarif, ist dagegen in allen Varianten gleich. 
In Variante I erfolgt der Mindestausgleich nach der Umsatzsteuerverteilung und nach der linearen Umverteilung im LFA. In Variante II wird der Mindestausgleich in die Umsatzsteuerverteilung integriert. Dagegen erfolgt in der Variante III der Mindestausgleich zwischen der Umsatzsteuerverteilung und dem eigentlichen dem eigentlichen Länderfinanzausgleich (WissenschaftLICHER BeIRAT [1992], S.79).

\section{Variante I und III}

Die Verteilung der Umsatzsteuern ist in Variante I und Variante III identisch und ausschließlich an den relativen Bevölkerungsanteilen orientiert (WISSENSCHAFTLICHER BeIRAT [1992], S.79):

$$
T_{s}^{C}=\kappa_{\mathcal{R}}^{s} T_{L d}^{C}
$$

\section{Variante II}

In dieser Variante wird die Mindestfinanzkraft innerhalb der Umsatzsteuerverteilung gesichert. Jedes Land, dessen regionale Finanzkraft weniger als $\pi_{u}^{C}$ Prozent der regionalen Ausgleichsmeßzahl erreicht, erhält eine Mindestauffüllung seiner regionalen Finanzkraft. Diese wird aus dem Umsatzsteueranteil der Bundesländer finanziert. ${ }^{33}$ Die entsprechende Zuweisung ist

$$
E A_{s}^{C}=\max \left[0 ; \pi_{E A}^{C} M A_{s}-M F_{s}^{C}\right]
$$

Für das weitere Vorgehen ist es sinnvoll, die Länder in zwei disjunkte Mengen einzuteilen. Die Länder mit positiven $E A_{s}^{C}$ werden als Menge $\mathcal{R}^{-}$und die restlichen Länder als Menge $\mathcal{R}^{+}$definiert:

$$
\mathcal{R}^{-}=\left\{s \mid E A_{s}^{C}>0, r \in \mathcal{R}\right\} \quad \mathcal{R}^{+}=\left\{s \mid E A_{s}^{C}=0, s \in \mathcal{R}\right\} .
$$

Die regionale Finanzkraftmeßzahl bei einer vollen Anrechnung der Gemeindeeinnahmen

$$
M F_{s}^{C}=T_{L d, s}^{1}+M F_{G m, s},
$$

enthält die Einnahmen der Länder vor der Umsatzsteuerverteilung $T_{L d, s}^{1}$. Im Gegensatz dazu, enthält die regionale Ausgleichsmeßzahl zusätzlich die Umsatzsteueranteile der Länder

\footnotetext{
${ }^{33} \mathrm{DaB}$ in der regionalen AusgleichsmeBzahl vor der Umsatzsteuerverteilung $M A_{s}$ bereits der Anteil aller Länder an den Umsatzsteuern enthalten ist, wird im Text des Gutachtens nicht ersichtlich. Man kann das allerdings aus den Berechnungen ersehen (siehe Wissenschaftlicher Beirat [1992], S.80 bzw. Tabelle 4).
} 
$T_{L d}^{C}{ }^{34}$. Damit ergibt sich als regionale Ausgleichsmeßzahl nach der Vorabverteilung der Umsatzsteuern $^{35}$

$$
M A_{s}=\kappa_{\mathcal{R}, F A}^{L d, s} T_{L d, \mathcal{R}}^{1}+\kappa_{\mathcal{R}, F A}^{G m, s} M F_{G m, \mathcal{R}}+\kappa_{\mathcal{R}}^{s} T_{L d}^{C} .
$$

Der verbleibende Rest des Anteils der Länder an den Umsatzsteuern wird nach Bevölkerungsrelationen auf die anderen Länder verteilt. Die gesamten Umsatzsteuereinnahmen in der Variante II sind dann

$$
T_{s}^{C}=\left\{\begin{array}{ll}
\pi_{E A}^{C} M A_{s}-M F_{s}^{C} & \text { für } s \in \mathcal{R}^{-} \\
\kappa_{\mathcal{R}^{+}}^{s}\left(T_{L d}^{C}-\sum_{r \in \mathcal{R}^{-}} E A_{r}^{C}\right) & \text { für } s \in \mathcal{R}^{+}
\end{array} \quad \text { mit } \pi_{E A}^{C}=\left(\frac{\pi^{S}-\pi^{F}}{1-\pi^{F}}\right)\right.
$$

mit $\pi^{F}$ als Ausgleichssatz im linearen LFA-tarif und $\pi^{S}$ als Mindestausgleichssatz im LFA.

\subsubsection{Horizontaler Finanzausgleich}

Die regionale Finanzkraftmeßzahl unter Einbezug der Umsatzsteuereinnahmen ist

$$
M F_{s}=T_{L d, s}^{F}+T_{G m, s}^{F} .
$$

Die Bevölkerungszahlen können, wahlweise, entweder veredelt oder nicht veredelt werden. Allgemein entspricht das

$$
E W_{s}^{F A}=\left(1+\pi_{s}^{E W}\right) E W_{s} .
$$

Die entsprechenden Gleichungen für die regionale Ausgleichsmeßzahl sind bei vollständiger Anrechnung der Gemeindefinanzkraft

$$
\begin{gathered}
M A_{s}=\kappa_{\mathcal{R}, F A}^{L d, s} M F_{L d, \mathcal{R}}+\kappa_{\mathcal{R}, F A}^{G m, s} M F_{G m, \mathcal{R}} \\
\text { mit } \quad M F_{L d, s}=T_{L d, s}^{F} \quad \text { und } \quad M F_{G m, s}=T_{G m, s}^{F}
\end{gathered}
$$

(vgl. Wissenschaftlicher Beirat [1992], S.78).

Zur genauen Berechnung des Länderfinanzausgleichs schlägt der Beirat drei alternative Verfahren vor. Jede dieser Varianten besitzt das gleiche Grundelement, nämlich einen linearen Umverteilungstarif.

\footnotetext{
${ }^{34}$ Nach Meinung des Beirats sind zur Ermittlung der Finanzkraft „alle Einnahmen einzubeziehen, die ökonomische Dispositionskraft vermitteln“. Ausgeschlossen werden Einnahmen, die „keinen Finanzierungszweck verfolgen oder in den Bundesländern (wie etwa Bußgelder) relativ gleichmäßig anfallen"oder "die Gegenleistungen für öffentliche Leistungen sind“, wie Gebühren und Beiträge. Zusätzlich sollten Steuern, die "lediglich von lokaler Bedeutung sind."nicht angerechnet werden. Alle anderen Gemeindeeinnahmen sind dagegen "voll in den Bund-Länder-Finanzausgleich einzubeziehen"(WISSENSCHAFTLICHER Beirat [1992], S.53-55). Ausnahmen sind die Einnahmen aus den Zuschlägen der Länder und Gemeinden und der Wegfall von Sonderbedarfen (Wissenschaftlicher Beirat [1992], S.100).

${ }^{35}$ Der Index $\mathcal{R}$ bezeichnet wiederum die Summe über alle Bundesländer aus der Menge $\mathcal{R}$.
} 
Variante I (WissenschaftLiChER Beirat [1992], S.79-80)

Die Finanzausgleichszahlungen bewirken einen Ausgleich von $\pi_{v}^{F}$ Prozent der Finanzkraftunterschiede und werden nach einem linearen Tarif ermittelt

$$
F A_{s}^{0}=\pi^{F}\left(M A_{s}-M F_{s}\right) \quad 0,5 \leq \pi^{F} \leq 0.6 .
$$

Dabei soll eine Mindestauffüllmenge in Höhe von $\pi^{S}$ (95) Prozent der regionalen Ausgleichsmeßzahl garantiert werden

$$
M F_{s}^{\min }=\pi^{S} \cdot M A_{s} .
$$

Der entsprechende Fehlbetrag

$$
F E_{s}=\max \left[M F_{s}^{\min }-\left(M F_{s}+F A_{s}^{0}\right) ; 0\right]
$$

kann durch Einsetzen weiter umgeformt werden und wird voll ausgeglichen:

$$
F Z_{s}=F E_{s}=\max \left[\left(\pi^{S}-\pi^{F}\right) M A_{s}-\left(1-\pi^{F}\right) M F_{s} ; 0\right] .
$$

Entsprechend ihrer Fehlbeträge kann man die Länder in die zwei folgenden Teilmengen einteilen:

$$
\mathcal{R}^{-}=\left\{s \mid F E_{s}>0, r \in \mathcal{R}\right\} \quad \mathcal{R}^{+}=\left\{s \mid F E_{s}=0, s \in \mathcal{R}\right\} .
$$

Finanziert werden die Finanzausgleichszuweisungen durch alle Länder deren Fehlbetrag gleich Null ist, und zwar proportional zu ihrer regionalen Finanzkraft, in der Ausgleichszuweisungen oder Ausgleichsbeiträge angerechnet werden. Dem entspricht der Ausdruck

$$
\begin{array}{rlr}
F B_{s} & =\frac{M F_{s}+F A_{s}^{0}}{\sum_{r \in \mathcal{R}^{+}}\left(M F_{\tau}+F A_{\tau}^{0}\right)} \sum_{r \in \mathcal{R}^{-}} F Z_{\tau} \quad \text { für } \mathcal{R}^{+} \\
& =\frac{\left(1-\pi^{F}\right) M F_{s}+\pi^{F} M A_{s}}{\sum_{r \in \mathcal{R}^{+}}\left[\left(1-\pi^{F}\right) M F_{s}+\pi^{F} M A_{s}\right]} \sum_{r \in \mathcal{R}^{-}} F Z_{r} \quad \text { für } \mathcal{R}^{+} .
\end{array}
$$

Als gesamte Finanzausgleichszahlungen der Länder aus $\mathcal{R}^{-}$erhält man dann

$$
\begin{aligned}
F A_{s} & =F A_{s}^{0}+F Z_{s} \\
& =\pi^{S} M A_{s}-M F_{s},
\end{aligned}
$$

und der Länder aus $\mathcal{R}^{+}$

$$
F A_{s}=\pi^{F}\left(M A_{s}-M F_{s}\right)-\frac{\left(1-\pi^{F}\right) M F_{s}+\pi^{F} M A_{s}}{\sum_{r \in \mathcal{R}^{+}}\left[\left(1-\pi^{F}\right) M F_{s}+\pi^{F} M A_{s}\right]} \sum_{r \in \mathcal{R}^{-}} F Z_{\tau}
$$


Variante II (Wissenschaftlicher Beirat [1992], S.80)

Da das Erreichen der Mindestfinanzkraft in dieser Variante durch die Mindestauffüllung in der Umsatzsteuerverteilung garantiert wird, wird im Rahmen des horizontalen Länderfinanzausgleichs nur mehr der lineare Umverteilungstarif verwendet. Demnach erhält man als LFA-Zuweisungen

$$
F A_{s}=\pi^{F}\left(M A_{s}-M F_{s}\right)
$$

Variante III (Wissenschaftlicher Beirat [1992], S.80-81)

Für diese Variante sieht der Beirat eine Mindestauffüllung der regionalen Finanzkraft zwischen der Umsatzsteuerverteilung und dem eigentlichen LFA vor. Die Mindestauffüllung kann dabei alternativ durch den Bund und die Länder (Variante IIIa) oder durch die finanzstarken Länder (Variante IIIb) finanziert werden.

Die zur Mindestfinanzkraft fehlenden Beträge

$$
F E_{s}=\max \left[\pi_{E A}^{C} M A_{s}-M F_{s} ; 0\right]
$$

werden unter Verwendung der Einteilung

$$
\mathcal{R}^{-}=\left\{s \mid F E_{s}>0, r \in \mathcal{R}\right\} \quad \mathcal{R}^{-}=\left\{s \mid F E_{s}=0, s \in \mathcal{R}\right\}
$$

in Variante IIIa von den Ländern ohne Fehlbeträge $\left(s \in \mathcal{R}^{+}\right)$, im Verhältnis ihrer Überschüsse über die Mindestfinanzkraft

$$
F B_{s}=\frac{M F_{s}-\pi_{E A_{A}}^{C} M A_{s}}{\sum_{r \in \mathcal{R}^{+}}\left(M F_{s}-\pi_{E A}^{C} M A_{s}\right)} \sum_{r \in \mathcal{R}^{-}} F Z^{r} \quad \forall s \in \mathcal{R}^{+}
$$

und in Variante IIIb durch den Bund (hier als Bundesergänzungszuweisungen modelliert) und die Länder im Verhältnis der Umsatzsteueranteile von Bund und Ländern aufgebracht. Die Aufteilung der Beiträge auf die Länder, die keine Fehlbeträge besitzen, erfolgt analog zur Darstellung in Variante IIIa in der Form

$$
F B_{s}=\frac{M F_{s}-\pi_{E A}^{C} M A_{s}}{\sum_{r \in \mathcal{R}^{+}}\left(M F_{s}-\pi_{E A^{C}}^{C} M A_{s}\right)} \sum_{r \in \mathcal{R}^{-}} F Z^{r} \quad \forall s \in \mathcal{R}^{+} .
$$

Die finanzschwachen Länder erhalten, zur Anhebung auf die Mindestfinanzkraft, Zuweisungen von den finanzstarken Länder

$$
F Z_{s}=\pi_{L d}^{F} F E_{s}=\pi_{L d}^{F}\left(\pi_{E A}^{C} M A_{s}-M F_{s}\right) \quad \forall r \in \mathcal{R}^{-}
$$


und Ergänzungszuweisungen vom Bund in Höhe von

$$
E Z_{s}=\left(1-\pi_{L d}^{F}\right) F E_{s}=\left(1-\pi_{L d}^{F}\right)\left(\pi_{E A}^{C} M A_{s}-M F_{s}\right) \quad \forall r \in \mathcal{R}^{-}
$$

$\pi_{L d}^{F}$ entspricht dabei dem Anteil der Mindestauffüllung, den die finanzstarken Länder leisten.

Die abschließende Umverteilung erfolgt wiederum gemäß

$$
\begin{gathered}
F A_{s}^{0}=\pi^{F}\left(M A_{s}^{F}-M F_{s}^{F}\right) \\
\operatorname{mit} M F_{s}^{F}=M F_{s}-F B_{s}+F Z_{s}+E Z_{s} \\
\quad \text { und } M A_{s}^{F}=M A_{s}+\kappa_{F A, \mathcal{R}}^{L d, s} E Z_{\mathcal{R}} .
\end{gathered}
$$

Die gesamten Finanzausgleichsbeträge sind dann

$$
F A_{s}=F A_{s}^{0}+F Z_{s}-F B_{s} .
$$

Für die Länder ohne Fehlbetrag $F E_{s}\left(r \in \mathcal{R}^{+}\right)$gilt demnach in beiden Varianten:

$$
\begin{aligned}
F A_{s} & =\pi^{F}\left(M A_{s}-M F_{s}^{F}\right)-F B_{s} \\
& =\pi^{F}\left(M A_{s}-M F_{s}\right)-\left(1-\pi^{F}\right) \frac{\left(M F_{s}-\pi_{E A}^{C} M A_{s}\right)}{\sum_{r \in \mathcal{R}^{+}}\left(M F_{\tau}-\pi_{E A}^{C} M A_{r}\right)} \sum_{r \in \mathcal{R}^{-}} F Z_{r}
\end{aligned}
$$

Für die finanzschwachen Länder $\left(\forall s \in \mathcal{R}^{-}\right)$folgt in Variante IIIa

$$
\begin{aligned}
F A_{s} & =F A_{s}^{0}+F Z_{s} \\
& =\left[\pi^{F}\left(1-\pi_{E A}^{C}\right)+\pi_{E A}^{C}\right] M A_{s}-M F_{s}
\end{aligned}
$$

und in Variante IIIb

$$
\begin{aligned}
F A_{s}= & F A_{s}^{0}+F Z_{s} \\
= & {\left[\pi^{F}\left(1-\pi_{E A}^{C}\right)+\pi_{L d}^{C} \pi_{E A}^{C}\right] M A_{s}-\pi_{L d}^{F} M F_{s} } \\
& \quad+\pi^{F} \kappa_{F A, \mathcal{R}}^{L d, s} E Z_{\mathcal{R}} .
\end{aligned}
$$

Die Bundesergänzungszuweisungen sollen in Variante IIIb die neuen Bundesländer auf die Garantiehöhe der Finanzkraft heben. Dagegen werden in den anderen Varianten die alten Bundesländer für ihre zusätzlichen Lasten durch die BEZ kompensiert. Weitere Ergänzungszuweisungen sollten nur bei Verschuldungs- und Strukturkrisen, und auch dann nur zweckgebunden, d.h. mit Verwendungsauflage, eingesetzt werden. Das Gesamtvolumen sollte nicht mehr automatisch an das Umsatzsteueraufkommen gebunden werden (WisSENSCHAFtLiChER BeIRAT [1992], S.110-112). 


\subsection{Der Vorschlag von Buhl/Pfingsten [1991]}

Buhl/Pfingsten [1991, 1990, 1986] und Buhl [1986] schlagen, ausgehend von den von ihnen entwickelten "Zehn Geboten für Finanzausgleichsverfahren", ein allgemeines Verfahren für einen Finanzausgleich vor, das nur zwei Umverteilungsschlüssel vorsieht.

Die LFA-Zuweisungen oder -Beiträge werden nach einem linearen Tarif berechnet. Sie werden auf Grundlage der Differenz zwischen der durchschnittlichen Finanzkraft (regionale Ausgleichsmeßzahl) und der regionalen Finanzkraft ermittelt, die zu $\pi_{v}^{F}$ Prozent ausgeglichen wird. Formalisiert entspricht das dem Ausdruck

$$
F A_{s}=\pi^{F}(\underbrace{\kappa_{\mathcal{R}}^{s} M F_{\mathcal{R}}}_{M A_{s}}-M F_{s}) .
$$

Die Bevölkerungsgrößen können dabei die veredelten Einwohnerzahlen sein. Die regionale Finanzkraft müßte wohl die normierten Gemeindeeinnahmen voll enthalten.

Die von außen kommenden Zuschüsse $E Z$ sollen nur nach Bedarfsverhältnissen (hier: Bevölkerungsanteilen) verteilt werden. Dies ergibt:

$$
E Z_{s}=\kappa_{\mathcal{R}}^{s} E Z
$$

Die gesamten Einnahmen der Region $s$ nach LFA und Ergänzungszuweisungen belaufen sich damit auf (siehe Buhl/Pfingsten [1991], S.484)

$$
\left(M F_{s}+F A_{s}+E Z_{s}\right)=\left(1-\pi^{F}\right) M F_{s}+\pi^{F} \kappa_{\mathcal{R}}^{s} M F_{\mathcal{R}}+\kappa_{\mathcal{R}}^{s} E Z .
$$

Die gesamte regionale Finanzkraft eines Landes nach Länderfinanzausgleich setzt sich zusammen aus der regionalen Finanzkraft vor Finanzausgleich $M F_{s}$, vermindert um die daraus geleisteten Zahlungen an andere Länder, zuzüglich der von den anderen Ländern empfangenen Zuweisungen und der Anteile an den Zuschüssen anderen Institutionen (hier: des Bundes).

\subsection{Der Vorschlag von Föttinger/Spahn [1993]}

FÖTtINGER/SPAhN ([1993], S.240-241) kritisieren die geltenden Regelungen und alle Neuvorschläge vor allem deshalb, weil diese durch die Zugrundelegung der Bevölkerungshöhe als Bedarfsgröße implizit unterstellen, daß kaum Kostenunterschiede in der Bereitstellung der öffentlichen Leistungen bestehen. Deshalb zielen die normalen Regelungen nur auf einen weitgehenden Ausgleich der Pro-Kopf zur Verfügung stehenden Finanzmittel 
ab. Ihrer Meinung nach gibt es jedoch erhebliche Unterschiede in den Kostenfunktionen der Produktion öffentlicher Güter, die dazu führen, daß bei annähernd gleicher Finanzausstattung unterschiedliche Mengen an öffentlichen Gütern bereitgestellt werden. Aus diesem Grund fordern sie, den Finanzausgleich an der realen regionalen Finanzkraft zu orientieren, d.h. unterschiedliche Kostenstrukturen zu berücksichtigen (FöTTINGER/SPAHN [1993], S.241). Grundsätzlich empfehlen sie die die Beibehaltung der „klassischen” Einteilung in Umsatzsteuerverteilung, Länderfinanzausgleich und einer „bedarfsorientierten Zusatzausstattung"(FöTtINGER/SPAHN [1993], S.241).

Die Verteilung der Umsatzsteuern soll sich nur an den Bevölkerungsrelationen orientieren (FötTinger/SPAHN [1993], S.241)

$$
T_{s}^{C}=\kappa_{\mathcal{R}}^{s} T_{L d}^{C} .
$$

Im Länderfinanzausgleich soll eine „reale Finanzkraft"berücksichtigt werden, die sich durch die Gewichtung der nominalen regionalen Finanzkraftmeßzahl $M F_{s}$ mit einem Korrekturfaktor $\alpha_{s}^{F}$ ergibt. Der Korrekturfaktor wird auf folgende Weise ermittelt (FöTTINGER/SPAHN [1993], S.242-243): Für jede Art $i, j \in \mathcal{I}$ der öffentlichen Leistungen, mit Prei$\operatorname{sen} q_{i}^{s}$, wird in der Region $s \in \mathcal{R}$ ein "Durchschnittspreis" $\bar{q}_{s}$ berechnet. Zusätzlich wird, in der hier präsentierten Form, der Konsistenz halber unterstellt, daß die Einwohnerzahlen nicht veredelt werden. Als Preisindex erhält man

$$
\begin{array}{cl}
\bar{q}_{i}=\sum_{r \in \mathcal{R}}\left(q_{i}^{s} \theta_{\mathcal{R}}^{r}\right) & \forall i \in \mathcal{I}, \\
\text { mit } & \theta_{\mathcal{R}}^{s}= \begin{cases}\kappa_{\mathcal{R}}^{s} & \text { in Variante I } \\
G_{s} / \sum_{\tau \in \mathcal{R}} G_{r} & \text { in Variante II . }\end{cases}
\end{array}
$$

In Variante I erfolgt die Gewichtung anhand der Bevölkerungsrelationen und in Variante II gemäß der relativen Höhe der öffentlichen Leistungen. ${ }^{36}$

Bildet man den relativen Anteil der Ausgaben für eine Art der öffentlichen Leistungen $i$ an allen öffentlichen Ausgaben

$$
\lambda_{i}^{s}=\frac{q_{i}^{s} G_{i}^{s}}{\sum_{j \in \mathcal{I}} q_{j}^{s} G_{j}^{s}} \quad \sum_{i \in \mathcal{I}} \lambda_{i}^{s}=1 \quad \forall i \in \mathcal{I},
$$

\footnotetext{
${ }^{36}$ FötTINGER/SPAHN ([1993], S.242, Fußnote 12) schlagen alternativ beide Gewichtungsfaktoren vor, wobei sie der Gewichtung mit Bevölkerungsverhältnissen den Vorzug geben, da diese, wie die Autoren betonen, gegenüber der Gewichtungen durch Mengen- oder Ausgabenrelationen, den Vorteil besitzt, daß sie "der direkten Einflußnahme der Budgetierenden entzogen ist ${ }^{\text {. }}$.
} 
dann erhält man durch Gewichtung mit $\lambda_{\mathcal{R}}^{s}$ den durchschnittlichen Anteil der Ausgabenart $i$ an den Budgets der Länder als

$$
\bar{\lambda}_{i}=\sum_{r \in \mathcal{R}}\left(\lambda_{i}^{r} \theta_{\mathcal{R}}^{r}\right) \quad \forall i \in \mathcal{I}
$$

Der endgültige Gewichtungsfaktor der nominalen regionalen Finanzkraft ist dann

$$
\alpha_{s}^{F}=1 / \sum_{i \in \mathcal{I}} \frac{q_{i}^{s}}{\bar{q}_{i}} \bar{\lambda}_{i} .
$$

Dies bedeutet im einzelnen, daß die regionale Finanzkraft um so geringer gewertet wird, je stärker die regionalen Ausgaben für die durchschnittliche Menge an öffentlichen Ausgaben von den länderdurchschnittlichen Ausgaben abweicht. In diesem Fall ist der Finanzbedarf des Landes relativ hoch.

Die LFA-Zuweisungen bzw. -beiträge ergeben sich mittels eines linearen Ausgleichssatzes $\pi^{F}$ aus der Differenz der realen regionalen Ausgleichsmeßzahl eines Landes mit seiner realen regionalen Finanzkraft und lauten (FöTTINGER/SPAHN [1993], S.243)

$$
F A_{s}=\pi^{F}\left(M A_{s}-\alpha_{s}^{F} M F_{s}\right) \quad \operatorname{mit} M A_{s}=\kappa_{\mathcal{R}}^{s} \sum_{\tau \in \mathcal{R}}\left(\alpha_{\tau}^{F} M S^{\tau}\right)
$$

\subsection{Der Vorschlag von Fuest/Lichtblau [1991]}

FUest/LichtblaU [1991] fordern eine sich weitgehend mit den Vorschlägen des Sachverständigenbeirats und Wissenschaftlichen Beirats deckende Neugestaltung des Finanzausgleichs. ${ }^{37}$

Die Umsatzsteuer soll nach Meinung der Autoren ausschließlich nach der Relation der Volkseinkommen (NSP zu Faktorkosten) verteilt und der Umsatzsteuervorwegausgleich ersatzlos gestrichen werden (FUEST/LichtBLAU [1991], S.50)

$$
T_{s}^{C}=T_{L d}^{C}\left(Y_{s} / \sum_{r \in \mathcal{R}} Y_{r}\right)
$$

Durch Abschaffung der Garantieklauseln und Einführung eines linearen (oder stetig progressiven) Tarifs sollen das Verfahren des LFA und die daraus entstehenden Vertei-

\footnotetext{
${ }^{37}$ Der Vorschlag zur Korrektur der Lohnsteuerzerlegung sieht eine zu je $50 \%$ nach dem Wohnort und dem Betriebsstättensitz durchgeführte Zerlegung der Steuraufkommen vor (Fuest/LichtBLAU [1991], S.52). Dies ist in dem hier verwendeten Ansatz jedoch nicht relevant, und wird daher nicht näher spezifiziert.
} 
lungswirkungen überschaubarer werden (Fuest/LichtBLAU [1991], S.51). ${ }^{38}$ Die LFAZuweisungen oder -Beiträge ergeben sich dann als

$$
F A_{s}=\pi^{F}\left(M A_{s}-M F_{s}\right) \quad \text { mit } \quad \pi^{F} \approx 0.6,
$$

mit $\pi^{F}$ als linearer Umverteilungssatz.

Da der Tarif jedoch keine Mindesthöhe der durchschnittlichen Finanzkraft garantiert, soll notfalls (!) der Bund durch allgemeine BEZ einspringen (FUEST/Lich TBLAU [1991], S.55). Diese könnten beispielsweise aus einem $\pi^{E Z}$-prozentigen Ausgleich des Fehlbetrages zwischen der regionalen Ausgleichsmeßzahl und der regionalen Finanzkraft nach LFA bestehen. Dies entspricht dem Ausdruck

$$
E Z_{s}=\max \left[\pi^{E Z} M A_{s}-\left(M F_{s}+F A_{s}\right) ; 0\right] .
$$

Eine zweite Form der BEZ soll nur als zweckgebundene Zuweisung für spezielle Ausgaben vergeben werden (FUEST/LichtBlaU [1991], S.55). Diese wird hier nicht näher spezifiziert, da die Autoren keine genaueren Vorgaben liefern.

\subsection{Der Vorschlag eines Verzichts auf die Umverteilung}

Ein völliger Verzicht auf die horizontalen Umverteilungselemente im Finanzausgleichssystem würde den Extremfall der Steuerverteilung darstellen, indem die Disparität zwischen den Bundesländern am höchsten bliebe.

Die Verteilung der Einkommens-, Körperschafts-, Produktions- und Gewerbesteuern wird nicht verändert, sondern weiterhin nach Maßgabe der gesetztlichen Regelungen durchgeführt.

Die Umsatzsteuer soll nur nach Bevölkerungsanteilen verteilt werden. Damit verändert sich die entsprechende Gleichung der Umsatzsteuereinnahmen (I.13) zu

$$
T_{s}^{C}=\kappa_{\mathcal{R}}^{s} T_{L d}^{C}
$$

Schließlich wird angenommen, daß weder ein Länderfinanzausgleich noch Bundesergänzungszuweisungen vorgesehen sind.

\footnotetext{
${ }^{38}$ Zusätzlich wird unterstellt, daß keine Veredelung stattfindet, obwohl sich die Autoren dazu nicht äußern.
} 


\section{Zusammenfassung}

Im Prinzip könnten die einzelnen Vorschläge aus den geltenden Regelungen dadurch gewonnen werden, daß nur die Werte der einzelnen Parameter verändert werden. Man könnte daher ein allgemeines Finanzausgleichssystem darstellen, aus dem sämtliche Vorschläge abgeleitet werden. Dazu müßten in der Darstellung der geltenden Regelungen einige $\mathrm{Pa}$ rameter zusätzlich eingeführt werden, wie z.B. ein Gewichtungsfaktor für die Finanzkraft $\alpha_{s}^{F}$, der in allen Vorschlägen außer dem von FöTTINGER/SPAHN [1993] gleich eins wäre. ${ }^{39}$ In der Tabelle (I.4) werden die Werte der einzelnen Parameter in den verschiedenen Vorschlägen dargestellt.

\footnotetext{
${ }^{39}$ Einige Besonderheiten, wie z.B. die Garantieklausel zur Einhaltung der Finanzkraftreihenfolge könnte man durch einen zusätzlichen Parameter mit dem Wert 1 einfangen. Wird diese Garantieklausel nicht verwendet, dann setzt man den Wert einfach auf Null. Diese zusätzlichen Parameter werden allerdings in der nachfolgenden Tabelle nicht berücksichtigt. Sie könnten jedoch ohne allzuviel Aufwand eingeführt werden.
} 
I Das bundesdeutsche Finanzausgleichssystem

\begin{tabular}{|c|c|c|c|c|c|c|c|c|c|c|}
\hline & & $<95^{a}$ & $>95^{b}$ & $\mathbf{W}-\mathbf{1}^{c}$ & $\mathbf{W}-2^{d}$ & $\mathbf{W}-3^{e}$ & $\mathbf{B} / \mathbf{P}^{f}$ & $\mathbf{F} / \mathbf{L}^{g}$ & $\mathbf{F} / \mathbf{S}^{h}$ & $\mathrm{Um}^{i}$ \\
\hline \multirow[t]{3}{*}{$\overline{\mathrm{UmSt}: T_{s}^{C}}$} & $\pi_{L d}^{C}$ & 0,37 & 0,44 & $?$ & $?$ & $?$ & 0,44 & $?$ & $?$ & $?$ \\
\hline & $\pi_{V A}^{C}$ & 0,75 & 0,75 & 1 & 0 & 1 & 0,75 & 1 & 1 & 1 \\
\hline & $\pi_{E A}^{C}$ & 0,92 & 0,92 & 0 & $\approx 0,88$ & 0 & 0,92 & 0 & 0 & 0 \\
\hline \multirow[t]{17}{*}{ LFA: $F A_{s}$} & $\pi_{G m}^{F A}$ & 0,5 & 0,5 & 1 & 1 & 1 & 1 & 1 & 1 & 0 \\
\hline & $\pi_{L d, s}^{E W}, \pi_{G m, s}^{E W}$ & $\geq 1$ & $\geq 1$ & $?$ & $?$ & $?$ & $?$ & 1 & $?$ & 0 \\
\hline & $\alpha_{s}^{F}$ & 1 & 1 & 1 & 1 & 1 & 1 & 1 & $\lesseqgtr$ & 0 \\
\hline & $\pi^{M}$ & 0,92 & 0,92 & 1 & 1 & 1 & 1 & 1 & 1 & 0 \\
\hline & $\pi^{V}$ & 1 & 1 & $\pi^{F}$ & $\pi^{F}$ & $\pi^{F}$ & $\pi^{F}$ & $\pi^{F}$ & $\pi^{F}$ & 0 \\
\hline & $\pi^{N}$ & 0,375 & 0,375 & 0 & 0 & 0 & 0 & 0 & 0 & 0 \\
\hline & $\pi^{S}$ & 0,95 & 0,95 & 0,95 & 0 & 0,95 & 0 & 0 & 0 & 0 \\
\hline & $\pi^{F}$ & {$\left[\pi^{\max }\right.$} & {$\left[\pi^{\max } ;\right.$} & 0,55 & 0,55 & 0,55 & $?$ & $\approx 0,6$ & $?$ & 0 \\
\hline & & $\left.\begin{array}{l}\pi^{m i t} \\
\pi^{m i n}\end{array}\right]$ & $\mid \begin{array}{l}\pi^{m i t} \\
\pi^{m i n}\end{array}$ & & & & & & & \\
\hline & $\pi^{\max }$ & 1 & 0,8 & $\pi^{F}$ & $\pi^{F}$ & $\pi^{F}$ & $\pi^{F}$ & $\pi^{F}$ & $\pi^{F}$ & 0 \\
\hline & $\pi^{m i t}$ & 0,7 & 0,66 & 0 & 0 & 0 & 0 & 0 & 0 & 0 \\
\hline & $\pi^{\min }$ & 0 & 0,15 & 0 & 0 & 0 & 0 & 0 & 0 & 0 \\
\hline & $\pi^{1}$ & 1,02 & 1,01 & 1 & 1 & 1 & 1 & 1 & 1 & 0 \\
\hline & $\pi^{2}$ & 1,1 & 1,1 & 1 & 1 & 1 & 1 & 1 & 1 & 0 \\
\hline & $\pi_{Z}^{F}$ & 1 & 0,5 & 1 & 0 & 1 & 0 & 0 & 0 & 0 \\
\hline & $\pi_{1}^{F}$ & 1 & 0,25 & 0 & 0 & 0 & 0 & 0 & 0 & 0 \\
\hline & $\pi_{L d}^{F}$ & 1 & 1 & 1 & 0,5 & 1 & 1 & 1 & 1 & 0 \\
\hline \multirow[t]{2}{*}{ BEZ: $E Z_{s}$} & $\pi_{\tau}^{E Z}$ & $\geq 0$ & $\geq 0$ & $\geq 0$ & $\geq 0$ & $\geq 0$ & $\geq 0$ & $\geq 0$ & $\geq 0$ & 0 \\
\hline & $\pi^{E Z}$ & 0,2 & 0,2 & $?$ & $?$ & $?$ & $?$ & ? & $?$ & 0 \\
\hline
\end{tabular}

${ }^{a}$ Regelungen vor 1995

${ }^{\circ}$ Regelungen nach 1995

${ }^{c}$ Wissenschaftlichen Beirat [1992], Variante I

${ }^{d}$ Vorschlag des Wissenschaftlichen Beirats [1992], Variante II

${ }^{e}$ Wissenschaftlichen Beirat [1992], Variante III

${ }^{f}$ Buhl/PFingsten [1991]

${ }^{g}$ FUEST/LichtBLAU [1991]

${ }^{h}$ FötTINGER/SPAHN [1993]

${ }^{i}$ Die Abschaffung der Umverteilungselemente.

Tabelle I.4: Vorschläge und Regelungen des Länderfinanzausgleichs 


\section{Kapitel II}

\section{Theoretische Grundlagen}

\section{Einleitung}

Nach der ausführlichen Darstellung des bundesdeutschen Finanzausgleichssystems werden nun die Effizienzaspekte von Finanzausgleichsregelungen mit Hilfe einer Partialanalyse analysiert, die im nächsten Kapitel durch eine allgemeine Gleichgewichtsanalyse ergänzt wird.

Bereits die Partialanalyse macht deutlich, daß Finanzausgleichsregelungen verzerrende Wirkungen auslösen können, deren Art und Ảusmaß zum einen von der genauen Gestaltung der jeweiligen Regelungen und zum anderen von der Struktur des föderalen Systems, d.h. vom Grad der regionalen Finanzautonomie abhängt.

Im einzelnen wird folgendes Vorgehen gewählt:

- In einem ersten Schritt wird die Budgetgleichung eines regionalen öffentlichen Haushalts unter die Lupe genommen. Dabei wird gezeigt, daß Finanzausgleichssysteme (FA-Systeme) die Anreize zur Ausschöpfung der regionalen Steuerquellen beeinflussen können.

Dies wird dann anhand des bundesdeutschen FA-Systems konkretisiert.

- Anschließend wird dieser Ansatz zu einem einfachen Entscheidungsmodell erweitert, in das die obige Budgetgleichung als Nebenbedingung eingeht. Die regionalen Regierungen werden in diesem zweiten Schritt als Maximierer der indirekten Nutzenfunktion ihres repräsentativen regionalen Haushalts modelliert.

Im Rahmen dieser Implementierung können regionale Entscheidungen über die Menge der bereitgestellten öffentlichen Leistungen und über die Höhe der Transfers an den privaten Haushalt, der Lump-Sum-Steuern oder sonstiger Steuersätze untersucht werden.

Mit Hilfe dieser Partialanalyse, in der weder Preiseffekte noch interregionale Interdependenzen berücksichtigt werden, können Auswirkungen von Finanzausgleichsregeln auf die relativen Güterpreise bzw. die optimalen Steuersätze bestimmt werden.

Die Ergebnisse der Partialanalyse sind, wie sich zeigen wird, vom Grad der regionalen Finanzautonomie und der genauen Gestaltung des Finanzausgleichssystems 
abhängig. Der Einfluß der Finanzautonomie schlägt sich in einer Dreiteilung der Analyse nieder:

1.) Als erstes wird angenommen, daß die Regionen keine Finanzautonomie besitzen und sich daher veränderte Einnahmen vollständig in Anpassungen der öffentlichen Leistungen niederschlagen.

Die in diesem Fall auftretenden preisverzerrenden Wirkungen von Finanzausgleichsregelungen werden unter Verwendung des Rationierungsansatzes von NEARY und ROBERTS [1980] herausgearbeitet.

2.) Als zweite Form der Finanzautonomie wird eine Ausgabenautonomie mit teilweiser Einnahmenautonomie implementiert.

Unter Ausgabenautonomie wird dabei die Möglichkeit einer Gebietskörperschaft ${ }^{1}$ verstanden, die Höhe ihrer öffentlichen Leistungen und ihrer Transfers frei zu wählen. Da explizit auch „negative” Transfers zugelassen sind, die einer Lump-Sum-Steuer entsprechen, wird zusätzlich von einer eingeschränkten Einnahmenautonomie gesprochen. Die Veränderung anderer Steuerarten ist den Regionen dagegen verwehrt.

Für diese Form der Modellierung werden mit Hilfe der Theorie der Finanzzuweisungen die Effekte unterschiedlicher Formen von FA-Regelungen untersucht. Unter Beachtung der Ergebnisse dieser Analyse wird anschließend das FA-System der Bundesrepublik genauer analysiert.

3.) Schließlich wird noch der Fall der vollständigen Finanzautonomie untersucht. Neben der freien Wahl der Einnahmenverwendung können in diesem Fall die regionalen Regierungen auch über ihre Einnahmenerzielung frei entscheiden, indem sie die Höhe ihrer Steuersätze bestimmen. Dies führt direkt zur Theorie der optimalen Besteuerung, die an dieser Stelle auch bezüglich der Auswirkungen von FA-Regelungen auf die Höhe der optimalen Steuersätze ergänzt wird.

\section{Partialanalyse: Regionale Budgetgleichungen}

Im folgenden Abschnitt wird eine einfache Untersuchung der regionalen öffentlichen Budgets durchgeführt, wie sie auf Musgrave [1961] und Bös [1978] zurückgeht. Dabei kann die Bedeutung der speziellen Ausgestaltung von Finanzausgleichsregelungen für die Anreize zur Ausschöpfung der regionalen Steuerquellen herausgearbeitet werden.

\footnotetext{
${ }^{1}$ Die Begriffe Region, Land und Gebietskörperschaft werden synonym verwendet. Im Fall der Bundesrepublik werden damit die Bundesländer - teilweise incl. aller Gemeinden - bezeichnet.
} 
Anschließend wird das bundesdeutsche Finanzausgleichssystem auf diese Anreizwirkungen hin genauer betrachtet.

\subsection{Analyse des öffentlichen Budgets}

Betrachtet man das Budget einer Gebietskörperschaft, dann kann man zeigen, welchen Einfluß steigende regionale Steueraufkommen bei vorhandenen Finanzausgleichsregelungen auf die Höhe der regionalen Einnahmen und damit auf die Höhe der regionalen Ausgaben besitzen.

Eine einfache Budgetgleichung, in der die öffentlichen Ausgaben für die öffentlichen Güter $G$ mit den Preisen $q_{G}$ durch die Einnahmen aus der Steuerverteilung $T$ und einem Länderfinanzausgleich $F A$ finanziert werden, besitze folgende Form:

$$
q_{G} G=T(S T)+F A(T(S T)),
$$

mit $S T$ als regionale Steueraufkommen, die die Grundlage zur Ermittlung der Steuerverteilungs- und LFA-Zuweisungen sind.

Ein marginaler Anstieg der regionalen Steueraufkommen $S T$ aufgrund exogener Einflüsse (z.B. Wirtschaftswachstum) führt zu einer unter- bzw. überproportionalen Erhöhung der regionalen öffentlichen Ausgaben, falls die dadurch bewirkte Veränderung der Einnahmen größer bzw. kleiner als eins ist. Die entsprechende Gleichung lautet

$$
\frac{\partial q_{G} G}{\partial S T}=\left(1+\frac{\partial F A}{\partial T}\right) \frac{\partial T}{\partial S T} \gtreqless 1 .
$$

Steigen die regionalen Ausgaben stärker als die regionalen Steueraufkommen (>1), so spricht Bös ([1978], S.106) in Anlehnung an Musgrave [1961] von einer Komplementaritätswirkung der Finanzausgleichsregelungen, ist er gleich eins, von Neutralität und ist er kleiner als eins, von einer Substitutionalitätswirkung. ${ }^{2}$

Bei einer substitutionalen Beziehung $(<1)$ zwischen Steueraufkommen und Finanzausgleichs-Zuweisungen kann das Land seine Ausgaben nur um einen Teil seiner eigenen Steueraufkommenszuwächse erhöhen. Damit steigen die own-cost des regionalen öffentlichen Gutes (Musgrave [1961], S.100). Dies senkt den Anreiz zur Ausschöpfung der

\footnotetext{
${ }^{2}$ Bös ([1978], S.105) bezeichnet dies sogar als Allokationseffekte in Form eines Einkommens- und Substitutionseffektes (siehe auch KuHN [1991/92], S.221). Diese Definition ist meines Erachtens miBverständlich, da Einkommens- und Substitutionseffekte in der ökonomischen Tradition im Zusammenhang mit Entscheidungen von Wirtschaftssubjekten definiert sind. Eine weitere Alternative wäre die Bezeichnung 'Einnahmen-Substitutions-Effekt', die von PEFFEKoven ([1980], S.623) eingeführt und von Fischer ([1988], S.35) aufgegriffen wurde.
} 
regionalen Steuerquellen, da ein Teil der zusätzlichen Aufkommen an andere Regionen oder die Zentralregierung abgegeben werden muß. Im Extremfall, in dem vollständige Substitutionalität besteht, bleiben die regionalen Einnahmen trotz höherer Steueraufkommen unverändert, und es besteht keinerlei Anreiz zur Ausschöpfung der regionalen Steuerquellen durch die jeweilige Gebietskörperschaft.

Besteht dagegen Komplementarität ( $>1)$, dann kann die Gebietskörperschaft ihre Ausgaben über den Zuwachs der eigenen Steueraufkommen hinaus ausdehnen, da sie zusätzliche Zuweisungen erhält. Dies entspricht einer zunehmenden Fremdfinanzierung der öffentlichen Ausgaben der Region und damit einer Verbilligung der öffentlichen Güter für das Land. In diesem Fall besteht für die Regionen ein Anreiz, ihre eigenen Steuerquellen möglichst auszuschöpfen, d.h. die Steueraufkommen zu erhöhen, da sie dadurch ihre regionalen Einnahmen überproportional erhöhen können.

Damit besitzt der Grad der Substitutionalität einen entscheidenden Einfluß auf die regionalen Anstrengungen zur Ausschöpfung ihrer Steuerquellen z.B. in Form einer strengen Verfolgung von Steuersündern. Die Frage nach dem Substitutionalitätscharakter der Finanzausgleichsregelungen $T(S T)$ und $F A(T(S T))$ ist letztlich nur unter genauer Kenntnis dieser Regeln zu beantworten. Aus diesem Grund wird als nächstes der Grad der Substitutionalität im bundesdeutschen Finanzausgleichssystem betrachtet. ${ }^{3}$

\subsection{Substitutionalität im bundesdeutschen Finanzausgleich}

Aufgrund der Komplexität der bundesdeutschen Finanzausgleichsregelungen (siehe Abschnitt 3.) ist es nicht möglich, die Substitutionalitätsbeziehungen in Form von Ableitungen der Finanzausgleichsformeln analytisch exakt zu ermitteln, und damit allgemeine Zusammenhänge zwischen Steueraufkommen und Einnahmen der Länder und Gemeinden abzubilden. Dies wäre im Prinzip notwendig, um die Anreizwirkungen des Finanzausgleichssystems in allgemeiner Form zu untersuchen.

Um sich dennoch ein Bild von den Anreizwirkungen machen zu können, wird hier pragmatisch vorgegangen und eine einfache empirische Betrachtung vorgenommen. Unter Verwendung des Datensatzes von GotTFried/WiEgard [1992] für die ersten drei Quartale von $1991^{4}$ werden die Veränderungen in den Einnahmen der Länder und Gemeinden ermittelt, die bei einer Erhöhung der Steueraufkommen um 1 Mio DM resultieren. Als Er-

\footnotetext{
${ }^{3}$ In der Literatur werden eine Reihe von Vorschlägen präsentiert, die speziell auf eine Verbesserung der Anreizwirkungen abzielen. Dies sind z.B. die Empfehlungen von Thurow [1970], LEGRAND [1975] oder auch Mathews [1975/76]. Ein Überblick über diese Vorschläge findet sich bei FisCHER [1988].

${ }^{4}$ Dieser wurde ergänzt um die Gewerbe- und Grundsteuereinnahmen der Ostländer.
} 
gebnis erhält man jeweils den Betrag in Mio DM, der den Ländern von einer zusätzlichen Mio DM an Steueraufkommen als Einnahmen zufließt. Ist dieser relativ groß, dann ist die Anreizwirkung zur Ausschöpfung der regionalen Steuerquellen recht hoch, ansonsten relativ gering. In den nachfolgenden Tabellen wird dies dokumentiert. In ihnen werden allerdings nur die Intervalle ausgewiesen, innerhalb der sich die aus einer Zunahme der Steueraufkommen ergebenden zusätzlichen Einnahmen aller Bundesländer bewegen.

\subsubsection{Umsatzsteuerverteilung}

Eine isolierte Betrachtung der Umsatzsteuerverteilung zeigt (Tabelle II.1), daß in allen Fällen in denen keine horizontalen Umverteilungselemente vorgesehen sind (Abschaffung des LFA, Wissenschaftlicher Beirat I und III sowie Fuest/Lichtblau), die Einnahmen aus der Umsatzsteuerverteilung nur von den Umsatzsteueraufkommen (dritte Spalte) beeinflußt werden. Von 1 Mio DM zusätzlicher Umsatzsteueraufkommen erhalten die Länder zwischen 0 und 0,11 Mio DM als zusätzliche Einnahmen. Die genaue Größe hängt von der relativen Bevölkerungsgröße bzw. bei FuEST/LichtBLAU vom relativen Volkseinkommen ab.

Eine Erhöhung der Aufkommen aus den sonstigen Ländersteuern (Spalte 2) senkt die Einnahmen aus der Umsatzsteuerverteilung teilweise um fast den gesamten Betrag. Ähnliches gilt bezüglich der Aufkommen aus den Einkommenssteuern (Spalte 3 bzw. 4), deren Einnahmen nach der Einkommenssteuerverteilung (0,575 bzw. 0,5 Mio DM von 1 Mio DM) in manchen Fällen fast vollständig durch sinkende Einnahmen aus der Umsatzsteuerverteilung nivelliert werden.

Höhere Einnahmen aus den Grund- und Gewerbesteuern werden nur in der II.Variante des Wissenschaftuichen Beirats für die Umsatzsteuerverteilung relevant, da sie dort in der Finanzkraft berücksichtigt werden. Ihre Auswirkung auf die Einnahmen aus der Umsatzsteuerverteilung ist zum Teil recht erheblich.

\subsubsection{Länderfinanzausgleich}

Ein Anstieg der Aufkommen der unterschiedlichen Steuerarten um 1 Mio DM reduziert in vielen Fällen die Einnahmen aus dem LFA recht deutlich. Es kann sich sogar eine fast vollständige Neutralisierung des Aufkommensanstiegs durch verminderte LFA-Einnahmen ergeben (siehe Tabelle II.2).

Es fällt u.a. auf, daß die LFA-Einnahmenverluste bei höheren Umsatzsteueraufkommen verhältnismäßig gering ausfallen. Dies liegt daran, daß als Folge der nivellierenden Effekte 


\begin{tabular}{|c|c|c|c|c|c|}
\hline \multirow[b]{2}{*}{ Vorschlag } & \multicolumn{5}{|c|}{$\begin{array}{c}\text { Intervalle der Veränderung der Zuweisungen } \\
\text { aus der Umsatzsteuerverteilung in Mio DM } \\
\text { bei } 1 \text { Mio DM zusätzlichen Aufkommen aus den }\end{array}$} \\
\hline & LSt. $^{a}$ & UmSt..$^{b}$ & EkSt. ${ }^{c}$ & $\mathrm{KSt}^{d}$ & GSt. ${ }^{e}$ \\
\hline vor 1995 ohne $\mathrm{OL}^{f}$ & {$[-0,98 ; 0,00]$} & {$[0,00 ; 0,07]$} & {$[-0,42 ; 0,00]$} & {$[-0,49 ; 0,00]$} & {$[-0,05 ; 0,00]$} \\
\hline vor $1995 \mathrm{mit} \mathrm{OL}^{g}$ & {$[-0,05 ; 0,00]$} & {$[0,00 ; 0,11]$} & {$[-0,02 ; 0,00]$} & {$[-0,02 ; 0,00]$} & 0,00 \\
\hline ab 1995 & {$[-0,42 ; 0,00]$} & {$[-0,49 ; 0,00]$} & {$[-0,39 ; 0,00]$} & {$[-0,46 ; 0,00]$} & {$[-0,04 ; 0,00]$} \\
\hline WISS.BEIRAT I & 0,00 & {$[0,00 ; 0,10]$} & 0,00 & 0,00 & 0,00 \\
\hline Wiss.BEIRAT II & {$[-0,99 ;-0,01]$} & {$[0,00 ; 0,10]$} & {$[-0,57 ;-0,01]$} & {$[-0,50 ;-0,01]$} & {$[-0,96 ;-0,01]$} \\
\hline Wiss.BEIRAT IIIa & 0,00 & {$[0,00 ; 0,10]$} & 0,00 & 0,00 & 0,00 \\
\hline Wiss.BEIRAT IIIb & 0,00 & {$[0,00 ; 0,10]$} & 0,00 & 0,00 & 0,00 \\
\hline Buhl/Pfingsten & {$[-0,91 ; 0,00]$} & {$[0,00 ; 0,16]$} & {$[-0,39 ; 0,00]$} & {$[-0,46 ; 0,00]$} & {$[-0,04 ; 0,00]$} \\
\hline FötTINGER/SPAHN & {$[-0,91 ; 0,00]$} & {$[0,00 ; 0,16]$} & {$[-0,39 ; 0,00]$} & {$[-0,46 ; 0,00]$} & {$[-0,04 ; 0,00]$} \\
\hline FuEst/Lichtblau & 0,00 & {$[0,01 ; 0,11]$} & 0,00 & 0,00 & 0,00 \\
\hline Abschaffung LFA & 0,00 & {$[0,00 ; 0,10]$} & 0,00 & 0,00 & 0,00 \\
\hline
\end{tabular}

${ }^{a}$ Ländersteuern: z.B. Vermögenssteuern.

${ }^{b}$ Umsatzsteuern.

${ }^{c}$ Lohnsteuer und veranlagte Einkommenssteuern.

${ }^{d}$ Kapitalertrags- und Körperschaftssteuern.

${ }^{e}$ Steuermeßbetrag der Grund- und Gewerbesteuer - nicht vom Aufkommen der Steuerart.

${ }^{f}$ Vor 1995 ohne Einbezug der Ostländer.

${ }^{g}$ Regelungen vor 1995 mit Einbezug der Ostländer.

Tabelle II.1: Veränderung der Einnahmen durch die Umsatzsteuerverteilung

der Umsatzsteuerverteilung die Länderfinanzkraft vor dem LFA nur um einen Bruchteil der zusätzlichen Umsatzsteueraufkommen steigt.

In dem Vorschlag von FÖTTINGER/SPAHN [1993] tritt unter Umständen noch ein zusätzlicher Effekt auf, der hier allerdings nicht dokumentiert wird. Sie empfehlen in einer Variante eine Gewichtung der Preise (Kosten) der öffentlichen Güter mit den relativen Mengen an öffentlichen Gütern. In diesem Fall wären die LFA-Zahlungen auch von der Menge der öffentlichen Güter abhängig. Eine Erhöhung der Nachfrage nach öffentlichen Gütern bewirkt dann eine Veränderung der LFA-Zuweisungen oder -Beiträge, der zwischen - 0,005 und -0,007 Geldeinheiten je Einheit Mengenzuwachs liegt.

Betrachtet man alle Vorschläge, dann sieht man aus der Tabelle II.2, daß in einer Reihe von Fällen ein Großteil der zusätzlichen Steueraufkommen durch geringere LFA-Zuweisungen oder höhere LFA-Beiträge neutralisiert wird. 


\begin{tabular}{||l||c|c|c|c||}
\hline \hline \multicolumn{1}{||l||}{} & \multicolumn{4}{|c||}{ Intervalle der Veränderung der LFA-Zuweisungen } \\
bei 1 Mio DM zusätzlicher Aufkommen aus den
\end{tabular}

${ }^{a}$ Ländersteuern: z.B. Vermögenssteuern.

${ }^{b}$ Umsatzsteuern.

${ }^{c}$ Lohnsteuer und veranlagte Einkommenssteuern.

${ }^{d}$ Kapitalertrags- und Körperschaftssteuern.

${ }^{e}$ Grund- und Gewerbesteuern.

${ }^{f}$ Vor 1995 ohne Einbezug der Ostländer.

${ }^{g}$ Regelungen vor 1995 unter Einbezug der Ostländer.

Tabelle II.2: Veränderung der Einnahmen durch den LFA

\subsubsection{Gesamte Einnahmenveränderung}

Für die Anreizwirkungen auf die regionale Steueranspannung sind allerdings die Gesamteffekte relevant, die sich aus den Einnahmenveränderungen der Steuerverteilung, des LFA und der BEZ sowie den -veränderungen aus den nicht der Steuerverteilung unterworfenen Steuerarten ergeben. In der folgenden Tabelle II.3 sind die Intervalle der Einnahmenveränderungen ausgewiesen, die sich nach Steuerverteilung, Länderfinanzausgleich und BEZ ergeben.

In überraschend vielen Fällen führen höhere regionale Steueraufkommen zu keinen oder nur zu einem marginalen Zuwachs der Einnahmen der Länderhaushalte. In einen Fall sinken die zusätzlichen Nettoeinnahmen sogar leicht. Die geringsten Erhöhungen der Einnahmen werden durch steigende Umsatzsteueraufkommen ausgelöst, da diese auf alle Länder verteilt werden. 


\begin{tabular}{|c|c|c|c|c|c|}
\hline \multirow[b]{2}{*}{ Vorschlag } & \multicolumn{5}{|c|}{$\begin{array}{l}\text { Intervalle der gesamten Einnahmenveränderung in Mio DM } \\
\text { bei } 1 \text { Mio DM zusätzlicher Aufkommen aus den }\end{array}$} \\
\hline & Ldst. $^{a}$ & $\mathrm{UmSt}^{b}$ & EkSt. ${ }^{c}$ & KSt. ${ }^{d}$ & GSt. ${ }^{e}$ \\
\hline vor 1995 ohne $\mathrm{OL}^{f}$ & {$[0,02 ; 1,00]$} & {$[0,00 ; 0,07]$} & {$[0,08 ; 0,58]$} & {$[0,01 ; 0,50]$} & {$[0,46 ; 1,00]$} \\
\hline 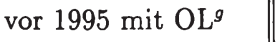 & {$[0,01 ; 0,23]$} & {$[0,00 ; 0,09]$} & {$[0,08 ; 0,19]$} & {$[0,00 ; 0,11]$} & {$[0,45 ; 0,71]$} \\
\hline ab 1995 & {$[0,01 ; 0,37]$} & {$[0,00 ; 0,10]$} & {$[0,08 ; 0,26]$} & {$[0,01 ; 0,19]$} & $0,00 ; 0,72]$ \\
\hline WISS.BEIRAT I & {$[0,23 ; 0,49]$} & {$[0,00 ; 0,10]$} & {$[0,13 ; 0,28]$} & {$[0,11 ; 0,24]$} & {$[0,01 ; 0,57]$} \\
\hline Wiss.BEIRAT II & {$[0,01 ; 0,47]$} & {$[0,00 ; 0,10]$} & {$[0,01 ; 0,27]$} & {$[0,01 ; 0,24]$} & {$[0,00 ; 0,55]$} \\
\hline WISS.BEIRAT IIIa & {$[0,22 ; 0,37]$} & {$[0,00 ; 0,10]$} & {$[0,11 ; 0,22]$} & {$[0,11 ; 0,19]$} & {$[0,01 ; 0,46]$} \\
\hline Wiss.BEIRAT IIIb & {$[0,34 ; 0,47]$} & {$[0,00 ; 0,10]$} & {$[0,20 ; 0,27]$} & {$[0,17 ; 0,24]$} & {$[0,26 ; 0,59]$} \\
\hline Buhl/Pfingsten & {$[0,02 ; 0,28]$} & {$[0,00 ; 0,10]$} & {$[0,02 ; 0,16]$} & {$[0,01 ; 0,14]$} & {$[0,02 ; 0,43]$} \\
\hline FÖTTINGER/SPAHN & {$[0,01 ; 0,25]$} & {$[0,00 ; 0,10]$} & {$[0,01 ; 0,15]$} & {$[0,01 ; 0,13]$} & {$[-0,02 ; 0,40]$} \\
\hline Fuest/Lichtblau & {$[0,41 ; 0,83]$} & {$[0,00 ; 0,10]$} & {$[0,23 ; 0,48]$} & {$[0,20 ; 0,41]$} & {$[0,40 ; 0,87]$} \\
\hline Abschaffung LFA & {$[1,00 ; 1,00]$} & {$[0,00 ; 0,10]$} & {$[0,58 ; 0,58]$} & {$[0,50 ; 0,50]$} & {$[0,94 ; 1,00]$} \\
\hline
\end{tabular}

${ }^{a}$ Ländersteuern: z.B. Vermögenssteuern.

${ }^{b}$ Umsatzsteuern.

${ }^{c}$ Lohnsteuer und veranlagte Einkommenssteuern.

${ }^{d}$ Kapitalertrags- und Körperschaftssteuern.

${ }^{e}$ Grund- und Gewerbesteuern.

${ }^{f}$ Vor 1995 ohne Einbezug der Ostländer.

${ }^{g}$ Regelungen vor 1995 unter Einbezug der Ostländer.

Tabelle II.3: Gesamte Einnahmenveränderung nach Steuerverteilung, LFA und BEZ

Das Referenzmodell bildet der Vorschlag der Abschaffung der umverteilenden Elemente des Finanzausgleichs, der dazu führt, daß den Ländern mit Ausnahme der Umsatzsteueraufkommen sämtliche regionalen Aufkommenszuwächse als zusätzliche Einnahmen zufallen.

Von den anderen Reformvorschlägen verursacht der von FUest und LichtblaU die geringsten negativen Anreizwirkungen, gefolgt von der Variante III b des WISSENSCHAFTLICHEN BEIRATS, in der allerdings die Finanzierung der zusätzlichen Bundeszuschüsse nicht berücksichtigt ist!

Allerdings sollte man bei der Interpretation der Tabellen recht vorsichtig sein, da die Intervalle sehr stark von den jeweiligen Daten abhängen. Berechnet man den LFA für ein anderes Jahr, dann könnten sich die Intervallgrenzen verändern. Dennoch kann man vermuten, daß sich auch in anderen Jahren ähnliche Intervalle ergeben. Die Einordnung der Länder innerhalb dieser Intervalle hängt von den Bevölkerungsgrößen und der relativen Fi- 
nanzkraft, im Vorschlag von FUEST/LichtBLAU vom Volkseinkommen und im Vorschlag von FötTinger/SPAHN auch von den öffentlichen Güterpreisen und Gütermengen ab.

\section{Damit läßt sich festhalten:}

Das bundesdeutsche Finanzausgleichssystem sowie die hier untersuchten Neuordnungsvorschläge führen zu erheblichen Abweichungen zwischen den Steueraufkommen eines Landes und/oder seinen Gemeinden und den für die Finanzsituation der jeweiligen Gebietskörperschaft relevanten Einnahmen. Dies kann sogar dazu führen, daß ein marginaler Anstieg der Steueraufkommen eines Landes zu keinem Zuwachs der Einnahmen aus dieser Steuer führt. Der Hauptteil der höheren Aufkommen wird dann an den Bund und die anderen Länder abgeführt.

Im Sinne der Definition von Musgrave [1961] oder Bös [1978] besteht deshalb weitgehend eine starke Substitutionalität zwischen Steueraufkommen und Finanzausgleichszuweisungen und daher nur ein sehr geringer Anreiz zur Ausnutzung der eigenen Steuerquellen (Steueranspannung). Können allerdings die Länder mangels Finanzautonomie ihre Steueranspannung nicht beeinflussen, so haben diese geringen Anreizwirkungen keine Auswirkungen auf ihr Verhalten.

Haben die Länder jedoch eine Einflußmöglichkeit auf ihre Steueranspannung, so könnte den Ländern nicht unbedingt daran gelegen sein, diese zu erhöhen. ${ }^{5}$ Sie können eventuell sogar darauf hoffen, daß ihnen ein Anstieg der Steueranspannung der anderen Länder zu höheren Einnahmen verhilft.

Diese Ergebnisse haben auch Konsequenzen auf Forderungen nach einer höheren Steuerautonomie der Länder. Damit diese Sinn macht, sollten die entsprechenden Einnahmen entweder gar nicht oder nur normiert in den Berechnungen der Finanzkraft im Länderfinanzausgleich angerechnet werden. Dies wird demnach konsequenterweise auch vom WISSENSCHAFTLICHEN BEIRAT [1992] empfohlen.

\footnotetext{
${ }^{5}$ Eine indirekte Möglichkeit, diese Steueranspannung zu verändern, bietet sich den Ländern aufgrund der von ihnen organisierten Steuerverwaltung. Je nach Schärfe der Verfolgung von Steuersündern oder Kontrolle von Unternehmen können die Steueraufkommen beeinflußt werden. (Daß dies nicht ganz unerhebliche Größenordnungen betrifft, zeigt der Fall um den Immobilienunternehmer Schneider.) Die geringste Anreizwirkung für die gesetzlichen Regelungen vor 1995, ohne Einbezug der Ostländer, ergab sich für Bremen und das Saarland. Dies könnte eine Erklärung für die Hinweise auf eine relativ geringe Steuerverfolgung im Saarland liefern. Von 1 Mio DM zusätzlichen Aufkommen aus den Lohnund veranlagten Einkommenssteuern (Körperschafts- und Kapitalertragssteuern) flossen dem Land nur 0,084 Mio DM (0,08 Mio DM) als zusätzliche Einnahmen zu.
} 


\subsection{Zwischenbemerkungen}

Wie sich in der einfachen Analyse des öffentlichen Budgets gezeigt hat, spielen die genauen Finanzausgleichsregelungen eine wesentliche Rolle für die Anreizwirkung zur Ausschöpfung der regionalen Steuerkraft.

Bezüglich des bundesdeutschen Finanzausgleichssystems wurde ein stark substitutionaler Charakter festgestellt. Dies läßt erwarten, daß sich eine stärkere Finanzautonomie der Bundesländer negativ auf die regionale Steueranspannung auswirkt.

Daher werden im folgenden die Konsequenzen verzerrender Finanzausgleichsregelungen bei unterschiedlichen Finanzautonomiegraden näher untersucht. Zu diesem Zweck wird ein einfaches regionales Entscheidungsmodell verwendet, in dem die regionale Regierung den indirekten Nutzen ihres repräsentativen privaten Haushalts maximiert.

\section{Exkurs: Grundlegendes Partialmodell}

An dieser Stelle soll die Grundstruktur des im weiteren verwendeten, einfachen regionalen Entscheidungsmodelles kurz dargestellt werden. Dies dient dazu, die vom jeweiligen Problem ablenkenden Erläuterungen zur Modellstruktur in den nachfolgenden Abschnitten möglichst kurz zu halten.

Für die Fragestellung nach den Effizienzwirkungen von Steuerverteilung, Länderfinanzausgleich und Ergänzungszuweisungen reicht es im Prinzip aus, nur diese drei Größen als mögliche Einnahmequellen der regionalen Regierungen zuzulassen, da bereits auf diese Weise wesentliche Einsichten in die resultierenden Effekte gewonnen werden können.

Die einzige wesentliche weitere Einnahmequelle wäre die Verschuldungsmöglichkeit. Diese wird hier nicht zugelassen, um das Modell möglichst einfach zu halten. Ein dynamisches oder zumindest Mehr-Perioden-Modell, das dazu notwendig wäre, würde an den grundsätzlichen Resultaten nicht allzuviel verändern (wenn es auch zusätzliche Einsichten ermöglichen würde). Dies gilt allerdings nicht für den Fall der fehlenden Finanzautonomie. Diese würde bei möglicher Kreditfinanzierung von öffentlichen Ausgaben durch die Länder im Prinzip aufgehoben. Zusätzlich würden intertemporale Verzerrungen auftreten, da die Verschuldungsmöglichkeit über veränderte Ausgabenströme und veränderte private Ausgabenmöglichkeiten die Steueraufkommen und damit die Steuereinnahmen beeinflußt. Gelingt es jedoch, effizienzverzerrende Einflüsse eines Finanzausgleichssystems bereits im statischen Ein-Perioden-Modell festzustellen, so reicht das aus, um zu zeigen, daß Finanzausgleich die allokative Effizienz beeinflußt. ${ }^{6}$

\footnotetext{
${ }^{6}$ Darüber hinaus sollen die hier entwickelten Aspekte auf die spätere Empirische Allgemeine Gleichge-
} 
Auf der Ausgabenseite genügt es, Lump-Sum-Transfers an die privaten Haushalte der Region und Ausgaben für regionale öffentliche Güter zu berücksichtigen. Da Transfers eine Umlenkung von öffentlichen Einnahmen in privaten Konsum darstellen, ist auf diese Weise die Entscheidung für eine öffentliche oder private Verausgabung von öffentlichen Einnahmen modellierbar. Dies entspricht einem einfachen Fall der Ausgabenautonomie.

Im Prinzip bleiben nun noch zwei Problemkreise bei der Modellierung des öfentlichen Entscheidungsproblems offen:

- Zum ersten stellt sich die Frage, ob eine Region überhaupt genügend Entscheidungsbefugnisse besitzt, um über die Verwendung ihrer Mittel selbst entscheiden (Grad der Ausgabenautonomie) oder durch die Wahl von Steuersätzen, ihre Einnahmen direkt beeinflussen zu können (Grad der Einnahmenautonomie).

- Zum zweiten ist zu klären, wie das Problem der Wahl der optimalen Menge des öffentlichen Gutes modelliert werden soll, insbesondere, ob eine Trennung zwischen öffentlicher und privater Entscheidung möglich oder geboten ist.

\subsection{Grad der Finanzautonomie der Regionen}

$\mathrm{Da}$ in der bundesdeutschen Finanzverfassung die Länder keine Steuergesetzgebungskompetenz (Gesetzgebungshoheit) besitzen, erscheint es sinnvoll, bei der Modellierung der regionalen öffentlichen Haushalte von fehlender Einnahmenautonomie auszugehen.

Allerdings sollte man darüber hinaus folgendes beachten:

- Im bundesdeutschen Finanzsystem besitzen die Länder weitgehende Ausgabenautonomie, die sich vor allem auf die verschiedenen öffentlichen Leistungen bezieht. Auch wenn in den Untersuchungen zum Finanzausgleich die meisten Autoren der Meinung sind, daß die Länder nicht in der Lage sind, ihre Einnahmen in den privaten Verbrauch umzulenken (siehe z.B. Bös [1978] oder WAHL/Frischmuth [1990]), ist dies m.E. in beschränktem Ausmaß teilweise möglich. Durch eine Variation von Transfers (Schulwegkostenfreiheit, Lehrmittelfreiheit, Landeserziehungsgeld etc.) an die privaten Haushalte können begrenzte Umschichtungen erreicht werden. Zudem können die Länder durch Veränderung der Zuweisungen die Finanzsituation der Gemeinden beeinflussen. Dies kann sich über veränderte Transfers (Sozialhilfesätze etc.) auf den privaten Verbrauch auswirken.

Dies könnte ein Argument für die Modellierung einer Ausgabenautonomie der Länder und Gemeinden sein. Gewichtiger ist jedoch die Möglichkeit, mit Hilfe der

wichtsanalyse übertragbar sein. Diese ist notwendigerweise als nichtdynamische Untersuchung konzipiert, da ein dynamisches Modell mit elf Ländern kaum noch rechen-, beherrsch- und interpretierbar ist. 
Berücksichtigung unterschiedlicher Grade von Ausgabenautonomie diskutieren zu können, welche Vor- oder Nachteile eine Erhöhung der regionalen Ausgabenautonomie gegenüber einer stark beschränkten Ausgabenautonomie besitzt.

- Geht man davon aus, daß die Länder durch Variation der Schlüsselzuweisungen an die Gemeinden, diese unter Umständen zu einer Anpassung der Gewerbe- und/oder Grundsteuerhebesätze, der Abgaben oder der Gebühren bewegen können, so könnte man durchaus auch von begrenzter Einnahmenautonomie der Länder sprechen, wenn man Gemeinden und Länder als eine Einheit modelliert. Will man darüber hinaus auch die Vorschläge zur Reform des Finanzausgleichssystems behandeln, die eine Erhöhung der Steuerautonomie der Länder fordern, so sollte in diesen Fällen ein Ansatz mit begrenzter Einnahmenautonomie gewählt werden.

Aus diesen Gründen und zum Zwecke der Erzielung allgemeiner Aussagen werden in der theoretischen Analyse drei Grade der Finanzautonomie, nämlich die Fälle einer fehlenden Autonomie, einer vorhandenen Ausgabenautonomie und einer vollen Finanzautonomie berücksichtigt. $^{7}$

Explizit sei an dieser Stelle darauf hingewiesen, daß in dem gewählten Modellrahmen die regionale Regierung nicht in der Lage ist, ihre Einnahmen über Einflußnahmen auf die Gestaltung der Finanzausgleichsregeln zu verändern. Die Entscheidungen über diese Regeln sind exogen vorgegeben.

\subsection{Trennung von privater und öffentlicher Entscheidung}

Besteht eine gewisse Autonomie der Regionen und somit ein Entscheidungsproblem über die optimale Nachfrage nach öffentlichen Gütern, so kann dieses Entscheidungsproblem im Prinzip auf zweierlei Arten modelliert werden.

Bei exogenen Steuersätzen könnte man annehmen, daß die regionale Regierung ihre Einnahmen vollständig als Lump-Sum-Transfers an den repräsentativen Haushalt ausschüttet. Dieser wählt dann seine nutzenoptimale Menge des öffentlichen Gutes $G$, der Freizeit

\footnotetext{
${ }^{7}$ Alternativ könnte man das Problem fehlender Ausgaben- und Einnahmenautonomie der Regionen auf einen Ansatz nationaler Ausgaben- und Einnahmenautonomie erweitern. In diesem Falle wäre es wieder möglich, Entscheidungen über die optimale Menge der regionalen öffentlichen Güter, von Transfers der Regionen an ihre Haushalte und die Bestimmung der optimalen Steuersätze als Entscheidungsproblem zu formulieren. Dieses würde dann daraus bestehen, daß die nationale Regierung durch Variation der entsprechenden Kontrollvariablen, hier z.B. des regionalen öffentlichen Gutes und eines Steuersatzes, die Wohlfahrt ihrer Regionen bzw. deren repräsentativer Haushalte maximiert.
} 
$F$ und des aggregierten privaten Konsumgutes $C .^{8}$ Dieses Vorgehen wird üblicherweise in Ansätzen zur Bestimmung der optimalen öffentlichen Gutmenge gewählt, wenn die Entscheidung von Optimalsteuerproblemen getrennt wird. Beispielsweise wählen WHALLEY/Trela [1986] dieses Vorgehen in ihrer Empirischen Allgemeinen Gleichgewichtsanalyse des kanadischen Finanzausgleichssystems.

Der entsprechende Ansatz besteht aus dem Optimierungsproblem

$$
\begin{array}{rl}
\max _{G, C, F} & H(G, C, F) \\
\text { u.d.NB.: } \quad p C+q_{G} G+w^{n} F=Y+Z,
\end{array}
$$

mit $p$ als Bruttopreis des privaten aggregierten Konsumgutes $C, q_{G}$ als Preis des öffentlichen Gutes $G, w^{n}$ als Opportunitätskosten der Freizeit in Form des entgangenen Nettolohnsatzes, $Z$ als exogen festgelegte Einnahmen aus den Finanzausgleichszuweisungen und $Y$ als sonstige mögliche Einkommen die sich aus den Einnahmen bei Verwendung der Zeitgrundausstattung $w^{n} E$ und den regionalen Transfers $\operatorname{Tr}$ zusammensetzen. $H(\cdot)$ sei die wohldefinierte Nutzenfunktion.

Werden die Einnahmen der regionalen öffentlichen Haushalte jedoch nicht vollständig in Form von Lump-Sum-Transfers an die privaten Haushalte weitergegeben, sondern hängen sie direkt oder indirekt von den regionalen Konsumentscheidungen ab, dann ist es geboten, die jeweilige Entscheidung zweistufig zu modellieren, und die Einnahmen der regionalen Regierung zu endogenisieren. Dies führt zu folgendem Ansatz, der im weiteren auch verwendet wird:

Die regionale Regierung bestimmt durch Maximierung der ihr bekannten indirekten Nutzenfunktion des repräsentativen privaten Haushalts das optimale Mix von $G$ und den Transfers $\operatorname{Tr}$ oder von $G$ und den jeweiligen Steuersätzen $\tau$. Damit wird unterstellt, daß die regionale Regierung keine eigenen, von den Präferenzen der Privaten abweichenden Ziele verfolgt. $^{9}$

\footnotetext{
${ }^{8}$ Eine derartige Modellierung ist aufgrund zweier Annahmen möglich. Zum ersten muß sich die regionale Regierung vollständig an die präferierten Mengen der privaten Haushalte halten (Median-Wählermodell oder repräsentativer privater Haushalt,) und zum zweiten wird unterstellt, daß der repräsentative Haushalt in seiner Entscheidung die Rückwirkung des Wirtschaftsprozesses auf die regionalen öffentlichen Einnahmen nicht berücksichtigt.

${ }^{9}$ Alternativ könnte man z.B. annahmen, daß die Regierung Einnahmenmaximierung betreibt. Dies würde einer Sichtweise der Neuen Politischen Ökonomie entsprechen, gemäß der die Regierungsmaßnahmen von der Bürokratie dominiert werden. Ein derartiger Ansatz wird beispielsweise von FilimoN u.a. [1982] verwendet und führt zu dem flypaper-Effekt bei Finanzzuweisungen.
} 
Definiert man die indirekte Nutzenfunktion ${ }^{10}$ des repräsentativen Konsumenten als

(II.3) $\nu\left(G ; p, w^{n}, Y\right)=\left\{\max _{F, C} H(G, F, C)\right.$ u.d.NB.: $\left.p C+w^{n} F=w^{n} E+T r=Y\right\}$,

mit $F$ als Freizeit und $E$ als Zeitgrundausstattung. ${ }^{11}$

Das entsprechende Optimierungskalkül der regionalen Regierung lautet dann

$$
\begin{aligned}
\max _{G, T \tau \text { oder } \tau} \nu\left(G ; p, w^{n}, Y\right) \\
\\
\text { u.d.NB.: } q_{G} G+T r=Z(G, T r),
\end{aligned}
$$

mit $Z$ als Zuweisungen aus dem Finanzausgleichssystem, die sich z.B. aus Steuerverteilungs-, LFA- und BEZ-Zuweisungen zusammensetzen. Die Steuersätze $\tau$ und die Transfers $\mathrm{Tr}$ sind in den Argumenten der indirekten Nutzenfunktion bereits enthalten, da der Konsumentenpreis $p=\left(1+\tau^{C}\right) q$, der Nettolohnsatz $w^{n}=w\left(1-\tau^{Y}\right)$ sowie das Einkommen $Y=w^{n} E+T r$ die entsprechenden Größen beinhalten. In diesem Ansatz wird angenommen, daß die jeweilige Regierung den Einfluß ihrer eigenen Ausgabenentscheidungen auf ihre Einnahmen aus dem Finanzausgleich $Z$ genau kennt.

Solange der regionalen Regierung Lump-Sum-Transfers oder Lump-Sum-Steuern und die öffentliche Gütermenge als Kontrollvariablen zur Verfügung stehen, ist der erste Ansatz äquivalent mit einem Ansatz in dem das öffentliche und private Entscheidungskalkül getrennt durchgeführt werden. ${ }^{12}$

Mit Hilfe dieses Partialmodells werden im weiteren die Auswirkungen von Finanzausgleichsregelungen auf die relativen Güterpreise ermittelt, und damit die durch die Regelungen ausgelösten Verzerrungen bestimmt.

\section{Fehlende Finanzautonomie}

Besitzt ein Land keine Möglichkeit, die eigenen Einnahmen und Ausgaben direkt durch die Variation eigener Steuersätze oder die Erhebung von zusätzlichen Steuern sowie einer

\footnotetext{
${ }^{10}$ Die indirekte Nutzenfunktion ist hier dadurch definiert, daß die privaten Konsummengen in die direkte Nutzenfunktion eingesetzt werden. Die öffentliche Gütermenge ist dagegen ein Argument der indirekten Nutzenfunktion. Die Verwendung dieser ungewöhnlichen Form der indirekten Nutzenfunktion ist bei kombinierten Steuersatz- und Mengenentscheidungen der öffentlichen Haushalte in Optimalsteuermodellen nicht unüblich (siehe z.B. AtKinson/Stiglitz [1980], S.496 oder Auerbach [1985], S.113).

${ }^{11}$ Die Form der privaten Budgetgleichung ergibt sich durch die Umformung des Lohneinkommens $w^{n} L$ in $w^{n} E-w^{n} F$.

${ }^{12}$ Siehe Atkinson/Stiglitz [1980], S.490-493.
} 
Umlenkung öffentlicher Einnahmen in den privaten Konsum zu verändern, dann besitzt das Land keine Finanzautonomie.

Man kann diesen Fall fehlender Finanzautonomie dahingehend interpretieren, daß das Land vollständig rationiert ist. Die Rationierung besteht darin, daß eine Einnahmenerhöhung nicht zu zusätzlichen Transfers an die privaten Haushalte führen darf. Diese Annahme ermöglicht die Verwendung von Rationierungsansätzen, wie sie grundlegend von NEARY/ROBERTS [1980] behandelt werden. ${ }^{13}$

Zur graphischen Verdeutlichung wird ein sehr einfacher Ansatz gewählt, in dem der regionale repräsentative Haushalt seinen Nutzen durch die Wahl des öffentlichen Gutes G und eines privaten composite commodity $C$ bestimmt.

In der Abbildung II.1 ist auf der Ordinate der private Konsum und auf der Abszisse die Menge des öffentlichen Gutes abgetragen. Die Budgetgerade $\overline{B B}$ besitzt die Steigung $-q_{G} / p$. Im Ausgangsgleichgewicht wurde der Punkt $A^{*}$ als Konsumpunkt gewählt.

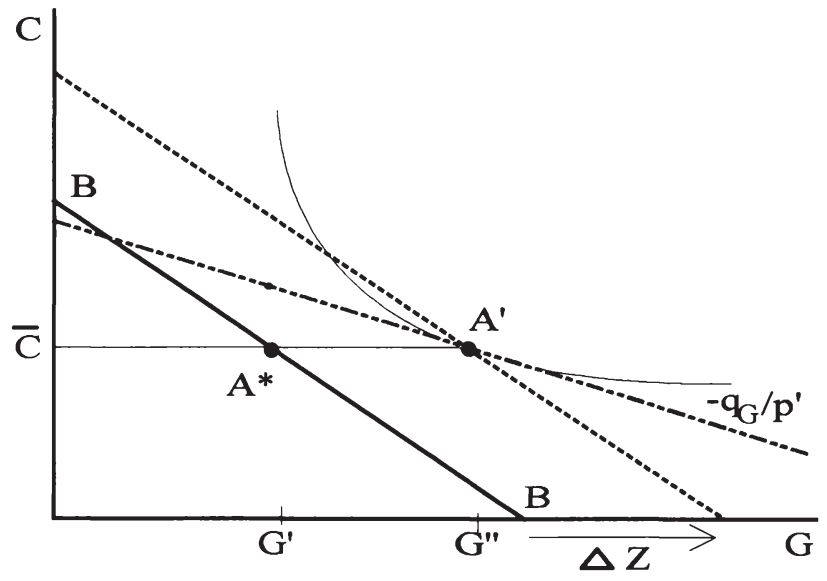

Abbildung II.1: Optimale Wahl bei Rationierung

Erhöhen sich nun die Einnahmen der Region um $\Delta Z$, und ist der repräsentative Haushalt bei gleichbleibendem Preis $p$ bezüglich der Wahl von $C$ in Höhe der alten Konsummenge $\bar{C}$ rationiert, so wird er $A^{\prime}$ wählen und den Konsum des öffentlichen Gutes auf $G^{\prime \prime}$ ausdehnen.

Dieses Ergebnis entstünde ebenfalls, wenn der Konsument durch die gestrichelte Budgetgerade mit der Steigung $-q_{G} / p^{\prime}$ restringiert wäre. Folglich ist eine Wahl mit dem

${ }^{13}$ Eine ausführliche Darstellung von Rationierungsansätzen findet sich bei WEBER [1995]. 
Preis $p^{\prime}$ für das rationierte Gut äquivalent zur "Wahl” bei Rationierung. Dieser Preis $p^{\prime}$ wird in der Literatur als "virtueller" Preis bezeichnet. ${ }^{14}$ Dieser kann im Prinzip aus dem Rationierungsniveau, den Preisen der nichtrationierten Güter und dem Nutzenniveau berechnet werden (siehe NEARY/RoberTs [1980], S.30). Mit Hilfe des virtuellen Preises kann grundsätzlich eine Slutsky-Zerlegung durchgeführt werden, um die auftretenden Substitutions- und Einkommenseffekte zu isolieren (siehe DeATon/Muellbauer [1988], S.112-113 oder NEARY/RoberTs [1980], S. 32-34). ${ }^{15}$

Die Rationierung führt demnach zu einer Veränderung der relativen Preise mit der Folge, daß bei einer Einkommenserhöhung das rationierte Gut relativ teurer wird (die Rationierung wird beschränkender). Es kommt somit zu einer virtuellen Preisverzerrung zugunsten des nicht-rationierten Gutes, das relativ billiger wird. Dies führt dazu, daß die Verwendung der zugeflossenen Mittel von der Verwendung bei Ausgabenautonomie der empfangenden Gebietskörperschaft abweicht und bei den Empfängerländern mehr öffentliche Leistungen angeboten werden als ohne Rationierung (vgl. auch WAHL/FrISCHMUTH ([1990], S.4-7), die allerdings nicht von Rationierung sprechen).

Das genaue Ausmaß dieser Effekte hängt von der Höhe der gesamten Finanzausgleichstransfers ab, da eine stärkere Verschiebung der Budgetgerade bei unveränderten Rationierungsmengen zu anderen virtuellen Preisen führt. Nur bei quasilinearen Präferenzen ist es möglich, daß die Steigungen der Tangenten an die Indifferenzkurven entlang der Rationierungslinie gleich bleiben (eine Definition quasilinearer Präferenzen bietet z.B. VARIAN [1992], S.164).

Zur formalen Darstellung wird das Grundmodell (Gleichungen (II.4) und (II.3)) um die Nebenbedingung ergänzt, daß sämtliche zusätzlichen Einnahmen für öffentliche Güter ausgegeben werden müssen, d.h. die Transfers dürfen nicht verändert werden. Dies führt zu dem Ansatz

$$
\begin{gathered}
\max _{G, T r} \nu\left(G ; p, w^{n}, Y\right) \\
\text { u.d.NB.: (1) } q_{G} G+T r=Z(G, T r) \\
\text { (2) } T r=\overline{T r} .
\end{gathered}
$$

Die entsprechende Lagrangefunktion

$$
\mathcal{L}=\nu\left(G ; p, w^{n}, Y\right)+\lambda\left(Z(G, T r)-q_{G} G-T r\right)+\mu(\overline{T r}-T r)
$$

\footnotetext{
${ }^{14}$ Das Konzept der virtuellen Preise geht auf Rothbarth [1940/41] zurück.

${ }^{15}$ Allerdings entsprechen diese Substitutions- und Einkommenseffekte nicht den durch die SlutskyGleichung erfaßbaren Effekten. Sie entsprechen keinen tatsächlichen Verhaltensänderungen, sondern nur dem Wunsch der Wirtschaftssubjekte, ihr Verhalten zu ändern. Daher entsprechen sie Nutzenverlusten bei den Wirtschaftssubjekten, die nicht in Mengenreaktionen erfaßbar sind.
} 
abgeleitet nach $\mathrm{G}$ führt zu

$$
\frac{\partial \nu}{\partial G}=\lambda\left(q_{G}-\frac{\partial Z(\cdot)}{\partial G}\right) .
$$

Im Optimum gilt $\partial \nu(\cdot) / \partial G=\partial H(\cdot) / \partial G$ (siehe AUERBACH [1985], S.113). Verbindet man dies mit der Optimalbedingung $\partial H(\cdot) / \partial C=\alpha p$, die aus der privaten Nutzenmaximierung (siehe Gleichung (II.2) auf Seite 67) resultiert, mit $\alpha$ als Grenznutzen des privaten Einkommens, so erhält man die Gleichung

$$
M R S_{C G}=-\frac{\partial H(\cdot) / \partial G}{\partial H(\cdot) / \partial C}=-\frac{\lambda\left(q_{G}-\partial Z(\cdot) / \partial G\right)}{\alpha p}
$$

In dieser Beziehung wird der Einfluß der Finanzzuweisungen auf die Optimalbedingungen deutlich. Da die Zuweisungen, falls ihre Höhe von den öffentlichen Ausgaben abhängt, die sozialen Kosten der öffentlichen Güter beeinflussen, besitzen sie eine preisverzerrende Wirkung. Je nachdem ob eine Substitutionalitätsbeziehung oder eine Komplementaritätsbeziehung zwischen den Zuweisungen und den öffentlichen Ausgaben besteht, treiben sie einen positiven oder negativen Keil zwischen die Grenzrate der Substitution und das relative Preisverhältnis des öffentlichen und privaten Gutes. Der zweite relevante Term ist die Relation zwischen dem Grenznutzen der öffentlichen Einnahmen $\lambda^{16}$ und dem privaten Grenznutzen des Einkommens $\alpha$. Ist $\lambda$ größer als $\alpha$, so weicht die Grenzrate der Substitution zwischen dem privaten und dem öffentlichen Gut positiv vom relativen Güterpreisverhältnis ab, d.h. die Region ist bereit für eine zusätzliche Einheit des öffentlichen Gutes mehr Einheiten des privaten Gutes aufzugeben, als das Preisverhältnis der Marktpreise signalisiert. ${ }^{17}$

Aus der Ableitung der Lagrangefunktion (II.6) nach der zweiten Kontrollvariable $\mathrm{Tr}$

$$
\frac{\partial \mathcal{L}(\cdot)}{\partial T r}=\frac{\partial \nu(\cdot)}{\partial Y} \frac{\partial Y}{\partial T r}-\lambda\left(1-\frac{\partial Z(\cdot)}{\partial T r}\right)-\mu=0 .
$$

erhält man wegen $\partial \nu(\cdot) / \partial Y=\alpha$ und wegen $\partial Y / \partial T r=1$ den Ausdruck

$$
\alpha=\lambda\left(1-\frac{\partial Z(\cdot)}{\partial T r}\right)+\mu .
$$

\footnotetext{
${ }^{16}$,the increase in a residents's utility that would result from a dollar falling from the sky into the governments's coffers" (ARNOTT/GRIESON [1981], S.29).

${ }^{17}$ Dies ist analog zu dem üblichen Ansatz einer Finanzierung der öffentlichen Ausgaben über Steuern, in dem Steueraufkommen gleich Steuereinnahmen sind. Dieser Ansatz geht in seiner Formalisierung auf Stiglitz/Dasgupta [1971] zurück. Eine kurze Darstellung enthält OAKLand [1987], S.495-496).
} 
Dies eingesetzt in die Gleichung (II.8) führt zur Grenzrate der Substitution zwischen dem öffentlichen und dem privaten Konsumgut

$$
M R S_{C G}=-\frac{\partial H(\cdot) / \partial G}{\partial H(\cdot) / \partial C}=-\frac{q_{G}-\partial Z(\cdot) / \partial G}{[(1-\partial Z(\cdot) / \partial T r)+\mu / \lambda] p} .
$$

Ist die Höhe der Finanzzweisungen von den jeweiligen öffentlichen Entscheidungen abhängig, dann entstehen Abweichungen der Grenzrate der Substitution von den Güterpreisverhältnissen. Inwieweit dies der Fall ist, hängt von den speziellen Finanzausgleichsregelungen ab. Für den bundesdeutschen Fall wird dies im Anschluß an den nächsten Abschnitt über die Theorie der Finanzzuweisungen näher untersucht.

Von besonderem Interesse ist außerdem der Einfluß der Rationierung auf die obige Gleichung (II.11). Ist die Rationierung bindend, was hier in jedem Fall zutrifft, dann treibt der Schattenpreis $\mu$ der Rationierung, der Lagrangemultiplikator der Rationierungsbedingung (2) in Gleichung (II.5), einen Keil zwischen die Grenzrate der Substitution und das Güterpreisverhältnis. Folglich führt eine fehlende Finanzautonomie zu Preisverzerrungen.

Verwendet man das Konzept der virtuellen Preise, dann kann man das Rationierungsproblem als Optimierungskalkül bei freier Entscheidung und virtuellen Preisen modellieren:

$$
\begin{aligned}
\max _{G, T r} & \nu\left(G ; p^{\prime}, w^{n}, Y\right) \\
& \text { u.d.NB.: } q_{G} G+T r=Z(G, T r) .
\end{aligned}
$$

Die Ableitung nach den Transfers $\operatorname{Tr}$ wird nun zu

$$
\frac{\partial \mathcal{L}(\cdot)}{\partial T r}=\frac{\partial \nu(\cdot)}{\partial Y} \frac{\partial Y}{\partial T r}-\lambda\left(1-\frac{\partial Z(\cdot)}{\partial T r}\right)=0 .
$$

Nach $\alpha$ aufgelöst lautet diese Gleichung

$$
\alpha=\lambda\left(1-\frac{\partial Z(\cdot)}{\partial T r}\right) .
$$

Daraus resultiert schließlich, da die Beziehung (II.8) unverändert bleibt, die modifizierte Relation der Grenzrate der Substitution

$$
M R S_{C G}=-\frac{\partial H(\cdot) / \partial G}{\partial H(\cdot) / \partial C}=-\frac{q_{G}-\partial Z(\cdot) / \partial G}{(1-\partial Z(\cdot) / \partial T r) p^{\prime}}
$$

Um in beiden Fällen eine äquivalente Lösung zu erreichen, müssen in beiden Ansätzen sowohl die Nutzenniveaus bei Konsum der Rationierungsmenge und voller Ausschöpfung des Budgets als auch die Grenzraten der Substitution gleich hoch sein. Dies führt durch 
Gleichsetzen der Nenner der rechten Seiten der Gleichungen (II.8) und (II.11b) zu folgender Bestimmungsgleichung für den virtuellen Preis (vgl. NEARY/RoBERTs [1980], S.31):

$$
p^{\prime}=\left[1+\frac{\mu}{\lambda(1-\partial Z(\cdot) / \partial T r)}\right] p,
$$

mit $\lambda$ als Grenznutzen der öffentlichen Einnahmen in der rationierten Situation und $\mu$ als Schattenpreis der Rationierungsbedingung. Da $\lambda$ größer gleich Null ist - bei Ausschluß von Randlösungen sogar größer Null ist ${ }^{18}$-, ist der virtuelle Preis immer dann größer (kleiner) als der normale Preis, wenn der Haushalt mehr (weniger) als die Rationierungsmenge vom restringierten Gut konsumieren möchte. In diesem Fall ist der Schattenpreis der Rationierungsmenge $(\mu)$ größer (kleiner) als Null. Zusätzlich wird der virtuelle Preis durch die Finanzausgleichsregelungen beeinflußt, falls es einen Zusammenhang zwischen Transfers und Zuweisungen gibt.

Weicht das virtuelle Preisverhältnis $q_{G} / p^{\prime}$ von dem normalen Preisen $q_{G} / p$ ab, so entstehen durch die Rationierung Preisverzerrungen. Das relativ billiger werdende Gut (hier das öffentliche Gut $G$ ) wird damit im Vergleich zum Normalfall vermehrt nachgefragt.

Ein Vorteil des Rationierungsansatzes liegt darin, daß bei Verwendung der virtuellen Preise die normalen Konzepte der Wohlfahrtsmessung verwendet werden können (vgl. NEARY/RobERTs [1980], S.39 für den Fall der Konsumentenrente). Im hier vorliegenden Fall bedeutet dies, daß z.B. die Hicksche Äquivalente Variation ( $H E V$ ) (vgl. z.B. VARIAN [1992], S.160-163) berechnet werden kann, indem die Differenz zwischen den Kompensationszahlungen, die mindestens notwendig sind, um den Entscheidungsträger bei alten Preisen auf das neue Nutzenniveau zu heben, und den minimalen Ausgaben im Ausgangspunkt ermittelt wird $\left(E V_{R}\right) .{ }^{19}$

Ein Vergleich der bei Rationierung resultierenden Wohlfahrtseffekte von Zuweisungen mit den Effekten ohne Rationierung zeigt, daß sich im Prinzip, unter Vernachlässigung von Rückwirkungen, bei Rationierung geringere Wohlfahrtsgewinne als in der nichtrationierten Situation ergeben (siehe Abbildung II.2). Ohne Rationierung wäre die HEV, vorausgesetzt an der Höhe der Zuweisungen wird nichts geändert, genau die Differenz zwischen den beiden Budgetgeraden $\overline{B B}$ und $\overline{B^{\prime} B^{\prime}}$, da die Zuweisungen nur als allgemeine Transfers wirken und keinerlei Substitutionseffekte auslösen $\left(E V_{O}\right)$.

Diese Analyse hat gezeigt, daß sogar im Falle fehlender Finanzautonomie wohlfahrtsreduzierende Effekte auftreten können. WAHL und FRISCHMUTH [1990] argumentieren nun,

\footnotetext{
${ }^{18}$ Dies ist in der Wahl des Maximierungskalküls im Prinzip bereits vorausgesetzt, da zu dessen Lösung anstelle des Kuhn-Tucker- der Lagrangeansatz verwendet wurde.

${ }^{19}$ Im Fall der HEV brauchen nicht einmal die virtuellen Preise zugrundegelegt werden.
} 


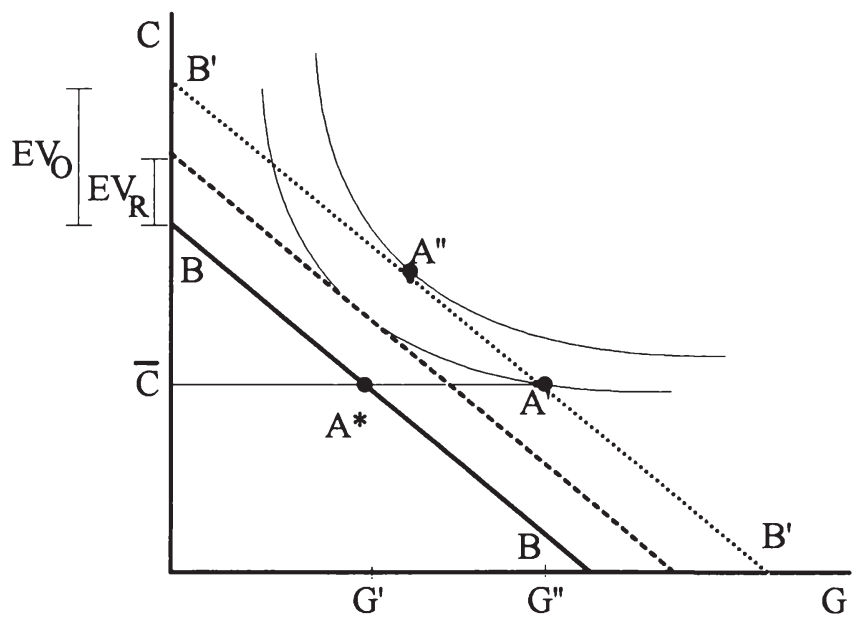

Abbildung II.2: HEV bei Rationierung

daß die Länder bei gleich hohen Zuweisungen und bei Ausgabenautonomie durch eine Umschichtung ihres Budgets ein höheres Nutzenniveau (z.B. Punkt A" in der Abbildung II.2) erreichen könnten als ohne Ausgabenautonomie. Darum fordern sie ebenso wie der WiSSenschaftliche Beirat [1992] eine Erhöhung der Steuerautonomie der Länder. Die obige Analyse stützt diese Forderung, soweit man von anderen Effizienzwirkungen einer Steuerautonomie absieht, die z.B. durch Steuersatzveränderungen hervorgerufen werden können.

Wie verändern sich diese Aussagen, falls die Regionen einen gewissen Grad an Finanzautonomie besitzen? Dieser Frage wird der nächste Abschnitt nachgehen.

\section{Ausgabenautonomie der Länder}

Die im Fall der Ausgabenautonomie zugelassene Reaktion der Länder auf veränderte Einnahmenströme kann in veränderten Ausgaben für öffentliche Güter oder, durch den Umweg über Lump-Sum-Transfers, für private Güter bestehen. Zur Vereinfachung der Analyse wird hier eine Kombination aus Ausgaben- und Einnahmenautonomie modelliert, in der die Transfers auch negativ werden können (Lump-Sum-Steuer), um zusätzlich eine Reduktion der privaten Konsumausgaben zuzulassen. Diese Form der Entscheidungsfreiheit wird traditionellerweise in der Theorie der Finanzzuweisungen untersucht (siehe 
z.B. Wilde [1968, 1971], Waldauer [1973], Oates [1972] oder Fischer [1988]). Für die hier vorliegende Problematik ist diese Theorie das richtige Instrumentarium, da der Einfluß der exogenen Veränderung der Einnahmen auf das Ausgabenverhalten zu untersuchen ist.

\subsection{Theorie der Finanzzuweisungen}

Die auftretenden Wohlfahrtseffekte hängen von der Art der Finanzzuweisungen ab, von denen eine ganze Reihe unterschieden werden können. ${ }^{20}$ An dieser Stelle werden allerdings nur vier Typen betrachtet, nämlich die allgemeinen Finanzzuweisungen, die Finanzzuweisungen mit Verwendungszweck, die Finanzzuweisungen mit Empfangsbedingung und die bedingten Finanzzuweisungen mit Eigenbeteiligung (matching grant). Abweichend von der üblichen graphischen Vorgehensweise, wie sie z.B. bei FISCHER [1988] zu finden ist, sollen sie weitgehend algebraisch untersucht werden. Auf diese Weise können Optimalbedingungen abgeleitet werden, die mit den in den anderen Teilen der Arbeit verwendeten Bedingungen exakt verglichen werden können. ${ }^{21}$ Im Gegensatz zum gebräuchlichen Vorgehen in der Literatur werden hier explizit auch solche Finanzzuweisungen analytisch untersucht, die zu unstetigen Budgetgeraden führen. ${ }^{22}$

Der Einfachheit halber werden grundsätzlich keine Finanzzuweisungen betrachtet, die zwischen verschiedenen öffentlichen Leistungen diskriminieren. Dies entspricht dem Charakter des anfangs präsentierten Modells, in dem nur ein öffentliches Gut definiert ist. Derartige Zuweisungen spielen im bundesdeutschen Finanzausgleichssystem auch nur eine untergeordnete Rolle und werden eigens geregelt (z.B. Gemeinschaftsaufgaben). Eine Ausnahme stellen einige Vorschläge und Regelungen der Bundesergänzungszuweisungen dar, die zum Teil nur zweckgebunden gewährt werden (sollen).

\footnotetext{
${ }^{20}$ Eine Darstellung der acht Grundtypen findet sich u.a. bei Musgrave/Musgrave/Kullmer [1987] oder OATEs [1972].

${ }^{21}$ Zur Vereinfachung und zum Zwecke der Übereinstimmung mit dem später entwickelten allgemeinen Modell werden Aspekte der asymmetrischen Information zwischen nationaler und regionaler Regierung außer Acht gelassen (siehe z.B. Johnson [1977] oder LEVAGGI [1992]).

${ }^{22}$ Die formalen Untersuchungen von Finanzzuweisungen vergleichen zumeist matching grants und allgemeine Zuweisungen, d.h., gerade die Typen von Zuweisungen, die nur lineare Budgetgeraden besitzen (z.B. JohNSON [1977] oder JURION [1979]). Finanzzuweisungen die unstetige Budgetgeraden erzeugen, werden nomalerweise nur graphisch oder empirisch untersucht. In empirischen Arbeiten ist dies relativ einfach möglich, da die Bestimmung der Optima bei nichtlinearen Budgetgeraden normalerweise mit Hilfe eines stufenweisen Maximierungsprozesses simuliert werden kann (siehe z.B. HaUSman [1981]). Dieses approximative Verfahren wird im empirischen Teil dieser Arbeit ebenfalls verwendet, da eine exakte Bestimmung der relevanten Schattenpreise an den Knick- oder Sprungstellen kaum möglich oder zumindest recht aufwendig ist (siehe Seite ?? bis ??).
} 
In der folgenden Analyse wird sich herausstellen, daß die spezifische Form, in der Finanzzuweisungen gewährt werden, durchaus unterschiedliche Wohlfahrtseffekte auslösen kann. ${ }^{23}$

\subsubsection{Allgemeine Zuweisungen ohne Auflagen}

Allgemeine Finanzzuweisungen sind Zuweisungen, deren Höhe exogen vorgegeben ist und deren Empfang und Verwendung an keine Bedingung geknüpft ist. Sie haben bei einer angenommenen Äquivalenz von Transfers an den repräsentativen Haushalt einer Region mit Zuweisungen an die jeweilige regionale Regierung (OATEs [1972] Kap.3, Appendix B ${ }^{24}$ nur Einkommenseffekte, da sie einer allgemeinen Einkommenssubvention entsprechen (Musgrave, Musgrave, Kullmer [1987], S.26).

Das formale Optimierungkalkül mit $Z$ als allgemeiner Zuweisung

$$
\begin{aligned}
\max _{G, T r} & \nu\left(G ; p, w^{n}, Y\right) \\
& \text { u.d.NB.: } q_{G} G+T r=Z
\end{aligned}
$$

führt analog zum Vorgehen auf den Seiten 70 und $72 \mathrm{zu}$

$$
M R S_{C G}=-\frac{\partial H(\cdot) / \partial G}{\partial H(\cdot) / \partial C}=-\frac{q_{G}}{p} .
$$

Die Grenzrate der Substitution zwischen $G$ und $C$ entspricht genau dem Preisverhältnis dieser Güter. Demnach verursachen die allgemeinen Zuweisungen keine Verzerrungen.

\subsubsection{Zweckgebundene Zuweisung ohne Eigenleistung}

Diese Art der Zuweisungen wird nur dann gewährt, wenn die jeweilige Region die zugewiesenen Mittel ausschließlich für das unterstützte Gut verwendet. Dies schließt jedoch nicht aus, daß die Empfängerregion ihre sonstigen Einnahmen für andere Zwecke verwendet. Demgemäß wird in Anlehnung an Fischer ([1988], S. 41) als einzige Bedingung formuliert, daß der Zuweisungsbetrag ausschließlich für das öffentliche Gut ausgegeben werden muß, jedoch eine Umlenkung von bisherigen Ausgaben $R$ für $G$ durch Transfers oder Steuerreduktionen in den Konsum des privaten Gutes $C$ möglich bleibt. Dies heißt, daß nicht mehr als die eigenen Einnahmen der Region $R$ für Transfers verwendet werden

\footnotetext{
${ }^{23}$ Darauf weist bereits Buchanan [1952] hin.

${ }^{24}$ Diese Äquivalenz sichert, daß nur Einkommenseffekte auftreten, und gilt dann, wenn Lump-SumTransfers an die privaten Haushalte genau gemäß den Steueranteilen der Haushalte verteilt werden (OATES [1972], S.112). Dies ist bei einem repräsentativen Haushalt immer erfüllt.
} 
dürfen $(T r \leq R)$. Außerdem ist die Höhe der Zuweisungen, wenn sie gewährt werden, exogen bestimmt.

Erweitert man das vereinfachte Grundmodell um sonstige Einnahmen des regionalen öffentlichen Haushalts $R$, so steht die regionale Regierung vor folgendem Maximierungsproblem:

$$
\begin{gathered}
\max _{G, T r} \nu\left(G ; p, w^{n}, Y\right) \\
\text { u.d.NB.: (1) } q_{G} G+T r=R+Z \\
\text { (2) } T r \leq R .
\end{gathered}
$$

Aus den entsprechenden Kuhn-Tucker-Bedingungen (siehe z.B. SiLBERBERG [1994], S.473)

$$
\begin{aligned}
& \frac{\partial \nu(\cdot)}{\partial G}-\lambda q_{G}=0 \\
& \frac{\partial \nu(\cdot)}{\partial T r}-\lambda-\mu \leq 0 \quad \text { wenn }<\text { dann } T r=0 \\
& R+Z-q_{G} G-T r=0 \\
& R-T r \geq 0 \quad \text { wenn }>0 \text { dann } \mu=0,
\end{aligned}
$$

mit $\lambda$ und $\mu$ als Lagrangemultiplikatoren der Nebenbedingungen (1) bzw. (2), erhält man nach Umformung der zweiten Marginalbedingung in

$$
\begin{aligned}
\frac{\partial \nu(\cdot)}{\partial Y} \frac{\partial Y}{\partial T r} & \leq \lambda+\mu \\
\Longrightarrow \alpha & \leq \lambda+\mu
\end{aligned}
$$

unter Vernachlässigung der Randlösung, d.h. für $\operatorname{Tr} \neq 0$, wegen

$$
M R S_{C G}=-\frac{\partial H(\cdot) / \partial G}{\partial H(\cdot) / \partial C}=-\frac{\lambda q_{G}}{\alpha p}
$$

schließlich

$$
M R S_{C G}=-\frac{\partial H(\cdot) / \partial G}{\partial H(\cdot) / \partial C}=- \begin{cases}\frac{\lambda q_{G}}{(\lambda+\mu) p} & \text { für } \operatorname{Tr}=R \\ \frac{q_{G}}{p} & \text { für } \operatorname{Tr}<R .\end{cases}
$$

Der Schattenpreis für die maximalen privaten Ausgaben $\mu$ steigt, wenn die Region mehr für den privaten Konsum ausgeben will, als ihr Budget ohne Zuweisungen beträgt, d.h. wenn sie ihre Transfers über die sonstigen Einnahmen $R$ hinaus ausdehnen möchte und infolgedessen die zweite Nebenbedingung bindend wird. In diesem Fall wird der private Konsum relativ teurer und es wird weniger $C$ und mehr $G$ nachgefragt als unter normalen Bedingungen. Unterhalb dieser Grenze gilt die normale unverzerrte Optimalbedingung. 


\subsubsection{Finanzzuweisungen mit Empfangsauflage/-bedingung}

Bei diesem Typ von Finanzzuweisungen sind die Zahlungen an die Erfüllung bestimmter Bedingungen, wie z.B. eines bestimmten Finanzbedarfs oder des Nichterreichens der durchschnittlichen Finanzkraft geknüpft. Die Höhe der Zuweisung ist jedoch unabhängig von den jeweiligen regionalen Entscheidungen und von der regionalen Finanzkraft. Im Hinblick auf die bundesdeutschen Finanzausgleichsregelungen wird hier der Fall untersucht, daß die Region nur dann Zuweisungen erhält, wenn ihr Steueraufkommen eine bestimmte Schranke nicht überschreitet. Alternativ könnte man auch annehmen, daß die Region nur dann Zuweisungen erhält, wenn ihr Versorgungsgrad an öffentlichen Gütern unterhalb einer bestimmten Schranke liegt. ${ }^{25}$

Um eine einfache graphischen Darstellung zu ermöglichen, wird der bisher verwendete Ansatz wie folgt modifiziert:

Der nationale Staat erhebt eine wertmäßige Steuer auf den Konsum des privaten Gutes, deren Steuersatz $\tau$ exogen festgelegt ist (z.B. durch nationale Gesetzgebung). Der Empfang der Zuweisungen durch die Region hängt von der Höhe der regionalen Konsumsteueraufkommen ab.

Betrachtet man die Abbildung II.3, so kann man sich, ausgehend von der ursprünglichen Budgetgerade $\overline{B B}$, einige Konsequenzen dieser Art der Zuweisungen verdeutlichen. Die Erhebung der proportionalen Konsumsteuer führt zu einer Drehung der Budgetgeraden zu $\overline{B^{\prime} B^{\prime}}$. Die Differenz zwischen der alten $(\overline{B B})$ und der neuen $\left(\overline{B^{\prime} B^{\prime}}\right)$ Budgetgeraden ist gerade das Steueraufkommen, das mit steigendem $C$ zunimmt.

Wird der Region nur dann eine Zuweisung von der nationalen Regierung gewährt, wenn das Steueraufkommen der Region unterhalb einer Mindesthöhe an Steuereinnahmen $T_{\max }$ liegt, so verschiebt sich die Budgetgerade unterhalb - bezogen auf die Höhe der Steuereinnahmen - dieses Minimalpunktes, d.h. rechts von $T_{m a x}$, um $Z$ nach oben. Ergebnis ist eine neue nichtstetige Budgetgerade $\left(\overline{B^{\prime} C D E}\right)$. Die Nutzenmaximierung der Region könnte dann aufgrund der Form der Geraden zu einem nichteindeutigen Ergebnis führen (Punkt $A$ und $D$ ), wenn die Indifferenzkurve z.B. die dargestellte Form besitzt.

Wird von der Region der Punkt $A$ gewählt, dann könnte man das Konzept der virtuellen Preise verwenden, um die Preisverzerrungen in diesem Punkt zu bestimmen, die durch die besondere Form der Finanzzuweisungen in diesem Punkt hervorgerufen wird.

\footnotetext{
${ }^{25}$ In der Literatur wird üblicherweise die Situation dargestellt, daß die Region nur dann Zuweisungen erhält, wenn sie eine bestimmte Mindestmenge an öffentlichen Gütern $G_{\max }$ bereitstellt (siehe z.B. Fischer [1988], S.39).
} 


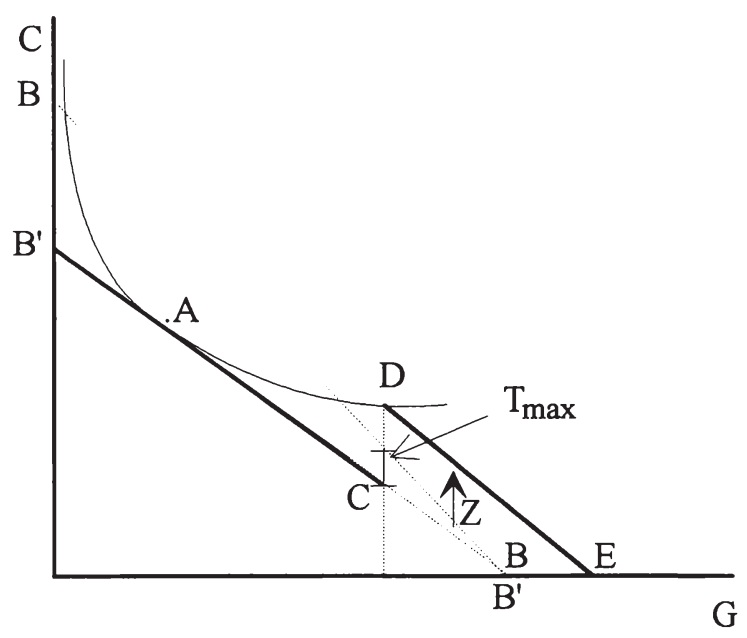

Abbildung II.3: Doppelter Tangentialpunkt bei Zuweisungen mit Empfangsauflage

$\mathrm{Daß}$ aus dieser Preisverzerrung Wohlfahrtsverluste resultieren, kann man sich in der Abbildung II.4 verdeutlichen. Ist die Beschränkung bindend, dann wird die optimale Wahl im Punkt $A$ getroffen. Könnte man dagegen die Beschränkung derart lockern, daß man den Budgetabschnitt zwischen $A$ und $E$ bis zum Punkt $F$ verlängert, so würde die optimale Wahl auf dem fiktiven Teil der Budgetgerade liegen (Punkt A'). Dies bedeutet, daß die Region durch die Beschränkung einen Nutzenverlust gegenüber dem Fall ohne Rationierung erleidet. Demnach führt das Preisverhältnis in der Optimalbedingung (II.20) zu einer zusätzlichen Preisverzerrung, wenn die zweite Nebenbedingung bindend ist.

Formal läßt sich dieser Fall wie folgt darstellen:

Die Regierung maximiere den indirekten Nutzen ihres repräsentativen privaten Haushalts bei gegebenem Budget, das in einer Fallunterscheidung in zwei unterschiedliche Segmente zerlegt wird. Die zur Ermittlung der Empfangsauflage relevanten Steueraufkommen seien allgemein $S T(G, T r)$. Da zum einen der Konsum des privaten Gutes als auch der von Freizeit und damit die Konsum- und Einkommenssteuern von den Transfers abhängen, und zum zweiten die öffentlichen Ausgaben in Form zusätzlicher Einkommenssteuereinnahmen etc. indirekt das regionale Steueraufkommen beeinflussen, sind die Steueraufkommen explizit von den regionalen Entscheidungen abhängig. Im folgenden wird nun unterstellt, 


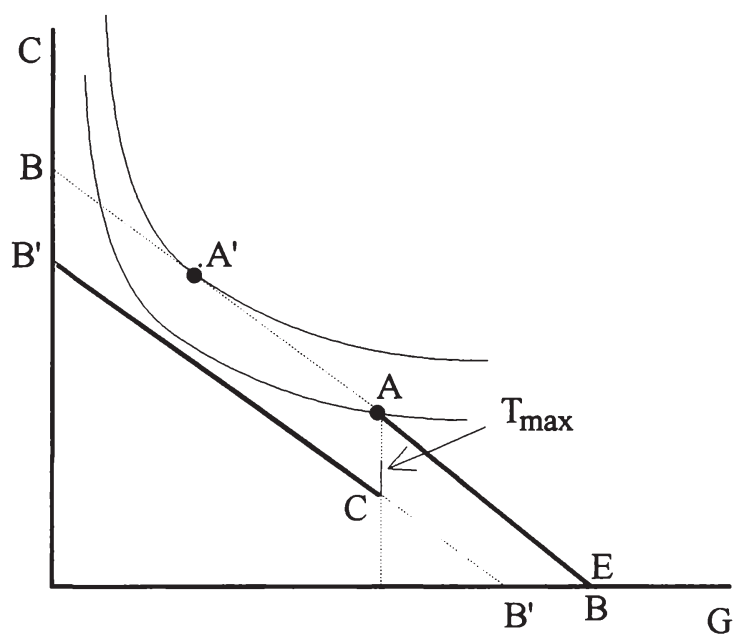

Abbildung II.4: Wohlfahrtsverlust bei Zuweisungen mit Empfangsauflage

daß die regionale Regierung diesen Zusammenhang zwischen ihren Steueraufkommen und ihren Ausgabenentscheidungen kennt und in ihrem Optimierungskalkül berücksichtigt. Im obigen Beispiel mit den Konsumsteuern wird dies direkt deutlich, da die Wahl der optimalen Konsummenge zugleich ein bestimmtes Steueraufkommen festlegt.

Liegen die Steuereinnahmen oberhalb (Fall (1)) der Grenze $T_{\max }$ besteht das regionale Budget nur aus den Einnahmen $R$. Ansonsten (Fall (2)) erhält die Region allgemeine Zuweisungen in Höhe von $Z$. Dies führt zu dem Ansatz

$$
\begin{aligned}
\max _{G, T r} \nu\left(G ; p, w^{n}, Y\right) \\
\text { u.d.NB.: } \begin{cases}(1) q_{G} G+T r=R & \text { für } S T(G, T r)>T_{\max } \\
(2) q_{G} G+T r=R+Z & \text { für } S T(G, T r) \leq T_{\max }\end{cases}
\end{aligned}
$$

mit $R$ als sonstige Einnahmen der regionalen Regierung.

Um dieses Optimierungsproblem mit Hilfe des Kuhn-Tucker-Ansatzes lösen zu können, wird es folgendermaßen umgeformt: ${ }^{26}$ Sind die regionalen Steueraufkommen höher als die

\footnotetext{
${ }^{26} \mathrm{Um}$ die Verwendung des Kuhn-Tucker-Ansatzes zu ermöglichen, wird im Fall (1) auch der Randpunkt der Budgetgeraden ohne $Z$ (in der Abbildung II.4 der Punkt C) einbezogen, der jedoch nicht realisierbar ist. Dies wirft allerdings kein Problem auf, da dieser Randpunkt aufgrund der Konvexität der Indifferenzkurven keiner nutzenmaximalen Wahl entsprechen kann.
} 
Obergrenze $T_{\max }$, so lautet der Ansatz für den Fall (1)

$$
\begin{aligned}
\max _{G, T r} \nu\left(G ; p, w^{n}, Y\right) & \\
\text { u.d.NB.: } & (1 a) R-q_{G} G-T r=0 \\
& \text { (1b) } S T(G, T r)-T_{\max } \geq 0 .
\end{aligned}
$$

Die Kuhn-Tucker-Bedingungen für Fall (1)

$$
\begin{array}{lr}
\frac{\partial \nu(\cdot)}{\partial G}-\lambda q_{G}+\mu \frac{\partial S T(\cdot)}{\partial G} \leq 0 & \text { wenn }<\text { dann } G=0 ; \\
\frac{\partial \nu(\cdot)}{\partial T r}-\lambda+\mu \frac{\partial S T(\cdot)}{\partial T r} \leq 0 & \text { wenn }<\text { dann } T r=0 \\
R-q_{G} G-T r=0 & \text { wenn }>0 \text { dann } \mu=0 \\
S T(G, T r)-T_{\max } \geq 0 &
\end{array}
$$

mit $\lambda$ und $\mu$ als Lagrange-Multiplikatoren der Nebenbedingungen (1a) und (1b). Da der Randpunkt der Budgetgerade bei $S T(G, T r)=T_{\max }$ in diesem Fall nicht realisierbar ist, ist die Nebenbedingung $(1 b)$ auf jeden Fall nicht bindend und damit gilt $\mu=0$. Nach Ausschluß der Randlösungen, d.h. für $G, T r \neq 0$ erhält man unter Verwendung der Marginalbedingung der privaten Nutzenmaximierung schließlich

$$
M R S_{C G}=-\frac{\partial H(\cdot) / \partial G}{\partial H(\cdot) / \partial C}=-\frac{q_{G}}{p} \quad \text { für } S T(G, T r)>T_{\max } .
$$

Sind dagegen die regionalen Steueraufkommen aus der Konsumsteuer geringer als $T_{\max }$, dann ist der entsprechende Optimierungsansatz im Fall (2):

$$
\begin{array}{r}
\max _{G, T r} \nu\left(G ; p, w^{n}, Y\right) \\
\text { u.d.NB.: }(2 a) R+Z-q_{G} G-T r=0 \\
\text { (2b) } T_{\max }-S T(G, T r) \geq 0 .
\end{array}
$$

Aus den entsprechenden Kuhn-Tucker-Bedingungen

$$
\begin{array}{lr}
\frac{\partial \nu(\cdot)}{\partial G}-\lambda q_{G}-\mu \frac{\partial S T(\cdot)}{\partial G} \leq 0 & \text { wenn }<\text { dann } G=0 \\
\frac{\partial \nu(\cdot)}{\partial T r}-\lambda-\mu \frac{\partial S T(\cdot)}{\partial T r} \leq 0 & \text { wenn }<\text { dann } T r=0 \\
R+Z-q_{G} G-T r=0 & \text { wenn }>0 \text { dann } \mu=0, \\
T_{\text {max }}-S T(G, T r) \geq 0 &
\end{array}
$$


erhält man nach Umformung der Bedingungen $\left(a^{\prime}\right)$ und $\left(b^{\prime}\right)$ und Ausschluß der Randlösungen, d.h. für $G, T r \neq 0$, die Ausdrücke

$$
\begin{aligned}
\frac{\partial H(\cdot)}{\partial G} & =\lambda q_{G}+\mu \frac{\partial S T(\cdot)}{\partial G} \\
\alpha & =\lambda+\mu \frac{\partial S T(\cdot)}{\partial T r} .
\end{aligned}
$$

Dies führt schließlich, wiederum unter Verwendung der Marginalbedingung des privaten Nutzenmaximierungskalküls $\partial H(\cdot) / \partial C=\alpha p, \mathrm{zu}$

$$
M R S_{C G}=-\frac{\partial H(\cdot) / \partial G}{\partial H(\cdot) / \partial C}=- \begin{cases}\frac{\lambda q_{G}+\mu(\partial S T(\cdot) / \partial G)}{[\lambda+\mu(\partial S T(\cdot) / \partial T r)] p} & \text { für } S T(G, T r)=T_{\max } \\ q_{G} / p & \text { für } S T(G, T r)<T_{\max } .\end{cases}
$$

Führt man die beiden Ergebnisse (Gleichung (II.17) und (II.19)) zusammen, erhält man als Marginalbedingungen für die Zuweisungen mit Empfangsauflage

$$
M R S_{C G}=-\frac{\partial H(\cdot) / \partial G}{\partial H(\cdot) / \partial C}=- \begin{cases}\frac{\lambda q_{G}+\mu(\partial S T(\cdot) / \partial G)}{[\lambda+\mu(\partial S T(\cdot) / \partial T r)] p} & \text { für } S T(G, T r)=T_{\text {max }} \\ q_{G} / p & \text { für } S T(G, T r) \neq T_{\max } .\end{cases}
$$

Für den Fall, daß die Beschränkung bindend wird $\left(S T(G, T r)=T_{\max }\right)$, ergeben sich Preisverzerrungen gegenüber dem Normalfall. Da der Multiplikator $\mu$ einen Schattenpreis für die Restriktion darstellt, der größer Null ist, wenn die Restriktion bindend ist, steigt der virtuelle Preis des öffentlichen und des privaten Gutes. Ob dann ein Gut und welches relativ billiger wird, hängt im einzelnen von der Höhe des Schattenpreises, von den Steueraufkommenstermen der öffentlichen Ausgaben $(\partial S T / \partial G)$ und der Transfers $(\partial S T / \partial T r)$ sowie dem Bruttopreis des privaten Gutes ab.

\subsubsection{Zuweisungen mit Eigenbeteiligung}

In der Literatur wird diese Art der Zuweisungen zumeist direkt an die von der Region bereitgestellte öffentliche Gütermenge gekoppelt (siehe z.B. OATES [1972], S.75-77). Im folgenden wird dieser Fall als Zuweisung mit direkter Eigenbeteiligung bezeichnet.

Im bundesdeutschen Finanzausgleichssystem hängt die Höhe von Finanzzuweisungen zum Teil von der Höhe der regionalen Finanzkraft ab. Diese regionale Finanzkraft wird ihrerseits indirekt durch die regionalen Entscheidungen beeinflußt. Höhere Ausgaben für 
öffentliche Güter führen z.B. zu höheren Einnahmen aus den Einkommenssteuern auf die in der Produktion der öffentlichen Güter erzielten Faktoreinkommen. Insofern beeinflußt die Wahl der öffentlichen Gütermenge indirekt die regionale Finanzkraft. Ebenso verändern variierte Transfers an die privaten Haushalte die Konsumausgaben und damit die Steueraufkommen der Regionen. Im folgenden wird angenommen, daß die regionalen Regierungen in ihrem Optimierungskalkül diese Zusammenhänge in etwa berücksichtigen. Dies bedingt, daß die finanzkraftabhängigen Zuweisungen den Charakter von Zuweisungen mit Eigenbeteiligung erhalten. Diese Art des indirekten Zusammenhangs zwischen Zuweisungshöhe und öffentlichen Ausgabenentscheidungen wird im weiteren als Zuweisung mit indirekter Eigenbeteiligung bezeichnet. Faßt man diese Zuordnung sehr weit, dann könnte man z.B. eine Veränderung der Bevölkerung durch Migration, wenn diese auf regionale Ausgabenentscheidungen zurückgeführt werden kann, ebenfalls als indirekte Eigenbeteiligung der Regionen an Finanzzuweisungen interpretieren, falls diese gemäß der relativen Bevölkerungsanteile vergeben werden.

\section{Zuweisungen mit direkter Eigenbeteiligung (matching grant)}

Diese z.B. von Musgrave/Musgrave/Kullmer ([1988], S.27) dargestellte Art der Finanzzuweisung (siehe auch Fischer [1988], S.43-47) sieht vor, daß die Höhe der Zuweisung von der Beteiligung der Region an der zusätzlichen Bereitstellung der öffentlichen Güter, also vom Ausmaß der finanziellen Selbstbeteiligung der Region an den Kosten der öffentlichen Güter abhängt.

Über

$$
\begin{aligned}
\max _{G, T r} \nu\left(G ; p, w^{n}, Y\right) \\
\text { u.d.NB.: } q_{G} G+T r=R+Z(G)
\end{aligned}
$$

erhält man

$$
\frac{\partial \nu(\cdot)}{\partial G}=\lambda\left(q_{G}-\frac{\partial Z(\cdot)}{\partial G}\right) \quad \text { und } \quad \frac{\partial \nu(\cdot)}{\partial Y} \frac{\partial Y}{\partial T r}=\lambda
$$

Aus der zweiten Gleichung ergibt sich $\alpha=\lambda$ und damit als Grenzrate der Substitution zwischen dem öffentlichen und privaten Gut

$$
M R S_{C G}=-\frac{\partial H(\cdot) / \partial G}{\partial H(\cdot) / \partial C}=-\frac{\lambda\left(q_{G}-\partial Z(\cdot) / \partial G\right)}{\alpha p}=\frac{q_{G}-\partial Z(\cdot) / \partial G}{p}
$$


Das öffentliche Gut wird relativ billiger, da es bezuschußt wird. Es wird daher relativ stärker nachgefragt als im Standardfall ohne Verzerrungen. ${ }^{27}$ Im Vergleich zu einer gleich hohen allgemeinen Zuweisung ohne Preisverzerrung entstehen dann Nutzenverluste durch die Preisveränderung und die daraus resultierenden Substitutionseffekte.

\section{Zuweisungen mit indirekter Eigenbeteiligung}

Die Höhe dieser Zuweisungen hängt nun in irgendeiner, nicht näher bestimmten Form von den regionalen Ausgabenentscheidungen ab. Dies führt zu folgender formalen Modifikation

$$
\begin{aligned}
& \max _{G, T r} \nu\left(G ; p, w^{n}, Y\right) \\
& \text { u.d.NB.: } q_{G} G+T r=R+Z(G, T r) .
\end{aligned}
$$

Aus den Marginalbedingungen

$$
\frac{\partial \nu(\cdot)}{\partial G}=\lambda\left(q_{G}-\frac{\partial Z(\cdot)}{\partial G}\right)
$$

und

$$
\frac{\partial \nu(\cdot)}{\partial Y} \frac{\partial Y}{\partial T r}=\lambda\left(1-\frac{\partial Z(\cdot)}{\partial T r}\right)
$$

kann man nach Umformung in

$$
\alpha=\lambda\left(1-\frac{\partial Z(\cdot)}{\partial T r}\right)
$$

die Beziehung zwischen der Grenzrate der Substitution und den Preisverhältnissen ermitteln:

$$
M R S_{C G}=-\frac{\partial H(\cdot) / \partial G}{\partial H(\cdot) / \partial C}=-\frac{\lambda\left(q_{G}-\partial Z(\cdot) / \partial G\right)}{\alpha p}=\frac{q_{G}-\partial Z(\cdot) / \partial G}{(1-\partial Z(\cdot) / \partial T r) p}
$$

Es ist unmittelbar erkennbar, daß eine indirekte Eigenbeteiligung ebenfalls zu Preisverzerrungen führen kann, wenn sich die verzerrenden Größen nicht gegenseitig aufheben.

\footnotetext{
${ }^{27}$ Dieses Ergebnis muß allerdings im N-Güter-Fall nicht mehr unbedingt gelten. Wie JURION [1979] zeigt, würde das subventionierte öffentliche Gut dann weniger nachgefragt als im Standardfall, wenn es eine Reihe von Komplementen für das subventionierte Gut in Form anderer öffentlicher Güter gäbe und dies dazu führt, daß das Substitut (hier das private Gut C) nicht von der Zuweisung profitiert.
} 


\subsubsection{Fliegenpapiereffekt (flypaper-effect)}

Die Ergebnisse können sich verändern, wenn der Fliegenpapiereffekt (flypaper-effect) auftritt. Dieses teilweise empirisch bestätigte Phänomen (siehe z.B. GramLICH [1977] oder Courant u.a. [1979] für die USA) besagt, daß eine Erhöhung der Zuweisungen zu einem im Vergleich zur obigen Darstellung überproportionalen Anstieg der öffentlichen Ausgaben führt (siehe z.B. Fischer [1990]).

Es gibt eine Reihe von möglichen Begründungen ${ }^{28}$, von denen zwei herausgegriffen werden sollen:

- Zum einen können unterschiedliche Ziele von Regierung und privaten Haushalten, wie z.B. Budgetmaximierung der Exekutive versus Nutzenmaximierung der Haushalte (MCGuire [1973]), eine Nichtäquivalenz zwischen Steuern/Transfers und Zuweisungen bewirken. In diesem Fall stellen die Regierungen die erhaltenen Zuweisungen den privaten Haushalten nicht in vollem Umfang für die Wahl zwischen $\mathrm{G}$ und $\mathrm{C}$ zur Verfügung. Dies kann auf asymmetrische Information zwischen Regierenden oder Wählern (OAtes [1972], Romer/Rosenthal [1980], Johnson [1977]) oder auf eine mangels sofort durchsetzbarer Alternativen entstehende, große Machtfülle der Regierung zurückgeführt werden (vgl. z.B. das Setter-Modell von Filimon, u.a. [1982]).

- Alternativ könnten die Individuen einer Art tax illusion unterliegen, indem sie Steuerveränderungen anders wahrnehmen als veränderte Zuweisungen (CoURANT u.a. [1979], OATEs [1977, 1979], WINER [1983] oder HewitT [1986]). Berücksichtigen die privaten Haushalte in ihren Entscheidungen nicht, daß sie die Zuweisungen mitfinanzieren müssen, so unterschätzen sie den tatsächlichen Steuerpreis der öffentlichen Ausgaben. Diese werden relativ billiger und vermehrt nachgefragt. ${ }^{29}$

Der flypaper-effecz führt bei allen Zuweisungsarten zu einer zusätzlichen Verzerrung des Preises des öffentlichen Gutes, der normalerweise aus einer Verringerung des Preises besteht. ${ }^{30}$

Für die Bundesrepublik besitzt der flypaper-effect vermutlich einige Bedeutung. Dies erklärt sich auf jeden Fall alleine schon durch die begrenzte Finanzautonomie der Länder, die eine Einnahmenerhöhung nur sehr eingeschränkt an die privaten Haushalte weitergeben können. Daher enthält der hier behandelte Fall fehlender Finanzautonomie den

\footnotetext{
${ }^{28}$ Einen Überblick der Argumente findet man bei KING [1984], S.102-113.

${ }^{29}$ Dieser in Partialmodellen entwickelte Ansatz wurde von LogAN ([1986], S.1311-1313) in ein Allgemeines Gleichgewichtsmodell übertragen.

${ }^{30}$ Dies ist natürlich abhängig von der genauen Implementierung. Doch selbst, wenn der Effekt durch Rationierung, wie bei Filimon u.a. [1982], modelliert wird, ergeben sich virtuelle Preisverzerrungen.
} 
Extremfall des flypaper-effect, nämlich den, daß überhaupt keine Weitergabe zusätzlicher Zuweisungen an die privaten Haushalte erfolgt. In der nachfolgenden Betrachtung des bundesdeutschen Finanzausgleichsystems wird der flypaper-effect nicht näher beachtet. Erst in später dargestellten EAG-Analyse ist er im Prinzip durch eine Modellierung berücksichtigt, die sich auf die Konsumentscheidung in ähnlicher Weise wie der flypaper-effect auswirkt.

Faßt man die Ergebnisse nochmals knapp zusammen, so ergeben sich als Hauptaussagen der Theorie der Finanzzuweisungen:

- Sprünge in den Budgetgeraden entstehen durch Empfangsauflagen. Daraus resultieren aber nur dann Preisverzerrungen, wenn die Optimallösung auf der Sprungstelle liegt (Rationierung).

- Die Steigung der Budgetgeraden ändert sich, wenn die Höhe der Zuweisungen an den Konsum des öffentlichen oder privaten Gutes gebunden ist, z.B. über die Höhe der Konsumsteuern, und mit dessen Höhe variiert (matching grants). Daraus resultieren Preisverzerrungen.

- Preisverzerrungen können auch durch den flypaper-effect hervorgerufen werden. I.d.R. führt dies zu einer Senkung des Preises des öffentlichen Gutes (direkt oder durch Rationierung).

- In allen anderen Fällen treten keine Preisverzerrungen auf.

Im folgenden sollen die bisherigen Aussagen und Überlegungen zur Theorie der Finanzzuweisungen auf das bundesdeutsche Finanzausgleichssystem übertragen werden.

\subsection{Finanzzuweisungen im bundesdeutschen Finanzausgleich}

Das bundesdeutsche Finanzausgleichssystem und die Reformvorschläge enthalten nur einige Zahlungsströme in Form von allgemeinen Zuweisungen. Nur die fixen Vorabzuweisungen im Rahmen der Bundesergänzungszuweisungen in den gesetzlichen Regelungen sind allgemeine Zuweisungen der reinen Form.

Zweckgebundene Zuweisungen sind in Form von BEZ nur von Fuest/LichtblaU [1991], Föttinger/SPAhn [1993] sowie dem Wissenschaftlichen Beirat [1992] vorgesehen.

Zuweisungen mit direkter Eigenbeteiligung und Zuweisungen mit Empfangsauflage gibt es in der reinen Form in keinem der Finanzausgleichssysteme. In einem 
Vorschlag von FöTTINGER/SPAHN [1993] ist allerdings die Höhe der öffentlichen Leistungen ein Kriterium zur Bestimmung der Finanzkraft und damit auch für die Höhe der LFA-Zuweisungen. Soweit Zuweisungen an eine Empfangsbedingung geknüpft sind, sind diese zumeist an eine Eigenbeteiligung geknüpft (s.u.).

Dagegen gibt es eine Reihe von Zuweisungen mit indirekter Eigenbeteiligung, wenn man annimmt, daß die Regionen in ihren Entscheidungen die Auswirkung ihres Ausgabenverhaltens auf die regionale Finanzkraft oder die Migration berücksichtigen. Ist dies nicht der Fall, dann sind diese Fälle in die Rubrik allgemeine Zuweisungen einzuordnen. Darunter fallen

- die Umsatzsteuerverteilung nach Bevölkerung, die in den gesetzlichen Regelungen die Vorabverteilung in Höhe von $75 \%$ der Länderanteile und in den meisten Reformvorschlägen die gesamte Umsatzsteuerverteilung betrifft,

- die Verteilung der Ergänzungsanteile und der Restverteilung in den bis 1995 geltenden Regelungen, wenn keine Ergänzungsanteile gewährt werden, die von einer Verteilung nach Bevölkerung abweichen,

- die Verteilung der Restverteilung, die in den seit 1.1.1995 geltenden Regelungen rein nach Bevölkerungsanteilen organisiert ist,

- die Umsatzsteuerverteilung nach den relativen Volkseinkommen, wie sie FUEST und LICHTBLAU [1991] vorschlagen wird,

- die Verteilung der Gewerbesteuerumlage und der Einkommenssteuern, die nach den regionalen Aufkommen vorgenommen werden, sowie

- die LFA-Zuweisungen und LFA-Beiträge in den Vorschlägen mit einstufigen, linearen Ausgleichstarifen (Föttinger/SPAhn [1993], Fuest/Lichtblau [1991], Buhl/Pfingsten [1991], Wissenschaftlicher Beirat [1991], Variante II).

Zuweisungen mit Empfangsbedingung und Eigenbeteiligung sind sämtliche von der Finanzkraft abhängige Zahlungsströme. Dies sind z.B.

- die Ergänzungsanteile in der Umsatzsteuerverteilung seit 1995 und in der Umsatzsteuerverteilung vor 1995, wenn diese im zweiten Fall nicht nach Bevölkerung verteilt werden,

- die Mindestzuweisungen in den Vorschlägen des Wissenschaftlichen Beirats [1991] und von Fuest/Lichtblau [1991],

- die Zuweisungen aufgrund der Garantieklauseln,

- die finanzkraftabhängigen BEZ in den gesetzlichen Regelungen sowie 
- die von den Westländern aus ihren Umsatzsteuereinnahmen zu leistenden Zahlungen in den Fonds Deutsche Einheit.

Zum Teil sind diese Zuweisungen auch in ihrer Höhe nach oben begrenzt, wie z.B. die Finanzausgleichszuweisungen an die finanzschwachen oder die Finanzbeiträge der finanzstarken Länder im bundesdeutschen Finanzausgleich.

Am Beispiel der Länderfinanzausgleichszuweisungen in den gesetzlichen Regelungen, die den Charakter von Zuweisungen mit Empfangsbedingung und Eigenbeteiligung aufweisen, sollen die Auswirkungen auf die Budgetgerade kurz demonstriert werden. Unter Vernachlässigung der Garantieklauseln und einer Reihe von Steuerarten und Sonderregeln kann man die Grundstrukutur des Länderfinanzausgleichs wie folgt darstellen:

In einem einfachen Modell, in dem Konsumsteuern explizit berücksichtigt werden, finanzieren sich öffentliche und private Ausgaben aus dem Einkommen $Y_{s}$, den sonstigen regionalen Steuern $T$ und den Finanzausgleichszuweisungen $F A_{s}$, die abhängig sind von den Nichtkonsumsteuern, den Konsumsteuern und den durchschnittlichen Steuereinnahmen der Länder $\bar{T}$. Setzt man $\bar{T}$ für $M A_{s}$ und $\tau q_{s} C_{s}+T$, für $M S_{s}$ in den Gleichungen für $F A Z_{s}$ (I.19) und für $F P_{s}$ (I.25) ein, so erhält man folgende Budgetgerade:

$$
q_{G}, G_{s}+(1+\tau) q_{s} C_{s}=Y_{s}+T_{s}+F A_{s}\left(\tau q_{s} C_{s}, T_{s}, \bar{T}\right)
$$

mit

$$
F A_{s}= \begin{cases}\text { (I) } 1,044 \bar{T}-\left(\tau q_{s} C_{s}+T_{s}\right) & \text { für } s \in \mathcal{S}_{3}^{+} \\ \text {(II) } 0,7\left[1,02 \bar{T}-\left(\tau q_{s} C_{s}+T_{s}\right)\right] & \text { für } s \in \mathcal{S}_{2}^{+} \\ \text {(III) } 0 & \text { für } s \in \mathcal{S}_{1}^{+} \\ \text {(IV) } 0,375\left[\bar{T}-\left(\tau q_{s} C_{s}+T_{s}\right)\right] & \text { für } s \in \mathcal{S}_{+}^{-} \\ \text {(V) } 0,03 \bar{T}+\left[0,92 \bar{T}-\left(\tau q_{s} C_{s}+T_{s}\right)\right] & \text { für } s \in \mathcal{S}_{-}^{-}\end{cases}
$$

Die Steigung der Budgetgeraden in ihren verschiedenen Segmenten, die sich durch Auflösung nach $C$, und Ableitung nach $G$, ergibt, ist dann:

$$
\frac{\partial C_{s}}{\partial G_{s}}= \begin{cases}-\frac{q_{G}}{p_{s}+\tau q_{s}} & \text { für (I) und }(V) \\ -\frac{q_{G_{s}}}{p_{s}+0,7 \tau q_{s}} & \text { für (II) } \\ -q_{G,} / p_{s} & \text { für (III) } \\ -\frac{q_{G_{s}}}{p_{s}+0,375 \tau^{C} q_{s}} & \text { für (IV) }\end{cases}
$$

Die Steigung, und damit das relative Preisverhältnis zwischen öffentlichem und privaten Gut, ist im Segment (III), dem ärmsten Teil der finanzstarken Länder, am größten und im 
Segment (I) und (V), den finanzstärksten und finanzschwächsten Ländern, am geringsten. Bis auf Segment (III) werden in allen anderen Budgetsegmenten zusätzliche Preisverzerrungen hervorgerufen, die im Abschnitt (I) und (V) am größten sind. Dies ist verständlich, da diese Länder bei einem geringen Zuwachs ihrer Steuereinnahmen die stärksten Einnahmeeinbußen aller Länder durch den LFA hinnehmen müssen.

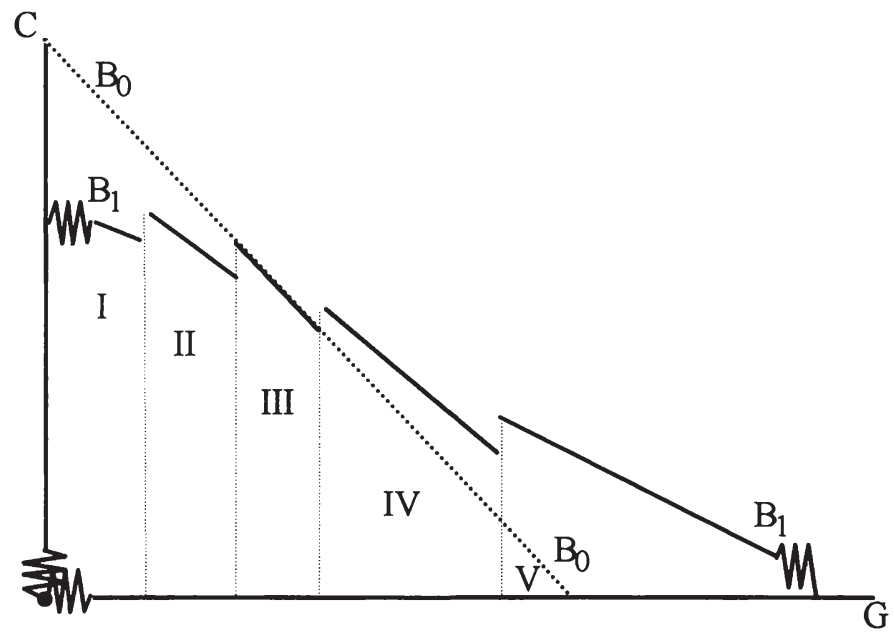

Abbildung II.5: Budgetsegmente bei LFA vor 1995, Ausschnitt

In Abbildung II.5 wird das graphisch verdeutlicht. Ausgehend von der ursprünglichen Budgetgerade ohne LFA $\overline{B_{0} B_{0}}$ sinkt ein Land unter dieses Budget, wenn es Steuereinnahmen oberhalb von $102 \%$ von $\bar{T}$ besitzt und steigt über dieses Budget, wenn es finanzschwach ist.

Die wirklichen Effekte könnte man nur durch Berechnung der tatsächlichen Budgetgerade ermitteln. Sie werden von den obigen Preisverzerrungen abweichen, da $G$, über seinen Einfluß auf die Einkommens- und Faktorsteuern sich indirekt selbst finanziert, und zudem die Garantieklauseln zu berücksichtigen sind.

In der vereinfachten Darstellung bleiben die Steigungen der Budgetgeraden in ihren verschiedenen Segmenten, in der Berechnung der $F A Z_{s}$ gegenüber den Regelungen vor 1995 unverändert. Für die Länder im Segment (I) sinkt die Anrechnung von $\tau^{C}$, da nur mehr $80 \%$ ihrer Finanzkraft oberhalb von $110 \%$ des Länderdurchschnitts angerechnet werden. Die Länder in Abschnitt (II), der jetzt schon bei $101 \%$ beginnt, werden nur noch zu $66 \%$ ihrer überschüssigen Finanzkraft belastet. Da nun auch die Länder im Finanzkraftbereich zwischen $100 \%$ und $101 \%$ zur Finanzierung der LFA-Zuweisungen herangezogen werden, 
verändert sich auch die Steigung ihrer Budgetgerade. Ihre überdurchschnittliche Finanzkraft wird zu $15 \%$ berücksichtigt. Daraus ergeben sich die Ableitungen der Budgetgeraden:

$$
\frac{\partial C_{s}}{\partial G_{S}}= \begin{cases}-\frac{q_{G}}{p_{s}+0,8 \tau^{C} q_{s}} & \text { für }(I) \text { und }(V) \\ -\frac{q_{G}}{p_{s}+0,66 \tau^{C} q_{s}} & \text { für }(I I) \\ -\frac{q_{G}}{p_{s}+0,15 \tau^{C} q_{s}} & \text { für }(I I I) \\ -\frac{q_{G}}{p_{s}+0,375 \tau^{C} q_{s}} & \text { für }(I V) \\ -\frac{q_{G}}{p_{s}+\tau^{C} q_{s}} & \text { für }(V) .\end{cases}
$$

Die niedrigste Steigung liegt nun bei den finanzschwächsten Ländern vor, deren privater Konsum den größten Preiszuwachs erleidet. Eine graphische Darstellung ist nicht notwendig, da sie der Abbildung II.5 sehr ähnlich ist.

\subsection{Zusammenfassende Bemerkungen zur Theorie der Finanz- zuweisungen}

Wie sich in vorangegangenen Analyse gezeigt hat, können mit Ausnahme der allgemeinen Zuweisungen bei allen Arten von Finanzzuweisungen preisverzerrende Effekte auftreten. Selbst bei allgemeinen Zuweisungen treten dann Verzerrungen auf, wenn ein flypapereffect existiert oder die Ausgabenautonomie der Länder beschränkt ist. Genaugenommen verlieren die Zuweisungen dann ihren allgemeinen Charakter.

Die Verzerrungen bestehen zum Teil in einer tatsächlichen Veränderung der relativen Preise (Drehung der Budgetgeraden) und zum Teil in einer Veränderung der virtuellen Preise, soweit die Entscheidungen auf den Sprungstellen der Budgetgeraden liegen.

Im bundesdeutschen Finanzausgleichssystem und den Neuordnungsvorschlägen können daher preisverzerrende Effekte auftreten, da in allen Alternativen verschiedene Arten von Zuweisungen auftreten. ${ }^{31}$ Allerdings können fast alle Zuweisungen mit Eigenbeteiligung nur über einen indirekten Einfluß des Ausgabenverhaltens auf die Steueraufkommen definiert werden, wie z.B. die Abhängigkeit der Umsatzsteueraufkommen von den Konsumentscheidungen.

\footnotetext{
${ }^{31}$ Dieses Ergebnis widerspricht dem von Fischer [1988], der die Finanzausgleichszuweisungen nur als allgemeine Zuweisungen charakterisierte.
} 


\section{Volle Finanzautonomie der Regionen}

Die eben dargestellte Theorie der Finanzzuweisungen ließe sich nun auch bei vollständiger Finanzautonomie der Regionen darstellen. Vollständige Finanzautonomie soll hier explizit als die Möglichkeit der regionalen Regierungen zur Variation einzelner Steuersätze definiert werden. Zur einfachen Abgrenzung gegenüber der Ausgabenautonomie werden keine Lump-Sum-Steuersätze betrachtet, da diese bereits in der Analyse der Ausgabenautonomie berücksichtigt wurden.

Die Auswirkungen der Finanzzuweisungen auf die Budgetgeraden und damit auf die relativen Preise wird als erstes untersucht (Abschnitt 6.1.). Bei Finanzautonomie treten zusätzlich verzerrende Wirkungen durch den Einfluß der Finanzausgleichsregelungen auf die Wahl der optimalen Steuersätze auf, die ebenfalls näher unter die Lupe genommen werden (Abschnitt 6.2.). Dies entspricht einer Untersuchung der Anreizwirkungen der Finanzausgleichsregelungen auf die Ausschöpfung der regionalen Steuerquellen (fiscal effort), wie sie in sehr einfacher Form bereits in der Analyse der Budgetgleichungen in Abschnitt 2. durchgeführt wurde.

\subsection{Verzerrungen der relativen Güterpreise}

Aus dem Optimierungsansatz

$$
\begin{aligned}
& \max _{G, \tau} \nu\left(G ; p, w^{n}, Y\right) \\
& \text { u.d.NB.: } q_{G} G+\operatorname{Tr}=R+Z(G, \tau)
\end{aligned}
$$

erhält man über die Ableitung der Lagrangefunktion nach $G$

$$
\frac{\partial \nu(\cdot)}{\partial G}=\lambda\left(q_{G}-\frac{\partial Z(\cdot)}{\partial G}\right)
$$

sowie dem aus dem privaten Nutzenmaximierungsansatz hergeleiteten Grenznutzen des privaten Konsums $\partial H(\cdot) / \partial C=\alpha p$ die Beziehung

$$
M R S_{C G}=-\frac{\partial H(\cdot) / \partial G}{\partial H(\cdot) / \partial C}=-\frac{\lambda\left(q_{G}-\partial Z(\cdot) / \partial G\right)}{\alpha p} .
$$

Den Ausdruck $\lambda / \alpha$ kann man genauer spezifizieren, indem man die Ableitung der Lagrangefunktion nach dem regionalen Steuersatz

$$
\frac{\partial \mathcal{L}(\cdot)}{\partial \tau}=\frac{\partial \nu(\cdot)}{\partial p} \frac{\partial p}{\partial \tau}+\frac{\partial \nu(\cdot)}{\partial w^{n}} \frac{\partial w^{n}}{\partial \tau}+\frac{\partial \nu(\cdot)}{\partial Y} \frac{\partial Y}{\partial \tau}+\lambda \frac{\partial Z(\cdot)}{\partial \tau}=0
$$


verwendet. Diese führt wegen $\partial \nu(\cdot) / \partial p=-\alpha C$ und wegen $\partial \nu(\cdot) / \partial w^{n}=-\alpha F$ (RoY's Identität - siehe z.B. VARIAN [1992], S.149) sowie wegen $\partial \nu(\cdot) / \partial Y=\alpha$ zu

$$
\alpha=\lambda\left(-\frac{\partial Z(\cdot) / \partial \tau}{\partial Y / \partial \tau-C(\partial p / \partial \tau)-F\left(\partial w^{n} / \partial \tau\right)}\right)
$$

mit $\alpha$ als privatem Grenznutzen der Einkommen. Schließlich erhält man die umgeformte Optimalbedingung

$$
M R S_{C G}=-\frac{\partial H(\cdot) / \partial G}{\partial H(\cdot) / \partial C}=\frac{q_{G}-\partial Z(\cdot) / \partial G}{p\left(-\frac{\partial Z(\cdot) / \partial \tau}{\partial Y / \partial \tau-C(\partial p / \partial \tau)-F\left(\partial w^{n} / \partial \tau\right)}\right)}
$$

Je nach Gestaltung der Finanzzuweisungen ergeben sich Preisverzerrungen. Zusätzlich entstehen Verzerrungen zwischen dem relativen Preis des privaten und des öffentlichen Gutes aufgrund der durch die Steuersatzvariationen veränderten privaten Einnahmen und Ausgaben (Nenner des Nenners).

\subsection{Verzerrungen der Optimalsteuersätze}

Man kann nun zusätzlich zeigen, daß die Art der Finanzzuweisungen eine entscheidende Rolle für die Höhe der optimalen Steuersätze spielen. Damit entsteht eine zweite Art der Verzerrung durch die Finanzausgleichsregelungen. Am Beispiel proportionaler Konsumsteuern wird dies im folgenden gezeigt.

Besteht die Steuerautonomie in Form frei wählbarer proportionaler Konsumsteuern, so erhält man für den Fall, daß die Region einen optimalen Konsumsteuersatz $\tau$ wählt, um ein bestimmtes Niveau an öffentlichen Einnahmen $R$ zu erzielen, folgenden Ansatz

$$
\begin{aligned}
& \max _{\tau} \nu(G ; p, w, Y) \\
& \text { u.d.NB.: } Z(S T)=\bar{R} \quad \text { mit } S T=\tau q C
\end{aligned}
$$

und

$$
\begin{gathered}
\nu(G ; p, w, Y)=\left\{\max _{C, F} U(G, C, F) \text { u.d.NB.: } p C+w F=Y\right\} \\
\text { mit } p=(1+\tau) q .
\end{gathered}
$$

Die Ableitung der entsprechenden Lagrangefunktion

$$
\mathcal{L}(\cdot)=\nu(\cdot)+\lambda[Z(S T)-\bar{R}]
$$


mit $\lambda$ als Grenznutzen der öffentlichen Einnahmen, nach dem Steuersatz

$$
\frac{\partial \mathcal{L}(\cdot)}{\partial \tau}=\frac{\partial \nu(\cdot)}{\partial p} \frac{\partial p}{\partial \tau}+\lambda \frac{\partial Z(\cdot)}{\partial S T}\left(q C+\tau q \frac{\partial C}{\partial p} \frac{\partial p}{\partial \tau}\right)=0
$$

führt wegen $\partial p / \partial \tau=q$ und unter Ausnutzung von RoY's Identität zu

$$
\alpha C=\lambda \frac{\partial Z(\cdot)}{\partial S T}\left(C+\tau q \frac{\partial C}{\partial p}\right)
$$

mit $\alpha$ als privatem Grenznutzen der Einkommen. Durch Erweiterung und Umstellung erhält man

$$
C\left(\alpha+\lambda \frac{\partial Z(\cdot)}{\partial S T} \tau q \frac{\partial C}{\partial Y}-\lambda \frac{\partial Z(\cdot)}{\partial S T}\right)=\lambda \frac{\partial Z(\cdot)}{\partial S T} \tau q\left(\frac{\partial C}{\partial p}+\frac{\partial C}{\partial Y} C\right) .
$$

Unter Verwendung der Slutsky-Gleichung mit $S_{C C}$ als Substitutionsterm führt dies zu

$$
C\left(\alpha+\lambda \frac{\partial Z(\cdot)}{\partial S T} \tau q \frac{\partial C}{\partial Y}-\lambda \frac{\partial Z(\cdot)}{\partial S T}\right)=\lambda \frac{\partial Z(\cdot)}{\partial S T} \tau q S_{C C} .
$$

Definiert man den sozialen Grenznutzen des privaten Einkommens $\gamma$ als privaten Grenznutzen plus den Grenznutzen, der aufgrund höherer privaten Einkommen zusätzlich erzielten Steuereinnahmen, d.h. als

$$
\gamma=\alpha+\lambda \frac{\partial Z(\cdot)}{\partial S T} \tau q \frac{\partial C}{\partial Y}
$$

dann erhält man schließlich

$$
\tau=-\left[\frac{\lambda(\partial Z(\cdot) / \partial S T)-\gamma}{\lambda(\partial Z(\cdot) / \partial S T)}\right] \frac{C}{q S_{C C}} .
$$

Der zweite Ausdruck auf der rechten Seite entspricht der inversen kompensierten Eigenpreiselastizität des privaten Konsums $\epsilon_{C C}$. Dies führt zur Bestimmungsgleichung für den optimalen Konsumsteuersatz (siehe z.B. AUERBACH [1985], S.87-88)

$$
\tau^{*}=-\frac{\lambda(\partial Z(\cdot) / \partial S T)-\gamma}{\lambda(\partial Z(\cdot) / \partial S T)} \frac{1}{\epsilon_{C C}}
$$

Der optimale Steuersatz ist umgekehrt proportional zur kompensierten Eigenpreiselastizität der Konsumnachfrage. Zusätzlich ist der optimale Steuersatz um so geringer, je größer der soziale Grenznutzen $\gamma$ einer zusätzlichen privaten Einnahmeeinheit im Vergleich zum Grenznutzen einer zusätzlichen öffentlichen Einnahmeeinheit $\lambda$ multipliziert 
mit dem Zusammenhang zwischen Zuweisungen und Steueraufkommen. ${ }^{32}$ Dies entspricht den Opportunitätskosten der Steuererhebung, die gemessen werden durch die relative marginale Nutzenveränderung einer Einkommenseinheit bei einer Umschichtung vom privaten in das öffentliche Budget. Je höher diese Opportunitätskosten der Steuererhebung sind, um so geringer ist der optimale Steuersatz.

Der Einfluß der Finanzausgleichsregelungen auf den optimalen Steuersatz $\tau^{*}$ ist in den Opportunitätskosten enthalten. Aus einer einfachen Umformung

$$
\tau^{*}=\left[1-\frac{\gamma}{\lambda(\partial Z(\cdot) / \partial S T)}\right] \frac{1}{-\epsilon_{C C}} .
$$

sieht man unmittelbar: Die Anreizwirkung zur Ausnutzung der eigenen Steuerquellen, d.h. die Höhe des optimalen Steuersatzes, sinkt mit steigendem Substitutionalitätscharakter der Finanzausgleichszuweisungen, d.h. mit sinkendem $\partial Z(\cdot) / \partial S T$. Dieses Ergebnis gilt unter der Annahme, daß $\partial Z(\cdot) / \partial S T$ keinen überkompensierenden gegenläufigen Einfluß auf $\alpha$ und $\lambda$ besitzt.

Man könnte vergleichbare Ergebnisse auch noch für andere Steuerarten herzuleiten versuchen und dabei zumindest ähnliche Aussagen bezüglich der Anreizwirkung der Finanzausgleichsregelungen ableiten. Um Platz zu sparen und dem Leser unnötige Ausschweifungen zu ersparen, soll das dargestellte Beispiel genügen. Dies macht auch deshalb Sinn, da in der Bundesrepublik für die Länder angesichts weitgehend fehlender Steuersetzungsrechte wohl keine Möglichkeit besteht, annähernd optimale Steuersätze zu wählen. Selbst das Hebesatzsetzungsrecht der Gemeinden auf die Gewerbe- und Grundsteuern, sowie die vom WissenschaftLIChEN BEIRAT [1992] u.a. vorgeschlagene verstärkte Steuerautonomie der Länder setzen einen so engen Spielraum bezüglich der Variation der jeweiligen Steuersätze, daß kaum davon ausgegangen werden kann, daß die Länder und Gemeinden tatsächlich optimale Steuersätze erheben können.

Bezüglich des bundesdeutschen Finanzausgleichssystems kann man dennoch bereits hier feststellen: Da der bundesdeutsche Finanzausgleich ebenso wie die meisten der Neugestaltungsvorschläge durch einen starken Substitutionalitätscharakter gekennzeichnet ist, ist die Anreizwirkung des bundesdeutschen Finanzausgleichssystems auf die Ausschöpfung der eigenen Steuerquellen (die Höhe der optimalen Steuersätze) sehr gering.

Dies sollte man beachten, wenn man den Ländern mehr Steuerautonomie zubilligen will. Wahrscheinlich wird das nur dann zu verstärkten Eigenanstrengungen der Länder zur

\footnotetext{
${ }^{32}$ Die allgemeine Herleitung der optimalen Steuersätze geht auf Diamond/MirrLeEs [1971] zurück. Eine der grundlegenden Arbeiten zur Darstellung der Optimalen Besteuerungsproblematik bei möglichem Steuerexport ist die von ARnotT/Grieson [1981].
} 
Ausschöpfung ihrer Steuerkraft führen, wenn die Anreizwirkung durch die Finanzausgleichsregelungen nicht zu stark gedämpft wird. Die Empfehlung des WissenschafTLICHEN BEIRATS [1992], die Einnahmen der Länder aus ihren selbstbestimmbaren Steuerarten nicht oder höchstens normiert in die Ermittlung der Finanzkraft im Länderfinanzausgleich einzubeziehen, würde dieser Folgerung entsprechen.

\section{Zusammenfassung der Partialanalyse}

In der bislang entwickelten Argumentation wurden erste Aspekte dargelegt, die in einer umfassenden Effizienzanalyse der geltenden Regelungen und der verschiedenen Vorschläge zum Finanzausgleich berücksichtigt werden sollten.

Finanzausgleichsregelungen besitzen demnach einen Einfluß auf die Optimalbedingungen der Nutzenkalküle, die sich in Form verzerrender Keile zwischen den relativen Güterpreisen und der Grenzrate der Substitution zwischen dem privaten und dem öffentlichen Gut oder einem verzerrenden Effekt auf die Höhe der optimalen Steuersätze bemerkbar machen können. Letzerer Zusammenhang entspricht einer Veränderung der Anreizwirkungen zur Ausschöpfung der regionalen Steuerquellen.

Die bundesdeutschen Finanzausgleichsregelungen sowie beinahe alle hier untersuchten Neuordnungsvorschläge sind durch einen hohen Grad an Substitutionalität zwischen den Steueraufkommen der Bundesländer und den Einnahmen der öffentlichen Haushalte des jeweiligen Landes gekennzeichnet. Dadurch setzen sie Anreize für die Länder, ihre Steueranspannung auf ein aus gesamtwirtschaftlicher Sicht suboptimales Niveau zu senken.

Der Einfluß des Finanzausgleichs auf die relativen Güterpreise hängt zum einen von den institutionellen Regelungen und zum anderen von den Auswirkungen der Ausgabenentscheidungen auf die regionalen Steueraufkommen ab (bei vollständiger Information - ansonsten auch vom Grad der Berücksichtigung dieser Aufkommenseffekte in den Entscheidungskalkülen). Eine Zerlegung der entsprechenden Effekte besitzt folgende Form:

$$
\frac{\partial Z(\cdot)}{\partial G}=\frac{\partial Z}{\partial G}+\frac{\partial Z}{\partial S T} \frac{\partial S T}{\partial G} \quad \text { bzw. } \quad \frac{\partial Z(\cdot)}{\partial T r}=\frac{\partial Z}{\partial S T} \frac{\partial S T}{\partial T r} .
$$

$\mathrm{Da}$ es keinen direkten Einfluß der Transfers auf die Höhe der Zuweisungen gibt, wird die entsprechende Ableitung in der obigen Gleichung nicht berücksichtigt.

Im Prinzip gilt jedoch: Höhere Ausgaben für öffentliche Güter oder höhere Transfers führen zu steigenden regionalen Steueraufkommen. Diese werden über die Finanzausgleichszuweisungen einnahmenrelevant. Allerdings hängt dies wiederum vom Grad der Substitutionalität der Finanzausgleichsregelungen ab. Ohne Finanzausgleichsregelungen 
oder bei Neutralität der Regelungen würden die Einnahmen genau mit der Höhe der Aufkommenserhöhung wachsen. Die öffentlichen Güterausgaben oder Transfers würden sich dann teilweise über höhere regionale Steuereinnahmen selbst finanzieren und die jeweiligen Güterpreise würden um diese Selbstfinanzierungsterme reduziert. Ist die Reduktion der beiden Preise nicht symmetrisch - was sehr wahrscheinlich der Fall ist -, dann entstehen Preisverzerrungen. Besteht Substitutionalität des Finanzausgleichs, so wird die Selbstfinanzierung abgeschwächt und damit die auftretende Preisverzerrung vermutlich reduziert.

In diesem Fall besitzt das bundesdeutsche Finanzausgleichssystem einen verzerrungsreduzierenden Einfluß auf die relativen Güterpreise. Die Selbstfinanzierung der öffentlichen Güter wird reduziert und damit der preissenkende Effekt von Steuern verringert. Damit bestimmt das Finanzausgleichssystem die Höhe der Selbstfinanzierungsterme $\partial Z(\cdot) / \partial G$ und folglich auch das Ausmaß der Preisverzerrung.

Besitzen die Regionen keine Finanzautonomie, dann entstehen zusätzliche Preisverzerrungen durch die Höhe der Zuweisungen, die mit zunehmender Größe den Schattenpreis der Rationierungsrestriktion erhöhen.

Preisverzerrungen lösen Substitutionseffekte aus, die letztlich zu einer Allokationsstörungen führen. Aus diesem Grund zeigt die hier durchgeführte Partialanalyse bereits Wohlfahrtswirkungen von Finanzausgleichsregelungen auf. Da sich in der Summe die durch den Finanzausgleich ausgelösten Einkommenseffekte aufheben werden, werden Substitutionseffekte die relevanten Größen zur Beurteilung von gesamtwirtschaftlichen Wohlfahrtseffekten sein. $\mathrm{Ob}$ jedoch die in der Partialanalyse festgestellten Substitutionseffekte bereits alle derartigen Effekte von Finanzausgleichsregelungen beschreiben, kann nur in einem allgemeinen Gleichgewichtsmodell festgestellt werden. Daher steht die Erweiterung der Analyse im Rahmen eines Totalmodells auch am Anfang des nächsten Kapitels.

Zur Frage der Beurteilung der Wohlfahrtsgewinne der einzelnen Länder müssen neben der Ermittlung der Substitutionseffekte zusätzlich die Höhe der Umverteilungszuweisungen (Transfers zwischen den Ländern) sowie noch weitere aus der Außenhandelsliteratur bekannte Größen untersucht werden (v.a. Terms-of-Trade-Veränderungen und Steuerexporte). Auch dies ist Inhalt des nächsten Kapitels und schließt sich an die Bestimmung der Substitutionseffekte an. 


\section{Kapitel III}

\section{Wohlfahrtseffekte}

Die bisherigen partialanalytischen Überlegungen zu den Effizienzwirkungen von Finanzausgleichssystemen sollen nun durch eine umfassendere Analyse ergänzt werden.

$\mathrm{Zu}$ diesem Zweck werden als Referenzgrößen die Bedingungen für ein Paretooptimum einer Gesellschaft mit $R$ Regionen, zwei Faktoren, $N$ Gütern, $N$ Produktionssektoren und Vorleistungen bestimmt. In einem zweiten Schritt werden die sich im Marktprozeß bei Staatstätigkeit ergebenden Optimalbedingungen hergeleitet und mit den Paretobedingungen verglichen. Dies soll Aufschluß über die durch die Finanzausgleichsregelungen und das Steuersystem hervorgerufenen Allokationsstörungen geben. In Anlehnung an das bisherige Vorgehen werden drei Grade der Finanzautonomie unterschieden und die in den verschiedenen Szenarien resultierenden Optimalbedingungen einander gegenübergestellt. Die auf diese Weise abgeleiteten Verzerrungen geben Hinweise auf Substitutionseffekte und (gesellschaftliche) Wohlfahrtsverluste. Allerdings sind diese Ergebnisse nur eine Orientierung für die tatsächlichen Wirkungen, da aufgrund der Second-Best-Problematik ein Abbau einzelner Substitutionseffekte nicht unbedingt wohlfahrtsverbessernd sein muß.

Im Anschluß an die Darstellung der staatlicherseits verursachten Verzerrungen werden Überlegungen bezüglich der Wohlfahrtsveränderungen einzelner Länder angestellt.

Das zugrundegelegte Modell orientiert sich an der bisherigen Darstellung und an dem später verwendeten Empirischen Allgemeinen Gleichgewichtsmodell. Die folgenden Überlegungen sollen damit zugleich als Vorbereitung zur Interpretation der Berechnungen in der EAG-Analyse dienen.

\section{Bedingungen für ein Pareto-Optimum}

Die Entwicklung und Darstellung der Pareto-Bedingungen erfolgt in enger Anlehnung an Rosenberg ([1993], 108-113), da das verwendete Modell weitgehend dieselbe Struktur wie das von FEHR/ROSENBERG/WIEGARD [1992] präsentierte EAG-Modell besitzt.

Das zur Ableitung der Marginalbedingungen verwendete Modell entspricht bezüglich der Güterstruktur dem bisher verwendeten Ansatz. Neben dem öffentlichen Gut $G_{\boldsymbol{s}}$ werden 
in jeder Region die privaten Konsumgüter $C_{r}$ und Freizeit $F_{s}$ konsumiert. Die Menge $\mathcal{N}$ bezeichnet den Typ des Gutes: privates Gut $C$ oder öffentliches Gut $G$. Der Index $i$ oder $j$ bezieht sich auf diesen Gütertyp.

Die Unternehmenssektoren $j$ produzieren ihre Outputgüter $Q_{j}^{s}$ unter Einsatz der Primärfaktoren Arbeit $L_{j}^{s}$ und Kapital $K_{j}^{s}$, sowie unter Einsatz eigener oder bezogener Outputgüter als Vorleistungen $V_{i j}^{r s}$. Es gibt demnach keine spezifischen Vorleistungsgüter, da jedes Outputgut sowohl als Vorleistungs- als auch als Endnachfragegut verwendet werden kann. Der Faktor Arbeit sei interregional immobil aber intersektoral mobil, während Kapital zwischen Sektoren und Regionen vollständig mobil ist.

Das Maximierungsproblem des allwissenden und wohlwollenden Planers

$$
\begin{array}{ll}
\max H_{s}\left(G_{s}, F_{s}, C_{r s} \mid r \in \mathcal{R}\right) & \\
\text { u.d.NB.: (1) } H_{h}\left(G_{h}, F_{h}, C_{r h} \mid r \in \mathcal{R}\right)-\bar{H}_{h}=0 & \forall h \in \mathcal{R}_{s} \\
\text { (2) } Q_{G}^{r}-f_{G}^{r}\left(K_{G}^{r}, L_{G}^{r}, V_{j G}^{k r} \mid \forall j \in \mathcal{N} ; k \in \mathcal{R}\right)=0 & \forall r \in \mathcal{R} \\
\text { (3) } Q_{C}^{r}-f_{C}^{r}\left(K_{C}^{r}, L_{C}^{r}, V_{j C}^{k r} \mid \forall j \in \mathcal{N} ; k \in \mathcal{R}\right)=0 & \forall r \in \mathcal{R} \\
\text { (4) } \bar{E}_{r}-F_{r}-\sum_{j \in \mathcal{N}} L_{j}^{r}=0 & \forall r \in \mathcal{R} \\
\text { (5) } \sum_{r \in \mathcal{R}} \bar{K}_{r}-\sum_{r \in \mathcal{R}} \sum_{j \in N} K_{j}^{r}=0 & \forall r \in \mathcal{R} \\
\text { (6) } Q_{G}^{r}-G_{r}-\sum_{k \in \mathcal{R}} \sum_{j \in \mathcal{N}} V_{G j}^{r k}=0 & \forall r \in \mathcal{R}, \\
\text { (7) } Q_{C}^{r}-\sum_{k \in \mathcal{R}} C_{\tau k}-\sum_{k \in \mathcal{R}} \sum_{j \in \mathcal{N}} V_{C j}^{r k}=0 &
\end{array}
$$

besteht nun darin, den Nutzen $H_{s}$ einer Region $s$ zu maximieren, ohne eine andere der restlichen $R_{s}{ }^{1}$ Regionen schlechter zu stellen. Die Konstanz der Nutzen dieser restlichen Regionen geht damit als Nebenbedingung (1) in das Optimierungskalkül ein. Weitere Nebenbedingungen sind die Produktionstechnologien, (2) und (3), sowie die Markträumungsbedingungen für die Faktor-, (4) (mit $\bar{E}_{\mathrm{r}}$ als Zeitgrundausstattung) und (5), und Gütermärkte, (6) und (7). Als Kontrollvariablen stehen dem Planer die Größen $\left(G_{s} \mid s \in \mathcal{R}\right),\left(F_{s} \mid s \in \mathcal{R}\right)$, $\left(C_{r s} \mid s, r \in \mathcal{R}\right),\left(Q_{i}^{s} \mid s \in \mathcal{R}, i \in \mathcal{N}\right),\left(K_{i}^{s} \mid s \in \mathcal{R}, i \in \mathcal{N}\right),\left(L_{i}^{s} \mid s \in \mathcal{R}, i \in \mathcal{N}\right)$ und $\left(V_{i j}^{r s} \mid r, s \in \mathcal{R}, i, j \in \mathcal{N}\right)$ zur Verfügung.

Die Ableitungen der Lagrangefunktion führt nach einigen Umformungen zu den notwendigen Bedingungen erster Ordnung. ${ }^{2}$

\footnotetext{
${ }^{1} R_{s}$ steht für alle Länder außer dem Land $s$.

${ }^{2}$ Wegen $N=2$ enthält das Gleichungssystem $8 \cdot R+5 \cdot R^{2}$ Unbekannte. Dies sind die jeweils $R$ Variablen
} 
$\mathrm{Zu}$ dem hier verfolgten Zweck ist es sinnvoll, die Bedingungen so darzustellen, daß im ersten Teil nur die intraregionalen Beziehungen berücksichtigt werden, und im zweiten Teil alle interregionalen Beziehungen zusammengestellt werden. Daraus resultieren, analog zu Rosenberg ([1993], S.109), als erstes die

(1) Bedingungen für die optimale Faktorallokation innerhalb einer Region

$$
\begin{aligned}
\frac{\partial f_{C}^{s} / \partial K_{C}^{s}}{\partial f_{C}^{s} / \partial L_{C}^{s}} & =\frac{\partial f_{G}^{s} / \partial K_{G}^{s}}{\partial f_{G}^{s} / \partial L_{G}^{s}} & & \forall s \in \mathcal{R}, \\
\frac{\partial f_{i}^{s}}{\partial V_{i i}^{s s}} & =1 & & \forall s \in \mathcal{R}, i \in \mathcal{N}
\end{aligned}
$$

Diese Gleichungen beziehen sich ausschließlich auf die Faktorallokation innerhalb einer Region. Wie üblich, muß die Grenzrate der technischen Substitution von Arbeit und Kapital in der Produktion der verschiedenen Güter einer (!) Region übereinstimmen (Bedingung (III.1a)). Solange dies nicht erfüllt ist, kann der Output durch intersektoralen Faktoraustausch gesteigert werden. Wie Rosenberg ([1993], S.110) betont, gilt diese Beziehung aufgrund der Immobilität des Faktors Arbeit nur innerhalb eines Landes.

Die Gleichung (III.1b) bezieht sich auf den Einsatz der selbstproduzierten Vorleistungen in die Produktion desselben Gutes. Sie beschreibt, daß im Optimum mit dem Einsatz einer Einheit eines Gutes genau eine Outputeinheit desselben Gutes produziert werden kann. Wäre das entsprechende Grenzprodukt kleiner als eins, so käme dieser Produktionsprozeß der Vernichtung von Gütern gleich. Dann wäre es empfehlenswert, diesen Produktionsbzw. Vernichtungsprozeß einzustellen. Wäre dagegen das entsprechende Grenzprodukt größer als eins, so könnte man durch den Produktionsprozeß größere Outputmengen erzeugen als Inputmengen eingesetzt werden. In diesem Fall wäre eine Ausdehnung der Produktion durch einen höheren Inputeinsatz so lange effizienzsteigernd bis das Grenzprodukt einer Faktoreinheit genau eins ergibt.

Die, laut Soнmen ([1976], S.136), bei Vorleistungen zusätzlich üblichen Marginalbedingungen bezüglich der Grenzrate der technischen Substitution werden an dieser Stelle nicht

$\overline{G_{r}}$ und $F_{r}$, die $R^{2}$ Variablen $C_{r s}$, die $N^{2} \cdot R^{2}$ Größen $V_{i j}^{r s}$ und die jeweils $N \cdot R$ Faktornachfragearten $L_{i}^{r}$ und $K_{i}^{r}$ sowie $N \cdot R$ Outputmengen $Q_{i}^{r}$, die allerdings durch Gleichsetzen der Nebenbedingungen der Produktionstechnologien (3) und (4) mit den Markräumungsbedingungen (5) und (6) eliminiert werden können. Die $6 R$ Nebenbedingungen ergeben sich aus den $R-1$ konstanten Nutzenniveaus, den $R$ Technologiebeschränkungen der öffentlichen Güter und $R$ Technologiebeschränkungen der privaten Güter (zusammen $N \cdot R$ ), den $R$ Arbeitsmarkt- und einer Kapitalmarkträumungsbedingung (zusammen $R+1$ ) sowie jeweils $R$ Gütermarkräumungsbedingungen der öffentlichen und privaten Güter (zusammen $N \cdot R$ ). Damit resultieren insgesamt $5 \cdot R^{2}+2 R$ Marginalbedingungen, die sich aus der Zahl der unabhängigen Variablen abzüglich der Menge der Nebenbedingungen ergeben. 
als Bedingungen für einen optimalen Faktoreinsatz formuliert. Sie werden vielmehr in die Beziehungen zwischen der Grenzrate der Substitution und der Grenzrate der Transformation integriert (Gleichungen (III.1c) und (III.1f)).

Zusätzlich zur Effizienz des Faktor- bzw. Vorleistungseinsatzes ist in jeder Region eine optimale Abstimmung von Produktionsstruktur und Nachfragestruktur sicherzustellen. Diesem Zweck dienen die folgenden aus dem obigen Optimierungsansatz abgeleiteten notwendigen

(2) Bedingungen für eine optimale Abstimmung von Produktions- und Nachfragestruktur innerhalb einer Region

$$
\begin{array}{ll}
\frac{\partial H^{s} / \partial G_{s}}{\partial H^{s} / \partial C_{s s}}=\frac{\partial f_{C}^{s} / \partial L_{C}^{s}}{\partial f_{G_{s}} / \partial L_{G_{s}}}=\frac{\partial f_{C}^{s} / \partial V_{i C}^{r s}}{\partial f_{G}^{s} / \partial V_{i G}^{r s}} & \forall s, r \in \mathcal{R}, \forall i \in \mathcal{N}, \\
\frac{\partial H^{s} / \partial F_{s}}{\partial H^{s} / \partial C_{s s}}=\frac{\partial f_{C}^{s}}{\partial L_{C}^{s}} & \forall s \in \mathcal{R} .
\end{array}
$$

Wie üblich sollen die Grenzraten der Substitution (MRS), d.h. die Tauschverhältnisse zwischen den einzelnen Gütern, genau der regionalen Rate der Transformation (MRT) dieser Güter entsprechen, und damit die Steigung der Indifferenzkurve der Region mit der Steigung der Produktionsmöglichkeitskurve übereinstimmen. Eine solche Beziehung gilt sowohl zwischen dem privaten Konsumgut (III.1c) und dem öffentlichen Gut als auch zwischen dem privaten Konsum und der Freizeit (III.1d). Die regionale Transformationsrate bezieht sich auf den Einsatz von Faktoren in der Inlandsproduktion. Sie wird an dieser Stelle nicht über den Kapitaleinsatz definiert, da dieser zur Formulierung der interregionalen Transformationsrate (FRT) benötigt wird.

Da ein Verzicht auf eine Arbeitseinheit im Prinzip die Produktion einer Einheit Freizeit bedeutet, ist das Grenzprodukt der Arbeit in der „Produktion” des Gutes Freizeit genau 1. Deshalb ist die MRT zwischen dem privaten Gut und der Freizeit nichts anderes als das Grenzprodukt des Faktors Arbeit in der Produktion des anderen Gutes. Die Bedingung (III.1d) kann demnach als Gleichheit der MRT mit der MRS zwischen dem privaten Konsumgut und der Freizeit interpretiert werden. ${ }^{3}$ Die Bedingung enthält keinen Bezug auf Vorleistungen, da zur "Produktion” von Freizeit kein Vorleistungseinsatz notwendig ist.

Nun müssen noch die Bedingungen für eine optimale interregionale Allokation von Gütern und Faktoren angegeben werden.

\footnotetext{
${ }^{3} \mathrm{Da}$ je Region nur ein repräsentativer Haushalt und nur ein von den Haushalten angebotener variabler Faktor (Arbeit) berücksichtigt wird, fallen alle anderen von TrESCH ([1981], S.34-35) aufgeführten Bedingungen weg.
} 
(3) Bedingungen für ein optimales interregionales Tauschgleichgewicht

$$
\begin{array}{lll}
\text { (III.1e) } & \frac{\partial H^{s} / \partial C_{s s}}{\partial H^{s} / \partial C_{r s}}=\frac{\partial H^{h} / \partial C_{s h}}{\partial H^{h} / \partial C_{r h}} & \forall r, h \in \mathcal{R}_{s}, \\
\text { (III.1f) } & \frac{\partial H^{s} / \partial C_{s s}}{\partial H^{s} / \partial C_{r s}}=\frac{\partial f_{C}^{r} / \partial K_{C}^{r}}{\partial f_{C}^{s} / \partial K_{C}^{s}}=\frac{\partial f_{C}^{r} / \partial V_{i C}^{h r}}{\partial f_{C}^{s} / \partial V_{i C}^{h s}} & \forall h \in \mathcal{R}, r \in \mathcal{R}_{s}, i \in \mathcal{N} .
\end{array}
$$

Die Gleichungen (III.1e) und (III.1f) beziehen sich auf den interregionalen Tausch und beschreiben die Bedingungen, unter denen bei interregionalem Handel der Güter alle Tauschgewinne ausgeschöpft werden. Solange die Grenzraten der Substitution zwischen zwei Gütern unterschiedlicher Herkunft nicht in allen Regionen übereinstimmen, wird es zu weiterem nutzenerhöhenden interregionalen Tausch kommen (Gleichung III.1e).

Der Ausdruck (III.1 $f$ ) bezeichnet die Bedingung für eine optimale internationale Kapitalund Vorleistungsgüterallokation. Erst, wenn die interregionale Grenzrate der Transformation (FRT) des Kapitaleinsatzes in der Produktion zweier nach Herkunft differenzierter Konsumgüter genau der MRS dieser Güter in jedem Empfängerland ist, besteht keine Möglichkeit mehr, durch Umschichtung des handelbaren Faktors Kapital Handelsgewinne zu erzielen. Die gleiche Argumentation greift bezüglich des Handels mit Vorleistungsgütern.

Für die ungewöhnliche Form der Gleichungen (III.1f) ist die ARMINGTON-Annahme verantwortlich, ohne die die übliche Bedingung für die optimale interregionale Kapitalallokation

$$
\frac{\partial f_{i}^{s}}{\partial K_{i}^{s}}=\frac{\partial f_{i}^{r}}{\partial K_{i}^{r}}
$$

resultieren würde: ${ }^{4}$ Zusätzlich entfiele in allen anderen Bedingungen der Index des Ursprungslandes der Güter. ${ }^{5}$

Für den weiteren Gang der Untersuchung ist es sinnvoll, die obigen Bedingungen etwas zu modifizieren. Auf diese Weise kann eine Beziehung zwischen dem regionalen öffentlichen Gut $G_{s}$ und der Freizeit $F_{s}$ gewonnen werden, die zur Darstellung der Verzerrungen durch ein Finanzausgleichssystem hilfreich sein kann.

\footnotetext{
${ }^{4}$ Bei Wegfall der ARMington-Annahme werden die Güter nicht mehr nach ihrem Ursprungsland differenziert. Dann gilt: $\partial H^{s} / \partial C_{s s}=\partial H^{s} / \partial C_{r s}$ und $\partial f_{i}^{r} / \partial V_{i i}^{s r}=\partial f_{i}^{s} / \partial V_{i i}^{s s}(\mathrm{vgl}$. FeHR/Rosenberg $/$ WIEGARD [1992], S.64).

${ }^{5}$ Eine ausführlichere Interpretation der Bedingungen bezüglich des Einsatzes der Vorleistungsgüter findet sich bei Sohmen [1976] und für ein EAG-Modell ähnlicher Struktur bei Rosenberg ([1993], S.111-113)
} 
Die entsprechende Marginalbedingung

$$
\frac{\partial H^{s} / \partial F_{s}}{\partial H^{s} / \partial G_{s}}=\frac{\partial f_{G}^{s}}{\partial L_{G}^{s}} \quad \forall s \in \mathcal{R}
$$

ist das Ergebnis der Division der Gleichung (III.1c) durch (III.1d). Die Interpretation erfolgt analog zu Gleichung (III.1d). Die MRS zwischen dem öffentlichen Gut und der Freizeit entspricht genau dem Grenzprodukt des Arbeitseinsatzes in der Produktion des öffentlichen Gutes.

Obwohl $G_{s}$ nicht interregional gehandelt wird besteht eine intraregionale MRS zwischen $C_{r s}$ und $G_{s}$, da der repräsentative Haushalt der Region $s$ sowohl $G_{s}$ als auch $C_{r s}$ konsumieren kann. Man kann nun unter Verwendung der Bedingungen für eine optimale inländische Konsum- und Produktionsstruktur zwischen $C_{s s}$ und $G_{s}$, den Gleichungen (III.1c), und den Bedingungen für eine optimale interregionale Konsum- und Produktionsstruktur zwischen $C_{r s}$ und $C_{s s}$, den Gleichungen (III.1f), eine Beziehung zwischen $G_{s}$ und $C_{r s}$ herleiten:

$$
\frac{\partial H^{s} / \partial G_{s}}{\partial H^{s} / \partial C_{r s}}=\frac{\partial f_{C}^{r} / \partial K_{C}^{r}}{\partial f_{G}^{s} / \partial K_{G}^{s}} \quad \forall r \in \mathcal{R}_{s}
$$

Diese Gleichung formuliert eine Gleichheit der Grenzrate der Substitution zwischen dem importierten Konsumgut und dem öffentlichen Gut mit der interregionalen Grenzrate der Transformation von Kapital in der Produktion beider Güter (FRT). Inhaltlich kann man dies so interpretieren, daß der inländische Haushalt $G_{s}$ gegen $C_{r s}$ tauschen möchte, dies aber nur indirekt über den Handel mit Kapital kann. Die dadurch induzierte Kapitalwanderung führt zu einer optimalen Abstimmung der Produktions- und der Konsumstruktur des öffentlichen Gutes und des importierten Konsumgutes. Insofern ist Kapitalmobilität ein Ersatz für den fehlenden Güterhandel und schöpft alle Tauschgewinne aus dem Handel aus. $^{6}$

\section{Optimalbedingungen bei Staatseingriffen}

Die hergeleiteten Pareto-Bedingungen entsprechen zugleich den Optimalbedingungen einer Marktökonomie ohne Marktversagen und ohne Staatseingriffe. Sie dienen als Referenzsystem zur Beurteilung der Effizienz einer Ökonomie, wenn sie auch in dieser Form

\footnotetext{
${ }^{6}$ Dies ist eine Umkehrung des Faktorpreisausgleichstheorems, gemäß dem Güterhandel unter bestimmten Bedingungen ein vollkommener Ersatz für fehlende Faktormobilität ist (siehe z.B. Gandolfo [1987a], S.86).
} 
aufgrund verzerrender Einflüsse nicht tatsächlich erreichbar sind. Im folgenden sollen diese Optimalbedingungen nun mit den in einer Marktökonomie mit Staatseingriffen resultierenden Marginalbedingungen verglichen werden, um erste Aussagen über allokativ verzerrende Effekte zu gewinnen.

\subsection{Optimalbedingungen bei Ausgabenautonomie}

Das zugrundeliegende Modell entspricht bezüglich der Nachfrageseite weitgehend dem im Kapitel II verwendeten Ansatz. Die aus Gleichung (II.2) auf Seite 67 bekannte Budgetgleichung wird ergänzt durch die Nettozinseinkünfte $r^{n} \cdot \bar{K}_{s}$, mit $r^{n}$ als Nettozinssatz nach Abzug der Zinssteuer. Zusätzlich wird die Zahl der Konsumgüter um die aus den Regionen $r$ bezogenen Güter erhöht. Dies führt dann zur indirekten Nutzenfunktion

$$
\begin{aligned}
\nu_{s}\left(G_{s} ; p_{r} \mid r \in \mathcal{R}, w_{s}^{n}, r^{n}, Y_{s}\right)=\left\{\max _{F_{s}, C_{r}} H_{s}\left(G_{s}, F_{s}, C_{r s} \mid r \in \mathcal{R}\right)\right. & \text { u.d.NB.: } \sum_{r} p_{r} C_{r s}+w_{s}^{n} F_{s} \\
& \left.=w_{s}^{n} \bar{E}_{s}+r^{n} \bar{K}_{s}+T r_{s}=Y_{s}\right\}, \\
\text { mit } \quad p_{r}=q_{r}\left(1+\tau^{C}\right), \quad r^{n}=\left(1-\tau^{Y}\right) r & \text { und } \quad w_{s}^{n}=\left(1-\tau^{Y}\right) w_{s} .
\end{aligned}
$$

Das entsprechende regionale Optimierungsproblem lautet

$$
\begin{aligned}
\max _{G_{s}, T_{s}} & \nu_{s}\left(G_{s} ; p_{r} \mid r \in \mathcal{R}, w_{s}^{n}, r^{n}, Y_{s}\right) \\
& \text { u.d.NB.: } q_{G_{s}} G_{s}+T r_{s}=T_{s}(\cdot)+F A_{s}(\cdot)+E Z_{s}(\cdot) \quad \forall s \in \mathcal{R},
\end{aligned}
$$

mit $T_{s}$ als Steuerverteilungs-, $F A_{s}$ als LFA- und $E Z_{s}$ als Ergänzungszusweisungen. ${ }^{7}$ Aus der Ableitung der Lagrangefunktion

$$
\mathcal{L}(\cdot)=\nu_{s}(\cdot)+\lambda\left[T_{s}(\cdot)+F A_{s}(\cdot)+E Z_{s}(\cdot)-q_{G} G_{s}+T r_{s}\right]
$$

erhält man

$$
\frac{\partial \nu_{s}}{\partial G_{s}}=\lambda\left(q_{G_{s}}-\frac{\partial T_{s}}{\partial G_{s}}-\frac{\partial F A_{s}}{\partial G_{s}}-\frac{\partial E Z_{s}}{\partial G_{s}}\right) .
$$

Definiert man den Anteil der Selbstfinanzierung der öffentlichen Ausgaben am Preis des öffentlichen Gutes in Form höherer, durch die Ausgaben bedingter Einnahmen der Region als $\delta_{q G \text {, mit }}$

$$
\delta_{q G_{s}}=\frac{\partial T_{s}}{\partial q_{G_{s}} G_{s}}+\frac{\partial F A_{s}}{\partial q_{G_{s}} G_{s}}+\frac{\partial E Z_{s}}{\partial q_{G_{s}} G_{s}}
$$

\footnotetext{
${ }^{7}$ Der Einfachheit halber werden im weiteren die Angaben $s \in \mathcal{R}$ immer dann weggelassen, wenn die angegebenen Beziehungen für alle Länder $s$ gelten.
} 
der sich aus den Ableitungen der Steuerverteilungs-, LFA- und BEZ-Zuweisungen zusammensetzt, dann kann man die obige Gleichung $(b)$ unter Beachtung von $\partial \nu_{s} / \partial G_{s}=$ $\partial H_{s} / \partial G_{s}$ umformen in

$$
\partial H_{s} / \partial G_{s}=\lambda q_{G_{0}}\left(1-\delta_{q G_{s}}\right)
$$

Verbindet man diesen Ausdruck mit der notwendigen Bedingung erster Ordnung der privaten Nutzenmaximierung

$$
\partial H_{s} / \partial C_{s s}=\alpha p_{s}
$$

mit $\alpha$ als Lagrangemultiplikator, dann erhält man schließlich

$$
\frac{\partial H_{s} / \partial G_{s}}{\partial H_{s} / \partial C_{s s}}=\frac{\lambda q_{G_{s}}\left(1-\delta_{q G_{s}}\right)}{\alpha p_{s}} .
$$

Die Beziehung $\lambda / \alpha$ gewinnt man aus der Ableitung der privaten Budgetgleichung nach den Transfers

$$
\sum_{r} \frac{\partial C_{r s}}{\partial T r_{s}}+w_{s}^{n} \frac{\partial F_{s}}{\partial T r_{s}}=1
$$

und aus der Ableitung der indirekten Nutzenfunktion nach den Transfers

$$
\frac{\partial \nu_{s}}{\partial T r_{s}}=\frac{\partial H_{s}}{\partial T r_{s}}=\left(\sum_{r} \frac{\partial H_{s}}{\partial C_{r s}} \frac{\partial C_{r s}}{\partial T r_{s}}+\frac{\partial H_{s}}{\partial F_{s}}+\frac{\partial F_{s}}{\partial T r_{s}}\right) \text {. }
$$

Dies ist wegen $(c)$, der Marginalbedingung des privaten Nutzenmaximierungskalküls

$$
\partial H_{s} / \partial F_{s}=\alpha w_{s}^{n}
$$

und (e) gleich dem Ausdruck

$$
\frac{\partial H_{s}}{\partial T r_{s}}=\alpha\left(\sum_{r} p_{r} \frac{\partial C_{r s}}{\partial T r_{s}}+w_{s}^{n} \frac{\partial F_{s}}{\partial T r_{s}}\right)=\alpha .
$$

Schließlich erhält man aus der Ableitung der Lagrangefunktion (a) die Gleichung

$$
\frac{\partial H_{s}}{\partial T r_{s}}=\alpha=\lambda\left(1-\frac{\partial T_{s}}{\partial T r_{s}}-\frac{\partial F A_{s}}{\partial T r_{s}}-\frac{\partial E Z_{s}}{\partial T r_{s}}\right) .
$$

Nach Definition des Selbstfinanzierungsanteils der Transfers $\delta_{T r}$, als

$$
\delta_{T r_{s}}=\frac{\partial T_{s}}{\partial T r_{s}}+\frac{\partial F A_{s}}{\partial T r_{s}}+\frac{\partial E Z_{s}}{\partial T r_{s}}
$$


wird $(f) \mathrm{zu}$

$$
\partial H_{s} / \partial T r_{s}=\alpha=\lambda\left(1-\delta_{T \tau_{s}}\right)
$$

Setzt man dies in die Gleichung $(d)$ ein, ergibt sich die Marginalbedingung

$$
\frac{\partial H_{s} / \partial G_{s}}{\partial H_{s} / \partial C_{s s}}=\frac{q_{G_{s}}\left(1-\delta_{q G_{s}}\right)}{p_{s}\left(1-\delta_{T_{s}}\right)},
$$

Die Konsumseite wird schließlich durch die Produktionsseite der Ökonomie ergänzt. Die Unternehmen des Sektors $j$ der Region $r$ verwenden die Produktionstechnologie $f_{j}^{s}(\cdot)$ zur Herstellung des sektoralen Outputs $Q_{j}^{s}$. Inputs in die Produktion sind die Primärfaktoren Arbeit $L_{j}^{s}$ und Kapital $K_{j}^{s}$ sowie die Vorleistungen $V_{i j}^{r s}$. Letztere werden zum Produzentenpreis $q_{i}^{\tau}$ aus dem Sektor $i$ der Region $r$ bezogen. Die Unternehmer verhalten sich als Gewinnmaximierer. Der Gewinn ist in der üblichen Profitgleichung als Erlös aus dem Verkauf des Outputs abzüglich der Inputkosten definiert. Die Faktorkosten für Kapital weichen aufgrund der abzuführenden Faktorsteuern $\tau_{j s}^{k}$ vom Faktorpreis (Zinssatz) $r$ ab. Der Arbeitseinsatz wird nicht besteuert. Die Produktionssteuern $\widehat{\tau}_{j s}^{Q}$ werden auf den Wert des Outputs zu Produktionskosten erhoben. ${ }^{8}$

Das Maximierungskalkül der Unternehmenssektoren $j$ der Region $s$ lautet demnach:

$$
\begin{gathered}
\max _{L_{j}^{s}, K_{j}^{s}, V_{i j}^{r s}} q_{j}^{s} Q_{j}^{s}-w_{j}^{s} L_{j}^{s}-\hat{r}_{j}^{s} K_{j}^{s}-\sum_{\tau} \sum_{i} q_{i}^{r} V_{i j}^{r s}-\widehat{\tau}_{j s}^{Q} q_{j}^{s} Q_{j}^{s} \\
\text { u.d.NB.: } f_{j}^{s}\left(L_{j}^{s}, K_{j}^{s}, V_{i j}^{r s} \mid r \in \mathcal{R}, i \in \mathcal{N}\right)=\bar{Q}_{j}^{s} \\
\text { mit } \hat{r}_{j}^{s}=r\left(1+\tau_{j s}^{K}\right) \text { und mit } \widehat{\tau}_{j s}^{Q}=\frac{\tau_{j s}^{Q}}{1+\tau_{j s}^{Q}} .
\end{gathered}
$$

Es führt zu den Optimalbedingungen:

$$
q_{j}^{s}\left(1-\widehat{\tau}_{j s}^{Q}\right) \frac{\partial f_{j}^{s}}{\partial K_{j}^{s}}=\hat{r}_{j}^{s}, \quad q_{j}^{s}\left(1-\widehat{\tau}_{j s}^{Q}\right) \frac{\partial f_{j}^{s}}{\partial L_{j}^{s}}=w^{s}, \quad q_{j}^{s}\left(1-\widehat{\tau}_{j s}^{Q}\right) \frac{\partial f_{j}^{s}}{\partial V_{i j}^{r s}}=q_{i}^{s} .
$$

Durch verschiedene Umstellungen erhält man die Optimalbedingungen der Konkurrenzwirtschaft bezüglich des intraregionalen Faktoreinsatzes:

(1) Optimale Faktorallokation

$$
\begin{gathered}
\partial f_{i}^{s} / \partial V_{i i}^{s s}=1 \\
\frac{1}{\left(1+\tau_{i s}^{K}\right)} \frac{\partial f_{i}^{s} / \partial K_{i}^{s}}{\partial f_{i}^{s} / \partial L_{i}^{s}}=\frac{1}{\left(1+\tau_{j s}^{K}\right)} \frac{\partial f_{j}^{s} / \partial K_{j}^{s}}{\partial f_{j}^{s} / \partial L_{j}^{s}} .
\end{gathered}
$$

\footnotetext{
${ }^{8}$ Auf diese Weise muß man nicht zwischen dem Herstellungspreis und dem Produzentenpreis unterscheiden, sondern kann auf die Verwendung des Herstellungspreises verzichten.
} 
Die Verzerrungen in der Faktorallokation werden durch sektoral und regional differenzierende Kapitalsteuern hervorgerufen. Eine einheitliche Steuer wäre demnach nicht verzerrend.

(2) Bedingungen für eine optimale Konsum- und Produktionsstruktur

Nimmt man eine konstante Zeitgrundausstattung der Region an, dann erhält als Marginalbedingungen aus den privaten und sektoralen Optimierungskalkülen die Ausdrücke

$$
\frac{\left(1+\tau^{C}\right)\left(1-\delta_{T r_{s}}\right)}{\left(1-\delta_{q G_{s}}\right)} \frac{\frac{\partial H_{s}}{\partial G_{s}}}{\frac{\partial H_{s}}{\partial C_{s s}}}=\frac{\left(1+\tau_{G_{s}}^{K}\right)\left(1-\hat{\tau}_{C s}^{Q}\right)}{\left(1+\tau_{C s}^{K}\right)\left(1-\widehat{\tau}_{G_{s}}^{Q}\right)} \frac{\frac{\partial f_{C}^{s}}{\partial K_{C}^{s}}}{\frac{\partial f_{G}^{s}}{\partial K_{G}^{s}}}=\frac{\left(1-\widehat{\tau}_{C s}^{Q}\right)}{\left(1-\widehat{\tau}_{G_{s}}^{Q}\right)} \frac{\frac{\partial f_{C}^{s}}{\partial V_{i C}^{+s}}}{\frac{\partial f_{G}^{s}}{\partial V_{i G}^{s}}}
$$

Demnach verursachen außer den differenzierten Faktor- und Produktionssteuern auch die Konsumsteuer und die Rückwirkungen von privaten und öffentlichen Ausgaben auf die Einnahmen der regionalen Regierung $\left(\delta_{q G}\right.$, bzw. $\left.\delta_{T r_{s}}\right)$ allokative Verzerrungen.

Zusätzlich ergibt sich

$$
\frac{\left(1+\tau^{C}\right)}{\left(1-\tau^{Y}\right)} \frac{\partial H_{s} / \partial F_{s}}{\partial H_{s} / \partial C_{s s}}=\left(1-\widehat{\tau}_{C s}^{Q}\right) \partial f_{C}^{s} / \partial L_{C}^{s} .
$$

Die Einkommenssteuer-, Konsumsteuer- und Produktionssteuersätze bewirken Allokationsstörungen, indem sie einen Keil zwischen die Grenzrate der Substitution und die Grenzrate der Transformation beider Güter treiben.

In Anlehnung an die modifizierte Beziehung (III.1 $d^{\prime}$ ) kann man durch Verwendung der obigen Gleichungen folgenden Zusammenhang zwischen $G_{s}$ und $F_{s}$ ermitteln:

$$
\frac{\left(1-\delta_{q G_{s}}\right)}{\left(1-\tau^{Y}\right)\left(1-\delta_{T r_{s}}\right)} \frac{\partial H_{s} / \partial F_{s}}{\partial H_{s} / \partial G_{s}}=\left(1-\hat{\tau}_{G_{s}}^{Q}\right) \partial f_{G}^{s} / \partial L_{G}^{s} .
$$

Demnach verhindern die Einkommenssteuer- und Produktionssteuersätze sowie die infolge höherer öffentlicher und privater Ausgaben auftretenden Steueraufkommensänderungen und deren Auswirkungen auf die Einnahmen eine optimale Abstimmung von Konsumund "Produktions"-struktur zwischen $F_{s}$ und $G_{s}$.

Die interregionalen Beziehungen führen zu den Marginalbedingungen für ein

(3) Optimales interregionales Tauschgleichgewicht

$$
\begin{gathered}
\frac{\partial H_{s} / \partial C_{s s}}{\partial H_{s} / \partial C_{r s}}=\frac{\partial H_{h} / \partial C_{s h}}{\partial H_{h} / \partial C_{r h}} \\
\frac{\partial H_{s} / \partial C_{s s}}{\partial H_{s} / \partial C_{r s}}=\frac{\left(1+\tau_{C s}^{K}\right)\left(1-\hat{\tau}_{C r}^{Q}\right)}{\left(1+\tau_{C r}^{K}\right)\left(1-\hat{\tau}_{C s}^{Q}\right)} \frac{\partial f_{C}^{r} / \partial K_{C}^{r}}{\partial f_{C}^{s} / \partial K_{C}^{s}}=\frac{\left(1-\hat{\tau}_{C r}^{Q}\right)}{\left(1-\hat{\tau}_{C s}^{Q}\right)} \frac{\partial f_{C}^{r} / \partial V_{i C}^{h r}}{\partial f_{C}^{s} / \partial V_{i C}^{h s}} .
\end{gathered}
$$


Die einzigen Verzerrungen im interregionalen Tausch werden durch die unterschiedliche Kapital- und Produktionsbesteuerung in den eng verwandten Sektoren beider Regionen hervorgerufen. Die Preisverzerrungen in den interregionalen Austauschbeziehungen könnten demnach durch eine interregionale steuerliche Gleichbehandlung der Produktion und des Kapitaleinsatzes in der Produktion der engen Substitutionsgüter auf Null reduziert werden.

Allerdings treten aufgrund der indirekten Beziehung zwischen $G_{s}$ und $C_{r s}$ über die Kapitalwanderung zusätzliche Verzerrungen im interregionalen Handel auf. Die entsprechende Gleichung

$$
\frac{\left(1+\tau^{C}\right)\left(1-\delta_{T \tau_{s}}\right)}{\left(1-\delta_{q G s}\right)} \frac{\partial H_{s} / \partial G_{s}}{\partial H_{s} / \partial C_{\tau s}}=\frac{\left(1+\hat{\tau}_{G s}^{K}\right)\left(1-\widehat{\tau}_{C r}^{Q}\right)}{\left(1+\hat{\tau}_{C r}^{K}\right)\left(1-\hat{\tau}_{G s}^{Q}\right)} \frac{\partial f_{C}^{\tau} / \partial V_{i C}^{h r}}{\partial f_{G}^{s} / \partial V_{i G}^{h s}}
$$

zeigt, daß interregionale Preisverzerrungen auch durch die Finanzausgleichsregeln hervorgerufen werden deren Einfluß in den Termen $\delta_{q G}$, und $\delta_{T r}$, enthalten ist.

\subsection{Optimalbedingungen ohne Finanzautonomie}

Bei unveränderten privaten und unternehmerischen Optimierungskalkülen bewirkt eine fehlende Finanzautonomie der Länder, daß die die öffentlichen Güter betreffenden Optimalbedingungen von den oben abgeleiteten Bedingungen (III.3c) und (III.3f) abweichen.

Die regionale Regierung ist bei fehlender Finanzautonomie in dem Sinn rationiert, daß sie sämtliche zusätzliche Einnahmen für das öffentliche Gut ausgeben muß. Entsprechend der bereits vertrauten Darstellung des Rationierungsansatzes führt dies zu dem Optimierungsproblem der regionalen Regierung mit folgender Form:

$$
\begin{gathered}
\max _{G_{s}, T r_{s}} \nu_{s}\left(G_{s} ; p_{r} \mid \forall r \in \mathcal{R}, w_{s}^{n}, r^{n}, Y_{s}\right) \\
\text { u.d.NB.: } \\
\text { (1) } q_{G_{s}} G_{s}+T r_{s}=T_{s}(\cdot)+F A_{s}(\cdot)+E Z_{s}(\cdot) \\
\text { (2) } T r_{s}=\overline{T r}_{s} .
\end{gathered}
$$

Bei Annahme einer konstanten Zeitgrundausstattung der Länder erhält man als Marginalbedingungen

$$
\begin{aligned}
& \frac{\left(1+\tau^{C}\right)\left[\left(1-\delta_{T_{s}}\right)+\mu_{s} / \lambda_{s}\right]}{\left(1-\delta_{q G_{s}}\right)} \frac{\partial H_{s} / \partial G_{s}}{\partial H_{s} / \partial C_{s s}}= \\
& =\frac{\left(1+\tau_{G s}^{K}\right)\left(1-\tau_{C s}^{Q}\right)}{\left(1+\tau_{C s}^{K}\right)\left(1-\tau_{G s}^{Q}\right)} \frac{\partial f_{C}^{s} / \partial K_{C}^{s}}{\partial f_{G}^{s} / \partial K_{G}^{s}}=\frac{\left(1-\hat{\tau}_{C s}^{Q}\right)}{\left(1-\hat{\tau}_{G s}^{Q}\right)} \frac{\partial f_{C}^{s} / \partial V_{i C}^{r s}}{\partial f_{G}^{s} / \partial V_{i G}^{r s}}
\end{aligned}
$$


$\mathrm{Zu}$ den von den Finanzausgleichsregelungen und den verschiedenen Steuersätzen verursachten Verzerrungen kommen zusätzliche Allokationsstörungen hinzu, die durch den Schattenpreis $\mu_{s}$ der Rationierung und den Grenznutzen der öffentlichen Einnahmen, $\lambda_{s}$, verursacht werden.

Ausgehend von den modifizierten Audrücken (III.3 $d^{\prime}$ ) und (III. $\left.3 f^{\prime}\right)$ kann man durch Verwendung der obigen Gleichungen eine Beziehung zwischen $F_{s}$ und $G_{s}$

$$
\frac{\left(1-\delta_{q G_{s}}\right)}{\left(1-\tau^{Y}\right)\left[\left(1-\delta_{T r_{s}}\right)+\mu_{s} / \lambda_{s}\right]} \frac{\partial H_{s} / \partial F_{s}}{\partial H_{s} / \partial G_{s}}=\left(1-\hat{\tau}_{G_{s}}^{Q}\right) \partial f_{G}^{s} / \partial L_{G}^{s}
$$

und zwischen $C_{r s}$ und $G_{s}$

$$
\frac{\left(1+\tau^{C}\right)\left[\left(1-\delta_{T_{s}}\right)+\mu_{s} / \lambda_{s}\right]}{\left(1-\delta_{q} G_{s}\right)} \frac{\partial H_{s} / \partial G_{s}}{\partial H_{s} / \partial C_{r s}}=\frac{\left(1-\hat{\tau}_{C r}^{Q}\right)}{\left(1-\widehat{\tau}_{G s}^{Q}\right)} \frac{\partial f_{C}^{r} / \partial V_{i C}^{h r}}{\partial f_{G}^{s} / \partial V_{i G}^{h s}}
$$

gewinnen. Es zeigt sich in beiden Fällen, daß sowohl der Schattenpreis der Rationierung $\mu_{s}$ als auch der Grenznutzen der öffentlichen Einnahmen $\lambda_{s}$ zusätzliche intra- und interregionale Verzerrungen verursachen.

Alternativ könnte man den obigen Ansatz auch mit Hilfe der virtuellen Preise formulieren. $\mathrm{Da}$ die Verzerrungen jedoch auch so deutlich werden, und außerdem der Rationierungsansatz mit mehreren privaten Gütern schwieriger zu formalisieren ist, wird die obige Darstellung beibehalten.

\subsection{Optimalbedingungen mit Finanzautonomie}

Gegenüber den Optimalbedingungen (III.3a) bis (III.3 $f^{\prime}$ ) verändert sich nur der Maximierungsansatz der regionalen Regierung, die nun in der Lage ist, den Nutzen ihres repräsentativen Haushalts mit Hilfe der Wahl der optimalen Menge des öffentlichen Gutes und eines optimalen Steuersatzes zu maximieren.

Die regionale Regierung wählt in diesem Fall ihre optimale Menge $G_{\boldsymbol{s}}$ und den optimalen Steuersatz $\tau_{s}$

$$
\begin{aligned}
\max _{G_{s}, \tau_{s}} & \nu_{s}\left(G_{s} ; p_{r} \mid \forall r \in \mathcal{R}, w_{s}^{n}, r^{n}, Y_{s}\right) \\
& \text { u.d.NB.: } q_{G_{s}} G_{s}+T r_{s}=T_{s}(\cdot)+F A_{s}(\cdot)+E Z_{s}(\cdot) .
\end{aligned}
$$

Von den Optimalbedingungen (III.3a) bis (III.3 $f^{\prime}$ ) müssen nur die Ausdrücke (III.3c) und die modifizierten Bedingungen (III.3 $\left.d^{\prime}\right)$ und (III.3 $f^{\prime}$ ) neu bestimmt werden. Alle anderen Gleichungen bleiben unverändert. 
Daraus kann man nach dem üblichen Vorgehen die entsprechenden Bedingungen für die optimale Abstimmung von Konsum- und Produktionsstruktur bestimmen. Sie lauten

$$
\left.\frac{\left(1+\tau^{C}\right) \alpha_{s} / \lambda_{s}}{\left(1-\delta_{q G_{s}}\right.}\right) \frac{\frac{\partial H_{s}}{\partial G_{s}}}{\frac{\partial H_{s}}{\partial C_{s s}}}=\frac{\left(1+\tau_{G s}^{K}\right)\left(1-\hat{\tau}_{C s}^{Q}\right)}{\left(1+\tau_{C s}^{K}\right)\left(1-\widehat{\tau}_{G s}^{Q}\right)} \frac{\frac{\partial f_{C}^{s}}{\partial K_{C}^{s}}}{\frac{\partial f_{G}^{s}}{\partial K_{G}^{s}}}=\frac{\left(1-\widehat{\tau}_{C s}^{Q}\right)}{\left(1-\widehat{\tau}_{G_{s}}^{Q}\right)} \frac{\frac{\partial f_{C}^{s}}{\partial V_{i C}^{T s}}}{\frac{\partial f_{G}^{s}}{\partial V_{i G}^{s}}}
$$

$$
\begin{gathered}
\frac{\left(1-\delta_{q G}\right)}{\left(1-\tau^{Y}\right) \alpha_{s} / \lambda_{s}} \frac{\partial H_{s} / \partial F_{s}}{\partial H_{s} / \partial G_{s}}=\left(1-\widehat{\tau}_{G_{s}}^{Q}\right) \partial f_{G}^{s} / \partial L_{G}^{s} \\
\text { sowie } \\
\frac{\left(1+\tau^{C}\right) \alpha_{s} / \lambda_{s}}{\left(1-\delta_{q G s}\right)} \frac{\partial H_{s} / \partial G_{s}}{\partial H_{s} / \partial C_{r s}}=\frac{\left(1+\hat{\tau}_{G s}^{K}\right)\left(1-\widehat{\tau}_{C r}^{Q}\right)}{\left(1+\hat{\tau}_{C r}^{K}\right)\left(1-\hat{\tau}_{G s}^{Q}\right)} \frac{\partial f_{C}^{r} / \partial K_{C}^{r}}{\partial f_{G}^{s} / \partial K_{G}^{s}} .
\end{gathered}
$$

Zur Bestimmung der tatsächlichen Preisverzerrung zwischen dem öffentlichen und dem privaten Gut muß, neben dem Verhältnis der Grenznutzen der privaten Einkommen $\alpha_{s}$ und der öffentlichen Einnahmen $\lambda_{s}$, zusätzlich die Abweichung der sozialen Grenzkosten der öffentlichen Ausgaben von deren Preis beachtet werden. ${ }^{9}$ Eine allgemeine Aussage über die Richtung der Preisverzerrung ist allerdings ohne genauere Kenntnis der Rückwirkungen von höheren Transfers und höheren öffentlichen Ausgaben auf die öffentlichen Einnahmen nicht möglich.

Das Verhältnis der Grenznutzen der privaten Einkommen und der öffentlichen Finnahmen kann durch die Ableitung der Lagrangefunktion nach dem regionalen Steuersatz $\tau$ aufgeschlüsselt werden. Es lautet:

$$
\alpha_{s} / \lambda_{s}=\frac{\partial T r_{s} / \partial \tau_{s}-\partial T_{s} / \partial \tau_{s}-\partial F A_{s} / \partial \tau_{s}}{\left(\partial Y_{s} / \partial \tau_{s}\right)-\sum_{r}\left(C_{s s} \partial p_{s} / \partial \tau_{s}\right)-F_{s} \partial w_{s}^{n} / \partial \tau_{s}}
$$

Die abgeleiteten Optimalbedingungen einer Marktökonomie mit Staatseingriffen erlauben eine erste Abschätzung der gesamtgesellschaftlichen Allokationseffekte, die das staatliche Handeln verursacht. Der Darstellung dieser verzerrenden Einflüsse sind die nächsten Abschnitte (3. und 3.5.) gewidmet.

\section{Allokationsverzerrungen durch Staatseingriffe}

Als erstes werden die Auswirkungen der modellierten Faktor-, Produktions-, Einkommensund Konsumsteuerarten auf die Optimalbedingungen beschrieben. Daran schließt sich eine Darstellung der Verzerrungen an, die durch die Finanzausgleichsregelungen verursacht werden.

\footnotetext{
${ }^{9}$ Eine Abweichung ergibt sich, wenn der Term $\left(1-\delta_{q G},\right)$ ungleich eins ist.
} 


\subsection{Produktionssteuern}

Die Produktionssteuern, die sektoral und regional differenziert ${ }^{10}$ und in den Produzentenpreisen bereits enthalten sind, sowie die Grenzkosten von öffentlichen und privaten Gütern beeinflussen, treiben einen Keil zwischen die MRS und die MRT in den regionalen ((III.3c), (III.3d) und (III.3 $\left.d^{\prime}\right)$ ) und die MRS und die FRT in den interregionalen Gleichgewichtsbedingungen ((III.3f) bzw. (III.3 $\left.\left.f^{\prime}\right)\right)$. Dagegen werden die Grenzraten der technischen Substitution nicht verzerrt, weil die Produktionssteuersätze nicht zwischen den Faktoren differenzieren.

\subsection{Faktorsteuern}

In der hier verwendeten Modellierung werden die Grund-, die Gewerbe-, die Kapitalertragsund die Körperschaftssteuern als Faktorsteuern auf den Kapitaleinsatz implementiert, die nach dem Quellenlandprinzip erhoben wird. ${ }^{11}$

Diese Steuern sind sektoral und regional differenziert. Dies ergibt sich aus der empirischen Bestimmung der sektoralen Steuersätze, die sich von Sektor zu Sektor und Region zu Region unterscheiden, da die Sektoren sich aus unterschiedlichen Unternehmenstypen mit unterschiedlichen Technologien und Strukturen zusammensetzen. Um zugleich die Struktur des Modells aufrechterhalten zu können, in denen die Sektoren Aggregate identischer Unternehmen sind, wird angenommen, daß die Steuern sektoral und regional differenziert sind. Im weiteren werden diese Faktorsteuern als "Kapitalsteuern” bezeichnet.

Für die Kapitalbereitstellung bei interregional mobilem Kapital sind die den Haushalten zufließenden Kapitaleinkünfte relevant. Die Quellen(land)besteuerung führt bei einer nach Sektoren und/oder Regionen differenzierenden Besteuerung des Kapitaleinsatzes zu einem einheitlichen Zinssatz auf das eingesetzte Kapital (vgl. Sins [1985], S.171). ${ }^{12}$ Die privaten Haushalte werden ihr Kapital dort zur Verfügung stellen, wo ihre Kapitalverzinsung am höchsten ist. Dies führt bei interregional mobilem Kapital, sogar bei differenzierten Faktorsteuersätzen, zu einem interregional einheitlichen Zinssatz nach Abzug der Faktorsteuern.

\footnotetext{
${ }^{10}$ Dies ergibt sich aufgrund der empirischen Bestimmung der Steuersätze, da die Sektoren Aggregate von Unternehmen mit unterschiedlichen Techniken und Strukturen sind.

${ }^{11}$ Eine genaue Begründung für die Behandlung dieser Steuern als Faktorsteuern wird im nächsten Kapitel nachgereicht.

${ }^{12}$ Unter "Zinssatz"wird im weiteren die Kapitalverzinsung nach Abzug der von den Unternehmen abzuführenden Faktorsteuern, also die den privaten Haushalten vor der Einkommensbesteuerung zufließenden Zinseinkünfte verstanden.
} 
Für die Kapitalnachfrage der Unternehmen ist jedoch nicht der (Netto-)Zinssatz, sondern der tatsächlich von ihnen zu bezahlende Bruttozinssatz relevant, da sie in ihrer Kalkulation zu dem interregional einheitlichen Zinssatz zusätzlich die Kapitalsteuern hinzurechnen müssen. Aus diesem Grund bewirken regional unterschiedliche Faktorsteuern auf Kapital eine Verzerrung der interregionalen Kapitalallokation in der Bedingung (III.3f). Der Kapitaleinsatz vergleichbarer Sektoren wird in der Region mit höherem Kapitalsteuersatz relativ teuerer.

Darüber hinaus bewirkt die Quellenbesteuerung des Faktors Kapital eine Verzerrung der Grenzrate der technischen Substitution zwischen den Sektoren einer Region, für den Fall, daß die Steuersätze sektoral differenziert sind und daher einen Keil zwischen die Bruttoverzinsung in den beiden Sektoren treiben (Gleichung (III.3b)).

Ebenso wird die optimale Abstimmung von Konsum- und Produktionsstruktur gestört, da die optimale Güteraufteilung nun über die Güterpreise auch von den sektoral differenzierten Kapitalsteuern beeinflußt wird. Dies führt zu einem Auseinanderdriften der MRS zweier Güter und der MRT des Kapitaleinsatzes in der Produktion dieser beiden Güter (siehe (III.3c) $)^{13}$. Dies gilt sowohl für die privaten als auch für die öffentlichen Güter (Bedingung (III.3d)), mit Ausnahme der Beziehungen zwischen Freizeit und dem öffentlichen bzw. dem heimischen privaten Gut, da für diese nur die Grenzproduktivität der Arbeit relevant ist. Darüber hinaus sind die Kapitalsteueraufkommen in den Beziehungen in den Termen $\delta_{q G}$, und $\delta_{T r}$, enthalten. Damit rufen die Kapitalsteuern zusätzliche Verzerrungen in den Bedingungen (III.3c), (III. $\left.3 d^{\prime}\right)$ und (III. $3 f^{\prime}$ ) hervor.

\subsection{Einkommenssteuern}

Die Einkommenssteuern bestehen in dem hier entwickelten Modell aus der Besteuerung der Einkommen von Arbeit und Kapital, demnach aus Lohnsteuer und veranlagter Einkommenssteuer (Steuer auf die Zinseinkünfte). Im Gegensatz zur bundesdeutschen Realität wird hierbei eine volle Doppelbesteuerung der Zinseinkünfte unterstellt.

Diese Steuerarten beeinflussen bei einem interregional immobilen Faktor Arbeit nur die intraregionale optimale Abstimmung der Konsum- und Produktionsstruktur in den Gleichungen (III.3d) und (III.3 $d^{\prime}$ ). Der Grund hierfür besteht darin, daß die Einkommenssteuer nur über den Nettolohnsatz entscheidungsrelevant wird. Die Einkommenssteuer treibt einen Keil zwischen den Preis der Freizeit und den Preis des privaten bzw. öffentlichen

\footnotetext{
${ }^{13} \mathrm{Im}$ folgenden werden die Verzerrungen, die in den Fällen der Ausgabenautonomie, fehlender Autonomie und voller Finanzautonomie in gleicher Form auftreten, stellvertretend auf den Fall der Ausgabenautonomie bezogen.
} 
Gutes und verzerrt damit die entsprechende Wahl. Wie Rosenberg ([1993], S.115-116) verdeutlicht, entsteht der verzerrende Einfluß der Lohnbesteuerung ausschließlich durch das variable Arbeitsangebot. Wäre es fix, hätte der Einkommenssteuersatz keinerlei allokationsverzerrenden Einfluß, da dann die Marginalbedingungen (III.3d) und (III.3 $d^{\prime}$ ) entfallen würden.

Da das Kapitalangebot fix ist und die Zinseinkünfte nach dem Wohnsitzlandprinzip besteuert werden besitzt der Einkommenssteuersatz keinen Einfluß auf die die Entscheidungen der privaten Haushalte darüber, welchem Sektor in welcher Region sie ihr Kapital zur Verfügung stellen. Für die privaten Haushalte sind vielmehr nur die Zinssätze $r$ und nicht die Nettozinssätze $r\left(1-\tau^{Y}\right)$ relevant, da die Besteuerung der Zinseinkünfte unabhängig vom Verwendungsort des Kapitals ist. Die Steuerschuld ist folglich unabhängig vom Einsatzort des Kapitals. Daher beeinflußt der Einkommenssteuersatz nicht die Kapitalverzinsung in den Unternehmen und bewirkt keine Verzerrung der Kapitalallokation.

\subsection{Konsumsteuern}

Die Konsumsteuern werden als allgemeine Konsumsteuer und nicht als Mehrwertsteuer modelliert. Die Steuersätze sind sektoral differenziert aber interregional einheitlich. Sie verzerren, durch ihren Einfluß auf die Konsumentenpreise und deren soziale Grenzkosten, die optimale Abstimmung der intraregionalen Konsum- und Produktionsstruktur (Gleichungen (III.3c) und (III.3d)). Der von ihnen zwischen Produzenten- und Konsumentenpreise geschobene Keil diskriminiert zwischen den unterschiedlichen Güterarten, da Freizeit und die öffentlichen Güter nicht besteuert werden.

\subsection{Effizienzwirkungen von Finanzausgleichsregelungen}

Der Einfluß der Finanzausgleichsregelungen ist in den Optimalbedingungen in den Größen $\delta_{q G_{s}}, \delta_{T r_{s}}$, bzw. $\alpha_{s} / \lambda_{s}$ (bei Finanzautonomie) erfaßt. ${ }^{14}$ Wenn diese Größen ungleich Null sind und sich nicht gegenseitig ausgleichen ${ }^{15}$, verursachen sie Allokationsstörungen in den Bedingungen (III.3c), (III.3 $\left.d^{\prime}\right),\left(\right.$ III.3 $\left.f^{\prime}\right),($ III. $4 c),\left(\right.$ III. $\left.4 d^{\prime}\right),\left(\right.$ III. $\left.4 f^{\prime}\right),\left(\right.$ III.5c), (III.5 $\left.d^{\prime}\right)$ und (III. $\left.5 f^{\prime}\right)$.

\footnotetext{
${ }^{14}$ Diese Größen enthalten u.a. die Ableitung der LFA-Einnahmen, der Einnahmen aus der Steuerverteilung und den BEZ nach den Steueraufkommen und nach einer der drei Kontrollvariablen, Transfers, Staatsausgaben oder Steuersätze. Die Steueraufkommen ihrerseits werden ebenfalls durch diese drei Kontrollvariablen beeinfluBt.

${ }^{15}$ Durch Rückgriff auf die Ergebnisse in den Abschnitten (2.2.) (auf Seite $58 \mathrm{ff}$.) können einige Aussagen bezüglich der Größenordnung der Effekte getroffen werden.
} 
Im einzelnen gilt:

- Finanzausgleichsregelungen beeinflussen die sozialen Grenzkosten der Bereitstellung der öffentlichen $\left(1-\delta_{q} G_{s}\right) q_{G}$, und privaten Güter $\left(1-\delta_{T r_{s}}\right) p_{s}$. Aus diesem Grund treiben sie in der intraregionalen Konsum- und Produktionsstruktur Verzerrungen durch den Keil, den sie zwischen die MRS und die MRT zwischen dem öffentlichen Gut und den privaten Gütern treiben. Insofern bewirken sie intraregionale Substitutionseffekte.

- Zusätzlich verzerren sie indirekt die interregionalen Austauschverhältnisse, indem sie den Handel des zur Produktion der öffentlichen Güter und privaten Konsumgüter unterschiedlicher Herkunft benötigten Kapitals beeinflussen. Daher treiben sie einen Keil zwischen die MRS und die „interregionale" Grenzrate der Transformation (FRT) der importierten Güter $C_{r s}$ und $G_{s}$.

- Berücksichtigt man allerdings zusätzliche Verzerrungen in Form von verschiedenen Steuerarten, wie sie in den Optimalbedingungen enthalten sind, dann können Finanzausgleichsregelungen unter Umständen auch zu einer Reduktion der Störungen beitragen, wenn sie die verzerrenden Einflüsse der Steuersätze reduzieren. Dies hängt im einzelnen von den jeweiligen Werten von $\delta_{q G}$, und $\delta_{T_{r}}$, ab. Insofern kann die Einführung eines Finanzausgleichssystems auch zu einer Effizienzsteigerung führen.

Die Stärke der Verzerrungen wird im wesentlichen durch den Grad der Abhängigkeit der öffentlichen Einnahmen von den einzelnen Steuerverteilungs- und Länderfinanzausgleichsregelungen bestimmt. Eine wesentliche Rolle für die Höhe der Allokationsstörungen spielt die Reaktion der regionalen Steueraufkommen auf eine Veränderung der öffentlichen Ausgaben, Transfers oder Steuersätze. Dieser Zusammenhang kann jedoch nur in einem ökonomischen Totalmodell untersucht und ermittelt werden, da nur dann die wesentlichen Rückwirkungen der staatlichen Kontrollvariablen adäquat berücksichtigt werden können.

Betrachtet man die gesetzlichen Regelungen und Gestaltungsvorschläge für den bundesdeutschen Finanzausgleich, so kann man diese Aussagen noch etwas präzisieren. Erste Berechnungen führen zu dem Resultat, daß zwei gegenläufige Effekte auftreten:

1.) Zum einen steigen infolge höherer Ausgaben der regionalen Regierung die regionalen Steueraufkommen. Dies würde bei ohne Finanzausgleich zu einem Anstieg der regionalen Einnahmen und damit zu einer teilweisen Selbstfinanzierung der öffentlichen Ausgaben führen. Ist $\delta_{q G}$, ungleich $\delta_{T r}$, führt dies zu einer Preisverzerrung.

2.) Zum zweiten bewirken Steuerverteilung, LFA und BEZ eine Veränderung dieser Beziehung, da die Steueraufkommenszuwächse nun nicht mehr unbedingt mit Einnahmensteigerungen identisch sind. 
Bei der im bundesdeutschen Finanzausgleich bestehenden hohen Substitutionalität (siehe Abschnitt 2.2. auf den Seiten $58 \mathrm{ff}$.) wird die Selbstfinanzierung durch die Finanzausgleichsregelung stark reduziert. Damit wird die Abweichung der sozialen Kosten der öffentlichen und privaten Ausgaben von den jeweiligen Güterpreisen vermindert. Inwieweit damit allerdings Verzerrungen abgebaut werden, hängt von den Veränderungen der Verhältnisse $\delta_{q G_{s}}$ und $\delta_{T r_{s}}$ ab.

Die bisherigen Ausführungen zeigen, welche Substitutionseffekte in einer Marktökonomie mit Staatstätigkeit auftreten. Diese führen dazu, daß die Allokation verzerrt wird, und gesellschaftliche Wohlfahrtsverluste gegenüber dem Pareto-Optimum auftreten. Eine Reduktion dieser Verzerrungen müßte demnach wohlfahrtserhöhend sein. Aufgrund der Second-Best-Problematik ist dies allerdings nur dann gesichert, wenn alle Verzerrungen gleichzeitig aufgehoben werden. Welchen Einfluß die Reduktion von Verzerrungen bei einer Vielzahl von bestehenden Allokationsstörungen (Steuern) letztlich besitzen, kann nur im Rahmen einer Empirischen Allgemeinen Gleichgewichtsanalyse untersucht werden.

Es ist allerdings durchaus möglich, daß sich die Wohlfahrt einzelner Regionen trotz gesamtwirtschaftlicher Effizienzverluste erhöht. Die Veränderung der relativen Wohlfahrtspositionen einzelner Länder, die nicht auf gesamtwirtschaftliche Wohlfahrtsverbesserungen zurückzuführen sind, werden im folgenden Abschnitt näher betrachtet.

\section{Wohlfahrtswirkungen}

Regionale Wohlfahrtsgewinne können im Prinzip auf drei Arten entstehen.

1. Eine Verringerung der Steuersatzdifferenzen zwischen den regionalen Nachfragekomponenten - der Güternachfrage oder der Faktornachfrage - oder eine Veränderung der Finanzausgleichsregelungen können intraregionale Substitutionseffekte reduzieren und damit regionale Wohlfahrtsverluste verringern. Dabei sollte man allerdings beachten, daß die Veränderung von Finanzausgleichsregelungen durchaus gegenläufige Auswirkungen auf die Effizienz besitzen kann. Beispielsweise kann eine Verringerung der Verzerrungen zwischen dem öffentlichen Gut und den privaten Konsumgütern, über ein Absinken von $\delta_{q G_{s}}$, zu einer Erhöhung der Verzerrung zwischen der MRS und der MRT des öffentlichen Gutes und der Freizeit führen.

Eine Reduktion der Verzerrungen vermindert die intraregionalen Substitutionseffekte und produzieren damit - aus first-best Sicht - gesamtgesellschaftliche und regionale Wohlfahrtsgewinne. In diesem Fall entspricht eine Erhöhung der regionalen Wohlfahrt auch einer gesamtgesellschaftlichen Wohlfahrtserhöhung, da sie nicht 
auf Kosten anderer Regionen zustande kommt. Dieser Fall wurde schon im vorhergehenden Abschnitt behandelt und kann daher hier vernachlässigt werden.

2) Interregionale Transfers bewirken eine Umverteilung von Einnahmen zwischen den Regionen und beeinflussen damit die Konsummöglichkeiten und die Wohlfahrt der betroffenen Länder. Gemäß dem zweiten Hauptsatz der Wohlfahrtsökonomie sind derartige Umverteilungen unter bestimmten Annahmen allokationsneutral, d.h. sie besitzen keinen Einfluß auf die gesamtgesellschaftliche Wohlfahrt (vgl. VARIAN [1992], S.326-329). Sie bewirken jedoch durch die Umschichtung der Einkommen eine Veränderung der relativen Wohlfahrtsposition der Länder.

3.) Verschiebungen im interregionalen Preisgefüge, beispielsweise durch die Einführung regional differenzierter Steuersätze, verändern die relative Kaufkraftposition der Regionen und lösen interregionale Substitutionseffekte aus, die zu Terms-of-TradeVeränderungen führen. Daraus ergibt sich eine Reduktion der gesamtwirtschaftlichen Wohlfahrt bei gleichzeitiger Verbesserung der Wohlfahrtsposition eines oder mehrerer Länder auf Kosten der Wohlfahrt anderer Regionen.

Die letzten beiden Fälle werden nun anhand der im Modell vorgesehenen institutionellen Regelungen etwas näher beleuchtet.

\subsection{Interregionale Wohlfahrtseffekte durch Transfers zwischen den Ländern}

Im hier vorliegenden Modell treten interregionale Transfers in Form von Steuerverteilungszuweisungen, LFA-Zuweisungen, Ergänzungszuweisungen und Steuerexporten auf. Von Steuerexporten spricht man dann, wenn eine Region Steuereinnahmen erzielt, die nicht aufgrund von Steuerzahlungen der eigenen regionalen Haushalte oder Unternehmen zustande kommen, sondern durch Wirtschaftssubjekte anderer Regionen finanziert werden. Die Region ist in diesem Fall in der Lage, die Last der Finanzierung ihrer Steuereinnahmen einer anderen Region aufzubürden, sie sozusagen zu exportieren. ${ }^{16}$ Zusätzlich können auch internationale Steuerexporte auftreten, die allerdings nicht nur interregionale Wohlfahrtseffekte, sondern auch nationale Wohlfahrtsveränderungen auslösen.

Alle diese Größen besitzen den Charakter von Transfers zwischen den Ländern und lösen entsprechende Effekte aus, die in der Außenhandelstheorie unter der Bezeichnung "Transfer-Problem" behandelt werden.

\footnotetext{
${ }^{16}$ Eine grundlegende Darstellung der Theorie des Steuerexports liefert Peffekoven [1975].
} 
Am einfachsten stellt sich dieses Transfer-Problem im Zwei-Länder-Zwei-Güter-Fall dar (siehe z.B. KRugman /ObstFeld [1991], S.107). Transferzahlungen eines Landes an eine andere Region führen zu einem Rückgang der inländischen und einem Anstieg der ausländischen Einnahmen. Demzufolge sinken die inländischen und wachsen die ausländischen Ausgaben. Besitzt nun das Geberland eine höhere marginale Ausgabenneigung bzgl. seiner Exportgüter als das Empfängerland, dann führt der Rückgang der Nachfrage des Geberlandes nach seinen eigenen Exportprodukten zu einer Verringerung der Weltnachfrage nach diesem Exportgut. Das Empfängerland der Transfers wird aufgrund seiner geringeren marginalen Ausgabenneigung nach Exportgütern des Geberlandes die ausgefallene Nachfrage nicht vollständig ausgleichen. Umgekehrt erhöht sich aufgrund der selben Argumentation die Nachfrage nach Exportgütern des Empfängerlandes, d.h. nach Importgütern des Geberlandes. Dies führt letztlich zu einem relativen Sinken der Exportpreise aufgrund der relativ zurückgehenden Weltnachfrage bei unverändertem Angebot. Damit entsteht neben einer Erhöhung der regionalen Einkommen eine zusätzliche Verbesserung der Terms of Trade für das Empfängerland der Transfers.

Nur unwesentlich komplizierter wird der Fall, wenn nun jedes Land ein nichthandelbares Gut produziert. Diese konkurrieren mit den Exportgütern um die inländischen Ressourcen. Ein Transfer eines Landes in ein anderes Land verringert dann die Nachfrage nach inländisch produzierten Gütern. Damit werden Ressourcen frei, wodurch deren Preise und dadurch auch die Güterpreise fallen. Das Empfängerland erhöht dagegen seine Nachfrage nach inländischen Gütern und verknappt damit seine Ressourcen. Die daraus entstehenden Faktorpreiserhöhungen führen zu steigenden Preisen der Importgüter des Geberlandes. Beide Effekte verschlechtern die ToT des Geberlandes (siehe KRUGMAN/OBSTFELD [1991]).

Obwohl durchaus Fälle konstruiert werden können, in denen das Geberland durch Transfers an andere Länder Wohlfahrtsgewinne erzielen kann, ist unter den hier verwendeten Modellbedingungen von der normalen Transferwirkung auszugehen. Sowohl die Datenlage, die eine Präferenz für Inlandsgüter vorgibt, als auch die Verwendung homothetischer Nutzenbäume mit „normalen” Substitutionsbeziehungen zwischen den Gütern spricht kaum für eine anomale Wirkung. ${ }^{17}$

Bezogen auf das hier verwendete Modell bedeutet das, daß die Nettotransfers zwischen den Ländern, d.h. die Summe der Finanzausgleichszahlungen und der Steuerexporte, die relevante Größe zur Bestimmung von entsprechenden Wohlfahrtseffekten ist. Zur Bestimmung der Nettotransfers werden auch die Nettosteuerexporte benötigt, die z.B. den An-

\footnotetext{
${ }^{17}$ BhAGWATI, BRECher, HatTa [1983]) haben demgegenüber Fälle mit Drei-Ländern konstruiert, in denen es durchaus zu Wohlfahrtsgewinnen des Geberlandes kommen kann. Siehe dazu auch MaJUMDAR/MitRa [1985].
} 
teilen der regionalen Grund- und Produktionssteuereinnahmen zusammensetzen, die von den Produzenten und Konsumenten anderer Regionen getragen werden.

Neben der Veränderung der regionalen Einnahmen, die interregionale Einkommenseffekte auslösen, führen Nettotransfers durch veränderte Nachfragen zu einer Veränderung der Terms-of-Trade und damit zu interregionalen Substitutionseffekten. Beide Effekte müßten sich insgesamt zugunsten der Wohlfahrt der Empfängerländer der Nettotransfers auswirken.

\subsection{Interregionale Wohlfahrtseffekte durch ToT-Veränderungen}

Interregionale Wohlfahrtseffekte entstehen aufgrund von Veränderungen der Terms-ofTrade (ToT). Diese können z.B. durch Aktivitäten von einzelnen Regionen entstehen, die die Möglichkeit besitzen, Steuersätze zu verändern oder Zölle zu erheben und diese auch in Anspruch nehmen, es sei denn, die jeweilige Region ist im Sinne der Außenhandelstheorie ein kleines Land, und kann daher die Preise, zu denen interregionaler Handel stattfindet, nicht verändern. ${ }^{18}$ In der hier durchgeführten Untersuchung sind die einzigen Handlungsparameter der regionalen Regierungen ihre Transfers an den repräsentativen regionalen Haushalt bzw. ihre regionalen Einkommenssteuerzuschläge. Da beide Größen keinen direkten Einfluß auf die Preise der gehandelten Güter besitzen, sind sie auch kein Instrument zur direkten Beeinflussung der Terms of Trade. Dazu würden sich in erster Linie Faktorsteuern, wie etwa Kapitalsteuern, eignen. ${ }^{19}$

\subsubsection{Allgemeine Auswirkungen von ToT-Veränderungen}

An dieser Stelle wird nicht näher auf Reaktionen der ToT bei Zöllen, Faktor- oder Produktionssteueränderungen eingegangen. Es soll nur allgemein der Effekt einer Verbesserung der ToT auf die Wohlfahrt eines Landes dargestellt werden. Ein geeignetes Instrumentarium dafür ist das für Heckscher-Ohlin-Modelle entwickelte Offerkurven-Diagramm. ${ }^{20}$

\footnotetext{
${ }^{18}$ Eine ausführliche Darstellung der entsprechenden Konzepte findet man z.B. bei GaNDoLfo [1987a].

${ }^{19}$ Die Effekte von Faktorsteuern diskutiert GeorgakopouLos [1974]. Ein Beispiel einer EAG-Analyse, in der der Einfluß von Faktorsteuern auf die ToT eine wesentliche Rolle spielen, liefert die Arbeit von HIRTE/WIEg ARD [1988].

${ }^{20}$ Eine ausführliche Herleitung, Darstellung und Anwendung dieser Methodik findet man z.B. bei GANDoLFo [1987a], S.45-51. Trotz der Unterschiede zwischen dem Heckscher-Ohlin-Modell und dem hier verwendeten Ansatz mit der Armington-Annahme kann das Offerkurvendiagramm auch hier verwendet werden. Die Unterschiede bestehen u.a. in unterschiedlichen Parametern der Nutzenfunktionen und Produktionstechnologien, in nichthandelbaren Gütern, variablen Vorleistungen, internationalen Tranfers, nicht ausgeglichenen Handelsbilanzen, einer größeren Zahl von Gütern, und einer bereits im Ausgangs-
} 


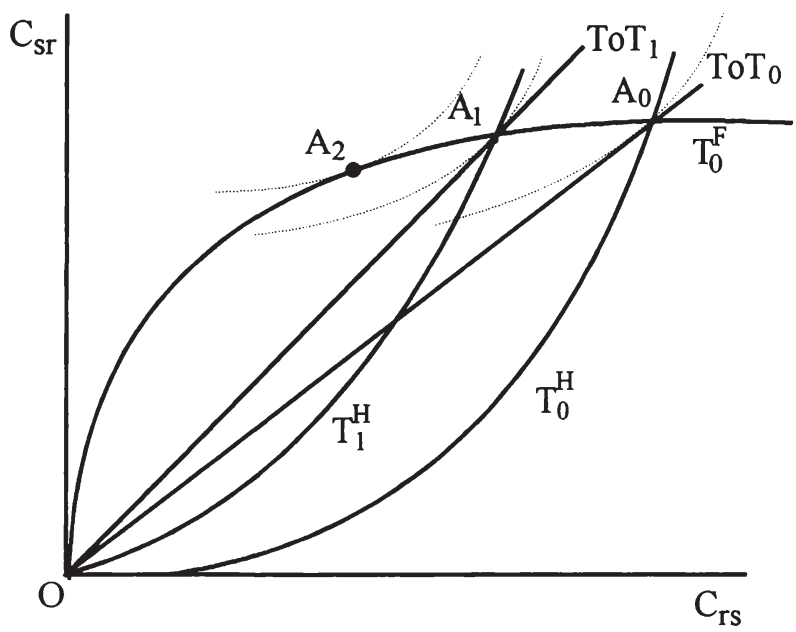

Abbildung III.1: Wohlfahrtseffekte bei Preiserhöhungen im Inland

Die Offerkurven in Abbildung III.1 geben an, wieviele Konsumgüter eine Region für ein zusätzliches importiertes Konsumgut herzugeben bereit ist. Im Rahmen des vorgestellten Ansatzes stehen den Lieferungen an die anderen Regionen Bezüge aus den anderen Regionen gegenüber. Deshalb wird anstelle von „Exporten' von "Lieferungen” und anstelle von "Importen” von „Bezügen” gesprochen.

Für die betrachtete Region werden die Bezüge, die zur Vereinfachung ein composite commodity der Bezüge aus den einzelnen Ländern seien, auf der Ordinate und die Lieferungen auf der Abszisse abgetragen. Die entsprechende heimische Offerkurve $\mathrm{T}_{0}^{H}$ steigt zunehmend, da der Nutzen der Bezüge für die Inländer mit zunehmender Menge abnimmt. Die aggregierte Offerkurve der anderen Länder (Rest der Bundesrepublik - RoD) $\mathrm{T}_{0}^{F}$ steigt abnehmend, da für RoD Bezüge und Lieferungen auf den anderen Achsen liegen. Der Schnittpunkt der beiden Kurven $A_{0}$ ist das Ausgangsgleichgewicht in dem Bezugsund Lieferungsnachfragen bzw. -angebote übereinstimmen. Dieser Punkt, der auch für die weitere Darstellung als Ausgangspunkt gilt, entspricht dem Freihandelsgleichgewicht, das durch die Pareto-Bedingungen bezüglich des interregionalen Handels (siehe (III.1e) und (III.1f ) ) charakterisiert ist. Die Gerade To $\mathrm{T}_{0}$, die durch den Ursprung des Koordinatensystems und diesen Punkt läuft, bestimmt das relative Austauschverhältnis von

gleichgewicht bestehenden verzerrenden Steuer- und Finanzausgleichsstruktur (siehe FEHR [1993], S.32). 
Bezügen und Lieferungen, d.h. die Terms of Trade (ToT). Diese Terms of Trade sind, bei Abwesenheit von Steuern, gerade das optimale Freihandelspreisverhältnis. Zusätzlich sind die Handelsindifferenzkurven als gestrichelte Kurven eingetragen. Da der Nutzen aus dem Handel steigt, wenn für die gleiche Menge von Bezügen weniger Güter geliefert werden müssen und für die gleiche Menge von Lieferungen mehr Güter bezogen werden können, wächst das heimische Nutzenniveau nach links oben.

Steigen nun die heimischen Preise, dann heißt das im Prinzip, daß die Region für dieselbe Menge an Bezügen jetzt weniger Güter liefern will. Folglich dreht sich die heimische Offerkurve nach links, mit dem Schnittpunkt der beiden Kurven $T_{1}^{H}$ und $T_{0}^{F}$ als neuem Gleichgewicht $\left(A_{1}\right)$. Insgesamt resultiert eine Wohlfahrtserhöhung für die betrachtete Region, die nun eine höhere Handelsindifferenzkurve erreicht (siehe z.B. GANDolFo [1987a], S.116-117). Es ist allerdings zu beachten, daß die Handelsgewinne nur bis zum Punkt $A_{2}$ wachsen. Steigen die ToT darüber hinaus, fallen die Handelsgewinne wieder.

Um den Einfluß der regionalen Entscheidung auf die ToT zu bestimmen, müßte man die Auswirkung der Politikveränderungen im einzelnen untersuchen. Eine Veränderung der intraregionalen Transfers bewirkt, unter den oben beschriebenen Annahmen, die im vorhergehenden Abschnitt dargestellten Preis- und ToT-Veränderungen. Die Preisreaktionen infolge einer Variation der Einkommenssteuerzuschläge können ebenfalls in etwa prognostiziert werden.

\subsubsection{Auswirkungen der Veränderung von Einkommenssteuerzuschlägen}

Eine Erhöhung der Einkommenssteuersätze durch regionale Zuschläge reduziert den relativen Preis der Freizeit. ${ }^{21}$ Folglich steigt die Freizeitnachfrage, es sei denn man befindet sich auf dem backward-bending-Ast der Arbeitsangebotskurve. Damit fällt das Arbeitsangebot im Inland. Aufgrund der dadurch ausgelösten Verknappung der Ressourcen steigt der relative Preis des Gutes, in dessen Produktion Arbeit besonders intensiv eingesetzt wird (siehe Gandolfo [1987a], S.174), d.i. das öffentliche Gut. Dies führt zur Substitution der öffentlichen Ausgaben durch den privaten Konsum. Bei einer höheren Präferenz für heimisch produzierte Konsumgüter steigt die Nachfrage nach diesen Gütern stärker als die nach importierten Gütern. Folglich steigt der relative Preis der heimischen Konsumgüter und damit die ToT des Inlandes. Der Anstieg der relativen Inlandspreise wird zusätzlich durch die Lohnsatzsteigerung verstärkt, die auch für die privaten, im Inland produzierten Güter eine Erhöhung der Produktionskosten bedeutet. Vermutlich ergibt sich daher bei einer Einkommenssteuererhöhung ein leichter Anstieg der regionalen ToT.

\footnotetext{
${ }^{21}$ Es wird implizit angenommen, daß die höheren Einnahmen als Lump-Sum-Transfers an den regionalen repräsentativen Haushalt vollständig zurücküberwiesen werden.
} 
Theoretisch wäre dies allerdings nur dann abgesichert, wenn Arbeit der in der Produktion der gehandelten Güter relativ intensiv eingesetzte Faktor wäre. ${ }^{22}$

\section{Zusammenfassung}

Betrachtet man die Ergebnisse der Überlegungen zu den Allokationsstörungen staatlicher Tätigkeiten, so kann man drei wesentliche Effekte herausstellen (siehe auch RosENBERG [1993], S.154).:

- Intraregionale Substitutionseffekte entstehen durch Verzerrungen gegenüber den inländischen Optimalbedingungen. Diese werden durch differenzierende Faktor-, Produktions- oder Konsumsteuern, durch Einkommenssteuern, die Abweichung der sozialen Grenzkosten der öffentlichen und privaten Güter von deren Preisen, die u.a. durch Finanzausgleich bedingt ist, sowie die Abweichung der Grenznutzen der öffentlichen Einnahmen von den Grenznutzen der privaten Einkommen, die ebenfalls durch Finanzausgleich beeinflußt wird, hervorgerufen. Damit besitzen die Finanzausgleichsregelungen einen Einfluß auf die Allokationsstörungen. Die Verminderung der Substitutionseffekte müßte daher regionale Wohlfahrtsgewinne erzeugen. Zwar können diese Aussagen nicht ohne weiteres in ein Second-Best-Modell übertragen werden, doch vermitteln sie vermutlich eine ungefähre Vorstellung von den tatsächlichen Effekten.

- Interregionale (internationale) Substitutionseffekte werden durch Verletzung der interregionalen Paretobedingungen (III.1e) bis (III.1 $f^{\prime}$ ) auf Seite 101 hervorgerufen. Diese gehen auf unterschiedliche Produktions- und/oder Kapitalsteuern zurück. Die Finanzausgleichsregelungen haben nur einen indirekten Einfluß auf die interregionale Allokation, da die öffentlichen Güter nicht getauscht werden. Über den Handel mit Kapital, der als Ersatz für den fehlenden Handel mit dem nichtgehandelten öffentlichen Gut dient, beeinflussen sie dennoch die interregionale Allokation. Eine Reduktion der interregionalen Substitutionseffekte müßte gesamtwirtschaftliche Wohlfahrtsgewinne nach sich ziehen. Inwieweit einzelne Regionen davon profitieren, hängt letztlich von der Veränderung der jeweiligen regionalen ToT ab.

\footnotetext{
${ }^{22}$ Bei dieser Folgerung sind eine Reihe von Problemen unterschlagen, die zu einer Anomalie der Offerkurve führen können. Dies sind insbesondere der Fall der nichtgehandelten Güter, der hier mit dem regionalen öffentlichen Gütern vorliegt, und der Fall, daß man sich auf dem backward-bending-Ast der Arbeitsangebotskurve befindet (siehe z.B. Gandolfo [1987a], Kapitel 7). Hier wird angenommen, daß eine Anomalie der Offerkurve nicht auftritt.
} 
- Interregionale Einkommens(Aufkommens)-Effekte entstehen durch Transfers zwischen den Ländern. Normalerweise erhöhen Transfers die Einkommen und damit die Konsummöglichkeiten der Empfängerländer. Bei einer höheren Präferenz für die heimischen Güter ergeben sich letztlich Wohlfahrtsgewinne für die Empfänger und -verluste für die Geberländer. Im hier vorliegenden Modell bestehen die Transfers aus den Zuweisungen, den LFA-Zuweisungen den Zuweisungen aus der Steuerverteilung und den Steuerexporten, soweit diese in der Steuerverteilung noch nicht erfaßt sind.

Wie bereits bezüglich der durch die Finanzausgleichsregelungen bedingten Verzerrungen angedeutet wurde, ist es nicht einfach, den Nettoeffekt einer Veränderung des Finanzausgleichsregimes auf die Substitutionseffekte zu bestimmen. Dies gilt erst recht, wenn man zusätzlich die ausgelösten Transferveränderungen betrachtet. Letztlich kann man die Höhe der Effekte und damit die gesamte Wohlfahrtsveränderung nur empirisch in einem umfassenden ökonomischen Modell mit integriertem Finanzausgleich bestimmen.

Eine der Größen, die bekannt sein müssen, um eine Vorstellung von der Größenordnung der hervorgerufenen Effekte zu bekommen, ist der Zusammenhang zwischen einer Veränderung der öffentlichen Ausgaben, Steuersätze oder Transfers und der Veränderung der Steueraufkommen. Die genaue Beziehung zwischen diesen Größen kann nur in einem allgemeinen Gleichgewichtsmodell bestimmt werden, weil dazu die Kenntnis der relevanten ökonomischen Zusammenhänge notwendig ist.

Dies sind zwei der Gründe, weshalb im folgenden eine Empirische Allgemeine Gleichgewichtsanalyse (EAG) für die Bundesrepublik Deutschland durchgeführt werden soll.

Das gewichtigste Argument zur Durchführung einer EAG-Analyse ist jedoch die SecondBest-Problematik. Die oben abgeleiteten Effizienzwirkungen treten letztlich nur unter First-Best-Gesichtspunkten in der dargestellten Form auf. Eine Reduktion (Verstärkung) der Störungen könnte jedoch insgesamt zu einer Wohlfahrtsreduktion (-verschlechterung) führen und damit genau die entgegengesetzten Effekte auslösen, die aufgrund der dargestellten Überlegungen erwartet werden müßten. Klarheit über die Richtung der gesamten Wohlfahrtswirkungen kann letztlich nur eine EAG-Analyse schaffen, da sie den Einfluß von Politikveränderungen bei weiterhin bestehenden Allokationsstörungen untersuchen kann. 
Georg Hirte - 978-3-631-75212-8

Downloaded from PubFactory at 01/11/2019 07:02:26AM

via free access 


\section{Kapitel IV}

\section{Das EAG-Modell}

\section{Einleitende Bemerkungen}

Aufbauend auf der Inzidenzanalyse von Harberger [1962] und der Entwicklung eines programmierbaren Fixpunktalgorithmus von SCARF [1967, 1973] $]^{1}$ haben SHOven und Whalley [1972, 1973, 1977] den Prototyp eines Empirischen Allgemeinen Gleichgewichtsmodells (EAG-Modell) zur Untersuchung von Inzidenzwirkungen vorgestellt. Dies war der Beginn einer rasanten Entwicklung, die mittlerweile zu einer nicht mehr überschaubaren Zahl von EAG-Analysen verschiedenster Art geführt hat. ${ }^{2}$

Im Prinzip bestehen EAG-Modelle walrasianischer Provenienz aus einem erweiterten mikroökonomisch fundierten Modell, das eine recht große Verwandtschaft zu normalen neoklassischen Ansätzen besitzt und sich daher deren theoretischen Erkenntnisse zunutze machen kann. Damit ist diese Methodik eine Erweiterung des normalen theoretischen Analyserahmens dort, wo dieser angesichts eines begrenztem analytischen Instrumentariums keine klaren Ergebnisse produzieren kann. Dies gilt insbesondere für den Fall alternierender Vorzeichen, zu deren eindeutiger Bestimmung eine empirische Untersuchung notwendig ist. ${ }^{3}$

Trotz der Orientierung an den üblichen neoklassischen Modellen, ist die EAG-Analyse nicht unumstritten, wie die lange Reihe von Aufsätzen zur Problematik dieser Methodik zeigt. ${ }^{4}$ Gerade der Versuch, immer komplexere und umfangreichere Modelle zu entwickeln

\footnotetext{
${ }^{1}$ Mittlerweile wurden eine Vielzahl anderer Algorithmen entwickelt, wie z.B. im Sammelband von ManNe [1985] deutlich wird. Die grundlegende Struktur des von Merrill verbesserten ScarfAlgorithmus inclusive einfacher Anwendungen beschreibt u.a. WIEg ARD [1985a].

${ }^{2}$ Siehe den Überblick in Shoven/Whalley [1984] oder WIEgard [1985b] sowie die Sammelbände von Scarf/Shoven [1984], Srinivasan/Whalley [1985] oder Piggott/Whalley [1985a, 1991].

${ }^{3}$ Eine grundlegende Darstellung der Methodik der EAG-Analyse und dabei entstehender Probleme bietet z.B. Hirte, Wiegard [1988]. Eine Untersuchung, in der theoretische Analysen und empirische Ergebnisse sehr ausführlich gegenübergestellt werden, bieten FEHR/RosENBERG/WIEGARD [1992, 1993] sowie Rosenberg [1993] im Zusammenhang mit Fehr [1993].

${ }^{4}$ Besonders ragt dabei die fundamentale Kritik von TAIT [1989] heraus. Weitere Problem werden von Borges [1986] oder Whalley [1988] diskutiert.
} 
(siehe z.B. PiggotT/Whalley [1985b]), deren Ergebnisse zunehmend weniger hinterfragt und ökonomisch begründet wurden (siehe z.B. WhALLEY/TrELA [1986]) führte angesichts der nach wie vor ungelösten Datenprobleme zu zunehmender Skepsis gegenüber diesen Modellen. Vor allem die Frage der Verläßlichkeit der Ergebnisse ist angesicht der Problematik der Datenlage und der Parameterschätzungen der Modelle Mittelpunkt heftiger Kritik. ${ }^{5}$

Wertet man die EAG-Analyse dagegen in erster Linie als Versuch, den Untersuchungsrahmen der ökonomischen Theorie zu erweitern, so zeigt sich, daß diese Methodik ungeachtet aller Kritik eine wichtige Ergänzung der ökonomischen Theorie darstellen kann. Insbesondere die detaillierte Implementierung institutioneller Regelungen in Verbindung mit einem ansonsten einfachen ökonomischen Modell ermöglicht es, die normalen theoretischen Erkenntnisse auch bei Berücksichtigung realer institutioneller Gegebenheiten zu verwenden oder gar zu erweitern. Insofern kann die Empirische Allgemeine Gleichgewichtsanalyse die Relevanz der ökonomischen Theorie für aktuelle Fragen unterstreichen und darüber hinaus auch dem Praktiker neue Einsichten in die aus institutionellen Regelungen entstehenden ökonomischen Probleme bieten.

Die Probleme von Finanzausgleichssystemen wurden bisher allerdings nur in der interregionalen EAG-Analyse von Whalley/Trela [1986] für Kanada untersucht. Allerdings implementieren sie den kanadischen Finanzausgleich nur rudimentär als System allgemeiner Zuweisungen der nationalen Regierung an die Regionen. Diese vereinfachte Struktur verdeckt jedoch die meisten der hier herausgearbeiteten Effizienzaspekte. Das eigentlich Interessante an ihrer Modellierung ist, daß ihre Arbeit eines der wenigen bislang vorliegenden interregionalen Modelle präsentiert. ${ }^{6}$ Dasselbe Modell wurde in einer Reihe weiterer Veröffentlichungen und Untersuchungen verwendet (JONES/WhALLEY/WIGLE [1985] und Jones/WhalLEY [1987, 1988, 1989] ) und stellt von daher sozusagen den Prototyp interregionaler Modelle dar. ${ }^{7}$

Da sich diese Modelle jedoch nur wenig von Mehr-Länder-Modellen unterscheiden, deren Anwendung sehr weit verbreitet ist ${ }^{8}$ ist es im Prinzip nicht notwendig zwischen interregionalen und internationalen Modellen zu unterscheiden. Dies wird z.B. durch die große Nähe des hier vorgestellten interregionalen Modells zu dem internationalen Modell von FEHR/ROSENBERG/WiEgaRD [1992, 1993] deutlich.

\footnotetext{
${ }^{5}$ Zur Frage der Robustheit von EAG-Analysen siehe z.B. HARrison u.a. [1993].

${ }^{6}$ Das erste, noch sehr einfach gehaltene interregionale EAG-Modell stellten KIMBELL/HARRISON [1984] vor.

${ }^{7}$ Z.B. orientiert sich die Struktur des ersten interregionalen Zwei-Länder Modells für die Bundesrepublik von Hirte/Genosko [1988/89] und HirTe [1990] an diesem Modell.

${ }^{8}$ Siehe z.B. WhaLLEY [1985].
} 
Das allgemeine Vorgehen der Empirischen Allgemeinen Gleichgewichtsanalyse besteht darin, ausgehend von einer simultanen Entwicklung des theoretischen Modells und der modellkonsistenten Datenbasis, die relevanten Parameter des Modells in der Kalibrierung zu schätzten. Zu diesem Zweck werden grundlegende Parameter, zumeist Elastizitäten, aus der Literatur gewählt und damit die restlichen Parameter kalibriert (berechnet). Dies geschieht so, daß man mit den ermittelten Parameterwerten in einer Kontrollrechnung (replication check) wieder genau die Datenbasis erhält. Damit entspricht die Kalibrierung einer Berechnung der Parameterwerte auf Grundlage einer einzigen Beobachtung und ist daher nicht unumstritten (siehe z.B. Mansur/Whalley [1984] und LAU [1984]). Im nächsten Schritt werden mit Hilfe eines der zahlreichen Algorithmen die Simulationen der Politikveränderungen durchgeführt. Anschließend beginnt die eigentliche ökonomische Arbeit, nämlich die Interpretation der Resultate, zu deren Hilfe eine Reihe von Sensitivitätsanalysen eingesetzt werden können und, um die Robustheit der Ergebnisse zu untersuchen, auch sollten.

Angelehnt an diese Kurzbeschreibung des allgemeinen Vorgehens wird im folgenden die EAG-Analyse durchgeführt.

\section{Theoretisches Modell}

Das Modell orientiert sich weitgehend an der in der vorangegangenen Analyse verwendeten Struktur. In den Grundzügen entspricht es zudem dem EAG-Modell von FEHR/ROSENBERG/WIEGARD [1992]. Abweichungen ergeben sich durch die differenziertere Steuerstruktur, die Berücksichtigung der regionalen öffentlichen Güter und des Finanzausgleichs.

Die Modifikationen resultieren aus der Absicht, die Effizienzaspekte von Finanzausgleichsregelungen genauer zu untersuchen. Beispielsweise werden sämtliche von der Steuerverteilung betroffenen Gemeinschaftssteuern modelliert. Um die Berechnung der Finanzkraft einigermaßen genau zu gestalten, werden zusätzlich Grundsteuern und die Ländersteuern ins Modell eingebaut. Das bundesdeutsche Finanzausgleichssystem wird exakt in der im Abschnitt 3. dargestellten Form implementiert. Die Besonderheit der gesetzlichen Finanzausgleichsregelung bedingt, daß alle Westländer berücksichtigt werden sollten. Die Ostländer werden dagegen, begründet durch die Datenlage und eine untereinander ähnliche Finanzkraftstruktur, als ein aggregiertes Land modelliert. Demnach besteht das Modell aus elf Ländern, einem detaillierten Finanzausgleichssystem sowie einer Reihe von Steuern, nämlich den Faktor-, Produktions-, Konsum- und Einkommenssteuern sowie einer Lump-Sum-Steuer als Aggregat der restlichen, modellbedingt nicht genauer implementierbaren Steuerarten. Zusätzlich werden öffentliche Güter berücksichtigt. 
Die Beziehungen zwischen den Regionen sind durch vollkommene Mobilität des homogenen Faktors Kapital ${ }^{9}$ und einem interregional immobilen Faktor Arbeit gekennzeichnet. Darüber hinaus bestehen finanzielle Zusammenhänge über den Finanzausgleich. Daraus ergeben sich nichtausgeglichene Handelsbilanzen, deren Saldo letztlich über die Übertragungsbilanz ausgeglichen wird.

Eine besondere Erwähnung bedarf an dieser Stelle die Modellierung der privaten Güter. Um eine differenziertere Struktur zu erhalten, in der auch intrasektoraler Handel zugelassen wird, wird die Armington-Annahme getroffen (ARmington [1969]). Darin wird davon ausgegangen, daß ein privates Gut $C$, das in jeder der beiden Regionen hergestellt wird, kein homogenes Gut ist, sondern sich aufgrund der Herkunft (die z.B. eine andere Marke bedeuten kann) voneinander unterscheidet. Von diesem privaten Gut gibt es daher pro Region ein zwar unterschiedliches aber in gewissen Sinn gleichartiges Produkt. Diese sind enge Substitute zueinander und konkurrieren miteinander auf dem Markt für den privaten Konsum. Deshalb gibt es bei elf Regionen insgesamt elf private Güter, die in jeder Region konsumiert werden können. In der hier verwendeten Notation werden diese privaten Güter mit $C_{r s}$ bezeichnet, wobei $r$ der Index für die jeweilige Herkunftsregion, d.h. die Güterart, und $s$ der Index für die jeweilige Verbrauchsregion ist.

Der Darstellung von FEHR ([1993], S.33) folgend orientiert sich die Präsentation des EAGModells an dem verwendeten Computerprogramm. Demnach bestimmen ausgehend von den vorgegebenen Faktorpreisen und Steuersätzen die Unternehmen ihre kostenminimale Einheitsnachfrage nach Primärfaktoren und Vorleistungsgütern. Dies führt über die Nullprofitbedingung zur Bestimmung der Produzentenpreise, die als Vorgabe für die Entscheidungen der repräsentativen Haushalte dienen (Abschnitt 2.1.). Weitere Entscheidungsgrundlagen der regionalen Haushalte sind die vorgegebenen Steuersätze sowie die nationalen Transfers und die Steuereinnahmen der Region sowie die sozialen Grenzkosten der öffentlichen Güter. Unter diesen Vorgaben wählen die repräsentativen privaten Haushalte ihre nutzenmaximalen Güternachfragen (Abschnitt 2.3.). Bei Markträumung bestimmen letztere die Outputmengen und damit über Multiplikation mit den Faktoreinheitsnachfragen die Faktornachfragen (Abschnitt 2.6.). Unter Verwendung dieser Angaben können schließlich die Steueraufkommen und über Berücksichtigung der Finanzausgleichsregelungen die Einnahmen der öffentlichen Haushalte berechnet werden (Abschnitt 2.4.). Als Ergebnis des beschriebenen Vorgehens erhält man die Überschußnachfragen auf den Faktormärkten sowie die Überschußnachfragen der regionalen Regierungen und des Staates. Mit Hilfe eines Lösungsalgorithmus kann man dann einen Gleichgewichtspreisvektor finden (Abschnitt 2.6.).

\footnotetext{
${ }^{9}$ Eine Modellierung mit interregional heterogenem Kapital präsentieren z.B. FeHR/RosenBERG/Wiegard [1992].
} 
Der Einfachheit halber wird folgende Vereinbarung bezüglich der Notation getroffen: Die Indizes $i, j$ beziehen sich auf die Güter $C$ und $G$ und die Indizes $r, s$ auf die jeweiligen Regionen. Soweit nichts anderes angegeben wird, laufen sie jeweils über die gesamte Menge $C, G$ bzw. $1, \cdots, R$.

\subsection{Regionale Produktionssektoren}

In jeder der elf Regionen werden die Güter in zwei Sektoren produziert. Im ersten Sektor wird das private Konsumgut der Region hergestellt, das frei handelbar ist. Dieses Gut kann in allen Produktionssektoren aller Regionen als Vorleistungsgut eingesetzt werden. Im zweiten Sektor wird das regionale öffentliche Gut produziert, das nur innerhalb der Region als öffentliches Konsumgut oder als als Vorleistungsgut verwendet werden kann. Es handelt sich demnach um ein nichthandelbares Gut. Zusätzlich beschäftigen die Unternehmenssektoren in der Herstellung ihrer Outputgüter die Primärfaktoren Kapital, das keinem Verschleiß unterliegt ${ }^{10}$, und Arbeit.

Die Sektoren sind darüber hinaus Wettbewerber und produzieren daher ohne Profite (Nullprofitbedingung). Ihr Verhalten ist durch Kostenminimierung gekennzeichnet, die als mehrstufiger Entscheidungsbaum modelliert ist. Die verwendeten Technologien sind Leontief- und CES-Produktionsfunktionen mit konstanten Skalenerträgen.

Entscheidungsgrundlage für die Unternehmen sind die exogen vorgegebenen Faktorlohnsätze sowie die jeweiligen Steuersätze. Daher sollen nun kurz die die Unternehmen betreffenden Steuerarten charakterisiert werden.

\subsubsection{Besteuerung der Produktionssektoren}

\section{(1) Produktionssteuern}

Die Produktionssteuern werden, abzüglich der Realsteuern, in der in den Input-OutputTabellen des Statistischen Bundesamtes ausgewiesenen Form modelliert. ${ }^{11}$ Analog zu HIRTE/WIEGARD [1988] sollen sie hier als sektorspezifische wertmäßige Produktionssteuern incl. Subventionen modelliert werden.

Da die Steuern auf den Output zu Herstellungspreisen bezogen sind, müßten mit dem Produzenten- und dem Herstellungspreis zwei Preiskonzepte auf der Unternehmensseite

\footnotetext{
${ }^{10}$ Damit sind explizit Abschreibungen und Ersatzinvestitionen ausgeschlossen.

${ }^{11} \mathrm{Zu}$ den Produktionssteuern zählen z.B. die Versicherungssteuer sowie die spezifische Verbrauchssteuern (Mineralölsteuer, Branntweinsteuer, Tabaksteuer). Eine vollständige Auflistung findet sich in STABA [1983a].
} 
verwendet werden. Um sich das zu ersparen, wird die Produktionssteuer als Steuer auf den Produzentenpreis (Bruttoproduktionswert inclusive Steuern) modelliert. Daraus ergibt sich die Steuerschuld der Sektoren aus der Gleichung

$$
S T_{j s}^{Q}=\frac{\tau_{j s}^{Q}}{1+\tau_{j s}^{Q}} q_{j}^{s} Q_{j}^{s},
$$

mit $\tau_{j s}^{Q}$ als Steuersatz auf die Herstellungskosten.

\section{(2) Kapitalsteuern}

Die Kapitalbesteuerung im Modell besteht aus drei Steuerarten. Zum ersten werden die Kapitalertrags- und die Körperschaftssteuern auf ausgeschüttete und nichtausgeschüttete Gewinne zu einer Kapitalsteuer in Form einer Steuer auf den Einsatz des Faktors Kapitals modelliert. Dies entspricht einer Quellen(land)besteuerung und führt zu interregional einheitlichen Zinssätzen nach Abzug der Faktorsteuern. ${ }^{12}$ Die Gewerbe- und die Grundsteuern werden jeweils separat ebenfalls als Steuer auf den Faktor Kapital modelliert. ${ }^{13}$

Für die Entscheidungen der Unternehmen sind jedoch die Bruttokapitallohnsätze $\widehat{r}_{j}^{s}$ relevant, die wie folgt definiert werden:

$$
\widehat{r}_{j}^{s}=r\left(1+\tau_{j s}^{K}+\tau_{j s}^{G w}+\tau_{j s}^{G r}\right) .
$$

$r$ ist der regional einheitliche Zinssatz, $\tau_{j s}^{K}$ der Kapitalsteuersatz der Kapitalertrags- und Körperschaftssteuer, $\tau_{j s}^{G w}$ der Gewerbe- und $\tau_{j s}^{G \tau}$ der Grundsteuersatz.

\subsubsection{Die Technologie und die Entscheidung der Sektoren}

Die beiden Unternehmenssektoren jeder Region produzieren mit Hilfe der Primärfaktoren Kapital und Arbeit sowie unter Einsatz von Zwischenprodukten jeweils ein Gut. Sie können dabei Kapital und Arbeit gegeneinander und die einzelnen privaten Produkte miteinander substituieren, d.h. sie haben bezüglich der privaten Vorleistungen variable Inputkoeffizienten.

Die Entscheidungsstruktur der Unternehmen kann nun, unter der Annahme, daß die Produktionstechnologie zwischen den primären Inputs, den privaten den Zwischenprodukten

\footnotetext{
${ }^{12}$ Eine Diskussion, unter welchen Annahmen eine Formulierung der Kapitalsteuern als Faktorsteuern möglich ist, führt SinN [1985]. Eine wesentliche Voraussetzung ist das Fehlen von Finanzierungsalternativen für die Unternehmen. Darüber hinaus fehlen hier Abschreibungen und Steuern auf den Kapitalbestand (vgl. SinN [1985], S.131 bzw. S.171).

${ }^{13}$ Eine genaue Begründung für dieses Vorgehen findet man bei GotTfried ([1992], S.103-108).
} 
und den öffentlichen Vorleistungen schwach separabel ist ${ }^{14}$, als Entscheidungsbaum modelliert werden (siehe Abbildung IV.1). ${ }^{15}$ Diese Entscheidungsstruktur erlaubt die Verwendung unterschiedlicher Substitutionselastizitäten auch bei einfachen Funktionen. ${ }^{16}$

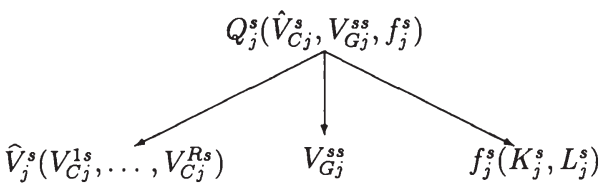

Abbildung IV.1: Struktur der Technologie

Ausgehend von der Gesamtproduktionsfunktion $Q_{j}^{s}$, deren funktionale Form eine fixe Aufteilung der Produktion auf eine Wertschöpfungsfunktion $f_{j}^{s}$, eine Vorleistungsfunktion des aggregierten privaten Gutes $\widehat{V}_{j}^{s}$ und einem fixen Vorleistungseinsatz des öffentlichen Gutes der Region vorgibt, wählen die Unternehmen in zwei getrennten Entscheidungsabläufen ihre kostenminimale Nachfrage nach den Primärfaktoren $L_{j}^{s}$ und $K_{J}^{s}$ und den einzelnen nach Herkunft unterschiedenen privaten Vorleistungsgütern $V_{C j}^{r s}$.

Die Produktionstechnologie der Sektoren sei dabei die folgende Leontief-Funktion:

$$
Q_{j}^{s}=\min \left[\frac{1}{a_{0 j}^{s}} f_{j}^{s}\left(K_{j}^{s}, L_{j}^{s}\right) ; \frac{\widehat{V}_{C j}^{s}\left(V_{C j}^{r s} \mid r \in \mathcal{R}\right)}{\widehat{a}_{j}^{s}} ; \frac{V_{G j}^{s s}}{a_{G j}^{s s}}\right]
$$

mit $\hat{a}_{j}^{s}$ als Vorleistungskoeffizient für den Einsatz des aggregierten privaten Zwischenproduktes $\hat{V}_{j}^{s}, a_{G j}^{s s}$ als Inputkoeffizient des öffentlichen Gutes und $a_{o j}^{s}$ als Koeffizient der Wertschöpfung.

\footnotetext{
${ }^{14}$ Das ist dann der Fall, wenn die Grenzrate der technischen Substitution zwischen zwei Faktoren einer Gruppe von der Veränderung des Faktoreinsatzes eines Faktors einer anderen Gruppe unabhängig ist (siehe Fuss/McFadden/Mundlock [1978], S.244 oder Diewert [1978], S.42).

${ }^{15}$ Zur Struktur der Produktionstechnologie mit variablen Zwischenprodukten siehe auch JoNES/Whalley [1989] oder Wigle [1988].

${ }^{16}$ Genauere Erläuterungen zu Baumstrukturen und deren Bedingungen finden sich bei KELLER [1976, 1977].
} 


\section{(1) Bestimmung der Primärfaktornachfragen}

Die Wertschöpfungsfunktion sei eine CES-Funktion ${ }^{17}$ :

$$
\left.f_{j}^{s}\left(L_{j}^{s}, K_{j}^{s}\right)=\phi_{j}^{s}\left[\delta_{j}^{s} L_{j}^{\frac{\sigma_{1 j}^{s}-1}{\sigma_{1 j}^{s}}}+\left(1-\delta_{j}^{s}\right) K_{j}^{s}\right]^{\frac{\frac{\sigma}{j}_{j}^{s}-1}{\sigma_{j}^{s}}}\right]^{\frac{\sigma_{j}^{s}}{\sigma_{j}^{j}-1}}
$$

mit $\sigma_{j}^{s}$ als Substitutionselastizität zwischen Arbeit und Kapital des Sektors $\mathrm{j}$ in der Region s, $\delta_{j}^{s}$ als Anteilsparameter des Faktors Arbeit am Output, $\phi_{j}^{s}$ als Skalenfaktor.

Aus dem entsprechenden Kostenminimierungskalkül bei vorgegebenem Outputniveau

$$
\begin{aligned}
& \min _{L_{j}^{s}, K_{j}^{s}} w_{s} L_{j}^{s}+\widehat{r}_{j}^{s} K_{j}^{s} \\
& \quad \text { u.d.NB.: } f_{j}^{s}\left(L_{j}^{s}, K_{j}^{s}\right)=a_{0 j}^{s} \bar{Q}_{j}^{s} \quad \forall j=C, G,
\end{aligned}
$$

ergeben sich die konditionalen Faktornachfragefunktionen nach den Primärfaktoren, aus denen nach Division durch die vorgegebenen Outputmenge $\bar{Q}_{j s}$ die Einheitsfaktornachfragefunktionen

$$
l_{j}^{s}\left(w_{s}, \hat{r}_{j}^{s}\right)=\frac{L_{j}^{s}}{\bar{Q}_{j}^{s}}=\frac{a_{0 j}^{s}}{\phi_{j}^{s}}\left[\delta_{j}^{s}+\left(1-\delta_{j}^{s}\right)\left(\frac{\delta_{j}^{s} \widehat{r}_{j}^{s}}{\left(1-\delta_{j}^{s}\right) w_{s}}\right)^{1-\sigma_{j}^{s}}\right]^{\frac{\sigma_{j}^{s}}{1-\sigma_{j}^{s}}}
$$

und

$$
k_{j}^{s}\left(w_{s}, \hat{r}_{j}^{s}\right)=\frac{L_{j}^{s}}{\bar{Q}_{j}^{s}}=\frac{a_{0 j}^{s}}{\phi_{j}^{s}}\left[\left(1-\delta_{1 j}^{s}\right)+\delta_{1 j}^{s}\left(\frac{\left(1-\delta_{1 j}^{s}\right) w_{s}}{\delta_{1 j}^{s} \widehat{r}_{j}^{s}}\right)^{1-\sigma_{1 j}^{s}}\right]^{\frac{\sigma_{1 j}^{s}}{1-\sigma_{1 j}^{i}}}
$$

hergeleitet werden können.

(2) Vorleistungsnachfrage nach den einzelnen privaten Gütern [2]

Die aus den einzelnen nach Herkunft unterschiedenen Vorleistungsprodukten zusammengesetzten Produktionsfunktionen sind ebenfalls CES-Funktionen:

$$
\left.\hat{V}_{j}^{s}\left(V_{C j}^{r s} \mid r \in \mathcal{R}\right)=\psi_{j}^{s}\left[\sum_{r=1}^{\mathcal{R}} d_{j}^{r s} V_{C j}^{r s}\right]^{\frac{\rho_{j}^{s}-1}{\rho_{j}^{s}}}\right]^{\frac{\rho_{j}^{s}}{\rho_{j}^{s}-1}} \quad \text { mit } \sum_{r=1}^{R} d_{j}^{r s}=1
$$

und $\rho_{j}^{s}$ als Substitutionselastizität zwischen den Vorleistungsgütern $V_{C j}^{r s}, \psi_{j}^{s}$ als Skalierungsfaktor sowie $d_{j}^{\text {rs }}$ als entsprechende Anteilsparameter.

\footnotetext{
${ }^{17}$ Die Verwendung von CES-Funktionen hat einige Vorteile. Sie haben sehr angenehme Eigenschaften wie Homogenität oder konstante Skalenerträge und sind dennoch bei Verwendung eines Entscheidungsbaumes relativ flexibel.
} 
Das Kostenminimierungskalkül

$$
\begin{aligned}
\min _{V_{C_{j}^{\prime}}^{r s}} & \sum_{r=1}^{R} q_{\tau} V_{C j}^{r s} \\
& \text { u.d.NB.: } \hat{V}_{j}^{s}\left(V_{C j}^{r s} \mid r \in \mathcal{R}\right)=\hat{a}_{j}^{s} \bar{Q}_{j}^{s}
\end{aligned}
$$

führt nach einigen Umformungen zu den Einheitsnachfragen nach den einzelnen Vorleistungsprodukten:

$$
a_{C j}^{\tau s}\left(q_{k} \mid k \in \mathcal{R}\right)=\frac{V_{C j}^{r s}}{\bar{Q}_{j}^{s}}=\frac{\hat{a}_{j}^{s}}{\psi_{j}^{s}}\left[d_{j}^{r s}+\sum_{k=1, k \neq s}^{R} d_{j}^{k s}\left(\frac{d_{j}^{r s} q_{k}}{d_{j}^{k s} q_{r}}\right)^{1-\rho_{j}^{s}}\right]^{\frac{\rho_{j}^{s}}{1-\rho_{j}^{s}}}
$$

Liegen allerding fixe Vorleistungskoeffizienten vor, dann ergibt sich die Vorleistungsnachfrage direkt aus der modifizierten Leontief-Produktionsfunktion

$$
Q_{j}^{s}=\min \left[\frac{1}{a_{0 j}^{s}} f_{j}^{s}\left(K_{j}^{s}, L_{j}^{s}\right) ; \frac{V_{G j}^{s s}}{a_{G j}^{s s}} ; \frac{V_{C j}^{r s}}{a_{C j}^{r s}} \mid \forall r \in \mathcal{R}\right] .
$$

\section{(3) Bestimmung der Produzentenpreise}

Aus der Nullprofitbedingung der Unternehmen

$$
q_{j}^{s} Q_{j}^{s}-w_{s} L_{j}^{s}(\cdot)-\widehat{r}_{j}^{s} K_{j}^{s}(\cdot)-\sum_{r \in \mathcal{R}} q_{r} V_{C j}^{r s}(\cdot)-q_{G s} V_{G j}^{s s}-S T_{s j}^{Q}=0
$$

können schließlich nach Division durch $Q_{j}^{s}$ die Produzentenpreise

$$
q_{j}^{s}=\left(1+\tau_{j s}^{Q}\right)\left(w_{s} l_{j}^{s}+\widehat{r}_{j}^{s} k_{j}^{s}+\sum_{r \in \mathcal{R}} q_{\tau} a_{C j}^{r s}(\cdot)+q_{G s} a_{G j}^{s s}\right)
$$

bestimmt werden, die in Vektorschreibweise folgende Form besitzt:

$$
\mathbf{q}=\left(\mathbf{E}^{*}-\mathbf{A}^{\prime}(\mathbf{q})\right)^{-1} \mathbf{W}
$$

mit $\mathbf{q}$ als Produzentenpreisvektor, $\mathbf{W}$ als Vektor der Summe der zu Faktorbruttopreisen bewerteten Faktoreinheitsnachfragen nach Arbeit $l_{j}^{s}$ und Kapital $\mathrm{k}_{j}^{s}$ und $E^{*}$ als Einheitsmatrix abzüglich der Terme $1 /\left(1+\tau_{j s}^{Q}\right)$ in der Hauptdiagonalen. $\mathbf{A}^{\prime}(q)$ ist die transponierte Matrix der Inputkoeffizienten der Vorleistungsmatrix, die von allen Produzentenpreisen abhängig sind.

Aufgrund der Nichtlinearität des Gleichungssystems können die Produzentenpreise nur mit Hilfe eines Algorithmus bestimmt werden, der die folgende Struktur besitzt. Ausgehend von einem beliebig, d.h. möglichst guten, vorgegebenen Produzentenpreisvektor 
werden die variablen privaten Vorleistungskoeffizienten berechnet, unter deren Verwendung über die Lösung der obigen Gleichung dann neue Produzentenpreise ermittelt werden können. Solange nun diese neu bestimmten Produzentenpreise von den zu Beginn vorgegebenen Preisen abweichen, wird das Verfahren unter Vorgabe der neu bestimmten Preise wiederholt. Am Ende ergeben sich sowohl die neuen Vorleistungskoeffizienten als auch die Produzentenpreise, die eine Entscheidungsgrundlage für die privaten Haushalte sind. ${ }^{18}$

\subsection{Regionaler privater und öffentlicher Bereich}

Die privaten Haushalte wählen über ihr Nutzenmaximierungskalkül die entsprechenden optimalen Nachfragemengen der einzelnen Güter bei vorgegebenen Einkommen, Steuern und Preisen.

Die Endnachfrage wird als Gesamtnachfrage der Regionen ermittelt in dem jede Region als repräsentativer Haushalt dargestellt wird. Damit dies möglich ist sei angenommen, daß die Individuen einer Region bezüglich ihrer Nachfragepräferenzen identisch sind, d.h. bezüglich der Güter identische Nutzenfunktionen besitzen ${ }^{19}$.

Die vom privaten Haushalt abzuführenden Steuern sind die Steuern auf Lohn- und Kapitaleinkünfte (als Lohn- und veranlagte Einkommenssteuer), sowie die jeweiligen Konsumsteuern (als vereinfachte Modellierung von Mehrwertsteuern).

\subsubsection{Besteuerung der Nachfrageseite}

\section{(1) Konsumbesteuerung im Modell}

In der Bundesrepublik wird eine Mehrwertsteuer vom Konsumtyp nach dem Vorsteuerabzugsverfahren und nach dem Ursprungslandprinzip ${ }^{20}$ sowie einige spezielle Verbrauchssteuern erhoben. Im hier vorliegenden Modell wird diese Mehrwertsteuer als reine Konsumsteuer nach dem Ursprungslandprinzip modelliert. ${ }^{21}$ Außerdem wird angenommen, daß die engen Substitutgüter steuerlich gleich behandelt werden und daher einem einheitlichen nationalen Konsumsteuersatz unterliegen. Demnach zahlen die Konsumenten

\footnotetext{
${ }^{18}$ Im Prinzip könnte man stattdessen auch jedes Standardverfahren, wie z.B. Newton-Verfahren, verwenden.

${ }^{19}$ Siehe dazu z.B. VARIAN [1992].

${ }^{20}$ Dies gilt innerhalb der Bundesrepublik. International wird das Bestimmungslandprinzip angewendet.

${ }^{21}$ Eine detaillierte Modellierung der bundesdeutschen Umsatzsteuer incl. der Steuerbefreiungen von Unternehmen findet sich z.B. bei GotTFRIED [1992].
} 
in den unterschiedlichen Regionen für dasselbe Gut $C_{r s}$ jeweils den gleichen Konsumentenpreis $p_{r}=\left(1+\tau^{C}\right) q_{r}$, dessen Steueranteil die Steueraufkommen des Herkunftlandes erhöht.

\section{(2) Einkommenssteuern}

Die Lohn- und die veranlagten Einkommenssteuern werden im vorliegenden Modell als Steuern auf Einkommen aus unselbstständiger Arbeit sowie auf Kapitaleinkünfte (synthetische Einkommenssteuer) modelliert.

Die Einkommenssteuer auf Kapitaleinkünfte wird gegenüber der in der Bundesrepublik geltenden Regelung dahingehend modifiziert, daß zum einen die Körperschaftssteuer auf ausgeschüttete Gewinne nicht in die Bemessungsgrundlage der Einkommenssteuer eingeht und zum anderen auch kein Steuerabzug der abgeführten Körperschaftssteuer auf ausgeschüttete Gewinne zugelassen wird. Es ergibt sich also der klassische Fall der vollen Doppelbesteuerung der privaten Kapitaleinkünfte, der allerdings wie einige Test zeigen, nur einen marginalen Einfluß auf die Simulationsergebnisse besitzt, andererseits jedoch erheblich einfacher programmiert werden kann.

Der progressive Verlauf des Einkommenssteuertarifes in der Bundesrepublik wird durch eine lineare (indirekt progressive) Einkommenssteuer als einfachstem Typ eines progressiven Steuertarifs simuliert. ${ }^{22}$ Im Gegensatz zu einer allgemeinen proportionalen Einkommenssteuer sind wegen des höheren Grenzsteuersatzes der linearen Einkommenssteuer (das gilt auch für alle anderen progressiven Verläufe) die Effizienzwirkungen einer progressiven Steuer größer (siehe dazu STöss [1991], S.28-32). ${ }^{23}$

Aufgrund der einfachen Modellstruktur, in der Pendeln nicht erlaubt ist, sind Wohnsitzland und Arbeitsstättenland bezüglich des Faktors Arbeit identisch. Demnach ergeben sich sowohl bei Anwendung des Wohnsitzland- als auch des Quellenlandprinzips hinsichtlich der Arbeitseinkommen keine Unterschiede. Dadurch wird die bei der Einkommenssteuerverteilung durchzuführende Lohnsteuerzerlegung nach dem Wohnortprinzip hinfällig. Dies vereinfacht die Berechnung der Einkommenssteuerverteilung erheblich.

Die weiter unten begründete Verwendung von repräsentativen Konsumenten erleichtert die Modellierung der Einkommenssteuern, da von jeglichen differenzierten Einkommens-

\footnotetext{
${ }^{22}$ Die lineare Einkommenssteuer wird deshalb als „indirekt progressiv"bezeichnet, da mit Anstieg der Bemessungsgrundlage nur der Durchschnittssatz nicht aber der (konstante) Grenzsteuersatz wächst. Z Igleich gilt jedoch, daß der Grenzsteuersatz größer als der Durchschnittssteuersatz ist. Zur alternativen Definition von Progression siehe z.B. Bös/Genser [1977] oder PollaCK [1980].

${ }^{23}$ Dies hängt letztlich von der Normalitätsannahme bezüglich des Arbeitsangebotes ab STöss [1991], S.32.
} 
wirkungen abgesehen werden kann. Bei Berücksichtigung mehrerer Konsumenten müßte ansonsten das Konzept des virtuellen Einkommens verwendet werden ${ }^{24}$.

Die entsprechende Modellierung der Einkommenssteuer lautet dann:

$$
S T_{s}^{E k}=\tau^{Y}\left(w_{s} L_{s}+r \bar{K}_{s}-A_{s}\right)
$$

mit $S T_{s}^{E k}$ als Einkommenssteueraufkommen der Länder vor der Steuerverteilung, $\tau^{Y}$ als entsprechender Grenzsteuersatz, $w_{s} L_{s}$ als Lohneinkommen des repräsentativen Konsumenten des Landes $s$ und $A_{s}$ als Freibetrag der Einkommenssteuer.

\section{(3) Sonstige Ländersteuern}

Die sonstigen Ländersteuern steuern einen nicht unwesentlichen Beitrag zu der Finanzkraft der Länder bei. Daher werden sie ebenfalls berücksichtigt. Da aber eine detaillierte Implementierung dieser Steuern, beispielsweise der Vermögens- und Erbschaftssteuern, zum Teil nur in einem dynamischen Modell möglich ist, werden sie hier nur rudimentär als exogene Lump-Sum-Steuern $T_{s}^{V}$ definiert.

\subsubsection{Endnachfrage der Regionen}

\section{(1) Abweichungen vom bisherigen Grundmodell}

Analog zu Whalley/Trela [1986] und Jones/Whalley [1989] soll das Entscheidungsproblem der Regionen als Entscheidungsbaum ${ }^{25}$ modelliert werden (siehe Abbildung IV.2), wobei das Kalkül des privaten repräsentativen Haushaltes um eine weitere Stufe ergänzt wird, in der der Haushalt bzw. die regionale Regierung den regionalen Nutzen durch die Wahl des öffentlichen Gutes und eines aggregierten privaten Konsumgutes maximiert.

Diese Modellierung weicht von dem bisher verwendeten Grundmodell in einem wesentlichen Punkt ab:

Um die Annahme der schwachen Separabilität zwischen dem regionalen öffentlichen Gut $G_{s}$ und den privaten Gütern verwenden zu können, darf die Rückwirkung des privaten Konsums auf den niedrigeren Ebenen des Entscheidungsbaumes auf die Steuereinnahmen

\footnotetext{
${ }^{24}$ Siehe Burtless/Hausman [1978], Hausman [1985] oder auch Stöss [1991], der eine Analyse der Wirkungen der Einkommenssteuern in einem EAG-Modell für die Bundesrepublik mit unterschiedlichen Haushaltsgruppen durchführte.

${ }^{25}$ Bezüglich der Voraussetzungen zur Verwendung dieser Entscheidungsstruktur insbesondere die Darstellung des Hickschen composite commodity Theorems, siehe Deaton/MUellBaUER ([1988], S.120-125) oder auch Varian [1992]. Eine der Voraussetzungen ist schwache Separabilität (Deaton/MuellbauER [1988], S.122).
} 
[1]

$[2]$

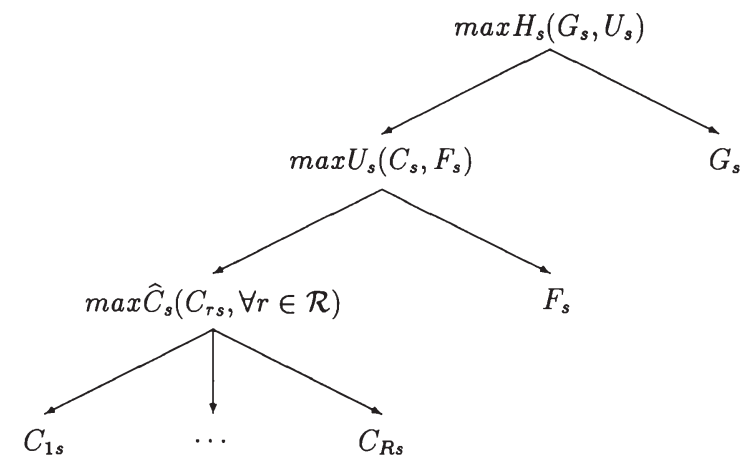

Abbildung IV.2: Nutzenbaum

der Region auf der obersten Ebene nicht in der Entscheidung berücksichtigt werden. Es wird daher angenommen, daß der regionale repräsentative Haushalt in seiner Entscheidung über den öffentlichen Konsum und die Transferhöhe die Auswirkungen seiner nachfolgenden privaten Konsumentscheidungen auf die Steueraufkommen der Region nicht berücksichtigt. Damit verändern sich die entsprechenden Ergebnisse des theoretischen Teils, ${ }^{26}$ Im Prinzip bedeutet das, daß die privaten Güter im Vergleich zur vollständigen Berücksichtigung der Rückwirkungen relativ teurer werden $(\alpha / \lambda=1$ statt $\alpha / \lambda<1)$, und damit eine Verzerrung gegenüber dem Grundmodell zugunsten des öffentlichen Konsums stattfindet, der nun relativ billiger wird. Interpretiert man dies als flypaper-effect, so ist diese Modellierung auch inhaltlich begründbar.

Da $\alpha=\lambda$ folgt aus dieser Form der Implementierung, daß die Trennung in eine öffentliche und private Entscheidung aufgehoben werden kann. Demzufolge wird auf der obersten Stufe [1] des Entscheidungsbaumes (siehe Abbildung IV.2) eine Entscheidung über den öffentlichen Konsum und den Konsum des aggregierten privaten Gutes (composite commodity) gefällt. Implizit wird damit auch die optimale Transferhöhe bestimmt.

Auf der zweiten Stufe [2] der Entscheidung wird die Konsummenge eines aggregiertes privates Konsumgutes und der Freizeit bestimmt. Voraussetzung für die Zulässigkeit dieses Vorgehens ist eine schwache Separabilität zwischen Freizeit und den einzelnen privaten Konsumgütern, die auch angenommen wird. ${ }^{27}$ In der dritten Stufe [3] des Entscheidungsprozesses wird schließlich der Nutzen über die nach Herkunft differenzierten

\footnotetext{
${ }^{26}$ Es fallen jeweils die Beziehungen $\partial Z(\cdot) / \partial T r$ oder $\delta_{T r}$ weg.

${ }^{27}$ Die Gültigkeit dieser Annahme ist allerdings umstritten. So stellt z.B. KAISER [1993] in einer empirischen Untersuchung für die Bundesrepublik einen derartigen Zusammenhang nur für einige Güter fest.
} 
privaten Konsumgütern maximiert. Im Gegensatz zu dem sonst üblichen Vorgehen wird nicht zuerst $z$ wischen einem heimischen und einem zusammengesetzten importierten Gut unterschieden, da davon ausgegangen wird, daß innerhalb der Bundesrepublik aufgrund der hohen Aggregationsstufe kaum eine besonders ausgeprägte Präferenz für Produkte des heimischen Bundeslandes besteht. Grundlage für die Entscheidungen sind jeweils die Konsumentenpreise bzw. Preisindizes. Die jeweiligen Budgets oder Teilbudgets sind Restriktionen der Maximierungsansätze.

\section{(2) Bestimmung der Konsumentenpreise}

Ausgehend von den Konsumentenpreisen für die privaten Güter, die vom Produzentenpreis aufgrund des Konsumsteuersatzes abweichen, werden nun die Preisindizes für das aggregierte private Konsumgut und anschließend für das aggregierte private Gut gebildet, die notwendig sind, um eine preisabhängige Entscheidung auf der zweiten und ersten Stufe des Nutzenbaumes fällen zu können. ${ }^{28}$

In der Stufe [1] wird eine CES-Nutzenfunktion ${ }^{29}$ verwendet, die zu folgendem Preisindex für das zusammengesetzte private Konsumgut führt:

$$
p_{C s}=\left[\sum_{r} \beta_{\tau s} p_{r}^{1-\mu_{3 s}}\right]^{1 /\left(1-\mu_{3 s}\right)}
$$

Der Preisindex für das private Gesamtkonsumgut auf der Stufe [1] lautet, da wieder eine CES-Funktion in Ebene [2] verwendet wird:

$$
p_{U}=\left[\left(1-\beta_{1 s}\right) p_{C s}^{1-\mu_{2 s}}+\beta_{s} w_{s}^{n^{1-\mu_{2 s}}}\right]^{1 /\left(1-\mu_{2 s}\right)} .
$$

$w_{s}^{n}=w_{s}\left(1-\tau^{Y}\right)$ entspricht dem Preis der Freizeit, der gleich dem Nettolohnsatz für eine Arbeitseinheit ist, da der Konsum einer Einheit von Freizeit gleichbedeutend mit einem Verzicht auf den Nettolohn einer Arbeitseinheit ist. Schließlich fehlt nur noch der Preis des öffentlichen Gutes. Da dieses keiner Konsumsteuer unterliegt sind Konsumenten- und Produzentenpreis $q_{G}$, identisch.

Nach Kenntnis der Preise können nun die einzelnen Entscheidungen getroffen werden. Dabei wird von Stufe [1] ausgegangen, die zugleich das Budget für das Optimierungskalkül auf der zweiten Stufe [2] bestimmt. Das nach den beiden Entscheidungen für den privaten Konsum verbleibende Budget stellt schließlich die Restriktion für die Nutzenmaximierung auf der untersten Ebene [3] dar.

\footnotetext{
${ }^{28}$ Die Bedingungen für die Existenz von Preisindizes sind dieselben wie für die Existenz zusammengesetzter Konsumgüter (siehe Keller [1976], S.178).

${ }^{29} \mathrm{Im}$ Prinzip wäre es wünschenswert eine LES-Funktion zu verwenden, da diese u.a. zu Engelkurven führen, die nicht durch den Ursprung gehen. Allerdings müßten dazu Mindestkonsummengen auf regionaler Ebene bestimmt werden, was nicht unproblematisch ist.
} 


\section{(3) Entscheidung über öffentliches und aggregiertes privates Gut [1]}

Auf der ersten Stufe wählt der repräsentative Haushalt seine Konsummenge des regionalen öffentlichen und des zusammengesetzten privaten Gutes (private Güter und Freizeit) unter der Annahme der Repräsentation der Präferenzen in Form einer CES-Nutzenfunktion und der Restriktion durch das volle Budget.

Das entsprechende regionale öffentliche Budget wird vollständig für die Bereitstellung der regionalen öffentlichen Güter $G_{s}$ und für Transfers an die privaten Haushalte der Regionen, $T r_{s}$, ausgegeben. Die Einnahmen ihrerseits setzen sich aus den Einnahmen aus Steuern nach der Steuerverteilung $T_{s}$, den staatlichen Finanzzuweisungen in Form von Ergänzungszuweisungen $E Z_{s}$ und/oder allgemeinen Zuweisungen ohne Eigenbeteiligung $Z_{s}$ und den Finanzausgleichszuweisungen oder -beiträgen $F A_{s}$ zusammen. Die entsprechende regionalstaatliche Budgetbeschränkung lautet:

$$
q_{G s} G_{s}+T r_{s}=T_{s}(\cdot)+F A_{s}(\cdot)+E Z_{s}(\cdot)+Z_{s}=Y_{s}^{R E G},
$$

Das private Budget des repräsentativen Haushaltes besteht aus dem vollen Einkommen $\hat{Y}_{s}^{p r}$ bei Verwendung der Zeitgrundausstattung für Arbeit, $w_{s} \bar{E}_{s}$, den Kapitaleinkünften, $r_{s} \bar{K}_{s}$, und den von der nationalen und regionalen Regierung erhaltenen Transfers, $T R_{s}$ bzw. $T r_{s}$. Davon werden die Einkommenssteuern $S T_{s}^{E k}$ und die Ländersteuern $S T_{s}^{V}$ abgezogen.

$$
\hat{Y}_{s}^{p r}=w_{s} E_{s}+r \bar{K}_{s}-S T_{s}^{E k}+T R_{s}+T r_{s}-S T_{s}^{V} .
$$

Unter Einsetzen der Einkommenssteuerschuld (Gleichung (IV.9)) erhält man schließlich das private verfügbare volle Einkommen

$$
\left(\text { IV.13) } Y_{s}^{p r}=\hat{Y}_{s}^{p r}-\tau^{Y} F_{s}=\left(1-\tau^{Y}\right)\left(w_{s} E_{s}+r \bar{K}_{s}\right)+\tau^{Y} A_{s}+T R_{s}+T r_{s}-S T_{s}^{V}\right. \text {, }
$$

das voll für den Konsum des aggregierten Gutes $U_{s}$ ausgegeben wird. Faßt man schließlich das öffentliche und das private Budget zusammen erhält man die regionale Budgetbeschränkung

$$
\begin{aligned}
q_{G} G_{s}+p_{U_{s}} U_{s}=\left(1-\tau^{Y}\right) & {\left[w_{s} E_{s}+r \bar{K}_{s}\right]+} \\
& +\tau^{Y} A_{s}+T R_{s}+T_{s}+F A_{s}+E Z_{s}+Z_{s}-S T_{s}^{V}=Y_{s}
\end{aligned}
$$

mit $Y_{s}$ als regionalem vollen Einkommen.

Maximiert man nun die regionale CES-Nutzenfunktion

$$
H_{s}\left(G_{s}, U_{s}\right)=\left[\left(1-\beta_{1 s}\right)^{\frac{1}{\mu_{1 s}}} U_{s}^{\frac{\mu_{1 s}-1}{\mu_{1 s}}}+\beta_{1 s}^{\frac{1}{\mu_{1 s}}} G_{s}^{\frac{\mu_{1 s}-1}{\mu_{1 s}}}\right]^{\frac{\mu_{1 s}}{\mu_{1 s}-1}}
$$


unter Beachtung der Budgetbedingung (IV.14) so gelangt man über die im Prinzip bereits bekannten Marginalbedingungen

$$
\frac{\partial H_{s} / \partial G_{s}}{\partial H_{s} / \partial U_{s}}=\frac{\left(q_{G_{s}}-\frac{\partial T_{s}}{\partial G_{s}}-\frac{\partial F A_{s}}{\partial G_{s}}-\frac{\partial E Z_{s}}{\partial G_{s}}\right)}{p_{U_{s}}}=\frac{q_{G_{s}}\left(1-\delta_{q G_{s}}\right)}{p_{U_{s}}}
$$

nach Definition des sozialen Preises von $G_{s}$

$$
\hat{q}_{G},=q_{G_{9}}\left(1-\delta_{q G_{s}}\right)
$$

zu folgenden Nachfragefunktionen nach dem regionalen öffentlichen Gut und der gesamten regionalen Privatnachfrage:

$$
\begin{aligned}
G_{s} & =\frac{\beta_{1 s} \hat{Y}_{s}}{\hat{q}_{G s}^{\mu_{1 s}} \Omega_{1 s}} \\
U_{s} & =\frac{\left(1-\beta_{1 s}\right) \hat{Y}_{s}}{p_{U s}^{\mu_{s} s} \Omega_{1 s}} \\
\operatorname{mit} \Omega_{1 s}=\left(1-\beta_{1 s}\right) p_{U_{s}}^{1-\mu_{1 s}} & +\beta_{1 s} \hat{q}_{G s}^{1-\mu_{1}} \text { und } \hat{Y}_{s}=Y_{s}-\widehat{q}_{G s} G_{s} .
\end{aligned}
$$

\section{(4) Bestimmung der sozialen Grenzkosten des öffentlichen Gutes}

Eine kleine Veränderung von $G_{s}$ kann dazu führen, daß sich die regionalen Steueraufkommen, die Berechnung und Höhe der FA-Zuweisungen und damit der soziale Preis von $G_{s}$ verändern. Die Region gerät dann auf ein anderes Segment der Budgetgerade mit einer entsprechend modifizierten Steigung. Daher müssen in jedem Programmdurchlauf die sozialen Grenzkosten neu bestimmt werden.

Anstelle der Verwendung von Schattenpreisen, die recht aufwendig zu berechnen wären, wird zu diesem Zweck das folgende Vorgehen verwendet: ${ }^{30}$

Im ersten Entscheidungsschritt wird die Steigung des Budgetsegments auf dem sich die Region bei gegebenen öffentlichen Gütermengen befindet (z.B. die Strecke $\overline{B D}$ in der Abbildung IV.3) als entscheidungsrelevantes Preisniveau angesehen.

Dieses Preisniveau dient dann als Steigung einer Budgetgerade, die gerade die Verlängerung des zugrundegelegten Budgetsegments ist und als Restriktion für das regionale Optimierungskalküls dient. Liegt der von der Region gewünschte Konsumpunkt auf dem realen Teil der Budgetgerade (z.B. Punkt $A^{*}$ ) wird angenommen, daß die sozialen Grenzkosten unverändert bleiben. Liegt sie dagegen auf dem fiktiven Teil (z.B. Punkt $A^{\prime}$ ), wird eine erneute Entscheidung durchgeführt. Als neuer relativer Preis wird nun die Steigung

\footnotetext{
${ }^{30}$ Dieses Verfahren ist bei der Berechnung des Arbeitsangebotes bei Einkommensteuersystemen, die zu geknickten Budgetgeraden führen üblich (siehe HAUSMAN [1985]).
} 


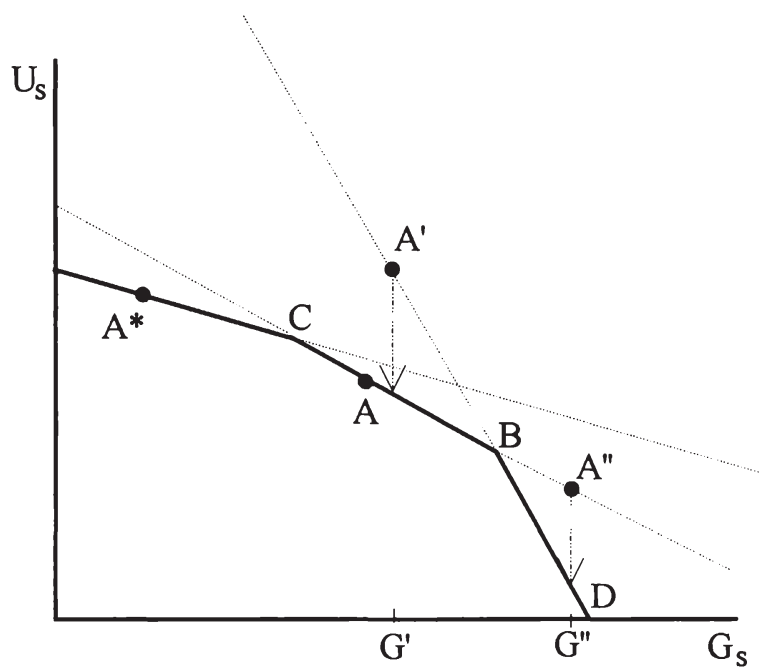

Abbildung IV.3: Bestimmung der sozialen Grenzkosten des öffentlichen Gutes

der Budgetgeraden $(\overline{B C})$ gewählt, die bei dem für die fiktive Gerade gewählten öffentlichen Konsummenge tatsächlich realisierbar ist. Diese neue Budgetgerade wird ebenfalls verlängert und dient als Grundlage für die erneute Durchführung der Optimierungsaufgabe. Dieses Verfahren wird solange angewendet, bis die Entscheidung auf dem realen Teil eines Budgetsegmentes liegt (z.B. $A^{*}$ ). Daraus können direkt die wahren sozialen Grenzkosten des öffentlichen Gutes bestimmt werden.

Tritt allerdings der Fall ein, daß kein solcher Punkt gefunden wird, da die optimale Wahl immer auf den virtuellen Teilen der Budgetgeraden liegen (z.B. $A^{\prime \prime}$ und $A^{\prime}$ ), dann liegt die Entscheidung auf dem Knickpunkt $(B)$ zwischen den beiden Budgetsegmenten $(\overline{B C})$ und $(\overline{B D})$ zwischen denen die Wahl von $G_{s}$ hin und herspringt. Dann braucht man die die sozialen Grenzkosten nicht zu berechnen, da durch den Knickpunkt direkt die Menge bestimmt wird. ${ }^{31}$

\section{(5) Entscheidung über Freizeit und das Gesamtkonsumgut [2]}

Auf der nächsten Stufe fällt die Konsumentscheidung über Freizeit $F_{s}$ und das zusammengesetzte private Gut $C_{s}$. Das Budget verringert sich nun um die Ausgaben für das regionale öffentliche Gut $q_{G} G_{s}$. Dies wird dadurch modelliert, daß die regionale Regie-

\footnotetext{
${ }^{31}$ Der virtuelle Preis würde zwischen den Preisen der beiden sich im Knickpunkt treffenden Budgetgeraden liegen
} 
rung den Rest ihres Budgets in Form von Transfers an den privaten Haushalt überweist:

$$
T r_{s}=Y_{s}^{R E G}-q_{G_{s}} G_{s} .
$$

Das sich ergebende private Budget wird vollständig für Freizeit $F_{s}$ und das composite commodity $C_{s}$ ausgegeben: ${ }^{32}$

(IV.19) $p_{s} C_{s}+w_{s}^{n} F_{s}=\left(1-\tau^{Y}\right)\left(w_{s} E_{s}+r \bar{K}_{s}\right)+\tau^{Y} A_{s}+T R_{s}+T r_{s}-S T_{s}^{V}=Y_{s}^{U}$.

Die Maximierung der entsprechende Nutzenfunktion vom CES-Typ

$$
U_{s}\left(C_{s}, F_{s}\right)=\left[\left(1-\beta_{2 s}\right)^{\frac{1}{\mu_{2 s}}} C_{s}^{\frac{\mu_{2 s}-1}{\mu_{2 s}}}+\beta_{2 s}^{\frac{1}{\mu_{2 s}}} F_{s}^{\frac{\mu_{2 s}-1}{\mu_{2 s}}}\right]^{\frac{\mu_{2 s}}{\mu_{2 s}-1}}
$$

unter der Einhaltung der Budgetrestriktion (IV.19) führt schließlich zu den Marshallschen Nachfragefunktionen nach Freizeit $F_{s}$ und dem privaten Gut $C_{s}$ :

$$
\begin{gathered}
F_{s}=\frac{\beta_{2 s} Y_{s}^{U}}{w_{s}^{n \mu_{2 s}} \Omega_{2 s}} \\
C_{s}=\frac{\left(1-\beta_{2 s}\right) Y_{s}^{U}}{p_{s}^{\mu_{2 s}} \Omega_{2 s}}, \\
\text { mit } \quad \Omega_{2 s}=\left(1-\beta_{2 s}\right) p_{s}^{1-\mu_{2 s}}+\beta_{2 s} w_{s}^{n^{1-\mu_{2 s}}} .
\end{gathered}
$$

\section{(6) Entscheidung über die einzelnen privaten Konsumgüter [3]}

Das nach der Wahl von $F_{s}$ und $C_{s}$ verbleibende verfügbare Geldeinkommen $Y_{s}^{C}$ (das verfügbare Faktoreinkommen zuzüglich der Transfers der nationalen Regierung $T R_{s}$ und der regionalen Regierung $T r_{s}$ an den Haushalt) verwenden die regionalen Haushalte für die einzelnen Konsumgüter. Die Budgetgleichung

$$
\sum_{r} p_{r} C_{r s}=\left(1-\tau^{Y}\right)\left(w_{s} L_{s}+r \bar{K}_{s}\right)+\tau^{Y} A_{s}+T R_{s}+T r_{s}-S T_{s}^{V}=Y_{s}^{C}
$$

geht als Restriktion in das Optimierungskalkül des privaten Haushalts ein. Die CESNutzenfunktion über die einzelnen privaten Konsumgüter hat die Form

$$
C_{s}\left(C_{r s} \mid r \in \mathcal{R}\right)=\left[\sum_{r} \beta_{r s}^{\frac{1}{\mu_{3 s}}} C_{r s}^{\frac{\mu_{3 s}-1}{\mu_{3 s}}}\right]^{\frac{\mu_{3 s}}{\mu_{3 s}-1}}
$$

mit $\mu_{3 s}$ als Substitutionselastizität und $\beta_{r s}$ als Budgetanteile der Güter.

Aus der Optimierung von (IV.24) unter der Budgetrestriktion (IV.23) resultieren die Nachfragefunktionen nach den einzelnen Produkten:

$$
C_{r s}=\frac{\beta_{r s} Y_{s}^{C}}{p_{r}^{\mu_{3 s}} \Omega_{3 s}} \quad \text { mit } \quad \Omega_{3 s}=\sum_{\tau} \beta_{r s} p_{r}^{1-\mu_{3} s} .
$$

\footnotetext{
${ }^{32}$ Freizeit wird nun mit dem Nettolohnsatz bewertet, da das auf der obersten Stufe um $\tau^{Y} w_{s} F_{s}$ verringerte Einkommen (Gleichung (IV.13)) die Kosten der Freizeit um diesen Betrag verringert.
} 


\subsection{Steuereinnahmen und öffentliche Haushalte}

Mit den so bestimmten Güternachfragen können nun die Faktornachfragen berechnet (Gleichung (IV.36)), daran anschließend die Steueraufkommen ermittelt und schließlich die Einnahmenaufteilung auf die einzelnen öffentlichen Haushalte durchgeführt werden. Um eine einheitliche Darstellung der Gleichgewichtsbedingungen zu erhalten, wird allerdings die Bestimmung der Faktornachfragen erst später dargestellt.

Die Steueraufkommen werden den Regionen zugerechnet, in deren Hoheitsgebiet die Steuern abgeführt werden. Demnach bestehen die Steueraufkommen einer Region aus:

$$
\begin{gathered}
S T_{s}=S T_{s}^{E k}+S T_{s}^{K}+S T_{s}^{V}+S T_{s}^{G w}+S T_{s}^{G r}+S T_{s}^{C}+S T_{s}^{Q} \\
\text { mit } \\
S T_{s}^{K}=\sum_{j} \tau_{j s}^{K} r K_{j}^{s} \\
S T_{s}^{G w}=\sum_{j} \tau_{j s}^{G w} r K_{j}^{s} \\
S T_{s}^{G r}=\sum_{j} \tau_{j s}^{G r} r K_{j}^{s} \\
S T_{s}^{C}=\sum_{r} \tau^{C} q_{s} C_{s \tau} \\
S T_{s}^{Q}=\sum_{j} \frac{\tau_{j s}^{Q}}{1+\tau_{j s}^{Q}} q_{j}^{s} Q_{j}^{s} .
\end{gathered}
$$

Über die im Abschnitt 3. dargestellten Finanzausgleichsregelungen werden diese Steueraufkommen dann auf die einzelnen Regionen und den nationalen Staat aufgeteilt.

Die in den Finanzausgleichsregelungen enthaltene Körperschafts- und Lohnsteuerzerlegung muß allerdings nicht durchgeführt werden. Da die Unternehmen im vorliegenden Modell nicht in Teilbetriebe gegliedert werden, entspricht die Modellierung der Besteuerung der Zinseinkünfte als Faktorsteuer auf Kapital, deren Aufkommen dem Land zufließt in dem das Unternehmen seinen Sitz hat, genau der Verteilung der Aufkommen nach dem Betriebsstättenprinzip. Diese Art der Modellierung ermöglicht es, die Einkommenssteuerverteilung im Rahmen des Finanzausgleichs vereinfacht darzustellen und auf eine explizite Körperschaftssteuerzerlegung zu verzichten. Diese wäre dann notwendig, wenn die Steuer ganz oder teilweise bei den Kapitalbesitzern erhoben würde, und damit nach dem Wohnsitzland abzuführen wäre.

Die Nichtberücksichtigung von Pendlern und die Erhebung der Lohnsteuer bei den Lohnbesitzern nach dem Wohnsitzland entspricht genau der Zurechnung der Lohnsteuer nach dem Wohnortprinzip und nimmt damit die Zerlegung der Lohnsteuer vorweg. 
Damit ergibt $\operatorname{sich}^{33}$

$$
\sum_{r} E Z_{r}(\cdot)+\sum_{r} Z_{r}+\sum_{r} T R_{s}=T_{G}^{C}(\cdot)+T_{G}^{Y}(\cdot)+T_{G}^{Q}+T_{G}^{U m}(\cdot)=T_{G}(\cdot)
$$

als Budget der nationalen Regierung. Die Steuereinnahmen $T_{G}(\cdot)$ werden von der nationalen Regierung für Zuweisungen $Z_{s}$ und Ergänzungszuweisungen $E Z_{s}$ an die regionalen öffentlichen Haushalte sowie Transfers $T R_{s}$ an die privaten Haushalte verwendet wird. Damit sind zugleich die regionalen Steuereinnahmen

$$
T_{s}(\cdot)=T_{s}^{C}(\cdot)+T_{s}^{Y}(\cdot)+T_{s}^{Q}+T_{s}^{U m}(\cdot)+T_{G m, s}^{G w}(\cdot)+T_{s}^{G r}(\cdot)
$$

bestimmt, die in das regionale öffentliche Budget $Y_{s}^{R E G}$ (Gleichung (IV.12)) eingehen.

\subsection{Regionaler Außenhandel}

Die interregionalen und internationalen Beziehungen der Regionen bestehen neben dem Austausch von Gütern und Vorleistungen, aus den interregionalen Transfers und Kapitalströmen in Form vom Kapitaleinkommen.

Zur Vereinfachung werden hier die zwei Arten von interregionalen und internationalen Beziehungen in eine regionale Handels- und Dienstleistungsbilanz $(H B)$, in der der Güterhandel und die Zinseinkünfte erfaßt sind, sowie eine regionale Übertragungsbilanz $(U B)$, in der die interregionalen Transfers enthalten sind, aufgespalten.

Die regionale Handels und Dienstleistungsbilanz ist:

$$
\begin{aligned}
& H B_{s}=\left(\sum_{r \neq s} p_{s} C_{s r}+\sum_{r \neq s} \sum_{j} q_{s} V_{C j}^{s r}(\mathbf{q})+X_{s}-\sum_{r \neq s} p_{r} C_{r}^{s}-\sum_{r \neq s} \sum_{j} q_{r} V_{C j}^{r s}(\mathbf{q})-M_{s}\right)+ \\
& +\left(r \bar{K}_{s}-r \sum_{j} K_{j s}\right),
\end{aligned}
$$

wobei die Nettozinszahlungen als Differenz zwischen den Kapitaleinkommen der Inländer und den von den inländischen Sektoren bezahlten Zinszahlungen berechnet werden.

Die Nettotransfers der jeweiligen Region mit dem nationalen Staat berechnen sich aus den erhaltenen Zuweisungen, Ergänzungszuweisungen und Transfers, den über die eigenen Steueraufkommen hinausgehenden Steuereinnahmen und den LFA-Zuweisungen und ergeben die regionale Übertragungsbilanz

$$
\begin{aligned}
U B_{s}=F A_{s}(\cdot)+Z_{s} & +E Z_{s}(\cdot)+T R_{s}+\left(T_{s}^{C}(\cdot)-S T_{s}^{C}\right)+ \\
& +\left(T_{s}^{Y}(\cdot)-S T_{s}^{E k}-S T_{s}^{K}\right)+\left(T_{s}^{Q}-S T_{s}^{Q}\right)+\left(T_{s}^{U m}(\cdot)-S T_{s}^{U m}\right) .
\end{aligned}
$$

\footnotetext{
${ }^{33}$ Soweit die Größen als Funktionen charakterisiert sind, bestimmen sie sich über das Finanzausgleichssystem.
} 
Die Summe der beiden Teilbilanzen muß im Gleichgewicht genau Null ergeben:

$$
H B_{s}+U B_{s}=0 \quad \forall s \in \mathcal{R} .
$$

\subsection{Nationaler Außenhandel}

Die Außenhandelsbeziehungen werden analog zu Whalley/Young [1984] und HiRTE/WIEGARD [1988] nur sehr rudimentär modelliert. Sie werden nicht aus Verhaltensfunktionen abgeleitet und gehen nicht in das Nutzenkalkül der Haushalte ein. Zusätzlich sei angenommen, daß jede Region nur eine Gutart importiert, die jeweils ihrem eigenen privaten Gut entspricht.

Die ausländischen Importangebots- und Exportnachfragefunktionen seien folgendermaßen spezifiziert (siehe HIRTE/WIEGARD [1988], S.178-180):

$$
X_{s}:=\left(\frac{q_{s}}{e}\right)^{\theta} X_{s}^{0} \quad \infty<\theta \leq-1
$$

bzw.

$$
M_{s}:=\left(\frac{q_{s}}{e}\right)^{\eta} M_{s}^{0} \quad 0 \leq \eta<\infty, .
$$

Dabei seien $\theta$ die ausländische Exportnachfrageelastizität, $\eta$ die ausländische Importangebotelastizität, $e$ der Wechselkurs zwischen inländischen und ausländischen Preisen und $\mathrm{X}_{s}^{0}$ bzw. $\mathrm{M}_{s}^{0}$ die Export- bzw. Importmengen im Basisjahr.

Die nationale Handelsbilanz lautet dann:

$$
\sum_{\tau \in \mathcal{R}}\left(q_{\tau} X_{\tau}-q_{r} M_{\tau}\right)=0
$$

Der Wechselkurs $e$, der als reine Finanzgröße keine reale Auswirkungen hat, läßt sich durch Einsetzen von (IV.28) und (IV.29) in die Handelsbilanzgleichung (IV.30) eliminieren. Dies führt nach einigen Umformungen zu folgenden veränderten ausländischen Exportnachfrage- und Importangebotsfunktionen: ${ }^{34}$

und

$$
X_{s}=\left[\frac{q_{s}^{\theta+1} X_{s}^{0}}{q_{s}^{\eta+1} M_{s}^{0}}\right]^{\frac{\theta}{\eta-\theta}} q_{s}^{\theta} X_{s}^{0}
$$

$$
M_{s}=\left[\frac{q_{s}^{\theta+1} X_{s}^{0}}{q_{s}^{\eta+1} M_{s}^{0}}\right]^{\frac{\eta}{\eta-\theta}} q_{s}^{\eta} M_{s}^{0} .
$$

\footnotetext{
${ }^{34}$ Bei den gewählten Funktionen wird ein gegenseitiger Handel mit den gleichen Gütern angenommen, ohne daß eine Erklärung für dieses cross-hauling geliefert wird.
} 


\subsection{Gleichgewichtsbedingungen}

Gleichgewichtsbedingungen des Modells sind die Markträumung aller Güter- und Faktormärkte, die Budgetrestriktionen der Regionen und die Budgetbeschränkung des Staates. Die Ausgeglichenheit der regionalen Leistungsbilanzen resultiert aus der Einhaltung der anderen Gleichgewichtsbedingungen und ist daher keine eigene Gleichgewichtsbedingung.

\section{Gütermärkte}

Für die privaten Konsumgüter erhält man

$$
Q_{C}^{s}=\sum_{r \in \mathcal{R}} C_{s r}+\sum_{r \in \mathcal{R}} \sum_{i=C}^{G} a_{C i}^{s r}(\mathbf{q}) Q_{i}^{s}+\left(X_{s}-M_{s}\right),
$$

und für das öffentliche Gut

$$
Q_{G}^{s}=\sum_{i=C}^{G} a_{G i}^{s s}(\mathbf{q}) Q_{i}^{s}+G_{s} .
$$

Unter Verwendung der Einheitsfaktornachfragen (IV.2) und (IV.3) sowie der Gleichgewichtsbedingungen für den Gütermarkt können letztere auf eine Abhängigkeit von den Faktorpreisen reduziert werden. Damit sind die Gütermärkte immer dann geräumt, wenn die Faktormärkte ausgeglichen sind. Folglich müssen die Gütermarktgleichgewichtsbedingungen nicht explizit berücksichtigt werden.

In Vektorschreibweise lauten die Gütermarktgleichgewichtsbedingungen

$$
\mathbf{Q}=(\mathbf{E}-\mathbf{A}(\mathbf{q}))^{-1}[\mathbf{C}+\mathbf{G}+\mathbf{E X}-\mathbf{I M}]
$$

mit $\mathbf{A}(\mathbf{q})$ als Inputkoeffizientenmatrix der von den Produzentenpreisen abhängigen Vorleistungen und $\mathbf{E}$ als Einheitsvektor. Die auf diese Weise bestimmten Produktionsmengen können nun durch Multiplikation mit den Faktoreinheitsnachfragen zur Bestimmung der Faktornachfragemengen eingesetzt werden, die damit ausschließlich von den Faktorpreisen abhängen: ${ }^{35}$

$$
K_{j}^{s}=k_{j}^{s} Q_{j}^{s} \quad \text { und } \quad L_{j}^{s}=l_{j}^{s} Q_{j}^{s}
$$

Damit ergeben sich die für die Bestimmung der Gleichgewichtspreise relevanten Überschußnachfragen:

\footnotetext{
${ }^{35} \mathrm{Zu}$ diesem Vorgehen siehe z.B. WIEGARD ([1985a], S.713).
} 
(1) Überschußnachfragen auf den Faktormärkten

$$
\begin{gathered}
z_{L}^{s}=\sum_{j=C}^{G} L_{j}^{s}-L_{s}=0 \quad \forall s \in \mathcal{R} . \\
z_{K}=\sum_{r \in \mathcal{R}} \sum_{j=C}^{G} K_{j}^{r}-\sum_{r \in \mathcal{R}} \bar{K}_{r}=0 .
\end{gathered}
$$

(2) Überschußnachfrage der nationalen Regierung

$$
z_{B}=T_{G}(\cdot)-\sum_{r \in \mathcal{R}} T R_{r}-\sum_{r \in \mathcal{R}} Z_{r}-\sum_{r \in \mathcal{R}} E Z_{r}=0
$$

(3) Überschußnachfragen der regionalen Regierungen

$$
z_{R}^{s}=T_{s}(\cdot)+Z_{s}+E Z_{s}(\cdot)+F A_{s}(\cdot)-q_{G_{s}} G_{s}-T r_{s}=0 \quad \forall s \in \mathcal{R} .
$$

Dieses reduzierte Gleichungssystem enthält nun $2 \cdot R+2$ Gleichungen bei $2 \cdot R+2$ Unbekannten, den regional unterschiedlichen Bruttolohnsätzen $w_{s}(R)$, dem einheitlichen Nettokapitallohnsatz $r$, sowie je nationaler und regionaler Regierung eine Variable zum Ausgleich des Budgets $(1+R)$.

Aufgrund der Gültigkeit des Walras-Gesetzes

$$
\sum_{s \in \mathcal{R}} w_{s} z_{L}^{s}+r z_{k}+z_{B}+\sum_{s \in \mathcal{R}} z_{R}^{s}=0
$$

ist das System nun allerdings bei der gleichen Anzahl von Gleichungen wie von Unbekannten überbestimmt, da eine Gleichung wegen des Walras-Gesetzes linear abhängig von allen anderen Gleichungen ist.

Werden die Budgetrestriktionen der öffentlichen Haushalte eingehalten und sind die Nachfrage- und Angebotsfunktionen nullhomogen in den Fakorpreisen und den Steuerparametern der Regierungen, so kann man jedoch die Preise normieren und damit die Zahl der Unbekannten reduzieren.

Nun kann man einen Preis als Numéraire festsetzen und mit Hilfe eines geeigneten Lösungsalgorithmus (z.B. Newton-Verfahren oder Fixpunktalgorithmen) den Gleichgewichtspreisvektor bestimmen. ${ }^{36}$

\footnotetext{
${ }^{36}$ Hier wurden alternativ die Prozeduren C05NF und E04FCF aus der NAG-Programmbibliothek verwendet.
} 


\section{Datensatz für das EAG-Modell}

Im Anschluß an dieses Modell wird nun die Erstellung des Datensatzes für die Bundesrepublik in den Grenzen nach 1990 erläutert.

Zur Berechnung der Inzidenzwirkungen des Finanzausgleichs in der alten BRD reicht ein Zwei-Länder-Modell, wie es in HiRTE/Genosko [1988/89] oder HiRTE [1990] vorgestellt wird, nicht aus, da die Regelungen des Finanzausgleichs teilweise derart gestaltet sind, daß sie erst bei einer Unterscheidung aller am Länderfinanzausgleich beteiligten Länder greifen. Mangels geeigneter Daten ist es derzeit jedoch nicht möglich, die neuen Bundesländer einzeln in das Modell zu integrieren. Aus diesem Grund wird die Bundesrepublik mit elf Ländern, den zehn Westländer und einem aggregierten Ostland, das alle Neuen Bundesländer und Berlin enthält, modelliert.

Für eine adäquate Untersuchung des Finanzausgleichs wird ein vollständiger interregionaler Datensatz benötigt, in dem auch alle interregionalen Verflechtungen, wie z.B. Vorleistungs- oder Konsumverflechtungen, enthalten sind. Zusätzlich muß dieser Datensatz vollständig mit dem zugrundeliegenden theoretischen Modell konsistent sein, d.h. er muß alle Gleichgewichtsbedingungen des Modells erfüllen.

Die Erstellung dieses 'optimalen' Datensatzes stößt allerdings auf erhebliche Schwierigkeiten, da ein großer Teil der benötigten Daten nicht in geeigneter Weise vorliegt, überhaupt nicht erhoben, nur für bestimmte Jahre bereitgestellt werden oder nach unterschiedlichen Konzepten ausgewiesen sind. Aus diesem Grund mußte der weitaus größte Teil der benötigten Werte durch zum Teil recht unbefriedigende Verfahren bestimmt werden. Deshalb soll der berechnete Datensatz nur mit größter Vorsicht verwendet werden und die damit erzielten Ergebnisse nur als Anhaltspunkte für tatsächliche Resultate dienen.

Im folgenden wird die recht aufwendige und problematische Erstellung des Datensatzes dargestellt. Dabei wird versucht, die Darstellung möglichst knapp zu halten, um allzu langwierige und langweilende technische Ausführungen zu vermeiden. Dennoch werden die Erläuterungen recht umfangreich sein, da eine derartige Vielzahl von Werten bestimmt werden muß, daß die folgenden Beschreibungen kaum noch kürzer gefaßt werden können, ohne deutlich an Verständlichkeit zu verlieren.

Ausgehend von den im ersten Schritt für die Westländer erstellten multiregionalen Verflechtungs-Tabellen wird in einem zweiten Schritt unter Verwendung eines Datensatzes für das aggregierte Ostland der Datenensatz für die gesamte Bundesrepublik gebildet. Schließlich erfolgt die Einbeziehung der Finanzbeziehungen zwischen den Ländern und eine Abgleichung mit den Gleichgewichtsbedingungen des theoretischen Modells, so daß ein modellkonsistenter Datensatz entsteht. 


\subsection{Struktur der benötigten Multiregionalen Tabelle}

Die benötigte multiregionale Tabelle der Güterverflechtungen und des Faktoreinsatzes muß folgende Elemente besitzen:

Zum ersten wird eine vollständige interregionale Vorleistungsverflechtung (1.Quadrant der Abbildung IV.1), sowie die Aufspaltung der Endnachfrage, die aus Konsumnachfrage, öffentlichem Verbrauch und Export abzüglich Import besteht, nach Regionen und Gütern (2.Quadrant) benötigt. Dies impliziert, daß Bezüge und Lieferungen zwischen den Ländern in einer vollständigen interregionalen Aufteilung ausgewiesen werden. Der dritte Quadrant erfaßt den Einsatz der Primärfaktoren der einzelnen Sektoren jeder Region. ${ }^{37}$

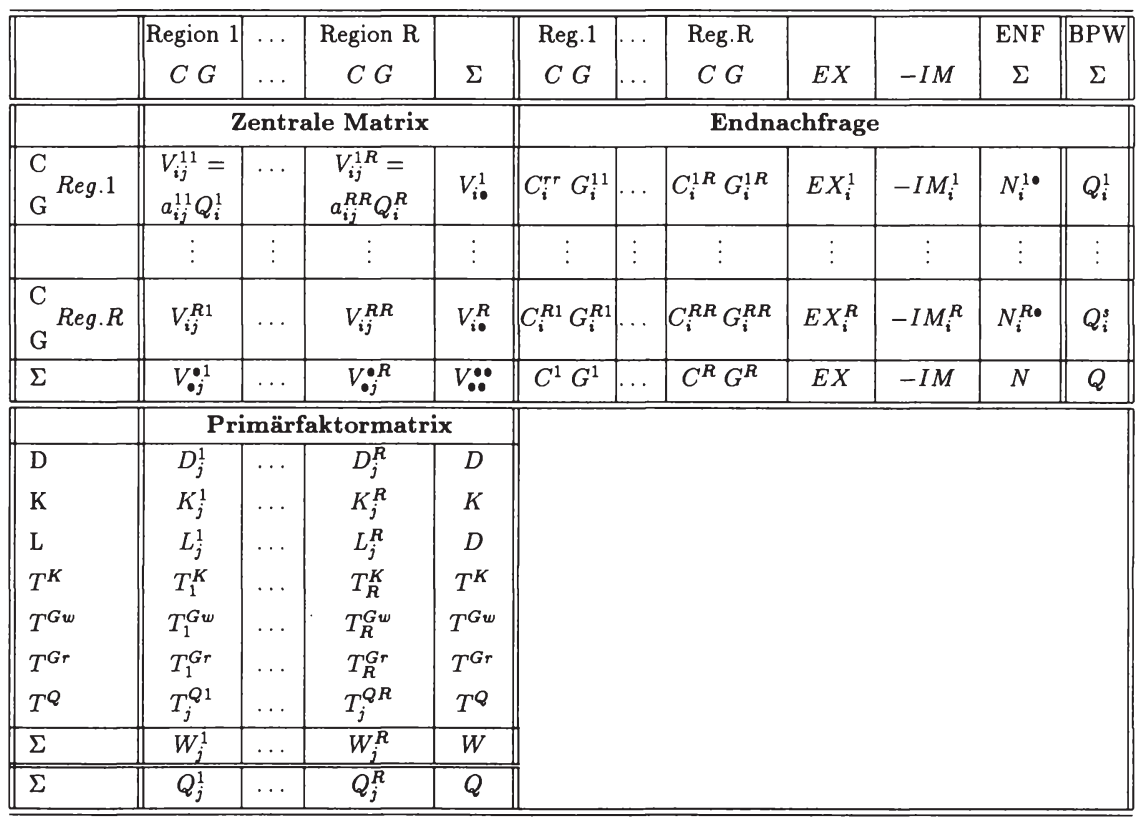

Tabelle IV.1: Interregionale Input-Outputtabelle der technolog. Verflechtung

Zum zweiten soll diese Tabelle nach dem funktionellen Prinzip erstellt sein. Dies ergibt sich aus dem theoretischen Modell, in dem Nachfragen nach einzelnen Gütern abgeleitet

\footnotetext{
${ }^{37}$ Die Struktur entspricht der einer Multiregionalen Input-Output-Tabelle, deren unterschiedliche Typen und Erstellungsweisen in Hewings und Jensen [1986] dargestellt sind. Siehe dazu auch NiJKamp/Rietveld/Snickars [1984, 1986] oder die Sammelbände von Adams/Glickman [1980] und ISSAEV, u.a. [1982].
} 
werden und nicht nach gemischten Gütern. Der Einfachheit halber werden als anbietende Sektoren dann ebenfalls nicht die Wirtschaftsbereiche modelliert - dies ergäbe eine Mischtabelle - sondern funktionell abgegrenzte Sektoren. ${ }^{38}$

Als Preiskonzept werden „Ab-Werk-Preise” gewählt, da bei diesem Konzept im Gegensatz zu dem Konzept der "Preise zu Anschaffungskosten” die Handelsleistungen nicht enthalten sind. Damit wird nur der Herstellungspreis des jeweiligen Gutes ausgewiesen, während die Handelsleistung als eigenes Gut dargestellt wird.

In diesem Zusammenhang sei darauf hingewiesen, daß gemäß der üblichen Konvention unterstellt wird, daß alle Faktor- und Güterpreise gleich eins sind und damit die Wertangaben der resultierenden Datenbasis zugleich Mengenangaben darstellen.

\subsection{Auswahl des Referenzjahres}

Eine weitere wichtige Entscheidung ist die des Referenzjahres des Datensatzes, bei der es zwischen Aktualität und Güte der Daten abzuwägen gilt. Je mehr originäre Werte verwendet werden können, um so genauer wird vermutlich der Datensatz werden. ${ }^{39}$ Zugleich soll der Datensatz möglichst aktuell sein, um Strukturbrüche zu umgehen. Betrachtet man die bisher für Länder der Bundesrepublik erstellten regionalen Input-Output-Tabellen (IOT), so zeigt sich, daß nur für einige Länder 'relativ' aktuelle Tabellen vorliegen, so für Hessen von $1980^{40}$, für Nordrhein-Westfalen von $1982^{41}$ und für Baden-Württemberg von 1980 , 1982, 1984 und 1986, wobei die baden-württembergische Tabelle für die Jahre 1980 und 1982 auch als interregionale Tabelle erstellt wurde. ${ }^{42}$ Es gibt eine ganze Reihe weiterer regionaler Input-Output-Tabellen, die allerdings sämtlich vor 1978 datiert sind und alle

\footnotetext{
${ }^{38}$ Es wäre mit Hilfe spezieller Tabellen durchaus möglich, auf der Inputseite eine funktionelle und auf der Outputseite eine institutionelle Gliederung zu wählen Dieses Vorgehen wählen BaLLaRd u.a. [1985] bei ihrem EAG-Modell der USA. In diesem Fall wäre es sogar möglich, eine unterschiedliche Zahl von Gütern und Wirtschaftsbereichen zu berücksichtigen. Das Problem der Zuordnung der Produkte auf die jeweiligen produzierenden Bereiche müßte mit Hilfe einer speziellen Zuordnungsmatrix gelöst werden, da jeder Bereich mehrere z.T. auch gleiche Güter produzieren wird.

${ }^{39}$ Allerdings zieht z.B. HüBLER [1979] dies in Frage, da „... Erhebungs- und Zufallsfehler bei der originären Datengewinnung auf Stichprobenbasis ... durchaus größer als die Schätzfehler bei der derivativen Methode sein..." können. Jedoch geht auch er letztlich davon aus, daß die originären Daten den derivativ ermittelten Werten vorzuziehen seien, „... da sich ... über Erhebungsfehler wenig sagen läßt... "(HÜBLER [1979], S.3).

${ }^{40}$ Siehe Gretz-Roth [1989] oder Gretz-Roth/Gretz [1986].

${ }^{41}$ StäGLin/Ludwig/SchintKe [1990].

${ }^{42}$ Die Tabellen für Baden-Württemberg sind in Münzenmaier [1986, 1988a, 1988b, 1989a und 1990] dargestellt. Die interregionalen Tabellen für Baden-Württemberg werden in Münzenmaier [1987a und 1989b] präsentiert.
} 
an institutionellen Prinzip orientiert sind.$^{43}$ Die einzige Tabelle mit multiregionaler Struktur hat CARLBerg [1979], für das Basisjahr 1970, für acht Regionen der Bundesrepublik erstellt und dabei auch interregionale Verflechtungen - allerdings nur aggregierte Bezüge und Lieferungen, nicht jedoch Vorleistungsverflechtungen - geschätzt.

Da die funktionellen Tabellen für Baden-Württemberg von 1980 und 1982 auch in interregionaler Form erstellt sind, zudem für Nordrhein-Westfalen eine institutionell gegliederte, regionale IOT für 1982 ausgewiesen ist ${ }^{44}$ und für Hessen eine IOT von 1980 vorliegt, empfiehlt es sich eines diese beiden Jahre als Basisjahr zu verwenden.

Darüber hinaus benötigt man zur Erstellung einer interregionalen Input-Output-Tabelle, die als Grundlage für den Datensatz dient, eine Outputtabelle. Für 1982 liegt eine solche für Baden-Württemberg und die Bundesrepublik vor (siehe MünzenmaIer [1987b]). Die Output-Tabellen der Bundesrepublik für die anderen Jahren sind dagegen nicht nach dem Input-Output-Prinzip sondern nach dem in der Volkswirtschaftlichen Gesamtrechnung (VGR) verwendeten Sozialproduktskonzept veröffentlicht und hier nicht verwendbar (STABA [1989a], Tabellen 2.2).

Schließlich gibt es eine Reihe von Übergangstabellen für Baden-Württemberg und die Bundesrepublik, in denen der Übergang der nach dem Konzept der VGR ausgewiesenen Sozialproduktswerte in Werte des institutionellen Input-Output-Konzeptes vorgenommen wird, nur für das Jahr 1982 (siehe z.B. Münzenmaier [1987b]).

Aus diesen Gründen wird das Jahr 1982 als Referenzjahr der Datenbasis gewählt.

\subsection{Grundsätzliche Vorgehensweise}

Bei der Erstellung des Datensatzes werden einige Annahmen getroffen und bestimmte Verfahren eingesetzt, die kurz dargestellt werden und im Prinzip aus den derivativen Verfahren entnommen sind ${ }^{45}$

\footnotetext{
${ }^{43}$ Einen Überblick über Input-Output-Tabellen bis 1974 findet man bei STÄGLIN ([1980] S.103-111 und [1982], S.15-21) oder bei MünZenmaier ([1984c], S.148-149). Für die Zeit bis 1982 bietet Rietveld [1982] eine Übersicht über multiregionale Modelle in der Bundesrepublik und anderen Ländern.

${ }^{44}$ Eine exakte Überführung der institutionellen in eine funktionell ausgerichtete Input-Output-Tabelle kann mit Hilfe eines Überleitungsmodells durchgeführt werden (siehe z.B. STAHMER $[1979,1984])$. Da dazu sowohl Output- (Make-) als auch Input-Tabellen (Use-Matrizen) des entsprechenden Landes benötigt werden, diese Tabellen jedoch nur für das jahr 1982 für Baden-Württemberg (MünZenmaIER [1987b]) ausgewiesen ist, kann eine funktionelle Tabelle nur näherungsweise abgeleitet werden.

${ }^{45}$ Die derivativen oder (Non-Survey)-Verfahren sind Methoden zur Ermittlung regionaler Input-OutputTabellen (IOT) aus vorgegebenen nationalen IOT oder IOT anderer Regionen mit Hilfe standardisierter Ansätze, wie z.B. die Annahme identischer Struktur oder das RAS-Verfahren. Eine ausführliche Darstel-
} 
- Grundsätzlich wird eine Ähnlichkeit der nationalen mit der regionalen technologischen Verflechtung unterstellt. ${ }^{46}$ Demnach werden die Koeffizienten der nationalen auf die regionale Input-Output-Tabelle übertragen, soweit sie nicht bereits regionalisiert vorliegen.

- Abweichungen von der vorgegebenen Gesamtsumme werden proportional auf die Länder verteilt. Es wird demnach angenommen, daß die Länder weitgehend die gleiche Strukur besitzen.

- Abweichungen der Zeilen- und Spaltensummen der geschätzten Felderwerte von den vorgegebenen Randsummen werden mit Hilfe des MODOP-Verfahrens, einer modifizierten RAS-Methode korrigiert. ${ }^{47}$

- Interregionale Daten werden mit Hilfe des Gravitiationsansatzes gewonnen. ${ }^{48}$

- Es wird unterstellt, daß in jeder Region die Zeilenkoeffizienten der Bezüge genau denen der Nachfrage nach heimischen Gütern entsprechen.

\subsection{Interregionale Tabelle für die Westländer}

\subsubsection{Randdaten der regionalen Input-Output-Tabelle}

Da die meisten Daten nur nach dem Sozialproduktskonzept der VGR vorliegen müssen sie in das Input-Output-Konzept übergeführt werden. Die Unterschiede zwischen beiden Konzepten, die z.B. von MüNZENMAIER ([1988b], S.125-127) zusammengefaßt sind, dokumentiert die Tabelle IV.2. ${ }^{49}$

lung der verschiedenen Ansätze bietet HüBLER [1979], während HEwings/JENSEN [1986] einen knappen Überblick geben.

${ }^{46}$ Die technologische Verflechtungsmatrix enthält in ihren Koeffizienten sämtliche in der Produktion oder in der Endnachfrage eingesetzten Güter, also auch die Bezüge und Importe einer Region.

${ }^{47}$ In der auf BACHARACH [1970] zurückgehenden RAS-Methode werden die Abweichungen durch eine abwechselnd nach Zeilen und Spalten durchgeführten proportionale Korrektur der Felderwerte korrigiert. Eine genaue Darstellung der RAS-Methode, die auch eine ausführliche Vorstellung des MODOPVerfahrens enthält, findet man bei HoLuB/SchNABL ([1985], S.96-101).

${ }^{48}$ Gravitationsmodelle gehören ebenfalls zu den derivativen Methoden. Im Prinzip werden in ihnen Güterströme zwischen Regionen aufgrund der Entfernung, der Transportkosten und der Größe der liefernden und beziehenden Region geschätzt. Eine Darstellung des Gravitationsansatzes incl. Kritik gibt GENOSKo [1992]. Bezogen auf die interregionalen Input-Output-Modelle diskutieren BATTEN/BoyCE [1986] dieses Verfahren.

${ }^{49}$ MAYER/REICH [1987] demonstrieren sehr detailliert diesen Übergang für die Bundesrepublik. 


\begin{tabular}{||l|c||}
\hline \hline Übergang von VGR auf I/O-Konzept ${ }^{a}$ \\
\hline \hline VGR-Daten \\
$+\quad$ firmeninterne Lieferungen und Leistungen \\
$-\quad$ Einstandswert der Handelsware \\
$-\quad$ Wiederkäuferumsätze mit Energie und Wasser \\
$-\quad$ veranlagte Frachtkosten des Transporthilfsgewerbes \\
$-\quad$ in Rechnung gestellte Frachtkosten des Versenders \\
$\pm \quad$ Übergang vom Inländer- zum Inlandskonzept \\
\hline$=\quad$ Daten nach dem institutionellen Input-Output-Konzept \\
\hline \hline
\end{tabular}

Tabelle IV.2: Schema für Übergang von VGR auf Input-Output-Konzept

${ }^{a}$ Quelle: GemSta Lä [1986]

\section{Bruttoproduktionswert}

Aus dem Bruttoproduktionswert (BPW) der VGR wird zuerst der BPW nach dem institutionellen und anschließend nach dem funktionellen Input-Output-Konzept abgeleitet. Die jeweilige Abweichungen des BPW der Sektoren nach dem institutionellen InputOutput-Konzept vom BPW nach dem Sozialproduktskonzept der VGR für die restliche Bundesrepublik (aus MAYER/REICH [1987]) nach Abzug der Größen von Baden-Württemberg und Hessen, die schon bekannt sind (siehe MüNZENMAIER, [1988b] oder GRETZRoth/Gretz [1986]), werden auf die einzelnen Sektoren der Länder gemäß ihrem relativen BPW-Anteil am BPW des gesamten Sektors der restlichen Bundesrepublik (RvD) verteilt. Damit wird unterstellt, daß die Abweichungen zwischen beiden Konzepten innerhalb eines Sektors für alle Länder, außer Baden-Württemberg und Hessen, gleich sind.

Für Baden-Württemberg sind die BPW Werte nach dem funktionellen Input-OutputPrinzip aus den schon berechneten IOT bekannt. Für Hessen werden sie durch Verwendung der Übergangskoeffizienten von 1980 für 1982 gewonnen. Der Übergang vom institutionellen zum funktionellen Input-Output-Konzept (I/O-Konzept) für die restlichen Länder erfolgt mit Hilfe der regionalen Output-(Make)-tabellen. Diese werden durch Verwendung der Koeffizienten der nationalen Make-Matrix (MüNZENMAIER [1987b]) gebildet, wobei soweit als möglich originäre Outputkoeffizienten der Länder abgeleitet und anstelle der nationalen Koeffizienten eingesetzt werden ${ }^{50}$. Setzt man die Werte des institutionellen Bruttoproduktonswertes als Spaltensummen in die Überleitungsmatrix ein, so führt die Multiplikation mit den Koeffizienten der inversen Outputtabelle diese in die funktionellen BPW-Werte über, die der Zeilensumme der Überleitungsmatrix entsprechen.

\footnotetext{
${ }^{50}$ Dies sind zumeist Werte für den Bausektor und das verarbeitende Gewerbe.
} 
Da jedoch die so ermittelten Werte in der Summe nicht mit den bundesdeutschen Werten übereinstimmen, werden sie mittels des MODOP-Verfahrens entsprechend korrigiert. Als Ergebnis erhält man die Tabelle (IV.3).

\begin{tabular}{||l|rrrrrrrrrrrr||}
\hline \hline \multicolumn{110}{|c||}{} & BPW nach funktionellem I/O-Konzept ${ }^{a}$ \\
\hline & S-H & HH & HB & NI & NRW & He & RP & Sa & BW & By & Be & BRD \\
\hline Sekt.1 & 6738 & 542 & 225 & 16127 & 13867 & 4408 & 5116 & 410 & 11514 & 18356 & 197 & 77500 \\
Sekt.2 & 4321 & 4748 & 1945 & 14864 & 60008 & 9006 & 5322 & 6164 & 12399 & 16788 & 2086 & 137651 \\
Sekt.3 & 39330 & 43124 & 18749 & 133088 & 390040 & 105197 & 83654 & 20961 & 229827 & 228709 & 43636 & 1336315 \\
Sekt.4 & 7385 & 6664 & 2191 & 21847 & 48476 & 16783 & 10505 & 3013 & 33421 & 35706 & 5717 & 191708 \\
Sekt.5 & 12635 & 42169 & 8722 & 35701 & 113808 & 42315 & 15774 & 5603 & 49447 & 54505 & 9303 & 389982 \\
Sekt.6 & 20288 & 36320 & 7600 & 56018 & 158651 & 71748 & 29615 & 8546 & 92114 & 114026 & 20138 & 615064 \\
Sekt.7 & 21401 & 13921 & 5432 & 53444 & 107920 & 37901 & 23468 & 6483 & 58198 & 69581 & 20091 & 417840 \\
$\Sigma$ & 112098 & 147488 & 44864 & 331089 & 892770 & 287358 & 173454 & 51180 & 486920 & 537671 & 101168 & 3166060 \\
\hline
\end{tabular}

Tabelle IV.3: BPW nach funktionellem I/O-Konzept

${ }^{a}$ Quellen: Münzenmaier (1988b) und eigene Berechnungen

\section{Wertschöpfung}

Die Daten der bereinigten Wertschöpfung werden unverändert aus der VGR übernommen (GemStaL ̈̈ [1986]). Eine nähere Erläuterung des Übergangs des VGR-zum InputOutput-Konzepts bedarf es bei der Bestimmung der Endnachfragekomponenten.

\section{Privater Konsum}

Die Angaben über die Bruttokonsumausgaben des VGR-Konzeptes (GEMSTAL $\ddot{A}$ [1986]) weichen vom dem nach dem Inlandskonzept ermittelten Daten der Input-Output-Rechnung genau um den Saldo der Reiseausgaben ab, wie die Tabelle IV.4 zeigt.

$\mathrm{Da}$ jedoch der Saldo der Reiseausgaben nicht von allen Bundesländern ausgewiesen oder soweit er intern ermittelt wird nicht von allen Bundesländer zur Veröffentlichung freigegeben wird, ist es notwendig ihn näherungsweise zu bestimmen.

Aus Angaben über die Käufe privater Haushalte von 1980 und dem Saldo der Reiseausgaben der Länder von 1980 (SchmidT [1986], S.186) wird der Anteil der Reiseausgaben an den Käufen für 1980 ermittelt. Dieser wird auf 1982 durch Multiplikation mit der Höhe der Käufe der privaten Haushalte 1982 hochgerechnet und damit eine erste Näherung für die Reiseausgaben 1982 der Länder ermittelt. Dieses Verfahren wird auch für die BRD durchgeführt. Nun zeigt sich, daß für die Gesamtwirtschaft der so ermittelte 


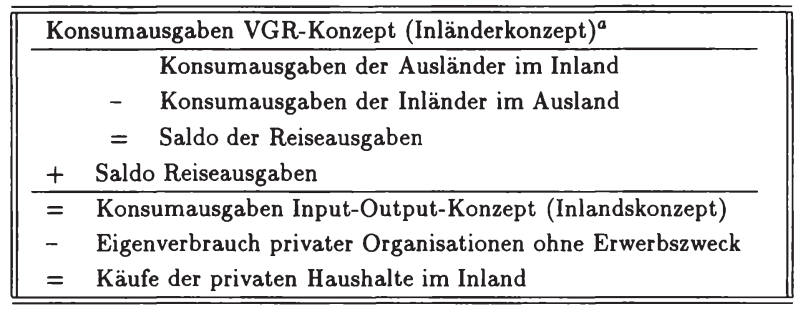

Tabelle IV.4: Schema des Übergangs von Konsum nach VGR auf IO-Konzept

${ }^{a}$ Quellen: Münzenmaier (1988b) und eigene Berechnungen

Wert von dem vorgegeben Wert abweicht. Aus diesem Grund wird ein Korrekturfaktor für den geschätzen Saldo für die Bundesrepublik ermittelt, der auf alle Länder übertragen wird (Annahme: gleiche Abweichung). Mit Hilfe dieses Übergangkoeffizienten werden die Salden nun neu geschätzt. Für die fünf Länder, deren Werte bekannt sind (Bayern, Berlin, BW, Bremen und Hessen ${ }^{51}$ ) werden die geschätzten durch die vorgegebenen Werte ersetzt. Anschließend wird durch proportionale Anpassung der nicht vorgegebenen Salden die Summe der Ländersalden an den Wert der BRD angepaßt.

\section{Überführung der vorgegebenen IOT in die gewünschte Form}

Nach Vorgabe der Bruttoproduktionswerte und der Wertschöpfung kann die Input-OutputTabelle von Hessen für 1980 relativ einfach mittels des MODOP-Verfahrens auf eine InputOutput-Tabelle für 1982 hochgerechnet werden. Dies ist zugleich einer der ersten Schritte zur Erstellung der Datenbasis.

Die institutionelle Input-Output-Tabelle (IOT) von Nordrhein-Westfalen für 1982 wird mit Hilfe der funktionellen Randdaten des BPW und der Wertschöpfung näherungsweise in eine funktionelle Tabelle übergeleitet. $\mathrm{Zu}$ diesem $\mathrm{Zweck}$ werden die institutionellen Feldwerte der IOT mit Hilfe des MODOP-Verfahrens an die funktionellen Randdaten angeglichen. Allerdings führt dies dazu, daß die Handelsleistungen nicht exakt umgebucht werden, was aber angesichts der später erfolgten Aggregation auf zwei Güter nicht allzu starke Verzerrungen hervorrufen dürfte.

\footnotetext{
${ }^{51}$ Für Hessen wurde angenommen, daß der Saldo der Reiseausgaben von 1982 genau den gleichen Anteil an den gesamten privaten Konsumausgaben nach dem VGR-Konzept hat wie 1980. Da die Werte für 1980 aus der Input-Output-Tabelle für Hessen (GRETZ-RoTH/GRETZ [1986]) bekannt waren, konnten daher die entsprechenden Werte für 1982 berechnet werden.
} 


\section{Öffentliche Endnachfrage}

Der öffentliche Verbrauch ist in der VGR gegeben und wird voll übernommen (GEMSTALÄ [1986]). Dabei wird unterstellt, daß der gesamte Staatsverbrauch nur für das öffentliche Gut ausgegeben wird.

\section{Export und Import}

Aus den Statistiken liegen für alle Länder Daten über die Exporte an Waren nach dem Spezialhandelskonzept und für Importe an Waren nach dem Generalhandelskonzept vor (STABA [1984b]). ${ }^{52}$ Eine Umrechnung auf die jeweiligen Werte nach dem Input-OutputKonzept erfordert die Kenntnis weiterer Daten. Dies sind Angaben über Export und Import von Dienstleistungen, über die Erwerbs- und Vermögenseinkommen der Inländer im Ausland und der Ausländer im Inland, über Waren- und Dienstleistungsströme aus und in die DDR, über die Reiseausgaben der Inländer im Ausland und der Ausländer im Inland, über die auf Importe erhobene Einfuhrumsatzsteuer und über Exporte und Importe auf und aus Lager.

Für Hessen (eigene Berechnungen), Baden-Württemberg (MünZENMAIER [1988b]) und Nordrhein-Westfalen (STÄGLIN/LUDWIG/SCHINTKE [1990]) liegen die endgültigen Exportund Import-Werte aus den Input-Output-Tabellen bereits vor.

Die Importe nach Generalhandel liegen für alle Länder vor (STABA [1984b]). Allerdings ist die Summe der Importe der Länder geringer als die Importe der Bundesrepublik, da Ersatzlieferungen und Rückwaren nicht erfaßt sind, und einige Werte nicht auf Länder zugeordnet werden konnten (vgl. STA BA [1984b]). Daher wird in einem ersten Schritt die Differenz zwischen den Importen der Bundesrepublik und der Summe der Einfuhren der Länder proportional gemäß den Anteilen der Importe der Länder auf diese verteilt.

Aus diesen Importen nach Generalhandelskonzept und zu cif-Preisen werden nun mit Hilfe der in der Tabellen IV.5 und IV.6 abgebildeten Überleitungen, die Importe nach dem Inlandskonzept berechnet.

In den einzelnen Übergängen werden allerdings, je nach Datenlage, nur Abschätzungen verwendet. An dieser Stelle wird nur auf zwei Aspekte näher eingegangen:

- Die Ermittlung der Importe von Dienstleistungen erfolgt durch Verwendung der bundesdeutschen Relation der Importe von Dienstleistungen zu den Importen von

\footnotetext{
${ }^{52}$ Generalhandels- und Spezialhandelskonzept unterscheiden sich im Falle der Importe aufgrund der Behandlung der Einfuhren auf Lager, die im ersteren Konzept vollständig und zwar im Zeitpunkt ihrer Einlagerung erfaßt werden. Im Spezialhandelskonzept werden dagegen Einfuhren auf Lager nur insoweit berücksichtigt, als sie nicht zur Wiederausfuhr bestimmt sind. Sie werden erst im Zeitpunkt ihrer Einfuhr aus dem Lager verbucht (vgl. z.B. STABA [1985a]).
} 


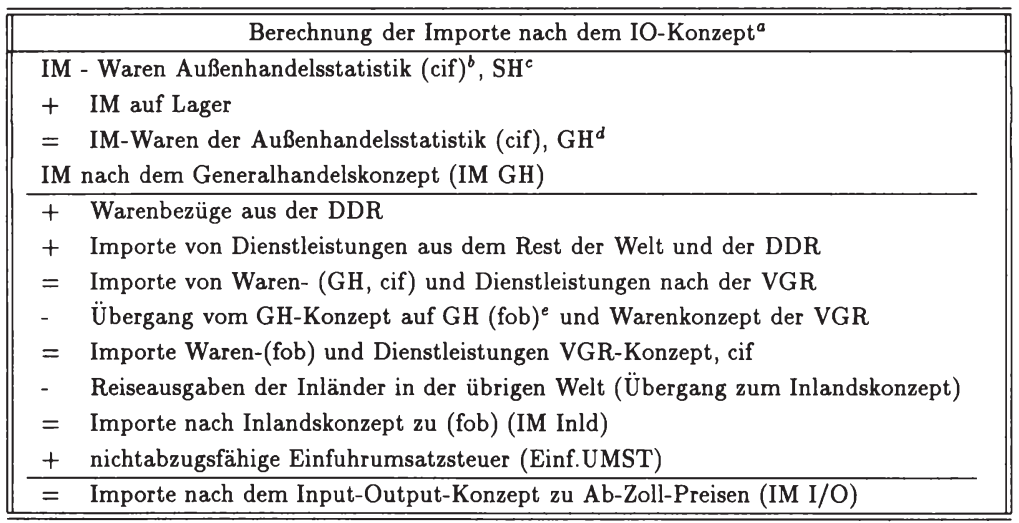

Tabelle IV.5: Berechnung der Importe nach dem IO-Konzept

${ }^{a}$ Vgl. STA BA [1992c], S.53.

${ }^{b}$ cif $=$ cost, insurance, freight. Die Preise der Importgüter enthalten Fracht- und Versicherungskosten ausländischer Transport- und Versicherungsunternehmer bis zur deutschen Grenze = Grenzübergangswert (vgl. z.B. STABA [1984], S.530.

${ }^{c}$ Spezialhandelskonzept.

${ }^{d}$ Generalhandelskonzept.

${ }^{e}$ free on board.

\begin{tabular}{||c||}
\hline \hline EX - Waren Außenhandelsstatistik (cif), SH \\
$-\quad$ EX aus Lager \\
$=\quad$ EX-Waren der Außenhandelsstatistik (cif), GH \\
EX nach dem Generalhandelskonzept (EX, cif, GH) \\
\hline$+\quad$ Warenlieferungen an die DDR \\
$+\quad$ Exporte von Dienstleistungen an den Rest der Welt und die DDR \\
$=\quad$ Exporte von Waren- (GH, cif) und Dienstleistungen nach der VGR \\
$-\quad$ Übergang vom GH-Konzept auf GH (fob) und Warenkonzept der VGR \\
$=\quad$ Exporte Waren-(fob) und Dienstleistungen VGR-Konzept, cif \\
$-\quad$ Reiseausgaben der Ausländer im Inland (Übergang zum Inlandskonzept) \\
\hline$=\quad$ Importe nach dem Input-Output-Konzept zu Ab-Zoll-Preisen (IM I/O) \\
\hline \hline
\end{tabular}

Tabelle IV.6: Berechnung der Exporte nach dem IO-Konzept

${ }^{a}$ Vgl. STA BA [1992], S.52-53. 
Waren nach cif-Preisen. Diese wird für die einzelnen Bundesländer verwendet und so aus den Warenimporten die Dienstleistungsimporte abgeschätzt. Für Bremen liegt allerdings der Originalwert der Dienstleistungsimporte vor (STATISTISCHES LANDESAMT BREMEN [1987]). Es wird angenommen, daß das Verhältnis der Dienstleistungsimporte zu den Warenimporten von Bremen auch für Hamburg gilt, da beide Länder sich aufgrund der großen Seehäfen hinsichtlich des Außenhandels in einer vergleichbaren Situation befinden.

- Die benötigten Angaben über die Reiseausgaben der Inländer in der übrigen Welt wird aus der Berechnung des Saldos der Reiseausgaben abgeleitet, der bereits bei der Ermittlung der privaten Nachfrage nach dem Input-Ouput-Konzept dargestellt wurde. Nun werden durch Multiplikation der Zahl der Übernachtungen von Ausländern in den jeweiligen Bundesländern mit den Preisen pro Übernachtung in der Bundesrepublik die Reiseausgaben der Ausländer im Inland abgeschätzt. ${ }^{53}$ Dabei wird unterstellt, daß in allen Ländern die bundesdurchschnittlichen (BRD ohne Bremen und Schleswig-Holstein) Ausgaben pro Übernachtung gleich sind und zum zweiten, daß keine Ausländer zu Tagesausflügen ins Bundesgebiet reisen, bzw. die daraus resultierenden Ausgaben im Vergleich zu den Übernachtungskosten relativ gering sind. ${ }^{54}$ Die Reiseausgaben von Inländern im Ausland, die zur Berechnung der Exporte benötigt werden, ergeben sich dann als Differenz der Reiseausgaben von Ausländern im Inland und dem Saldo der Reiseausgaben.

Bezüglich der Berechnung der Exporte wird im Prinzip analog vorgegangen, mit Ausnahme zweier grundlegender Unterschiede. Zum einen sind fast ausschließlich Daten nach dem Spezialhandelskonzept vorgegeben, die mit Hilfe von Angaben über Exporte aus Lager in das Generalhandelskonzept übergeführt werden müssen und zum anderen gibt es anstelle der Einfuhrumsatzsteuer nur die nichtabzugsfähige Umsatzsteuer auf Exporte, die jedoch sehr gering ist. Als Ergebnis des Übergangs erhält man daher nur die Bruttoexporte (incl. nichtabzugsfähiger Umsatzsteuer).

\subsubsection{Primärfaktormatrix}

Die Berechnung der fehlenden Wertschöpfungsquadranten erfolgt durch Multiplikation der Matrix der Wertschöpungswerte aus der VGR mit den inversen Zeilenkoeffizienten der Ouptputtabelle (vgl. STABA [1979]). Die sich so ergebenden ersten Abschätzungen der Wertschöpfungsmatrizen werden mittels MODOP für jede einzelne Wertschöpfungskomponente an die bundesdeutschen Werte angeglichen.

\footnotetext{
${ }^{53}$ Die Daten stammen aus STABA [1984b].

${ }^{54}$ Zusätzlich werden alle anderen Ausgaben, die sich aus Bewirtung etc. ergeben vernachlässigt.
} 
Die endgültige Form erhält die Wertschöpfungsmatrix allerdings erst nach einigen Modifikationen, die sich weitgehend an GoTTFRIED ([1992] S.116-146) orientieren.

Nach Berechnung der Vorleistungsmatrix steht die nichtabzugsfähige Umsatzsteuer fest. Diese wird proportional auf die einzelnen Komponenten der Wertschöpfung übertragen und so in die Wertschöpfungsmatrix integriert.

Nun werden die Kapitalsteuern eigens ausgewiesen. Analog zu GotTfried ([1992], S.135136) wird unterstellt, daß sie in gleichen Verhältnissen, wie die Größe in der sie eingangs enthalten sind, in den einzelnen Sektoren auftreten. Demnach wird die Grund- und Gewerbesteuer proportional zur Produktionssteuer auf die einzelnen Sektoren aufgeteilt und aus der Produktionssteuer subtrahiert. Die Kapitalertrags- und Körperschaftssteuer werden proportional zu den Gewinnen aus Unternehmertätigkeit- und Vermögen auf die Sektoren verteilt und von diesen abgezogen. ${ }^{55}$

Da in der vorläufigen Wertschöpfungsmatrix noch Abschreibungen enthalten sind, ist es notwendig diese als Verwendung der anderen Primärfaktoren aufzufassen. Die Abschreibungen bilden zugleich eine wichtige Hilfsgröße bei der Ermittlung des Kapitaleinsatzes in die Produktion des öffentlichen Sektors. Da im theoretischen Modell davon ausgegangen wird, daß auch der öffentliche Sektor in seiner Produktion Kapital einsetzt, dieses aber in den Input-Output-Tabellen nicht als Einkommen aus Unternehmertätigkeit und Vermögen (Kapital) klassifiziert ist, wird hier analog zu GoTTFRIED ([1992], S.137-139) vorgegangen. Bei diesem Vorgehen sollen die bestehenden Kapital-Arbeits-Einsatzverhältnisse in den Sektoren ebenso aufrechterhalten werden wie die Arbeitseinsatzrelationen zwischen den verschiedenen Sektoren. Zugleich soll das regionale Verhältnis von Kapital zu Arbeitseinsatz unverändert bleiben.

Da im theoretischen Modell das gesamte bestehende Kapital zur Produktion eingesetzt wird, entspricht es dem regionalen Kapitalstock. Demgemäß werden nun die Werte für die regionalen Anlagevermögen als regionales Kapitalangebot eingesetzt und entsprechend der Relation von Kapitaleinsatz zu Arbeitseinsatz aus der IOT daraus das regionale Arbeitsvolumen generiert. Zusätzlich wird der regionale Abschreibungsanteil nach dem Verhältnis der Abschreibungen aus der Wertschöpfungsmatrix und dem Anlagevermögen gebildet und daraus die regionalen Abschreibungen der Primärfaktormatrix gebildet.

Mit Hilfe der Relation von Kapital und Arbeit in jedem Sektor sowie der Arbeit zwischen den Sektoren wird nun der regionale Kapital- und Arbeitseinsatz auf die einzelnen Sektoren verteilt.

\footnotetext{
${ }^{55}$ Berücksichtigt man zwei Sektoren, so werden sie vollständig von Sektor eins aufgebracht, da der öffentliche Sektor keine Einkommen aus Unternehmertätigkeit und Vermögen erzielt.
} 
Anschließend wird der staatliche Kapitaleinsatz bestimmt, indem das Verhältnis aus Abschreibungen zu staatlichem Anlagevermögen dazu benutzt wird, aus den gegebenen $\mathrm{Ab}$ schreibungen den öffentlichen Kapitalstock zu bestimmen. Dieser wird von den Arbeitseinkommen subtrahiert und in das öffentliche Kapitaleinsatzfeld umgesetzt. Schließlich werden noch die Abschreibungen dem Kapitaleinsatz hinzuaddiert.

Damit hat man die vollständigen regionalen Primärfaktormatrizen berechnet.

\subsubsection{Vorleistungsmatrix}

Nach der Berechnung der Bruttowertschöpfung und des Bruttoproduktionswertes kann nun durch Subtraktion der Wertschöpfung vom BPW die Spaltensumme der Vorleistungsmatrix gewonnen werden.

Zur weiteren Ermittlung der noch unbekannten Vorleistungsmatrizen, die von BadenWürttemberg, Hessen und Nordrhein-Westfalen sind bereits bekannt, werden die Gütergruppen in die 58 Produktionsbereiche der bundesdeutschen Input-Output-Tabelle gegliedert. Auf diese Weise ist es zumindest teilweise möglich, die Besonderheiten der einzelnen Länder zu berücksichtigen.

Im allgemeinen wird wie folgt vorgegangen:

Soweit die Werte der BPW und Bruttowertschöpfung aus der VGR vorliegen, können aus ihnen die Spaltensumme der Vorleistungen nach dem VGR-Konzept der einzelnen Sektoren berechnet werden. ${ }^{56}$ Unterstellt man, daß die Relation zwischen VGR-Konzept und Input-Output-Konzept innerhalb eines der sieben Hauptsektoren ${ }^{57}$ für jedes der Untersektoren konstant ist, so können aus den VGR-Vorleistungssummen durch Verwendung der Übergangskoeffizienten der Hauptsektoren, die Vorleistungsspaltensummen nach dem Input-Output-Konzept der 58 Sektoren berechnet werden.

Soweit die entsprechenden VGR-Werte nicht vorliegen, werden Angaben über die Umsätze verwendet, und unterstellt, daß sich die Proportionen zwischen den Sektoren zwischen Umsatz und Vorleistungen nach dem IO-Konzept nicht verändern. Die auch dann noch fehlenden Werte der einzelnen Teilsektoren werden durch bundesdeutsche Relationen dieser Sektoren, bezogen auf die noch unbekannten Werte der Teilsektoren, ermittelt.

\footnotetext{
${ }^{56}$ Für das Saarland und Bayern sind Bruttowertschöpfungswerte für 34 Sektoren ausgewiesen (STAtistisches Amt des Saarlandes [1990a] und Bayerisches Landesamt [1990]). Für die anderen Länder für 12 Sektoren. Zusätzlich sind recht detaillierte Umsatzwerte aus den Umsatzsteuerstatistiken für das Verarbeitende Gewerbe, veröffentlicht. Bezüglich der anderen Sektoren liegen je nach Bundesland unterschiedlichste Umsatzzahlen, die als Ersatz für die fehlenden BPW verwendet werden.

${ }^{57}$ Land- und Forstwirtschaft, Bergbau und Energie, Produzierendes Gewerbe, Baugewerbe, Handel und Verkehr, übrige marktbestimmte Dienstleistungen und nicht marktbestimmte Dienstleistungen.
} 
Schließlich werden aus den so vorgegebenen Spaltensummen der Vorleistungsmatrix durch Multiplikation mit den bundesdeutschen Spaltenkoeffizienten die einzelnen Felder der differenzierten Vorleistungsmatrix ermittelt. Durch Addition der einzelnen Teilsektoren erhält man daraus eine erste Abschätzung der Zentralen Matrix mit sieben Sektoren.

In einem letzten Schritt wird diese erste Näherung der Vorleistungsquadranten mit Hilfe des MODOP-Verfahrens korrigiert. Als Vorgaben für das MODOP-Verfahren können für Berlin die Zeilen- und Spaltensummen der Vorleistungsmatrix vorgegeben werden, da diese als Residuen nach Berechnung der Wertschöpfungsmatrix und der Endnachfrage berechnet werden können. Für die anderen Länder sind jedoch die Salden aus den Lieferungen und Bezügen nicht gegeben. Daher können für sie keine Zeilensummen der Vorleistungsmatrix vorgegeben werden. Für diese Länder sind jedoch die Spaltensummen der Vorleistungsmatrix bekannt und zudem ist die Summe der einzelnen Felderwerte über alle Länder, d.i. die bundesdeutsche Vorleistungsmatrix, gegeben. Daher kann für jede Spalte der Vorleistungsquadranten mit Hilfe des MODOP-Verfahrens eine Aufteilung der bundesdeutschen Werte auf die einzelnen Länder vorgenommen werden. Durch Zusammensetzen dieser Einzelwerte erhält man schließlich eine vollständige zentrale Matrix für jedes Land.

\subsubsection{Endnachfragematrix}

\section{Aufspaltung des privaten Konsums}

Die genauen Werte der Konsumnachfrage nach den einzelnen Gütern können nur näherungsweise abgeschätzt werden. Für alle Länder hat man Angaben über den Konsum im Inland nach Lieferbereichen. Zusätzlich existieren für die gesamte Bundesrepublik sowie Berlin(West) Angaben über den Konsum nach Verwendungszwecken. Die Konsumnachfrage für Berlin(West) kann relativ leicht unter Verwendung der bundesdeutschen Zuordnungstabelle zwischen Konsum nach Verwendungszwecken und Konsum nach Gütergruppen berechnet werden (siehe STABA [1989a], S.400-401).

Für die anderen Länder, mit Ausnahme von Hessen und Baden-Württemberg, ist dies nicht so ohne weiteres möglich, da die Lieferbereiche nicht nach Gütergruppen gegliedert sind. Für diese Länder wird angenommen, daß die Umbuchung, die für die Bundesrepublik einen Übergang von Lieferbereichen in Gütergruppen ermöglicht in den selben Proportionen auch für die Länder zutrifft. Damit erhält man für alle Länder eine Konsumnachfrage nach den sieben Sektoren aus denen durch Verwenden der bundesdeutschen Umsatzsteuersätze die Nettokonsumnachfrage der Input-Output-Tabelle ermittelt werden kann. 


\section{Berechnung der Investitionen}

Investitionen werden zwar in der Datenbasis nicht berücksichtigt. Dennoch muß ihre Höhe abgeschätzt werden, da sie zum einen den privaten Konsum erhöht, zu dem sie addiert werden, und zum anderen für die Bestimmung des Saldos von Lieferungen und Bezügen bekannt sein muß. Grundsätzlich erfolgt die Berechnung der Investitionen nach dem Prinzip der Gleichheit von nationalen und regionalen Strukuren. Ausgehend von gegebenen Ausrüstungs- und Bauinvestitionen können dann die Investitionen der Länder berechnet werden. Durch Hinzufügen, der mit Hilfe bundesdeutscher Anteile der Vorratsinvestitionen an den anderen Investitionsarten gewonnen Vorratsinvestitionen erhält man schließlich die gesamten Investitionen aus denen, analog zum Vorgehen beim privaten Verbrauch schließlich durch Herausrechnen der Umsatzsteuern die Investitionen der Input-Output-Tabellen bestimmt werden.

\section{Aufspaltung der Exporte und Importe}

Aus der Auswertung der Außenhandelsstatistik können in etwa die Relationen der Güterexund -Importe der einzelnen Sektoren am gesamten Warenhandel ermittelt werden. ${ }^{58} \mathrm{Da}$ Gut 7 nicht gehandelt wird, muß dann zusätzlich der Dienstleistungshandel auf Gut 5 und Gut 6 aufgeteilt werden. Dies erfolgt weitgehend nach bundesdeutschen Anteilen.

Aus der bundesdeutschen Importtabelle können dann, wieder unter der Annahme identischer nationaler und regionaler Importstrukturen die Importe auf die einzelnen Nachfragekategorien aufgeteilt werden.

Zugleich erfolgt eine Anpassung des Datensatzes an das theoretische Modell. In diesem wird davon ausgegangen, das das öffentliche Gut $\mathrm{G}$ als regionales öffentliches Gut nicht gehandelt wird, und nicht von anderen Nachfragern als der jeweiligen Regierung konsumiert werden kann. Aus diesem Grund werden alle Endnachfragen nach Gut 7 in der Nachfrage $\mathrm{G}$ zusammengefaßt. Zieht man die Importmatrix von den Input-Output-Tabellen ab, so erhält man die Zentrale Matrix und die Endnachfrage nach der Bundesverflechtung.

\section{Saldo von Lieferungen und Bezügen}

Mit der Berechnung der Wertschöpfungsmatrix, der Vorleistungsmatrix, der privaten Endnachfrage, von Exporten und Importen, der öffentlichen Nachfrage und der Investitionen kann nun der Saldo der Lieferungen und Bezüge durch Abzug diese Größen vom Bruttoproduktionswert bestimmt werden. Für Hessen und Baden-Württemberg sind die Lieferungen und Bezüge aus den Input-Output-Tabellen getrennt ausgewiesen.

\footnotetext{
${ }^{58}$ Die weiteren Korrekturen werden nicht näher dargestellt.
} 


\section{Korrektur der Endnachfragematrix}

Nach Aggregation der so erstellten Multiregionalen Input-Output-Tabelle von sieben auf zwei Gütergruppen erhält man eine erste Näherung für den Datensatz. Allerdings sind zwei Bedingungen des theoretischen Modells noch nicht erfüllt: Zum einen stimmen die Summen der Primärfaktormatrix und der Endnachfragematrix nicht überein, und zum anderen ist die Handelsbilanz nicht ausgeglichen, da die Exporte von den Importen abweichen.

Dies erfordert eine Korrektur der gesamten Tabelle im Endnachfragebereich, da die Wertschöpfungsmatrix bereits feststeht und eine Veränderung der Vorleistungsmatrix die Gesamtdifferenz nicht verändern kann. Die Korrektur wird zuerst bezüglich der aggregierten nationalen Tabelle durchgeführt und anschließend proportional zu den nationalen Veränderungen auf die Tabellen der einzelnen Länder übertragen.

In einem ersten Schritt wird die Summe der Importe an die der Exporte angeglichen. Dies bedeutet zugleich eine Erhöhung der Importe von Gut 1, da Gut 2 nicht gehandelt ist. Diese Korrektur bewirkt nun aber, daß die Summe der Endnachfrage incl. Importe noch stärker von der Primärfaktormatrix abweicht.

Die nun noch bestehende Abweichung zwischen Spalten- und Zeilensumme bei Gut 1 kann nur durch eine Veränderung der Differenz zwischen privatem Konsum und Exporten bzw. Importe verringert werden, da die Exporte gleich den Importen sein müssen. Allerdings soll die Korrektur das Verhältnis von Konsum zu Exporten (Importe) nicht verändern, so daß letztlich durch Anpassung des Konsums, bei gewahrten Relationen zwischen Konsum und Exporten (Importen) die Abweichung beseitigt wird. Als erstes wird die entsprechende Korrektur für die nationale IOT-Tabelle durchgeführt und anschließend proportional auf den privaten Konsum sowie die Ex- und Importe der Länder übertragen. Die noch verbleibende Differenz zwischen Zeilen- und Spaltensumme beim privaten Gut in den regionalen Tabellen wird zum Saldo der Lieferungen und Bezüge addiert.

Damit hat man nun regionale Tabellen, in denen die Gleichgewichtsbedingungen des Systems weitgehend erfüllt sind. Nun müssen nur noch die interregionalen Beziehungen genauer aufgeschlüsselt werden.

\section{Lieferungen und Bezüge}

Über Lieferungen und Bezüge liegen nur wenige Grundinformationen vor. Aus den bisherigen Berechnungen sind die nach Güterarten differenzierten Salden der Lieferungen minus Bezüge bekannt. Nur für Hessen und Baden-Württemberg sind Lieferungen und Bezüge getrennt ausgewiesen. Zusätzlich weist Münzenmaier [1989] eine vollständige Bezugs- bzw. Lieferungsmatrix des Restes der Bundesrepublik mit Baden-Württemberg aus. 
Weitere Originärdaten sind nur für Warenlieferungen und Warenbezüge zwischen Berlin und der restlichen Bundesrepublik bekannt (STATISTISCHes LANDESAMT BERLIN [1983, 1984]). Diese Werte werden zur Berechnung der technologischen IOT vorgegeben. Im Anschluß daran können die IOT der andern Länder und nochfolgend die Bezüge bzw. Lieferungen aller Länder mit Hilfe des Gravitationsmodells von Carlberg ([1979], S.123137) geschätzt werden.

Das Gravitationsmodell von Carlberg [1979]: Das Standardverfahren zur Berechnung von Güterströmen zwischen zwei Räumen ist der Gravitationsansatz, auch wenn sein ökonomischer Gehalt stark bezweifelt werden kann (siehe. z.B. GENosKo [1992]). Es ist jedoch unbestritten, daß dieses Verfahren relativ brauchbare Schätzungen für Güterströme liefert.

Der Einsatz dieser Methode zur Berechnung interregionaler Güterströme geht auf LEONTIEF und STrout [1963] zurück. Das hier verwendete Verfahren wurde von Carlberg ([1979], S.123-137) entwickelt und von ihm zur Berechnung der interregionalen Güterströme zwischen den Ländern der Bundesrepublik eingesetzt.

Die interregionalen Güterströme hängen in diesem Ansatz von mehreren Aspekten ab:

- Je größer die Produktion eines Gutes in einer Region ist, um so höher sind die Lieferungen dieses Gutes aus dieser Region in andere Gebiete (siehe CARLBERG [1979], S.123).

- Je stärker eine Region auf ein Gut spezialisiert ist, um so größer ist der Anteil der Lieferungen dieses Gutes an den gesamten Lieferungen der Region (vgl. CARLBERG [1979], S.123).

- Je mehr eine Region produziert und verbraucht, um so größer sind ihre Importe, da sie mehr Vorleistungen aber auch mehr Endprodukte benötigt (vgl. CARLBERG [1979], S.123).

- Je größer die Distanz zwischen zwei Regionen ist, um so höher sind die Transportbzw. Kommunikationskosten des Handels. Aus diesem Grund sinkt der Handel mit zunehmender Entfernung (Siehe CARLBERG [1979], S.123).

Dies sind die wesentlichen Grundgedanken des Modells von CARLBERG, in dem die Lieferungen eines Gutes $i$ aus Region $r$ nach Region $\mathbf{s}, X_{i}^{r s}$, proportional zum Angebot des Gutes $i$ in der Region $Q_{i}^{r}$, zur Spezialisierung der Lieferregion auf das Gut $i$, d.h. dem Anteil der Produktion des Gutes $i$ an der Gesamtproduktion der Region $Q_{i}^{r} / Q^{r}$, proportional zur gesamten Produktion in der Empfangsregion $Q^{s}$ und umgekehrt proportional 
zur Distanz zwischen den Regionen $d_{r s}$ ist:

$$
X_{i}^{r s}=\beta_{i} Q_{i}^{r} \frac{Q_{i}^{r}}{Q^{r}} Q^{s} d_{r s}^{-\alpha_{i}} \quad \forall i \text { und } r, s .
$$

mit $\beta_{i}$ als Korrekturgröße und $\alpha_{i}$ als Gravitationskoeffizient, der den Einfluß der Entfernung auf die Güterströme angibt.

Schätzung der Gravitationskonstanten: Durch Umformung erhält man die Regressionsgleichung, die durch Logarithmisierung linearisiert werden kann:

$$
\frac{X_{i}^{r s} Q^{r}}{\left(Q_{i}^{r}\right)^{2} Q^{s}}=\beta_{i} d_{r s}^{-\alpha_{i}} e_{i} \quad \forall i \text { und } r, s
$$

mit $e_{i}$ als Störgröße.

Mit Hilfe dieser Gleichung werden nun die Gravitationskoeffizienten $\alpha_{i}$ geschätzt. Als Datengrundlage dienen die Werte aus den IOT von Baden-Württemberg für 1972, 1974 zu Preisen von 1970, 1978, 1980, 1982, 1984 und 1986 (MünZenMaIER [1982, 1984, 1985, 1986b, 1987, 1988b, 1989a, 1989b, 1990]), die von Hessen für 1980 (GRETZ-RoTh / Gretz [1986]) und 1982 (eigene Berechnungen), sowie die von Berlin von 1982 (eigene Berechnungen).

Verwendet man jeweils die Lieferungen der betrachteten Region an sich selbst, der Region an den Rest der Bundesrepublik, des Restes der Bundesrepublik an die Region und des Restes der Bundesrepublik an sich selbst erhält man auf diese Weise 36 Beobachtungen, die in einer kombinierte Zeitreihen- und Querschnittsanalyse verwendet werden können. ${ }^{59}$ Die Entfernungsangaben zwischen den Regionen werden als mittlere $\mathrm{km}$-Angaben aus CARlberg ([1979], S.134) genommen, die bei Aggregation über die Länder mit ihrem jeweiligen BPW gewichtet werden (vgl. CARLBERG [1979], S.131).

Die Schätzungen werden für einen Datensatz durchgeführt, in dem die Eigenlieferungen vom jeweiligen Rest der Bundesrepublik an sich selbst nicht berücksichtigt. Dieser Ansatz

\footnotetext{
${ }^{59}$ Einige Probleme werden dabei jedoch unberücksichtigt gelassen. Zum ersten werden die Daten von 1972 nicht verwendet, da sie teilweise in der Systematik von denen der anderen Jahre abweichen. Dagegen werden die Werte von 1974 berücksichtigt, obwohl sie auf Preisen von 1970 beruhen. Dies führt dazu, daß Lieferungen für diesen Zeitraum zu niedrig ausgewiesen sind. Die Werte für Hessen von 1982 und Berlin von 1982 werden verwendet, obwohl sie zum Teil auf eigenen Berechnungen beruhen, da sie vermutlich nicht allzu stark von den IOT-Werten der amtlichen Statistiken abweichen dürften. Die Hessische Tabelle von 1980 beruht z.T. auf einer proportionalen Korrektur der Werte von 1982, so daß das umgekehrte Verfahren zu nicht allzu starken Abweichungen führen dürfte. Für Berlin sind zumindest die Warenlieferungen und -Bezüge zwischen Berlin und dem Rest der Bundesrepublik weitgehend Originalwerte aus den Güterstatistiken.
} 
wird deshalb gewählt, da sich in der Aggregation "Rest der Bundesrepublik" die jeweiligen Lieferungen und Bezüge der im Aggregat enthaltenen Bundesländer gegenseitig aufheben, und damit bezüglich der Eigenlieferungen stark verzerrt sind. Die Anzahl der Beobachtungen reduziert sich dann auf $28 .{ }^{60}$

Als Schätzergebnisse für die Gravitationskonstanten $\alpha_{i}$ erhält man die in der Tabelle IV.7 zusammengestellten Werte:

\begin{tabular}{||c|ccc||}
\hline \hline & $-\alpha_{i}$ & \multicolumn{1}{c||}{$\mathrm{T}$} & adj. $\mathrm{r}^{2}$ \\
\hline Sektor 1 & -2.39018 & -37.967 & 0.9856 \\
Sektor 2 & -2.02076 & -17.724 & 0.9372 \\
Sektor 3 & -1.11104 & -8.439 & 0.7698 \\
Sektor 4 & -3.30034 & -23.445 & 0.9631 \\
Sektor 5 & -2.62321 & -21.599 & 0.9569 \\
Sektor 6 & -3.07596 & -15.372 & 0.9289 \\
\hline \hline
\end{tabular}

Tabelle IV.7: Schätzergebnisse des Gravitationsmodells 1982

Wie aufgrund der Datenlage (Klumpenschätzung) zu erwarten ist, sind alle Ergebnisse hochsignifikant.

Lieferungen/Bezüge nach dem Gravitationsmodell: Unter Verwendung der geschätzten Gravitationskonstanten $\alpha_{i}$ kann man nun die Lieferungen der einzelnen Bundesländer abschätzen. Sie ermitteln sich für jedes der sechs Gütergruppen aus dem obigen Gravitationsmodell in Gleichung (IV.42). Diese Lieferungen werden dann zu ein der Lieferung des aggregierten Gutes zusammengefaßt. Anschließend werden diese Schätzungen an die Randwerte der Lieferungen und Bezüge aus den Input-Output-Tabellen durch das MODOP-Verfahren angepaßt.

Da allerdings für die einzelnen Länder, mit Ausnahme von Hessen, Berlin und BadenWürttemberg, nur die Salden der Lieferungen und Bezüge gegeben sind, müssen zuerst die Randwerte abgeschätzt werden. Dazu werden verschieden Verfahren verwendet.

Aus der Summe der geschätzten Lieferungen und dem vorgegebenen Saldo der Lieferungen und Bezüge werden die Bezüge als Residuen gewonnen. Auf die gleiche Weise werden alternativ die Lieferungen, unter Vorgabe der geschätzten Bezüge, als Residuen berechnet. Das Mittel aus beiden Verfahren ergibt die Summe der Lieferungen bzw. Bezüge, die als

\footnotetext{
${ }^{60}$ Ein weiteres Problem ist die einseitige Ausrichtung auf Baden-Württemberg, da sich nicht weniger als 18 der 28 Beobachtungen auf Baden-Württemberg beziehen. Damit erhält man im Prinzip eine Klumpenschätzung. Dies läßt sich angesichts der Datenlage jedoch nicht verhindern.
} 
Randwerte zur Bestimmung der Lieferungen und Bezüge des jeweiligen Gutes zwischen den einzelnen Länder eingesetzt werden.

Nun kann mit Hilfe von MODOP die Anpassung der einzelnen geschätzten Felderwerte der Lieferungen jeder Region in alle Regionen durchgeführt werden. Als Ergebnis erhält man für jedes Land eine Lieferungsmatrix bezüglich der Empfangsländer der jeweiligen Güter. Aus dieser kann durch Transponieren die Bezugsmatrix der einzelnen Länder gewonnen werden, die ebenfalls nur nach Gütern und Herkunftsregion differenziert ist.

Um jedoch eine vollständige Bezugsmatrix zu erhalten, müssen die Bezüge noch nach Verwendungskategorie aufgespalten werden. Zu diesem Zweck wird angenommen, daß die Bezüge eines Gutes genauso wie der Eigenverbrauch dieses Gutes in der Empfangsregion auf die einzelnen Nachfragekomponenten (Vorleistungen, Konsum) aufgeteilt werden. Daraus läßt sich die vollständige Bezugsmatrix und schließlich nach Addition der Importmatrix die vollständige multiregionale Tabelle bestimmen.

\subsubsection{Sonstige Größen}

Nun fehlen noch einige zusätzliche Daten. Dies sind neben den Steueraufkommen, die Zuweisungen und Transfers zwischen den Ländern, durch die die Einhaltung der Budgetgleichungen und der regionalen Handelsbilanzen gesichert werden.

\section{Einkommenssteuern}

Die Einkommenssteueraufkommen der Länder sind vorgegeben STA BA [1989b]. Geht man außerdem von einer einheitlichen Besteuerung in der Bundesrepublik aus und unterstellt einen konstanten Grenzsteuersatz, so kann man nach Vorgabe eines Grenzsteuersatzes die Steuerfreibeträge jeder Region bestimmen. Als Grenzsteuersatz wird der Wert $24,5 \%$ aus FEHR ([1993], S.82) übernommen. ${ }^{61}$

\section{Konsumsteuern}

Die Umsatzsteueraufkommen der Bundesrepublik für 1982 (STABA [1989b]) dienen zur Ermittlung des Konsumsteuersatzes für das Gut 1. Gut 2 wird nicht konsumiert und gilt als steuerfrei, da nur reine Konsumsteuern modelliert sind. Es wird demnach das Umsatzsteueraufkommen mit den Aufkommen aus der Konsumbesteuerung gleichgesetzt und

\footnotetext{
${ }^{61}$ Die Berechnungen in FEHR ([1993], S.82) gelten für 1981. Es wird hier unterstellt, daß sich die Grenzsteuersätze auch für 1982 nicht verändern. Die Berechnung geht von dem ,gewichtete[n] Durchschnitt der Daten von Alleinstehenden und Verheirateten mit zwei Kindern aus der Tabelle 4 in MCKEE u.a. ([1986], S.67)" aus.
} 
daher der nicht unbeträchtliche Anteil der nichtabzugsfähigen Umsatzsteuer der Konsumsteuer zugeschlagen. Dies geschieht mit der Absicht, die im Finanzausgleich durchgeführte Finanzkraftberechnung nicht allzu stark zu verändern.

Allerdings führt diese Art der Berechnung der Konsumsteuern über einheitliche Steuersätze dazu, daß die Aufkommen der Länder, die sich aus den Lieferungen dieser Länder sowie deren Eigenkonsum ergeben, von den tatsächlichen Aufkommen abweichen. Diese Abweichungen sind jedoch nicht allzu groß, und werden daher nicht weiter beachtet.

\section{Produktionssteuern}

Aus der Primärfaktormatrix wird die Summe der Produktionssteueraufkommen je Region ermittelt. Den Ländern stehen von diesen die tatsächlichen Verbrauchssteuern aus den Steuerstatistken zu (STABA [1983a, 1989b]), um die Finanzkraftberechnung im Finanzausgleich nicht zu verfälschen. Die Differenz zwischen den regionalen Verbrauchssteuern und den Produktionssteuern erhält der Bund.

\section{Faktorsteuern}

Die Kapitalertrags- und Körperschaftssteuern sowie die Gewerbe- und Grundsteuern werden nach dem in der Ermittlung der Primärfaktormatrix verwendeten Verfahren bestimmt. Sie liegen damit nach Erstellung der interregionalen Güterverflechtungstabelle bereits fest. Allerdings werden zusätzlich die Hebesätze der Realsteuern benötigt. Für sie werden die länderdurchschnittlichen Hebesätze aus STABA ([1985b], S.455) verwendet.

\section{Finanzausgleich}

Steuerverteilung, Länderfinanzausgleich und Bundesergänzungszuweisungen werden ana$\log z u$ den geltenden Regelungen aus den Steueraufkommen bestimmt. Diese Bestimmungen sind ausführlich in Kapitel 3. dargestellt.

\subsubsection{Ergänzung um das aggregierte Ostland}

Die bisherige Beschreibung des Datensatzes bezieht sich ausschließlich auf die alten Bundesländer, wobei der letzte Teil über die sonstigen Größen weitgehend auch für die gesamte Bundesrepublik gilt.

Im folgenden wird nun die Erstellung des Datensatzes für den Osten beschrieben, die sich teilweise erheblich von der für die alten Bundesländer unterscheidet.

Da die Ostländer nach ihrem Beitritt zur Bundesrepublik einen Strukturbruch erlitten haben, können die tatsächlichen Daten von 1982 nicht verwendet werden, um Effekte für 
die Zeit nach der Einigung abzuschätzten. Aus diesem Grund wird ein fiktiver Datensatz für 1982 gewonnen, der sich an den 1991 geltenden Relationen der Daten für die Neuen Länder zu den Werten der Westländer orientiert. Grundsätzlich wird dabei so vorgegangen, daß diese Relationen durch Multiplikation mit den Werten des für 1982 erstellten Datensatzes der Westländer auf 1982 heruntergerechnet werden.

\section{Wertschöpfung}

Das Bruttoanlagevermögen der Neuen Länder ist für 1991 zu Preisen von 1990 gegeben (STIlle, u.a. [1992], S.163). Dieses wird mit Hilfe der Westrelation, zwischen 1990 und 1991, auf die Preise von 1991 umgerechnet. Durch das Verhältnis des Bruttoanlagevermögens West '91 (STABA [1994], S.703) zu '82 wird das Bruttoanlagevermögen Ost auf 1982 heruntergerechnet. Dasselbe gilt für das Bruttoanlagevermögen des Staates und die Abschreibungen des Sektors 1, die Einkommen aus Unternehmertätigkeit und Vermögen für Sektor 1 sowie die Einkommen aus selbstständiger Arbeit für beide Sektoren. ${ }^{62}$

Die Produktionssteuern der Sektoren werden proportional zu denen des Westens bestimmt. Dadurch erhält man bereits eine erste Primärfaktormatrix mit Abschreibungen, Kapital-, Arbeitseinsatz und Produktionssteuern abzüglich Subventionen.

Die Abschreibungen des Gutes 2 werden analog zu den Abschreibungen im Westen ermittelt. Um zu vermeiden, daß der Kapitaleinsatz negativ wird, wird er aus der Summe von Kapitaleinsatz und Abschreibungen berechnet. Multipliziert man mit diesem Wert das Verhältnis des staatlichen Anlagevermögens zum Anlagevermögen aller Unternehmen ergibt sich der Kapitaleinsatz des öffentlichen Sektors. Zieht man diesen vom Arbeitseinsatz ab, so erhält man die korrigierte Faktormatrix.

Durch Übertragung der relativen Steueraufkommen der Neuen Bundesländer incl. Westberlins von 1991 auf 1982 werden dann die Steueraufkommen der Kapitalertrags-, Körperschafts-, Gewerbe- und Grundsteuern des Ostens in 1982 errechnet. ${ }^{63}$ Diese werden im Sektor 1 vom Kapitaleinsatz und den Produktionssteuern abgezogen. Damit ist die gesamte Matrix des primären Faktoreinsatzes berechnet.

\section{BPW und Vorleistungen}

Zur Berechnung des BPW und der Vorleistungen wird unterstellt, daß die Vorleistungsanteile der Unternehmen im Osten und im Westen gleich sind. ${ }^{64}$ Daher werden die Spal-

\footnotetext{
${ }^{62}$ Die Daten stammen aus STABA [1992a], S.59 und S.126, sowie DIW [1993], S.270.

${ }^{63}$ Die Daten stammen aus STABA ([1992b], S.51-57).

${ }^{64}$ Dies stützt sich zumindest bezüglich des Verarbeitenden Gewerbes auf Erkenntnisse des Statistischen Bundesamtes (Essig/STrohm [1993], S.187), das aufgrund der Kostenstrukturerhebung 1991 im Verar-
} 
tensumme der Vorleistungen und die Bruttoproduktionswerte dadurch gewonnen, daß für den Sektor 1 das Verhältnis zwischen Wertschöpfung und BPW im Westen auf den Osten übertragen wird. Ein ähnliches Vorgehen für den Sektor 2 würde dazu führen, daß deren Bruttoproduktionswert so niedrig wird, daß BPW abzüglich Staatskonsum negativ werden würde. Daher wird pragmatisch angenommen, daß der Anteil der Wertschöpfung im öffentlichen Sektor niedriger liegt als im Westen und der Einfachheit halber die Vorleistungen gleich der Wertschöpfung gesetzt. Die Vorleistungen werden proportional zu den westlichen Vorleistungskoeffizienten auf die beiden Sektoren verteilt. Dies ist bei diesem hohen Aggregationsniveau eine sicherlich zu vertretende Annahme. Als Ergebnis erhält man die vollständige Vorleistungsmatrix und die Bruttoproduktionswerte für die Neuen Länder.

\section{Endnachfrage}

Privater Konsum: Aus den VGR-Daten für 1991 (DIW [1993], STABA [1994], EsSIG/STrohm [1993]) wird der private Konsum durch eine Auswertung der Verbrauchsstatistiken der einzelnen Konsumgüter für drei Haushaltstypen in West- und Ost ermittelt. ${ }^{65}$

Durch Verwendung der Übergangstabelle vom Verbrauch nach Verwendungsarten auf den Verbrauch nach Güterarten im Westen (STABA [1989a], S.400-401), wird dann für 1991 der Ostkonsum der beiden Güterarten ermittelt. Mit Hilfe des Anteils des Westverbrauchs 1982 zum Westverbrauch 1991 wird schließlich der Netto-Ostverbrauch 1992 nach dem Input-Output-Konzept berechnet.

Ex- und Importe: Die vorgegebenen Exporte- und Importwerte aus der VGR werden proportional zum Westen korrigiert. Dabei müssen bei den Exporten zuerst die Reiseausgaben abgezogen werden und bei den Importen hinzugezählt werden.

Die ermittelten Werte für 1991 nach dem Input-Output-Konzept werden ebenfalls proportional zum Westen für 1982 heruntergerechnet. Zusätzlich wird die im Durchschitt auf die Exporte liegende Umsatzsteuer im Westen als Durchschnittssatz auf die Exporte auf den Osten übertragen und so die Umsatzsteuer der Exporte ermittelt.

beitenden Gewerbe davon ausgeht, daß sich die Vorleistungsanteile des Ostens „allmählich” an die des Westens annähern.

${ }^{65}$ Die Daten über den Verbrauch der drei Haushaltstypen stammen aus STA BA ([1993], S.558-560 und S.564-566), die über die relative Häufigkeit der drei Haushaltstypen im Osten und Westen aus STILLE, u.a. [1992]. 
Investitionen und öffentlicher Konsum: Die Investitionen und der öffentliche Verbrauch des Ostens werden gemäß der Westveränderung zwischen 1991 und 1982 korrigiert. Entsprechend dem durchschnittlichen Umsatzsteueranteils des Westens wird dann für den Osten die Umsatzsteuer auf Investitionen ermittelt. ${ }^{66}$

Lieferungen und Bezüge: Führt man zuerst die notwendige Korrektur der IOT Ost durch, indem man die Importe an die Exporte angleicht, ${ }^{67}$ so kann man nun den Saldo von Lieferungen und Bezügen bestimmen. Dieser dient als Vorgabe zur Abschätzung der Lieferungen und Bezüge des Ostens.

Die Angaben der Lieferungen und Bezüge im Warenverkehr zwischen dem gesamten Osten und den einzelnen Westländern für 1991 (LANDESAMT NRW [1993]. S.760-763) werden proportional so korrigiert, daß ihre Summe genau der Vorgabe der Warenlieferungen und -Bezüge aus der VGR (STABA [1993], S.667) entspricht.

Nun wird angenommen, daß die Struktur der Lieferungen und Bezüge der Dienstleistungen genau denen im Warenverkehr entspricht und die entsprechenden Koeffizienten mit der vorgegebenen Summe der Dienstleistungslieferungen bzw. -Bezüge (STA BA [1993], S.667) multipliziert.

Anschließend wird der Saldo der Lieferungen und Bezüge auf den Saldo der vorläufigen Input-Output-Tabelle von 1982 heruntergerechnet und proportional die Bezüge und Lieferungen zwischen den einzelnen Westländer und dem Osten korrigiert. Nach Abzug einer Importmatrix des Ostens, die mit Westkoeffizienten erstellt ist, wird schließlich die vollständige Bezugsmatrix für den Osten ermittelt. Dabei wird wieder mit der Annahme gearbeitet, daß der Verbrauch der Bezüge gleich dem der heimischen Produkte ist. Entsprechend werden die Lieferungen der Westländer aufgespalten. Ergebnis ist eine Verflechtung der Ost- mit den Westländern.

Im Osten werden nun noch der Konsum und die Investitionen zum privaten Konsum aggregiert und der Konsum des Gutes 2 dem Staatsverbrauch zugerechnet.

Für die Westländer bedeuten die sehr hohen Lieferungen an den Osten eine Verringerung der eigenen Konsummöglichkeiten. Aus diesem Grund wird ihr eigener Konsum entsprechend verringert und zugleich die Konsumsteuersätze erhöht, so daß für den Westteil, nach Abzug von Westberlin, wieder die ursprünglich gegebenen Umsatzsteueraufkommen resultieren.

\footnotetext{
${ }^{66}$ Vorgabe sind die VGR-Daten aus DIW [1993], StaBA [1994] oder Essig/Strohm [1993].

${ }^{67}$ Anstelle einer recht komplizierten Umrechnung durch Abzug der DDR-Exporte und -Importe von 1982 von den Westländern und Zurechnung dieser zu Lieferungen und Bezügen wird einfach unterstellt, daß sich an den westlichen Exporten und Importen durch Berücksichtigung des Ostens nichts ändert.
} 


\section{Steueraufkommen und Finanzausgleich}

Grundsätzlich werden die Steueraufkommen des Ostens proportional zu 1991 auf 1982 heruntergerechnet, wobei das Verhältnis zwischen West- und Ost gewahrt bleibt.

Die Finanzbeziehungen werden ansonsten analog zur Beschreibung für den Westteil ermittelt. Derselbe Einkommenssteuergrenzsatz wie im Westen führt bei gegebenen Einkommensteueraufkommen zur Bestimmung des Steuerfreibetrages. Die Anwendung des bundesdeutschen Umsatzsteuersatzes dient der Ermittlung der Konsumsteueraufkommen und der Ausgleich der Budgetgleichungen und der interregionalen Handelsbeziehungen in West- und Ost wird zur Bestimmung der Transfers und Zuweisungen an Ost- und Westländer verwendet.

\section{Zuweisungen und Transfers als Residuen}

Die Zuweisungen und Transfers der nationalen Regierung werden aus den Gleichgewichtsbedingungen des Modells abgeleitet. Sie ergeben sich aus den interregionalen Leistungsbilanzen und den regionalen, nationalen und privaten Budgetgleichungen. Die Transfers der nationalen Regierung an die privaten Haushalte gleichen die Budgetbeschränkungen der privaten Haushalte aus, deren restliche Werte sich aus den Faktoreinkommen und den Ausgaben für die Güter ergeben. Die Zuweisungen gleichen die regionale Leistungsbilanz und gleichzeitig die regionalen und nationalen Regierungsbudgets aus. Es zeigt sich, daß diese residuale Bestimmung von Zuweisungen und Transfers zu sehr hohen Zuweisungen und Transfers an den Osten führt.

An dieser Stelle sei nochmals darauf hingewiesen, daß der gewonnene Datensatz, vor allem aufgrund des Einbezugs der Neuen Bundesländer, nur eine sehr ungenaue Näherung der Situation darstellt. Er sollte daher nur mit äußerster Vorsicht und in erster Linie nur zu einer sehr groben Abschätzung von Vorzeichen verwendet werden.

\section{Berechnung der Modelparameter: Kalibrierung}

Um mit dem vorgestellten Modell und dem Datensatz Berechnungen durchführen zu können, müssen noch die Parameter des Modells, dies sind im wesentlichen die Parameter der Nutzen- und Produktionsfunktionen, bestimmt werden.

Das übliche Vorgehen bei der Ermittlung der Parameter der Verhaltensfunktionen ist die Kalibrierung dieser Parameter. Da Zeitreihen der zugrundeliegenden Datensätze kaum zur Verfügung stehen, werden die Parameter unter Vorgabe der wichtigsten Elastizitätswerte 
direkt aus der Datenbasis berechnet. Die so ermittelten Parameterwerte generieren bei Berechnung des Gleichgewichtes genau wieder die Datenbasis. Diese Eigenschaft wird zur Kontrolle der Berechnung ausgenutzt. ${ }^{68}$

Eine wichtige Entscheidung bei diesem Vorgehen ist die Wahl der vorzugebenden Elastizitäten. Diese sind soweit als möglich bereits in der Literatur geschätzte Elastizitäten, wie Importnachfrageelastizitäten, Substitutionselastizitäten, Arbeitsangebotselastizitäten. Allerdings sollte beachtet werden, daß die Schätzungen sehr häufig stark differieren, oder für die Bundesrepublik gar nicht vorgenommen wurden.

\subsection{Vorgabe der Elastizitäten}

Bezüglich der Produktionsfunktionen werden die jeweiligen Substitutionselastizitäten vorgegeben: Zum einen die zwischen der Primärfaktoren Arbeit und Kapital und zum anderen die zwischen den einzelnen privaten Vorleistungsgütern.

Die hier verwendeten Werte der Substitutionselastizitäten zwischen Kapital und Arbeit $\sigma_{j}^{s}$ $(0,8$ und 0,95) finden sich bei DeardorfF/Stern [1986] (siehe auch Grais/DeMelo/ Urata [1986]). Für die Vorleistungselastizitäten gibt es nur sehr wenig Schätzungen für die Bundesrepublik, die sich zum Teil nur auf zusammengesetzte Zwischenproduktnachfragen beziehen wie z.B. NAKAMURA [1984 und 1986], LÄCHLER [1985] oder RUTNER [1984]. Für die Substitutionselastizität zwischen den privaten Vorleistungsgütern unterschiedlicher Herkunft in beiden Sektoren werden die sektorspezifischen Elastizitäten zwischen heimischen und importierten Vorleistungen verwendet, wie sie HARRISON u.a. ([1991], S.100-101) für die Bundesrepublik präsentieren $\left(\rho_{2 j}^{s}=1,7\right)$. Für die Bezugsnachfrageelastizität des Konsums wird der in HARRISON u.a. [1993] für die EU angegebene Wert der Importnachfrageelastizität verwendet $\left(\epsilon_{s}=-1,1\right) \cdot{ }^{69}$

Der Wert für die unkompensierte Arbeitsangebotselastizität $\varepsilon_{s}=0,15,{ }^{70}$ und der für das Verhältnis von Zeitgrundausstattung zu Arbeitszeit $\zeta_{s}=1,75$ stammen aus BALlaRD, u.a. ([1985], S.135).

\footnotetext{
${ }^{68}$ Nähere Ausführungen zur Problematik der Kalibrierung finden sich bei MansuR/Whalley [1984]. Eine Diskussion des Vorgehens der Kalibrierung liefert im AnschluB daran LaU [1984].

${ }^{69}$ Dies entspricht dem Vorgehen von PiggotT/Whalley [1985a], die vorschlagen, die unkompensierte Eigenpreiselastiziät als Ersatz für die Substitutionselastizität zu verwenden, da letztere bezüglich der Nachfrage zwischen heimischen und importierten Gütern nur selten geschätzt werden. GoLDSTEIN/KHAN [1990], S.1049 präsentieren dazu Schätzungen für die Bundesrepublik, die zwischen -0,72 (1969) und $-1,48$ (1970) liegen.

${ }^{70}$ Für die Bundesrepublik liegen Schätzungen von WEBER [1996] vor, die in etwa um diesen Wert schwanken.
} 


\begin{tabular}{||l|c|c|c||}
\hline \hline \multicolumn{2}{|c|}{ Bereich } & \multicolumn{2}{c||}{$\begin{array}{c}\text { Parameter } \\
\text { exogen vorgegeben }\end{array}$} \\
& $K, L$ & $\sigma=0,8,0,95$ & $\delta_{j}^{s}, \phi_{j}^{s}$ \\
& $V_{C j}^{r s}, \forall r \in \mathcal{R}$ & $\rho_{j}^{s}=1,7$ & $d_{j}^{s}$ \\
\hline \hline Produktion & $G_{s}, U_{s}$ & $\mu_{1 s}=0,5$ & $\beta_{1 s}$ \\
& $C_{s}, F_{s}$ & $\zeta_{s}=1,75, \epsilon_{s}=0,15$ & $\mu_{2 s}, \beta_{2 s}$ \\
& $C_{r s}, \forall r \in \mathcal{R}$ & $\varepsilon_{s}=-1,1$ & $\mu_{3 s} \beta_{r s}$ \\
\hline Außenhandel & & $\eta=0,12 \theta=-10,0$ & \\
\hline \hline
\end{tabular}

Tabelle IV.8: Vorgegebene Elastizitäten und Kalibrierung

Die Substitutionselastizitäten zwischen dem aggregierten privaten und dem öffentlichen Gut wird ebenfalls vorgegeben. Für sie wird der Wert 0,5 für die Substitutionselastizität des öffentlichen Gutes aus Whalley/Trela ([1986], S.128) verwendet, der u.a. auf Schätzungen von POMmEREHNE/SCHNEIDER [1978] zurückgeht.

Die Außenhandelselastizitäten werden so gewählt, daß die in STERN, Francis, SchuHMACHER [1976] angegebene Eigenpreiselastizität der Exportnachfrage von -1,1 erreicht wird. Da dies mit einer unendlichen $\mathrm{Zahl}$ von Kombinationen von $\theta$ und $\eta$ möglich ist, wird angenommen, daß die Elastizitäten einer höheren Preissensitivität der deutschen Exporte als der Importe entsprechen sollen. Dies ist bei $\theta=-10$ und $\eta=0,12$ der Fall.

Einen Überblick über die vorgegebenen Elastizitäten bietet die Tabelle IV.8.

\subsection{Berechnung der Parameter}

\subsubsection{Produktionssektor}

Die Kalibrierung des Produktionssektors wird weitgehend analog zu HirTe/Wiegard [1988] oder HiRTE/GENOSKo [1988/89] durchgeführt.

\section{(1) Wertschöpfungsfunktion}

Bei vorgegebenen Substitutionselastizitäten müssen die Technologieparameter $\phi_{j}^{\text {s }}$ und die Anteilsparameter $\delta_{j}^{s}$ berechnet werden. 
Über die Bedingungen erster Ordnung für eine kostenminimalen Faktoreinsatz erhält man durch Auflösen :

$$
\delta_{j}^{s}=\frac{w_{s} L_{j}^{S^{\frac{1}{\sigma_{1 j}^{3}}}}}{\hat{r}_{j}^{s} K_{j}^{\sigma^{\frac{1}{\sigma 1}}}+w_{s} L_{j}^{s}}
$$

Bei vorgegebenen $\sigma_{j}^{s}$, aus der Datenbasis bekannten $\mathrm{K}_{j}^{s}, \mathrm{~L}_{j}^{s}, \tau_{j s}^{K}, \tau_{j s}^{G w}, \tau_{j s}^{G \tau}$ und gemäß Vereinbarung gegebenen Preisen $\mathrm{w}_{s}$ bzw. $r$ kann $\delta_{j}^{s}$ berechnet werden. Der Technologieparameter $\phi_{j}^{s}$ läßt sich dann leicht aus der Wertschöpfungsfunktion (IV.1) auf Seite 130 berechnen:

$$
\phi_{j}^{s}=a_{j s}^{0} Q_{j}^{s} /\left[\delta_{j}^{s} L_{j}^{s} \frac{\sigma_{j}^{s}-1}{\sigma_{j}^{s}}+\left(1-\delta_{j}^{s}\right) K_{j}^{\frac{\sigma_{j}^{s}-1}{\sigma_{j}^{s}}}\right]^{\frac{\sigma_{s}^{s}}{\sigma_{j}^{s}-1}}
$$

\section{(2) Parameter der disaggregierten Vorleistungsfunktion}

Nach Vorgabe der Substitutionselastizitäten $\rho_{j}^{s}$ und aus der Summierung der notwendigen Bedingung erster Ordnung des Kostenminimierungsproblems (Gleichung (IV.5) auf Seite 131) erhält man als Anteilsparameter

$$
d_{j}^{r s}=\frac{q_{r} V_{C j}^{r s^{\frac{1}{\rho_{j}^{j}}}}}{\sum_{k} q_{k} V_{C j}^{k s}} .
$$

Bei gegebenen $V_{C j}^{r s}, q_{r}$ und $\rho_{C j}^{s}$ sind diese Anteilsparameter $d_{j}^{r s}$ genau bestimmbar.

Den Skalenparamter $\psi_{j}^{s}$ kann man dann einfach durch Umstellung der Vorleistungsnachfragefunktion (IV.6) von Seite 131 berechnen:

$$
\psi_{j}^{s}=\frac{\hat{a}_{j}^{s} \bar{Q}_{j}^{s}}{\left.\left[\sum_{r} d_{j}^{r} V_{C j}^{r s}\right]^{\frac{\rho_{j}^{s}-1}{\rho_{j}^{s}}}\right]^{\frac{\rho_{j}^{s}}{\rho_{j}^{s}-1}}} .
$$

\section{(3) Leontief-Technologie}

Die Anteilsparameter der Vorleistungen und der Wertschöpfung ergeben sich einfach durch Division der Summe des Vorleistungseinsatzes des Sektors durch den Gesamtoutput bzw. 
durch Division der Summe des Primärfaktoreinsatzes (incl. Kapital- und Produktionssteuern) durch den Gesamtoutput:

$$
\hat{a}_{j}^{s}=\frac{\sum_{r} V_{C j}^{r s}}{Q_{j}^{s}} \quad a_{G j}^{s s}=\frac{V_{G j}^{s s}}{Q_{j}^{s}}
$$

bzw.

$$
a_{0 j}^{s}=\frac{L_{j}^{s}+K_{j}^{s}+T_{j s}^{Q}+T_{j s}^{G w}+T_{j s}^{G r}+T_{j s}^{K}}{Q_{j}^{s}} .
$$

\subsubsection{Haushaltssektor}

Beginnend mit dem untersten Ast, der Nachfrage zwischen den einzelnen, nach Herkunft unterschiedenen Produkten werden von unten nach oben die jeweiligen Parameter der entsprechenden Nutzenfunktionen, die indirekten Nutzenfunktionen und die Preisindizes berechnet.

(1) Parameter der Nachfragefunktion nach den einzelnen Konsumgütern Die Preiselastizität der Bezugsnachfrage nach $C_{r s}$ ist:

$$
\varepsilon_{r s}=\frac{\partial C_{r s} / C_{r s}}{\partial p_{r} / p_{r}}
$$

Leitet man die Güternachfragefunktionen (IV.25) auf Seite 140 nach $p_{r}$ ab, erhält man

$$
\frac{\partial C_{r s}}{\partial p_{r}}=-\frac{\beta_{r s} Y_{s}^{C} \mu_{3 s}}{p_{r}^{\mu_{3 s}+1} \Omega_{3 s}}-\frac{\beta_{r s} Y_{s}^{C}}{p_{r}\left(\Omega_{3 s}\right)^{2}}\left(1-\mu_{3 s}\right) \beta_{r s} p_{r}^{-\mu_{3} s}
$$

Setzt man dies wieder in die Nachfragefunktionen (IV.25) (Seite 140) ein ergibt sich über

und nach Umstellung zu

$$
\varepsilon_{r s}=-\mu_{3 s}-\frac{\left(1-\mu_{3 s}\right) \beta_{r s} p_{r}^{\left(1-\mu_{3 s}\right)}}{\Omega_{3 s}}
$$

schließlich

$$
\frac{p_{r} C_{r s}}{Y_{s}^{C}}=\frac{\beta_{r s} p_{r}^{\left(1-\mu_{3 s}\right)}}{\Omega_{3 s}}
$$

$$
\mu_{3 s}=\frac{\left(\varepsilon_{r s} Y_{s}^{C}+p_{r} C_{r s} / Y_{s}^{C}\right)}{p_{r} C_{r s}-Y_{s}^{C}} .
$$

Aus der Datenbasis sind $C_{r s}, Y_{s}^{C}, p_{r}$ bekannt und somit kann $\mu_{3 s}$ berechnet werden. Die noch fehlenden $\beta_{r s}$ können nach einigen Umformungen aus dem Quotienten der Nachfragefunktionen (IV.25) wie folgt ermittelt werden:

$$
\beta_{r s}=\frac{p_{\tau}^{\mu_{3 s}} C_{r s}}{\sum_{r \in \mathcal{R}} p_{\tau}^{\mu_{3 s}} C_{r s}} .
$$




\section{(2) Parameter der Nachfrage zwischen Freizeit und C}

Da Substitutionselastizitäten zwischen Freizeit und Konsum kaum geschätzt werden können, werden zur Berechnung der Parameter der Gesamtnutzenfunktion die leichter ermittelbaren unkompensierten Arbeitsangebotselastizitäten $\epsilon_{s}$ vorgegeben. Diese werden zuerst umgewandelt in die Lohnelastizität der Freizeitnachfrage $\epsilon_{s}^{F}$ und anschließend über die Ableitung der Freizeitnachfrage $F_{s}$ nach dem Lohn die Substitutionselastizität zwischen Freizeit und dem zusammengesetzten Gut ermittelt. Durch Einsetzen dieses Wertes und des über die indirekte Nutzenfunktion ermittelten Preisindexes für dieses zusammengesetzte Gut in das nach $\beta_{2 s}$ aufgelöste Verhältnis der Nachfragefunktionen nach Freizeit und der Endnachfrage erhält man $\beta_{2 s}$.

$$
\epsilon_{s}=\frac{\partial L_{s} / L_{s}}{\partial w_{s}^{n} / w_{s}^{n}} \quad \text { und } \quad \epsilon_{s}^{F}=\frac{\partial F_{s} / F_{s}}{\partial w_{s}^{n} / w_{s}^{n}}
$$

Da $\partial \bar{E}_{s} / w_{s}^{n}=0$, und das Verhältnis von Arbeitszeit $L_{s}$ zu Zeitgrundausstattung $\bar{E}_{s}$ als $\zeta=\bar{E}_{s} / L_{s}$ definiert wird, erhäält man

$$
\epsilon_{s}^{F}=\frac{\partial\left(\bar{E}_{s}-L_{s}\right) /\left(\bar{E}_{s}-L_{s}\right)}{\partial w_{s}^{n} / w_{s}^{n}}=-\frac{\partial L_{s} /\left(\bar{E}_{s}-L_{s}\right)}{\partial w_{s}^{n} / w_{s}^{n}}=-\frac{\epsilon_{s}}{\left(\zeta_{s}-1\right)} .
$$

Die Substitutionselastizität $\mu_{2 s}$ ergibt sich dann über die Ableitung der Freizeitnachfrage (Gleichung (IV.21) auf Seite 140)

$$
\frac{\partial F_{s}}{\partial w_{s}^{n}}=-\frac{\mu_{s} F_{s}}{w_{s}^{n}}+\frac{\beta_{s} \bar{E}_{s}}{w_{s}^{n} \mu_{2 s} \Omega_{2 s}}-\frac{\beta_{2 s} F_{s}\left(1-\mu_{2 s}\right)}{\Omega_{2 s}} .
$$

Eingesetzt in (IV.45) führt dies zu

$$
\epsilon_{s}^{F}=-\mu_{2 s}+\frac{\beta_{2 s} w_{s}^{n\left(1-\mu_{2 s}\right)} \bar{E}_{s}}{F_{s} \Omega_{2 s}}-\frac{\beta_{s}\left(1-\mu_{2 s}\right) w_{s}^{n}\left(1-\mu_{2 s}\right)}{\Omega_{2 s}} .
$$

Definiert man $a_{s}^{F}$ als Anteil der Ausgaben für Freizeit am vollen Einkommen

$$
a_{s}^{F}=\frac{w_{s}^{n} F_{s}}{Y_{s}}=\frac{\beta_{2 s} w_{s}^{n}\left(1-\mu_{2 s}\right)}{\Omega_{2 s}}
$$

und $a_{s}^{E}$ als den Anteil des Wertes der Zeitgrundausstattung am vollen Einkommen

$$
a_{s}^{E}=\frac{w_{s} \bar{E}_{s}}{Y_{s}}
$$

dann kann man (IV.46) auflösen und erhält die Substitutionselastizität zwischen Freizeit und dem aggregierten privaten Konsumgut:

$$
\mu_{2 s}=\frac{a_{s}^{E}-a_{s}^{F}-\epsilon_{s}^{F}}{1-a_{s}^{F}} .
$$


Da alle Variablen aus der Datenbasis bekannt sind, ist $\mu_{2 s}$ ermittelbar.

Nun fehlen nur noch die Budgetparamter $\beta_{2 s}$, die durch Auflösung des Quotienten aus Freizeitnachfrage (IV.21) und Konsumnachfrage (IV.22) (auf Seite 140) hergeleitet werden können:

$$
\beta_{2 s}=\frac{w_{s}^{n_{2 s}} F_{s}}{p_{C}^{s^{\mu_{2} s}} C_{s}+w_{s}^{n^{\mu_{s}}} F_{s}} .
$$

In dieser Gleichung ist $C_{s}$ unbekannt, das aus der indirekten Nutzenfunktion

$$
C_{s}=Y_{s}^{C} \Omega_{3 s}^{\frac{1}{\left(\mu_{3 s}-1\right)}}
$$

berechnet werden kann.

\section{(3) Parameter der Nachfrage zwischen dem öffentlichen und privaten Gut}

Der Budgetparameter der Nutzenfunktionen zwischen dem composite commodity $U_{s}$ und dem regionalen öffentlichen Gut $G_{\boldsymbol{s}}$ kann, unter Vorgabe einer entsprechenden Substitutionselastizität $\mu_{1 s}$, aus der Nutzenmaximierung (IV.15) (Seite 137) und über die notwendigen Bedingungen erster Ordnung (IV.16) von Seite 138 berechnet werden. Als Ergebnis erhält man

$$
\beta_{s}=\frac{\hat{q}_{G_{s}}^{\mu_{1 s}} G_{s} / p_{U_{s}}^{\mu_{1 s}} C_{s}}{1+\left[\hat{q}_{G s}^{\mu_{1 s}} G_{s} / p_{U_{s}}^{\mu_{1 s}} U_{s}\right]} .
$$

Dabei ist $p_{s}$ aus der Formel (IV.11) auf Seite 136 bekannt aber $U_{s}$ noch unbekannt, da $U_{s}$ nicht im Datensatz auftaucht. Über die indirekte Nutzenfunktion

$$
U_{s}=Y_{s}^{U} \Omega_{2 s}^{\frac{1}{\left(\mu_{2 s}-1\right)}} .
$$

kann nun unter Verwendung des Konsumgüterpreisindexes aus Gleichung (IV.10) auf Seite 136 und den anderen Größen, die alle aus der Datenbasis gegeben sind, der Budgetanteilsparameter $\beta 1 s$ bestimmt werden.

Damit sind nun alle Parameter des Modells bestimmt. Man kann jetzt unter Verwendung des theoretischen Modells und der Datenbasis die gewünschten Simulationen durchführen. 


\section{Kapitel V}

\section{Simulationen}

\section{Einführung zu den Berechnungen}

In diesem Kapitel werden die Ergebnisse der Empirischen Allgemeinen Gleichgewichtsanalyse (EAG) der alternativen Finanzausgleichsregelungen präsentiert und ökonomisch interpretiert.

In den Berechnungen werden drei Szenarien untersucht.

- Als erstes werden die Auswirkungen des Übergangs von den vor 1995 geltenden Finanzausgleichsregelungen zu dem ab 1995 eingeführten Finanzausgleichsregime betrachtet. Das Hauptaugenmerk liegt bei diesen Berechnungen auf dem Einbezug der Ostländer in das bundesdeutsche Finanzausgleichssystem.

- In einer zweiten Serie von Berechnungen werden die Auswirkungen eines Wechsels der Finanzausgleichsregelungen nach dem Jahr 1995 untersucht. Diese Simulationen ermöglichen einen Vergleich der unterschiedlichen Neuregelungsmodelle im Hinblick auf ihre Wohlfahrts- und Verteilungswirkungen.

- Schließlich werden die Wohlfahrtseffekte einer stärkeren Finanzautonomie der Bundesländer, in Form eines regionalen Einkommenssteuerzuschlages, berechnet.

Der Präsentation der Ergebnisse wird ein Abschnitt vorangestellt, in dem einige der in der EAG-Analyse verwendeteten Konzepte erläutert werden.

\section{1. Überprüfung des EAG-Modells}

Aufgrund der Komplexität des in Kapitel IV vorgestellten EAG-Modells ist eine theoretische Analyse der Güte des theoretischen Modells und der resultierenden Ergebnisse nicht mehr möglich. Allerdings können einige einfache Kontrollmechanismen zumindest grobe Umsetzungsfehler bei der Programmierung des Modells aufdecken. Drei solcher Kontrollmechanismen sind im Programm endogenisiert. Nach jeder Iteration erfolgt eine Überprüfung des Walras-Gesetzes, das nicht verletzt sein darf. Am Ende jeder Simulation 
müssen sämtliche Gleichgewichtsbedingungen des Modells, sowie die privaten Budgetgleichungen und die Nullprofitbedingungen erfüllt sein. Dies wird im Modellablauf kontrolliert. Schließlich wird eine Kontrollrechnung replication check durchgeführt, in der die kalibrierten Parameter vorgegeben werden. Resultiert daraus der vorgegebene Datensatz, kann zumindest von der Konsistenz der Berechnungen in der Kalibrierung und im Modell ausgegangen werden. Darüber hinaus erzeugen die Simulationen in Verbindung mit den Sensitivitätsanalysen eine Reihe von Ergebnissen, die theoretisch nachvollziehbar sind. Auch dies ist ein Indikator für die Richtigkeit der Modellspezifikation. ${ }^{1}$

Als Ergebnisse der Simulationen erhält man jeweils einen vollständigen neuen Datensatz mit einer sehr hohen Anzahl von Einzeldaten. Um sich nicht in diesem Datendickicht $z u$ verlieren, und sich auf einige wesentliche Resultate beschränken zu könnnen, ist es notwendig, einige Kriterien zur Beurteilung der Ergebnisse zu spezifizieren.

\subsection{Grundsätzliche Vorgehensweise in den Simulationen}

Die Simulation verlaufen immer nach demselben Schema:

Ausgehend vom ursprünglichen Gleichgewicht, bei gegebener Finanzausgleichsregelung, wird ein neues Finanzausgleichssystem eingeführt. Dies führt über veränderte Zahlungsströme zwischen den Ländern schließlich zu einer Veränderung der Faktorpreise und der staatlichen Handlungsparameter, wodurch Rückwirkungen auf die anderen Größen des Systems incl. der Finanzausgleichszahlungen ausgelöst werden. Solange die Faktorüberschußnachfragen und die staatlichen Budgets nicht ausgeglichen sind, kommt es immer wieder zu neuen Preisveränderungen mit entsprechenden Folgen für das System. Dieser Prozeß kommt erst zum stoppen, wenn ein Preisvektor gefunden ist, der zu einem Ausgleich sämtlicher Gleichgewichtsbedingungen führt. Die aus diesem Preisvektor abgeleiteten neuen Werte der ökonomischen Variablen - Faktornachfragen, Güternachfragen, Finanzausgleichszahlungen etc. - stellen das neue Gleichgewicht nach Durchführung der Reform dar. Der Vergleich dieser Werte mit denen des Ausgangsgleichgewichts offenbart die durch die Reform ausgelösten Veränderungen. Das Vorgehen entspricht damit einer komperativ-statischen Analyse.

\footnotetext{
${ }^{1}$ Im Prinzip könnte man, wie FeHR ([1993], S.96-97) demonstriert, durch eine starke Vereinfachung des Modells, die Übereinstimmung der Resultate mit den theoretischen Erwartungen untersuchen. Da das vorliegende Modell eine Struktur besitzt, die der des Modells von FeHr/Rosenberg/Wiegard [1992] sehr ähnlich ist, soll, mit dem Hinweis auf die Kontrollberechnungen bei FEHR [1993], davon ausgegangen werden, $\mathrm{da} B$ auch die mit dem hier vorliegenden Modell durchgeführten Berechnungen weitgehend theoretisch abgesichert sind.
} 
Welcher der staatlichen Handlungsparameter endogen das Budget ausgleicht, hängt von dem zugrundegelegten Inzidenzkonzept ab. Hier wird in fast allen Simulationen das Konzept der Budgetinzidenz verwendet, in dem die Ausgabenseite bei konstanter Steuersatzstruktur an veränderte öffentliche Einnahmen angepaßt wird. Über die Aufteilung der so entstehenden regionalen öffentlichen Ausgaben auf öffentliche Güter und Transfers wird dann mittels eines Nutzenkalküls entschieden. Der Ausgleich der Ausgaben auf nationaler Ebene erfolgt durch eine Anpassung der Summe der Zuweisungen an die Regionen, die proportional zu den ursprünglichen Zuweisungen auf die einzelnen Regionen aufgeteilt werden.

Will man dagegen die Ausgaben konstant halten, und daher die Einnahmenseite an veränderte LFA - und/oder Steuereinnahmen anpassen, dann entspricht dies dem Konzept der Differentialinzidenz. ${ }^{2}$ Eine Konstanz der Ausgaben entspricht sinnvollerweise einer Konstanz der realen Ausgaben, da ansonsten jede Preisveränderung die Höhe der öffentlichen Güternachfrage verändert. Wie üblich werden zu diesem Zweck die Ausgaben einer Region oder des Staates mit einem regionalen bzw. nationalen LaspeyresKonsumpreisindex gewichtet. ${ }^{3}$

Im Konzept der differentiellen Inzidenz wird vorausgesetzt, daß die öffentlichen Haushalte Steuersätze variieren können, um ihre Einnahmenseite auszugleichen. Da dies in der vorliegenden Untersuchung nur im Falle der Finanzautonomie der Fall ist, wird hier das Konzept der differentiellen Inzidenz nur bei der Untersuchung einer höheren Steuerautonomie verwendet. ${ }^{4}$

\subsection{Konzepte zur Beurteilung der Ergebnisse}

Im folgenden werden einige Konzepte dargestellt, die zur Beurteilung der gesamten Wohlfahrts- und Verteilungswirkungen dienen und/oder Hilfen zu einer detaillierteren Analyse der Resultate geben.

\footnotetext{
${ }^{2}$ Dieser Ansatz geht auf MuSgrave [1959] zurück.

${ }^{3}$ Der Laspeyres-Index ist nicht unumstritten, da er einen Preisanstieg überschätzt. Der Paasche-Index ist allerdings im Programmablauf nicht verwendbar, da die benötigten Mengenveränderungen erst gegen Ende einer Iteration berechnet werden können. Siehe vor allem SHoven/WHALLEy [1977].

${ }^{4}$ Dieses Vorgehen ist allerdings nicht mit einer Bestimmung von Optimalsteuersätzen zu verwechseln, da die Steuersätze in der Höhe bestimmt werden, daß gerade das regionalstaatliche Budget ausgeglichen wird. Sie entspringen damit keiner nutzenmaximalen Wahl. Ein EAG-Modell zur Bestimmung optimaler Steuersätze präsentiert z.B. YoRK [1993].
} 


\subsubsection{Wohlfahrtsmessung}

Unter der Vielzahl möglicher Wohlfahrtsmaße haben sich diejenigen weitgehend durchgesetzt, in denen die Nutzenveränderung in Form eines Einkommensäquivalentes gemessen wird. Dies sind vor allem die Hicksche Äquivalente Variation (HEV) und die Einkommenskompensierende Variation (HCV) (siehe z.B. VARIAN [1992], S.160-163). ${ }^{5}$ In dieser Arbeit wird der HEV der Vorzug gegeben, da sie die Nutzenveränderung anhand der Verwendung der urspünglichen Preise mißt. Aufgrund identischer Ausgangspreise in den verschiedenen Simulationen ermöglicht dies einen unproblematischen Vergleich der Ergebnisse unterschiedlicher Berechnungen.

Die HEV ist ein einkommensäquivalentes Maß für eine Nutzenveränderung und mißt, mit wieviel Einkommen der betrachtete Haushalt kompensiert werden muß, um bei dem ursprünglichen Preisniveau das gleiche Nutzenniveau zu erreichen, das er mit den neuen Preisen erzielen würde (siehe z.B.VARIAN [1992]). Sie kann bei Verwendung von CESFunktionen (siehe GotTFRIED [1992], S.211-212) wie folgt definiert werden:

$$
H E V_{s}=\frac{H_{s}^{1}-H_{s}^{0}}{H_{s}^{0}} Y_{s} \text {. }
$$

Neben den Wohlfahrtsgewinnen der einzelnen Länder interessieren jedoch vor allem gesamtgesellschaftliche Wohlfahrtswirkungen der unterschiedlichen Finanzausgleichsregelungen. Zu diesem Zweck wird angenommen, daß die Summe der HEV's ein brauchbarer Indikator der gesamtwirtschaftlichen Wohlfahrtseffekte darstellt. ${ }^{6}$

Allerdings ist, aufgrund des BOADWAY-Paradoxons (siehe BoADWAY $[1974])^{7}$, keineswegs gesichert, daß ein gesamtgesellschaftlicher Wohlfahrtsgewinn auch einer nationalen Pareto-Verbesserung entspricht. Damit dies der Fall ist, müßte grundsätzlich eine Kompensation der „Verlierer” einer politischen Reform durch die „Gewinner” möglich sein. Im Rahmen einer EAG-Analyse ist daher in jedem Einzelfall zu überprüfen, ob eine positive HEV-Summe auch tatsächlich eine Pareto-Verbesserung impliziert. Unter Verwendung des Verfahrens von FEHR/ROSENBERG/WIEGARD ([1992], S.82) wurden hier entsprechende Untersuchungen durchgeführt. ${ }^{8}$ Ergebnis dieser Berechnungen war, daß in allen kontrollierten Fällen eine positive Summe der regionalen HEV's auch einer Pareto-Verbesserung

\footnotetext{
${ }^{5}$ Eine ausführliche Diskussion alternativer Wohlfahrtsmaße wird beispielsweise in AHLHEIM/Rose [1989] geführt.

${ }^{6}$ Dieses Vorgehen ist jedoch sehr umstritten, da eine Aggregation der HEV's unzulässige interpersonelle Nutzenvergleiche anstellt (siehe dazu Morey ([1984], S.167) oder AhLheim/Rose [1984], S.345) Diese Position wird von von EberT ([1987], S.24, S.45) zurückgewiesen, der unter bestimmten Annahmen die Zulässigkeit der Aggregation zeigt.

${ }^{7}$ Siehe auch EBERT ([1987], Kap.4) oder STöss ([1991], S.112-114).

${ }^{8}$ Dabei wird wie folgt vorgegangen: Ausgehend vom dem neuen Gleichgewicht, nach Durchführung der
} 
entsprach. Deshalb wird im weiteren angenommen, daß eine gesellschaftliche Wohlfahrtsverbesserung (eine positive Summe der HEV's) auch einer Pareto-Verbesserung entspricht.

\subsubsection{Verteilungsmessung}

Als Maß für die Verteilungwirkungen wird der Gini-Koeffizient verwendet. Je näher dieser dem Wert eins ist, um so ungleicher ist die Verteilung. Da dieses Maß eine Gesamtgröße ist, ist es durchaus möglich, daß eine Umverteilung für ein Land zu einem stärkeren Abweichen vom Durchschnitt führt, wenn dafür andere Länder näher am Durchschnitt liegen.

\section{Hilfsgrößen zur detaillierteren Untersuchung}

Die theoretische Analyse der Wohlfahrtswirkungen in Kapitel III hat drei Arten von Effekten isoliert. Neben den intraregionalen Substitutionseffekten sind dies die internationalen Substitutions- und die interregionalen Einkommenseffekte. Zur Interpretation der Ergebnisse wäre es wünschenswert, diese Effekte auch numerisch zu trennen, um ihre Einflüsse genauer bestimmen zu können.

Zur Berechnung der Substitutionseffekte werden die Einkommenseffekte dadurch eliminiert, daß die Nettotransfers der Länder, die sie nach Durchführung der Reform erhalten, durch ein Ausgleichssystem zurücküberwiesen werden, und die Simulation wiederholt wird. ${ }^{9}$ Als Ergebnis erhält man die Substitutionseffekte der Politikveränderung. Allerdings sollte dabei beachtet werden, daß in dieser Berechnung der Substitutionseffekte, anders als in Partialmodellen, die Substitutionseffekte ihrerseits über veränderte Nettotransfers neue Einkommenseffekte auslösen. Von daher entsprechen die ermittelten Substitutionseffekte nicht denen partialanalytischer Untersuchungen.

Zur Bestimmung der internationalen Substitutionseffekte werden die Terms of Trade verwendet. Analog zu FEHR ([1993], S.103-104) werden die sich in den Berechnungen der Wirkungen von Substitutionseffekten ergebenden Terms of Trade (ToT $T_{S E}$ ) als die ToT interpretiert, die durch die Substitutionseffekte verursacht werden. Die Vorzeichen der intraregionalen Substitutionseffekte ergeben sich dann aus der Differenz der

Reform, leisten die Gewinner einer Reform Transfers an die Verlierer, die diese genau auf Nutzenniveau vor der Reform anheben. Gibt es danach noch immer Gewinner, kann man das Reformprogramm als Pareto-Verbesserung ansehen.

${ }^{9}$ Dieses Verfahren wurde von FEHR entwickelt und dargestellt (FEHR ([1993], S.103-104)). 
Wohlfahrtswirkungen der Substitutionseffekte $\left(\mathrm{HEV}_{S E}\right)$ und der prozentualen $\mathrm{ToT}_{S E^{-}}$ Veränderung. Die exakte Größenordnung der intraregionalen oder interregionalen Substitutionseffekte kann allerdings nicht näher spezifiziert werden.

Die interregionalen Einkommenseffekte werden durch Ermittlung der Nettotransfers unter Berücksichtigung von Steuerexporten abgeschätzt. Ihr Einfluß auf die Terms of Trade ergibt sich aus der Differenz zwischen den ToT der jeweiligen Hauptberechnung $\left(T_{0} T_{G E}\right)$ und den ToT $T_{S E}$. Die Berechnung der Steuerexporte bedingt, daß zusätzlich internationale Einkommenseffekte isoliert werden können, die durch Steuerexporte an das Ausland ausgelöst werden.

\subsection{Terms of Trade Definition}

In der vorliegenden Arbeit wird die Terms of Trade Definition der net-barter terms of trade verwendet, in der die Ex- und Importpreisindizes anstelle von Mengenindizes berücksichtigt werden. Zusätzlich wird der Laspeyres-Preisindex verwendet, in den die ursprünglichen Mengen als Gewichte eingehen. Dies ermöglicht eine bessere Vergleichbarkeit alternativer Ergebnisse. Da im vorliegenden Modell keine Einfuhrumsatzsteuern erhoben werden und keine Transportkosten existieren etc. und daher die Weltmarktpreise den Inlandspreisen entsprechen, werden die Im- und Exporte mit den Produzentenpreisen in der ToT-Berechnung berücksichtigt. ${ }^{10}$ Folglich erhält man als Definition der Terms of Trade:

$$
T o T_{s}=\frac{\frac{\sum_{r}\left(p_{s}\right)_{1}\left(C_{s r}\right)_{0}+\sum_{r}\left(q_{s}\right)_{1} \sum_{j}\left(V_{C j}^{s r}\right)_{0}+\left(q_{s}\right)_{1}\left(X_{s}\right)_{0}}{\sum_{r}\left(p_{r}\right)_{0}\left(C_{s r}\right)_{0}+\sum_{r}\left(q_{s}\right)_{0} \sum_{j}\left(V_{C j}^{s r}\right)_{0}+\left(q_{s}\right)_{0}\left(X_{s}\right)_{0}}}{\frac{\sum_{r}\left(p_{s}\right)_{1}\left(C_{r s}\right)_{0}+\sum_{r}\left(q_{r}\right)_{1} \sum_{j}\left(V_{C j}^{r s}\right)_{0}+\left(q_{s}\right)_{1}\left(M_{s}\right)_{0}}{\sum_{r}\left(p_{r}\right)_{0}\left(C_{r s}\right)_{0}+\sum_{r}\left(q_{r}\right)_{0} \sum_{j}\left(V_{C j}^{r s}\right)_{0}+\left(q_{s}\right)_{0}\left(M_{s}\right)_{0}}} .
$$

\subsection{Steuerexporte und interregionale Transfers}

Um die interregionalen und internationalen Einkommenseffekte genauer bestimmen zu können, ist es wünschenswert, die Transfers zwischen den Ländern zu ermitteln. Normalerweise würde man dazu die Transferbilanz betrachten und im Falle eines Nettoüberschusses von positiven Nettotransfers ausgehen. Allerdings enthalten die so berechneten Nettotransfers, bei vorhandenen Steuerexporten, keineswegs alle erhaltenen oder geleisteten interregionalen und internationale Zahlungen. Vielmehr müßte man auch die Steuerexporte, als Steuerzahlungen des Auslandes an das Inland, zu den Nettotransfers hinzuzählen, da sie interregionale oder internationale Einkommenseffekte auslösen.

\footnotetext{
${ }^{10}$ Eine ausführliche Diskussion alternativer Terms-of-Trade-Konzepte findet sich bei GuTMANN [1981]. Eine Begründung für die Auswahl der hier verwendeten Terms-of-Trade-Definition bietet FEHR [1993].
} 
Die Steuerexporte bestehen aus den von den anderen Ländern erhaltenen Steuerzahlungen, abzüglich der an die anderen Länder geleisteten Steuerzahlungen der Region. Sie umfassen neben den Konsumsteuerexporten auch die überwälzten Faktor- und Produktionssteuerexporte. Unter Verwendung der von GoTTFRIED ([1992], Kap.2) entwickelten formalen Herleitung der effektiven Umsatzsteuersätze werden die in den Produzentenpreisen enthaltenen effektiven Produktions- und Faktorsteuersätze berechnet. Nimmt man an, daß die Steuern voll auf die Endnachfrager überwälzt werden, dann kann man mit Hilfe der effektiven Steuersätze die durch die Endnachfrager geleisteten Steuerzahlungen ermitteln, und damit die Steuerexporte bestimmen. Die effektiven Faktor- und Produktionssteuersätze werden im folgenden abgeleitet.

\subsubsection{Effektiver Kapitalsteuergehalt der Produzentenpreise}

Die Bestimmungsgleichung für die Produzentenpreise (IV.8)

$$
\mathbf{q}=\left(\mathbf{E}^{*}-\mathbf{A}^{\prime}(\mathbf{q})\right)^{-1} \mathbf{W}
$$

kann man durch Aufspaltung des Vektors der Faktoreinheitskosten in einen Vektor der Nettofaktorkosten $\mathbf{W}^{n}$ und einen Vektor der Steuerkosten $\mathbf{W}^{t}$, der die Elemente $\tau_{j s}^{k} r k_{j}^{s}$ für alle Güter und Regionen enthält, umformen in

$$
\mathbf{q}=\left(\mathbf{E}^{*}-\mathbf{A}^{\prime}(\mathbf{q})\right)^{-1}\left(\mathbf{W}^{n}+\mathbf{W}^{t}\right) \text {. }
$$

Daraus erhält man

$$
\mathbf{q}-\left(\mathbf{E}^{*}-\mathbf{A}^{\prime}(\mathbf{q})\right)^{-1} \mathbf{W}^{t}=\left(\mathbf{E}^{*}-\mathbf{A}^{\prime}(\mathbf{q})\right)^{-1} \mathbf{W}^{n}=\tilde{\mathbf{q}}
$$

Die linke Seite der Gleichung entspricht dem Produzentenpreisvektor abzüglich der Kapitalsteuerkosten, die rechte Seite dem Nettoproduzentenpreis q̃. Die Differenz aus dem Produzentenpreis und dem Nettoproduzentenpreis gibt dann den Steuerbetrag pro Einheit des Endnachfragegutes an

$$
\tilde{\mathbf{t}}^{K}=\left(\mathbf{E}^{*}-\mathbf{A}^{\prime}(\mathbf{q})\right)^{-1} \mathbf{W}^{t}=\mathbf{q}-\tilde{\mathbf{q}},
$$

aus dem die effektiven Kapitalsteueranteile in den Nettoproduzentenpreisen ermittelt werden können

$$
\tilde{\tau}_{j s}^{e K}=\frac{q_{j}^{s}-\tilde{q}_{j}^{s}}{\tilde{q}_{j}^{s}} .
$$

Als Steuersatz auf den (Brutto-)Produzentenpreis ergibt sich daraus

$$
\tau_{j s}^{e K}=\frac{\tilde{\tau}_{j s}^{e K}}{1+\tilde{\tau}_{j s}^{e K}} q_{j}^{s} .
$$




\subsubsection{Effektiver Produktionssteuergehalt der Produzentenpreise}

In der Bestimmungsgleichung für die Produzentenpreise (IV.8)

$$
\mathbf{q}=\left(\mathbf{E}^{*}-\mathbf{A}^{\prime}(\mathbf{q})\right)^{-1} \mathbf{W}
$$

besteht die Matrix $\mathbf{E}^{*}$ aus dem Einheitsvektor $\mathbf{E}$, abzüglich der Produktionssteuerterme $1 /\left(1+\tau_{j s}^{Q}\right)$ in der Diagonalen. Nach Definition des Vektors $t^{Q}$ als Vektor der Produktionssteuerterme $1 /\left(1+\tau_{j s}^{Q}\right)$ erhält man:

$$
\mathbf{q}=\left(\mathbf{E}-\mathbf{A}^{\prime}(\mathbf{q})-\mathbf{E} \mathbf{t}^{Q}\right)^{-1} \mathbf{W} .
$$

Der entsprechende Produzentenpreisvektor nach Abzug der Produktionssteuern ist

$$
\tilde{\mathbf{q}}=\left(\mathbf{E}-\mathbf{A}^{\prime}(\mathbf{q})\right)^{-1} \mathbf{W} \text {. }
$$

$\mathrm{Da} \mathbf{W}$ in beiden Fällen gleich ist, folgt daraus:

$$
\left(\mathbf{E}-\mathbf{A}^{\prime}(\mathbf{q})-\mathbf{E} \mathbf{t}^{Q}\right) \mathbf{q}=\left(\mathbf{E}-\mathbf{A}^{\prime}(\mathbf{q})\right) \tilde{\mathbf{q}}
$$

Dies führt über

$$
\left(\mathbf{E}-\mathbf{A}^{\prime}(\mathbf{q})\right) \mathbf{q}-\mathbf{E} \mathbf{t}^{Q} \mathbf{q}=\left(\mathbf{E}-\mathbf{A}^{\prime}(\mathbf{q})\right) \tilde{\mathbf{q}}
$$

und

$$
\left(\mathbf{E}-\mathbf{A}^{\prime}(\mathbf{q})\right)(\mathbf{q}-\tilde{\mathbf{q}})=\mathbf{E} \mathbf{t}^{Q} \mathbf{q}
$$

$\mathrm{zu}$

(a)

$$
\tilde{\mathbf{t}}^{Q}=(\mathbf{q}-\tilde{\mathbf{q}})=\left(\mathbf{E}-\mathbf{A}^{\prime}(\mathbf{q})\right)^{-1} \mathbf{E} \mathbf{t}^{Q} \mathbf{q}
$$

mit $\tilde{\mathrm{t}}^{Q}$ als Vektor der effektiven Produktionssteuerbeträge pro Besteuerungseinheit.

Da der Produzentenpreis incl. Produktionssteuer, $q_{j}^{s}$, gleich dem neuen Produzentenpreis abzüglich der Produktionssteuer, aber incl. effektiver Steuersätze, $\tilde{q}_{j}^{s}$, sein soll, gilt

$$
\left(1+\tau_{j s}^{e Q}\right) \tilde{q}_{j}^{s}=q_{j}^{s} .
$$

Zugleich folgt aus (a)

$$
\tilde{t}_{j s}^{Q}=q_{j}^{s}-\tilde{q}_{j}^{s}
$$


Daraus ergibt sich

$$
\tilde{\tau}_{j s}^{e Q}=\frac{\tilde{t}_{j s}^{Q}}{\tilde{q}_{j}^{s}}=\frac{q_{j}^{s}-\tilde{q}_{j}^{s}}{\tilde{q}_{j}^{s}},
$$

als effektive Steuersätze bezogen auf den Herstellungspreis $\tilde{q}_{j}^{s}$. Bezogen auf den (Brutto-) produzentenpreis erhält man:

$$
\tau_{j s}^{e Q}=\frac{\tilde{\tau}_{j s}^{e Q}}{1+\tilde{\tau}_{j s}^{e Q}} q_{j}^{s} .
$$

\subsubsection{Nettotransfers}

Zur Ermittlung der interregionalen Transfers werden die Einnahmen der Region aus den Steuern (Steuerverteilung $T_{s}$ ), den Zuweisungen (Länderfinanzausgleich $F A_{s}$, Ergänzungszuweisungen $E Z_{s}$ und Zuweisungen $\left.Z_{s}\right)$ sowie den Transfers $\left(T R_{s}\right)$ aufaddiert, und von diesen die Steuerzahlungen der Inländer subtrahiert. Letztere werden mit Hilfe der Konsumsteuersätze sowie der effektiven Produktions- und Faktorsteuersätze berechnet. Die Differenz zwischen den Einnahmen aus der Steuerverteilung und den Steuerzahlungen der Inländer entspricht den regionalen Steuerexporten.

Als Nettoransfers $T R_{s}^{n}$ erhält man schließlich ${ }^{11}$ :

$$
\begin{aligned}
T R_{s}^{n} & =F A_{s}(\cdot)+E Z_{s}(\cdot)+Z_{s}+T R_{s}+T_{s}\left(j-\sum_{r}\left(\tau^{C}+\tau_{C s}^{e Q}+\tau_{C s}^{e K}\right) q_{r} C_{r s}-\right. \\
& -\left(\tau_{C s}^{e Q}+\tau_{C s}^{e K}\right) q_{s} I M_{s}-\left(\tau_{G s}^{e Q}+\tau_{G s}^{e K}\right) q_{G s} G_{s}
\end{aligned}
$$

\subsection{Sensitivitätsanalysen}

Da die Parameter und einige der Elastizitäten des Modells nicht auf statistischen Schätzungen beruhen, ist es notwendig, die Robustheit der Ergebnisse bezüglich einer Variation der entsprechenden Werte zu untersuchen. Zu diesem Zweck werden Sensitivitätsanalysen durchgeführt, die darin bestehen, daß die zentralen Parameter variiert und die daraus resultierenden Ergebnisse miteinander verglichen werden. Unterscheiden sich die Resultate nur geringfügig, kann davon ausgegangen werden, daß die Ergebnisse der EAG-Analyse einigermaßen robust sind. Schwanken sie dagegen sehr stark, oder ändern sich sogar die

\footnotetext{
${ }^{11}$ In dieser Gleichung sind Steuerzahlungen für Importe enthalten, die sich auf die effektiven Steuersätze des Inlandes beziehen. Damit wird implizit unterstellt, daß die effektiven Steuersätze des Auslandes mit denen des Inlandes identisch sind.
} 
Vorzeichen, dann kann man, ohne Kenntnis der „wahren” Parameter, keine fundierte Aussagen über die Auswirkungen der jeweiligen Politikreform machen.

Die Sensitivitätsanalysen können noch einem weiteren Zweck dienen. Die Variationen einzelner Werte (z.B. Arbeitsangebotselastizitäten) oder eine leichte Veränderung der Modellstruktur (z.B. Kapitalmobilität) können die Interpretationen erleichtern. Auf diese Weise läßt sich der Einfluß einzelner Reaktionen, z.B. Arbeitsangebotsentscheidung, oder die Rolle bestimmter Modellteile, z.B. Kapitalmobilität, genauer untersuchen.

Da es kaum möglich ist, die unendliche Vielzahl von Rechnungen vorzunehmen, die zu einer umfassenden Sensitivitätsanalyse notwendig wären, werden nur einige Berechnungen durchgeführt. ${ }^{12}$ Von diesen werden diejenigen präsentiert, die die höchsten Abweichungen von den Resultaten des Standardfalles produzieren und/oder den Einfluß einzelner Elastizitäten demonstrieren.

Die in dieser Arbeit vorgenommenen Sensitivitätsanalysen beziehen sich auf die Variation der

- Substitutionselastizitäten zwischen dem öffentlichen Gut und dem aggregierten privaten Gut,

- Substitutionselastizitäten zwischen Freizeit und Konsum, die aus den unkompensierten Arbeitsangebotselastizitäten abgeleitet werden,

- Bezugsnachfrageelastizitäten, aus denen die Substitutionselastizitäten zwischen den einzelnen, regionalen Gütern abgeleitet werden,

- Exportnachfrage- und Importangebotselastizitäten des Auslandes,

- Substitutionselastizitäten zwischen Kapital und Arbeit,

- und der Substitutionselastizitäten zwischen den einzelnen Vorleistungsgütern.

Zusätzlich erfolgt eine Erweiterung des Modells durch eine Einschränkung der Kapitalmobilität.

Damit sind die wesentlichen Konzepte dargestellt, die zur Interpretation der Ergebnisse hilfreich sein können. Im folgenden werden nun die Resultate präsentiert und erläutert.

${ }^{12}$ Eine kurze Darstellung der verfügbaren Sensitivitätsverfahren findet sich in FEHR ([1993], S.138-141). 


\section{Die Ergebnisse im Einzelnen}

\subsection{Vorbemerkungen zu den Berechnungen}

Als Leitfaden zur Bewertung der Ergebnisse sollen folgende Fragen dienen:

(1) Ist es möglich, durch eine Reform der Finanzausgleichsregelungen gesamtwirtschaftliche Wohlfahrtsgewinne zu erzielen?

(2) Wie verändert sich die Wohlfahrtsposition der einzelnen Ländern in den verschiedenen Reformpaketen?

(3) Wie eng ist der Zusammenhang zwischen Nettotransfers und Wohlfahrtsgewinnen, bzw. welchen Einfluß besitzen die Substitutionseffekte auf die Ergebnisse? Gibt es nennenswerte Substitutionseffekte, dann ist zu fragen wodurch diese verursacht werden.

(4) Verändern die unterschiedlichen Finanzausgleichsregelungen die sozialen Grenzkosten der öffentlichen Güter, und inwieweit spielen die sozialen Grenzkosten eine Rolle für die Wohlfahrtseffekte? Gibt es nur einen geringen Einfluß der sozialen Grenzkosten, dann können die durch die sozialen Grenzkosten verursachten Verzerrungen bei zukünftigen Überlegungen außer acht gelassen werden.

(5) Sind die First-round-Berechnungen eine brauchbare Näherung für die endgültigen Finanzausgleichszuweisungen und für die Wohlfahrtseffekte? Wenn ja, braucht man zu einer Abschätzung der Wohlfahrtseffekte nur auf die First-round-Berechnungen zurückzugreifen.

(6) Welche Umverteilungswirkungen ergeben sich aus den einzelnen Vorschlägen? Korrespondieren die endgültigen Wirkungen mit den prognostizierten Umverteilungen, dann genügen die First-round-Berechnungen zu einer entsprechenden Beurteilung der Verteilungswirkungen der einzelnen Reformprogramme.

Einige Vermutungen über die auftretenden Effekte können aufgrund der bisherigen Überlegungen aufgestellt werden:

Neben den Einkommenseffekten, die durch die Nettotransferveränderungen hervorgerufen werden, treten Substitutionseffekte auf. Da sich die regionalen Nettotransferveränderungen in der Summe weitgehend aufheben, dürften gesamtgesellschaftliche Wohlfahrtsgewinne hauptsächlich auf Substitutionseffekte zurückgehen. Dies gilt jedoch nicht in jedem Fall, weil zusätzlich internationale Steuerexporte auftreten können, die ebenfalls die gesellschaftliche Wohlfahrtsposition verändern. 
Intraregionale Substitutionseffekte werden primär durch die Veränderung der sozialen Grenzkosten der öffentlichen Güter ausgelöst, die sich aus der Abschaffung der Umverteilungsregelungen ergeben. Die direkte Abhängigkeit der öffentlichen Einnahmen von den Finanzausgleichsregelungen wird in der Größe $\delta_{q G}$, erfaßt. Für die auftretenden Verzerrungen ist allerdings der Multiplikator $m_{G_{s}}=\left(1-\delta_{q G_{s}}\right)$ relevant, der durch Multiplikation mit dem öffentlichen Güterpreis $q_{G}$, die sozialen Grenzkosten der öffentlichen Güter ergibt. Ist der Multiplikator größer (kleiner) als eins sind die sozialen Grenzkosten höher (niedriger) als der Preis des öffentlichen Gutes. Dieser Multiplikator ist jeweils in der letzten Spalte der Tabellen ausgewiesen.

Eine Erhöhung von $m_{G}$, bewirkt eine Verringerung der Verzerrung zwischen dem öffentlichen und inländischen privaten Konsum (Gleichung (III.3c) auf Seite 106), aber eine Erhöhung der Verzerrung zwischen dem öffentlichen Konsum und der Freizeit (Gleichung (III.3 $d^{\prime}$ ) auf Seite 106). Zugleich verringern sich die indirekten interregionalen Verzerrungen zwischen dem regionalen öffentlichen Konsum und dem bezogenen Konsumgütern (Gleichung (III.3 $f^{\prime}$ ) auf Seite 107). Welche dieser Effekte letztlich dominiert, ist nicht eindeutig zu bestimmen. In der letzten Spalte der Tabellen ist der Wert der Veränderung des Multiplikators $m_{G}$, abgedruckt. Immer, wenn das Vorzeichen der Veränderung nicht mit dem Vorzeichen der $\mathrm{HEV}_{S E}$ übereinstimmt, wird der Multiplikatorwert eingerahmt. In diesem Fall würde der Einfluß der Beziehung zwischen dem öffentlichen Gut und der Freizeit dominieren, falls der Multiplikator das Vorzeichen der $\mathrm{HEV}_{S E}$ bestimmt.

Sekundär werden intraregionale Substitutionseffekte durch Preiserhöhungen(-Senkungen) und stärkere (geringer) Preisschwankungen erhöht (verringert), die die in Form diskriminierender Steuersätze bestehenden Verzerrungen verstärken (abschwächen).

Interregionale Substitutionseffekte sekundärer Art werden dann über stärkere Preisdifferenzen zwischen den Ländern oder höhere Preise der gehandelten Güter gesteigert.

\subsection{Einbezug der Ostländer zum 1.1.1995}

Der Einbezug der Ostländer führt zu einer erheblichen Umverteilung zugunsten der Ostländer. Aufgrund der hohen Transferveränderung ist zu erwarten, daß eventuell auftretende gegenläufige Substitutionseffekte die Einkommenseffekte nicht überkompensieren können. Daher sollten die Transferveränderungen sowohl die Vorzeichen als auch die Größenordnung der Wohlfahrtseffekte und der Terms-of-Trade-Veränderungen bestimmen. Entsprechend müßte die Wohlfahrt der Ostländer zunehmen, da sie als einziges Land eine positive Nettotransferveränderung erfahren. Dagegen müßte sich die Wohlfahrtsposition aller anderen Länder verschlechtern. 


\subsection{1. Übergang bei Beibehaltung der alten Regelungen}

Die Simulation des Einbezugs der Ostländer bei geltenden Finanzausgleichsregelungen — verändert werden nur der Umsatzsteueranteil des Bundes und der Vervielfältiger der Gewerbesteuerumlage - wird in Tabelle V.1 präsentiert.

Insgesamt steigt die gesamtgesellschaftliche Wohlfahrt (vorletzte Zeile, Spalte 2). Der Grund dafür sind zusätzliche Steuerexporte an das Ausland infolge von Preisveränderungen, die in der 3.Spalte der vorletzten Zeile als Nettotransferveränderung in Mio DM ausgewiesen sind. Da die Differenz zwischen den gesamten Wohlfahrtseffekten und der Nettotransferveränderung jedoch negativ ist, müßten relativ hohe Wohlfahrtsverluste aufgrund von Substitutionseffekten auftreten.

Wie erwartet, dominiert die Veränderung der Transferströme (in Spalte 4 ausgewiesen) sämtliche anderen Effekte und bestimmt eindeutig sowohl die Vorzeichen als auch die Größenordnung der Hickschen Äquivalen Variation (Spalte 2 und 3). Da sämtliche Westländer nun geringere Transfers erhalten oder höhere Beiträge leisten müssen, nimmt deren Wohlfahrt ab. Die Wohlfahrtsgewinne der Ostländer, als einzige Nettottransferempfänger, sind beträchtlich.

Die Substitutionseffekte (Spalte 5) fallen im Vergleich zu den Einkommenseffekten (Spalte 4) zwar recht gering aus, erreichen aber dennoch eine beachtliche Höhe. Ihre Abschätzung ist für die meisten Länder relativ gut gelungen, wie die Addition der prozentualen Veränderung der Nettotransfers (Spalte vier) mit der prozentualen Angabe der HEV $\mathrm{HE}_{S E}$ (Spalte fünf) zeigt, da diese relativ genau die gesamte prozentuale Wohlfahrtsveränderung $\left(\mathrm{HEV}_{G E}\right.$ in Spalte drei) ergibt. Allerdings gilt das für die Ostländer, Hamburg und Bremen nicht. Für diese Länder wird die $\mathrm{HEV}_{S E}$ überschätzt, da in der isolierten Substitutionseffektberechnung gerade für diese Länder erhebliche Veränderungen der Finanzausgleichszuweisungen entstehen, die die Substitutionseffekte dominieren und zu einer Überschätzung der Substitutionseffekte führen. (Für Hamburg dreht sich dadurch sogar das erwartete Vorzeichen um!) Dieser Effekt ist auch für das positive Vorzeichen der Summe der $\mathrm{HEV}_{S E}$ verantwortlich.

Die in der letzten Spalte ausgewiesene Veränderung des Multiplikators der öffentlichen Güterpreise, $m_{G_{s}}$, zeigt eine weitgehende Übereinstimmung zwischen der Veränderung dieser Werte und der Richtung der Substitutionseffekte. Dies wäre dann zu erwarten, wenn

- erstens die Verzerrungen zwischen den öffentlichen Gütern und den privaten Konsumgütern die zwischen den öffentlichen Gütern und der Freizeit dominiert,

- und zweitens diese Verzerrung einen dominanten Einfluß auf die gesamten Substitutionseffekte besitzt. 


\begin{tabular}{|c|c|c|c|c|c|c|c|c|c|}
\hline \multirow[t]{3}{*}{ Land } & \multicolumn{2}{|c|}{$\mathrm{HEV}^{a}$} & \multirow{2}{*}{$\begin{array}{c}\Delta \mathrm{TR}^{b} \\
\text { in } \%\end{array}$} & \multirow{2}{*}{$\begin{array}{r}\mathrm{HEV}_{S E}{ }^{c} \\
\text { in \% }\end{array}$} & \multirow{2}{*}{$\begin{array}{r}\mathrm{ToT}_{G E}{ }^{d} \\
\text { in \% }\end{array}$} & \multirow{2}{*}{$\begin{array}{r}\mathrm{ToT}_{S E}{ }^{e} \\
\text { in } \%\end{array}$} & \multicolumn{2}{|c|}{$\mathrm{FA}^{f}$} & \multirow{2}{*}{$\begin{array}{r}\Delta m_{G_{s}}{ }^{g} \\
\text { in \% }\end{array}$} \\
\hline & Mio & in $\%$ & & & & & neu $\%^{h}$ & f.r. $\%^{i}$ & \\
\hline & $\mathrm{DM}$ & $\mathrm{REV}^{j}$ & REV & $\mathrm{REV}^{k}$ & $\mathrm{ToT}^{l}$ & $\mathrm{ToT}^{m}$ & REV & REV & $m_{G}^{0}$ \\
\hline $\mathrm{SH}$ & -713 & $-10,3$ & $-7,4$ & $-3,6$ & $-0,19$ & $-0,09$ & $-2,4$ & $-3,2$ & $-0,44$ \\
\hline $\mathrm{HH}$ & -1720 & $-26,0$ & $-22,4$ & 0,4 & $-0,33$ & 0,01 & $-18,8$ & $-18,8$ & 0,58 \\
\hline $\mathrm{HB}$ & -295 & $-11,8$ & $-12,6$ & 2,2 & $-0,10$ & 0,03 & $-6,5$ & $-6,8$ & $-0,02$ \\
\hline NS & -1881 & $-9,7$ & $-7,7$ & $-2,0$ & $-0,16$ & $-0,04$ & $-2,8$ & $-3,5$ & $-0,47$ \\
\hline NW & -3753 & $-7,9$ & $-7,1$ & $-1,0$ & $-0,09$ & $-0,01$ & $-3,7$ & $-4,3$ & $-0,20$ \\
\hline $\mathrm{HE}$ & -1473 & $-9,3$ & $-9,4$ & 0,2 & $-0,08$ & 0,01 & $-4,3$ & $-4,8$ & 0,90 \\
\hline $\mathrm{RP}$ & -795 & $-8,1$ & $-6,8$ & $-1,6$ & $-0,07$ & $-0,02$ & $-3,3$ & $-3,8$ & $-0,25$ \\
\hline SA & -316 & $-11,0$ & $-10,1$ & $-1,3$ & $-0,10$ & $-0,01$ & $-6,2$ & $-6,9$ & $-0,05$ \\
\hline BW & -2171 & $-8,2$ & $-7,3$ & $-1,0$ & $-0,09$ & $-0,01$ & $-4,4$ & $-5,0$ & 0,66 \\
\hline BY & -2127 & $-7,0$ & $-6,1$ & $-1,1$ & $-0,10$ & $-0,02$ & $-2,1$ & $-2,8$ & 0,39 \\
\hline $\mathrm{OL}$ & 15897 & 106,6 & 95,5 & 12,9 & 3,02 & 0,33 & 126.2 & 128,8 & 0,34 \\
\hline$\sum$ & 655 & & $1055^{n}$ & $86^{\circ}$ & & & & & \\
\hline \multicolumn{3}{|c|}{ Gini-Koeff. vor StV ${ }^{p}$} & 0,180 & n.StV & 0,120 & n.LFA & 0,065 & n.BEZ & 0,059 \\
\hline
\end{tabular}

Tabelle V.1: Übergang mit alter Regelung, Ausgabenautonomie

$q$

${ }^{a}$ Gesamte HEV.

${ }^{b}$ Prozentuale Veränderung der Nettotransfers.

${ }^{c}$ HEV der Substitutionseffektberechnung.

${ }^{d}$ Gesamte ToT.

${ }^{e}$ ToT der Substitutionseffektberechnung.

${ }^{f}$ Veränderung von Finanzausgleich + Steuereinnahmen+ Ergänzungszuweisungen.

${ }^{g}$ Prozentuale Veränderung des Multiplikators der sozialen Grenzkosten der öffentlichen Güter.

${ }^{h}$ Prozentuale Veränderung, endgültig .

${ }^{i}$ Prozentuale Veränderung gemäß First-round-Berechnung.

${ }^{j}$ In Prozent des öffentlichen Budgets ohne Zuweisungen vor der Reform.

${ }^{k} \mathrm{HEV}$ in Prozent des öffentlichen Budgets vor der Reform.

'Prozentuale Preisveränderung der gesamten ToT.

${ }^{m}$ Prozentuale Preisveränderung der ToT des Substitutionseffektes.

${ }^{n}$ Internationale Nettotransfers in Mio DM.

${ }^{\circ}$ Summe der HEV der Substitutionseffekte in Mio DM.

${ }^{P}$ Gini-Koeffizient vor (v.) und nach (n.) der Steuerverteilung (StV), nach LFA und nach BEZ.

${ }^{q}$ Wie in allen weiteren Tabellen zu den Standardfällen werden Abweichungen von den Vorzeichen der HEV fett gedruckt. Abweichungen der für die Berechnung der Substitutionseffekte relevanten Größen von den $\mathrm{HEV}_{S E}$ werden eingerahmt. 
Dies kann an dieser Stelle jedoch nicht näher untersucht werden. Immerhin deutet das trotz relativ hoher Werte für $m_{G}$, gegenläufige Vorzeichen zwischen der Veränderung von $m_{G}$, und der $\mathrm{HEV}_{S E}$ für Baden-Württemberg und Bayern darauf hin, daß der Einfluß der Selbstfinanzierungsterme der öffentlichen Güter auf die Wohlfahrt nicht allzugroß ist. Da alle Länder gleiche Steuersätze besitzen, müßten die Einflüsse von $m_{G_{s}}$ auf die Höhe der Substitutionseffekte in allen Ländern zumindest in die gleiche Richtung weisen.

In der achten und neunten Spalte sind die Veränderung der LFA, der BEZ und der Steuerexporte im neuen Gleichgewicht und in einer First-round-Berechnung abgedruckt. ${ }^{13}$ Wie eine genauere Betrachtung dieser Werte zeigt, bieten die First-round-Werte eine relativ gute Näherung für die endgültigen Finanzausgleichsveränderungen, allerdings nicht für die Reaktion der Nettotransfers. Dies kann über ein stark verändertes Ausgabenverhalten des Bundes begründet werden. Dieser ist aufgrund gesunkener Einnahmen, v.a. als Folge des niedrigeren Umsatzsteueranteils, gezwungen, seine Zuweisungen an die Bundesländer proportional zu kürzen. Daher ist die Veränderung der Nettotransfers sämtlicher Westländer deutlich stärker negativ als die Veränderung der Finanzausgleichszuweisungen. Die Ostländer erhalten Nettottransferszuwächse, die deutlich unter den zu erwartenden zusätzlichen Finanzausgleichszahlungen liegen.

Der Gini-Koeffizient liegt im mittleren Bereich des entsprechenden Wertes aller Vorschläge. Demnach würde eine Beibehaltung der alten gesetzlichen Regelungen im Vergleich zu den anderen Vorschlägen zu eher durchschnittlichen Finanzkraftunterschieden zwischen den Ländern führen.

Vergleicht man diese Ergebnisse mit denen bei Rationierung (fehlende Ausgabenautonomie), dann zeigt sich, daß sämtliche Länder eine geringere Wohlfahrt erreichen, wenn sie bezüglich der öffentlichen Entscheidungen rationiert sind (siehe Tabelle V.2). Damit werden genau die theoretischen Aussagen bestätigt, die Nutzenverluste infolge von Rationierung voraussagen. Eindrucksvoll wird dies auch durch den Rückgang der $H_{E V}$ der einzelnen Länder bestätigt. Nur die $\mathrm{HEV}_{S E}$ der Ostländer entspricht nicht den Erwartungen. Dies liegt an starken Nettotransferveränderungen, die in der Berechnung der Substitutionseffekte auftreten und die reinen Substitutionseffekte überlagern. Wie allerdings eine

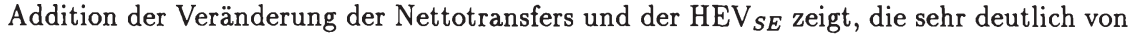
der gesamten HEV abweicht, werden die Substitutionseffekte für die Ostländer deutlich überschätzt. Die Summe der beiden Größen für Hamburg und Bremen weicht ebenfalls deutlich von der HEV ab. Dies ist ebenfalls auf stark veränderte Nettotransferströme

\footnotetext{
${ }^{13}$ Die First-round-Werte ergeben sich über die Berechnung der Transfers mit den neuen Finanzausgleichsregelungen unter Verwendung der Daten der Datenbasis und entsprechen im Prinzip den üblichen Vorausschätzungen der Steuerverteilungs-,LFA- und Bundesergänzungszuweisungen. Allerdings kommt hier noch eine Abschätzung der Steuerexporte hinzu.
} 


\begin{tabular}{|c|c|c|c|c|c|c|c|c|c|}
\hline \multirow[t]{3}{*}{ Land } & \multicolumn{2}{|c|}{$\mathrm{HEV}$} & \multirow{2}{*}{$\begin{array}{l}\Delta \mathrm{TR} \\
\text { in } \%\end{array}$} & \multirow{2}{*}{$\begin{array}{r}\mathrm{HEV}_{S E} \\
\text { in } \%\end{array}$} & \multirow{2}{*}{$\begin{array}{r}\mathrm{ToT}_{G E} \\
\text { in \% }\end{array}$} & \multirow{2}{*}{$\begin{array}{r}\text { ToT }_{S E} \\
\text { in \% }\end{array}$} & \multicolumn{2}{|c|}{$\mathrm{FA}$} & \multirow{3}{*}{$\begin{array}{r}\Delta m_{G_{s}} \\
\text { in \% } \\
m_{G_{s}}^{0}\end{array}$} \\
\hline & Mio & in $\%$ & & & & & neu $\%$ & f.r.\% & \\
\hline & DM & REV & REV & REV & ToT & ToT & REV & REV & \\
\hline $\mathrm{SH}$ & -728 & $-10,5$ & $-7,0$ & $-4,0$ & $-0,25$ & $-0,11$ & $-2,1$ & $-3,2$ & $-0,44$ \\
\hline $\mathrm{HH}$ & -1858 & $-28,1$ & $-21,8$ & $-0,3$ & $-0,32$ & 0,00 & -17.9 & $-18,8$ & 0,58 \\
\hline $\mathrm{HB}$ & -304 & $-12,2$ & $-12,1$ & 1,6 & $-0,11$ & 0,02 & $-5,9$ & $-6,8$ & $-0,02$ \\
\hline NS & -1942 & $-10,0$ & $-7,4$ & $-2,4$ & $-0,20$ & $-0,05$ & $-2,4$ & $-3,5$ & $-0,47$ \\
\hline NW & -3798 & $-8,0$ & $-6,8$ & $-1,2$ & -0 , & $-0,02$ & $-3,3$ & $-4,3$ & $-0,20$ \\
\hline $\mathrm{HE}$ & -1497 & $-9,5$ & $-9,2$ & 1 & $-l$ & 1 & 9 & 4,8 & 0,90 \\
\hline $\mathrm{RP}$ & -930 & $-8,4$ & $-6,6$ & $-1,8$ & $-0,09$ & $-0,03$ & $-3,1$ & $-3,8$ & $-0,25$ \\
\hline SA & -326 & $-11,4$ & $-9,6$ & $-1,8$ & $-0,11$ & $-0,02$ & $-5,5$ & $-6,9$ & $-0,05$ \\
\hline BW & -2183 & $-8,2$ & $-7,1$ & $-1,1$ & $-0,09$ & $-0,01$ & $-4,1$ & $-5,0$ & 0,66 \\
\hline BY & -2122 & $-7,1$ & $-5,9$ & $-1,3$ & -0 , & $-0,02$ & $-1,8$ & $-2,8$ & 0,39 \\
\hline $\mathrm{OL}$ & 14603 & 98,0 & 92,9 & 15,6 & 3,30 & 0,43 & 122.5 & 128,8 & 0,34 \\
\hline$\sum$ & -993 & & 1114 & 109 & & & & & \\
\hline \multicolumn{3}{|c|}{ Gini-Koeff. vor StV } & 0,177 & n.StV & 0,118 & n.LFA & 0,064 & n.BEZ & 0,058 \\
\hline
\end{tabular}

Tabelle V.2: Übergang mit alter Regelung, keine Autonomie

zurückzuführen. Für alle anderen Länder gibt es dagegen eine weitgehende Übereinstimmung zwischen der Summe aus den Nettotransferveränderungen und der HEV $\mathrm{HE}_{\text {mit den }}$ regionalen Wohlfahrtsgewinnen $\left(\mathrm{HEV}_{G E}\right)$.

Die starke Nettotransferveränderung bei der Berechnung der Substitutionseffekte führt dazu, daß erneut positive gesellschaftliche Wohlfahrtsgewinne infolge veränderter Substitutionseffekte ausgewiesen werden, obwohl diese negativ sein müßten. Die Zerlegung in Einkommens- und Substitutionseffekte ist daher ebenfalls nur für einige Länder gelungen. Insgesamt müßten die durch die Substitutionseffekte verursachten Wohlfahrtsverluste bei Rationierung erheblich höher sein als bei Ausgabenautonomie, da die gesamte Wohlfahrtsveränderung trotz positiver Nettotransferveränderungen deutlich negativ ist. Die auftretenden Substitutionseffekte müßten daher die Einkommenseffekte dominieren.

Ein Großteil dieser Substitutionseffekte dürfte auf die Rationierung zurückzuführen sein, deren verzerrender Einfluß um so höher ist, je größer die Finanzausgleichszuweisungen ausfallen. Angesichts der hier auftretenden immensen Wirkungen, ist eine Erhöhung der regionalen Ausgabenautonomie dringend zu empfehlen.

Die Folgen der Rationierung werden zusätzlich durch sekundäre Substitutionseffekte verstärkt, die allerdings ähnlich auch bei Ausgabenautonomie autreten. Die starken Umschichtungen führen dazu, daß sich die regionalen Preise verändern und noch dazu er- 
heblich auseinanderdriften. Höhere Preise und wachsende Preisdifferenzen verstärken die Auswirkungen vorhandener Verzerrungen. Infolgedessen werden die intraregionalen und internationalen Substitutionseffekte verstärkt und regionale und nationale Wohlfahrtsverluste erzeugt.

Bei Rationierung erhalten die Ostländer wesentliche geringere Finanzausgleichszuweisungen. Die Abweichung der tatsächlichen Finanzausgleichszuweisungen und Steuerexporte von den entsprechenden First-round-Werten wird daher größer.

Von den durchgeführten Sensitivitätsanalysen wird an dieser Stelle nur eine exemplarisch dargestellt, da auch in allen anderen alternativen Berechnungen die obigen Ergebnisse weitgehend bestätigt wurden. Im hier dargestellten Fall wird mit einer höheren Bezugnachfrageelastizität gerechnet $(-1,7$ statt $-1,1)$. Dieser alternative Elastizitätswert bewegt sich an der oberen Intervallgrenze der in der Literatur ausgewiesenen Schätzungen von Importnachfrageelastizitäten.

\begin{tabular}{||c|r|r|r|r||r|r|r|r||}
\hline \hline & \multicolumn{3}{|c||}{ Standard: $\varepsilon=-1,1$} & \multicolumn{5}{|c||}{$\varepsilon=-1,7$} \\
Land & HEV $_{G E}$ & $\Delta$ TR & HEV $_{S E}$ & ToT $_{G E}$ & HEV $_{G E}$ & $\Delta \mathrm{TR}$ & $\mathrm{HEV}_{S E}$ & ToT $_{G E}$ \\
\hline SH & $-10,3$ & $-7,4$ & $-3,6$ & $-0,19$ & $-10,3$ & $-7,6$ & $-3,5$ & $-0,19$ \\
HH & $-26,0$ & $-22,4$ & 0,4 & $-0,33$ & $-25,7$ & $-22,5$ & 0,4 & $-0,30$ \\
HB & $-11,8$ & $-12,6$ & 2,2 & $-0,10$ & $-11,7$ & $-12,6$ & 2,1 & $-0,10$ \\
NS & $-9,7$ & $-7,7$ & $-2,0$ & $-0,16$ & $-9,7$ & $-7,9$ & $-1,9$ & $-0,15$ \\
NW & $-7,9$ & $-7,1$ & $-1,0$ & $-0,09$ & $-7,9$ & $-7,1$ & $-1,0$ & $-0,09$ \\
HE & $-9,3$ & $-9,4$ & 0,2 & $-0,08$ & $-9,4$ & $-9,4$ & 0,2 & $-0,03$ \\
RP & $-8,1$ & $-6,8$ & $-1,6$ & $-0,07$ & $-8,1$ & $-6,9$ & $-1,5$ & $-0,07$ \\
SA & $-11,0$ & $-10,1$ & $-1,3$ & $-0,10$ & $-11,0$ & $-10,2$ & $-1,2$ & $-0,10$ \\
BW & $-8,2$ & $-7,3$ & $-1,0$ & $-0,09$ & $-8,2$ & $-7,3$ & $-1,0$ & $-0,09$ \\
BY & $-7,0$ & $-6,1$ & $-1,1$ & $-0,10$ & $-7,0$ & $-6,1$ & $-1,1$ & $-0,10$ \\
OL & 106,6 & 95,5 & 12,9 & 3,02 & 106,9 & 96,1 & 12,3 & 2,84 \\
\hline$\sum$ & 655 & & & & 687 & & & \\
\hline \hline
\end{tabular}

Tabelle V.3: Übergang mit alten Regelungen, Ausgabenautonomie, Variation der Bezugnachfrageelastizität

Der Fall einer höheren Bezugnachfrageelastizität illustriert die Zuverlässigkeit des Modells in einem weiteren Punkt. Es ist zu erwarten, daß bei einem Anstieg der Elastizität die Haushalte stärker auf Preisänderungen reagieren. Deshalb können Preisänderungen in einer Region nicht mehr in voller Höhe an andere Regionen weitergegeben werden, was zu einer Verringerung der Unterschiede in den regionalen Preisniveaus führen müßte. Da 
die Terms of Trade daher nicht mehr so stark steigen oder fallen, müßten auch die Wohlfahrtsgewinne oder -verluste geringer werden. Dies ist, wie aus der Tabelle V.3 ersichtlich ist, auch das Resultat der Berechnung. Allerdings gilt das nur für die $\mathrm{HEV}_{S E}$, da bei der Gesamtwohlfahrtsberechnung Nettotransferveränderungen auftreten, die die erwarteten Effekte überkompensieren können. Die auftretenden Veränderungen sind jedoch sehr gering. Demzufolge kommt den Bezugsnachfrageelastizitäten keine allzugroße Bedeutung für die Wohlfahrtseffekte zu, d.h. die Resultate sind robust bezüglich Variationen dieser Elastizitäten.

\subsection{2. Übergang mit den neuen Regelungen}

Simuliert man den vollen Einbezug der Ostländer in das bundesdeutsche Finanzausgleichssystem anhand des tatsächlich durchgeführten gleichzeitigen Wechsels der Finanzausgleichsregime, dann wird aus der Tabelle V.4 deutlich, daß die obigen Aussagen auch für diesen Übergang gelten.

\begin{tabular}{|c|c|c|c|c|c|c|c|c|c|}
\hline \multirow[t]{3}{*}{ Land } & \multicolumn{2}{|c|}{ HEV } & \multirow{3}{*}{$\begin{array}{c}\Delta \mathrm{TR} \\
\text { in } \% \\
\mathrm{REV}\end{array}$} & \multirow{3}{*}{$\begin{array}{r}\mathrm{HEV}_{S E} \\
\text { in \% } \\
\mathrm{REV} \\
\end{array}$} & \multirow{3}{*}{$\begin{array}{r}\text { ToT }_{G E} \\
\text { in } \% \\
\text { ToT }\end{array}$} & \multirow{3}{*}{$\begin{array}{r}\text { ToT }_{S E} \\
\text { in \% } \\
\text { ToT }\end{array}$} & \multicolumn{2}{|c|}{$\mathrm{FA}$} & \multirow{3}{*}{$\begin{array}{r}\Delta m_{G} \\
\text { in } \% \\
m_{G_{s}}\end{array}$} \\
\hline & Mio & in $\%$ & & & & & neu $\%$ & f.r. $\%$ & \\
\hline & $\mathrm{DM}$ & REV & & & & & REV & REV & \\
\hline $\mathrm{SH}$ & -929 & $-13,4$ & $-9,2$ & $-4,7$ & $-0,32$ & $-0,01$ & $-4,9$ & $-6,1$ & 0,04 \\
\hline $\mathrm{HH}$ & -749 & $-11,3$ & $-11,7$ & 2,8 & $-0,15$ & 0,04 & $-5,6$ & $-6,1$ & 0,54 \\
\hline HB & -183 & $-7,3$ & $-9,3$ & 3,2 & $-0,06$ & 0,04 & $-1,9$ & $-2,6$ & 0,07 \\
\hline NS & -2375 & $-12,3$ & $-9,4$ & $-2,6$ & $-0,25$ & $-0,06$ & $-4,8$ & $-5,7$ & 0,01 \\
\hline NW & -4422 & $-9,3$ & $-8,4$ & $-1,1$ & $-0,11$ & $-0,01$ & $-5,0$ & $-5,7$ & $-0,24$ \\
\hline $\mathrm{HE}$ & -1723 & $-10,9$ & $-11,0$ & 0,4 & $-0,06$ & 0,01 & $-5,6$ & $-6,2$ & 0,81 \\
\hline $\mathrm{RP}$ & -1049 & $-10,7$ & $-8,9$ & $-2,2$ & $-0,11$ & $-0,03$ & $-5,7$ & $-6,7$ & 0,15 \\
\hline $\mathrm{SA}$ & -281 & $-9,8$ & $-9,2$ & $-1,1$ & $-0,07$ & $-0,01$ & $-5,0$ & $-5,8$ & 0,03 \\
\hline BW & -2561 & $-9,6$ & $-8,6$ & $-1,1$ & $-0,11$ & $-0,01$ & $-5,6$ & $-6,4$ & 0,53 \\
\hline BY & -3027 & $-10,0$ & $-8,6$ & $-1,4$ & $-0,16$ & $-0,02$ & $-4,8$ & $-5,6$ & 0,29 \\
\hline OL & 17832 & 119,6 & 108,9 & 13,9 & 3,40 & 0,36 & 140.2 & 143,0 & 0,38 \\
\hline$\sum$ & 535 & & 1215 & 68 & & & & & \\
\hline \multicolumn{3}{|c|}{ Gini-Koeff. vor StV } & 0,179 & n.StV & 0,122 & n.LFA & 0,081 & n.BEZ & 0,074 \\
\hline
\end{tabular}

Tabelle V.4: Übergang mit neuer Regelung, Ausgabenautonomie

Ein genauerer Vergleich der beiden Finanzausgleichsregime bringt folgende Resultate: Die Anwendung der neuen Regeln ergibt höhere internationale Steuerexporte (Summe der dritten Spalte) bei gleichzeitig geringerem gesamtgesellschaftlichen Wohlfahrtsgewinn, 
der sich in erster Linie durch höhere Preise sowie stärkere Preisdifferenzen und demzufolge gestiegenen Substitutionseffekten ergibt. Eindeutig besser schneiden die Ostländer und Hamburg ab. Die ersteren erhalten höhere Zahlungen, die zweiten müssen geringere Zahlungen leisten. Für alle anderen Länder mit absolut hohen HEV-Veränderungen gilt, daß diese bei Anwendung der neuen Regel noch größer werden, da die entsprechenden Zahlungsströme zunehmen.

Besteht Rationierung (Tabelle V.5), entsteht ein gesellschaftlicher Wohlfahrtsverlust von annähernd 1,4 Mrd DM, der wiederum durch starke Preisanstiege erklärt werden kann. Bis auf die $H_{E V}$ Ostländer, die bei der Berechnung der Substitutionseffekte höhere Transfers erhalten, sinken die $H_{E V}$ für alle Länder. Dies entspricht der erwarteten Reaktion bei Rationierung.

Für alle Berechnungen des Übergangs gilt, daß die First-round-Berechnungen der Veränderungen der Finanzausgleichsströme die Vorzeichen der Wohlfahrtsgewinne widerspiegeln. Nicht ganz so genau reflektieren sie die relative Größenordnung der Wohlfahrtseffekte zwischen den Ländern. Dennoch sind die First-round-Werte zumindest ein grober Indikator für die Größenordnung der Wohlfahrtseffekte, die allerdings teilweise doppelt so hoch sind, wie die berechneten Veränderungen der Finanzausgleichsströme. Wesentlich genauer entsprechen sich die First-round-Berechnungen und die endgültigen Finanzausgleichszuweisungen und Steuerexporte.

Die Simulation liefert eine eindrucksvolle Bestätigung der theoretischen Ergebnisse bezüglich der Rationierung. Da die Verluste bei Rationierung im Vergleich mit Ausgabenautonomie - bei konstanten Preisen - um so größer sind je höher die Einkommensveränderung ist, müßten durch den Übergang relativ hohe Wohlfahrtsdifferenzen entstehen. Dies wird in den Berechnungen bestätigt. Demzufolge wäre eine erhöhte Ausgabenautonomie der Länder empfehlenswert.

Der Vergleich der Ergebnisse der Übergangsberechnungen zeigt, daß die Verteilung bei Beibehaltung der alten Regelungen näher an der Gleichverteilung der öffentlichen Einnahmen liegt, als bei Einführung der Neuregelung (Gini-Koeffizient ist 0,059 gegenüber 0,074). Die gesamtwirtschaftlichen Wohlfahrtsgewinne bei Ausgabenautonomie sind dagegen annähernd vergleichbar, wenn auch bei der Neuregelung die Nettotransferveränderungen wesentlich höher sind. In beiden Fällen führt erhöhte Ausgabenautonomie, erwartungsgemäß, zu deutlichen Wohlfahrtsverlusten. 


\begin{tabular}{|c|c|c|c|c|c|c|c|c|c|}
\hline \multirow[t]{3}{*}{ Land } & \multicolumn{2}{|c|}{$\mathrm{HEV}$} & \multirow{2}{*}{$\begin{array}{l}\Delta \mathrm{TR} \\
\text { in } \%\end{array}$} & \multirow{2}{*}{$\begin{array}{r}\mathrm{HEV}_{S E} \\
\text { in \% }\end{array}$} & \multirow{2}{*}{$\begin{array}{r}\text { To }_{G E} \\
\text { in \% }\end{array}$} & \multirow{2}{*}{$\begin{array}{r}\mathrm{ToT}_{S E} \\
\text { in \% }\end{array}$} & \multicolumn{2}{|c|}{ FA } & \multirow{3}{*}{$\begin{array}{c}\Delta m_{G_{s}} \\
\text { in \% } \\
m_{G}^{0}\end{array}$} \\
\hline & Mio & in $\%$ & & & & & neu $\%$ & f.r. $\%$ & \\
\hline & $\mathrm{DM}$ & REV & & REV & ToT & ToT & REV & REV & \\
\hline $\mathrm{SH}$ & -946 & $-13,7$ & $-8,6$ & $-5,2$ & $-0,38$ & $-0,15$ & $-4,4$ & $-6,1$ & 0,04 \\
\hline $\mathrm{HH}$ & -773 & $-11,7$ & $-11,6$ & 2,6 & $-0,16$ & 0,04 & $-5,2$ & $-6,1$ & 0,54 \\
\hline HB & -188 & $-7,5$ & $-9,1$ & 3,1 & $-0,08$ & 0,04 & $-1,6$ & $-2,6$ & 0,07 \\
\hline NS & -2440 & $-12,6$ & $-9,0$ & $-3,1$ & $-0,29$ & $-0,07$ & $-4,3$ & $-5,7$ & 0,01 \\
\hline NW & -4467 & $-0,4$ & $-8,0$ & $-1,4$ & $-0,12$ & $-0,02$ & $-4,5$ & $-5,7$ & 0,11 \\
\hline $\mathrm{HE}$ & -1760 & $-11,2$ & $-10,8$ & 0,3 & $-0,06$ & 0,01 & $-5,2$ & $-6,2$ & 0,84 \\
\hline $\mathrm{RP}$ & -1085 & $-11,0$ & $-8,6$ & $-2,5$ & $-0,13$ & $-0,04$ & $-5,3$ & $-6,7$ & 0,15 \\
\hline SA & -289 & $-10,1$ & $-8,7$ & $-1,5$ & $-0,08$ & $-0,01$ & $-4,4$ & $-5,8$ & 0,04 \\
\hline BW & -2604 & $-9,8$ & $-8,5$ & $-1,2$ & $-0,11$ & $-0,01$ & $-5,5$ & $-6,4$ & 0,60 \\
\hline BY & -3073 & $-1,2$ & $-8,4$ & $-1,7$ & $-0,17$ & $-0,03$ & $-4,4$ & $-5,6$ & 0,45 \\
\hline OL & 16204 & 108,7 & 105,8 & 17,0 & 3,72 & 0,49 & 135,9 & 143,0 & 0,39 \\
\hline$\sum$ & -1421 & & 1114 & 87 & & & & & \\
\hline \multicolumn{3}{|c|}{ Gini-Koeff. vor StV } & 0,178 & n.StV & 0,120 & n.LFA & 0,081 & n.BEZ & 0,073 \\
\hline
\end{tabular}

Tabelle V.5: Übergang mit neuer Regelung, keine Autonomie

\subsection{Wechsel der Finanzausgleichsregelungen nach 1995}

In den folgenden Simulationen werden die Wohlfahrts- und Verteilungswirkungen der Einführung der verschiedenen Neuordnungsvorschläge untersucht. Um ein realistisches Szenario zu erhalten, wird davon ausgegangen, daß die einzelnen Vorschläge erst nach dem vollständigen Einbezug der Ostländer relevant werden. Daher wird als neue Datenbasis der Datensatz verwendet, der aus den Berechnungen des Übergangs von den alten zu den neuen gesetzlichen Regelungen resultiert.

Alle Empfehlungen werden unter Verwendung des Konzeptes der Budgetinzidenz berechnet. Eine Veränderung der Einnahmen wird demnach durch eine Anpassung der Ausgabenseite des jeweiligen regionalen öffentlichen Haushaltes ausgeglichen.

\subsubsection{Wissenschaftlicher Beirat [1992]}

\section{(1) Ergebnisse zum Vorschlag des Wissenschaftlichen Beirats}

Der Vorschlag des Wissenschaftlichen Beirats [1992] sieht außer der Umgestaltung der Finanzausgleichsregelungen auch eine erweiterte Finanzautonomie der Länder vor. Dieser zweite Aspekt wird vorerst nicht berücksichtigt. Vielmehr werden die Vorschläge 
zur Neuordnung der Finanzausgleichsregelungen isoliert untersucht. Auf diese Weise werden die Effekte der geänderten Regelungen nicht von den Auswirkungen verzerrender Steuersatzveränderungen überlagert. Darüber hinaus läßt sich der Vorschlag des Beirats hinsichtlich der Finanzausgleichsregelungen auf diese Weise leichter mit den Empfehlungen anderer Autoren vergleichen.

\begin{tabular}{|c|c|c|c|c|c|c|c|c|c|}
\hline \multirow[t]{3}{*}{ Land } & \multicolumn{2}{|c|}{ HEV } & \multirow{3}{*}{$\begin{array}{l}\Delta \mathrm{TR} \\
\text { in } \% \\
\mathrm{REV}\end{array}$} & \multirow{3}{*}{$\begin{array}{r}\mathrm{HEV}_{S E} \\
\text { in \% } \\
\mathrm{REV}\end{array}$} & \multirow{3}{*}{$\begin{array}{r}\text { ToT } T_{G E} \\
\text { in \% } \\
\text { ToT } \\
\end{array}$} & \multirow{3}{*}{$\begin{array}{r}\text { ToT }_{S E} \\
\text { in \% } \\
\text { ToT }\end{array}$} & \multicolumn{2}{|c|}{ FA } & \multirow{3}{*}{$\begin{array}{c}\Delta m_{G}, \\
\text { in \% } \\
m_{G_{s}}^{0}\end{array}$} \\
\hline & Mio & in $\%$ & & & & & neu $\%$ & f.r. $\%$ & \\
\hline & $\mathrm{DM}$ & REV & & & & & REV & REV & \\
\hline $\mathrm{SH}$ & -248 & $-3,7$ & $-2,7$ & $-1,1$ & $-0,06$ & $-0,01$ & $-2,9$ & $-3,7$ & $-0,14$ \\
\hline $\mathrm{HH}$ & -33 & $-0,5$ & $-1,3$ & 1,0 & $-0,24$ & $-0,23$ & $-0,2$ & $-0,7$ & $-0,05$ \\
\hline HB & -165 & $-6,6$ & $-6,4$ & 0,4 & $-0,15$ & \begin{tabular}{|l|}
$-0,08$ \\
\end{tabular} & $-5,9$ & $-6,3$ & $-0,12$ \\
\hline NS & -638 & $-3,4$ & $-2,8$ & $-0,5$ & $-0,01$ & 0,04 & $-2,7$ & $-3,4$ & $-0,14$ \\
\hline NW & -53 & $-0,1$ & $-0,2$ & 0,1 & $-0,08$ & $-0,08$ & 0,3 & $-0,4$ & 0,32 \\
\hline $\mathrm{HE}$ & 105 & 0,7 & 0,1 & 0,5 & $-0,11$ & $-0,12$ & 1,1 & $-0,4$ & $-0,01$ \\
\hline $\mathrm{RP}$ & -141 & $-1,5$ & $-1,1$ & $-0,3$ & $\mathbf{0 , 1 1}$ & 0,13 & $-0,9$ & $-1,6$ & $-0,10$ \\
\hline SA & -213 & $-7,7$ & $-6,7$ & $-0,6$ & $\mathbf{0 , 1 3}$ & 0,23 & $-6,9$ & $-7,5$ & $-0,49$ \\
\hline BW & 476 & 1,9 & 1,5 & 0,3 & $-0,01$ & $-0,03$ & 2,2 & 1,5 & $-0,40$ \\
\hline BY & -214 & $-0,7$ & $-0,7$ & 0,0 & $-0,02$ & 0,00 & $-0,2$ & $-0,9$ & 0,14 \\
\hline $\mathrm{OL}$ & 1144 & 3,0 & 3,3 & $-0,2$ & 1,07 & 0,83 & 3,9 & 3,8 & 0,11 \\
\hline$\sum$ & 22 & & 161 & -12 & & & & & \\
\hline \multicolumn{3}{|c|}{ Gini-Koeff. vor StV } & 0,179 & n.StV & 0,142 & n.LFA & 0,072 & n.BEZ & 0,076 \\
\hline
\end{tabular}

Tabelle V.6: Wiss. Beirat I, Ausgabenautonomie

In allen vier Varianten des Vorschlags des Beirats (Tabellen V.6, V.7, V.8, und V.9) liegen die gesamtwirtschaftlichen Wohlfahrtseffekte nahe bei Null.

In fast allen Fällen stimmen Größenordnung und Vorzeichen von Nettotransfers und HEV's überein. In zwei Fällen (HE und RP) in der Variante IV besitzen Nettotransferveränderungen und Wohlfahrtseffekte unterschiedliche Vorzeichen. $\mathrm{Da}$ in beiden Fällen die Nettotransferveränderungen sehr gering sind, können sie von den Substitutionseffekten dominiert werden.

Im einzelnen sind die Ergebnisse der verschiedenen Varianten recht unterschiedlich. Der größten Schwankung sind die Gewinne von Baden-Württemberg und den Ostländern unterworfen. Erstere liegen zwischen 1,1 Mrd DM und 9 Mio DM; letztere zwischen 370 Mio und 1,1 Mrd DM. Die größten Differenzen ergeben sich zwischen der vierten und allen anderen Varianten der Empfehlung, da die Finanzierung der Bundesbeteiligung in Variante IV gleichmäßiger von den Ländern getragen wird, als eine stärkere Umverteilung der 


\begin{tabular}{|c|c|c|c|c|c|c|c|c|c|}
\hline \multirow[t]{3}{*}{ Land } & \multicolumn{2}{|c|}{ HEV } & \multirow{3}{*}{$\begin{array}{l}\Delta \mathrm{TR} \\
\text { in } \% \\
\mathrm{REV}\end{array}$} & \multirow{3}{*}{$\begin{array}{r}\mathrm{HEV}_{S E} \\
\text { in \% } \\
\mathrm{REV}\end{array}$} & \multirow{3}{*}{$\begin{array}{r}\text { ToT } \mathrm{T}_{G E} \\
\text { in } \% \\
\text { ToT }\end{array}$} & \multirow{3}{*}{$\begin{array}{r}\text { ToT }_{S E} \\
\text { in \% } \\
\text { ToT } \\
\end{array}$} & \multicolumn{2}{|c|}{ FA } & \multirow{3}{*}{$\begin{array}{c}\Delta m_{G} \\
\text { in } \% \\
m_{G_{0}}^{0}\end{array}$} \\
\hline & Mio & in $\%$ & & & & & neu $\%$ & f.r. $\%$ & \\
\hline & $\mathrm{DM}$ & REV & & & & & REV & REV & \\
\hline $\mathrm{SH}$ & -225 & $-3,4$ & $-2,5$ & $-0,9$ & $-0,05$ & 0,00 & 2,6 & 1,9 & $-0,09$ \\
\hline $\mathrm{HH}$ & -111 & $-1,7$ & $-2,1$ & 0,7 & $-0,26$ & $-0,23$ & 1,0 & 0,5 & $-0,01$ \\
\hline $\mathrm{HB}$ & -92 & $-3,7$ & $-3,9$ & 0,5 & $-0,12$ & $-0,08$ & $-0,6$ & $-1,1$ & $-0,07$ \\
\hline NS & -363 & $-2,0$ & $-1,6$ & $-0,3$ & 0,02 & 0,04 & 2,9 & 2,2 & $-0,09$ \\
\hline NW & 276 & 0,6 & 0,4 & 0,2 & $-0,08$ & \begin{tabular}{|c|}
$-0,08$ \\
\end{tabular} & 3,7 & 3,0 & 0,36 \\
\hline $\mathrm{HE}$ & 24 & 0,2 & $-0,3$ & 0,4 & $-0,10$ & $-0,12$ & 3,7 & 3,0 & 0,05 \\
\hline $\mathrm{RP}$ & 16 & 0,2 & $-0,2$ & 0,0 & 0,13 & 0,13 & 3,8 & 3,1 & \begin{tabular}{|l|}
$-0,06$ \\
\end{tabular} \\
\hline $\mathrm{SA}$ & -110 & $-4,0$ & $-3,5$ & $-0,3$ & 0,18 & 0,23 & $-0,3$ & $-0,9$ & $-0,40$ \\
\hline BW & 236 & 0,9 & 0,7 & 0,3 & $-0,02$ & $-0,03$ & 3,7 & 3,0 & 0,45 \\
\hline BY & -4 & 0,0 & $-0,1$ & 0,0 & 0,00 & 0,00 & 3,5 & 2,8 & 0,19 \\
\hline OL & 367 & 1,0 & 1,5 & $-0,5$ & 0,92 & 0,81 & 7,2 & 7,1 & 0,14 \\
\hline$\sum$ & 14 & & 124 & -13 & & & & & \\
\hline \multicolumn{3}{|c|}{ Gini-Koeff. vor StV } & 0,179 & n.StV & 0,142 & n.LFA & 0,088 & n.BEZ & 0,069 \\
\hline
\end{tabular}

Tabelle V.7: Wiss. BeIRat IV, Ausgabenautonomie

\begin{tabular}{|c|c|c|c|c|c|c|c|c|c|}
\hline \multirow[t]{3}{*}{ Land } & \multicolumn{2}{|c|}{$\mathrm{HEV}$} & \multirow{3}{*}{$\begin{array}{l}\Delta \mathrm{TR} \\
\text { in } \% \\
\mathrm{REV} \\
\end{array}$} & \multirow{3}{*}{$\begin{array}{r}\mathrm{HEV}_{S E} \\
\text { in \% } \\
\mathrm{REV}\end{array}$} & \multirow{3}{*}{$\begin{array}{r}\text { ToT }_{G E} \\
\text { in \% } \\
\text { ToT }\end{array}$} & \multirow{3}{*}{$\begin{array}{r}\text { ToT }_{S E} \\
\text { in \% } \\
\text { ToT }\end{array}$} & \multicolumn{2}{|c|}{ FA } & \multirow{3}{*}{$\begin{array}{c}\Delta m_{G} \\
\text { in \% } \\
m_{G s}^{0}\end{array}$} \\
\hline & Mio & in $\%$ & & & & & neu $\%$ & f.r. $\%$ & \\
\hline & $\mathrm{DM}$ & REV & & & & & REV & REV & \\
\hline $\mathrm{SH}$ & -375 & $-5,7$ & $-3,9$ & $-1,6$ & $-0,12$ & $-0,02$ & $-4,6$ & $-5,5$ & 0,32 \\
\hline $\mathrm{HH}$ & 267 & 4,2 & 1,9 & 2,1 & $-0,18$ & $-0,22$ & 4,0 & 3,4 & 0,81 \\
\hline HB & -208 & $-8,4$ & $-8,3$ & 0,9 & $-0,17$ & $-0,07$ & $-7,5$ & $-7,8$ & 0,58 \\
\hline NS & -923 & $-5,0$ & $-3,9$ & $-0,8$ & $-0,04$ & 0,03 & $-4,0$ & $-4,8$ & 0,55 \\
\hline NW & -132 & $-0,3$ & $-0,4$ & 0,1 & $-0,09$ & $-0,08$ & 0,2 & $-0,6$ & 1,39 \\
\hline $\mathrm{HE}$ & 245 & 1,6 & 0,7 & 0,8 & $-0,10$ & $-0,12$ & 2,0 & 1,3 & 0,81 \\
\hline $\mathrm{RP}$ & -571 & $-6,1$ & $-4,8$ & $-0,8$ & 0,14 & 0,12 & $-5,2$ & $-5,8$ & 0,39 \\
\hline SA & -145 & $-5,3$ & $-4,6$ & $-0,4$ & 0,16 & 0,22 & $-4,4$ & $-5,1$ & 0,35 \\
\hline BW & 1112 & 4,4 & 3,5 & 0,7 & 0,02 & $-0,02$ & 4,6 & 3,9 & 1,96 \\
\hline BY & -439 & $-1,5$ & $-1,3$ & $-0,1$ & $-0,04$ & 0,00 & $-0,9$ & $-1,6$ & 1,11 \\
\hline $\mathrm{OL}$ & 1129 & 3,0 & 3,7 & $-0,5$ & 1,07 & 0,80 & 4,0 & 3,8 & 0,29 \\
\hline$\sum$ & -41 & & 212 & -37 & & & & & \\
\hline \multicolumn{3}{|c|}{ Gini-Koeff. vor StV } & 0,179 & n.StV & 0,109 & n.LFA & 0,075 & n.BEZ & 0,084 \\
\hline
\end{tabular}

Tabelle V.8: Wiss. Beirat II, Ausgabenautonomie 


\begin{tabular}{|c|c|c|c|c|c|c|c|c|c|}
\hline \multirow[t]{3}{*}{ Land } & \multicolumn{2}{|c|}{ HEV } & \multirow{3}{*}{$\begin{array}{l}\Delta \mathrm{TR} \\
\text { in } \% \\
\mathrm{REV}\end{array}$} & \multirow{3}{*}{$\begin{array}{r}\mathrm{HEV}_{S E} \\
\text { in \% } \\
\mathrm{REV}\end{array}$} & \multirow{3}{*}{$\begin{array}{r}\mathrm{ToT}_{G E} \\
\text { in } \% \\
\mathrm{ToT}\end{array}$} & \multirow{3}{*}{$\begin{array}{r}\mathrm{ToT}_{S E} \\
\text { in \% } \\
\text { ToT }\end{array}$} & \multicolumn{2}{|c|}{$\mathrm{FA}$} & \multirow{3}{*}{$\begin{array}{c}\Delta m_{G}, \\
\text { in } \% \\
m_{G}^{0}\end{array}$} \\
\hline & Mio & in $\%$ & & & & & neu $\%$ & f.r. $\%$ & \\
\hline & $\mathrm{DM}$ & REV & & & & & REV & REV & \\
\hline $\mathrm{SH}$ & -127 & $-1,9$ & $-1,4$ & $-0,6$ & $-0,02$ & $-0,01$ & $-1,2$ & 2,0 & 0,57 \\
\hline $\mathrm{HH}$ & -175 & $-2,7$ & $-2,6$ & 0,2 & $-0,27$ & $-0,24$ & $-2,1$ & $-2,6$ & 0,70 \\
\hline HB & -122 & $-4,9$ & $-4,6$ & 0,1 & $-0,14$ & $-0,08$ & $-4,2$ & $-4,7$ & 0,78 \\
\hline NS & -278 & $-1,5$ & $-1,2$ & $-0,3$ & 0,03 & 0,04 & $-0,9$ & $-1,6$ & 0,84 \\
\hline NW & -85 & $-0,2$ & $-0,2$ & 0,0 & $-0,09$ & $-0,08$ & 0,2 & $-0,5$ & 1,89 \\
\hline $\mathrm{HE}$ & -19 & $-0,1$ & $-0,4$ & 0,2 & $-0,12$ & $-0,12$ & 0,3 & $-0,4$ & 1,20 \\
\hline $\mathrm{RP}$ & -36 & $-0,4$ & $-0,3$ & $-0,1$ & $\mathbf{0 , 1 3}$ & 0,13 & 0,1 & $-0,6$ & 0,61 \\
\hline $\mathrm{SA}$ & -115 & $-4,2$ & $-3,6$ & $-0,4$ & 0,18 & 0,23 & $-3,6$ & $-4,2$ & 0,76 \\
\hline BW & 9 & 0,0 & 0,0 & 0,0 & $-0,03$ & $-0,03$ & 0,4 & $-0,3$ & 1,82 \\
\hline BY & -140 & $-0,5$ & $-0,4$ & $-0,1$ & $-0,01$ & $\overrightarrow{0,00}$ & 0,0 & $-0,7$ & $-1,53$ \\
\hline $\mathrm{OL}$ & 1146 & 3,0 & 3,0 & 0,2 & 1,07 & 0,86 & 3,8 & 3,8 & 0,36 \\
\hline$\sum$ & 57 & & 111 & 2 & & & & & \\
\hline \multicolumn{3}{|c|}{ Gini-Koeff. vor StV } & 0,179 & n.StV & 0,142 & n.LFA & 0,065 & n.BEZ & 0,069 \\
\hline
\end{tabular}

Tabelle V.9: Wiss. BeIRAT III, Ausgabenautonomie

Länder untereinander. Daher differieren die Wohlfahrtswirkungen in Variante IV nicht so stark.

Die veränderten Substitutionseffekte bewirken kaum Wohlfahrtsveränderungen. Nur in der zweiten Variante übersteigen sie für zwei Länder $1 \%$ des öffentlichen Budgets. Für fast alle Länder verlaufen die Wohlfahrtsgewinne aus den Substitutionseffekten $\left(\mathrm{HEV}_{S E}\right)$ entgegengesetzt zur Veränderung der Terms of Trade. Dies kann entweder auf hohe intraregionale Substitutionseffekte oder auf interregionale Einkommenseffekte zurückgeführt werden. In der Regel treten starke intraregionale Substitutionseffekte auf, da ein Anstieg des regionalen Preisniveaus die intraregionalen Substitutionseffekte erhöht. Interessanterweise gibt es keinen eindeutigen Zusammenhang zwischen der Veränderung der sozialen Grenzkosten der öffentlichen Güter und der $\operatorname{HEV}_{S E}$.

Bei nahezu konstantem Arbeitsangebot müßten die, von der Veränderung der $m_{G}$, bezüglich der Freizeit ausgelösten Substitutionseffekte niedriger sein, da eine Preisverzerrung geringere Substitutionseffekte auslöst als bei flexiblem Arbeitsangebot. Dies müßte zu einem verstärkten Zusammenhang zwischen der Richtung der Multiplikatorveränderung und der Richtung der $\mathrm{HEV}_{S E}$ führen. In der Tabelle V.10, in der die Ergebnisse mit variablem und fixem Arbeitsangebot gegenübergestellt werden, läßt sich dies deutlich sehen. Die Resultate zeigen, daß sich sich der positive Zusammenhang zwischen der Veränderung 
von $m_{G}$, und der $\mathrm{HEV}_{S E}$ verstärkt. Bis auf zwei Ausnahmen besteht bei fixem Arbeitsangebot ein positiver Zusammenhang zwischen der Veränderung des Multiplikators $m_{G}$, und der $\mathrm{HEV}_{S E}$. Es zeigt sich damit, daß der Multiplikator durchaus einen Einfluß auf die gesamten Substitutionseffekte ausübt. In den beiden Fällen in denen der Zusammenhang nicht gilt, ist die Veränderung des Multiplikators sehr gering, so daß er in diesen Ländern (HH und NRW) durch andere Effekte (Einkommenseffekte) überkompensiert werden kann.

\begin{tabular}{|c|c|c|c|c|c|c|}
\hline \multirow[b]{2}{*}{ Land } & \multicolumn{3}{|c|}{ Variabl. Arbeitsangebot } & \multicolumn{3}{|c|}{ „Fixes” Arbeitsangebot } \\
\hline & \begin{tabular}{r|}
$\mathrm{HEV}_{S E} \%$ \\
$\mathrm{REV}$
\end{tabular} & $\begin{array}{r}\mathrm{ToT}_{S E} \% \\
\mathrm{ToT} \\
\end{array}$ & $\begin{array}{r}\Delta m_{G_{s}} \\
\text { in } \% m_{G_{s}}^{0}\end{array}$ & \begin{tabular}{r|}
$\mathrm{HEV}_{S E} \%$ \\
$\mathrm{REV}$
\end{tabular} & $\begin{array}{r}\text { ToT }_{S E} \% \\
\text { ToT } \\
\end{array}$ & $\begin{array}{r}\Delta m_{G}, \\
\text { in } \% m_{G}^{0}\end{array}$ \\
\hline SH & $-1,1$ & $-0,01$ & $-0,45$ & $-1,0$ & 0.01 & $-0,14$ \\
\hline $\mathrm{HH}$ & 1,0 & $-0,23$ & $-0,21$ & 0,6 & $-0,24$ & \begin{tabular}{|l|}
$-0,05$ \\
\end{tabular} \\
\hline $\mathrm{HB}$ & 0,4 & $-0,08$ & $-0,53$ & $-0,4$ & $-0,08$ & $-0,17$ \\
\hline NS & $-0,5$ & 0,04 & $-0,52$ & $-0,6$ & 0,04 & $-0,14$ \\
\hline NW & 0,1 & $-0,08$ & $-0,21$ & 0,1 & $-0,08$ & 0,32 \\
\hline $\mathrm{HE}$ & 0,5 & $-0,12$ & $-0,38$ & 0,5 & 0,04 & $-0,01$ \\
\hline $\mathrm{RP}$ & $-0,3$ & 0,13 & $-0,27$ & $-0,3$ & $-0,12$ & $-0,10$ \\
\hline SA & $-0,6$ & 0,23 & $-1,14$ & $-1,1$ & 0,13 & $-0,49$ \\
\hline BW & 0,3 & $-0,03$ & $-0,02$ & 0,5 & 0,23 & 0,40 \\
\hline $\mathrm{BY}$ & 0,0 & 0,00 & $-0,35$ & 0,0 & 0,00 & 0,14 \\
\hline $\mathrm{OL}$ & $-0,2$ & 0,83 & 0,61 & 0,1 & 0,85 & 0,11 \\
\hline
\end{tabular}

Tabelle V.10: Wiss. Beirat I, Ausgabenautonomie, mit und ohne Freizeitwahl

Die Finanzkraft der Länder ist nach erfolgtem Länderfinanzausgleich stärker gleichverteilt als nach Erhalt der Ergänzungszuweisungen. Dies gilt allerdings nicht für die vierte Variante, da die Zuschüsse des Bundes im LFA dort als Ergänzungszuweisungen gewertet werden.

Die Unterschiede zwischen den Ergebnissen bei Ausgabenautonomie und fehlender Finanzautonomie (Tabellen V.11, V.12, V.13 und V.14) sind in allen Fällen mit eindeutigen Wohlfahrtseffekten sehr gering (unter $10 \%$ der Gesamteffekte und teilweise erheblich darunter). Überraschenderweise gilt nicht immer, daß die Wohlfahrtsgewinne bei Rationierung niedriger und die Wohlfahrtsverluste höher sind als bei Ausgabenautonomie. Dies kann zum Teil durch überkompensierende, gegenläufige Transferveränderungen erklärt werden. Da insgesamt die Auswirkungen der Ausgabenautonomie sehr klein sind, kann man davon ausgehen, daß sich die sozialen Grenzkosten der öffentlichen Güter nur wenig auf die Wohlfahrtseffekte auswirken. Dies verdeutlichen auch die geringen Unterschiede der $\mathrm{HEV}_{S E}$ zwischen den Berechnungen mit und ohne Ausgabenautonomie. 


\begin{tabular}{|c|c|c|c|c|c|c|c|c|c|}
\hline \multirow[t]{3}{*}{ Land } & \multicolumn{2}{|c|}{$\mathrm{HEV}$} & \multirow{2}{*}{$\begin{array}{l}\Delta \mathrm{TR} \\
\text { in } \%\end{array}$} & \multirow{2}{*}{$\begin{array}{r}\mathrm{HEV}_{S E} \\
\text { in \% }\end{array}$} & \multirow{2}{*}{$\begin{array}{r}\mathrm{ToT}_{G E} \\
\text { in } \%\end{array}$} & \multirow{2}{*}{$\begin{array}{r}\text { ToT }_{S E} \\
\text { in } \%\end{array}$} & \multicolumn{2}{|c|}{$\overline{F A}$} & \multirow{3}{*}{$\begin{array}{r}\Delta m_{G_{s}} \\
\text { in } \% \\
m_{G \text { s }}^{0}\end{array}$} \\
\hline & Mio & in $\%$ & & & & & neu $\%$ & f.r. $\%$ & \\
\hline & $\mathrm{DM}$ & REV & REV & REV & ToT & ToT & REV & REV & \\
\hline $\mathrm{SH}$ & -253 & $-3,8$ & $-2,6$ & $-1,2$ & $-0,07$ & $-0,01$ & $-2,8$ & $-3,7$ & $-0,14$ \\
\hline $\mathrm{HH}$ & -31 & $-0,5$ & $-1,4$ & 1,1 & $-0,24$ & $-0,23$ & $-0,1$ & $-0,7$ & $-0,05$ \\
\hline HB & -171 & $-6,9$ & $-6,3$ & 0,2 & $-0,15$ & $-0,08$ & $-5,6$ & $-6,3$ & \begin{tabular}{|l|}
$-0,12$ \\
\end{tabular} \\
\hline NS & -653 & $-3,5$ & $-2,7$ & $-0,6$ & $-0,01$ & 0,04 & $-2,6$ & $-3,4$ & $-0,14$ \\
\hline NW & -42 & $-0,1$ & $-0,2$ & 0,1 & $-0,08$ & $-0,08$ & 0,3 & $-0,4$ & 0,32 \\
\hline $\mathrm{HE}$ & 110 & 0,7 & 0,1 & 0,6 & $-0,11$ & $-0,12$ & 1,1 & 0,4 & \begin{tabular}{|l|}
$-0,01$ \\
\end{tabular} \\
\hline $\mathrm{RP}$ & -143 & $-1,5$ & $-1,1$ & $-0,3$ & 0,11 & 0,12 & $-0,9$ & $-1,6$ & $-0,10$ \\
\hline $\mathrm{SA}$ & -223 & $-8,1$ & $-6,5$ & $-0,8$ & 0,13 & 0,22 & $-6,6$ & $-7,5$ & $-0,49$ \\
\hline BW & 484 & 1,9 & 1,5 & 0,4 & $-0,01$ & $-0,03$ & 2,1 & 1,5 & 0,40 \\
\hline BY & -211 & $-0,7$ & $-0,7$ & 0,0 & $-0,02$ & 0,00 & $-0,2$ & $-0,9$ & 0,14 \\
\hline $\mathrm{OL}$ & 1132 & 3,0 & 3,3 & $-0,1$ & 1,09 & 0,84 & 3,8 & 3,8 & 0,11 \\
\hline$\sum$ & 0 & & 178 & -9 & & & & & \\
\hline
\end{tabular}

Tabelle V.11: Wiss. Beirat I, keine Autonomie

\begin{tabular}{|c|c|c|c|c|c|c|c|c|c|}
\hline \multirow[t]{3}{*}{ Land } & \multicolumn{2}{|c|}{$\mathrm{HEV}$} & \multirow{3}{*}{$\begin{array}{l}\Delta \mathrm{TR} \\
\text { in } \% \\
\mathrm{REV}\end{array}$} & \multirow{3}{*}{$\begin{array}{r}\mathrm{HEV}_{S E} \\
\text { in \% } \\
\mathrm{REV}\end{array}$} & \multirow{3}{*}{$\begin{array}{r}\mathrm{ToT}_{G E} \\
\text { in } \% \\
\text { ToT }\end{array}$} & \multirow{3}{*}{$\begin{array}{r}\mathrm{ToT}_{S E} \\
\text { in \% } \\
\text { ToT }\end{array}$} & \multicolumn{2}{|c|}{ FA } & \multirow{3}{*}{$\begin{array}{c}\Delta m_{G}, \\
\text { in } \% \\
m_{G s}^{0}\end{array}$} \\
\hline & Mio & in $\%$ & & & & & neu \% & f.r. $\%$ & \\
\hline & DM & REV & & & & & REV & REV & \\
\hline $\mathrm{SH}$ & -377 & $-5,7$ & $-3,7$ & $-1,8$ & $-0,13$ & $-0,03$ & $-4,4$ & $-5,5$ & 0,24 \\
\hline $\mathrm{HH}$ & 270 & 4,2 & 1,7 & 2,3 & $-0,18$ & $-0,21$ & 3,9 & 3,4 & $-0,07$ \\
\hline HB & -211 & $-8,5$ & $-7,9$ & 0,4 & $-0,16$ & $-0,08$ & $-7,0$ & $-7,8$ & 0,19 \\
\hline NS & -930 & $-5,0$ & $-3,8$ & $-0,9$ & $-0,05$ & 0,03 & $-3,8$ & $-4,8$ & 0,28 \\
\hline NW & -138 & $-0,3$ & $-0,4$ & 0,1 & $-0,08$ & $-0,08$ & 0,1 & $-0,6$ & 0,30 \\
\hline $\mathrm{HE}$ & 249 & 1,6 & 0,6 & 1,0 & $-0,10$ & $-0,11$ & 1,9 & 1,3 & $-0,04$ \\
\hline $\mathrm{RP}$ & -580 & $-6,2$ & $-4,7$ & $-1,0$ & 0,03 & 0,11 & $-4,9$ & $-5,8$ & 0,23 \\
\hline SA & -147 & $-5,3$ & $-4,4$ & $-0,6$ & 0,16 & 0,23 & $-4,1$ & $-5,1$ & 0,16 \\
\hline BW & 1109 & 4,3 & 3,5 & 0,8 & 0,02 & $-0,02$ & 4,5 & 3,9 & 0,34 \\
\hline BY & -435 & $-1,6$ & $-1,3$ & 0,0 & $-0,04$ & 0,00 & $-0,9$ & $-1,6$ & 0,16 \\
\hline OL & 1121 & 3,0 & 3,8 & $-0,5$ & 1,09 & 0,80 & 3,9 & 3,8 & 0,17 \\
\hline$\sum$ & -89 & & 234 & -31 & & & & & \\
\hline
\end{tabular}

Tabelle V.12: Wiss. BeIRAT II, keine Autonomie 


\begin{tabular}{|c|c|c|c|c|c|c|c|c|c|}
\hline \multirow[t]{3}{*}{ Land } & \multicolumn{2}{|c|}{$\mathrm{HEV}$} & $\Delta \mathrm{TR}$ & $\mathrm{HEV}_{S E}$ & $\mathrm{ToT}_{G E}$ & ToT $_{S E}$ & \multicolumn{2}{|c|}{ FA } & \multirow{3}{*}{$\begin{array}{c}\Delta m_{G}, \\
\text { in } \% \\
m_{G}^{0}\end{array}$} \\
\hline & Mio & in $\%$ & in $\%$ & in $\%$ & in $\%$ & in $\%$ & neu $\%$ & f.r. $\%$ & \\
\hline & $\mathrm{DM}$ & REV & REV & REV & ToT & ToT & REV & REV & \\
\hline $\mathrm{SH}$ & -128 & $-1,9$ & $-1,3$ & $-0,6$ & $-0,02$ & 0,00 & $-1,2$ & $-2,0$ & 0,00 \\
\hline $\mathrm{HH}$ & -177 & $-2,8$ & $-2,6$ & 0,2 & $-0,27$ & $-0,24$ & $-2,0$ & $-2,6$ & 0,06 \\
\hline HB & -125 & $-5,0$ & $-4,4$ & $-0,1$ & $-0,13$ & $-0,08$ & $-4,0$ & $-4,7$ & 0,00 \\
\hline NS & -282 & $-1,5$ & $-1,2$ & $-0,3$ & $-0,02$ & 0,04 & $-0,8$ & $-1,6$ & $-0,00$ \\
\hline NW & -77 & $-0,2$ & $-0,2$ & 0,0 & $-0,09$ & $-0,08$ & 0,2 & $-0,5$ & 0,43 \\
\hline $\mathrm{HE}$ & -17 & $-0,1$ & $-0,4$ & 0,2 & $-0,12$ & $-0,12$ & 0,3 & $-0,4$ & 0,15 \\
\hline $\mathrm{RP}$ & -35 & $-0,4$ & $-0,3$ & $-0,1$ & 0,13 & 0,13 & 0,1 & $-0,6$ & 0,01 \\
\hline SA & -118 & $-4,3$ & $-3,5$ & $-0,5$ & 0,18 & 0,23 & $-3,4$ & $-4,2$ & $-0,25$ \\
\hline BW & 16 & 0,0 & 0,0 & 0,0 & 0,03 & $-0,03$ & 0,4 & $-0,3$ & 0,55 \\
\hline BY & -137 & $-0,5$ & $-0,4$ & $-0,1$ & $-0,01$ & 0,00 & 0,0 & $-0,7$ & 0,27 \\
\hline $\mathrm{OL}$ & 1135 & 3,0 & 2,9 & 0,3 & 1,09 & 0,87 & 3,7 & 3,8 & 0,11 \\
\hline$\sum$ & 54 & & 120 & 8 & & & & & \\
\hline
\end{tabular}

Tabelle V.13: Wiss. BeIrat III, keine Autonomie

\begin{tabular}{|c|c|c|c|c|c|c|c|c|c|}
\hline \multirow[t]{3}{*}{ Land } & \multicolumn{2}{|c|}{ HEV } & \multirow{3}{*}{$\begin{array}{l}\Delta \mathrm{TR} \\
\text { in } \% \\
\mathrm{REV}\end{array}$} & \multirow{3}{*}{$\begin{array}{r}\mathrm{HEV}_{S E} \\
\text { in \% } \\
\mathrm{REV}\end{array}$} & \multirow{3}{*}{$\begin{array}{r}\mathrm{ToT}_{G E} \\
\text { in \% } \\
\mathrm{ToT} \\
\end{array}$} & \multirow{3}{*}{$\begin{array}{r}\text { ToT }_{S E} \\
\text { in \% } \\
\text { ToT }\end{array}$} & \multicolumn{2}{|c|}{ FA } & \multirow{3}{*}{$\begin{array}{c}\Delta m_{G} \\
\text { in } \% \\
m_{G_{s}}^{0}\end{array}$} \\
\hline & Mio & in $\%$ & & & & & neu $\%$ & f.r. $\%$ & \\
\hline & $\mathrm{DM}$ & REV & & & & & REV & REV & \\
\hline $\mathrm{SH}$ & -229 & $-3,5$ & $-2,4$ & 0,5 & $-0,06$ & 0,03 & 2,7 & 1,9 & $-0,09$ \\
\hline $\mathrm{HH}$ & -111 & $-1,7$ & $-2,2$ & $-0,3$ & $-0,25$ & $-0,25$ & $\mathbf{1 , 1}$ & 0,5 & $-0,01$ \\
\hline $\mathrm{HB}$ & -94 & $-3,8$ & $-3,8$ & $-0,8$ & $-0,12$ & $-0,09$ & $-0,5$ & $-1,1$ & -0.07 \\
\hline NS & -370 & $-2,0$ & $-1,5$ & 0,1 & 0,02 & 0,05 & 3,0 & 2,2 & \begin{tabular}{|l|}
-0.09 \\
\end{tabular} \\
\hline NW & 279 & 0,6 & 0,4 & $-0,6$ & $-0,08$ & $-0,09$ & 3,7 & 3,0 & 0.36 \\
\hline $\mathrm{HE}$ & 25 & 0,2 & $-0,3$ & 0,2 & $-0,12$ & $-0,12$ & 3,9 & 3,0 & 0,05 \\
\hline $\mathrm{RP}$ & 16 & 0,2 & 0,2 & $-0,6$ & 0,13 & 0,12 & 3,1 & 3,1 & $-0,06$ \\
\hline $\mathrm{SA}$ & -114 & $-4,1$ & $-3,4$ & $-0,5$ & 0,18 & 0,23 & $-0,1$ & $-0,9$ & $-0,46$ \\
\hline BW & 238 & 0,9 & 0,6 & $-0,6$ & $-0,02$ & $-0,04$ & 3,6 & 3,0 & 0,45 \\
\hline BY & -2 & 0,0 & $-0,1$ & $-0,4$ & 0,00 & $-0,01$ & 3,5 & 2,8 & 0,19 \\
\hline $\mathrm{OL}$ & 366 & 1,0 & 1,6 & 1,6 & 0,93 & 0,97 & 7,2 & 7,1 & 0,14 \\
\hline$\sum$ & 4 & & 138 & 40 & & & & & \\
\hline
\end{tabular}

Tabelle V.14: Wiss. Beirat IV, keine Autonomie 
Die Werte der First-round-Berechnungen der Finanzausgleichstransfers ergeben in allen Varianten recht gute Näherungen an die relative Größenordnung der gesamten Finanzausgleichstransfers. Sie weichen allerdings in Einzelfällen um mehrere hundert Prozent von den tatsächlichen Werten ab. Bei höheren Beträgen differieren beide Berechnungsarten dagegen um höchstens $20 \%$.

Die gesamten Wohlfahrtseffekte entsprechen in den Varianten I bis III in Vorzeichen und Größenordnung den First-round-Berechnungen der Finanzausgleichstransfers. Daher sind die First-round-Werte eine gute Näherung für die tatsächlich auftretenden Wohlfahrtsgewinne der einzelnen Länder. Allerdings enthalten die als Finanzausgleichstransfers angegebenen Werte auch die Steuerexporte, die in den üblichen Finanzausgleichsberechnungen nicht ermittelt werden. Nur in der Variante IV gibt es so gut wie keine Übereinstimmung zwischen den First-round-Finanzausgleichszahlungen und den gesamten Wohlfahrtseffekten. Teilweise weichen sogar die Vorzeichen voneinander ab. Die Nichtbeachtung der Veränderung der nationalen Zuweisungen und Transfers in den First-round-Berechnungen ist die Ursache für diese Divergenz. Nur wenn man bei der Ermittlung der Finanzausgleichszahlungen auch die Veränderung sonstiger öffentlicher Zahlungsströme mitberücksichtigt, die eine Reaktion auf veränderte Einnahmen des Bundes darstellen, kann man durch First-round-Berechnungen die tatsächlichen Wohlfahrtseffekte abschätzen. Dies ist z.B. bei einer Kredit- oder Steuerfinanzierung der zusätzlichen Lasten des Bundes nicht mehr ohne weiteres möglich. Reagiert der Bund dagegen durch eine Verringerung der sonstigen Zuweisungen oder Transfers, wie in der hier durchgeführten Simulation, so kann man dies, a priori, in etwa abschätzen und entsprechend aussagekräftige First-roundBerechnungen anstellen.

\section{(2) Sensitivitätsanalysen zum Vorschlag des Wissenschaftlichen Beirats}

\section{(a) Substitutionselastizitäten zwischen Kapital und Arbeit}

Durch Variation der Substitutionselastizitäten zwischen Kapital und Arbeit kann die Bedeutung dieser Elastizitäten für die Ergebnisse ermittelt werden.

Niedrigere Substitutionselastizitäten bedeuten stärker gekrümmte Isoquanten der Wertschöpfungsfunktion. Veränderungen der Faktorpreisrelationen lösen daher nur geringe Substitutionsprozesse aus. Da Substitutionen normalerweise dämpfend auf die ursprünglichen Faktorpreisveränderungen einwirken, werden die Preisveränderungen bei niedrigeren Elastizitäten vermutlich höher ausfallen. Demnach werden Produzenten- und Konsumentenpreise stärker differieren als bei hohen Substitutionselastizitäten. Dadurch werden Substitutionseffekte und Wohlfahrtseffekte verstärkt. 


\begin{tabular}{|c|c|c|c|c|c|c|c|c|c|}
\hline \multirow[t]{3}{*}{ Land } & \multicolumn{2}{|c|}{$\mathrm{HEV}$} & \multirow{2}{*}{$\begin{array}{l}\Delta \mathrm{TR} \\
\text { in } \%\end{array}$} & \multirow{2}{*}{$\begin{array}{r}\mathrm{HEV}_{S E} \\
\text { in \% }\end{array}$} & \multirow{2}{*}{$\begin{array}{r}\mathrm{ToT}_{G E} \\
\text { in \% }\end{array}$} & \multirow{2}{*}{$\begin{array}{r}\text { ToT }_{S E} \\
\text { in \% }\end{array}$} & \multicolumn{2}{|c|}{$\mathrm{FA}$} & \multirow{3}{*}{$\begin{array}{r}\Delta m_{G} \\
\text { in } \% \\
m_{G}^{0}\end{array}$} \\
\hline & Mio & in $\%$ & & & & & neu $\%$ & f.r.\% & \\
\hline & DM & REV & REV & REV & ToT & ToT & REV & REV & \\
\hline $\mathrm{SH}$ & -249 & $-3,7$ & $-2,5$ & $-1,2$ & $-0,07$ & $-0,02$ & $-2,9$ & $-3,7$ & $-0,14$ \\
\hline $\mathrm{HH}$ & -32 & $-0,5$ & $-1,4$ & 1,2 & $-0,24$ & $-0,22$ & $-0,2$ & $-0,7$ & $-0,56$ \\
\hline HB & -165 & $-6,6$ & $-6,5$ & 0,5 & $-0,15$ & $-0,07$ & $-5,9$ & $-6,3$ & $-0,12$ \\
\hline NS & -640 & $-3,4$ & $-2,7$ & $-0,6$ & $-0,01$ & 0,03 & $-2,7$ & $-3,4$ & $-0,14$ \\
\hline NW & -57 & $-0,1$ & $-0,2$ & 0,1 & $-0,08$ & $-0,08$ & 0,3 & $-0,4$ & 0,32 \\
\hline $\mathrm{HE}$ & 105 & 0,7 & 0,1 & 0,6 & $-0,11$ & $-0,12$ & 1,1 & 0,4 & $-0,01$ \\
\hline $\mathrm{RP}$ & -143 & $-1,5$ & $-1,1$ & $-0,3$ & 0,10 & 0,12 & $-0,9$ & $-1,6$ & $-0,10$ \\
\hline SA & -213 & $-7,7$ & $-6,6$ & $-0,7$ & 0,12 & 0,22 & $-6,9$ & $-7,5$ & $-0,49$ \\
\hline BW & 471 & 1,8 & 1,4 & 0,4 & $-0,01$ & $-0,03$ & 2,2 & 1,5 & 0,40 \\
\hline BY & -218 & $-0,7$ & $-0,7$ & 0,0 & $-0,02$ & 0,00 & $-0,2$ & $-0,9$ & 0,14 \\
\hline $\mathrm{OL}$ & 1135 & 3,0 & 3,5 & $-0,2$ & 1,09 & 0,81 & 3,9 & 3,8 & 0,11 \\
\hline$\Sigma$ & -5 & & 205 & -9 & & & & & \\
\hline
\end{tabular}

Tabelle V.15: Wiss. Beirat, Variante I, Ausgabenautonomie, Variation der Substitutionselastizitäten zwischen Kapital und Arbeit

Simuliert man den Vorschlag des Wissenschaftlichen Beirats [1992], Variante I, mit niedrigeren Substitutionselastizitäten zwischen Kapital und Arbeit, hier $\sigma=0,2$, so zeigt

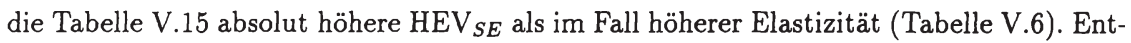
sprechend der oben angeführten Vermutung, entstehen bei niedrigerer Substitutionselastizität höhere Gewinne und Verluste durch die Substitutionseffekte $\left(\mathrm{HEV}_{S E}\right)$. Allerdings sind die Unterschiede relativ gering, so daß letztlich der beschriebene Mechanismus nur einen marginalen Einfluß auf die Ergebnisse besitzt. Bedingt, durch zusätzlich auftretende Nettotransferveränderungen, die zu einer Nivellierung der sich ergebenden stärkeren Differenzen der $\mathrm{HEV}_{S E}$ führen, ergeben sich jedoch kaum Veränderungen der gesamten Wohlfahrt der einzelnen Länder und der Gesamtwirtschaft.

\section{(b) Fehlende Kapitalmobilität}

Hebt man die Annahme der vollkommenen Kapitalmobilität auf, und ersetzt sie durch vollkommene interregionale Immobilität von Kapital, so erweitert sich das Modell um die Faktornachfragefunktionen für Kapital in den einzelnen Regionen. Im Algorithmus sind dann elf regionale Kapitallohnsätze $\mathrm{zu}$ bestimmen.

Welche Wirkungen sind bei Immobilität von Kapital zu erwarten? Da die Unternehmen einer Region nur noch auf das regionale Kapitalangebot zurückgreifen können, bewirkt ein 


\begin{tabular}{|c|c|c|c|c|c|c|c|c|c|}
\hline \multirow[t]{3}{*}{ Land } & \multicolumn{2}{|c|}{$\mathrm{HEV}$} & \multirow{3}{*}{$\begin{array}{l}\Delta \mathrm{TR} \\
\text { in } \% \\
\mathrm{REV}\end{array}$} & \multirow{3}{*}{$\begin{array}{r}\mathrm{HEV}_{S E} \\
\text { in \% } \\
\mathrm{REV}\end{array}$} & \multirow{3}{*}{$\begin{array}{r}\text { ToT }_{G E} \\
\text { in } \% \\
\text { ToT }\end{array}$} & \multirow{3}{*}{$\begin{array}{r}\text { ToT } \\
\text { in } \% \\
\text { ToT }\end{array}$} & \multicolumn{2}{|c|}{$\mathrm{FA}$} & \multirow{3}{*}{$\begin{array}{c}\Delta m_{G}, \\
\text { in \% } \\
m_{G_{s}}^{0}\end{array}$} \\
\hline & Mio & in $\%$ & & & & & neu $\%$ & f.r. $\%$ & \\
\hline & $\mathrm{DM}$ & REV & & & & & REV & REV & \\
\hline $\mathrm{SH}$ & -241 & $-3,6$ & $-2,6$ & $-1,0$ & $-0,10$ & $-0,04$ & $-2,9$ & $-3,7$ & $-0,14$ \\
\hline $\mathrm{HH}$ & -39 & $-0,6$ & $-1,1$ & 0,7 & $-0,25$ & $-0,24$ & $-0,1$ & $-0,7$ & $-0,05$ \\
\hline HB & -164 & $-6,6$ & $-6,2$ & 0,1 & $-0,14$ & $-0,08$ & $-5,8$ & $-6,3$ & $-0,12$ \\
\hline NS & -629 & $-3,3$ & $-2,7$ & $-0,6$ & $-0,02$ & 0,03 & $-2,6$ & $-3,4$ & $-0,14$ \\
\hline NW & -15 & 0,0 & $-0,1$ & 0,1 & $-0,09$ & $-0,09$ & 0,3 & $-0,4$ & 0,32 \\
\hline $\mathrm{HE}$ & 118 & 0,8 & 0,3 & 0,5 & $-0,11$ & $-0,12$ & 1,1 & 0,4 & $-0,01$ \\
\hline $\mathrm{RP}$ & -132 & $-1,4$ & $-1,1$ & $-0,3$ & 0,10 & 0,12 & $-0,9$ & $-1,6$ & $-0,10$ \\
\hline SA & -210 & $-7,6$ & $-6,6$ & $-0,6$ & 0,15 & 0,24 & $-6,9$ & $-7,5$ & $-0,49$ \\
\hline BW & 517 & 2,0 & 1,5 & 0,4 & $-0,02$ & $-0,04$ & 2,2 & 1,5 & 0,40 \\
\hline BY & -185 & $-0,6$ & $-0,6$ & 0,0 & $-0,03$ & $-0,02$ & $-0,2$ & $-0,9$ & 0,14 \\
\hline $\mathrm{OL}$ & 1076 & 2,8 & 3,0 & 0,1 & 1,31 & 1,08 & 3,7 & 3,8 & 0,11 \\
\hline$\Sigma$ & 96 & & 176 & 64 & & & & & \\
\hline
\end{tabular}

Tabelle V.16: Wiss. BEIRAT, Variante I, Ausgabenautonomie, ohne Kapitalmobilität

Rückgang der regionalen Produktion einen Angebotsüberschuß von Kapital. Als dessen Folge wird der Faktorpreis von Kapital und letztlich auch die Güterpreise, insbesondere die Preise der relativ kapitalintensiv produzierten Güter, sinken. Dieser Rückgang wird deutlich stärker ausfallen als bei Kapitalmobilität, da der Angebotsüberschuß nicht durch Kapitalzuwanderung, sondern ausschließlich über einen Anstieg der Kapitalentlohnung reduziert werden kann. Insgesamt ergeben sich stärkere Faktorpreisausschläge mit höheren Preisveränderungen als bei Kapitalmobilität. Damit werden auftretende Substitutionseffekte tendenziell verstärkt.

Besonders deutlich werden die Faktorpreise der Länder steigen, die relativ hohe, zusätzliche Nettotransfers erhalten, und die derjenigen Länder fallen, deren Nettotransfers sinken. $\mathrm{Da}$ in allen Ländern die gehandelten Güter relativ kapitalintensiv produzierten werden, werden sich tendenziell die ToT der Länder mit gestiegenen (gefallenen) Nettotransfers verbessern (verschlechtern). Insgesamt ist daher, bei immobilem Kapital, eine stärkere Differenzierung der ToT und damit der Wohlfahrtseffekte zu erwarten.

Überraschenderweise entsprechen die Ergebnisse des Vorschlags des WissenschaftLICHEN Beirats [1992], Variante I, nicht diesen Erwartungen. Aus der Tabelle V.16 ist ersichtlich, daß die Unterschiede zwischen beiden Modellvarianten sehr gering sind. Dennoch zeigt eine genauere Betrachtung der Resultate, daß zum einen die Wohlfahrtseffekte bei immobilem Kapital, etwas näher beieinander liegen und zum zweiten, die ToT 
ebenfalls weniger stark auseinanderfallen. Letzteres gilt, obwohl die Kapitallohnsätze sehr stark zwischen den Regionen differenziert sind. Die Begründung liefern die Nettotransferveränderungen, die sich dämpfend auf die ToT auswirken. Da die Länder mit hohen Kapitallohnsätzen und damit auch hohen Güterpreisen höhere Steuereinnahmen erzielen, die zu einem Anstieg der regionalen Finanzkraft führen, erhalten sie nun geringere Finanzausgleichszuweisungen. Der entsprechende Einnahmenrückgang verringert, über zurückgehende Preise, die ursprüngliche ToT-Verbesserung.

Insgesamt ist der Einfluß der unterschiedlichen Grade von Kapitalmobilität nur sehr gering. Dies ist nicht verwunderlich, da die Veränderung der Finanzausgleichsregelungen Verzerrung auslöst, von der der Kapitaleinsatz direkt betroffen ist.

\section{(c) Variation der Vorleistungselastizitäten}

Bei einer höheren Substitutionselastizität der Vorleistungsgüter sind geringere Preis- und ToT-Differenzen zwischen den einzelnen Ländern zu erwarten. Die nicht direkt die Vorleistungsgüter betreffenden Substitutionseffekte müßten dann niedriger sein als bei einer geringeren Elastiziät. Die direkten Substitutionseffekte sind dagegen höher, da eine Veränderung der Preisrelationen relativ starke Substitutionen zwischen den verschiedenen Vorleistungsgütern hervorrufen. Weil jedoch der Vorleistungseinsatz nicht direkt durch die Finanzausgleichsregelungen verzerrt wird, ist anzunehmen, daß der letze Effekt nicht dominiert. Demnach wäre insgesamt eine Verringerung der Wohlfahrtsunterschiede zu erwarten. Dies wird durch die Werte der $\mathrm{HEV}_{S E}$ in der Tabelle V.17 bestätigt, die etwas näher beieinanderliegen, als bei einer geringeren Elastizität. Dennoch sind die Unterschiede nur minimal, so daß insgesamt davon ausgegangen werden kann, daß die Einflüsse der Substitutionselastizitäten der Vorleistungen recht unbedeutend sind.

\subsubsection{Buhl/Pfingsten [1991]}

\section{(1) Ergebnisse des Vorschlags von Buhl/Pfingsten [1991]}

Das von Buhl/Pfingsten [1991] empfohlene Verfahren führt zu einer höheren Gleichverteilung - Gini-Koeffizient ist 0,055 - als die bisher betrachteten Regelungen. Dies ist eine Folge der sehr hohen Umverteilungstransfers zwischen den Ländern, die zudem zu höheren Wohlfahrtsgewinnen der Nettoempfänger und höheren Wohlfahrtsverlusten der Nettozahler führen.

Insgesamt erhöht sich die gesellschaftliche Wohlfahrt (siehe Tabelle V.18). Dies ist auf gestiegene Steuerexporte an das Ausland sowie auf leichte Gewinne aus der Verringerung der Substitutionseffekte zurückzuführen. 


\begin{tabular}{|c|c|c|c|c|c|c|c|c|c|}
\hline \multirow[t]{3}{*}{ Land } & \multicolumn{2}{|c|}{$\mathrm{HEV}$} & \multirow{3}{*}{$\begin{array}{c}\text { TR } \\
\text { in } \% \\
\text { REV }\end{array}$} & \multirow{3}{*}{$\begin{array}{r}\mathrm{HEV}_{S E} \\
\text { in \% } \\
\mathrm{REV}\end{array}$} & \multirow{3}{*}{$\begin{array}{r}\text { ToT } \\
\text { in } \% \\
\text { ToT } \\
\end{array}$} & \multirow{3}{*}{$\begin{array}{r}\text { ToT }_{S E} \\
\text { in \% } \\
\text { ToT }\end{array}$} & \multicolumn{2}{|c|}{ FA } & \multirow{3}{*}{$\begin{array}{c}\Delta m_{G s} \\
\text { in \% } \\
m_{G}^{0}\end{array}$} \\
\hline & Mio & in $\%$ & & & & & neu $\%$ & f.r. $\%$ & \\
\hline & $\mathrm{DM}$ & REV & & & & & REV & REV & \\
\hline $\mathrm{SH}$ & -247 & $-3,7$ & $-2,8$ & $-0,9$ & $-0,05$ & 0,00 & $-2,9$ & $-3,7$ & $-0,14$ \\
\hline $\mathrm{HH}$ & -33 & $-0,5$ & $-1,2$ & 0,8 & $-0,23$ & $-0,02$ & $-0,2$ & $-0,7$ & $-0,06$ \\
\hline HB & -162 & $-6,5$ & $-6,4$ & 0,3 & $-0,14$ & $-0,08$ & $-5,9$ & $-6,3$ & $-0,12$ \\
\hline NS & -633 & $-3,4$ & $-2,8$ & $-0,5$ & 0,00 & 0,04 & $-2,7$ & $-3,4$ & $-0,14$ \\
\hline NW & -63 & $-0,1$ & $-0,2$ & 0,1 & $-0,08$ & $-0,08$ & 0,3 & $-0,4$ & 0,32 \\
\hline $\mathrm{HE}$ & 101 & 0,7 & 0,2 & 0,5 & $-0,11$ & $-0,12$ & 1,1 & 0,4 & $-0,01$ \\
\hline RP & -141 & $-1,5$ & $-1,5$ & $-0,3$ & 0,10 & 0,12 & 1,0 & $-0,7$ & $-0,10$ \\
\hline $\mathrm{SA}$ & -211 & $-7,7$ & $-6,8$ & $-0,6$ & 0,13 & 0,22 & $-6,9$ & $-7,5$ & $-0,49$ \\
\hline BW & 466 & 1,8 & 1,5 & 0,3 & $-0,01$ & $-0,03$ & 2,2 & 1,5 & 0,40 \\
\hline BY & -215 & $-0,7$ & $-0,7$ & 0,0 & $-0,02$ & 0,00 & $-0,2$ & $-0,9$ & 0,13 \\
\hline $\mathrm{OL}$ & 1169 & 3,1 & 3,4 & 0,1 & 1,03 & 0,82 & 4,0 & 3,9 & 0,11 \\
\hline$\Sigma$ & 31 & 148 & -9 & & & & & & \\
\hline
\end{tabular}

Tabelle V.17: Wiss. Beirat, Variante I, Ausgabenautonomie, Variation der Substitutionselastiziäten des Vorleistungseinsatzes, $\rho=3,0$

\begin{tabular}{|c|c|c|c|c|c|c|c|c|c|}
\hline \multirow[t]{3}{*}{ Land } & \multicolumn{2}{|c|}{ HEV } & \multirow{2}{*}{$\begin{array}{l}\Delta \mathrm{TR} \\
\text { in } \%\end{array}$} & \multirow{3}{*}{$\begin{array}{r}\mathrm{HEV}_{S E} \\
\text { in \% } \\
\mathrm{REV}\end{array}$} & \multirow{3}{*}{$\begin{array}{r}\mathrm{ToT}_{G E} \\
\text { in } \% \\
\mathrm{ToT} \\
\end{array}$} & \multirow{3}{*}{$\begin{array}{r}\text { ToT }_{S E} \\
\text { in } \% \\
\text { ToT }\end{array}$} & \multicolumn{2}{|c|}{ FA } & \multirow{3}{*}{$\begin{array}{c}\Delta m_{G_{s}} \\
\text { in \% } \\
m_{G_{s}}^{0}\end{array}$} \\
\hline & Mio & in $\%$ & & & & & neu $\%$ & f.r. $\%$ & \\
\hline & DM & REV & REV & & & & REV & REV & \\
\hline $\mathrm{SH}$ & 84 & 1,3 & 0,8 & 0,1 & 0,06 & 0,02 & 1,6 & 0,9 & 0,19 \\
\hline $\mathrm{HH}$ & -606 & $-9,4$ & $-7,0$ & $-1,3$ & $-0,36$ & $-0,26$ & $-7,9$ & $-8,3$ & 0,15 \\
\hline $\mathrm{HB}$ & -60 & $-2,4$ & $-1,9$ & $-0,4$ & $-0,11$ & $-0,09$ & $-1,8$ & $-2,4$ & 0,16 \\
\hline NS & 339 & 1,8 & 1,5 & 0,1 & 0,08 & 0,05 & 2,2 & 1,4 & 0,20 \\
\hline NW & -617 & $-1,3$ & $-1,1$ & $-0,3$ & $-0,10$ & $-0,09$ & $-1,0$ & $-1,7$ & 0,41 \\
\hline $\mathrm{HE}$ & -465 & $-3,0$ & $-2,5$ & $-0,5$ & $-0,15$ & $-0,13$ & $-2,5$ & $-3,3$ & 0,26 \\
\hline $\mathrm{RP}$ & 110 & 1,2 & 0,8 & 0,0 & 0,16 & 0,13 & 1,3 & 0,5 & 0,16 \\
\hline SA & 39 & 1,4 & 1,1 & 0,0 & 0,28 & 0,24 & 1,5 & 0,8 & 0,07 \\
\hline BW & -1350 & $-5,2$ & $-4,4$ & $-0,8$ & $-0,10$ & $-0,01$ & $-4,8$ & $-5,5$ & 0,48 \\
\hline BY & -264 & $-0,9$ & $-0,8$ & $-0,3$ & 0,00 & 0,00 & $-0,6$ & $-1,3$ & 0,36 \\
\hline $\mathrm{OL}$ & 2988 & 7,8 & 6,4 & 1,7 & 1,43 & 0,97 & 8,3 & 8,8 & 0,09 \\
\hline$\sum$ & 196 & & 101 & 37 & & & & & \\
\hline \multicolumn{3}{|c|}{ Gini-Koeff. vor StV } & 0,179 & n.StV & 0,150 & n.LFA & 0,055 & n.BEZ & 0,055 \\
\hline
\end{tabular}

Tabelle V.18: Buhl/Pfingsten, Ausgabenautonomie 
Die Substitutionseffekte sind sehr gering und übersteigen nur für die Ostländer und Hamburg $1 \%$ der öffentlichen Einnahmen. In beiden Fällen verändern sich die Terms of Trade der Substitutionseffekte sehr stark, so daß die Wohlfahrtsgewinne aus den Substitutionseffekten weitgehend durch die Veränderung der ToT erklärt werden können. Allerdings gilt dies für drei Länder nicht. Für diese Länder - Rheinland-Pfalz, Saarland und Bayern - werden die Nettotransfers in der Berechnung der Substitutionseffekte verändert und bestimmen letztlich die Vorzeichen der $\mathrm{HEV}_{S E}$. Dies zeigt wieder, daß eine exakte Trennung von Einkommens- und Substitutionseffekten nicht möglich ist, da die Substitutionseffekte selbst Einkommenseffekte auslösen, die die reinen Substitutionseffekte überlagern können. Es zeigt sich jedoch auch, daß die reinen Substitutionseffekte sehr gering sind und von den, durch sie hervorgerufenen und nicht allzugroßen Einkommensveränderungen dominiert werden können.

\begin{tabular}{|c|c|c|c|c|c|c|c|c|c|}
\hline \multirow[t]{3}{*}{ Land } & \multicolumn{2}{|c|}{ HEV } & \multirow{3}{*}{$\begin{array}{c}\Delta \mathrm{TR} \\
\text { in } \% \\
\mathrm{REV}\end{array}$} & \multirow{3}{*}{$\begin{array}{r}\mathrm{HEV}_{S E} \\
\text { in \% } \\
\mathrm{REV}\end{array}$} & \multirow{3}{*}{$\begin{array}{r}\text { ToT }_{G E} \\
\text { in \% } \\
\text { ToT }\end{array}$} & \multirow{3}{*}{$\begin{array}{r}\mathrm{ToT}_{S E} \\
\text { in \% } \\
\text { ToT }\end{array}$} & \multicolumn{2}{|c|}{ FA } & \multirow{3}{*}{$\begin{array}{c}\Delta m_{G} \\
\text { in \% } \\
m_{G}^{0}\end{array}$} \\
\hline & Mio & in $\%$ & & & & & neu $\%$ & f.r. $\%$ & \\
\hline & DM & REV & & & & & REV & REV & \\
\hline $\mathrm{SH}$ & 86 & 1,3 & 0,8 & 0,1 & 0,06 & 0,02 & 1,6 & 0,9 & 0,19 \\
\hline $\mathrm{HH}$ & -620 & $-9,7$ & $-6,6$ & $-1,8$ & $-0,36$ & $-0,27$ & $-7,4$ & $-8,3$ & 0,15 \\
\hline $\mathrm{HB}$ & -61 & $-2,4$ & $-1,7$ & $-0,5$ & $-0,12$ & $-0,09$ & $-1,7$ & $-2,4$ & 0,16 \\
\hline NS & 340 & 1,8 & 1,4 & 0,1 & 0,08 & 0,05 & 2,1 & 1,4 & 0,20 \\
\hline NW & -608 & $-1,3$ & $-1,0$ & $-0,4$ & $-0,10$ & $-0,09$ & $-0,9$ & $-1,7$ & 0,41 \\
\hline $\mathrm{HE}$ & -466 & $-3,0$ & $-2,4$ & $-0,6$ & $-0,15$ & $-0,13$ & $-2,4$ & $-3,2$ & 0,26 \\
\hline $\mathrm{RP}$ & 112 & 1,2 & 0,8 & 0,0 & 0,16 & 0,13 & 1,3 & 0,5 & 0,16 \\
\hline SA & 39 & 1,4 & 1,1 & 0,0 & 0,27 & 0,24 & 1,5 & 0,8 & 0,07 \\
\hline BW & -1360 & $-5,3$ & $-4,3$ & $-0,9$ & $-0,09$ & $-0,04$ & $-4,6$ & $-5,5$ & 0,48 \\
\hline BY & -260 & $-0,9$ & $-0,7$ & $-0,3$ & $-0,01$ & $-0,01$ & $-0,6$ & $-1,3$ & 0,36 \\
\hline $\mathrm{OL}$ & 2939 & 7,7 & 6,1 & 1,9 & 1,48 & 1,00 & 8,0 & 8,8 & 0,09 \\
\hline$\sum$ & 141 & & 97 & 56 & & & & & \\
\hline \multicolumn{3}{|c|}{ Gini-Koeff. vor StV } & 0,179 & n.StV & 0,149 & n.LFA & 0,055 & n.BEZ & 0,055 \\
\hline
\end{tabular}

Tabelle V.19: Buhl/Pfingsten, keine Autonomie

Die Summe der Substitutionseffekte ist positiv. Demnach müßte eine Erhöhung des Multiplikators $m_{G_{s}}$, tendenziell, eine Verringerung der Verzerrungen bewirken. Das heißt, daß die Verzerrung zwischen dem öffentlichen Gut und dem Freizeitkonsum, die erhöht wird, durch die verringerten Verzerrungen zwischen dem öffentlichen Gut und dem regionalen privaten Gut sowie zwischen dem öffentlichen Gut und den bezogenen Gütern überkompensiert werden. Allerdings ist der Abbau der Verzerrungen insgesamt nur sehr gering. 
Die Veränderung der Finanzausgleichseinnahmen in Spalte acht entspricht weitgehend der Nettotransferveränderung und der Wohlfahrtsveränderung, zumindest in bezug auf die Vorzeichen und die Größenordnung. Obwohl die First-round-Berechnungen nicht unerheblich von den endgültigen Finanzausgleichseinnahmen abweichen, entsprechen sie doch in ihren Vorzeichen und ihrer Größenordnung den gesamten Wohlfahrtseffekten. Demnach liefern die First-round-Berechnungen der Finanzausgleichszahlungen eine brauchbare Näherung für die Wohlfahrtswirkungen.

Sowohl bei Ausgabenautonomie als auch bei Rationierung (fehlender Ausgabenautonomie, Tabelle V.19) entsteht ein gesellschaftlicher Wohlfahrtsgewinn, wenn man den Vorschlag von Buhl/PFingsten einführt. Der Gewinn ist bei fehlender Ausgabenautonomie erwartungsgemäß niedriger als bei Rationierung, auch wenn die Unterschiede nur sehr gering sind. Für die einzelnen Länder kann man jedoch keinen eindeutigen Trend erkennen. Offensichtlich gibt es Effekte, die die durch die Rationierung ausgelösten wohlfahrtsverringernden Substitutionseffekte abschwächen oder überkompensieren. Dies bestehen in erster Linie in veränderten Nettotransfers.

\section{(2) Sensitivitätsanalyse zu Buhl/Pfingsten: Fixes Arbeitsangebot}

Versucht man, die Bedeutung der Arbeitsangebotsentscheidung auf die Ergebnisse herauszuarbeiten, empfiehlt sich eine Sensitivitätsanalyse mit fixem Arbeitsangebot als Vergleichssituation. Diesen Fall könnte man durch Weglassen eines Astes des Nutzenbaumes simulieren. Hier wird jedoch ein anderes Vorgehen gewählt, das im Prinzip die gleiche Situation erzeugt. Setzt man die unkompensierte Arbeitsangebotselastizität auf den Wert 0 und die Variable $\zeta$ auf den Wert 1.001, so erhält man ein beinahe fixes Arbeitsangebot in Form einer kompensierten Arbeitsangebotselastizität von ungefähr $0 .{ }^{14}$

Betrachtet man die Ergebnisse des Vorschlags von Buhl/Pfingsten [1991] (siehe Tabelle V.20), so zeigen sich in den Ergebnissen für quasifixes und variables Arbeitsangebot nur geringe Unterschiede. Ein Vergleich der Nettotransferveränderung mit der Summe der $\mathrm{HEV}_{G E}$ und der Summe der $\mathrm{HEV}_{S E}$ zeigt, daß die letztere bei quasifixem Arbeitsangebot negativ sein müßte. Insgesamt sinkt die gesellschaftliche Wohlfahrt bei annäherend gleichen internationalen Steuerexporten. Folglich müssen die Substitutionseffekte eine wesentliche Rolle spielen. Die zusätzlich auftretenden internationalen ToT-Effekte sind in allen Fällen sehr gering, und besitzen daher nur einen marginalen Einfluß.

Da bei quasifixem Arbeitsangebot die Faktorpreise und damit die Güterpreise stärker ausschlagen, werden die intraregionalen Substitutionseffekte höher sein. Dies dürfte die

\footnotetext{
${ }^{14} \mathrm{Da}$ diese Modellierung minimale Arbeitsangebotsreaktionen zuläßt, wird hier von quasifixem Arbeitsangebot gesprochen.
} 


\begin{tabular}{|c|c|c|c|c|c|c|c|c|c|}
\hline \multirow[t]{3}{*}{ Land } & \multicolumn{2}{|c|}{$\mathrm{HEV}$} & \multirow{3}{*}{$\begin{array}{l}\Delta \mathrm{TR} \\
\text { in } \% \\
\mathrm{REV}\end{array}$} & \multirow{2}{*}{$\begin{array}{r}\mathrm{HEV}_{S E} \\
\text { in \% }\end{array}$} & \multirow{2}{*}{$\begin{array}{r}\text { To }_{G E} \\
\text { in \% }\end{array}$} & \multirow{2}{*}{$\begin{array}{r}\mathrm{ToT}_{S E} \\
\text { in \% }\end{array}$} & \multicolumn{2}{|c|}{$\mathrm{FA}$} & \multirow{3}{*}{$\begin{array}{c}\Delta m_{G} \\
\text { in } \% \\
m_{G}^{0}\end{array}$} \\
\hline & Mio & in $\%$ & & & & & neu $\%$ & f.r. $\%$ & \\
\hline & DM & REV & & REV & ToT & ToT & REV & REV & \\
\hline $\mathrm{SH}$ & 69 & 1,0 & 0,8 & 0,1 & 0,03 & 0,02 & 1,6 & 0,9 & 0,19 \\
\hline $\mathrm{HH}$ & -575 & $-8,9$ & $-6,7$ & $-1,6$ & $-0,31$ & $-0,25$ & $-7,6$ & $-8,3$ & 0,15 \\
\hline HB & -60 & $-2,4$ & $-1,8$ & $-0,3$ & $-0,10$ & $-0,09$ & $-1,7$ & $-2,4$ & 0,16 \\
\hline NS & 304 & 1,6 & 1,3 & 0,3 & 0,06 & 0,05 & 2,1 & 1,4 & 0,20 \\
\hline NW & -652 & $-1,4$ & $-1,1$ & $-0,3$ & $-0,10$ & $-0,09$ & $-1,0$ & $-1,7$ & 0,41 \\
\hline $\mathrm{HE}$ & -474 & $-3,1$ & $-2,5$ & $-0,5$ & $-0,14$ & $-0,13$ & $-2,5$ & $-3,3$ & 0,26 \\
\hline RP & 92 & 1,0 & 0,7 & 0,1 & 0,14 & 0,13 & 1,2 & 0,5 & 0,16 \\
\hline SA & 35 & 1,2 & 0,9 & 0,2 & 0,26 & 0,24 & 1,5 & 0,8 & 0,07 \\
\hline BW & -1361 & $-5,2$ & $-4,2$ & $-1,0$ & $-0,08$ & $-0,04$ & $-4,7$ & $-5,5$ & 0,48 \\
\hline BY & -298 & $-1,0$ & $-0,8$ & $-0,2$ & $-0,01$ & 0,00 & $-0,6$ & $-1,3$ & 0,36 \\
\hline $\mathrm{OL}$ & 2969 & 7,8 & 6,4 & 1,7 & 1,34 & 0,95 & 8,3 & 8,8 & 0,09 \\
\hline$\Sigma$ & 49 & & 95 & 60 & & & & & \\
\hline
\end{tabular}

Tabelle V.20: Buhl/Pfingsten Ausgabenautonomie, mit fixem Arbeitsangebot

Erklärung für die Zunahme der Substitutionseffekte und die Abnahme der gesellschaftlichen Wohlfahrt sein.

Insgesamt zeigen die geringen Unterschiede jedoch, daß die Effekte einer Einführung des Vorschlags von BUHL/PFINGsTEN offensichtlich robust gegen Veränderungen der Arbeitsangebotselastizitäten sind.

\subsubsection{Föttinger/Spahn [1993]}

(1) Berechnungen zum Vorschlag von Föttinger/Spahn [1993]

FötTINGER/SPAHN [1993] präsentieren einen Vorschlag, in dem die reale Finanzkraft als Grundlage für Ausgleichszahlungen dienen soll. Aus den Kosten (Preisen) der öffentlichen Güter gebildete Kostenindizes werden zur Indexierung der Finanzkraft herangezogen. Die Kostenindizes werden mit Hilfe der mit den Bevölkerungszahlen gewichteten öffentlichen Güterpreise aller Regionen abgeleitet (Variante I). Als Alternative zur Gewichtung mit den Bevölkerungsgrößen schlagen FötTinger/SpAHN [1993] als Gewichtungsfaktor die Menge des Verbrauchs an öffentlichen Gütern vor (Variante II). In Variante I (Tabelle V.21) entstehen sehr hohe Wohlfahrtseffekte, die sich durch die erheblichen Nettotransferveränderungen erklären lassen, die letztlich eine sehr starke Gleichverteilung nach LFA bewirken (der Gini-Koeffizient ist 0,013) Die Reaktion der Nettotransfers bestimmen die 


\begin{tabular}{|c|c|c|c|c|c|c|c|c|c|}
\hline \multirow[t]{3}{*}{ Land } & \multicolumn{2}{|c|}{$\mathrm{HEV}$} & \multirow{3}{*}{$\begin{array}{l}\Delta \mathrm{TR} \\
\text { in } \% \\
\mathrm{REV}\end{array}$} & \multirow{3}{*}{$\begin{array}{r}\mathrm{HEV}_{S E} \\
\text { in \% } \\
\mathrm{REV} \\
\end{array}$} & \multirow{3}{*}{$\begin{array}{r}\mathrm{ToT}_{G E} \\
\text { in } \% \\
\text { ToT }\end{array}$} & \multirow{3}{*}{$\begin{array}{r}\text { ToT }_{S E} \\
\text { in } \% \\
\text { ToT }\end{array}$} & \multicolumn{2}{|c|}{ FA } & \multirow{3}{*}{$\begin{array}{c}\Delta m_{G}, \\
\text { in } \% \\
m_{G \text { g }}^{0}\end{array}$} \\
\hline & Mio & in $\%$ & & & & & neu $\%$ & f.r. $\%$ & \\
\hline & DM & REV & & & & & REV & REV & \\
\hline $\mathrm{SH}$ & 426 & 6,4 & 3,9 & 1,3 & 0,25 & 0,06 & 5,8 & 5,0 & 0,20 \\
\hline $\mathrm{HH}$ & -2118 & $-33,1$ & $-24,1$ & $-3,9$ & $-0,64$ & $-0,29$ & -28.6 & $-27,5$ & 0,24 \\
\hline $\mathrm{HB}$ & -539 & $-21,6$ & $-17,2$ & $-2,1$ & $-0,32$ & $-0,11$ & -20.0 & $-19,2$ & 0,18 \\
\hline NS & 1265 & 6,8 & 5,0 & 0,6 & 0,22 & 0,06 & 6,3 & 5,4 & 0,20 \\
\hline NW & -826 & $-1,8$ & $-1,3$ & $-0,6$ & $-0,11$ & $-0,09$ & $-1,5$ & $-2,1$ & 0,72 \\
\hline $\mathrm{HE}$ & -356 & $-2,3$ & $-1,6$ & $-1,0$ & $-0,12$ & $-0,13$ & $-2,1$ & $-2,7$ & 0,43 \\
\hline $\mathrm{RP}$ & 318 & 3,4 & 2,6 & 0,1 & 0,19 & 0,14 & 3,3 & 2,4 & 0,19 \\
\hline SA & 188 & 6,8 & 5,8 & 0,1 & 0,35 & 0,25 & 6,5 & 5,6 & 0,09 \\
\hline BW & -1212 & $-4,7$ & $-3,7$ & $-0,9$ & $-0,09$ & $-0,04$ & $-4,3$ & $-4,8$ & 0,90 \\
\hline BY & 6 & 0,0 & 0,1 & $-0,4$ & 0,02 & $-0,01$ & 0,2 & $-0,5$ & 0,54 \\
\hline $\mathrm{OL}$ & 3244 & 8,5 & 5,7 & 2,4 & 1,51 & 1,02 & 8,4 & 8,7 & 0,08 \\
\hline$\sum$ & 396 & & -8 & 65 & & & & & \\
\hline \multicolumn{3}{|c|}{ Gini-Koeff. vor StV } & 0,180 & n.StV & 0,143 & n.LFA & 0,014 & n.BEZ & 0,013 \\
\hline
\end{tabular}

Tabelle V.21: Föttinger/Spahn I, Ausgabenautonomie

Wohlfahrtseffekte sowohl in ihren Vorzeichen als auch ihrer Größenordnung. Die Veränderung der Terms of Trade und die HEV der Substitutionseffekte haben, mit Ausnahme von Bayern, dessen Nettotransfers nahe bei Null liegen, dieselbe Richtung wie die Gesamtwohlfahrtseffekte.

Der gesamtgesellschaftliche Wohlfahrtsgewinn liegt immerhin bei annähernd 400 Mio DM. Da die Nettotransfers aus dem Ausland -8 Mio DM betragen, kann der Gewinn nur aus den Substitutionseffekten und den unterschiedlichen Grenznutzen der Einkommen entstehen. Die Summe der HEV's der Substitutionseffekte beläuft sich auf 65 Mio DM, wodurch ein Teil der gesamtgesellschaftlichen Gewinne erklärt wird.

Die First-round-Berechnungen in der vorletzten Spalte ergeben eine Annäherung an die Wohlfahrtseffekte und endgültigen Finanzausgleichszahlungen, die relativ genau ist.

Die Ergebnisse der Variante I zeigen im Vergleich zur Gewichtung mit den öffentlichen Gütern (Variante II, Tabelle V.21) erheblich höhere Umverteilungswirkungen (GiniKoeffizient von 0,987 gegenüber 0,972 ) und wesentliche höhere Wohlfahrtseffekte. Dies betrifft vor allem die Ostländer, die im ersten Vorschlag einen Wohlfahrtsgewinn von annäherend 3 Mrd. DM erzielen, dagegen im zweiten Fall Wohlfahrtsverluste erleiden. Die First-round-Aufkommensberechnungen sind in beiden Fällen gleich. Allerdings sind die endgültigen Veränderungen der Finanzausgleichszuweisungen (Spalte 8) sehr verschieden. 


\begin{tabular}{|c|c|c|c|c|c|c|c|c|c|}
\hline \multirow[t]{3}{*}{ Land } & \multicolumn{2}{|c|}{$\overline{\mathrm{HEV}}$} & \multirow{3}{*}{$\begin{array}{l}\Delta \mathrm{TR} \\
\text { in \% } \\
\mathrm{REV}\end{array}$} & \multirow{3}{*}{$\begin{array}{r}\mathrm{HEV}_{S E} \\
\text { in \% } \\
\mathrm{REV}\end{array}$} & \multirow{3}{*}{$\begin{array}{r}\mathrm{ToT}_{G E} \\
\text { in } \% \\
\text { ToT }\end{array}$} & \multirow{3}{*}{$\begin{array}{r}\text { ToT }_{S E} \\
\text { in \% } \\
\text { ToT }\end{array}$} & \multicolumn{2}{|c|}{$\overline{F A}$} & \multirow{3}{*}{$\begin{array}{c}\Delta m_{G}, \\
\text { in } \% \\
m_{G_{s}}^{0}\end{array}$} \\
\hline & Mio & in $\%$ & & & & & neu $\%$ & f.r. $\%$ & \\
\hline & DM & REV & & & & & REV & REV & \\
\hline $\mathrm{SH}$ & 376 & 5,7 & 3,4 & 1,4 & 0,22 & 0,06 & 5,4 & 5,0 & 0,03 \\
\hline $\mathrm{HH}$ & -1524 & $-23,8$ & $-17,2$ & $-3,5$ & $-0,52$ & $-0,29$ & $-20,8$ & $-27,4$ & 0,08 \\
\hline HB & -444 & $-17,8$ & $-14,1$ & $-2,1$ & $-0,28$ & $-0,11$ & $-16,6$ & $-19,2$ & 0,04 \\
\hline NS & 1136 & 6,1 & 4,5 & 0,8 & 0,20 & 0,07 & 5,8 & 5,4 & 0,01 \\
\hline NW & 110 & 0,2 & 0,4 & $-0,2$ & $-0,08$ & $-0,09$ & 0,6 & $-2,1$ & 0,45 \\
\hline $\mathrm{HE}$ & 71 & 0,5 & 0,8 & $-0,7$ & $-0,10$ & $-0,13$ & 0,6 & $-2,7$ & 0,19 \\
\hline $\mathrm{RP}$ & 332 & 3,5 & 2,8 & 0,4 & 0,18 & 0,14 & 3,7 & 2,4 & 0,04 \\
\hline SA & 126 & 4,6 & 4,0 & 0,2 & 0,30 & 0,24 & 4,6 & 5,6 & $-0,18$ \\
\hline BW & -173 & $-0,7$ & $-0,3$ & $-0,4$ & $-0,04$ & $-0,04$ & $-0,3$ & $-4,8$ & 0,62 \\
\hline BY & 417 & 1,4 & 1,3 & $-0,1$ & 0,03 & 0,00 & 1,7 & $-0,5$ & 0,29 \\
\hline $\mathrm{OL}$ & -225 & $-0,6$ & $-1,9$ & 1,0 & 0,82 & 0,92 & $-0,3$ & 8,7 & 0,14 \\
\hline$\sum$ & 202 & & -135 & 49 & & & & & \\
\hline \multicolumn{3}{|c|}{ Gini-Koeff. vor StV } & 0,180 & n.StV & 0,143 & n.LFA & 0,037 & n.BEZ & 0,031 \\
\hline
\end{tabular}

Tabelle V.22: FöTtinger/Spahn II, Ausgabenautonomie

In Variante I entsprechen sie fast genau den First-round-Berechnungen. Die First-roundBerechnungen stellen in Variante I eine bezüglich der Vorzeichen und Größenordnung recht gute Abschätzung der Nettotransferveränderung (Spalte 4) dar. Demgegenüber kehrt sich in Variante II die Nettotransferveränderung für einige Länder sogar um. Am stärksten betrifft das die Ostländer, die bei der Gewichtung mit den öffentlichen Gütern, trotz positiver $\mathrm{HEV}_{S E}$, Wohlfahrtsverluste erleiden. Diese gehen auf negative Nettotransfers zurück.

Wodurch lassen sich diese unterschiedlichen Ergebnisse erklären? Eine Betrachtung der Ergebnisse bei Rationierung kann darüber Aufschluß geben. Die in Tabelle V.23 präsentierten Werte gelten nämlich bei fehlender Ausgabenautonomie für beide Varianten. Die Unterschiede bewegen sich im Nachkommabereich. Folglich müssen die bei Ausgabenautonomie auftretenden überraschenden Differenzen zwischen beiden Varianten auf die Reagibilität bezüglich der öffentlichen Ausgaben zurückzuführen sein.

Bleiben die Ausgaben für den privaten Konsum konstant, so verändern sich auch die Einnahmen aus den Steuern nur wenig. Bei Rationierung sind daher die Steuereinnahmen in beiden Modellen in etwa gleich. ${ }^{15}$ Besteht dagegen Ausgabenautonomie, so führt die stärkere Reagibilität der öffentlichen Hand über ausgelöste Nachfrageanpassungen zu

\footnotetext{
${ }^{15}$ Überraschenderweise sind die Gewichtungsfaktoren ebenfalls fast gleich, obwohl sie in einem Fall aus
} 


\begin{tabular}{|c|c|c|c|c|c|c|c|c|c|}
\hline \multirow[t]{3}{*}{ Land } & \multicolumn{2}{|c|}{$\mathrm{HEV}$} & \multirow{2}{*}{$\begin{array}{l}\Delta \mathrm{TR} \\
\text { in } \%\end{array}$} & \multirow{2}{*}{$\begin{array}{r}\mathrm{HEV}_{S E} \\
\text { in \% }\end{array}$} & \multirow{2}{*}{$\begin{array}{r}\mathrm{ToT}_{G E} \\
\text { in } \%\end{array}$} & \multirow{2}{*}{$\begin{array}{r}\mathrm{ToT}_{S E} \\
\text { in \% }\end{array}$} & \multicolumn{2}{|c|}{$\mathrm{FA}$} & \multirow{3}{*}{$\begin{array}{c}\Delta m_{G} \\
\text { in } \% \\
m_{G_{s}}^{0}\end{array}$} \\
\hline & Mio & in $\%$ & & & & & neu $\%$ & f.r. $\%$ & \\
\hline & DM & REV & REV & REV & ToT & ToT & REV & REV & \\
\hline $\mathrm{SH}$ & 420 & 6,3 & 3,7 & 2,2 & 0,25 & 0,09 & 5,6 & 5,0 & 0,20 \\
\hline $\mathrm{HH}$ & -2319 & $-36,3$ & $-22,5$ & $-12,8$ & $-0,62$ & $-0,40$ & -27.9 & $-27,5$ & 0,24 \\
\hline HB & -573 & $-23,0$ & $-15,8$ & $-5,8$ & $-0,31$ & $-0,15$ & $-18,8$ & $-19,2$ & 0,18 \\
\hline NS & 1238 & 6,6 & 4,8 & 1,4 & 0,21 & 0,08 & 6,0 & 5,4 & 0,20 \\
\hline NW & -839 & $-1,8$ & $-1,3$ & $-2,3$ & $-0,11$ & $-0,12$ & $-1,5$ & $-2,1$ & 0,72 \\
\hline $\mathrm{HE}$ & -368 & $-2,4$ & $-1,5$ & $-3,5$ & $-0,12$ & $-0,15$ & $-2,1$ & $-2,7$ & 0,43 \\
\hline $\mathrm{RP}$ & 314 & 3,4 & 2,5 & 0,2 & 0,19 & 0,15 & 3,1 & 2,4 & 0,19 \\
\hline SA & 184 & 6,7 & 5,6 & 2,4 & 0,35 & 0,29 & 6,1 & 5,6 & 0,09 \\
\hline BW & -1227 & $-4,8$ & $-3,6$ & $-4,5$ & $-0,09$ & $-0,08$ & $-4,2$ & $-4,8$ & 0,90 \\
\hline BY & -4 & 0,0 & 0,1 & $-1,5$ & 0,01 & $-0,02$ & 0,2 & $-0,5$ & 0,54 \\
\hline $\mathrm{OL}$ & 3190 & 8,4 & 5,3 & 10,0 & 1,55 & 1,68 & 8,1 & 8,7 & 0,08 \\
\hline$\sum$ & 19 & & -30 & 147 & & & & & \\
\hline \multicolumn{3}{|c|}{ Gini-Koeff. vor StV } & 0,176 & n.StV & 0,140 & n.LFA & 0,014 & n.BEZ & 0,014 \\
\hline
\end{tabular}

Tabelle V.23: Föttinger/Spahn, keine Ausgabenautonomie (beide Fälle)

veränderten Steuereinnahmen. Bei Ausgabenautonomie erhöhen die Empfangsländer von Finanzausgleichszuweisungen ihren privaten Konsum stärker und den öffentlichen Konsum weniger stark, als bei Rationierung. Da in der Variante II der Kehrwert der öffentlichen Gütermengen als Gewichtungsfaktor zur Bestimmung der realen Finanzkraft eingesetzt wird, wird die Finanzkraft der Empfangsländer höher eingeschätzt als bei Rationierung. Folglich erhalten sie geringere LFA-Zuweisungen. Für die Nettozahler im Finanzausgleich gilt dieser Zusammenhang genau umgekehrt. Sie müssen letztlich geringere Beiträge leisten.

Aufgrund der unterschiedlichen Nettotransfers entstehen Preisdifferenzen zwischen den beiden Vorschlägen, die letztlich zu differierenden Substitutionseffekten führen. In der Variante I sinken die Preisniveaus in zehn der elf Länder stärker als in der Variante II. Dies führt zu geringeren intraregionalen Preisschwankungen und schließlich zu einer Verringerung der intraregionalen Substitutionseffekte, die damit geringer sind als in der Variante II. Ein Vergleich der Nettotransferveränderungen mit den $\mathrm{HEV}_{G E}$ zeigt, daß sich die Substitutionseffekte in beiden Varianten allerdings nur wenig unterscheiden, und der beschriebene Effekt zwar vorhanden ist, aber nur einen geringen Einfluß besitzt.

den Bevölkerungsrelationen und im anderen Fall aus der Menge der öffentlichen Güter bestehen. 
Der größere Teil der unterschiedlichen Gesamtwohfahrtseffekte beruht auf den internationalen Steuerexporten. Dieser Größe ist allerdings aufgrund der rudimentären Außenhandelsmodellierung mit Vorsicht zu begegnen.

In den Tabellen werden nicht unbeachtliche Substitutionseffekte ausgewiesen, die nur zum Teil auf die hohen Veränderungen der Multiplikatoren $\left(m_{G_{s}}\right)$ zurückgeführt werden können. Betrachtet man die Resultate genauer, dann zeigt sich, daß die Summe der prozentualen Veränderung der Nettotransfers (Einkommenseffekte) und der HEV der Substitutionseffekte bei einigen Ländern nicht einmal annähernd mit den Gesamteffekten übereinstimmt. Dies deutet darauf hin, daß die isolierte Berechnung der Substitutionseffekte zu sehr großen Veränderungen der Finanzausgleichszahlungen führt. Die entsprechenden Nettotransferveränderungen sind beispielsweise für die Ostländer größer als die Veränderung der gesamten Nettotransfers. Die daraus entstehenden Einkommenseffekte verstärken die Substitutionseffekte. Demnach ist die Zerlegung in ihrem quantitativen Ergebnis nicht sehr zufriedenstellend. Dennoch ist sie bezüglich der Richtung der einzelnen Effekte sehr aussagekräftig. Die ermittelten Vorzeichen der Wohlfahrtsgewinne aus der Berechnung der Substitutionseffekte entsprechen, in jedem Fall, den Vorzeichen der Differenz der Veränderung der Nettotransfers und der $\mathrm{HEV}_{G E}$.

\section{(2) Sensitivitätsanalyse: Fixes Arbeitsangebot}

Die Berechnungen zum Vorschlag von FötTINGER/SPAHN [1993], Variante II, ergeben ein recht buntes Bild (Tabelle V.24). Die regionalen HEV $_{S E}$ zeigen keine einheitliche Tendenz. Dies gilt ebenso für die Gesamtwohlfahrtseffekte. Darüber hinaus gibt es eine nicht unbeträchtliche Veränderung der Nettotransferströme. Dennoch verändert sich an den Größenordnungen und Vorzeichen im Prinzip nichts. Auch hier besitzen unterschiedliche Arbeitsangebotselastizitäten offensichtlich keinen wesentlichen Einfluß.

\subsubsection{Fuest/Lichtblau [1991]}

\section{(1) Berechnungen zum Vorschlag von Fuest/Lichtblau [1991]}

Die Verteilung der Umsatzsteueranteile der Länder anhand der relativen Volkseinkommen ist die Besonderheit der Empfehlung von Fuest/Lichtblau [1991]. Dies führt dazu, daß die relativ reichen Länder höhere Umsatzsteuereinnahmen erzielen, die im LFA nicht vollständig nivelliert werden. Daher erhöhen sich ihre gesamten Einnahmen im Vergleich zu den gesetzlichen Regelungen (siehe Spalte 8 und 9 der Tabelle V.25). Die einkommensschwachen Länder erhalten dagegen deutlich niedrigere Finanzmittel aus der Steuervereilung und dem LFA. 


\begin{tabular}{||r|r|r|r|r|r|r|r|r|r||}
\hline \hline Land & \multicolumn{2}{|c|}{ HEV } & \multicolumn{1}{c|}{$\Delta$ TR } & $\mathrm{HEV}_{S E}$ & ToT $_{G E}$ & ToT $_{S E}$ & \multicolumn{2}{|c|}{ FA } & $\Delta m_{G_{s}}$ \\
& Mio & in $\%$ & in $\%$ & in $\%$ & in $\%$ & in $\%$ & neu \% & f.r. $\%$ & in \% \\
& DM & REV & REV & REV & ToT & ToT & REV & REV & $m_{G s}^{0}$ \\
\hline SH & 348 & 5,2 & 3,6 & 1,2 & 0,13 & 0,04 & 5,4 & 5,0 & 0,24 \\
HH & -1421 & $-22,1$ & $-16,0$ & $-4,5$ & $-0,39$ & $-0,27$ & $-19,7$ & $-27,4$ & 0,27 \\
HB & -430 & $-17,3$ & $-12,9$ & $-3,3$ & $-0,20$ & $-0,11$ & $-15,6$ & $-19,2$ & 0,21 \\
NS & 1072 & 5,8 & 4,3 & 1,0 & 0,14 & 0,06 & 5,7 & 5,4 & 0,25 \\
NW & 102 & 0,2 & 0,2 & $-0,1$ & $-0,08$ & $-0,09$ & 0,6 & $-2,1$ & 0,79 \\
HE & 56 & 0,4 & 0,5 & $-0,3$ & $-0,11$ & $-0,13$ & 0,6 & $-2,7$ & 0,49 \\
RP & 320 & 3,4 & 2,6 & 0,6 & 0,16 & 0,14 & 3,6 & 2,4 & 0,22 \\
SA & 123 & 4,5 & 3,6 & 0,6 & 0,28 & 0,25 & 4,5 & 5,6 & 0,16 \\
BW & -173 & $-0,7$ & $-0,4$ & $-0,2$ & $-0,04$ & $-0,04$ & $-0,3$ & $-4,8$ & 0,97 \\
BY & 400 & 1,4 & 1,1 & 0,1 & 0,02 & 0,00 & 1,6 & $-0,5$ & 0,60 \\
OL & -267 & $-0,7$ & $-1,3$ & 0,5 & 0,80 & 0,88 & $-0,2$ & 8,7 & 0,13 \\
\hline$\Sigma$ & 131 & & -79 & 72 & & & & & \\
\hline \hline
\end{tabular}

Tabelle V.24: Föttinger/SPAhn II, Ausgabenautonomie, fixes Arbeitsangebot

\begin{tabular}{|c|c|c|c|c|c|c|c|c|c|}
\hline \multirow[t]{3}{*}{ Land } & \multicolumn{2}{|c|}{$\mathrm{HEV}$} & \multirow{3}{*}{$\begin{array}{l}\Delta \mathrm{TR} \\
\text { in } \% \\
\mathrm{REV}\end{array}$} & \multirow{3}{*}{$\begin{array}{r}\mathrm{HEV}_{S E} \\
\text { in \% } \\
\mathrm{REV}\end{array}$} & \multirow{3}{*}{$\begin{array}{r}\mathrm{ToT}_{G E} \\
\text { in \% } \\
\text { ToT }\end{array}$} & \multirow{3}{*}{$\begin{array}{r}\text { ToT }_{S E} \\
\text { in } \% \\
\text { ToT } \\
\end{array}$} & \multicolumn{2}{|c|}{ FA } & \multirow{3}{*}{$\begin{array}{c}\Delta m_{G}, \\
\text { in \% } \\
m_{G}^{0},\end{array}$} \\
\hline & Mio & in $\%$ & & & & & neu $\%$ & f.r. $\%$ & \\
\hline & DM & REV & & & & & REV & REV & \\
\hline $\mathrm{SH}$ & -77 & $-1,1$ & $-1,2$ & 0,0 & 0,03 & 0,02 & 1,8 & 1,2 & 0,24 \\
\hline $\mathrm{HH}$ & -468 & $-7,3$ & $-6,1$ & $-0,8$ & $-0,31$ & $-0,25$ & $-5,6$ & $-5,9$ & 0,27 \\
\hline HB & -328 & $-13,1$ & $-11,5$ & $-0,9$ & $-0,22$ & $-0,09$ & -11.3 & $-11,4$ & 0,21 \\
\hline NS & -54 & $-0,3$ & $-0,5$ & 0,2 & 0,07 & 0,05 & 2,0 & 1,4 & 0,25 \\
\hline NW & 1236 & 2,7 & 2,3 & 0,4 & $-0,05$ & $-0,08$ & 4,3 & 3,7 & 0,78 \\
\hline $\mathrm{HE}$ & 749 & 4,8 & 4,0 & 0,7 & $-0,07$ & $-0,19$ & 6,4 & 5,8 & 0,49 \\
\hline $\mathrm{RP}$ & 204 & 2,2 & 2,0 & 0,4 & 0,14 & 0,13 & 4,2 & 3,6 & 0,22 \\
\hline $\mathrm{SA}$ & -92 & $-3,3$ & $-2,7$ & $-0,2$ & 0,17 & 0,23 & $-1,1$ & $-1,6$ & 0,16 \\
\hline $\mathrm{BW}$ & 1247 & 4,8 & 4,1 & 0,7 & 0,02 & $-0,02$ & 6,2 & 5,7 & 0,97 \\
\hline BY & 843 & 2,8 & 2,4 & 0,5 & 0,04 & 0,01 & 4,6 & 4,1 & 0,60 \\
\hline OL & -3377 & $-8,8$ & $-7,4$ & $-1,7$ & 0,21 & 0,70 & $-5,5$ & $-6,1$ & 0,13 \\
\hline$\sum$ & -117 & & -82 & -27 & & & & & \\
\hline \multicolumn{3}{|c|}{ Gini-Koeff. vor StV } & 0,180 & $\mathrm{n} . \mathrm{StV}$ & 0,168 & n.LFA & 0,079 & n.BEZ & 0,067 \\
\hline
\end{tabular}

Tabelle V.25: Fuest/LichtBlaU, Ausgabenautonomie 
Für vier der elf Länder sinken die Preisniveaus und damit ihre intraregionalen Substitutionseffekte, während sie sich für die anderen Länder verstärken. Obwohl in diesem Vorschlag relativ geringe Preisschwankungen zwischen den Regionen auftreten, die zu einer Verringerung interregionaler Substitutionseffekte beitragen, bewirken die intraregionalen Substitutionseffekte offenbar eine Verschlechterung der gesellschaftlichen Wohlfahrt (Summe der $\mathrm{HEV}_{S E}$ ).

Die First-round-Berechnungen der Veränderung der Finanzausgleichszahlungen stellen eine gute Näherung an die endgültigen Veränderungen dieser Größen dar. Allerdings weichen die Vorzeichen der Nettotransfers von Schleswig-Holstein und Niedersachsen von dem jeweiligen Vorzeichen der Finanzausgleichsveränderungen ab. Dies zeigt, daß gerade bei relativ geringen Finanzausgleichszuweisungen, die Berücksichtigung aller anderen nationaler oder interregionaler Finanzströme notwendig ist, um die Wohlfahrtseffekte abschätzen zu können.

Die gesamte Wohlfahrt verringert sich durch die Einführung des Vorschlags von FUEST/sc Lichtblau [1991], weitgehend bedingt durch den negativen Saldo der Nettotransferveränderung gegenüber dem Rest der Welt und durch die hohen intraregionalen Substitutionseffekte.

\begin{tabular}{|c|c|c|c|c|c|c|c|c|c|}
\hline \multirow[t]{3}{*}{ Land } & \multicolumn{2}{|c|}{ HEV } & \multirow{3}{*}{\begin{tabular}{|c|}
$\Delta \mathrm{TR}$ \\
in $\%$ \\
$\mathrm{REV}$
\end{tabular}} & \multirow{3}{*}{$\begin{array}{r}\mathrm{HEV}_{S E} \\
\text { in \% } \\
\mathrm{REV}\end{array}$} & \multirow{3}{*}{$\begin{array}{r}\mathrm{ToT}_{G E} \\
\text { in } \% \\
\mathrm{ToT} \\
\end{array}$} & \multirow{3}{*}{$\begin{array}{r}\text { ToT }_{S E} \\
\text { in \% } \\
\text { ToT }\end{array}$} & \multicolumn{2}{|c|}{ FA } & \multirow{3}{*}{$\begin{array}{c}\Delta m_{G,} \\
\text { in \% } \\
m_{G_{g}}^{0}\end{array}$} \\
\hline & Mio & in $\%$ & & & & & neu $\%$ & f.r.\% & \\
\hline & DM & REV & & & & & REV & REV & \\
\hline $\mathrm{SH}$ & -87 & $-1,3$ & $-1,3$ & 0,1 & 0,03 & 0,03 & 1,8 & 1,2 & 0,24 \\
\hline $\mathrm{HH}$ & -488 & $-7,6$ & $-6,1$ & $-0,8$ & $-0,30$ & $-0,25$ & $-5,4$ & $-6,0$ & 0,27 \\
\hline $\mathrm{HB}$ & -351 & $-14,1$ & $-11,1$ & $-1,3$ & $-0,21$ & $-0,10$ & $-10,9$ & $-11,4$ & 0,21 \\
\hline NS & -73 & $-0,4$ & $-0,6$ & 0,2 & 0,08 & 0,06 & 2,0 & 1,4 & 0,25 \\
\hline NW & 1212 & 2,7 & 2,2 & 0,5 & $-0,05$ & $-0,08$ & 4,2 & 3,7 & 0,79 \\
\hline $\mathrm{HE}$ & 738 & 4,8 & 3,9 & 0,8 & $-0,07$ & $-0,12$ & 6,3 & 5,8 & 0,49 \\
\hline $\mathrm{RP}$ & 201 & 2,1 & 1,9 & 0,5 & 0,15 & 0,14 & 4,1 & 3,6 & 0,22 \\
\hline SA & -97 & $-3,5$ & $-2,7$ & $-0,2$ & 0,17 & 0,23 & $-1,0$ & $-1,6$ & 0,16 \\
\hline BW & 1221 & 4,8 & 4,1 & 0,8 & 0,02 & \begin{tabular}{|l|}
$-0,02$ \\
\end{tabular} & 6,1 & 5,6 & 0,97 \\
\hline BY & 828 & 2,8 & 2,3 & 0,6 & 0,04 & 0,01 & 4,6 & 4,1 & 0,60 \\
\hline $\mathrm{OL}$ & -3466 & $-9,1$ & $-7,1$ & $-2,0$ & 0,15 & 0,68 & $-5,2$ & $-6,1$ & 0,14 \\
\hline$\sum$ & -362 & & -79 & -45 & & & & & \\
\hline \multicolumn{3}{|c|}{ Gini-Koeff. vor StV } & 0,180 & n.StV & 0,168 & n.LFA & 0,078 & n.BEZ & 0,066 \\
\hline
\end{tabular}

Tabelle V.26: Fuest/Lichtblau, keine Autonomie 
Die Simulation mit Rationierung (fehlender Finanzautonomie, Tabelle V.26) führt, erwartungsgemäß, zu einem eindeutigen gesellschaftlichen Wohlfahrtsverlust, der deutlich höher liegt als bei Autonomie.

(2) Sensitivitätsanalyse: Variation der Substitutionselastizität zwischen Arbeit und Kapital

Die Ergebnisse des Vorschlags von Fuest/Lichtblau [1991] mit unterschiedlichen Substitutionselastizitäten zwischen Kapital und Arbeit (siehe Tabelle V.27) zeigen nur marginale Unterschiede zum Standardfall (Tabelle V.25). Insgesamt ergeben sich leichte gesell-

\begin{tabular}{|c|c|c|c|c|c|c|c|c|c|}
\hline \multirow[t]{3}{*}{ Land } & \multicolumn{2}{|c|}{$\mathrm{HEV}$} & \multirow{3}{*}{$\begin{array}{l}\Delta \mathrm{TR} \\
\text { in } \% \\
\mathrm{REV}\end{array}$} & \multirow{3}{*}{$\begin{array}{r}\mathrm{HEV}_{S E} \\
\text { in \% } \\
\mathrm{REV}\end{array}$} & \multirow{3}{*}{$\begin{array}{r}\mathrm{ToT}_{G E} \\
\text { in } \% \\
\text { ToT }\end{array}$} & \multirow{3}{*}{$\begin{array}{r}\mathrm{To}_{S E} \\
\text { in } \% \\
\mathrm{ToT} \\
\end{array}$} & \multicolumn{2}{|c|}{ FA } & \multirow{3}{*}{$\begin{array}{c}\Delta m_{G s} \\
\text { in } \% \\
m_{G}^{0}\end{array}$} \\
\hline & Mio & in $\%$ & & & & & neu $\%$ & f.r. $\%$ & \\
\hline & $\mathrm{DM}$ & REV & & & & & REV & REV & \\
\hline $\mathrm{SH}$ & -74 & $-1,1$ & $-1,3$ & 0,1 & 0,03 & 0,03 & 1,7 & 1,2 & 0,24 \\
\hline $\mathrm{HH}$ & -497 & $-7,3$ & $-5,9$ & $-1,0$ & $-0,32$ & $-0,25$ & $-5,6$ & $-5,9$ & 0,27 \\
\hline HB & -328 & $-13,1$ & $-11,3$ & $-1,1$ & $-0,23$ & $-0,09$ & $-11,3$ & $-11,4$ & 0,21 \\
\hline NS & -46 & $-0,2$ & $-0,6$ & 0,2 & 0,08 & 0,06 & 1,9 & 1,4 & 0,25 \\
\hline NW & 1248 & 2,7 & 2,3 & 0,5 & $-0,05$ & $-0,08$ & 4,3 & 3,7 & 0,79 \\
\hline $\mathrm{HE}$ & 758 & 4,9 & 3,9 & 0,7 & $-0,06$ & $-0,12$ & 6,4 & 5,8 & 0,49 \\
\hline $\mathrm{RP}$ & 204 & 2,2 & 1,9 & 0,4 & 0,14 & 0,13 & 4,1 & 3,6 & 0,22 \\
\hline SA & -92 & $-3,3$ & $-2,6$ & $-0,3$ & 0,16 & 0,23 & $-1,2$ & $-1,6$ & 0,16 \\
\hline BW & 1255 & 4,8 & 4,1 & 0,8 & 0,03 & $-0,02$ & 6,3 & 5,7 & 0,97 \\
\hline BY & 851 & 2,9 & 2,4 & 0,5 & 0,04 & 0,01 & 4,6 & 4,1 & 0,61 \\
\hline $\mathrm{OL}$ & -3355 & $-8,8$ & $-7,4$ & $-1,7$ & 0,16 & 0,71 & $-5,6$ & $-6,1$ & 0,13 \\
\hline$\sum$ & -48 & & -54 & 1 & & & & & \\
\hline
\end{tabular}

Tabelle V.27: Fuest/Lichtblau Ausgabenautonomie, Variation der Substitutionselastizitäten zwischen Kapital und Arbeit

schaftliche Wohlfahrtsgewinne, die zum Teil auf verringerte Steuerimporte zurückgeführt werden können.

Die bei relativ geringen Substitutionselastizitäten zu erwartenden stärkeren Preisausschläge treten auf. Darauf beruhen vermutlich die insgesamt leicht höheren Substitutionseffekte. Das Bild bezüglich der regionalen Substitutionseffekte ist allerdings nicht eindeutig. Dies ist u.a. darauf zurückzuführen, daß die Preisniveaus in sechs der Länder fallen und in fünf steigen. Folglich sinken die intraregionalen Substitutionseffekte der ersteren und steigen die der letzteren Länder. 
Die Resultate der Berechnungen zum Vorschlag von Fuest/LichtblaU [1991] zeigen, daß die Ergebnisse recht robust gegenüber Parametervariationen sind. Da der Multiplikator $m_{G}$, die einzige, verzerrende Effekte hervorrufende Größe ist, die direkt verändert wird, ist zu erwarten, daß die öffentliche Konsumentscheidung einen sehr großen Einfluß auf die Substitutionseffekte besitzt. Die Berechnungen bestätigt das eindrucksvoll, da die unterschiedlichen Modellierungen der öffentlichen Entscheidung, die weitaus größten Differenzen in den Resultaten erzeugen.

\subsubsection{Abschaffung der horizontalen Umverteilung}

Die Abschaffung der horizontalen Umverteilungsregelungen in der Steuerverteilung, LFA und der BEZ, nach 1995, erzeugt sehr hohe Veränderungen der Nettotransfers. Diese führen dazu, daß die reicheren (ärmeren) Bundesländer höhere (niedrigere) Einnahmen erzielen. Die dadurch ausgelösten interregionalen Einkommenseffekte verursachen entsprechende Wohlfahrtsgewinn(verluste).

\begin{tabular}{|c|c|c|c|c|c|c|c|c|c|}
\hline \multirow[t]{3}{*}{ Land } & \multicolumn{2}{|c|}{$\mathrm{HEV}$} & \multirow{2}{*}{$\begin{array}{l}\Delta \mathrm{TR} \\
\text { in } \%\end{array}$} & \multirow{2}{*}{$\begin{array}{r}\mathrm{HEV}_{S E} \\
\text { in \% }\end{array}$} & \multirow{2}{*}{$\begin{array}{r}\mathrm{ToT}_{G E} \\
\text { in } \%\end{array}$} & \multirow{2}{*}{$\begin{array}{r}\text { ToT }_{S E} \\
\text { in \% }\end{array}$} & \multicolumn{2}{|c|}{ FA } & \multirow{3}{*}{$\begin{array}{c}\Delta m_{G}, \\
\text { in } \% \\
m_{G s}^{0}\end{array}$} \\
\hline & Mio & in $\%$ & & & & & neu $\%$ & f.r. $\%$ & \\
\hline & $\mathrm{DM}$ & REV & REV & REV & ToT & ToT & REV & REV & \\
\hline $\mathrm{SH}$ & 167 & 2,5 & 1,6 & 1,4 & 0,06 & 0,06 & 4,2 & 3,8 & $-0,70$ \\
\hline $\mathrm{HH}$ & 1306 & 20,3 & 16,2 & 0,7 & 0,03 & $-0,23$ & 17,3 & 17,0 & $-0,53$ \\
\hline $\mathrm{HB}$ & 11 & 0,4 & 1,1 & $-1,7$ & $-0,09$ & $-0,10$ & $-0,1$ & $-0,4$ & $-0,62$ \\
\hline NS & 625 & 3,3 & 2,4 & 0,8 & 0,01 & 0,07 & 4,1 & 3,6 & $-0,71$ \\
\hline NW & 4896 & 10,5 & 9,2 & 1,3 & 0,04 & $-0,07$ & 11,1 & 10,7 & $-0,33$ \\
\hline $\mathrm{HE}$ & 2224 & 14,3 & 13,0 & 1,0 & $-0,03$ & $-0,12$ & 14,6 & 14,2 & $-0,77$ \\
\hline $\mathrm{RP}$ & 462 & 4,9 & 4,5 & 1,0 & 0,13 & 0,14 & 6,2 & 5,8 & $-0,61$ \\
\hline SA & -158 & $-5,7$ & $-4,1$ & $-0,6$ & 0,06 & 0,22 & $-4,0$ & $-4,4$ & $-1,53$ \\
\hline BW & 4968 & 19,0 & 16,6 & 2,5 & 0,19 & 0,00 & 19,6 & 19,2 & $-0,44$ \\
\hline BY & 2601 & 8,8 & 7,6 & 1,2 & 0,10 & 0,01 & 9,5 & 9,0 & $-0,54$ \\
\hline $\mathrm{OL}$ & -18606 & $-48,6$ & $-41,2$ & $-5,5$ & $-2,46$ & 0,44 & $-45,4$ & $-45,2$ & $-0,36$ \\
\hline$\sum$ & -1504 & & -883 & -81 & & & & & \\
\hline \multicolumn{3}{|c|}{ Gini-Koeff. vor StV } & 0,181 & n.StV & 0,144 & n.LFA & 0,144 & n.BEZ & 0,144 \\
\hline
\end{tabular}

Tabelle V.28: Abschaffung der Umverteilung, Ausgabenautonomie

Eine weitere Folge der veränderten regionalen Einkommen sind Preiserhöhungen in den reicheren, und Preissenkungen in den ärmeren Ländern, aus denen sich ToT-Reaktionen 


\begin{tabular}{|c|c|c|c|c|c|c|c|c|c|}
\hline \multirow[t]{3}{*}{ Land } & \multicolumn{2}{|c|}{$\mathrm{HEV}$} & \multirow{3}{*}{$\begin{array}{l}\Delta \mathrm{TR} \\
\text { in } \% \\
\mathrm{REV}\end{array}$} & \multirow{3}{*}{$\begin{array}{r}\mathrm{HEV}_{S E} \\
\text { in \% } \\
\mathrm{REV}\end{array}$} & \multirow{3}{*}{$\begin{array}{r}\text { ToT }_{G E} \\
\text { in } \% \\
\text { ToT } \\
\end{array}$} & \multirow{3}{*}{$\begin{array}{r}\text { ToT }_{S E} \\
\text { in \% } \\
\text { ToT }\end{array}$} & \multicolumn{2}{|c|}{ FA } & \multirow{3}{*}{$\begin{array}{r}\Delta m_{G}, \\
\text { in \% } \\
m_{G}^{0},\end{array}$} \\
\hline & Mio & in $\%$ & & & & & neu $\%$ & f.r.\% & \\
\hline & DM & REV & & & & & REV & REV & \\
\hline $\mathrm{SH}$ & 154 & 2,3 & 1,4 & 1,6 & 0,09 & 0,07 & 4,2 & 3,8 & $-0,70$ \\
\hline $\mathrm{HH}$ & 1316 & 20,6 & 16,2 & 0,8 & 0,05 & $-0,23$ & 17,3 & 17,0 & $-0,53$ \\
\hline HB & 13 & & 1,0 & $-1,8$ & $-0,07$ & $-0,10$ & $-0,1$ & $-0,4$ & $-0,62$ \\
\hline NS & 624 & 3,4 & 2,2 & 0,9 & 0,15 & 0,07 & 4,0 & 3,6 & $-0,71$ \\
\hline NW & 4806 & 10,5 & 9,3 & 1,3 & 0,05 & $-0,07$ & 11,1 & 10,7 & $-0,33$ \\
\hline $\mathrm{HE}$ & 2190 & 14,3 & 13,1 & 0,8 & $-0,02$ & $-0,12$ & 14,6 & 14,2 & $-0,77$ \\
\hline $\mathrm{RP}$ & 457 & 4,9 & 4,0 & 1,0 & 0,14 & 0,14 & 6,2 & 5,8 & $-0,61$ \\
\hline $\mathrm{SA}$ & -175 & $-6,4$ & $-4,3$ & $-0,5$ & 0,06 & 0,22 & $-4,0$ & $-4,4$ & $-1,53$ \\
\hline BW & 4715 & 18,4 & 16,9 & 2,4 & 0,19 & $-0,01$ & 19,6 & 19,2 & $-0,43$ \\
\hline BY & 2571 & 8,8 & 7,6 & 1,2 & 0,12 & 0,02 & 9,5 & 9,0 & $-0,54$ \\
\hline $\mathrm{OL}$ & -22127 & $-58,1$ & $-41,4$ & $-5,7$ & $-2,86$ & 0,40 & $-45,4$ & $-45,2$ & $-0,37$ \\
\hline$\sum$ & -5456 & & -959 & -171 & & & & & \\
\hline \multicolumn{3}{|c|}{ Gini-Koeff. vor StV } & 0,185 & n.StV & 0,147 & n.LFA & 0,147 & n.BEZ & 0,147 \\
\hline
\end{tabular}

Tabelle V.29: Abschaffung der Umverteilung, keine Autonomie

ergeben. Allerdings weisen die Preisveränderungen, und damit auch die ToT-Veränderungen, nicht in allen Fällen, in die aufgrund der Nettotransferveränderung zu erwartende Richtung. Wie die Zerlegung der ToT (Spalte 6 und 7 der Tabelle V.28) zeigt, liegt dies an sehr starken gegenläufigen $\mathrm{ToT}_{S E}$. Diese lösen Substitutionseffekte aus, welche die durch die Nettotransferveränderung ausgelösten Preisentwicklungen konterkarieren.

Die Substitutionseffekte werden insgesamt stark unterschätzt, wie ein Vergleich der Summe der $\mathrm{HEV}_{G E}$ mit den Nettotransferveränderungen und den $\mathrm{HEV}_{S E}$ verdeutlicht. Die Substitutionseffekte werden durch Nettotransferveränderungen dominiert, die aufgrund einer Abnahme sämtlicher regionaler Preisniveaus gesunkenen intraregionalen Substitutionseffekte überkompensieren. Dagegen treten bei der Berechnung der Gesamteffekte erhebliche zusätzliche Substitutionseffekte auf, da im Vergleich zur Substitutionseffektberechnung nun sehr starke Preisdifferenzen zwischen den einzelnen Ländern auftreten, die zu einer Zunahme der ToT-Schwankungen und der Schwankungen der Wohlfahrtseffekte führen. Zusätzlich erhöhen sich damit interregionale Substitutionseffekte.

Insgesamt zeigt sich, daß die Nettotransferveränderungen die gesamten Wohlfahrtseffekte dominieren, und eine brauchbare Näherung an diese abgeben. Überraschenderweise gilt dies erst recht für die First-round-Berechnungen, deren Bestimmung die Wohlfahrtswirkungen fast exakt erfaßt. 
Bei der Größenordnung der Nettotransfers verwundert es nicht, daß nun auch die Wohlfahrtsverluste aus der Rationierung voll durchschlagen (siehe Tabelle V.29). Dies führt vor allem für die Ostländer nochmals zu einem Zuwachs der Wohlfahrtsverluste im Vergleich mit der Situation bei Ausgabenautonomie. Erwartungsgemäß nimmt auch der gesamtgesellschaftliche Wohlfahrtsverlust zu.

Die Isolierung der Substitutionseffekte ist auch hier nicht sehr erfolgreich, wie ein Vergleich der Summe von Nettotransferveränderung und HEV der Substitutionseffekte mit den Gesamteffekten zeigt. Es kann vielmehr vermutet werden, daß die tatsächlichen Substitutionseffekte durch die berechneten Substitutionseffekte deutlich unterschätzt werden.

$\mathrm{Da}$ die Nettotransferveränderungen ein so hohes Ausmaß annehmen, kann davon ausgegangen werden, daß sie sämtliche anderen Effekte sehr deutlich dominieren. Dies zeigen auch die Sensitivitätsanalysen, die die ebenso die anderen Ergebnisse bestätigen und daher nicht gesondert dargestellt werden.

\subsubsection{Ein Vergleich der Vorschläge}

Vergleicht man die einzelnen Vorschläge zur Neuordnung des Finanzausgleichs, so zeigt sich, daß letztlich die intraregionalen- und internationalen Substitutionseffekte die Unterschiede in den Ergebnissen bestimmen. Eine genauere Untersuchung der Preisentwicklungen offenbart, daß eine Reihung der Vorschläge, gemäß der Höhe der nationalen Wohlfahrtsgewinne aus den Substitutionseffekten, genau der Reihenfolge der Vorschläge nach dem Ausmaß der Preissenkung entspricht. Je stärker die Preissenkungen der Länder ausfallen und je mehr Länder ihre Preise relativ senken, um so höher sind die nationalen Wohlfahrtsgewinne.

In der dritten Spalte der Tabelle V.30 ist die Differenz zwischen der Summe der Nettotransferveränderungen und der Summe der $\operatorname{HEV}_{G E}$ ausgewiesen. Sie dient als Näherung für die Wohlfahrtswirkungen der Substitutionseffekte. Wie man sieht, gibt es einen deutlichen Trend. Die Vorschläge, in denen in der Mehrzahl der Länder die Preisniveaus sinken - die Zahl der entsprechenden Länder ist in der letzten Spalte aufgeführt —, besitzen positive Wohlfahrtsgewinne aus den Substitutionseffekten. Diese entstehen, da in den Ländern die Preise und Preisdifferenzen sinken und damit die intraregionalen Substitutionseffekte gegenüber der Ausgangssituation abnehmen. In allen anderen Ländern steigen die Preise und die Schwankungen der Preise, woraus sich höhere Substitutionseffekte ergeben.

Die Vorschläge von Föttinger/SPAHN und der von Buhl/PFingSTEN unterscheiden sich durch die Stärke der Preisveränderungen. Immerhin in zehn Ländern sinken die Preise in der Variante I des Vorschlags von FöTtinger/SPAHN am stärksten. Folglich 


\begin{tabular}{||c|r|r|r|l||}
\hline \hline Vorschlag & $\mathrm{HEV}_{G E}$ & $\begin{array}{l}\mathrm{HEV}_{G E} \\
-\Delta N T R^{a}\end{array}$ & $\begin{array}{l}\text { nat. } \\
\text { Preisindex }\end{array}$ & $\begin{array}{l}\text { reg. } \\
\text { Preisindex }\end{array}$ \\
\hline Fött./Spahn I & 396 & 404 & 0,9961 & 10 \\
Fött./Spahn II & 202 & 335 & 0,9955 & 11 \\
Buhl/Pfingsten & 196 & 95 & 1,0001 & 10 \\
Fuest/Lichtblau & -117 & -35 & 0,9998 & 4 \\
Wiss.Beirat III & 57 & -54 & 1,0018 & 0 \\
Wiss.Beirat IV & 14 & -110 & 1,0027 & 0 \\
Wiss.Beirat I & 22 & -139 & 1,0032 & 0 \\
Wiss.Beirat II & -41 & -171 & 1,0047 & 0 \\
Abschaffung & -1504 & -621 & 0,9927 & 9 \\
\hline \hline
\end{tabular}

Tabelle V.30: Vergleich der Vorschläge zur Neuregelung bei Ausgabenautonomie

${ }^{a}$ Als Näherung für die $\mathrm{HEV}_{S E}$

${ }^{b}$ Nationaler Preisindex.

${ }^{c}$ Zahl der Länder, in denen der regionale Preisindex fällt.

Wohlfahrtseffekten der anderen Vorschläge lassen sich im einzelnen durch die Stärke der Preisveränderungen begründen. Offensichtlich besitzen die durch die Multiplikatoren der öffentlichen Güter $m_{G}$, ausgelösten Substitutionseffekte nur einen marginalen Einfluß und werden von den sekundären Substitutionseffekten, die durch Preisveränderungen ausgelöst werden dominiert.

Dies gilt nicht für den Vorschlag einer Abschaffung der horizontalen Umverteilungselemente. Neben relativ stark sinkenden Multiplikatoren $m_{G_{s}}$, die tendenziell zu negativen Substitutionseffekten führen, kommt es zu fallenden Preisen in den meisten Ländern. Dennoch ergeben sich relativ starke Preisdifferenzen zwischen den Ländern, die mit entsprechend hohen interregionalen Substitutionseffekten einhergehen. Dieser Vorschlag führt insgesamt zu den größten Wohlfahrtsverlusten und den größten Substitutionseffekten. Er ist daher unter Wohlfahrtsgesichtspunkten eindeutig abzulehnen.

Wie die Ergebnisse zeigen, ist der Grad der Gleichverteilung nicht irrelevant. Gerade die Vorschläge von Föttinger/SPAHN und Buhl/PFingsten erzielen die höchsten Wohlfahrtsgewinne, während der Vorschlag mit der geringsten Gleichverteilung (Abschaffung der horizontalen Umverteilungselemente) die stärksten Wohlfahrtsverluste erzeugt. Dies könnte sich jedoch dann ändern, wenn den Ländern eine höhere Finanzautonomie zugebilligt würde. 


\subsection{Finanzautonomie: Wissenschaftlicher Beirat [1992]}

Der Vorschlag des Wissenschaftlichen Beirats [1992] sieht eine eingeschränkte Steuerautonomie der Länder vor. Hier wird die Ausgestaltung in Form eines Einkommenssteuerzuschlages des Bundes und der Länder genauer betrachtet, da dieser in dem vorgegebenen Modellrahmen relativ gut untersucht werden kann.

\subsubsection{Einkommenssteuerzuschläge}

\section{(1) Berechnungen zur Finanzautonomie mit Einkommenssteuer}

Gemäß dem Vorschlag des Beirats werden vorab die Einkommenssteuersätze des Bundes gesenkt. Anstelle der vorgeschlagenen fünf-prozentigen Senkung wird hier eine Senkung der Grenzsteuersätze um 4,5\% vorgenommen. Bund und Länder können dann durch die Erhebung eigener Zuschläge oder Abschläge auf die nationalen Einkommenssteuersätze, ihre eigene Steuerpolitik durchführen. Die Einnahmen werden nicht zur Finanzkraft der Länder hinzugerechnet und verbleiben bei der steuersetztenden Gebietskörperschaft. Zugleich wird das gesetzliche Verfahren durch die Einführung der Regelungen aus der Empfehlung des Wissenschaftlichen Beirats [1992], Variante I, ersetzt.

Die Simulation dieses Regimewechsels wird mit Hilfe der differentiellen Inzidenz durchgeführt. Dieses Vorgehen simuliert eine aufkommensneutrale Politikveränderung. Die regionalen Einkommenssteuerzuschläge und der Einkommenssteuerzuschlag des Bundes werden so angepaßt, daß die Einnahmen der öffentlichen Haushalte, preisbereinigt, konstant bleiben. ${ }^{16}$

Die Reduktion der nationalen Einkommenssteuersätze ${ }^{17}$ verringert die Finanzkraft der, bezogen auf die Einkommenssteueraufkommen Pro-Kopf, relativ reichen Länder sehr viel stärker als die Finanzkraft der relativ armen Länder. Demnach sinken die Beiträge der reicheren Länder im LFA. Zugleich erhöhen sich ihre Anteile aus der Umsatzsteuerverteilung. Obwohl sie andererseits geringere Zuweisungen aus der Einkommenssteuerverteilung erhalten, können die reicheren Länder ihre regionalen Einkommenssteuerzuschläge so gestalten, daß die gesamten Einkommensteuersätze auf die regionalen Einkommen unter den ursprünglichen nationalen Grenzsteuersätzen liegen. Geringere Beiträge im Finanzausgleich und geringere regionale Zuschläge auf die Grenzsteuersätze führen zu Wohlfahrts-

\footnotetext{
${ }^{16}$ Dieses Vorgehen isoliert allerdings bei mehreren Ländern nicht die Substitutionseffekte. Es treten nach wie vor interregionale und internationale Einkommenseffekte durch Nettotransferveränderungen auf.

${ }^{17}$ Genaugenommen werden im Simulationsablauf die nationalen Grenzsteuersätze gesenkt und die $\mathrm{Zu}$ schläge auf den Grenzsteuersatz erhoben. Der Einfachheit halber wird im weiteren nicht auf diese Unterscheidung geachtet.
} 


\begin{tabular}{|c|c|c|c|c|c|c|c|c|c|c|}
\hline \multirow[t]{3}{*}{ Land } & \multicolumn{2}{|c|}{$\overline{\mathrm{HEV}}$} & \multirow{3}{*}{$\begin{array}{c}\Delta \mathrm{TR} \\
\text { in } \% \\
\mathrm{REV}\end{array}$} & \multirow{3}{*}{$\begin{array}{r}\mathrm{HEV}_{S E} \\
\text { in \% } \\
\mathrm{REV}\end{array}$} & \multirow{3}{*}{$\begin{array}{r}\text { ToT }_{G E} \\
\text { in \% } \\
\text { ToT }\end{array}$} & \multirow{3}{*}{$\begin{array}{r}\text { ToT }_{S E} \\
\text { in \% } \\
\text { ToT }\end{array}$} & \multicolumn{2}{|c|}{$\overline{F A}$} & \multirow{3}{*}{$\begin{array}{r}\Delta m_{G,} \\
\text { in \% } \\
m_{G,}^{0}\end{array}$} & \multirow{3}{*}{$\begin{array}{r}\Delta \tau_{s}^{Y a} \\
-0,045^{b}\end{array}$} \\
\hline & Mio & in $\%$ & & & & & neu $\%$ & f.r. $\%$ & & \\
\hline & $\mathrm{DM}$ & REV & & & & & REV & REV & & \\
\hline $\mathrm{SH}$ & -214 & $-3,2$ & $-2,2$ & $-0,7$ & 0,02 & 0,02 & $-2,2$ & $-3,7$ & $-0,14$ & 0,0102 \\
\hline $\mathrm{HH}$ & 65 & 1,0 & 1,4 & 0,0 & $-0,29$ & $-0,26$ & 2,2 & $-0,7$ & $-0,06$ & $-0,0030$ \\
\hline HB & -158 & $-6,3$ & $-3,5$ & $-2,3$ & $-0,05$ & $-0,07$ & $-4,5$ & $-6,3$ & $-0,12$ & 0,0204 \\
\hline NS & -596 & $-3,2$ & $-2,0$ & $-0,8$ & 0,05 & 0,05 & $-2,1$ & $-3,4$ & $-0,14$ & 0,0105 \\
\hline NW & 468 & 1,0 & 0,6 & 0,6 & $-0,10$ & $-0,10$ & 1,7 & $-0,4$ & 0,32 & $-0,0042$ \\
\hline $\mathrm{HE}$ & 308 & 2,0 & 1,5 & 0,6 & $-0,14$ & $-0,13$ & 2,6 & 0,4 & $-0,01$ & $-0,0050$ \\
\hline $\mathrm{RP}$ & -74 & $-0,8$ & $-0,9$ & 0,0 & 0,14 & 0,14 & $-0,1$ & $-1,6$ & $-0,10$ & 0,0030 \\
\hline SA & -219 & $-7,9$ & $-5,0$ & $-3,0$ & 0,36 & 0,30 & $-6,3$ & $-7,5$ & $-0,49$ & 0,0327 \\
\hline BW & 911 & 3,6 & 2,2 & 1,4 & $-0,05$ & $-0,04$ & 3,8 & 1,5 & 0,40 & $-0,0105$ \\
\hline BY & 71 & 0,2 & 0,2 & 0,3 & $-0,01$ & $-0,01$ & 0,9 & $-0,9$ & 0,14 & $-0,0014$ \\
\hline OL & -849 & $-2,2$ & $-1,1$ & $-1,3$ & 1,13 & 0,97 & $-2,0$ & 3,8 & 0,11 & 0,0224 \\
\hline$\sum$ & -286 & & 58 & -29 & & & & & & $0,0211^{c}$ \\
\hline \multicolumn{3}{|c|}{ Gini-Koeff. vor StV } & 0,167 & n.StV & 0,132 & n.LFA & 0,069 & n.BEZ & 0,073 & \\
\hline
\end{tabular}

Tabelle V.31: Wiss. BeIrat, I, Steuerautonomie bei gesenktem $\tau^{Y}$ und diff.Inzidenz

${ }^{\circ}$ Veränderung der Einkommenssteuersätze gegenüber dem Datensatz.

${ }^{b}$ Senkung der nationalen Einkommenssteuersätze um 0,45 Prozent.

${ }^{c}$ Einkommenssteuerzuschlag des Bundes.

gewinnen der reicheren Länder im Vergleich zur Simulation bei Ausgabenautonomie und Budgetinzidenz. Die ärmeren Länder müssen dagegen mit Wohlfahrtsverlusten rechnen.

Die Resultate, die in der Tabelle V.31 ausgewiesen sind, bestätigen diese Vermutungen. In der letzten Spalte ist die Differenz zwischen dem gesamten regionalen Grenzsteuersatz, bestehend aus dem nationalen Grenzsteuersatz und den regionalen und nationalen $\mathrm{Zu}$ schlägen, und den ursprünglichen nationalen Grenzsteuersätzen angegeben. Ein negativer Wert bedeutet eine Senkung des gesamten regionalen Grenzsteuersatzes. Alle Länder mit positiven (negativen) Finanzausgleichsveränderungen besitzen negative (positive) Grenzsteuersatzveränderungen und erzielen Wohlfahrtsgewinne(verluste).

Die interessantere Frage ist jedoch, wie diese Umgestaltung die nationale Wohlfahrt beeinflußt?

Zum einen führen regional differenziertere Einkommenssteuersätze zu einer stärkeren Differenzierung der regionalen Güterpreise und damit auch der ToT. Höhere Grenzsteuersätze verringern den relativen Preis der Freizeit $\left(w_{s}\left(1-\tau_{s}^{Y}\right) / p_{s}\right)$. Dies führt zu einem relativ 
höheren Freizeitkonsum, sinkendem Arbeitsangebot und höheren Lohnsätzen. Letztlich erhöhen sich dadurch die Güterpreise und die ToT. Mit Ausnahme der ToT-Anpassungen von Bremen entsprechen alle ToT-Veränderungen diesen Erwartungen. Die stärkere Spreizung der ToT verstärkt interregionale Substitutionseffekte.

Zusätzlich führen veränderte Einkommenssteuersätze zu intraregionalen Substitutionseffekten, da die Verzerrungen zwischen der optimalen Abstimmung der Konsum- und Produktionsstruktur bezüglich Freizeit und dem privaten Konsum, sowie in bezug auf Freizeit und das öffentlichen Gut, zunehmen (fallen), wenn die regionale Grenzsteuerbelastung steigt (sinkt). Diese intraregionalen Substitutionseffekte verstärken die Wohlfahrtsgewinne der steuersenkenden und steigern die -verluste der steuererhöhenden Länder. In der Summe ergeben sich gesellschaftliche Wohlfahrtsverluste, die ausschließlich auf Substitutionseffekte zurückgeführt werden können.

Die Trennung in Einkommens- und Substitutionseffekte ist nicht ganz geglückt. Dies liegt in erster Linie an den unterschiedlichen Einkommenssteuerzuschlägen der Bundesländer in der Gesamtberechnung und der Simulation der Substitutionseffekte. In der isolierten Berechnung der Substitutionseffekte sind die regionalen Einkommenssteuerzuschläge wesentlich weniger differenziert als in der Hauptsimulation. Folglich fallen die intra- und interregionalen Substitutionseffekte niedriger aus.

Die als First-round-Berechnungen ermittelten Werte für die Veränderung der Finanzausgleichszahlungen und Steuerexporte stellen nur teilweise eine brauchbare Abschätzung für die Wohlfahrtseffekte dar. In einigen Fällen sind sie den Wohlfahrtsgewinnen genau entgegengesetzt.

Ein Vergleich mit den Berechnungen bei Ausgabenautonomie (Tabelle V.6, Seite 197) und bei fehlender Ausgabenautonomie (Tabelle V.11, Seite Seite 201) zeigt, überraschenderweise, daß die gesellschaftliche Wohlfahrt bei Finanzautonomie durch Einkommenssteuerzuschläge erheblich niedriger ist, ja sogar sinkt. Dies deutet darauf hin, daß die Substitutionseffekte sehr starke Wohlfahrtswirkungen auslösen, die die Wohlfahrtsgewinne aus einer stärkeren Autonomie überkompensieren.

\section{(2) Sensitivitätsanalysen zur Finanzautonomie mit Einkommenssteuern}

\section{(a) Fixes Arbeitsangebot}

Sehr deutliche Veränderungen ergeben sich bei der Empfehlung des WissEnschaftuICHEN BEIRATS [1992], wenn man quasifixes Arbeitsangebot unterstellt. Wie aus der Tabelle V.32 ersichtlich ist, kehrt sich der Gesamtwohlfahrtseffekt sogar um. Dies ist im Prinzip auf die geringere Reagibilität der Freizeitwahl zurückzuführen. In der Argumentation zur Simulation mit variablem Arbeitsangebot spielten zunehmendẹ Verzerrungen 
zwischen Freizeit und dem privaten Konsum eine wesentliche Rolle. Diese führten dazu, daß sich die Güterpreise und ToT in Richtung der gesamten regionalen Grenzsteuersatzveränderung anpassen.

Bei Annahme eines quasifixen Arbeitsangebotes können Steuersatzveränderungen dagegen keine Veränderung des Arbeitsangebotes und damit keine Reaktion der Güterpreise und der ToT hervorrufen. Demnach differieren die interregionalen Preise bei quasifixem Arbeitsangebot sehr viel geringer. Dadurch werden interregionale Substitutionseffekte reduziert. Insgesamt erhält man sogar eine Umkehr der gesellschaftlichen Wohlfahrtseffekte, die etwa in Höhe der Steuerexporte, leicht zunehmen.

\begin{tabular}{|c|c|c|c|c|c|c|c|c|c|c|}
\hline \multirow[t]{3}{*}{ Land } & \multicolumn{2}{|c|}{ HEV } & \multirow{3}{*}{$\begin{array}{l}\Delta \mathrm{TR} \\
\text { in } \% \\
\mathrm{REV}\end{array}$} & \multirow{3}{*}{$\begin{array}{r}\mathrm{HEV}_{S E} \\
\text { in \% } \\
\mathrm{REV}\end{array}$} & \multirow{3}{*}{\begin{tabular}{|r|} 
ToT $_{G E}$ \\
in $\%$ \\
ToT \\
\end{tabular}} & \multirow{3}{*}{$\begin{array}{r}\text { ToT }_{S E} \\
\text { in \% } \\
\text { ToT }\end{array}$} & \multicolumn{2}{|c|}{ FA } & \multirow{3}{*}{$\begin{array}{c}\Delta m_{G s} \\
\text { in \% } \\
m_{G}^{0}\end{array}$} & \multirow{3}{*}{$\begin{array}{r}\Delta \tau_{s}^{Y} \\
-0,045\end{array}$} \\
\hline & Mio & in $\%$ & & & & & $\%$ & f.r. $\%$ & & \\
\hline & $\mathrm{DM}$ & REV & & & & & REV & REV & & \\
\hline $\mathrm{SH}$ & -196 & $-2,9$ & $-2,1$ & $-0,7$ & 0,01 & 0,01 & $-2,2$ & $-3,7$ & $-0,14$ & 0,0087 \\
\hline $\mathrm{HH}$ & 119 & 1,9 & 0,9 & 0,8 & $-0,24$ & $-0,24$ & 2,2 & $-0,7$ & $-0,06$ & $-0,0039$ \\
\hline $\mathrm{HB}$ & -140 & $-5,6$ & $-4,8$ & $-0,6$ & $-0,09$ & $-0,09$ & $-4,9$ & $-6,3$ & $-0,12$ & 0,0169 \\
\hline NS & -524 & $-2,8$ & $-2,2$ & $-0,5$ & 0,04 & 0,04 & $-2,2$ & $-3,4$ & $-0,14$ & 0,0088 \\
\hline NW & 605 & 1,3 & 1,0 & 0,3 & $-0,08$ & $-0,08$ & 1,7 & $-0,4$ & 0,32 & $-0,0048$ \\
\hline $\mathrm{HE}$ & 351 & 2,3 & 1,6 & 0,6 & $-0,12$ & $-0,12$ & 2,7 & 0,4 & $-0,01$ & $-0,0056$ \\
\hline $\mathrm{RP}$ & -56 & $-0,6$ & $-0,4$ & $-0,1$ & 0,13 & 0,13 & 0,0 & $-1,6$ & $-0,10$ & 0,0020 \\
\hline SA & -216 & $-7,8$ & $-6,3$ & $-1,1$ & 0,23 & 0,23 & $-6,9$ & $-7,5$ & $-0,49$ & 0,0282 \\
\hline BW & 963 & 3,7 & 2,9 & 0,7 & $-0,03$ & $-0,03$ & 4,0 & 1,5 & 0,40 & $-0,0107$ \\
\hline BY & 151 & 0,5 & 0,4 & 0,1 & 0,00 & 0,00 & 1,0 & $-0,9$ & 0,14 & $-0,0021$ \\
\hline $\mathrm{OL}$ & -1036 & $-2,7$ & $-1,8$ & $-0,7$ & 0,80 & 0,80 & $-2,0$ & 3,8 & 0,11 & 0,0172 \\
\hline$\Sigma$ & 21 & & 34 & 30 & & & & & & \\
\hline
\end{tabular}

Tabelle V.32: Wiss. BEIRAT I, diff.Inzidenz, Finanzautonomie, mit $\tau_{L d}^{Y}$ bei quasifixem Arbeitsangebot

\section{(b) Keine nationale Einkommenssteuersenkung}

Eine alternative Lösung zur Einführung einer Finanzautonomie über Einkommenssteuerzuschläge wäre die der Beibehaltung der nationalen Steuersätze. Dazu sei angenommen, daß die nationalen Grenzsteuersätze gegenüber der Ausgangsposition unverändert bleiben, der Bund und die Länder jedoch zusätzlich $\mathrm{Zu}$ - oder Abschläge auf die Grenzsteuersätze erheben können.

Dieser Vorschlag wird ebenfalls mit Hilfe der differentiellen Inzidenz simuliert. Die Ergebnisse kann man recht gut mit dem Fall der Ausgabenautonomie vergleichen, in dem die 
Budgetinzidenz verwendet wurde, da wie bereits angemerkt, die Simulation der Ausgabenautonomie mit Budgetinzidenz einer Finanzautonomie mit Lump-Sum-Steuern entspricht.

Vergleicht man die Resultate bei differentieller Inzidenz und Budgetinzidenz, so ist zu erwarten, daß die Finanzautonomie mit regional differenzierten Einkommenssteuerzuschlägen über stärkere Preisdifferenausschlägen zu höheren Substitutionseffekten führt und daher geringere Wohlfahrtsgewinne erzeugt, als die Ausgabenautonomie über eine Transferanpassung. Betrachtet man die Tabellen V.33 und V.6, dann stellt man jedoch fest, daß die gesamtgesellschaftlichen Wohlfahrtsgewinne im Falle der Finanzautonomie mit Einkommenssteuerzuschlägen höher sind, als mit Lump-Sum-Steuern.

\begin{tabular}{|c|c|c|c|c|c|c|c|c|c|}
\hline \multirow[t]{3}{*}{ Land } & \multicolumn{2}{|c|}{$\mathrm{HEV}$} & $\Delta \mathrm{TR}$ & $\mathrm{HEV}_{S E}$ & $\mathrm{ToT}_{G E}$ & ToT $_{S E}$ & $\mathrm{~F}$ & & \multirow{3}{*}{$\tau_{s}^{Y}$} \\
\hline & Mio & in $\%$ & in $\%$ & in $\%$ & in $\%$ & in $\%$ & neu $\%$ & f.r. $\%$ & \\
\hline & $\mathrm{DM}$ & $\mathrm{REV}$ & REV & REV & ToT & ToT & REV & REV & \\
\hline $\mathrm{SH}$ & -210 & $-3,2$ & $-2,4$ & $-0,8$ & 0,06 & 0,05 & $-2,8$ & $-3,7$ & 0,0096 \\
\hline $\mathrm{HH}$ & -10 & $-0,2$ & $-0,2$ & $-0,2$ & $-0,22$ & $-0,22$ & $-0,1$ & $-0,7$ & 0,0003 \\
\hline HB & -156 & $-6,3$ & $-3,8$ & $-2,4$ & $-0,01$ & $-0,05$ & $-5,2$ & $-6,3$ & 0,0198 \\
\hline NS & -523 & $-2,8$ & $-2,1$ & $-0,8$ & 0,10 & 0,08 & $-2,5$ & $-3,4$ & 0,0092 \\
\hline NW & 69 & 0,2 & $-0,1$ & 0,1 & $-0,08$ & $-0,08$ & 0,3 & $-0,4$ & $-0,0029$ \\
\hline $\mathrm{HE}$ & 143 & 0,9 & 0,6 & 0,1 & $-0,12$ & $-0,12$ & 1,1 & 0,4 & $-0,0031$ \\
\hline $\mathrm{RP}$ & -99 & $-1,1$ & $-0,9$ & $-0,4$ & 0,15 & 0,15 & $-0,9$ & $-1,6$ & 0,0031 \\
\hline $\mathrm{SA}$ & -197 & $-7,1$ & $-4,2$ & $-2,9$ & 0,36 & 0,31 & $-6,1$ & $-7,5$ & 0,0288 \\
\hline BW & 549 & 2,2 & 1,2 & 0,6 & $-0,03$ & $-0,03$ & 2,0 & 1,5 & $-0,0078$ \\
\hline BY & -98 & $-0,3$ & $-0,4$ & $-0,1$ & 0,02 & 0,01 & $-0,2$ & $-0,9$ & $-0,0004$ \\
\hline $\mathrm{OL}$ & 1162 & 3,1 & 1,3 & 2,0 & 0,35 & 0,49 & 3,4 & 3,8 & $-0,0385$ \\
\hline$\sum$ & 631 & & -111 & & & & & & 0,0006 \\
\hline
\end{tabular}

Tabelle V.33: Wiss. BeIRAT, I, Steuerautonomie bei fixen $\tau^{Y}$ und diff.Inzidenz

Der Grund für die Wohlfahrtsgewinne bei Steuersatzänderungen muß in einer deutlichen Reduktion der Substitutionseffekte liegen. Da regional differenzierte Einkommenssteuersätze keine primären interregionalen Verzerrungen bewirken, können ihre interregional verzerrenden Einflüsse nur indirekt über eine Veränderung der Preisdifferenzen zwischen den Ländern zum Tragen kommen. Wie die Ergebnisse nun zeigen, führen regional differenzierte Einkommenssteuerzuschläge, im relevanten Fall, allerdings zu geringeren Differenzen der regionalen ToT. In erster Linie spielen die Ostländer eine entscheidende Rolle. Sie können nun aufgrund höherer Finanzausgleichszahlungen bzw. Nettotransfers Abschläge auf die Einkommenssteuersätze erheben. Demnach steigt ihr Nettolohnsatz und damit der relative Preis der Freizeit. Dies führt zu einem erhöhten Arbeitsangebot, zu sinkenden 
Lohnsätzen und schließlich zu Preissenkungen in den Ostländern. Letztlich reduzieren sich ihre ToT im Vergleich zur Simulation ohne Einkommenssteuervariationen. Allein die geringere Zunahme der Preise und ToT der Ostländer reduziert die Preisdifferenzen zwischen den Ländern sehr deutlich und führt zu einem geringeren durchschnittlichen Preisniveau aller Länder. Geringere Preisdifferenzen bewirken dann eine Abnahme der interregionalen Substitutionseffekte.

Darüber hinaus nehmen für die großen Länder, mit Ausnahme von Bayern und Niedersachsen, die intraregionalen Substitutionseffekte zusätzlich deshalb ab, weil sie Abschläge auf die Einkommenssteuersätze erheben können. Dadurch reduzieren sich die Preiskeile zwischen dem öffentlichen Konsum und Freizeit und zwischen dem privaten Konsum und Freizeit. Darüber hinaus sinkt ihr Preisniveau und damit auch alle anderen intraregionalen Substitutionseffekte.

Offensichtlich werden die dadurch entstehenden Wohlfahrtsgewinne nicht durch die steigenden Substitutionseffekte in den anderen Ländern überkompensiert. Hinzu kommt, daß Bayern und Hamburg Zuschläge erheben, die unter einer Promille liegen, und daher nur minimale Verzerrungen hervorrufen. ${ }^{18}$

Insgesamt ergeben sich somit Wohlfahrtsgewinne aus dem Abbau der interregionalen Substitutionseffekte und der intraregionalen Substitutionseffekte in den größeren Ländern.

Man kann damit festhalten: Eine Erhöhung der regionalen Finanzautonomie durch die Möglichkeit, Zuschläge auf die Einkommenssteuersätze zu erheben, ist wohlfahrtserhöhend. Allerdings gilt dies nicht mehr unbedingt, wenn zugleich der einheitliche Einkommenssteuersatz gesenkt wird. Dann würden regional deutlich stärker differenzierten Steuersätzen und damit recht hohe Substitutionseffekte ausgelöst werden. Darüber hinaus verändern sich bei einer Senkung der nationalen Steuersätze und Einführung von regionalen $\mathrm{Zu}$ schlägen die Finanzausgleichszahlungen und Nettotransfers sehr stark. Für die Ostländer würde das letztlich bedeuten, daß ihre Einnahmen im Finanzausgleich deutlich geringer sind, als in allen anderen Vorschlägen.

\subsection{Zusammenfassung der Ergebnisse}

In den bisher dargestellten Berechnungen zeigen sich einige grundsätzliche Tendenzen:

Bezüglich nationaler Wohlfahrtsgewinne spielen die internationalen Steuerexportveränderungen nicht die dominierende Rolle, die die Nettotransferveränderungen bei der Bestim-

\footnotetext{
${ }^{18}$ Bayern erhebt zwar Abschläge, die jedoch in Verbindung mit den nationalen Zuschlag von 0,0006 zu einem Anstieg der regionalen Grenzsteuerbelastung führen.
} 
mung der regionalen Wohlfahrtswirkungen ausüben. Die Gesamteffekte werden zumeist durch die intraregionalen und interregionalen Substitutionseffekte bestimmt. ${ }^{19}$

Aus diesen Ausführungen kann man als zentrale Ergebnisse festhalten:

Erstens: Die Berechnung der Finanzausgleichszahlungen, ohne Berücksichtigung von Rückwirkungen, reicht zur Bestimmung der Wohlfahrtseffekte nicht aus. Ergänzt man die reinen Finanzausgleichsströme durch First-round-Berechnungen der Steuerexporte und der vermuteten Veränderungen im Finanzgebaren des Bundes, so erhält man First-roundAbschätzungen der Nettotransfers. Die Veränderung dieser Nettotransfers können sogar eine andere Richtung aufweisen, als die erwartete Reaktion der Finanzausgleichszuweisungen. In den meisten Fällen können die so berechnten First-round-Nettotransferveränderungen als gute Näherung an die endgültigen Nettotransferentwicklungen und an die regionalen Wohlfahrtseffekte angesehen werden.

Zweitens: Die Selbstfinanzierungsterme der öffentlichen Güter lösen nur relativ geringe Verzerrungen aus. Ein möglicher Grund besteht darin, daß sich die einzelnen Komponenten gegenseitig nivellieren. Eine Erhöhung der öffentlichen Ausgaben erhöht zwar die regionalen Steuereinnahmen, vermindert aber zugleich die Einnahmen aus dem LFA und den Ergänzungszuweisungen. Darüber hinaus verlaufen die Verzerrungen zwischen den öffentlichen Gütern und den privaten Konsumkategorien gegenläufig. In der Hauptsache ist die geringe Bedeutung des Selbstfinanzierungstermes darauf zurückzuführen, daß dieser einen sehr geringen Wert besitzt (unter $1 \%$ der öffentlichen Güterpreise). Geht man von fehlender Ausgabenautonomie der Länder aus, üben die sozialen Grenzkosten der öffentlichen Güter keinen Einfluß auf die Entscheidung auf. Demnach sind diese Verzerrungen dann nicht relevant.

Drittens: Die Umverteilungsmaßnahmen des Finanzausgleichssystems rufen sehr starke Preisveränderungen hervor, die die vorhandenen Verzerrungen verstärken oder vermindern, und damit sekundäre Substitutionseffekte auslösen. Die Umverteilungsmaßnahmen sind damit nicht allokationsneutral. Dies gilt selbst dann, wenn die Länder ihre notwendigen Budgetanpassungen ausschließlich über Lump-Sum-Transfers vornehmen. Daher sind die gesamtgesellschaftlichen Wohlfahrtseffekte der einzelnen Vorschläge nur in einer umfassenden empirischen Analyse, z.B. einer EAG-Analyse bestimmbar. Die vorliegenden Ergebnisse deuten darauf hin, daß eine stärkere Gleichverteilung Wohlfahrtsgewinne bewirken kann. Dies ist auf weniger stark differierende Preise zurückzuführen.

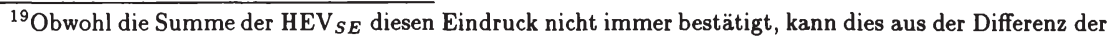
Summe der Nettotransferveränderungen mit der Summe der $\mathrm{HEV}_{G E}$ abgeleitet werden. Diese Differenz kann letztlich nur durch Substitutionseffekte erklärt werden, da die internationalen ToT-Effekte nur sehr gering sind.
} 
Viertens: Eine Erhöhung der Finanzautonomie der Länder ist in allen Fällen gesellschaftlich wohlfahrtserhöhend. In der Tendenz gilt dies auch für die Wohlfahrt der einzelnen Länder. Allerdings gilt diese Aussage nur dann ohne Einschränkungen, wenn eine höhere Finanzautonomie nicht mit einer gleichzeitigen Senkung der nationalen Steuersätze verbunden ist. Überraschenderweise kann die Einführung regional differenzierter Einkommensteuerzuschläge im Vergleich zur Lump-Sum-Steuer-Finanzierung (als Ausgabenautonomie modelliert) für die Gesellschaft wohlfahrtssteigernd wirken. Dies kann durch die Second-best-Problematik erklärt werden.

Fünftens: Der Einbezug der Ostländer in das bundesdeutsche Finanzausgleichssystem ist gesellschaftlich wohlfahrtserhöhend, wenn tatsächlich Ausgabenautonomie besteht. Dieses Ergebnis ist allerdings nur mit Vorsicht zu behandeln, da die Wohlfahrtsgewinne auf Steuerexporte zurückzuführen sind. Inwieweit diese von der rudimentären Außenhandelsmodellierung beeinflußt sind, können nur entsprechende Modellerweiterungen erhellen. 
Georg Hirte - 978-3-631-75212-8

Downloaded from PubFactory at 01/11/2019 07:02:26AM

via free access 


\section{Kapitel VI}

\section{Schlußbemerkungen}

Die theoretische und empirische Analyse des bundesdeutschen Finanzausgleichssystems verdeutlicht einige Gesichtspunkte, die nochmals zusammenfassend dargestellt werden sollen.

Ergebnis der theoretischen Analyse war, daß Finanzausgleichssysteme Selbstfinanzierungseffekte auslösen, die zu einem Abweichen der sozialen Grenzkosten der öffentlichen und der privaten Güter von deren jeweiligen Preisen führen können. Infolgedessen wird die intraregional optimale Abstimmung der Produktions- und Konsumstruktur zwischen dem öffentlichen Gut und den inländischen privaten Gütern (Konsumgut bzw. Freizeit) verzerrt. Die (indirekt) interregional optimale Konsum- und Produktionsstruktur zwischen dem öffentlichen Gut und den bezogenen Konsumgütern wird ebenfalls gestört. Damit entstehen intraregionale und interregionale Substitutionseffekte. Diese Effekte wurden in der wissenschaftlichen Diskussion bislang nicht beachtet. Für die Frage der optimalen Gestaltung von Finanzausgleichssystemen ist sie gleichwohl, zumindest theoretisch, von Belang.

Ein zweites zentrales Ergebnis der theoretischen Analyse war, daß bei fehlender Finanzautonomie der Länder Wohlfahrtsverluste infolge der Rationierung entstehen. Und drittens zeigte eine genauere Betrachtung des bundesdeutschen Finanzausgleichssystems, daß dieses nicht nur allgemeine Zuweisungen enthält, sondern eine Reihe anderer Zuweisungsarten mit entsprechenden verzerrenden Wirkungen aufweist.

Die empirische Untersuchung in Form einer Empirischen Allgemeinen Gleichgewichtsanalyse konnte allerdings nicht bestätigen, daß die Selbstfinanzierungseffekte ein wesentliche Rolle spielen. Die berechneten Selbstfinanzierungsterme der öffentlichen Güter belaufen sich fast ausnahmslos auf weniger als ein Prozent der Güterpreise. Allein deshalb können sie keinen besonders starken Einfluß besitzen. Die theoretische Analyse hat zudem gezeigt, daß sich die Einflüsse des Steuer- und Finanzausgleichssystems auf die Kosten der öffentlichen Güter gegenseitig nivellieren. In ähnlicher Form gilt das auch für die verschiedenen, durch die Selbstfinanzierungsterme hervorgerufenen Verzerrungen.

Das zweite zentrale Ergebnis der empirischen Analysen ist, daß Umverteilungsmaßnahmen im Rahmen des Finanzausgleichssystems nicht unbedingt allokationsneutral sind. 
Vielmehr verursachen diese eine Reihe von Preisveränderungen und damit Verstärkungen oder Abschwächungen der Substitutionseffekte. Damit kann die Frage der gesellschaftlichen Wohlfahrtswirkungen der Substitutionseffekte, wenn überhaupt, nur mit Hilfe einer EAG-Analyse beantwortet werden. Diese Arbeit liefert dazu einen Beitrag, der deutlich macht, daß merkliche Wohlfahrtswirkungen auftreten können. Ungeachtet aller Skepsis gegenüber einer solchen empirischen Untersuchung dürfte unbestritten sein, daß die ausgelösten Veränderungen der sekundären Substitutionseffekte durchaus relevant werden können. Dies ist eines der zentralen Ergebnisse der EAG-Studie.

In einem weiteren Punkt haben die Simulationen theoretische Ergebnisse präzisiert. In der Analyse im theoretischen Teil wurde deutlich, daß die Rationierung der Gebietskörperschaften, in Form fehlender Einnahmen- und Ausgabenautonomie, im Vergleich mit einer Ausgabenautonomie zu Wohlfahrtsverlusten führt. Dies gilt natürlich auch bezüglich einer vollen Finanzautonomie, wenn diese in Form von Lump-Sum-Steuern organisiert ist. Die Berechnungen des empirischen Teils reproduzierten diese Ergebnisse. In allen Fällen liegen die gesellschaftlichen Wohlfahrtsgewinne bei Rationierung deutlich unter denen der Ausgabenautonomie, die allerdings in den Simulationen im Prinzip als Finanzautonomie modelliert wurde. ${ }^{1}$ Teilweise sind die Unterschiede so groß, daß die Rationierung zu einer Umkehrung der Vorzeichen der gesellschaftlichen Wohlfahrtseffekte führte. Dies verdeutlicht, daß für die Wohlfahrtswirkungen von Finanzausgleichssystemen, der Grad der Finanzautonomie eine nicht unbedeutende Rolle spielt. Insofern ist die Frage nach einem optimalen Grad der Finanzautonomie zu diskutieren. Die Ergebnisse der EAG-Studie geben deutliche Hinweise darauf, daß eine Erhöhung der regionalen Ausgabenautonomie unter Effizienzgesichtspunkten wünschenswert wäre.

Nun stellen allerdings Lump-Sum-Steuern keinen allzu realistischen Fall der Steuerautonomie dar. Realistischere Szenarien wären z.B. Einkommenssteuer- oder Körperschaftssteuerzuschläge, wie sie der WissensChaftLiChE BEIRAT [1992] vorgeschlagen hat. Die Einführung regional differenzierter Steuersätze verursacht allerdings, das ist nicht zuletzt seit der Harmonisierungsdebatte in der EU deutlich geworden, interregionale, aber auch intraregionale Substitutionseffekte. Dem stehen Wohlfahrtsgewinne aus einer höheren Finanzautonomie entgegen. Nur die empirische Analyse kann klären, welche dieser Größen überwiegt.

Der empirische Vergleich von Lump-Sum-Steuern mit regionalen Einkommenssteuerzuschlägen, als Instrument größerer Finanzautonomie, zeigte, daß letztere zu höheren Wohlfahrtsgewinnen führen können, wenn die Zuschlagssatzdifferenzen nicht allzugroß werden.

\footnotetext{
${ }^{1}$ Die Länder konnten ihre Ausgaben mit Hilfe von Lump-Sum-Transfers an die regionalen Einnahmen anpassen. Da diese jedoch auch negative Werte annehmen dürfen, entspricht dies einer Finanzautonomie.
} 
Der Grund liegt darin, daß als Folge differenzierter Einkommenssteuerzuschläge, unter bestimmten Bedingungen, vorhandene Substitutionseffekte reduziert werden und dadurch die gesellschaftliche Wohlfahrt steigt; ein Ergebnis, daß in einer (höchstens) Secondbest-Welt nicht unbedingt überrascht. ${ }^{2}$ Von daher ist solchen Vorschlägen, die vor der Einführung einer regionalen Steuerautonomie nationale Steuersenkungen vorsehen, mit Skepsis zu begegnen.

Aus der EAG-Analyse ist darüber hinaus deutlich geworden, daß die üblichen Berechnungen der Finanzausgleichsströme, nicht in jedem Fall einen verläßlichen Indikator für Wohlfahrtsgewinne darstellen. Vielmehr müßten zusätzlich interregionale und internationale Steuerexporte und Refinanzierungsmaßnahmen des Bundes berücksichtigt werden. Letztere allerdings nur, wenn sie die Bundesländer unterschiedlich treffen. Im konkreten Fall hieße das z.B., daß bei Berechnungen der Finanzausgleichszahlungsströme auch die Budgetanpassungen des Bundes berücksichtigt werden sollten, die notwendig sind, um die verminderten Einnahmen des Bundes auszugleichen. ${ }^{3}$

Selbst wenn es gelänge, die Nettotransferveränderungen annähernd genau zu ermitteln, müssen damit noch keineswegs die tatsächlichen Wohlfahrtswirkungen bekannt sein. Bei regionalen Steuerzuschlägen ist das von vorneherein klar, da erhebliche zusätzliche Substitutionseffekte auftreten können. Die empirische Analyse hat gezeigt, daß auch in den anderen Vorschlägen deutliche Abweichungen zwischen Nettotransferveränderungen und Wohlfahrtseffekten bestehen können. Zwar ist in den meisten Fällen eine Übereinstimmung zwischen Vorzeichen und Größenordnung beider Größen ermittelt worden, doch gibt es davon Ausnahmen. Beispielsweise wird dieser Zusammenhang bei fehlender Ausgabenautonomie schwächer, da eine Rationierung die bestehenden Substitutionseffekte verstärkt. Demnach kann die Berechnung der First-round-Finanzausgleichszuweisungen keinen zuverlässigen Eindruck über die Wohlfahrtswirkungen geben. Erst eine EAG-Analyse ist in der Lage, zu zeigen, in welchem Vorschlag und unter welchen Bedingungen die Ermittlung der First-round-Größe die Wohlfahrtsveränderungen ausreichend charakterisiert.

\footnotetext{
${ }^{2}$ Im Rahmen der empirischen Analyse wurden auch Auswirkungen regionaler Körperschaftsteuerzuschläge simuliert. Diese Ergebnisse werden allerdings hier nicht aufgeführt, da eine wirklich erhellende Untersuchung dieser Problematik in einem dynamischen Modell durchgeführt werden müßte. Es zeigte sich, daß in der statischen Welt regional differenzierte Kapitalsteuersätze im Vergleich mit Lump-SumSteuern ebenfalls Wohlfahrtsgewinne erzeugen können. Dieser Zusammenhang ist noch stärker als bei Einkommenssteuerzuschlägen.

${ }^{3} \mathrm{DaB}$ dies äußerst schwierig ist, da nicht jede Veränderung des Bundeshaushalts eindeutig irgendeinem Grund zuordenbar ist, dürfte klar sein. Es ändert aber nichts an der grundsätzlichen Bedeutung der Reaktionen des Bundes auf veränderte Einnahmen aus dem Finanzausgleich.
} 
Man könnte diesen Aspekten noch eine Reihe weiterer Punkte hinzufügen, die jedoch mehr oder weniger deutlich bereits alle in den vorhergehenden Kapiteln angesprochen wurden. Darum soll es dabei bewendet bleiben.

Sinn und Zweck dieser Arbeit war es, die Effizienzaspekte von Finanzausgleichsregelungen unter die Lupe zu nehmen, und am Beispiel des Finanzausgleichssystems der Bundesrepublik Deutschland die einzelnen im theoretischen Teil hergeleiteten Aspekte auf ihren empirischen Gehalt hin zu untersuchen. Als Instrumentarium wurde dazu eine Empirische Allgemeine Gleichgewichtsanalyse walrasianischer Provenienz eingesetzt. Ein Nebenprodukt der Untersuchung sollte es sein, zu verdeutlichen, daß diese Methode sinnvoll eingesetzt werden kann, um theoretische Ergebnisse zu reproduzieren und um der Theorie Hilfestellungen zu geben, zu erkennen, welche der theoretisch festgestellten Effekte dominieren und für die Wirkungen wesentlich sind. In diesem Sinne ist die Empirische Allgemeine Gleichgewichtsanalyse als ergänzendes Hilfsmittel der reinen analytischen Theorie zu sehen.

Im Hinblick auf die Diskussion über das bundesdeutsche Finanzausgleichssystem sei davor gewarnt, die ausgewiesenen Resultate als Fakten zu verwenden. Die produzierten Ergebnisse sind keine auf Heller und Pfennig exakten Berechnungen der tatsächlich auftretenden Wohlfahrtswirkungen. Soweit sie robust gegenüber Modellvariationen oder $\mathrm{Pa}$ rameteränderungen sind, ist lediglich zu vermuten, daß Richtung und Größenordnung der Effekte nicht aus der Luft gegriffen sind und in etwa zutreffen. Dies mag wohl die an politischen Handlungsanweisungen Interessierten enttäuschen, liegt aber im Wesen der verwendeten Analysetechnik.

Einige der neueren Vorschläge zur Neugestaltung des bundesdeutschen Finanzausgleichssystems, wie z.B. HombURG [1993] oder RICHTER/WELlisch [1993], wurden in dieser Arbeit nicht berücksichtigt. Dies ist in der Art der Vorschläge begründet, für die eine statische Untersuchung nicht adäquat ist. Vielmehr sollten sie einer dynamischen Analyse unterworfen werden, ebenso wie eine genauere Betrachtung der Auswirkungen regionaler Steuerautonomie in Form von Zuschlägen auf die Körperschaftssteuer. Dynamische EAG-Analysen könnten einen Beitrag leisten, die daraus entstehenden Wohlfahrtseffekte und Umverteilungswirkungen zu klären. Wenn bereits eine statische EAG-Analyse im Prinzip nur von einem Team durchführbar ist, so ist eine dynamische Empirische Allgemeine Gleichgewichtsanalyse erst recht nur von einer Gruppe von Wissenschaftlern zu bewältigen. Dies könnte eine der Herausforderungen auf der weiteren Suche nach optimalen Finanzausgleichssystemen darstellen. 


\section{Abkürzungsverzeichnis}

$\begin{array}{ll}\text { Bd } & \text { Bund } \\ \text { BE } & \text { Berlin(West) } \\ \text { BEZ } & \text { Bundesergänzungszuweisungen } \\ \text { BSP } & \text { Bruttosozialprodukt } \\ \text { BPW } & \text { Bruttoproduktionswert } \\ \text { BW } & \text { Baden-Württemberg } \\ \text { BY } & \text { Bayern } \\ \text { CES } & \text { Constant Elasticity of Substitution } \\ \text { cif } & \text { cost, insurance and freight } \\ \text { DIW } & \text { Deutsches Institut für Wirtschaftsforschung } \\ \text { fob } & \text { free on board } \\ \text { FRT } & \text { interregionale Grenzrate } \\ \text { FZ } & \text { Finanzzuweisungen } \\ \text { EAG } & \text { Empirische Allgemeine Gleichgewichts[-analyse] } \\ \text { ENF } & \text { Endnachfrage } \\ \text { EX } & \text { Exporte } \\ \text { FAG } & \text { Finanzausgleichsgesetz } \\ \text { FKPG } & \text { Gesetz zur Umsetzung des Föderalen Konsolidierungsprogramms } \\ \text { GE } & \text { Gesamteffekt } \\ \text { GG } & \text { Grundgesetz } \\ \text { Gm } & \text { Gemeinden } \\ \text { GH } & \text { Generalhandelskonzept } \\ \text { HB } & \text { Bremen } \\ \text { HE } & \text { Hessen } \\ \text { HEV } & \text { Hicksche Äquivalente Variation } \\ \text { HH } & \text { Hamburg } \\ \text { i.d.R. } & \text { in der Regel } \\ \text { IM } & \text { Importe } \\ \text { I/O } & \text { Input-Output } \\ \text { IOT } & \text { Input-Output-Tabelle } \\ \text { Ld } & \text { Länder } \\ \text { LES } & \text { Linear Expenditure System } \\ \text { LFA } & \text { Länderfinanzausgleich } \\ & \\ \text { HE }\end{array}$


MODOP Modifiziertes RAS-Verfahren

MRS Grenzrate der Substitution

MRT Grenzrate der Transformation

MRTS Grenzrate der technischen Substitution

NRW Nordrhein-Westfalen

NS Niedersachsen

NSP Nettosozialprodukt

NW Nordrhein-Westfalen

OL Ostländer

RP Rheinland-Pfalz

SA Saarland

SE Substitutionseffekt

SH Schleswig-Holstein oder Spezialhandelskonzept

StandOG Standortsicherungsgesetz

STABA Statistisches Bundesamt

StV Steuerverteilung

RvD Rest von Deutschland

ToT Terms of Trade

u.a.O. und andere Orte

u.d.. unter der Nebenbedingung

VGR Volkswirtschaftliche Gesamtrechnungen 


\section{Verzeichnis der Variablen}

\section{Variablen des FA-Systems in Kapitel I}

As Freibetrag der Einkommenssteuer in der Region $s$.

$E A_{s}^{C} \quad$ Ergänzungsanteile an das Land $s$ aus der Umsatzsteuerverteilung.

$E A_{s}^{C, 0} \quad$ Ergänzungsanteile an das Land $s$ aus der Umsatzsteuerverteilung, erste Näherung.

$E A_{s}^{E W}$ Mindestanteil an den Ergänzungsanteilen, die dem Land $s$ aufgrund seiner Bevölkerungsrelation zusteht.

$E A_{s}^{M A} \quad$ Mindestauffüllquote aus den Ergänzungsanteilen, die dem Land $s$ aufgrund seiner Finanzkraft zusteht.

$E W_{s} \quad$ Zahl der Einwohner der Region $s$.

$E W_{G m, s}^{F A}$ Im LFA angerechnete Bevölkerungsgröße der Gemeinden des Landes $s$.

$E W_{L d, s}^{F A}$ Im LFA berücksichtigte Einwohnerzahl des Landes $s$.

$E Z_{s} \quad$ Bundesergänzungszuweisungen (BEZ).

$E Z_{s}^{F} \quad$ Fixe Bundesergänzungszuweisungen.

$F A_{s} \quad$ Endgültige LFA-Zuweisungen.

$F A_{s}^{0} \quad$ LFA-Zuweisungen nach dem linearen Umverteilungstarif.

$F A Z_{s} \quad$ Länderfinanzausgleichszuweisungen - ohne Garantieklausel - an das Land $s$.

$F B_{s} \quad$ LFA-Beiträge.

$F B_{s}^{1} \quad$ LFA-Beiträge; erste Näherung.

$F B_{s}^{2} \quad$ LFA-Beiträge; zweite Näherung.

$F B_{s}^{3} \quad$ LFA-Beiträge; dritte Näherung.

$F B_{s}^{3} \quad$ LFA-Beiträge; vierte Näherung.

$F B E_{s}^{1} \quad$ Beiträge der finanzstarken Länder zum Fehlbetragsausgeleich (im LFA nach 1995).

$F E_{s} \quad$ Fehlbetrag zur garantierten Finanzkraft im LFA.

$F E_{s}^{1} \quad$ Fehlbetrag der finanzstarken Länder im LFA; erste Garantieklausel.

$F E_{s}^{2} \quad$ Fehlbetrag der finanzstarken Länder im LFA; zweite Garantieklausel.

$F E_{s}^{3} \quad$ Fehlbetragsausgleich an die finanzstarken Länder im LFA (ab 1995).

$F P_{s} \quad$ Finanzpflichtige Finanzkraft im LFA.

$F P_{s}^{1} \quad$ LFA vor 1995: Finanzpflichtige Finanzkraft im LFA bei $\pi_{0}^{m i t}$ und $\pi_{0}^{m i n}$.

LFA nach 1995: Maximale Finanzpflichtigkeit des Landes $s$.

$F P_{s}^{2} \quad$ Finanzpflichtige Finanzkraft im LFA bei $\pi^{m i t}=1$ und $\pi_{0}^{m i n}$. 
$F Z_{s} \quad$ LFA-Zuweisungen.

$F Z_{s}^{1} \quad$ LFA-Zuweisungen an das Land $s\left(=F A Z_{s}+F Z Z_{s}\right)$; erste Näherung.

$F Z_{s}^{2} \quad$ LFA-Zuweisungen an das Land $s\left(=F A Z_{s}+F Z Z_{s}\right)$; zweite Näherung.

$F Z_{s}^{3} \quad$ LFA-Zuweisungen an das Land $s\left(=F A Z_{s}+F Z Z_{s}\right)$; dritte Näherung.

$F Z Z_{s} \quad$ Zusätzliche LFA-Zuweisungen an das Land $s$ aufgrund der Garantieklausel.

FT Fiktive Steueraufkommen, die in der Berechnung der Finanzkraft verwendet werden.

$F T_{s}^{G r} \quad$ Fiktive Grundsteuereinnahmen der Gemeinden des Landes $s$.

$F T_{s}^{G w} \quad$ Fiktive Gewerbesteuereinnahmen der Gemeinden des Landes $s$.

$G_{i}^{s} \quad$ Regionale Nachfrage nach dem öffentlichen Gut $i$.

$h_{s}^{G r} \quad$ Durchschnittlicher regionaler Grundsteuerhebesatz.

$h_{s}^{G w} \quad$ Durchschnittlicher regionaler Gewerbesteuerhebesatz.

$h^{U m} \quad$ Vervielfältiger der Gewerbesteuereinnahmen der Gemeinden (LFA).

I Menge der verschiedenen öffentlichen Güter.

$K_{s} \quad$ Menge des Kapitalangebotes der Region $s$.

$L_{s} \quad$ Menge des Arbeitsangebotes der Region $s$.

$M A_{s} \quad$ Ausgleichsmeßzahl des Landes $s$ (regionale Ausgleichsmeßzahl).

$M A_{G m, s}$ Ausgleichsmeßzahl der Gemeindesn des Landes $s$.

$M A_{L d, s}$ Ausgleichsmeßzahl des Landes $s$.

$M F_{s} \quad$ Finanzkraftmeßzahl des Landes $s$ (regionale Finanzkraftmeßzahl.)

$M F_{L d, s} \quad$ Finanzkraftmeßzahl des Landes $s$.

$M F_{G m, s}$ Finanzkraftmeßzahl der Gemeinden des Landes $s$.

$\bar{q}_{i} \quad$ Durchschnittlicher Preisindex der öffentlichen Güterart $i$

$q_{i}^{s} \quad$ Preis der öffentlichen Güterart $i$ in der Region $s$

$\mathcal{R} \quad$ Menge aller Bundesländer.

$\mathcal{R}^{+} \quad$ Menge der finanzstarken Länder.

$\mathcal{R}_{1}^{+} \quad$ Menge der am wenigsten finanzstarken Länder (im LFA).

$\mathcal{R}_{2}^{+} \quad$ Menge der mittleren finanzstarken Länder (im LFA).

$\mathcal{R}_{3}^{+} \quad$ Menge der finanzstärksten Länder (im LFA).

$\mathcal{R}_{+}^{+} \quad$ Finanzstärkere Länder aus der Menge der finanzstarken Länder.

$\mathcal{R}_{-}^{+} \quad$ Finanzschwächere Länder aus der Menge der finanzstarken Länder.

$\mathcal{R}_{+}^{+,} \quad$ Finanzstarke Länder ohne Fehlbetrag; zweite Garantieklausel.

$\mathcal{R}_{+}^{+,-} \quad$ Finanzstarke Länder ohne Fehlbetrag; zweite Garantieklausel.

$\mathcal{S}_{+}^{+,-} \quad$ Finanzstarke Länder mit Fehlbetrag; zweite Garantieklausel.

$\mathcal{R}^{-} \quad$ Menge der finanzschwachen Westländer.

$\mathcal{R}_{-}^{-} \quad$ In der Umsatzsteuerverteilung: Länder, deren Mindestauffüllquote größer als deren Mindestanteile sind. 
Im LFA: Finanzschwache Länder, deren Finanzkraft in der Garantieklausel zusätzlich aufgebessert wird.

$\mathcal{R}_{+}^{-} \quad$ In der Umsatzsteuerverteilung: Länder, deren Mindestauffüllquote kleiner als deren Mindestanteile sind.

Im LFA: Finanzschwache Länder, für die die Garantieklausel nicht relevant wird.

$r \quad$ Einheitlicher Kapitallohnsatz.

$R E_{s}^{C} \quad$ Verteilung der Restbeträge an das Land $s$ nach Bevölkerung (Umsatzsteuerverteilung).

$R G_{s}^{C} \quad$ Verteilung der Restbeträge an das Land $s$ gemäß der Garantieklausel (Umsatzsteuerverteilung).

$R V_{s}^{C} \quad$ Gesamte Einnahmen des Landes $s$ aus der Verteilung der Restbeträge (Umsatzsteuerverteilung).

$\mathcal{S} \quad$ Menge aller Westländer.

$\mathcal{S}^{+} \quad$ Menge der finanzstarken Westländer.

$\mathcal{S}_{1}^{+} \quad$ Menge der am wenigsten finanzstarken Länder (im LFA).

$\mathcal{S}_{2}^{+} \quad$ Menge der mittleren finanzstarken Länder (im LFA).

$\mathcal{S}_{3}^{+} \quad$ Menge der finanzstärksten Länder (im LFA).

$\mathcal{S}_{+}^{+} \quad$ Finanzstärkere Länder aus der Menge der finanzstarken Länder.

$\mathcal{S}_{-}^{+} \quad$ Finanzschwächere Länder aus der Menge der finanzstarken Länder.

$\mathcal{S}_{+}^{+,+} \quad$ Finanzstarke Länder ohne Fehlbetrag; zweite Garantieklausel.

$\mathcal{S}_{+}^{+,-} \quad$ Finanzstarke Länder ohne Fehlbetrag; zweite Garantieklausel.

$\mathcal{S}_{+}^{+,-} \quad$ Finanzstarke Länder mit Fehlbetrag; zweite Garantieklausel.

$\mathcal{S}^{-} \quad$ Menge der finanzschwachen Westländer.

$\mathcal{S}_{-}^{-} \quad$ In der Umsatzsteuerverteilung: Länder, deren Mindestauffüllquote größer als deren Mindestanteile sind.

Im LFA: Finanzschwache Länder, deren Finanzkraft in der Garantieklausel zusätzlich aufgebessert wird.

$\mathcal{S}_{+}^{-} \quad$ In der Umsatzsteuerverteilung: Länder, deren Mindestauffüllquote kleiner als deren Mindestanteile sind.

Im LFA: Finanzschwache Länder, für die die Garantieklausel nicht relevant wird.

$\mathcal{S}=\quad$ Gruppe der finanzschwachen Länder, die zusätzliche Finanzzuweisungen im LFA gemäß der Garantieklausel für die finanzschwachen Länder erhalten.

$\mathcal{S}^{\mp} \quad$ Gruppe der finanzschwachen Länder, die keine zusätzlichen Finanzzuweisungen im LFA erhalten. 
$S B_{s} \quad$ Sonderlasten des Landes $s$, die in der Berechnung der Finanzkraftmeßzal berücksichtigt werden.

ST Steueraufkommen der Regionen.

$S T^{C} \quad$ Gesamte Aufkommen aus den Umsatzsteuern.

$S T_{s}^{C} \quad$ Gesamte Aufkommen des Landes $s$ aus den Umsatzsteuern.

$S T^{E k} \quad$ Gesamte Aufkommen aus den Lohn- und veranlagten Einkommenssteuern.

$S T_{s}^{E k, Z}$ Gesamte Aufkommen des Landes $s$ aus den Lohn- und veranlagten Einkommenssteuern (ohne Zuschläge).

$S T_{s}^{E k, Z}$ Gesamte Aufkommen des Landes $s$ aus den Lohn- und veranlagten Einkommenssteuern, incl. den Einnahmen aus den regionalen Einkommenssteuerzuschlägen.

$S T_{s}^{G w} \quad$ Gewerbesteueraufkommen der Gemeinden der Region s.

$\widehat{S T}_{s}^{G w} \quad$ Steuermeßbetrag der regionalen Gewerbesteuern.

$S T^{K} \quad$ Gesamte Aufkommen aus den Körperschafts- und Kapitalertragssteuern (Kapitalsteuern).

$S T_{s}^{K} \quad$ Aufkommen des Landes $s$ aus den Körperschafts- und Kapitalertragssteuern (Kapitalsteuern).

$S T^{U m} \quad$ Summe der Gewerbesteuerumlagen.

$S T_{s}^{U m} \quad$ Gewerbesteuerumlage der Gemeinden der Region s.

$T \quad$ Steuereinnahmen nach der Steuerverteilung.

$T_{B d}^{C} \quad$ Anteil des Bundes an den Umsatzsteuern.

$\hat{T}_{L d}^{C} \quad$ Anteil der Länder an den Umsatzsteuern.

$T_{L d}^{C} \quad$ Anteil der Westländer an den Umsatzsteuern.

$T_{s}^{C} \quad$ Einnahmen des Landes $s$ aus den Umsatzsteuern.

$T_{L d, s}^{F} A$ Einnahmen des Landes $s$, die in der Berechnung der Finanzkraftmeßzahl berücksichtigt werden. (nach Abzug der Sonderbedarfe

$T_{L d, s}^{F} \quad$ Einnahmen des Landes $s$, die in der Berechnung der Finanzkraftmeßzahl berücksichtigt werden. (ohne Berücksichtigung der Sonderbedarfe).

$T_{G m, s}^{F} \quad$ Einnahmen der Gemeinden des Landes $s$, die in der Berechnung der Finanzkraftmeßzahl berücksichtigt werden.

$T_{G m, s}^{G w} \quad$ Einnahmen der Gemeinden aus der Gewerbesteuer.

$T_{s}^{G r} \quad$ Einnahmen der Gemeinden des Landes $s$ aus den Grundsteuern.

$T_{s}^{G w} \quad$ Gesamte Einnahmen der Gebietskörperschaften des Landes $s$ (regionale Einnahmen) aus der Gewerbesteuer.

$T_{L d}^{U m} \quad$ Anteil der Länder an der Gewerbesteuerumlage.

$T_{s}^{R E G} \quad$ Einnahmen des Landes $s$ aus den Ländersteuern. 
$T_{s}^{U m} \quad$ Einnahmen des Landes $s$ aus der Gewerbesteuerumlage.

$T_{G m}^{Y} \quad$ Gemeindeanteil an den Einkommenssteuern.

$T_{L d}^{Y} \quad$ Länderanteil an den Einkommenssteuern.

$T_{G m, s}^{Y} \quad$ Einnahmen des Landes $s$ aus allen Einkommenssteuerarten.

$T_{L d, s}^{Y} \quad$ Einnahmen des Landes $s$ aus allen Einkommenssteuerarten.

$T_{s}^{Y} \quad$ Gesamte Einnahmen der Gebietskörperschaften des Landes $s$ (regionale Einnahmen) aus allen Einkommenssteuerarten.

$t_{L d, s}^{1} \quad$ Pro-Kopf-Einnahmen des Landes $s$ aus den Ländersteuern, der Gewerbesteuerumlage und den Einkommenssteuern.

$\bar{t}_{L d}^{1} \quad$ Durchschnittliche Pro-Kopf-Einnahmen der Länder aus den Ländersteuern, der Gewerbesteuerumlage und den Einkommenssteuern.

$t_{L d, s}^{2} \quad$ Pro-Kopf-Einnahmen des Landes $s$ vor der Umsatzsteuerverteilung zuzüglich der Umsatzsteueranteile (vor der Garantieklausel).

$\bar{t}_{L d, s}^{2} \quad$ Durchschnittliche Pro-Kopf-Einnahmen der Länder vor der Umsatzsteuerverteilung zuzüglich der Umsatzsteueranteile (vor der Garantieklausel).

$t_{L d, s}^{3} \quad$ Pro-Kopf-Finanzkraft der finanzstarken Länder incl. Finanzbeiträge im LFA.

$t_{L d, s}^{3} \quad$ Durchschnittliche Pro-Kopf-Finanzkraft der finanzstarken Länder incl. Finanzbeiträge im LFA.

$V A_{s}^{C} \quad$ Einnahmen des Landes $s$ aus der Vorabverteilung der Umsatzsteuern.

$w_{s} \quad$ Faktorlohnsatz für Arbeit.

$Y_{s} \quad$ Nettosozialprodukt zu Faktorkosten.

$Z A^{C} \quad$ Zusätzlicher Beitrag der Westländer aus ihrem Umsatzsteueranteil für den Fonds 'Deutsche Einheit'.

$Z A_{s}^{C} \quad$ Zusätzlicher Beitrag des Landes $s$ aus seinem Umsatzsteueranteil zum Fonds 'Deutsche Einheit'.

$\alpha_{s}^{F} \quad$ Korrekturfaktor zur Berechnung der realen Finanzkraft.

$\kappa_{p}^{C} \quad$ Proportionaler Kürzungsfaktor in der Berechnung der Ergänzungsanteile (Umsatzsteuerverteilung).

$\kappa_{\mathcal{R}, F A}^{L d, s} \quad$ Bevölkerungsanteil des Landes $s$ an der Gesamtbevölkerung (im LFA).

$\kappa_{\mathcal{R}, F A}^{G m, s}$ Bevölkerungsanteil der Gemeinden des Landes $s$ an der Gesamtbevölkerung (im LFA).

$\kappa_{\mathcal{R}}^{s} \quad$ Bevölkerungsanteil des Landes $s$ an der Bevölkerung aller Länder aus der Menge $\mathcal{R}$.

$\kappa_{\mathcal{S}}^{s} \quad$ Bevölkerungsanteil des Landes $s$ an der Bevölkerung der Länder aus der Menge $\mathcal{S}$.

$\bar{\lambda}_{i} \quad$ Durchschnittlicher Anteil der Ausgaben für das öffentliche Gut vom Typ $i$ an den Gesamtausgaben für öffentliche Güter, über alle Länder. 
$\lambda_{i}^{s} \quad$ Gewichtete Anteile der öffentlichen Ausgaben für das öffentliche Gut vom Typ $i$ an den regionalen Gesamtausgaben.

$\pi^{1} \quad$ Anrechnungssatz der Finanzkraft bei der Ermittlung der Finanzpflichtigkeit (oberstes Segment).

$\pi^{2} \quad$ Anrechnungssatz der Finanzkraft bei der Ermittlung der Finanzpflichtigkeit (mittleres Segment).

$\pi_{L d}^{C} \quad$ Anteilssatz der Länder an den Umsatzsteuern.

$\pi_{L d}^{F} \quad$ Anteil der finanzstarken Länder an den LFA-Zuweisungen an die finanzschwachen Länder.

$\pi_{V A}^{C} \quad$ Anteilssatz zur Berechnung des Anteils der Umsatzsteuern, der in der Vorabverteilung verteilt wird.

$\pi_{E A}^{C} \quad$ Mindestgarantiesatz in der Verteilung der Ergänzungsanteile.

$\pi_{G m}^{E k} \quad$ Anteilssatz der Gemeinden an den Lohn- und veranlagten Einkommenssteuern.

$\pi^{E Z} \quad$ Anteil der Ergänzungsanteile am Bundesanteil der Umsatzsteuer.

$\pi_{s}^{E Z} \quad$ Verteilungssatz der Ergänzungsanteile an das Land $s$.

$\pi_{v}^{E Z} \quad$ Mindestauffüllungssatz durch die Ergänzungszuweisungen.

$\pi_{L d, s}^{E W} \quad$ Veredelungsfaktor der Bevölkerungszahl des Landes $s$.

$\pi_{G m, s}^{E W} \quad$ Veredelungsfaktor der Bevölkerungszahlen der Gemeinden des Landes $s$.

$\pi_{G m}^{F A} \quad$ Anrechnungsfaktor der Gemeindeeinnahmen bei der Berechnung der regionalen Finanzkraft.

$\pi^{F} \quad$ Ausgleichssatz der Finanzkraft im linearen LFA-Tarif.

$\pi_{h}^{G r} \quad$ Vervielfältiger der Grundsteuereinnahmen der Gemeinden (LFA).

$\pi^{M} \quad$ Prozentsatz der durchschnittlichen Finanzkraft der im LFA ausgeglichen wird (vor der Garantieklausel).

$\pi^{\max } \quad$ Anrechnungsfaktor der Finanzkraft (bei starker Überschreitung der durchschnittlichen Finanzkraft) bei der Ermittlung der Finanzpflichtigkeit im LFA.

$\pi_{0}^{\max } \quad$ Vorgegebener Anrechnungsfaktor der Finanzkraft (bei starker Überschreitung der durchschnittlichen Finanzkraft) bei der Ermittlung der Finanzpflichtigkeit im LFA.

$\pi^{m i t} \quad$ Anrechnungsfaktor der Finanzkraft (im mittleren Segment der finanzstarken Länder) bei der Ermittlung der Finanzplichtigkeit im LFA.

$\pi_{0}^{m i t} \quad$ Vorgegebener Anrechnungsfaktor der Finanzkraft (im mittleren Segment der finanzstarken Länder) bei der Ermittlung der Finanzpflichtigkeit im LFA.

$\pi^{\min } \quad$ Anrechnungsfaktor der Finanzkraft (im untersten Segment der finanzstarken Länder) bei der Ermittlung der Finanzpflichtigkeit im LFA.

$\pi_{0}^{\min } \quad$ Vorgegebener Anrechnungsfaktor der Finanzkraft (im untersten Segment der finanzstarken Länder) bei der Ermittlung der Finanzpflichtigkeit im LFA. 
$\pi^{N} \quad$ Ausgleichssatz der finanzstärkeren der finanzschwachen Länder im LFA.

$\pi^{S} \quad$ Im LFA den finanzschwachen Ländern garantierter Anteil an der durchschnittlichen Finanzkraft.

$\pi_{h}^{U m} \quad$ Vervielfältiger der Gewerbesteuerumlage.

$\pi_{L d}^{U m} \quad$ Anteilssatz der Länder an der Gewerbesteuerumlage.

$\pi^{Y} \quad$ Höchstsatz für die Summe aus nationalem und regionalem Einkommenssteuerzuschlag.

$\pi_{L d}^{Y} \quad$ Anteilssatz der Länder an den Einkommenssteuern.

$\theta_{\mathcal{R}}^{s} \quad$ Gewichtungfaktor des Preisindex für die öffentlichen Leistungen im Vorschlag von FöTTINGER/SPAHN.

$\tau^{Y} \quad$ Nationaler Grenzsteuersatz der Einkommenssteuer.

$\tau_{B d}^{Y} \quad$ Nationaler Zuschlagssatz zum Grenzsteuersatz der Einkommenssteuer.

$\tau_{L d, s}^{Y} \quad$ Regionaler Zuschlagssatz zum Grenzsteuersatz der Einkommenssteuer.

\section{Variablen des EAG-Modells in Kapitel III}

A Inputkoeffizientenmatrix.

$\hat{a}_{j}^{s} \quad$ Anteil der aggregierten Vorleistungsfunktion am Output des Sektors $j$ der Region $s$.

$a_{C j}^{r s} \quad$ Inputkoeffizient des Gutes $C$ aus der Region $r$ in der der Produktion des Sektors $j$ in der Region $s$.

$a_{G j}^{s s} \quad$ Inputkoeffizient des regionalen öffentlichen Gutes in der Produktion des Sektors $j$ in der Region $s$.

$a_{0 j}^{s} \quad$ Anteil der Wertschöpfung am Output des Gutes $j$ der Region $s$.

$a_{s}^{E} \quad$ Anteil des Wertes der Zeitgrundausstattung am möglichen Einkommen.

$a_{s}^{F} \quad$ Anteil der Freizeitausgaben am möglichen Einkommen.

$A_{l l} \quad$ Freibetrag der Einkommenssteuer in der Region $s$.

C Vektor der Konsumnachfrage.

$C_{s} \quad$ Nachfrage des repräsentativen Haushalts der Region $s$ nach dem aggregierten privaten Konsumgut.

$C_{r s} \quad$ Konsumnachfrage in der Region $s$ nach Produkten aus der Region $r$.

$d_{r s} \quad$ Distanz zwischen den Regionen (in der Gravitationsgleichung).

$d_{h, s} \quad$ Opportunitätskosten der Wanderung des Individuums $h$ aus der Region $s$.

$d_{j}^{r s} \quad$ Anteilsparameter der Vorleistungseinsatzes des Gutes $C$ aus der Region $r$ in der Produktion des Sektors $j$ in der Region $s$.

e Wechselkurs.

E Einheitsvektor. 
$\mathbf{E}^{*} \quad$ Einheitsmatrix, von der in der Diagonalen die Terme $\left[1 /\left(1+\tau_{j s}^{Q}\right)\right]$ abgezogen werden.

$E_{s} \quad$ Zeitgrundausstattung in der Region $s$.

EX Exporte.

$E Z_{s} \quad$ Einnahmen der Region $s$ aus Ergänzungszuweisungen.

$F \quad$ "Fremde" Regionen.

$F_{s} \quad$ Nachfrage des repräsentativen Haushalts der Region $s$ nach Freizeit.

$f_{j}^{s}(\cdot) \quad$ Wertschöpfungsfunktion des Sektors $j$ in der Region $s$.

$F A_{s} \quad$ Einnahmen der Region $s$ aus dem LFA.

$\overline{f k} \quad$ Durchschnittliche Pro-Kopf-Finanzkraft aller Länder.

$f k_{s} \quad$ Pro-Kopf-Finanzkraft des Landes $s$.

G Vektor der Nachfragen nach den regionalen öffentlichen Gütern.

$g \quad$ Gini-Koeffizient.

$G_{s} \quad$ Nachfrage des repräsentativen Haushalts der Region $s$ nach dem regionalen öffentlichen Gut.

$H B_{s} \quad$ Handelsbilanz der Region $s$.

$\bar{K}_{s} \quad$ Kapitalangebot der Region $s$.

$K_{j}^{s} \quad$ Einsatz von Kapital in der Produktion des Gutes $j$ der Region $s$.

$k_{j}^{s} \quad$ Einsatz von Kapital pro Einheit Output des Gutes $j$ der Region $s$.

$K B_{s} \quad$ Kapitalbilanz der Region $s$.

$L_{s} \quad$ Arbeitsangebot der Region $s$.

$L_{j}^{s} \quad$ Einsatz von Arbeit in der Produktion des Gutes $j$ der Region $s$.

$l_{j}^{s}$

IM Importe

M Importvektor.

$M_{s} \quad$ Importe der Region $s$.

$m_{G} \quad$ Multiplikator der sozialen Grenzkosten der öffentlichen Güter $m_{G_{s}}=(1-$ $\left.\delta_{G_{s}} / q_{G_{s}}\right)$.

$p_{r} \quad$ Konsumentenpreis des Gutes aus der Region $r$ in allen anderen Regionen.

$p_{C} \quad$ Preisindex des aggregierten privaten Konsumgutes.

$p_{U_{s}} \quad$ Preisindex des aggregierten privaten Gutes (Konsum und Freizeit).

Qs Gesamtproduktion einer Region $s$.

$Q_{j}^{s} \quad$ Outputmenge des Sektors $j$ der Region $s$.

Q Vektor der Produktionsmengen.

q Vektor der Produzentenpreise.

$q_{j}^{s} \quad$ Produzentenpreis des Gutes $j$ aus der Region $s$.

$q_{r} \quad$ Produzentenpreis des privaten Gutes $C$ aus Region $r$.

$r \quad$ (Netto-)kapitallohnsatz (national einheitlich). 
$\hat{r}_{j}^{s} \quad$ Bruttokapitalentlohnung im Sektor $j$ der Region $s$ (z.B. $r=r\left(1+\tau_{j s}^{K}+\tau_{j s}^{G w}+\right.$ $\left.\tau_{j s}^{G r}\right)$.

$S T_{s} \quad$ Gesamte Steueraufkommen der Region $s$.

$S T_{s}^{C} \quad$ Konsumsteueraufkommen der Region $s$.

$S T_{s}^{E k} \quad$ Einkommenssteuerschuld der privaten Haushalts der Region $s$.

$S T_{s}^{G \tau} \quad$ Grundsteueraufkommen der Region $s$.

$S T_{s}^{G w} \quad$ Gewerbesteueraufkommen der Region $s$.

$S T_{s}^{K} \quad$ Kapitalsteueraufkommen der Region $s$.

$S T_{s}^{Q} \quad$ Produktionssteueraufkommen der Region $s$.

$S T_{j s}^{Q} \quad$ Produktionssteuerschuld des Sektors $j$ der Region $s$.

$T_{G}^{C} \quad$ Konsumsteuereinnahmen der nationalen Regierung.

$T_{G}^{Q} \quad$ Produktionssteuereinnahmen der nationalen Regierung.

$T_{G}^{Y} \quad$ Steuereinnahmen der nationalen Regierung aus allen Einkommenssteuerarten.

$T_{G}^{U m} \quad$ Einnahmen der nationalen Regierung aus der Gewerbesteuerumlage.

$T_{s} \quad$ Gesamte Steuereinnahmen der Region $s$ nach der Steuerverteilung.

$T_{s}^{C} \quad$ Regionale Einnahmen aus den Konsumsteuern.

$T_{G m, s}^{G w} \quad$ Einnahmen der Gemeinden der Region $s$ aus den Gewerbesteuern.

$T_{s}^{G \tau} \quad$ Regionale Einnahmen aus den Grundsteuern.

$T_{s}^{Q} \quad$ Regionale Einnahmen aus den Produktionssteuern.

$T_{s}^{U m} \quad$ Regionale Einnahmen aus der Gewerbesteuerumlage.

$T_{s}^{Y} \quad$ Regionale Einnahmen aus allen Einkommenssteuerarten.

$T_{s}^{V} \quad$ Regionale Aufkommen und Einnahmen aus den Vermögenssteuern.

$\mathrm{Tr}_{s} \quad$ Regionale Transfers an den repräsentativen Haushalt der Region $s$.

$T R_{s} \quad$ Transfers der nationalen Regierung an den repräsentativen Haushalt der Region $s$.

$U_{s} \quad$ Nachfrage des repräsentativen Haushalts der Region $s$ nach den privaten aggregierten Gut.

Nutzenfunktion über die privaten Güter $C$ und $F$.

$U B_{s} \quad$ Übertragungsbilanz der Region $s$.

$\bar{V}_{j}^{s} \quad$ Einsatz des aggregiertes Vorleistungsgutes in der Produktion des Gutes $j$ in der Region $s$.

Aggregierte Vorleistungsfunktion des Sektors $j$ der Region $s$.

$V_{G j}^{s s} \quad$ Vorleistungseinsatz des regionalen öffentlichen Gutes in der Produktion des Gutes $j$ in der Region $s$.

$V_{C j}^{r s} \quad$ Vorleistungseinsatz des Gutes $C$ aus der Region $r$ in der Produktion des Gutes $j$ in der Region $s$.

$w_{s} \quad$ Arbeitslohnsatz in der Region $s$. 
$w_{s}^{n} \quad$ Nettoarbeitslohnsatz in der Region $s: w_{s}=w_{\boldsymbol{s}}\left(1-\tau^{Y}\right)$.

X Vektor der Exporte.

$X_{s} \quad$ Exporte der Region $s$.

$X_{i}^{r s} \quad$ Lieferungen des Gutes $i$ aus der Region $r$ in die Region $s$.

$Y_{s} \quad$ Volles Einkommen der Region $s$.

$Y_{s}^{R E G} \quad$ Regionales öffentliches Budget.

$Y_{s}^{p r} \quad$ Privates mögliches Einkommen des repräsentativen Haushalts der Region $s$.

$Y_{s}^{C} \quad$ Privates Geldeinkommen in der Region $s$.

$Y_{s}^{h} \quad$ Einkommen des Individuums $\mathrm{h}$.

$Y_{s}^{U} \quad$ Mögliches privates Einkommen, das zum Konsum von U zur Verfügung steht.

$Z_{s} \quad$ Einnahmen der Region $s$ aus Zuweisungen.

$z_{B} \quad$ Überschußnachfrage der nationalen Regierung.

$z_{K} \quad$ Überschußnachfrage nach Kapital.

$z_{L}^{s} \quad$ Überschußnachfrage nach Arbeit.

$z_{R}^{s} \quad$ Überschußnachfrage der regionalen Regierung.

$\alpha_{s} \quad$ Lagrangemultiplikator: Grenznutzen des privaten Einkommens.

$\alpha_{i} \quad$ Gravitationskoeffizient.

$\beta_{i} \quad$ Korrekturgröße der Gravitationsgleichung.

$\beta_{1 s} \quad$ Anteilsparameter des öffentlichen Gutes am regionalen Gesamtnutzen.

$\beta_{2 s} \quad$ Anteilsparameter von Freizeit am Nutzen aus aggregierten privaten Gut in Region $s$.

$\beta_{r s} \quad$ Anteilsparamter der Konsumgüter aus der Region $r$ am zusammengesetzten Konsumgut der Region $s$.

$\delta_{j}^{s} \quad$ Anteilsparameter des Arbeitseinsatzes in der Wertschöpfungsfunktion im Sektor $j$ der Region $s$.

$\eta \quad$ Ausländische Importangebotselastizität.

$\epsilon_{s} \quad$ Unkompensierte Arbeitsangebotselastizität.

$\epsilon_{s}^{F} \quad$ Lohnelastizität der Freizeitnachfrage.

$\varepsilon_{r s} \quad$ Preiselastizität der Bezugsnachfrage der Region $s$ nach dem privaten Konsumgut aus der Region $r$.

$\lambda \quad$ Lagrangemultiplikator: Grenznutzen des öffentlichen Einkommens.

$\lambda_{1, s} \quad$ Parameter der Migrationsentscheidung.

$\lambda_{2,9} \quad$ Parameter der Migrationsentscheidung.

$\mu_{1 s} \quad$ Substitutionselastizität zwischen dem regionalen öffentlichen Gut und dem aggregierten privaten Gut in der Region $s$.

$\mu_{2 s} \quad$ Substitutionselastizität zwischen dem aggregierten privaten Konsumgut und Freizeit in der Region $s$. 
$\mu_{3 s} \quad$ Substitutionselastizität der Region $s$ zwischen den einzelnen privaten Konsumgütern unterschiedlicher Herkunft.

$\nu_{s} \quad$ Indirekte Nutzenfunktion des repräsentativen Konsumenten der Region s.

$\psi_{j}^{s} \quad$ Technologiekoeffizient der Vorleistungsfunktion des Sektors $j$ in der Region $s$.

$\phi_{j}^{s} \quad$ Technologiekoeffizient der Wertschöpfungsfunktion des Sektors $j$ in der Region $s$.

$\rho_{j}^{s} \quad$ Substitutionselastizität zwischen den Vorleistungsgütern $C$ mit verschiedener Herkunft in der Produktion des Sektors $j$ in der Region $s$.

$\sigma_{1 j}^{s} \quad$ Substitutionselastizität zwischen Kapital und Arbeit im Sektor $j$ der Region $s$.

$\theta \quad$ Ausländische Exportnachfrageelastizität.

$\tau^{C} \quad$ Konsumsteuersatz.

$\tau_{j s}^{e K} \quad$ Effektiver Kapitalsteuersatz auf den Produzentenpreis des Gutes $j$ der Region $s$.

$\tilde{\tau}_{j s}^{e K} \quad$ Effektiver Kapitalsteuersatz auf den Nettoproduzentenpreis des Gutes $j$ der Region $s$.

$\tau_{j s}^{e Q} \quad$ Effektiver Produktionssteuersatz auf den Produzentenpreis des Gutes $j$ der Region $s$.

$\tilde{\tau}_{j s}^{e Q} \quad$ Effektiver Produktionssteuersatz auf den Nettoproduzentenpreis des Gutes $j$ der Region $s$.

$\tau_{j s}^{G r} \quad$ Grundsteuersatz im Sektor $j$ der Region $s$.

$\tau_{j s}^{G w} \quad$ Gewerbesteuersatz im Sektor $j$ der Region $s$.

$\tau_{j s}^{K} \quad$ Kapitalsteuersatz im Sektor $j$ der Region $s$.

$\tau_{j s}^{Q} \quad$ Produktionssteuersatz im Sektors $j$ der Region $s$ auf den Herstellungspreis.

$\tau^{Y} \quad$ Einkommenssteuersatz.

$\zeta \quad$ Verhältnis von Zeitgrundausstattung zu Arbeitszeit $(E / L)$.

$\Omega_{s} \quad$ Ausdrücke in den Nachfragefunktionen, die die Preise alternativer Güter enthalten. 
Georg Hirte - 978-3-631-75212-8

Downloaded from PubFactory at 01/11/2019 07:02:26AM

via free access 


\section{Literaturverzeichnis}

Adams, F.G.; N.J. Guickman [1980], Modeling the Multiregional Economic System: Perspectives of the Eighties, Lexington, Mass.

AнN, E. [1988], A General Equilibrium Model of Interjurisdictional Tax Competition, Dissertation, Ann Arbor.

Анцhеiм, M.; M. Rose [1989], Messung individueller Wohlfahrt, Heidelberg.

Armington, P.S. [1969], A Theory of Demand for Products: Distinguished by Place of Production, International Monetary Fund Staff Papers 16, S.156-167.

ARnotT, R.; R.E. Grieson [1981], Optimal Fiscal Policy for a State or Local Government, Journal of Urban Economics 9, S.23-48.

Atkinson, A.B.; J.E. Stiglitz [1980], Lectures on Public Economics, New York.

Auerbach, A.J. [1985], The Theory of Excess Burden and Optimal Taxation, in: A.J. Auerbach; M. Feldstein (Hrsg.), Handbook of Public Economics, Vol I, Amsterdam u.a.O., S.61-127.

BACHARACH, M. [1970], Biproportional Matrices and Input-Output-Change, Cambridge.

Ballard, Ch.L.; D. Fullerton; J.B. Shoven; J. Whalley [1985], A General Equilibrium Model for Tax Policy Evaluation, Chicago.

Bartsch, P.; H. Probst [1988], Ansätze zu einer Reform des Länderfinanzausgleichs, Wirtschaftsdienst 68/X, S.533-4.940.

BatTen, D.F.; D.E. Boyce [1986], Spatial Interaction, Transportation, and Interregional Commodity Flow Models, in: P. NiJkamp (Hrsg.), Handbook of Regional and Urban Economics, Volume I: Regional Economics, Amsterdam, New York u.a.O., S.357-406.

Bayerisches Landesamt für Statistik und Datenverarbeitung [1990], Statistisches Berichte P I 2 - j/88: Entstehung, Verwendung und Verteilung des Sozialprodukts in Bayern 1970 bis 1988, Berechnungsstand März 1989, München.

Bhagwati, J.N.; R.A. Brecher; T. Hatta [1983], The Generalized Theory of Transfers and Welfare: Bilateral Transfers in a Multilateral World, American Economic Review 73, S.606-618.

Boadway, R.W. [1974], The Welfare Functions of Cost-Benefit Analysis, Economic Journal 84, S.926-939.

Borges, A. [1986], Applied General Equilibrium Models: An Assessment ot their Usefulness for Policy Analysis, OECD Economic Studies 7, 7-43. 
Bös, D. [1971], Eine ökonomische Theorie des Finanzausgleichs, Wien, New York.

Bös, D. [1978], Zur Theorie des Finanzausgleichs, in: W. Dreissig (Hrsg.), Probleme des Finanzausgleichs I, (Schriften des Vereins für Socialpolitik, N.F. 96/I), Berlin, S.45-133.

Bös, D.; B. Genser [1977], Steuertariflehre, in: W. Albers (Hrsg.), Handwörterbuch der Wirtschaftswissenschaften, 7.Bd., Stuttgart u.a.O., S.412-427.

Bradford, D.; W.E. OAtes [1971a], The Analysis of Revenue Sharing in a New Approach to Collective Fiscal Decisions, Quaterly Journal of Economics 85, S.416-439.

Brennan, G.; J.M. Buchanan [1980], The Power to Tax. Analytical Foundations of a Fiscal Constitution, Cambridge.

Breuel, B. [1987], Neuordnung des Länderfinanzausgleichs: Gleichwertigkeit der Lebensverhältnisse nicht gefährden, Wirtschafsdienst 67, S.330-333.

Breuel, B. [1988], Der Vorschlag Niedersachsens, Wirtschaftsdienst 68, S.230-234.

Buchanan, J.M. [1952], Federal Grants and Resource Allocation, Journal of Political Economy 60, S.208-217.

BuHL, H.U. [1986], Ein finanzwissenschaftlich-methodischer Diskussionsbeitrag zur Neuordnung des Länderfinanzausgleichs, Finanzarchiv N.F. 44, S. 476-483.

Buhl, H.U.; A. Pfingsten [1986], Eigenschaften und Verfahren für einen angemessenen Länderfinanzausgleich in der Bundesrepublik Deutschland, Finanzarchiv N.F. 44, S.98-109.

Buhl, H.U.; A. Pfingsten [1990], On the Distribution of Public Funds, European Journal of Political Economy 6, S. 363-376.

Buhl, H.U.; A. Pfingsten [1991], Zehn Gebote für Finanzausgleichsverfahren und ihre Implikationen, Wirtschaftsdienst 71/IX, 481-484.

Bundesrat [1988], Entwurf eines Gesetzes zur Änderung des Bundessozialhiflegesetzes und des Gesetzes über den Finanzausgleich zwischen Bund und Ländern, Gesetzesantrag der Länder Bremen, Hamburg, Niedersachsen, Nordrhein-Westfalen, Saarland und Schleswig-Holstein, Bundesrat-Drucksache 124/88 vom 17.3.1988.

Burtless, G.; J.A. Hausman [1978], The Effects of Taxation on Labor Supply: Evaluating the Gary Negative Income Tax Experiment, Journal of Political Economy 86, S.1103-1130.

BVerfGE 72: Entscheidungen des Bundesverfassungsgericht [1987], 72.Band, Tübingen, S.330-436.

BVerfGE 86: Entscheidungen des Bundesverfassungsgericht [1992], 86.Band, Tübingen, S.198-279.

Carlberg, M. [1979], Ein interregionales, multisektorales Wachstumsmodell - dargestellt für die Bundesrepublik Deutschland, Göttingen. 
Courant, P.N.; E.M. Gramlich; D.L. Rubinfeld [1979], The Stimulative Effects of Intergovernmental Grants: Or Why Money Sticks Where It Hits, in: P. MiEsZKowski, W.H. OAKLAND (Hrsg.), Fiscal Federalism and Grants-in-Aid, Washington D.C., S.5-21.

Deardorff, A.; R.Stern [1986], The Michigan Model of World Production and Trade, Cambridge.

Deaton, A.S.; J. Muellbauer [1988], Economic and Consumer Behavior, Cambridge.

DIW: Deutsches Institut für Wirtschaftsforschung [1993], DIW-Wochenbericht 18-19/93.

Diamond, P.A.; J.A. Mirrlees [1971], Optimal Taxation and Public Production I: Production Efficency, American Economic Review 61, S.8-27.

Diamond, P.A.; J.A. Mirlees [1971], Optimal Taxation and Public Production II: Tax Rules, American Economic Review 61, 261-278.

DohnANYI, K. von [1986], Der Verfassungsstreit um den Finanzausgleich: Für einen stadtstaatengerechten Finanzausgleich, Wirtschaftsdienst 66/II, S.59-62.

EBERT, U. [1987], Beiträge zur Wohlfahrtsökonomie, Berlin.

Essig, H.; W. Sтrонм [1993], Volkswirtschaftliche Gesamtrechnungen 1992. Vorläufiges Ergebnis, Wirtschaft und Statistik 3/1993, S.167-190.

FÄrBER, G. [1992], Länderfinanzausgleich und Gemeindefinanzen - Anmerkungen zu einigen häufig übersehenen Tatsachen, in: K. BонR (Hrsg.), Föderalismus - demokratische Struktur für Deutschland und Europa, München, S.103-122.

FärBER, G. [1993], Reform des Länderfinanzausgleichs, Wirtschaftsdienst 1993/VI, S.305313.

Fenr, H. [1993], Umsatzsteuerharmonisierung in der EG. Eine Empirische allgemeine Gleichgewichtsanalyse, Teil B: Modellaufbau und Simulationsergebnisse, Regensburg.

Fehr, H.; Ch. Rosenberg; W. Wiegard [1992], Quantitative Aspekte einer Harmonisierung der Umsatzsteuern in der EG, in: Karl-Heinrich HANSMEYER (Hrsg.), Ausgewählte Probleme der EG-Finanzen, (Schriften des Vereins für Socialpolitik, N.F. Bd.216), Berlin, S.11-113.

Fehr, H.; Ch. Rosenberg; W. Wiegard [1993], Value Added Taxation in the EC after 1992: Some Applied General Equilibrium Calculations, EUROPEAN ECONOMIC REVIEW, 37, S.1483-1506.

Filimon, R.; T. Romer; H. Rosenthal [1982], Asymmetric Information and Agenda Control, Journal of Public Economics 17, S.51-70.

Fischer, H. [1988], Finanzzuweisungen, Berlin.

Fischer, H. [1990], Der "Fliegenpapier"-Effekt, Das Wirtschaftsstudium 15, S.293-295. 
FISHER, R.C. [1981], Expenditure Incentives of Intergovernmental Grants, in: V. HENDERSON (Hrsg.), Research in Urban Economics: A Research Annual, Bd.1, Greenwich, Conneticut, S.201-218.

FÖTTINGER, W.; P.B. SPAHN [1993], Für einen kostenorientierten Länderfinanzausgleich, Wirtschaftsdienst 1993/V, S.237-246.

FreY, D. [1988], Kapitel I Die Finanzverfassung der Bundesrepublik Deutschland, in: V. ARnold; O.-E. GesKe (Hrsg.), Öffentliche Finanzwirtschaft, München, S.11-4.94.

Fuest, W.; K. Lichtblau [1991], Finanzausgleich im vereinten Deutschland, ( Beiträge zur Wirtschafts- und Sozialpolitik 192, Institut der deutschen Wirtschaft), Köln.

Fuss, M.; D. McFadden; Y. Mundlock [1978], A Survey of Functional Forms in The Economic Analysis of Production, in: M. Fuss; D. MCFAdDEN (Hrsg.), Production Economics: A Dual Approach to Theory and Application, Bd.1: Theory of Production, Amsterdam, S.219-268.

Gandolfo, G. [1987a], International Economics I: The Pure Theory of International Trade, Berlin u.a.O.

Gandolfo, G. [1987b], International Economics II: International Monetary Theory and Open-Economy Macroeconomics, Berlin u.a.O.

GemStaL $\ddot{A}:$ Gemeinschaftsveröffentlichung der Statistischen Landesämter [1986], Volkswirtschaftliche Gesamtrechnungen der Länder Heft 15: Entstehung, Verteilung und Verwendung des Sozialprodukts in den Ländern der Bundesrepublik Deutschland, Revidierte Ergebnisse 1970 bis 1985, Stuttgart.

Genosko, J. [1992], Gravitations- und Potentialmodelle, Diskussionsbeiträge der Wirtschaftswissenschaftlichen Fakultät Ingolstadt, Nr.22., Ingolstadt.

Georgakopoulos, Th. [1974], The 'Excess Burden' controversy in a Trading World: A Case for Special Indirect Taxes, Public Finance 29, S.152-167.

Gerking, S.D.; J.H. Mutti [1982], Possibilities for the Exportation of Production Taxes. A General Equilibrium Analysis, Journal of Public Economics 16, S.233-252.

Geske, O.-E. [1986], Konturen eines neuen Länderfinanzausgleichs, Wirtschaftsdienst 66/VII, S.399-403.

Goetz, Ch.J. [1971], Discussion, American Economic Review, 61, S.463.

Goetz, Ch.J.; Ch.R. MCKnew, Jr. [1972], Paradoxical Results in a Public Choice Model of Alternative Government Grant Forms, in: J.M. BuChanAN, R.D. Tollison (Hrsg.), Theory of Public Choice, Ann Arbor, S.224-235.

Goldstein, M; M.S. Khan [1990], Income and Price Effects in Foreign Trade, in: R.W. JoNES; P.B. KENEN (Hrsg.), Handbook of International Economics, Volume II: International Monetary Economics and Finance, S.1041-1105. 
GoвRECHT, H. [1987], Neuordnung des Länderfinanzausgleichs: Gerechtigkeit für die Stadtstaaten, Wirtschaftsdienst 67/VII, S. 335-337.

GotTfried, P. [1992], Die verdeckten Effizienzwirkungen der Umsatzsteuer. Eine Empirische Allgemeine Gleichgewichtsanaylse, Frankfurt a.M. u.a.O.

GottFried, P.; W. Wiegard [1992], Finanzausgleich zum Selberrechnen, in: E. WeGNER (Hrsg.), Finanzausgleich im vereinten Deutschland, Marburg, (Probleme der Einheit 9), S.133-168.

Grais, W.; J. DeMelo; S. Urata [1986], A General Equilibrium Estimation of the Effects of Reductions in Tariffs and Quantitative Restrictions in Turkey in 1978, in: T.N. Srinivasan; J. Whalley (Hrsg.), General Equilibrium Trade Policy Modeling, Cambridge, Mass., S.61-88.

Gramlich, E.M. [1977], Intergovernmental Grants: A Review of the Empirical Literature, in: Wallace E. OAtes (Hrsg.), The Political Economy of Fiscal Federalism, Lexington, Mass., S.219-239.

Gretz-Roth, V. [1989], Input-Output-Analysen für Hessen - Methodische Konzepte und empirische Ergebnisse, Allgemeines Statistisches Archiv 73, S.346-366.

Gretz-Roth, V.; W. Gretz [1986], Regionale Input-Output-Tabelle Hessen 1980, Wiesbaden.

Groenewegen, P. [1988], Taxation and Decentralisation: A Reconsideration of the Costs and Benefits of a Decentralised Tax System, Working Papers in Economics, University of Sydney.

Gutmann, P. [1981], The Measurement of Terms of Trade Effects, Review of Income and Wealth 27, S.433-453.

HANSMEYER, K.-H.; M. KoPS [1985], Interdependenzen im passiven Finanzausgleich, in: D. Cansier; D. Kath (Hrsg.), Öffentliche Finanzen, Kredit und Kapital, Berlin, S.4-32.

HANSmeyeR, K.-H.; M. KoPS [1990], Die Gliederung der Länder in einem vereinten Deutschland, Wirtschaftsdienst 70, S.234-239.

HARBERger, A.C. [1962], The Incidence of the Corporation Income Tax, Journal of Political Economy 70, S.215-240.

Harrison, G.W.; T.F. RUtherford; I. Wooton [1991], An Empirical Database for a General Equilibrium Model of the European Community, in: J. PIgGoTT; J. WhALLEY (Hrsg.), Applied General Equilibrium, Heidelberg, S.95-120.

Harrison, G.W.; R. Jones; L.J. Kimbell; R. Wigle [1993], Journal of Policy Modeling, 15, S.99-115.

Hausman, J.A. [1981], Labor Supply in: H.J. Aaron, J.A. Pechman (Hrsg.), How Taxes affect Economic Bahavior Washington D.C., S.27-72. 
Hausman, J.A. [1985], Taxes and Labor Supply, in: A.J. Auerbach; M. Feldstein (Hrsg.), Handbook of Public Economics, Bd.1, Amsterdam u.a.O., S.213-263.

Haverkamp, F. [1988], Die Finanzbeziehungen zwischen Ländern und Gemeinden, in: V. Arnold, O.-E. Geske (Hrsg.), Öffentliche Finanzwirtschaft, München, S.55120.

HeilmanN, M. [1992], Vorschläge zur Neuordnung des Bund-Länder-Finanzausgleichs im vereinten Deutschland - eine kritische Bestandsaufnahme, in: E. WEgNer (Hrsg.), Finanzausgleich im vereinten Deutschland, Marburg, (Probleme der Einheit 9), S.45-106.

HenkE, K.-D. [1985], Neuordnung des Finanzausgleichs im föderativen System der Bundesrepublik Deutschland, in: H. Giersch (Hrsg.), Wie es zu schaffen ist. Agenda für die deutsche Wirtschaftspolitik, Stuttgart, S.153-175.

Hewings, G.J.D.; R.C.Jensen [1986], Regional, Interregional and Multiregional InputOutput-Analysis, in: P. NiJKAmP (Hrsg.), Handbook of Regional and Urban Economics, Volume I: Regional Economics, Amsterdam, New York, u.a.O., S.295-355.

Hewings, G.J.D.; R.C. JENSEN [1988], Emerging Challenges in Regional Input-Output Analysis, Annals of Regional Science 22, S.43-4.93.

HEwitT, D. [1986], Fiscal Illusion From Grants and the Level of State and Federal Expenditures, National Tax Journal 39, S.471-483.

HirTe, G. [1990], Ein empirisches allgemeines Gleichgewichtsmodell mit Zwei-Ländern zur Inzidenzanalyse von Finanzzuweisungen, Bd.11, Ingolstadt, Diskussionsbeiträge der Wirtschaftswissenschaftlichen Fakultät Ingolstadt.

HirTe, G. [1991] Inzidenzanalyse von Finanzzuweisungen: Eine empirische allgemeine Gleichgewichtsanalyse, Diskussionsbeiträge der Wirtschaftswissenschaftlichen Fakultät Ingolstadt, Bd.12, Ingolstadt.

Hirte, G.; J. GENosko [1988/89], Regionalisierte empirische allgemeine Gleichgewichtsanalyse: Eine Einführung mit einem einfachen Beispiel für die Bundesrepublik Deutschland, Jahrbuch für Regionalökonomie 9/10, S.32-4.96.

Hirte, G.; W. Wiegard [1988], An Introduction to Applied General Equilibrium Tax Modelling. With a Preliminary Approach to the Reform of Factor Taxes in the FRG, in: Dieter Bös, Manfred Rose, Christian SEIDL (Hrsg.), Welfare and Efficiency in Public Economics, Berlin, S.167-203.

Holub, H.-W.; H.SchNaBL [1985], Input-Output-Rechnung: Input-Output-Tabellen. Eine Einführung, München, Wien.

Homburg, St. [1993], Eine Theorie des Länderfinanzausgleichs: Finanzausgleich und Produktionseffizienz, Finanzarchiv N.F. 50, S.458-486. 
Homburg, St. [1994], Anreizwirkungen des deutschen Finanzausgleichs, Finanzarchiv N.F. 50, S.312-330.

HüBLER, O. [1979], Regionale Sektorstrukturen: Verfahren zur Schätzung und Auswertung regionaler Input-Output-Beziehungen, Berlin.

Hummel, M.; W. Leibfritz [1987], Die Stadtstaaten im Länderfinanzausgleich, ifoschnelldienst 20/87, S.7-19.

Issaev, B.; P. NijkamP; P. Rietveld; F. SNiCKaRs [1982], Multiregional Economic Modeling: Practice and Prospect, Amsterdam, u.a.O.

Johnson, M.B. [1977], Recognizing the Competitive Nature of General Revenue Sharing Grants, Southern Economic Journal 44, S.143-147.

Jones, R.C. [1988], Applied General Equilibrium Analysis of Customs Unions and Interregional Labour Mobility, Ph.D., London, Ontario.

Jones, R.; J.Whalley [1987], Regional Balance Sheets of Gains and Losses from National Policies, Working Paper $8708 \mathrm{C}$, London, Ontario.

Jones, R.; J.Whalley [1988], Regional Effects of Taxes in Canada: An Applied General Equlibrium Approach, Journal of Public Economics 37, S.1-28.

Jones, R.; J.Whalley [1989], A Canadian Regional General Equilibrium Model and some Applications, Journal of Urban Economics 25, S.368-404.

Jones, R.; R. Wigle; J. Whalley [1985], Regional Impacts of Tariffs in Canada: Preliminary Results from a Small Dimensional Numerical General Equilibrium Model, in: J. Piggotт; J. Whalley (Hrsg.), New Developments in Applied General Equilibrium Analysis, Cambridgt, S.139-202.

JuRION, B.J. [1979], Matching Grants and Unconditional Grants: The Case With N Goods, Public Finance 34, S.234-244.

KaISER, H. [1993], Testing for the Separability between Commodity Demand and Labour Supply in West Germany, Empirical Economics 18, S.57-73.

Keller, W.J. [1976], A Nested CES-Type Utility Function and its Demand and PriceIndex Functions, European Economic Review 7, S. 175-186.

Keller, W.J. [1977], Savings, Leisure, Consumption and Taxes: The Houshold Expenditure System, European Economic Review 9, S.151-167.

Kenyon, D.A.; J. Kincaid (Hrsg.) [1988], Competition among States and Local Governments. Efficiency and Equity in American Federalism, Washington, D.C.

KING, D.N. [1984], Fiscal Tiers: The Economics of Multi-Level Government, London, Boston, Sydney.

Kimbell, L.; G. Harrison [1984], On the Solution of General Equilibrium Models, Economic Modeling 3, S.197-212. 
Klanberg, F.; A. Prinz [1988], Was taugt der Albrecht-Vorschlag?, Wirtschaftsdienst 68, Heft VII, S.291-297.

Krugman, P.R.; M. Obstfeld [1991], International Economics. Theory and Policy, New York.

Kuнn, T. [1991/92], Zuweisungen und Allokation im kommunalen Finanzausgleich, Finanzarchiv N.F. 49, S.215-236.

LÄCHLER, U. [1985], The Elasticity of Substitution between Imported and Domestically Produced Goods in Germany, Weltwirtschaftliches Archiv (Review of World Economics) 121 , S.74-96.

Landesamt NRW: Landesamt für Datenverarbeitung und Statistik Nordrhein-Westfalen [1993], Statistisches Jahrbuch Nordrhein-Westfalen 1993, Düsseldorf.

LaU, L.J. [1984], Comments, in: H.E. Scarf, J.B. Shoven (Hrsg.), Applied General Equilibrium Analysis, Cambridge, S.127-137.

LeGrand, J. [1975], Fiscal Equity and Central Government Grants to Local Authorities, The Economic Journal, 85, S.531-4.947.

LENK, Th. [1992], Entwicklung eines Kriterienrasters für eine gesetzliche Regelung des Länderfinanzausgleichs am Beispiel der Bundesrepublik Deutschland, DER ÖFFENTLiChe Sektor - Forschungsmemorandeum, 18, S.64-85.

Leontief, W.W.; A.M.Strout (1963), Multiregional Input-Output Analysis, in: T. Barna (Hrsg.), Structural Interdependence and Economic Development, London, S.119-150.

Levaggi, R. [1991], Fiscal Federalism and Grants-in-Aid. The Problem of Asymmetrical Information, Aldershot u.a.O.

Logan, R.E. [1986], Fiscal Illusion and the Grantor Government, Journal of Political Economy 94, S.1304-1318.

Majumdar, M.; T.Mitra [1985], A Result on the Tranfer Problem in International Trade Theory, Journal of International Economics 19, S.161-170.

Manne, A.S. (Hrsg.) [1985], Economic Equilibrium: Model Formulation and Solution, Amsterdam.

Mansur, A.; J. Whalley [1984], Numerical Specification of Applied General Equilibrium Models: Estimation, Calibration, and Data, in: H.E. ScARF, J.B. Shoven (Hrsg.), Applied General Equilibrium Analysis, Cambridge, S.69-127.

Mathews, R.L. [1975/76], Fiscal Equalisation in Australia: The Methodology of the Grants Commission, Finanzarchiv N.F. 34, S.66-85.

MAYER, H.; U.-P. REICH [1987], Bruttodarstellung des Handels in der Input-OutputRechnung, Wirtschaft und Statistik 8/1987, S.601-609. 
MCKeE, M.; J. Visser; P. SAUnders [1986], Marginal Tax on the Use of Labor and Capital in OECD Countries, OECD Economic Studies 7, S.45-101.

MichaLK, J. [1989], Die Garantieklauseln im Länderfinanzaugleich, Wirtschaftsdienst 69/IX, S. 446-453.

MofFITT, R.A. [1984], The Effects of Grants-in-Aids on State and Local Expenditures: The Case of AFDC, Journal of Public Economics, 23, S.279-305.

Morey, E. [1984], Confuser Surplus, American Economic Review 74, S.163-173.

MünZenmaIER, W. [1982], Die wirtschaftliche Verflechtung des Landes Baden-Württemberg - Konzeption und Methode der Input-Output-Tabellen 1972, Jahrbücher für Statistik und Landeskunde von Baden-Württemberg 27, S.67-91.

MünZEnmaIER, W. [1984a], Intersektoral-interregionale Input-Output-Tabelle 1974. Eine Berechnung der Verflechtungsbeziehungen zwischen Baden-Württemberg und dem übrigen Bundesgebiet auf der Basis einer multiregionalen Input-Output-Tabelle, Allgemeines Statistisches Archiv 68, S.281-306.

MüNZENMAIER, W. [1984b], Erweiterung der regionalen Input-Output-Analyse durch Einbeziehung interregionaler Ströme: Notwendigkeit und Realisierungsmöglichkeiten dargestellt am Beispiel der Exportabhängigkeit Baden-Württembergs, Jahrbücher für Statistik und Landeskunde von Baden-Württemberg 29., S.166-179.

MünZEnMAIER, W. [1984c], Konzeption der Sektorenabgrenzung in der regionalen InputOutput-Rechnung der Bundesrepublik Deutschland, in: Statistisches Bundesamt (Hrsg.), Darstellungskonzepte der Input-Output-Rechnung, Stuttgart, Mainz, S.145166.

MünZenmaier, W. [1985], Konzeption und Methode der Input-Output-Tabellen für Baden-Württemberg 1978, Jahrbücher für Statistik und Landeskunde von BadenWürttemberg 30, S.146-165.

MünZenmaier, W. [1986], Input-Output-Tabellen 1980, Baden-Württemberg in Wort und Zahl 34, S.24-30.

MünZenmaIER, W. [1987a], Interregional Input-Output-Analysis for the Federal Republic of Germany - The Compilation of an Interregional - Interindustrial System of Economic Flows between two Regions, and some Consequences for Regional Analysis, Jahrbuch für Regionalwissenschaft 8, S.15-39.

MünZenmaIER, W. [1987b], Zur Bedeutung statistischer Einheiten für die Integration regionaler Input-Output-Tabellen in Systeme Volkswirtschaftlicher Gesamtrechnungen, Jahrbücher für Statistik und Landeskunde von Baden-Württemberg 32, S.454.98 .

MüNZENMAIER, W. [1988a], Input-Output-Tabellen 1982, Baden-Württemberg in Wort und Zahl 36, S.109-115. 
MüNZENMAIER, W. [1988b], Berechnungsmethoden für die Input-Output-Tabellen BadenWürttemberg 1982, Jahrbücher für Statistik und Landeskunde von Baden-Württemberg 33, S.123-132.

MüNZenMAIER, W. [1989a], Input-Output-Tabellen 1984, Baden-Württemberg in Wort und Zahl 37, S.279-285.

MÜNZEnMAIER, W. [1989b], Interregionale und intersektorale Verflechtungsbeziehungen: Berechnung einer interregionalen Input-Output-Tabelle für Baden-Württemberg und das übrige Bundesgebiet 1982, Jahrbücher für Statistik und Landeskunde von Baden-Württemberg 34, S.113-125.

MüNZENMAIER, W. [1990], Input-Output-Tabellen 1986, Baden-Württemberg in Wort und Zahl 38, S.120-124.

Musgrave, R.A. [1959], The Theory of Public Finance: A Study in Public Economy, New York.

Musgrave, R.A. [1961], Approaches to a Fiscal Theory of Political Federalism, in: National Bureau of Economic Research (NBER), Public Finances: Needs, Sources, ans Utilization, Princeton, S.97-122.

Musgrave, R.A.; P.B. Musgrave; L. Kullmer [1987], Die öffentlichen Finanzen in Theorie und Praxis, 3., völlig überarbeitete Auflage, Tübingen.

Nakamura, S. [1984], An Inter-Industry Tranlog Model of Prices and Technical Change for the West German Economy, Berlin.

Nakamura, S. [1986], A Dynamic Multisectoral Model of Production, Investment and Prices Based on Flexible Cost Functions, DIW Vierteljahreshefte 3/86, S.110-122.

NAPP, H.-G. [1994], Kummunale Finanzautonomie und ihre Bedeutung für eine effiziente lokale Finanzwirtschaft, Frankfurt.

NEARY J.P.; K.W.S. RoBerTs [1980], The Theory of Household Behaviour under Rationing, European Economic Review 13, S.25-42.

NiJkamp, P. (Hrsg.) [1986], Handbook of Regional and Urban Economics, Volume I: Regional Economics, Amsterdam, New York, u.a.O.

Nijkamp, P.; P. Rietveld; F. Snickars [1984], A Survey of Multiregional Economic Models, in: G. Bahrenberg; M.M. Fischer; P. NiJKamp (Hrsg.), Recent Developments in Spatial Data Analysis: Methodology, Measurement, Models, Aldershot, U.K., S.93-115.

NiJkamp, P.; P. RietVeld; F. Snickars [1986], Regional and Multiregional Economic Models: A Survey, in: P. NiJKAmP (Hrsg.), Handbook of Regional and Urban Economics, Volume I: Regional Economics, Amsterdam, New York, u.a.O., S.257-294. 
OAKLand, W.E. [1987], Theory of Public Goods, in: A.J. AuerbaCh; M. Feldstein (Hrsg.), Handbook of Public Economics: Volume II, Amsterdam, New York u.a.O., S.485-4.935.

OAtes, W.E. [1972], Fiscal Federalism, New York.

OAtes, W.E. [1975], Financing the New Federalism: Revenue Sharing, Conditional Grants, and Taxation, Baltimore.

OAtes, W.E. [1977], The Political Economy of Fiscal Federalism, Lexington.

OAtes, W.E. [1979], Lump-Sum Intergovernmental Grants Have Price Effects, in: P. Mieszkowski, W.H. OAKLAnd (Hrsg.), Fiscal Federalism and Grants-in-Aid, Washington D.C., S.23-30.

PALM, G. [1986], Die Eigenanstrengungen der Länder müssen honoriert werden, Wirtschaftsdienst 66/II, S.64-66.

PALM, G. [1987], Neuordnung des Länderfinanzausgleichs: Der Bund ist Partei geworden, Wirtschaftsdienst 67, S.333-335.

Peffekoven, R. [1975], Zur Theorie des Steuerexports, Tübingen.

Peffekoven, R. [1980], Finanzausgleich I: Wirtschaftstheoretische Grundlagen, in: W.

AlBers (Hrsg.), Handwörterbuch der Wirtschaftswissenschaften, Bd.II, Stuttgart u.a., S.608-636.

Peffekoven, R. [1985], Zur Problematik der Umsatzsteuerverteilung, in: D. CANSIER, D.Kath (Hrsg.), Öffentliche Finanzen, Kredit und Kapital, Berlin, S.53-79.

Peffekoven, R. [1987], Zur Neuordnung des Länderfinanzausgleichs, Finanzarchiv N.F. $45, \mathrm{~S} .181-228$.

Peffekoven, R. [1988], Berücksichtigung der Seehafenlasten im Länderfinanzausgleich, Finanzarchiv N.F.46, S.397-415.

Peffekoven, R. [1990], Finanzausgleich und Sonderbedarfe. Thema und vier Variationen, in: F.X. BEA; W. KitTerer (Hrsg.), Finanzwissenschaft im Dienste der Wirtschaftspolitik, Tübingen, S.323-341.

Peffekoven, R. [1992], Das Urteil des Bundesverfassungsgerichts zum Länderfinanzausgleich, Wirtschaftsdienst 72/VII, S.349-352.

Peffekoven, R. [1994], Reform des Finanzausgleichs - eine vertane Chance, Finanzarchiv N.F. 51, S.281-311.

Piggott, J.; J. Whalley (Hrsg.) [1985a], New Developments in Applied General Equilibrium Analysis, Cambridge.

PiggotT, J.; J. Whalley [1985b], UK Tax Policy and Applied General Equilibrium Analysis, Cambridge.

Piggott, J.; J. Whalley [1991], Applied General Equilibrium, Heidelberg. 
Pollack, H. [1980], Steuertarife, in: N. Andel; F. Haller; F. Neumark (Hrsg.), Handbuch der Finanzwissenschaft, Bd.2, 3.Aufl, Tübingen S.240-266.

Pommerehne, W.W.; F.Schneider [1978], Fiscal Illusion, Political Institutions, and Local Public Spending Kyklos 31, S.381-408.

Posser, D. [1986], Die Bundesergänzungszuweisungen nach objektiven Kriterien verteilen, Wirtschaftsdienst 66/II, S.62-64.

Renner, P. [1982], Finanzausgleich unter den Ländern und Bundesergänzungs- zuweisungen, in: Der Bundesminister der Finanzen (Hrsg.), Die Finanzbeziehnungen zwischen Bund, Ländern und Gemeinden aus finanzverfassungsrechtlicher und finanzwirtschaftlicher Sicht, Bonn S.328-384.

Richter, W.F.; D. Wellisch [1993], Allokative Theorie eines interregionalen Finanzausgleichs, Finanzarchiv N.F. 50, S.433-457.

Rietveld, P. [1982], A Survey of Multiregonal Economic Models, in: B. Issaev; P. NiJKamP; P. Rietveld; F. Snickars (Hrsg.) Multiregional Economic Modeling: Practice and Prospect, Amsterdam u.a., S.231-332.

Romer, T.; H. Rosenthal [1980], An Institutional Theory of the Effect of Intergovernmental Grants, National Tax Journal 33, S.451-458.

Rosenberg, Ch. [1993], Umsatzsteuerharmonisierung in der EG. Eine Empirische allgemeine Gleichgewichtsanalyse, Teil A: Institutionelle Regelungen und theoretische Grundlagen, Regensburg.

RothbaRTh, E. [1940/41], The Measurement of Changes in Real Income under Conditions of Rationing, Review of Economic Studies 8, S.100-107.

Rutner, D. [1984], Faktorsubstitution in den Produktionssektoren der Bundesrepublik Deutschland: Eine ökonometrische Analyse anhand des Translog-Modells, 19611980, Frankfurt a.M.

SACHVERSTÄNDIGENRAT zur Begutachtung der gesamtwirtschaftlichen Entwicklung , Für Wachstumsorientierung - gegen lähmenden Verteilungsstreit, Jahresgutachten 1992/93, Stuttgart.

SCARF, H.E. [1967], On the Computation of Equilibrium Prices, in: W.Fellner, u.a. (Hrsg.), Ten Economic Studies in the Tradition of Irving Fisher, New York, S.207230.

Scarf, H.E. (mit T. HANSEN) [1973], The Computation of Economic Equilibria, New Haven.

Scarf, H.E.; J.B. Shoven (Hrsg.) [1984], Applied General Equilibrium Analysis, Cambridge.

SснміDT, G. [1986], Der private Verbrauch im Rahmen der volkswirtschaftliche Gesamtrechnungen der Länder, Bayern in Zahlen, 5/1986, S.152-156. 
Shoven, J.B.; J.Whalley [1972], A General Equilibrium Calculation of the Effects of Differential Taxation of Income from Capital in the USA, Journal of Public Econoics 1, S.281-321.

Shoven, J.B.; J.Whalley [1973], General Equilibrium with Taxes: A Computational Procedure and an Existence Proof, The Review of Economic Studies 60, S.475-489.

Shoven, J.B.; J.Whalley [1977], Equal Yield Tax Alternatives. General Equilibrium Computational Techniques, Journal of Public Economics 8, S.211-224.

Shoven, J.B.; J.Whalley [1984], Applied General Equilibrium Models of Taxation and International Trade, Journal of Economic Literature 22, S.1007-1051.

Silberberg, E. [1990], The Structure of Economics. A Mathematical Analysis, 2te Auflage, New York u.a.

SinN, H.-W. [1985], Kapitaleinkommensbesteuerung, Tübingen.

Soнmen, E. [1976], Allokationstheorie und Wirtschaftspolitik, Tübingen.

Srinivasan, T.N. ; J. Whalley (Hrsg.) [1986], General Equilibrium Trade Policy Modeling, Cambridge, Mass.

STÄGLIN, R. [1980], Zur Input-Output Rechnung in der Bundesrepublik Deutschland: Eine Bestandsaufnahme, in: J. Frohn; R. STÄGlin (Hrsg.), Empirische Wirtschaftsforschung: Konzeptionen, Verfahren und Ergebnisse, Festschrift für Rolf Krengel, Berlin, S.95-130.

STÄGLIN, R. [1982], Überblick über die Aktivitäten auf dem Gebiet der Input-OutputRechnung in der Bundesrepublik-Deutschland, in: R. Krengel (Hrsg.), Die Weiterentwicklung der Input-Output-Rechnung in der Bundesrepublik

Deutschland, Göttingen, S.7-4.96.

STÄGlin, R.; I. LUdWIG; J. SchinTKe [1990], Input-Output-Relationen für NordrheinWestfalen als Grundlage eines Strukturvergleiches mit der Bundesrepublik, Vierteljahreshefte zur Wirtschaftsforschung 1990, S.201-242.

STAHMER, C. [1979], Verbindung von Ergebnissen der herkömmlichen Sozialproduktsberechnung und der Input-Output-Rechnung in der Bundesrepublik Deutschland: Das Überleitungsmodell des Statistischen Bundesamtes, Allgemeines Statistisches Archiv 63, S.340-385.

STAHMER, C. [1984], Überlegungen zu einem System von Input-Output-Tabellen für die Bundesrepublik Deutschland, in: Statistisches Bundesamt (Hrsg.), Darstellungskonzepte der Input-Output-Rechnung Stuttgart, Mainz, S.41-71.

Statistisches Amt Des SaARlandes [1990a], Statistische Berichte P I 1-s 85-88.

STABA: Statistisches Bundesamt [1981], Statistisches Jahrbuch 1980 für die Bundesrepublik Deutschland, Stuttgart. 
STABA: Statistisches Bundesamt [1982], Statistisches Jahrbuch 1981 für die Bundesrepublik Deutschland, Stuttgart.

STABA: Statistisches Bundesamt [1983a], Fachserie 14: Finanzen und Steuern, Reihe 4., Steuerhaushalt, 4.Vierteljahr und Jahr 1982, Stuttgart.

STABA: Statistisches Bundesamt [1983b], Statistisches Jahrbuch 1982 für die Bundesrepublik Deutschland, Stuttgart.

STA BA: Statistisches Bundesamt (Hrsg.) [1984a], Darstellungskonzepte der Input-Output-Rechnung, Stuttgart, Mainz.

STA BA: Statistisches Bundesamt [1984b], Statistisches Jahrbuch für die Bundesrepublik Deutschland 1983, Stuttgart.

STA BA: Statistisches Bundesamt [1985a], Fachserie 18: Volkswirtschaftliche Gesamtrechnungen, Reihe S.8: Revidierte Ergebnisse 1960 bis 1984, Stuttgart.

STABA: Statistisches Bundesamt [1985b], Statistisches Jahrbuch für die Bundesrepublik Deutschland 1984, Stuttgart.

STABA: Statistisches Bundesamt [1987b], Statistisches Jahrbuch 1986 für die Bundesrepublik Deutschland, Stuttgart.

STABA: Statistisches Bundesamt [1988], Statistisches Jahrbuch 1987 für die Bundesrepublik Deutschland, Stuttgart.

STA BA: Statistisches Bundesamt [1989a], Fachserie 18: Volkswirtschaftliche Gesantrechnungen, Reihe S.12: Ergebnisse der Input-Output-Rechnung 1970-1986, Stuttgart.

STABA: Statistisches Bundesamt [1989b], Fachserie 14: Finanzen und Steuern, Reihe 4.S.1., Kassenmäßige Steuereinnahmen 1977 bis 1987, Stuttgart.

STA BA: Statistisches Bundesamt [1989c], Statistisches Jahrbuch 1988 für die Bundesrepublik Deutschland, Stuttgart.

STA BA: Statistisches Bundesamt [1992a], Fachserie 18: Volkswirtschaftliche Gesamtrechnungen, Reihe 1.2.: Stuttgart.

Sta BA: Statistisches Bundesamt [1992b], Fachserie 14: Finanzen und Steuern, Reihe 4: Steuerhaushalt, 4.Vierteljahr und Jahr 1992, Stuttgart. für die Bundesrepublik Deutschland, Stuttgart.

STA BA: Statistisches Bundesamt [1992c], Fachserie 18: Volkswirtschaftliche Gesamtrechnungen, Reihe 13: Konten und Standardtabellen 1991, Stuttgart.

STABA: Statistisches Bundesamt [1993], Statistisches Jahrbuch 1992 für die Bundesrepublik Deutschland, Stuttgart.

STABA: Statistisches Bundesamt [1994], Statistisches Jahrbuch 1993 für die Bundesrepublik Deutschland, Stuttgart.

Statistisches Landesamt Berlin [1983], Statistisches Jahrbuch 1983, Berlin. 
Statistisches Landesamt Berlin [1984], Statistisches Jahrbuch 1984, Berlin.

Statistisches Landesamt Bremen [1987], Freie Hansestadt Bremen: Statistisches Handbuch 1981-1985, Bremen.

Stern, R.; J. Francis; B. Schuhmacher [1976], Price Elasticities in International Trade. An Annotated Bibliography, London.

Stiglitz, J.E.; P.S. DASGUPTA [1971], Review of Economic Studies 38, S.151-174.

Stille, F. u.a. [1992], Strukturwandel im Prozeß der deutschen Vereinigung. Analysen der strukturellen Entwicklung, Strukturberichterstattung 1992, DIW, Beiträge zur Strukturforschung Bd. 136, Berlin.

STöss, E. [1991], Effizienzwirkungen direkter und indirekter Steuern. Eine Empirische Allgemeine Gleichgewichtsanalyse, Regensburg.

TAIT, A. [1989], Not so General Equilibrium and not so Optimal Taxation, Public Finance 44, S.171-182.

TAUBE, R. [1990], Ein Vorschlag zur Reform des Länderfinanzausgleichs, Wirtschaftsdienst 70, S.372-380.

Thurow, L. [1970], Aid to State and Local Governments, National Tax Journal 23.

Tiebout, Ch.M. [1956], A Pure Theory of Local Expenditures, Journal of Political Economics 64, S.416-424.

Tresch, R.W. [1981], Public Finance: A Normative Theory, Plano, Texas.

VARIAN, H.R. [1992], Microeconomic Analysis, Third Edition, New York, London.

Wahl, J.; B. Frischmuth [1990], Finanzausgleich zur Angleichung der Lebensverhältnisse in den Bundesländern, Passau, unveröffentlichtes Manuskript.

Waldauer, Ch. (1973), Grant Structures and their Effects on aided government Expenditures: An Indifference Curve Analysis, Public Finance 28, S.212-226.

Weber, R. [1995], Das Arbeitsangebot verheirateter Männer. Eine theoretische und empirische Analyse, Frankfurt a.M.

Whalley, J. [1985], Trade Liberalization among Major World Trading Areas, Cambridge, Mass., London.

Whalley, J.; I. Trela [1986], Regional Aspects of Confederation, Toronto.

Whalley, J. [1988], Lessons from General Equilibrium Models, in: H.AARON, H.GALPher, J.A.Pechman (Hrsg.), Uneasy Compromise. Problems of a Hybrid IncomeConsumption Tax, Washington, D.C., S.15-4.90.

Whalley, J.; B. Young [1984], External Sector 'Closing' Rules in Applied Equilibrium Models, Journal of International Economics 16, S.123-138.

WIEgARD, W. [1980], Distortionary Taxation in a Federal Economy, Jahrbücher für Nationalökomie und Statistik 40, S.183-206. 
Wiegard, W. [1985a], Die Algorithmen von Scarf und Merrill zur numerischen Berechnung allgemeiner Gleichgewichte: Eine Einführung mit Beispielen aus der Steuerpolitik, Zeitschrift für Wirtschafts- und Sozialwissenschaften 6, S.709-742.

WiEgarD, W. [1985b], Empirische Allgemeine Gleichgewichtsanalyse: Von der Theorie zur Empirie, List-Forum 13, 159-176.

Wigle, R. [1988], General Equilibrium Evaluation of Canada-U.S. Trade Liberalization in a Global Context, Canadian Journal of Economics 21, S.539-4.964.

Wildasin, D.E. [1984], The Welfare Effects of Intergovernmental Grants in an Economy with Distorting Local Taxes: A Simple General Equilibrium Analysis, Journal of Public Economics 25, S.103-125.

Wilde, J.A. [1971], Grants-in-Aid: The Analysis of Design and Response, National Tax Journal 24, S.143-155.

WineR, St.L. [1983], Some Evidence of the Effects of the Separation of Spending on Taxing Decisions, Journal of Political Economy 91, S.126-140.

Wissenschaftlicher Beirat beim Bundesminister der Finanzen [1992], Gutachten zum Länderfinanzausgleich in der Bundesrepublik Deutschland, Bonn.

YoRK, H.L. [1993], An Applied General Equilbrium Model of International Tax Competition among the Group of Seven Countries, Journal of Policy Modeling 15, S.653-671.

Zimmermann, H.; K. Henke [1994], Finanzwissenschaft. Eine Einführung in die Lehre von der öffentlichen Finanzwirtschaft, München.

\section{Gesetzestexte und Urteile}

Achtes Gesetz zur Änderung des Gesetzes über den Finanzausgleich zwischen Bund und Ländern [FAG 1987], vom 18.12.1987, Bundesgesetzblatt 1987, Teil I, S.2764-2766.

Gesetz über den Finanzausgleich zwischen Bund und Ländern [FAG 1988], vom 11.2.1988, Bundesgesetzblatt 1988, Teil I, S.95-99.

Gesetz über den Finanzausgleich zwischen Bund und Ländern [FAG 1969], vom 28.8.1969, Bundesgesetzblatt 1969, Teil I, S.1432-1436.

Gesetz über Maßnahmen zur Bewältigung der finanziellen Erblasten im Zusammenhang mit der Herstellung der Einheit Deutschlands, zur langfristigen Sicherung des Aufbaus in den neuen Ländern, zur Neuordnung des bundestaatlichen Finanzausgleichs und zur Entlastung der öffentlichen Haushalte (Gesetz zur Umsetzung des Föderalen Konsolidierungsprogramms - FKPG) vom 26.6.1993 [FAG 1993], Bundesgesetzblatt 1993, Teil I, S.944-991.

Gesetz zur Verbesserung der steuerlichen Bedingungen zur Sicherung des Wirtschaftsstandorts Deutschland im Europäischen Binnenmartk (Standortsicherungsgesetz StandOG), vom 13.9.1993, Bundesgesetzblatt 1993, Teil I, S.1569-1593. 


\section{FINANZWISSENSCHAFTLICHE SCHRIFTEN}

Band 1 Werner Steden: Finanzpolitik und Einkommensverteilung. Ein Wachstums- und Konjunkturmodell der Bundesrepublik Deutschland. 1979.

Band 2 Rainer Hagemann: Kommunale Finanzplanung im föderativen Staat. 1976.

Band 3 Klaus Scherer: Maßstäbe zur Beurteilung von konjunkturellen Wirkungen des öffentlichen Haushalts. 1977.

Band 4 Brita Steinbach: "Formula Flexibility" - Kritische Analyse und Vergleich mit diskretionärer Konjunkturpolitik. 1977.

Band 5 Hans-Georg Petersen: Personelle Einkommensbesteuerung und Inflation. Eine theoretisch-empirische Analyse der Lohn- und veranlagten Einkommensteuer in der Bundesrepublik Deutschland. 1977.

Band 6 Friedemann Tetsch: Raumwirkungen des Finanzsystems der Bundesrepublik Deutschland. Eine Untersuchung der Auswirkungen der Finanzreform von 1969 auf die Einnahmenposition der untergeordneten Gebietskörperschaften und ihrer regionalpolitischen Zieladäquanz. 1978.

Band 7 Wilhelm Pfähler: Normative Theorie der fiskalischen Besteuerung. Ein methodologischer und theoretischer Beitrag zur Integration der normativen Besteuerungstheorie in der Wohlfahrtstheorie. 1978.

Band 8 Wolfgang Wiegard: Optimale Schattenpreise und Produktionsprogramme für öffentliche Unternehmen. Second-Best Modelle im finanzwirtschaftlichen Staatsbereich. 1978.

Band 9 Hans P. Fischer: Die Finanzierung des Umweltschutzes im Rahmen einer rationalen Umweltpolitik. 1978.

Band 10 Rainer Paulenz: Der Einsatz finanzpolitischer Instrumente in der Forschungs- und Entwicklungspolitik. 1978.

Band 11 Hans-Joachim Hauser: Verteilungswirkungen der Staatsverschuldung. Eine kreislauftheoretische Inzidenzbetrachtung. 1979.

Band 12 Gunnar Schwarting: Kommunale Investitionen. Theoretische und empirische Untersuchungen der Bestimmungsgründe kommunaler Investitionstätigkeit in NordrheinWestfalen 1965-1972. 1979.

Band 13 Hans-Joachim Conrad: Stadt-Umland-Wanderung und Finanzwirtschaft der Kernstädte. Amerikanische Erfahrungen, grundsätzliche Zusammenhänge und eine Fallstudie für das Ballungsgebiet Frankfurt am Main. 1980.

Band 14 Cay Folkers: Vermögensverteilung und staatliche Aktivität. Zur Theorie distributiver Prozesse im Interventionsstaat. 1981.

Band 15 Helmut Fischer: US-amerikanische Exportförderung durch die DISC-Gesetzgebung. 1981.

Band 16 Günter Ott: Einkommensumverteilungen in der gesetzlichen Krankenversicherung. Eine quantitative Analyse. 1981.

Band 17 Johann Hermann von Oehsen: Optimale Besteuerung. (Optimal Taxation). 1982.

Band 18 Richard Kössler: Sozialversicherungsprinzip und Staatszuschüsse in der gesetzlichen Rentenversicherung. 1982.

Band 19 Hinrich Steffen: Zum Handlungs- und Entscheidungsspielraum der kommunalen Investitionspolitik in der Bundesrepublik Deutschland. 1983.

Band 20 Manfred Scheuer: Wirkungen einer Auslandsverschuldung des Staates bei flexiblen Wechselkursen. 1983. 
Band 21 Christian Schiler: Staatsausgaben und crowding-out-Effekte. Zur Effizienz einer Finanzpolitik keynesianischer Provenienz. 1983.

Band 22 Hannelore Weck: Schattenwirtschaft: Eine Möglichkeit zur Einschränkung der öffentlichen Verwaltung? Eine ökonomische Analyse. 1983.

Band 23 Wolfgang Schmitt: Steuern als Mittel der Einkommenspolitik. Eine Ergänzung der Stabilitätspolitik? 1984.

Band 24 Wolfgang Laux: Erhöhung staatswirtschaftlicher Effizienz durch budgetäre Selbstbeschränkung? Zur Idee einer verfassungsmäßig verankerten Ausgabengrenze. 1984.

Band 25 Brita Steinbach-van der Veen: Steuerinzidenz. Methodologische Grundlagen und empirisch-statistische Probleme von Länderstudien. 1985.

Band 26 Albert Peters: Ökonomische Kriterien für eine Aufgabenverteilung in der Marktwirtschaft. Eine deskriptive und normative Betrachtung für den Allokationsbereich. 1985.

Band 27 Achim Zeidler: Möglichkeiten zur Fortsetzung der Gemeindefinanzreform. Eine theoretische und empirische Analyse. 1985.

Band 28 Peter Bartsch: Zur Theorie der längerfristigen Wirkungen 'expansiver' Fiskalpolitik. Eine dynamische Analyse unter besonderer Berücksichtigung der staatlichen Budgetbeschränkung und ausgewählter Möglichkeiten der öffentlichen Defizitfinanzierung. 1986.

Band 29 Konrad Beiwinkel: Wehrgerechtigkeit als finanzpolitisches Verteilungsproblem. Möglichkeiten einer Kompensation von Wehrungerechtigkeit durch monetäre Transfers. 1986.

Band 30 Wolfgang Kitterer: Effizienz- und Verteilungswirkungen des Steuersystems. 1986.

Band 31 Heinz Dieter Hessler: Theorie und Politik der Personalsteuern. Eine Kritik ihrer Einkommens- und Vermögensbegriffe mit Blick auf die Leistungsfähigkeitstheorie. 1994.

Band 32 Wolfgang Scherf: Die beschäftigungspolitische und fiskalische Problematik der Arbeitgeberbeiträge zur Rentenversicherung. Eine Auseinandersetzung mit der Kritik an der lohnbezogenen Beitragsbemessung. 1987.

Band 33 Andreas Mästle: Die Steuerunion. Probleme der Harmonisierung spezifischer Gütersteuern. 1987.

Band 34 Günter Ott: Internationale Verteilungswirkungen im Finanzausgleich der Europäischen Gemeinschaften. 1987.

Band 35 Heinz Haller: Zur Frage der zweckmäßigen Gestalt gemeindlicher Steuern. Ein Diskussionsbeitrag zur Gemeindesteuerreform. 1987.

Band 36 Thomas Kuhn: Schlüsselzuweisungen und fiskalische Ungleichheit. Eine theoretische Analyse der Verteilung von Schlüsselzuweisungen an Kommunen. 1988.

Band 37 Walter Hahn: Steuerpolitische Willensbildungsprozesse in der Europäischen Gemeinschaft. Das Beispiel der Umsatzssteuer-Harmonisierung. 1988.

Band 38 Ulrike Hardt: Kommunale Finanzkraft. Die Problematik einer objektiven Bestimmung kommunaler Einnahmemöglichkeiten in der gemeindlichen Haushaltsplanung und im kommunalen Finanzausgleich. 1988.

Band 39 Jochen Michaelis: Optimale Finanzpolitik im Modell überlappender Generationen. 1989.

Band 40 Bernd Raffelhüschen: Anreizwirkungen der sozialen Alterssicherung. Eine dynamische Simulationsanalyse. 1989.

Band 41 Berend Diekmann: Die Anleihe- und Darlehenstransaktionen der Europäischen Gemeinschaften. 1990.

Band 42 Helmut Kaiser: Konsumnachfrage, Arbeitsangebot und optimale Haushaltsbesteuerung. Theoretische Ergebnisse und mikroökonometrische Simulation für die Bundesrepublik Deutschland. 1990. 
Band 43 Rüdiger von Kleist: Das Gramm-Rudman-Hollings-Gesetz. Ein gescheiterter Versuch der Haushaltskonsolidierung. 1991.

Band 44 Rolf Hagedorn: Steuerhinterziehung und Finanzpolitik. Ein theoretischer Beitrag unter besonderer Berücksichtigung der Hinterziehung von Zinserträgen. 1991.

Band 45 Cornelia S. Behrens: Intertemporale Verteilungswirkungen in der gesetzlichen Krankenversicherung der Bundesrepublik Deutschland. 1991.

Band 46 Peter Saile: Ein ökonomischer Ansatz der Theorie der intermediären Finanzgewalten Die Kirchen als Parafisci. 1992.

Band 47 Peter Gottfried: Die verdeckten Effizienzwirkungen der Umsatzsteuer. Eine empirische allgemeine Gleichgewichtsanalyse. 1992.

Band 48 Andreas Burger: Umweltorientierte Beschäftigungsprogramme. Eine Effizienzanalyse am Beispiel des "Sondervermögens Arbeit und Umwelt". 1992.

Band 49 Jeanette Malchow: Die Zuordnung verteilungspolitischer Kompetenzen in der Europäischen Gemeinschaft. Eine Untersuchung aufgrund einer Fortentwicklung der ökonomischen Theorie des Föderalismus. 1992.

Band 50 Barbara Seidel: Die Einbindung der Bundesrepublik Deutschland in die Europäischen Gemeinschaften als Problem des Finanzausgleichs. 1992.

Band 51 Ralph Wiechers: Markt und Macht im Rundfunk. Zur Stellung der öffentlich-rechtlichen Rundfunkanstalten im dualen Rundfunksystem der Bundesrepublik Deutschland. 1992.

Band 52 Klaus Eckhardt: Probleme einer Umweltpolitik mit Abgaben. 1993.

Band 53 Oliver Schwarzkopf: Die Problematik unterschiedlicher Körperschaftsteuersysteme innerhalb der EG. 1993.

Band 54 Thorsten Giersch: Bergson-Wohlfahrtsfunktion und normative Ökonomie. 1993.

Band 55 Li-Fang Chou: Selbstbeteiligung bei Arzneimitteln aus ordnungspolitischer Sicht. Das Beispiel der Bundesrepublik Deutschland. 1993.

Band 56 Harald Schlee: Einkommensteuerliche Behandlung von Transferzahlungen. Zur Neuordnung der Familienbesteuerung sowie der Besteuerung von Versicherungsleistungen und Sozialtransfers. 1994.

Band 57 Alexander Spermann: Kommunales Krisenmanagement. Reaktionen baden-württembergischer Stadtkreise auf steigende Sozialhilfekosten und Einnahmenausfälle (198092). 1993.

Band 58 Otto Roloff / Sibylle Brander / Ingo Barens / Claudia Wesselbaum: Direktinvestitionen und internationale Steuerkonkurrenz. 1994.

Band 59 Claudia Wesselbaum-Neugebauer: Internationale Steuerbelastungsvergleiche. 1994.

Band 60 Stephanie Miera: Kcmmunales Finanzsystem und Bevölkerungsentwicklung. Eine Analyse des kommunalen Finanzsystems vor dem Hintergrund der sich abzeichnenden Bevölkerungsentwicklung am Beispiel Niedersachsens unter besonderer Berücksichtigung des Landkreises Wolfenbüttel und seiner Gemeinden. 1994.

Band 61 Wolfgang Scherf: Die Bedeutung des kaldorianischen Verteilungsmechanismus für die gesamtwirtschaftlichen Wirkungen der staatlichen Neuverschuldung. 1994.

Band 62 Rainer Volk: Vergleich der Vergünstigungseffekte der verschiedenen investitionsfördernden Maßnahmen. 1994.

Band 63 Hans-Georg Napp: Kommunale Finanzautonomie und ihre Bedeutung für eine effiziente lokale Finanzwirtschaft. 1994. 2., unveränderte Auflage 1994.

Band 64 Bernd Rahmann / Uwe Steinborn / Günter Vornholz: Empirische Analyse der Autonomie lokaler Finanzwirtschaften in der Europäischen Gemeinschaft. 1994. 
Band 65 Carsten Kühl: Strategien zur Finanzierung der Altlastensanierung. 1994.

Band 66 Stephan Boll: Intergenerationale Umverteilungswirkungen der Fiskalpolitik in der Bundesrepublik Deutschland. Ein Ansatz mit Hilfe des Generational Accounting. 1994.

Band 67 Karl Justus Bernhard Neumärker: Finanzverfassung und Staatsgewalt in der Demokratie. Ein Beitrag zur konstitutionellen Finanztheorie. 1995.

Band 68 Christian Haslbeck: Zentrale versus dezentrale Internalisierung externer Effekte bei unvollständiger Information. 1995.

Band 69 Regina Müller: Horizontale oder vertikale Transfers zur Durchsetzung eines horizontalen Finanzausgleichs. 1995.

Band 70 Christian Hockenjos: Öffentliche Sportförderung in der Bundesrepublik Deutschland. Darstellung und finanztheoretische Analyse. 1995.

Band 71 Manfred Rosenstock: Die Kontrolle und Harmonisierung nationaler Beihilfen durch die Kommission der Europäischen Gemeinschaften. 1995.

Band 72 Christian Rüsch: Wohnungsbau- und Wohneigentumspolitik im Rahmen der Einkommensteuer. Eine Analyse unter steuersystematischen, verteilungspolitischen und fiskalischen Aspekten. 1996.

Band 73 Stephan Winters: Die kollektive Vorsorge für den Pflegefall im Alter. Eine Untersuchung am Beispiel der gesetzlichen Pflegeversicherung in den Niederlanden. 1996.

Band 74 Knut Blind: Allokationsineffizienzen auf Sicherheitsmärkten: Ursachen und Lösungsmöglichkeiten. Fallstudie: Informationssicherheit in Kommunikationssystemen. 1996.

Band 75 Barbara Petrick-Rump: Ökonomische Wirkungen von Steueramnestien. Untersuchung konkreter Erfahrungen ausgewählter Länder mit dem Einsatz von Steueramnestien anhand eines effizienten Steueramnestieprogramms. 1996.

Band 76 Georg Hirte: Effizienzwirkungen von Finanzausgleichsregelungen. Eine Empirische Allgemeine Gleichgewichtsanalyse für die Bundesrepublik Deutschland. 1996. 


\title{
Thilo Müller-Overheu
}

\section{Der bundesstaatliche Finanzausgleich im Rahmen der deutschen Einheit}

\author{
Frankfurt/M., Berlin, Bern, New York, Paris, Wien, 1994. 167 S., 39 Tab. \\ Europäische Hochschulschriften: Reihe 5, Volks- und Betriebswirtschaft. Bd. 1480 \\ ISBN 3-631-46695-1 br. DM 53.--
}

Der bundesstaatliche Finanzausgleich ist eines der zentralen Themen der gegenwärtigen Föderalismusdiskussion. Die vorliegende Arbeit verfolgt das Ziel, auf der Basis einer Analyse der gegenwärtigen Finanzstrukturen der alten und jungen Bundesländer funktionale Zusammenhänge des bundesstaatlichen Finanzausgleichssystems unter den veränderten Parametern der deutschen Einheit darzustellen. Die zentrale Fragestellung lautet dabei: In welchem Umfang ist das gegenwärtig praktizierte Finanzausgleichssystem übertragbar auf die erweiterte Ländergesamtheit unter Berücksichtigung des gravierenden ökonomischen Gefälles unter den Ländern, und welche Anforderungen an zukünftige Ausgleichssysteme ergeben sich aus der veränderten Länderkonstellation und den unterschiedlichen Haushaltslagen der Länder unter der Maßgabe der "Einheitlichkeit der Lebensverhältnisse" in Deutschland? Aus dem Inhalt: Föderalismus in Deutschland . Der Einheitlichkeitsbegriff im Grundgesetz . Der Finanzausgleich . Die öffentlichen Haushalte · Die Länderfinanzkraft · Steuerschätzung vom Mai 1992 . Simulation unterschiedlicher Finanzausgleichssysteme für 1991 und 1995

Peter Lang Europäischer Verlag der Wissenschaften

Frankfurt a.M. - Berlin - Bern - New York - Paris - Wien

Auslieferung: Verlag Peter Lang AG, Jupiterstr. 15, CH-3000 Bern 15

Telefon (004131) 9402121, Telefax (004131) 9402131

- Preisănderungen vorbehalten - *inklusive Mehrwertsteuer 
Georg Hirte - 978-3-631-75212-8

Downloaded from PubFactory at 01/11/2019 07:02:26AM

via free access 